\title{
NERI Research Project
}

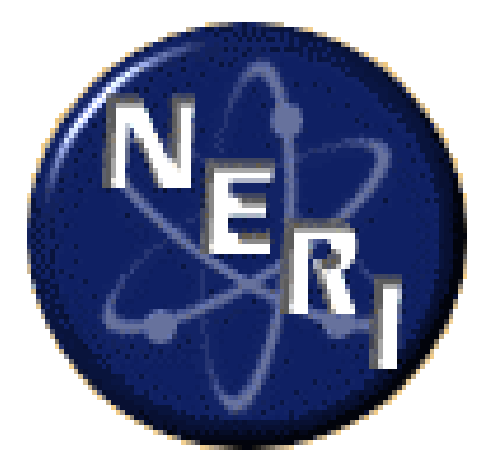

\section{Development of Advanced Technologies to Reduce Design, Fabrication and Construction Costs for Future Nuclear Power Plants}

\author{
Year Three Activities Report \\ \&
}

Final Technical Report

November 30, 2002

DOE Project DE-FCO-99SF21898/A002

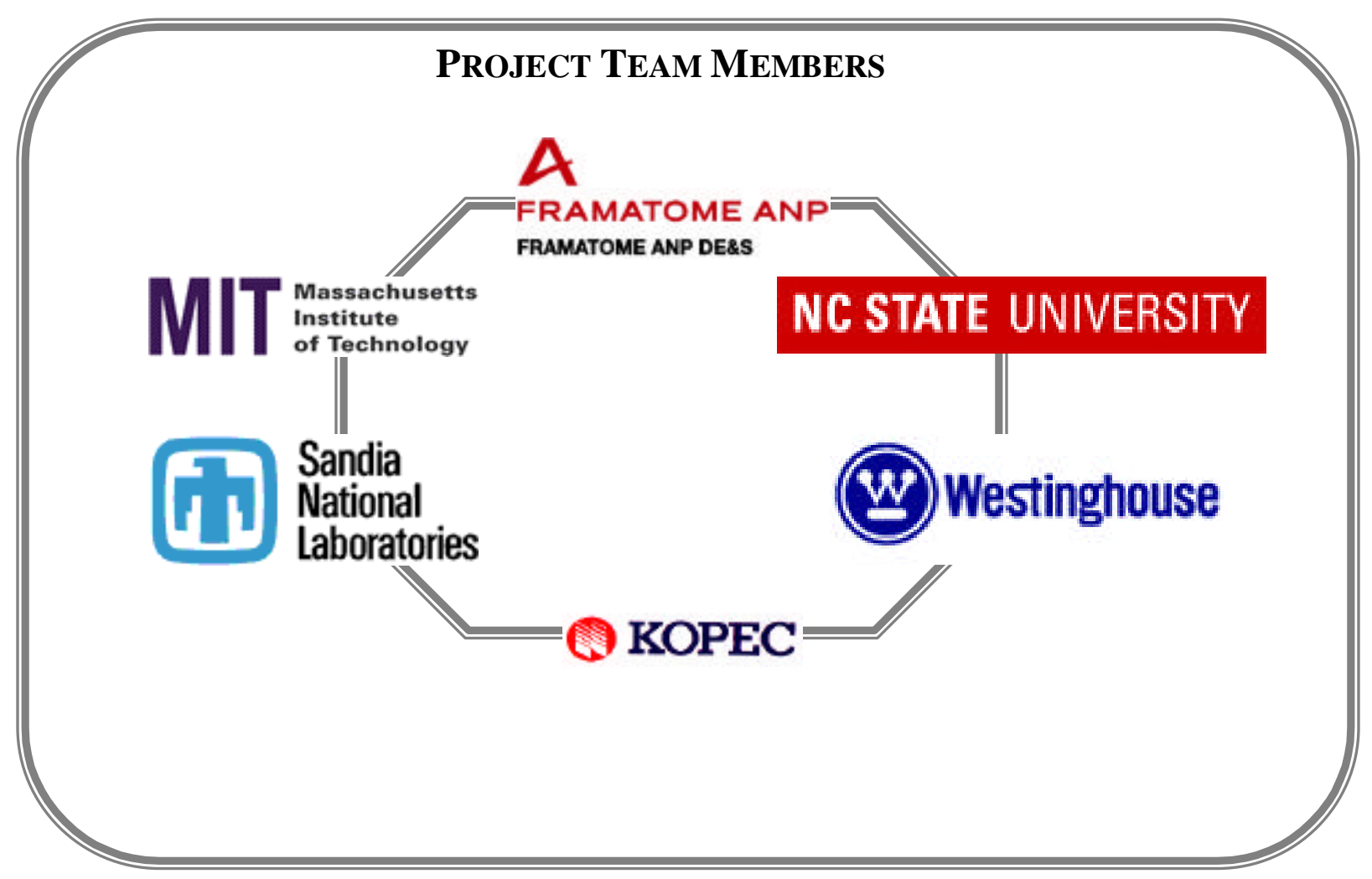




\title{
Development of Advanced Technologies to Reduce Design, Fabrication and Construction Costs for Future Nuclear Power Plants
}

\author{
Year Three Progress Report \\ $\&$ \\ Final Technical Report
}

Project Report, November 2002

\author{
Principal Investigator \\ Camillo A. DiNunzio, Framatome ANP DE\&S
}

\section{Cosponsors}

Dr. Abhinav Gupta, Assistant Professor, NCSU

Dr. Michael Golay, Professor, MIT

Dr. Vincent Luk, Sandia National Laboratories

Rich Turk, Westinghouse Electric Company Nuclear Systems

Charles Morrow, Sandia National Laboratories

Geum-Taek Jin, Korea Power Engineering Company, Inc. 


\section{DISCLAIMER OF WARRANTIES AND LIMITATION OF LIABILITIES}

THIS DOCUMENT WAS PREPARED BY THE ORGANIZATION (S) NAMED BELOW AS AN ACCOUNT OF WORK SPONSORED OR COSPONSORED BY THE DEPARTMENT OF ENERGY, (DOE). NEITHER DOE, IT'S SUB-CONTRACTORS, THE ORGANIZATION (S) BELOW, NOR ANY PERSON ACTING ON BEHALF OF ANY OF THEM:

(A) MAKES ANY WARRANTY OR REPRESENTATION WHATSOEVER, EXPRESS OR IMPLIED, (I) WITH RESPECT TO THE USE OF ANY INFORMATION, APPARATUS, METHOD, PROCESS, OR SIMILAR ITEM DISCLOSED IN THIS DOCUMENT, INCLUDING MERCHANTABILITY AND FITNESS FOR A PARTICULAR PURPOSE, OR (II) THAT SUCH USE DOES NOT INFRINGE ON OR INTERFERE WITH PRIVATELY OWNED RIGHTS, INCLUDING ANY PARTY'S INTELLECTUAL PROPERTY, OR (III) THAT THIS DOCUMENT IS SUITABLE TO ANY PARTICULAR USER'S CIRCUMSTANCE; OR

(B) ASSUMES RESPONSIBILITY FOR ANY DAMAGES OR OTHER LIABILITY WHATSOEVER (INCLUDING ANY CONSEQUENTIAL DAMAGES, EVEN IF THE DOE OR ANY DOE REPRESENTATIVE HAS BEEN ADVISED OF THE POSSIBILITY OF SUCH DAMAGES) RESULTING FROM YOUR SELECTION OR USE OF THIS DOCUMENT OR ANY INFORMATION, APPARATUS, METHOD, PROCESS, OR SIMILAR ITEM DISCLOSED IN THIS DOCUMENT.

ORGANIZATION (S) THAT PREPARED THIS DOCUMENT

Framatome ANP DE\&S

Massachusetts Institute of Technology

North Carolina State University

Sandia National Laboratories

Westinghouse Electric Company Nuclear Systems

Korea Power Engineering Company, Inc.

This is a yearly project report to the Department of Energy for the Nuclear Energy Research Initiative (NERI) program work. This report is intended as an informal report of continuing research, a meeting, or a topical study. 


\section{CITATIONS}

This document was prepared by:

Camillo A. DiNunzio

Principal Investigator

Framatome ANP DE\&S

400 Donald Lynch Blvd.

Marlborough, MA 01752

Dr. Michael Golay

Dr. Abhinav Gupta

Professor of Nuclear Engineering

Assistant Professor

Massachusetts Institute of Technology

North Carolina State University

77 Massachusetts Avenue

Cambridge, MA 02139

413 Mann Hall

Raleigh, NC 27695-7908

Dr. Vincent Luk

Charles Morrow

International Nuclear Safety Dept.

Sandia National Laboratories

Albuquerque, NM 87185-0744

Advanced Nuclear Concepts

Sandia National Laboratories

Mr. Richard S. Turk

Albuquerque, NM 87185-1178

Westinghouse Electric Company

Nuclear Systems

2000 Day Hill Road

Windsor, CT 06095

Geum-Taek Jin

Korea Power Engineering Co.

360-9 Mabuk-ri, Guseong-eup

Yongin-si, Gyeonggi-do

449-713, Korea

This document describes research sponsored by the Department of Energy and was performed, in part at Sandia National Laboratories. Sandia is a multi-program laboratory operated by Sandia Corporation, a Lockheed Martin Company, for the U.S. Department of Energy under Contract DE-AC04-94AL85000. 
TABLE OF CONTENTS

SECTION $\quad \underline{\text { PAGE }}$

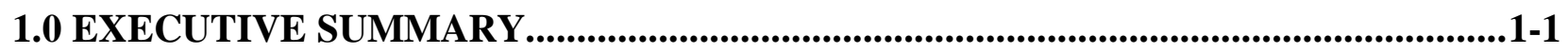

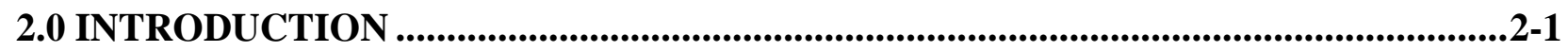

2.1 Background \& Needs........................................................................................2-2

2.2 Vision.................................................................................................2-3

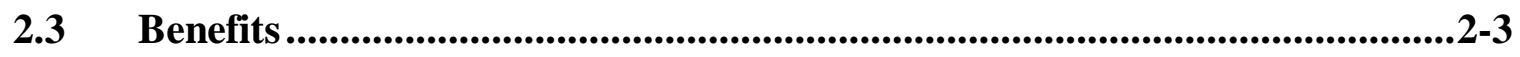

3.0 PROJECT GOALS, ORGANIZATION, AND APPROACH.....................................3-1

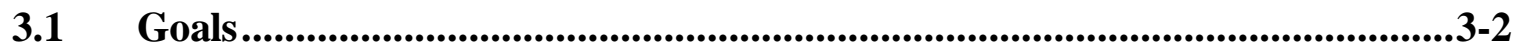

3.2 Organization ...........................................................................................................3-2

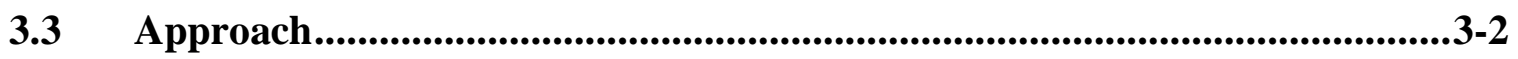

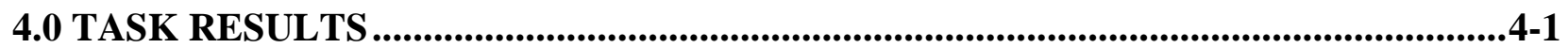

4.1 Decision Support Systems In Seismic Design Of Structures,

Systems, And Components ...........................................................................4-3

4.2 Bayesian Belief Network (BBN)-Based Advisory System

Development For Steam Generator Replacement Project

Management..................................................................................................4-22

4.3 System Dynamics Model For Nuclear Power Plant Design

And Construction Project Management ....................................................4-74

4.4 Solid Modeling "Design To Analysis" Tool ..................................................4-145

4.5 4D Visualization Modeling ...................................................................4-181

\subsection{MODEL DEVELOPMENT}

5.1 Product Model......................................................................5-1

5.2 Productivity Model.............................................................5-3

5.3 Process Model..................................................................5-3

APPENDIX A

List Of Project Participants

APPENDIX B

System Dynamics Model Model Variables, Definitions, And Equations

APPENDIX C

References 


\subsection{EXECUTIVE SUMMARY}

This report presents a summation of the third and final year of a three-year investigation into methods and technologies for substantially reducing the capital costs and total schedule for future nuclear plants. In addition, this is the final technical report for the three-year period of studies.

During the first and second years, the studies involved the following:

?? Examining potential impact of information technology on the physical construction activities (note: we examined information technology impacts on the design side in year one)

?? Identifying capital cost and schedule reduction strategies.

?? Evaluating potential project management concepts as advanced tools to help assure on budget and on schedule performance of a complex project such as a nuclear plant

?? Examining Cost Risk modeling as a mechanism to help assess the viability of potential cost and schedule reduction techniques

?? Exploring mechanisms to take out excess conservatism and reduce error while shrinking the time between design and analysis activities

?? Evaluating methods to remove excess seismic margins and simplifying containment construction.

The interim results and the final output of the product supports Generation IV reactor development as well as assisting in the near-term deployment of advanced reactors.

More specifically, this year's work focused on the following initiatives:

1. Containment and Structural Simplifications

Developing a decision support system (DSS) for evaluating this trade-off was developed. A case study for simulation based design, was developed i.e. use of formal optimization tools and their limitations in an automated process.

2. Project Management Cost and Schedule Modeling

Developing two advisory systems for project management:1) System Dynamics model that uses the deterministic approach and 2) Bayesian Belief Network (BBN) model that uses the probabilistic approach.

3. Solid Modeling-"Design to Analysis" Tool

Developing a preliminary version of a "design-to-analysis" tool that converts solid models of a pressurizer and its piping systems in a CAD package into a finite element mesh ready for analysis purposes.

4. $4 \mathrm{D}$ Visualization Modeling

Identifying strategies used to reduce costs associated with the Design, Procurement, Construction, Installation and/or test phases of a nuclear plant project, using "4D visualization." 
- Collecting construction data of a nuclear plant and reviewed the schedule reduction method

- Developing 4D visualization Model; Link design model with construction schedule for whole power block (Containment, Auxiliary and Turbine Building)

- Preparing4D Simulation movie clips for each building and whole power block.

The results of the three effort provided innovative tools for reducing the overall costs associated with the design and construction of a nuclear power plant. The designers of a new plant are responsible for the committed costs and schedule associated with a new design.

Much of our year one work focused on applying concepts to reduce costs and schedule from a design perspective.

The second year work moved more into the execution of a design in evaluating and proposing the use of tools to support the balance of activities after a design is complete.

Year Three work continued with enhancements to the models that were developed in Year Two. In general, we extended the work of years one and two and integrated the insights into the models.. This work should be able to provide some certainty to our claims of being able to reduce cost and schedule.

These tools will also ensure that designs are built consistent with the safety analyses and thus ensuring that the promise to the public for safe nuclear technology is met at an affordable cost. Therefore, we have been examining how new technologies might be brought to bear on ensuring that schedules can be kept and that costs can be contained. 


\subsection{INTRODUCTION}




\subsection{Background \& Needs}

The purchase of an advanced nuclear power plant is initially based upon the assessment of the total capital cost on a dollar per installed kilowatt electric coupled with the planned operations and maintenance costs on a operating cost per kilowatt electric $(\mathrm{kW})$ generated. Both these metrics are the yardsticks by which nuclear power plants are compared against market alternatives of gas fired generation or coal-fired generation. While the existing fleet of nuclear power plants in the United States operates cost effectively and competitively in a partially deregulated market, the decision to invest in new nuclear generation is contingent upon not only the capital cost per $\mathrm{kW}$ installed, but also the confidence with which the schedule of delivery can be completed. The prior work sponsored jointly by the Department of Energy and the Electric Power Research Institute in the Advanced Light Water Reactor (ALWR) program produced reactor plant designs that were optimized for operations and maintenance expenditure, but not capital cost or schedule as the research initiatives were conducted when the electric power system had not experienced the impact of deregulation.

Deregulation has since altered the analysis of new power plant ordering to balance both capital costs and operations and maintenance costs since in the new energy market, there will not be programmatic support for investment decisions other than the economics of the power generation source and its fuel. Figure 2-1 below illustrates the decision logic to be satisfied for new nuclear plant orders.

Figure 2-1 - Requirements for New Plant Construction

\begin{tabular}{|c} 
Licensability $\rightarrow$ Constructability $\rightarrow \begin{array}{l}\text { Proven } \\
\text { Schedule }\end{array} \rightarrow \begin{array}{l}\text { Risk is } \\
\text { Understood }\end{array} \rightarrow \begin{array}{l}\text { Investment Can } \\
\text { Be Made }\end{array}$ \\
Pesign $\rightarrow$ Procure $\rightarrow$ Construct \\
Analytical Models \\
Materials Models $\quad \begin{array}{l}\text { Engineering Models } \\
\text { Labor \& Productivity Models }\end{array}$
\end{tabular}


Therefore, the nuclear industry faces two major hurdles to overcome, a lower competitively priced nuclear unit coupled with a schedule that is competitive and most importantly, believable to the prospective investor.

The ALWR designs represent potential starting points for examining capital cost reductions, however these designs are burdened by the considerations of a regulated environment in which the design bases considerations date to the dawn of the nuclear power era when the technology was new and therefore design margins were created to offset the lack of experience. So, there is much to examine for the basic design of a next generation nuclear plant. However, design of a competitive plant is merely a pre-requisite to the next stage that is the construction of a plant. Promising reactor designs would have to be built using the same strategies that were found to be less than adequate for controlling costs in the 1970's and 80's.

Therefore, delivery of a reactor plant within the three constraints of quality, cost and schedule is not currently achievable in a deregulated market. The challenge is therefore to re-define the rules of the game to make success possible. Work must be done to link prior industry research that has examined specific incremental improvement strategies with applications of information technology and new thinking on coordination of businesses to produce dramatic reductions in the capital cost of new reactor plants. This project's focal point for keeping score of potential improvements is on the total cost and cycle time associated with the Design, Procure, Construct, Install, and Test (DPCIT) cycle. By examining each of these activities in building a plant, we expect to apply new thinking and improvement techniques that have been developed in other industries.

\section{$2.2 \quad$ Vision}

The DPCIT project represents a merger of information technologies and supply chain management principles with design and construction improvements. The project will focus on process and along the way adopt the appropriate tools to execute the process. Figure 2-2 below provides a visual image of the intent of the DPCIT project.

Figure 2-2 - Project Overview

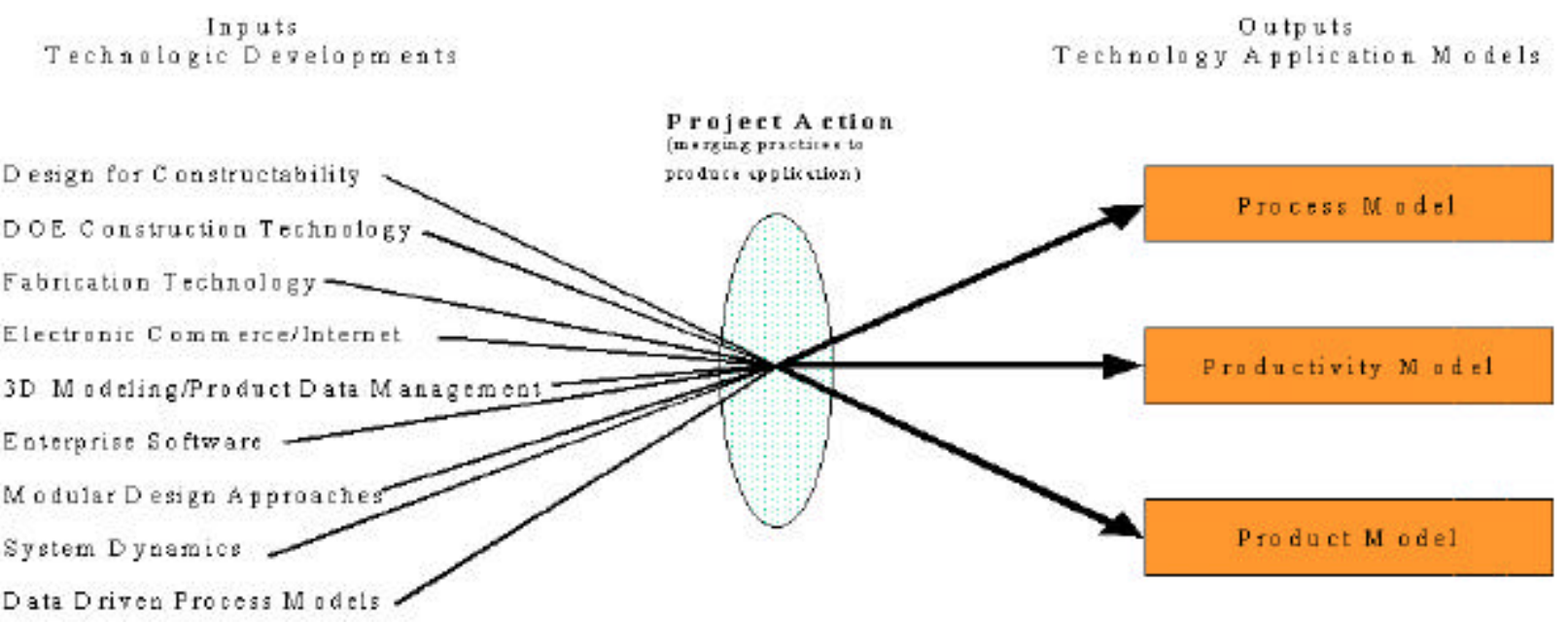

\subsection{Benefits}

The benefits of our approach and broad review of non-nuclear technology advances coupled with improvements within the nuclear industry and related NERI projects are: 
1. to identify the recipe for successful development of the next generation reactor plants in terms of their cost structure,

2. provide the ability to be constructed swiftly and confidently, and

3. identify the fundamental changes in practices needed to accommodate the competitive energy marketplace for new reactor designs.

The project addressed key improvement technologies that can be applied to both reduce a nuclear plant's costs and addressed the issues of schedule and capital at risk. 


\subsection{PROJECT GOALS, ORGANIZATION, AND APPROACH}




\subsection{Goals}

The goal of the DPCIT project team is to identify those methods that can deliver a $40 \%$ reduction in capital cost and schedule for a future nuclear power plant. Given the starting point of the ALWR program's benchmark of $\$ 1500$ per $\mathrm{kW}$ installed and a 60 -month construction schedule, the targeted reductions translate into a power plant built for $\$ 900$ per $\mathrm{kW}$ and 36 months from first concrete to fuel load. Ideally, these techniques and innovations would be able to be demonstrated for the near term reactor plants and then incorporated into the planned Generation IV reactor plants which are under conceptual and detailed development. Most of the innovations planned in this project should be applicable to most reactor technologies since the concepts under consideration are not linked to a specific reactor design, though the proposed reductions will be demonstrated using pressurized water reactor technology.

\subsection{Organization}

Framatome ANP DE\&S leads the team with an assigned Project Manager who has the accountability for all budgetary and contractual matters between Framatome ANP DE\&S and the DOE as well as the sub-contract organizations. Since Sandia National Laboratory (SNL) funding is provided separately, there is no management of the SNL funds.

Project team members are provided from the following organizations:

Massachusetts Institute of Technology, Nuclear Engineering Department

North Carolina State University, Civil Engineering Department

Sandia National Laboratories, Advanced Nuclear Concepts and International Nuclear Safety Department

Westinghouse Electric Company Nuclear Systems

Korea Power Engineering Company, Inc.

\subsection{Approach}

Given the diverse nature of the team members and their own interests and individual strengths, the project has been organized with tasks assigned to specific team members. Generally, each team member was assigned a task or set of tasks with the outcomes being brought together via the annual and final technical reports as well as incorporating insights into the Product, Productivity, and Process models. 


\subsection{TASK RESULTS}




\section{Organization of This Section}

Section 4 contains the write-ups of the activities undertaken during the $3^{\text {rd }}$ project year for this three-year project. Each write up stands on its own.. Section 5 provides the integrated view of how the various research efforts are connected to achieve the overall project goals. Our major objectives of shrinking cycle time for the entire project duration as well as shrinking cost have been under examination as a series of efforts this year. In particular we are concerned not only about reducing the sums of costs and schedule, but also providing some assurance that these targets can be met in an actual situation by developing project management approaches more capable of dealing with change than prior US construction experience. We think this is particularly important since the bulk of the experienced nuclear construction professionals are close to retirement age for use on the next US nuclear plant planned for construction.

This project year's work had several main themes:

\section{2) Containment and Structural Simplifications}

Developing a decision support system (DSS) for evaluating this trade-off was developed. A case study for simulation based design, was developed i.e. use of formal optimization tools and their limitations in an automated process.

\section{Project Management Cost and Schedule Modeling}

Developing two advisory systems for project management:1) System Dynamics model that uses the deterministic approach and 2) Bayesian Belief Network $(\mathrm{BBN})$ model that uses the probabilistic approach.

\section{2) Capital Cost Model Development and Testing}

Developing a capital costing model that addressed the uncertainty in the potential savings, both in cost and in project duration from other research activities within this project, and developing . a tool with which to rank other options in terms of their ability to reduce capital costs.

es Solid Modeling-"Design to Analysis" Tool

Developing a preliminary version of a "design-to-analysis" tool that converts solid models of a pressurizer and its piping systems in a CAD package into a finite element mesh ready for analysis purposes.

\section{Visualization Modeling}

? Identifying strategies used to reduce costs associated with the Design, Procurement, Construction, Installation and/or test phases of a nuclear plant project, using "4D visualization."

? Collecting construction data of a nuclear plant and reviewed the schedule reduction method

? Developing 4D visualization Model; Link design model with construction schedule for whole power block (Containment, Auxiliary and Turbine Building)

? Preparing4D Simulation movie clips for each building and whole power block. 


\subsection{DECISION SUPPORT SYSTEMS IN SEISMIC DESIGN OF STRUCTURES, SYSTEMS, AND COMPONENTS}

\subsubsection{Summary of Accomplishments in Year 1 and Year 2:}

esesllustrated excessive seismic margins in:

? concrete containment - excessively low allowable value of shear stress.

? pipe and pipe support loads - uncoupled piping analysis is excessively

conservative.

? qualification requirements for electrical cabinets and equipment - generic \& excessively conservative amplification factors used irrespective of equipment location.

esectlect and categorized cost data for piping and pipe support in existing plants.

Eestudied the concept of composite construction for internal concrete structures wherein concrete is poured in between steel skin plates

\subsubsection{Introduction}

We continued our work on developing computational design tools for simplification of structural systems. While reduced loads are likely to result in the development of simpler alternative structural systems, a trade-off exists between the reduction of seismic margins and risk. Any decision regarding structural simplification should be targeted towards addressing this trade-off. During the year 3 of this project, we worked on developing a decision support system (DSS) for evaluating this trade-off. We conducted a case study for simulation-based design, i.e. use of formal optimization tools and their limitations in an automated process.

Often a decision maker does not have a full understanding of the problem and knowledge of system parameters at the onset of the design process. A better understanding of the problem, however, may be gained through trial-and-error. One means of facilitating this process is through an alternative decision-making paradigm in which a computer-based decision support system (DSS) is used. A DSS can support a collection of analytical and computational techniques, schemes for uncertainty propagation, and optimization tools. Object-oriented languages such as Java provide the flexibility to not only interface with commercial and regulatory scale software but also to monitor intermediate results for review by human decision makers. A decision maker selects the schemes, software, and parameters for exploration as well as the design and serviceability requirements for compliance. Professional judgment can be used to provide feedback.

To begin with, it appears that any design should be optimized over the DPCIT cycle. A traditional use of optimization is to find the "optimal" solution. This solution, however, is rarely the best solution to the real problem because some objectives and constraints may not have been explicitly stated in the problem formulation. The omissions may be due to errors, the unquantifiable nature of certain issue or issues that were not identified at the point of model formulation. Traditionally, this has been a major limitation of optimization applications. Modeling to Generate Alternative (MGA) techniques use optimization to 
generate a small set of very different solutions. MGA is an extension of single and multiple-objective mathematical programming techniques with an emphasis on generating a set of alternatives that are "good" but "as different as possible." By generating these good yet different solutions, a decision maker can explore alternatives that satisfy unmodeled objectives to varying degrees. The decision maker may assess these alternatives either subjectively or quantitatively. We use MGA in the development of DSS, which employs genetic algorithms for optimization and searching alternatives. High performance distributed computing is used in the computationally intensive DSS applications.

\subsubsection{Decision Support Systems}

We conduct a case study for application of the DSS to the design of snubbers and their locations in a piping system. Such an illustration is undertaken primarily because in our opinion a DSS forms a key element of the process envisioned for DPCIT project. We use the genetic algorithms (GAs) to not only arrive at the most optimal solution but also evaluate the design trade-off. We then use the MGA technique to evaluate alternative designs that are near optimal but different.

Seismic design and qualification of secondary systems such as power plant piping, like other complex decision problems, involves many tradeoffs among competing criteria. At all stages in a power plant life cycle, structural engineers are faced with formidable challenges of complex decision-making. In the context of seismic design and performance evaluation, these may relate to technical as well as practical feasibility, cost, and redundancy. Although some of these challenges can be quantified and formally analyzed, others may call for outside opinions from experts or engineering judgment. Key features of the decisionmaking process include identification of alternatives for solving the given problem. Then, whether an alternative is satisfactory typically requires analysis by one or more computer models, e.g., redundancy versus cost analyses, and the expertise and judgment of the engineer.

Optimization for seismic support locations in piping systems is a highly complex and iterative problem that is time and cost intensive. A large number of possibilities may exist, making enumeration impractical. The current practice of designing piping support locations is primarily heuristic, relying heavily on professional experience. Engineers use the knowledge learned from observed performances and past failures to arrive at the most appropriate locations. In practical applications, the use of mathematical optimization or other formal search techniques may lead to "optimal" structural systems that are either infeasible or unnecessarily costly in the sense that they are not conducive to construction, maintenance or serviceability. In this scenario, the optimization model produces a single solution that is "best," but only with respect to the objectives and constraints that appear in the model: the unmodeled issues, such as downstream maintenance or outage costs, may not have been factored into the search process. To produce a truly optimal solution, then, would require that the judgment and expertise of an experienced designer somehow be embedded in the model. These difficulties in modeling are a key limitation, not of optimization techniques per se, but of the way in which they are routinely used.

The objectives of the present study are realized through a prototype decision support system (DSS) that draws on the complementary strengths of the engineer and the computer in a joint-cognitive system. Genetic algorithms (GA) are used as part of a formal procedure for generating alternative solutions using a methodology referred to as "modeling to generate alternatives - MGA." MGA uses optimization to generate a set of solutions that 
are similar in objective space - "near optimal," but very different in decision space - "as different as possible". The computational performance requirements of the DSS are addressed by using an existing framework for high performance distributed computing on workstation clusters. One such object-oriented framework, Vitri, has been developed at NC State University (Gupta et al. 2002). It includes basic support for distributed computing and communication, and distributed GA implementations. While the original development of the basic GA algorithms for a structural engineering DSS was conducted under a National Science Foundation grant, the present study evaluates its role in the application to seismic design of a real-life piping and pipe supports.

\subsubsection{Design of Pipe Supports for Seismic Loads}

Conventionally, the initial design of a piping system and its supports is drawn primarily from the standpoint of withstanding loads calculated from thermal, hydraulic, and deadweight considerations.

In piping systems that experience significant thermal stresses, additional supports are likely to increase the thermal stresses due to increased constraints. At the same time, additional supports are needed to withstand earthquake loads. Supports called snubbers are used to satisfy these competing requirements. Snubbers have a locking mechanism that activates only during pipe vibrations, thereby avoiding additional constraints against thermal loads in normal operation. Snubbers can be either hydraulic or mechanical devices that require periodic maintenance. Regulations dictate that the integrity of critical piping systems and pipe supports be maintained at all times to withstand future earthquakes safely. It should be noted that pipe vibrations due to hydraulic loads such as water hammer might cause snubbers to lock and activate during normal operation. Emergency plant outages arise whenever a snubber is found to have failed or malfunctioned. In addition to having high capital cost, the lifetime cost of snubbers is excessively high especially when the cost of replacement and loss of revenue during an unscheduled outage are considered together with maintenance cost. In piping systems that have negligible thermal stresses, a designer can avoid the use of snubbers for withstanding seismic loads by using rigid or hanger type supports. While the capital and maintenance cost of such supports is significantly less than that of snubbers, the other issues related to lifetime cost and downtime remains the same.

The key elements of piping system design for withstanding seismic loads are determining the number of supports, the type of supports (capacities), and their locations. Some of the considerations in this process are:

? minimization of number of supports.

? minimization of high capacity (and also high cost) supports to reduce the maintenance and replacement costs.

? selection based on analysis for (a) identification and elimination of cases with overstressed piping, (b) evaluating support loads and their acceptability against support capacities, and (c) evaluating maximum pipe displacements and their acceptability with respect to serviceability limits if any.

An additional consideration that is not quantified in present practice is related to the desire to place a supports directly under heavy equipment such as valves that may be located on the piping system. Further, a piping system that is very flexible is undesirable with respect to vibrations encountered during everyday operations, i.e., it is desired that the fundamental frequency of vibration be above $1 \mathrm{~Hz}$. Typical values for piping system frequencies are on 
the order of $5 \mathrm{~Hz}$ or greater. Other considerations are the forces and moments transferred by the piping at anchors to the attached equipment or building. Whether or not these forces and moments are acceptable depends upon the strength and stability requirements of the equipment or building. A designer may wish to change either the piping design or improve the stability of the attached equipment whenever the transferred forces and moments are found to be unacceptable. Additional constraints may also exist depending upon the particular problem or a given solution.

\subsubsection{Pipe Support Optimization - Representative Piping System}

Let us consider a simple non-existent but representative piping system for application of the proposed GA technique and to study the optimization for capital and lifetime cost. Gupta et al. (2002) describes this piping system in detail and used it in the development and verification of the various GA algorithms employed in structural engineering DSS. For simplicity, a $46 \mathrm{~cm} \sim(18$ inch) outer diameter straight pipe having $3.5 \mathrm{~cm} \sim(1.375$ inch $)$ wall thickness is considered. As shown in Figure 4.1-1, the two ends of the straight pipe are considered to be anchored and several lumped masses representing valves and other equipment are located on the pipe. In Figure 4.1-1, the numbers under the lumped masses show their corresponding mass in kilograms. The pipe is discretized into beam elements with 46 nodes including the two anchors. Each node on the pipe is allowed only two degrees of freedom, namely, the vertical displacement and the in-plane rotation. The piping system is modeled using commercial piping analysis program PIPESTRESS (DST 1997). Seismic analysis is performed for a response spectrum input in the vertical direction corresponding to El Centro, 1940 S00E earthquake that is applied uniformly at all the supports. Table 4.1-1 shows the list of supports that are considered for the piping system, along with their cost and support capacities. The objective function employed by Gupta et al. (2002) for pipe support optimization is given by

$$
\begin{aligned}
& \max \stackrel{?}{\stackrel{?}{?}} \frac{1}{a ? ? ? b ? ? ? c ? ? ? d C ? e\left|n_{\text {sup }} ? n_{\text {req }}\right|} \stackrel{?}{?} \text { ? }
\end{aligned}
$$

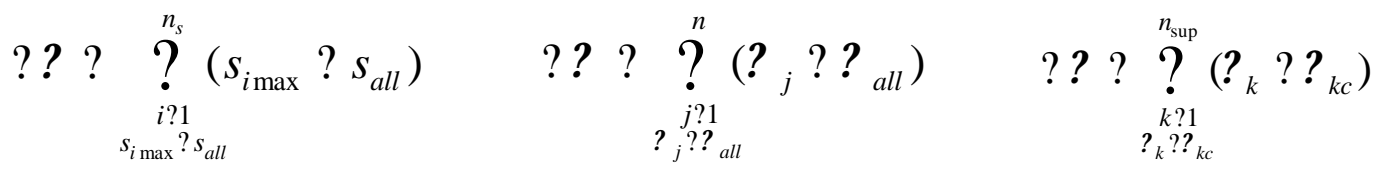

where $a, b, c, d, e$ are multipliers that are assigned values according to the relative importance of the constraints; $s_{i}$ is the stress at node $i ; s_{\text {all }}$ is the allowable stress; $n$ is the total number of nodes in the piping system; $n_{s}$ is the total number of spans in the system; $s_{\text {jmax }}$ is the maximum displacement to span ratio in $\operatorname{span} j ; s_{\text {all }}$ is the allowable displacement to span ratio; $?_{k}$ and $?_{k c}$ are the support load and support capacity, respectively, at the $k^{\text {th }}$ support location; $n_{\text {sup }}$ is the total number of supports in the system; and $n_{\text {req }}$ is the number of supports specified for a given solution. The allowable stresses are selected based on the ASME Boiler and Pressure Vessel Code Section III Class 2 (ASME 1995). It should be noted that the allowable for shear force and end-moments at the two anchors are not included explicitly in the optimization. These allowable are governed by the strength and stability requirements of the building or equipment to which the piping is attached. The decision on whether or not the shear and end-moment calculated for a particular solution is 
acceptable is left to the designer. The purpose is to provide flexibility to either modify the piping design or increase the stability of attached equipment.

The capital cost for various support types can be directly considered in the formulation because such costs are readily available. The capital cost is given by

$$
C ? \stackrel{?_{\text {sup }}}{n^{2} 1} c_{k}
$$

where $c_{k}$ is the capital cost of the $k^{\text {th }}$ support and $n_{\text {sup }}$ is the number of supports. Figure 4.1-2 shows the results obtained by using the above equation. It can be seen that the minimum capital cost solutions also have minimum number of supports.

The lifetime cost includes the cost of replacement and outage in addition to capital and maintenance costs. Revenue losses due to an unscheduled outage are much higher than maintenance and capital costs. Since specific estimates of lifetime costs are unavailable, lifetime cost is assumed to be proportional to a certain power of the support capacities, i.e.,

$$
C ? \stackrel{?}{?_{k ? 1}^{n_{\text {sup }}}} c_{k}^{f}
$$

where $c_{k}$ is the capacity of the $k^{\text {th }}$ support. We consider $f=1.2$ even though actual values of $f$ are expected to be much higher. The results generated by using this cost function in Equation 4.1-1 are shown in Figure 4.1-1. In contrast to the results shown in Figure 4.1-2, it can also be observed that there is a trade-off between the lifetime cost and the number of supports even though we considered a relatively small value of $f$. The optimal solutions are shown in Figure 4.1-4.

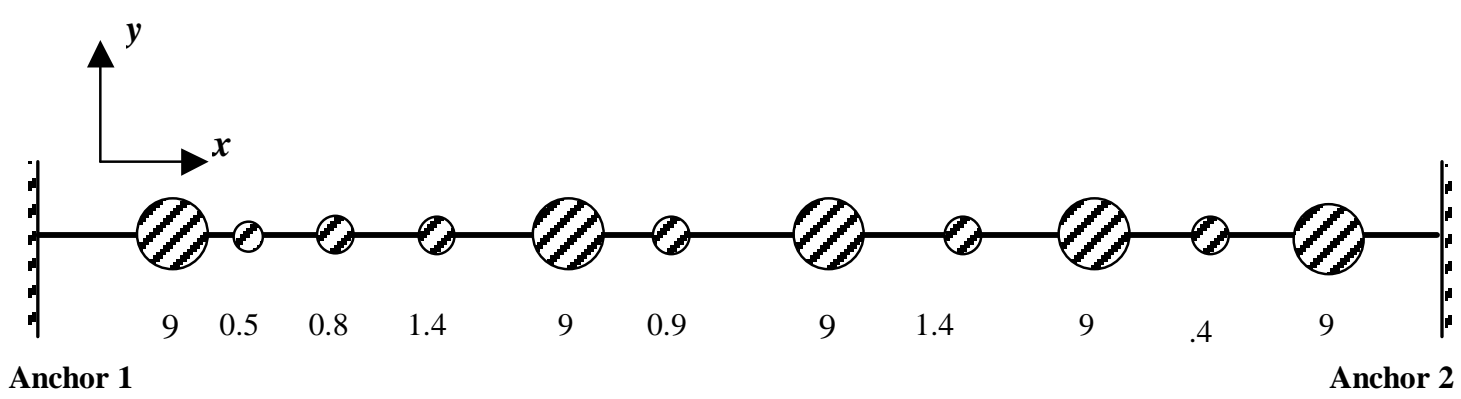

Figure 4.1-1: Simple Representative Piping With Lumped Masses 


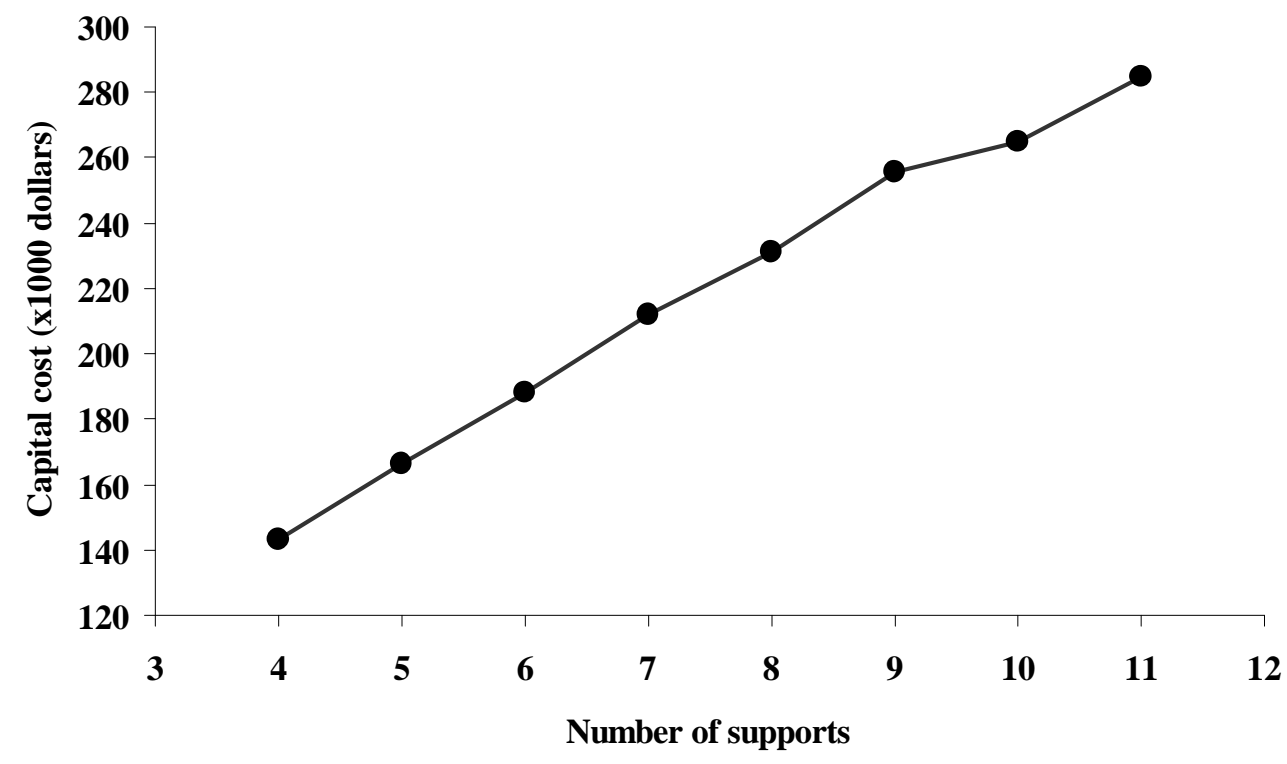

Figure 4.1-2: Trade-Off Curve Between Capital Cost And Number Of Supports, Simple Piping System

Table 4.1-1: Cost And Capacity Of Supports

\begin{tabular}{c|c|c}
\hline \hline S1. No. & Support Capacity $(\mathrm{N})$ & Cost $\left(\times 10^{3}\right.$ dollars $)$ \\
\hline 0 & 0 & 0.0 \\
1 & 8,802 & 15.0 \\
2 & 9,198 & 15.1 \\
3 & 16,020 & 21.0 \\
4 & 38,314 & 21.5 \\
5 & 61,188 & 24.0 \\
6 & 85,218 & 24.6 \\
7 & 89,445 & 24.8 \\
8 & 104,353 & 32.0 \\
9 & 165,985 & 35.5 \\
10 & 275,900 & 36.0 \\
11 & 313,058 & 42.0 \\
\hline \hline
\end{tabular}




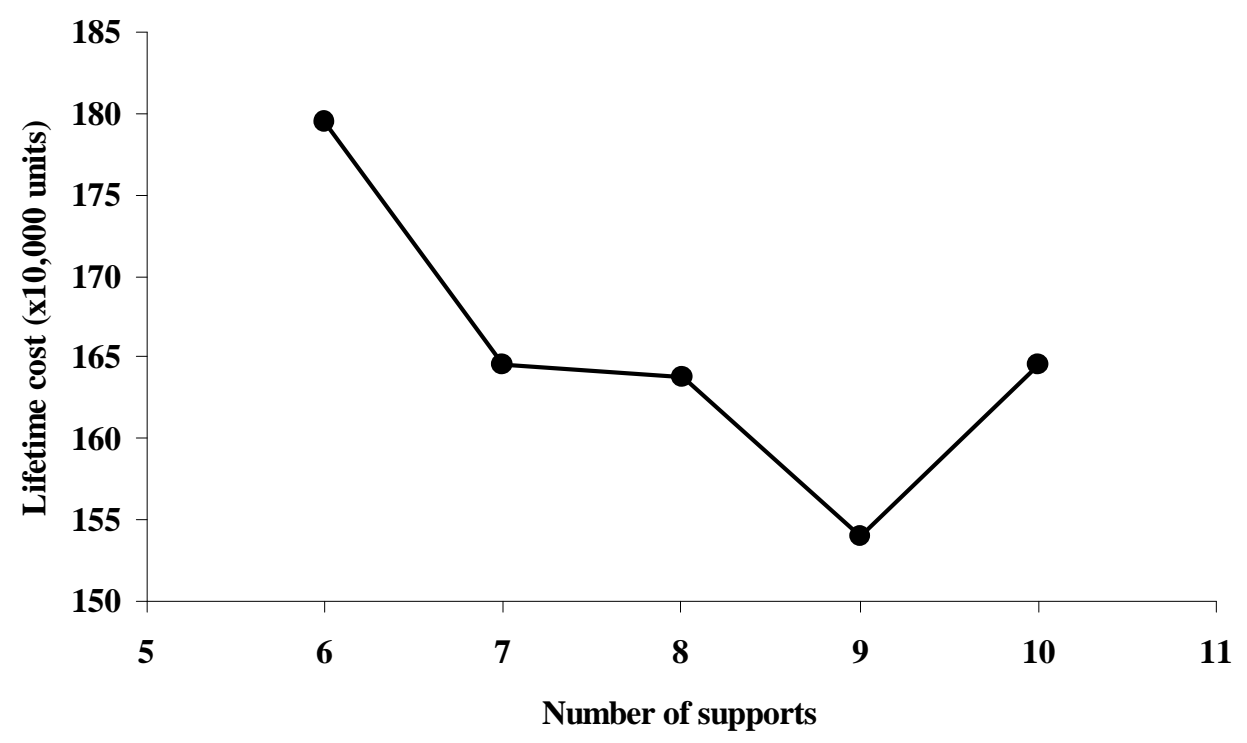

Figure 4.1-3: Trade-Off Curve Between Lifetime Cost And Number Of Supports, Simple Piping
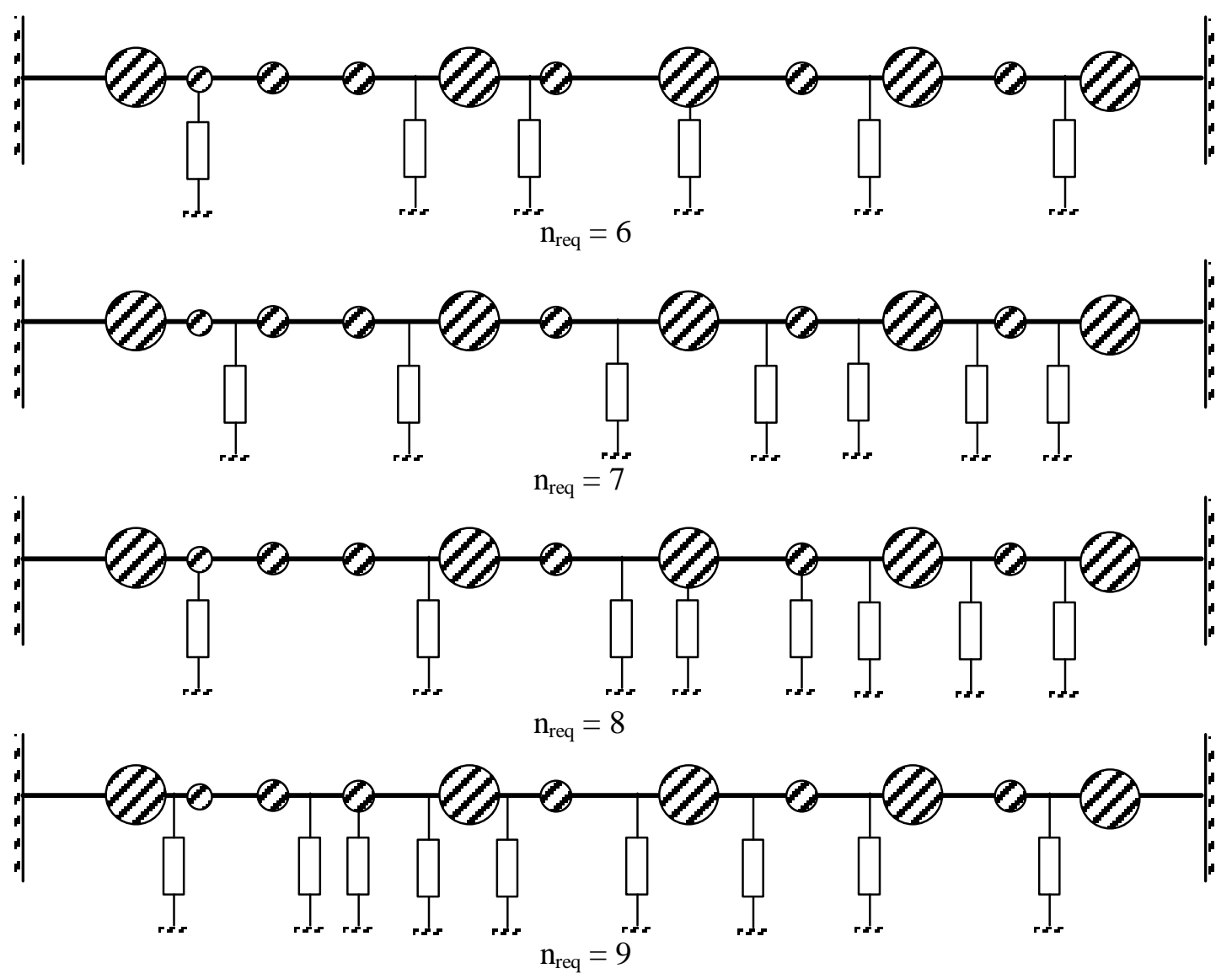

Figure 4.1-4: Optimal Solutions For Lifetime Cost 


\subsubsection{Modeling to Generate Alternatives (MGA)}

A traditional use of optimization is to create and run a model to find the "optimal" solution. This solution, however, is rarely the best solution to the real problem because some objectives and constraints may not have been explicitly stated in the problem formulation. The omissions may be due to errors, unquantifiable nature of certain issues or issues that were not identified at the point of model formulation. Traditionally, this has been a major limitation of optimization applications. Modeling to Generate Alternative (MGA) techniques (Baugh et al. 1997, Brill et al. 1990) use optimization to generate a small set of very different solutions. MGA is an extension of single and multiple-objective mathematical programming techniques with an emphasis on generating a set of alternatives that are "good" but "as different as possible." By generating these good yet different solutions, a decision maker can explore alternatives that satisfy unmodeled objectives to varying degrees. These alternatives may be assessed by the decision maker either subjectively or quantitatively. Consider a single-objective model with a solution $A$ shown in Figure 4.1-5. Adding an additional consideration would result in a tradeoff curve, perhaps the one shown in Figure 4.1-6, where solution $B$ might represent a decision maker's most desirable compromise between objectives $Z_{1}$ and $Z_{2}$. Yet, had $Z_{2}$ been omitted from the model, the solution represented by $B$ would appear to be inferior to $A$. Likewise, although it appears inferior, solution $C$ might be non-dominated and preferred by a decision maker in the presence of a third objective, $Z_{3}$.

This idea can be further demonstrated with a simple example from linear programming. Consider the following formulation of a design optimization problem:

\begin{tabular}{|c|}
\hline $\begin{array}{l}\text { Maximize: } \\
\text { Subject to: } \\
x ? y ? 30\end{array}$ \\
\hline$y ? 20$ \\
\hline$x, y ? 0$ \\
\hline
\end{tabular}

The decision space for the problem is shown as the shaded region in Figure 4.1-7 and the slope of the objective function can be seen from a typical contour line at an arbitrary value, 80. Mathematical optimization correctly produces $z=50$ at point $A$ as the solution. Thus, all other things being equal, the design would be $\langle x, y\rangle$ ? $\langle 10,20\rangle$.

The premise of MGA, however, is that all other things may not be equal, and that there may be features not completely captured by the model. When those issues are considered, point A may be less desirable overall than a point, call it $\mathrm{B}$, originally deemed inferior by the model. The issues involved in finding B and establishing the computer assistance needed in this process are discussed below.

? Generating all feasible solutions suggests that one has no confidence in the model - one would expect that point B optimizes the objective function nearly as well as A, so only these good solutions need to be examined. This idea is illustrated in Figure 4.1-8, where only those solutions that are within $10 \%$ of the optimal are retained. 
? Available solutions should represent a cross-section of good solutions, so that, if B is not actually among them, perhaps one of them is close enough from which to begin "tinkering."

? Since a decision maker can reasonably consider only a small number of designs, a subset of these good solutions should be presented for inspection.

For the problem defined above, the first alternative can be found by changing the formulation to maximize a difference metric $\mathrm{d}$, instead of $z$ and imposing the constraint, $\mathrm{x}+2 \mathrm{y}=0.9(50)$.

The difference metric $\mathrm{d}$ for this problem can be defined as $\mathrm{d}^{2} ¥(x-10)^{2}+(y-20)^{2}$, which is the distance between the two points in the decision space. Baugh et al. (1997) illustrate the application of MGA to topological truss optimization.

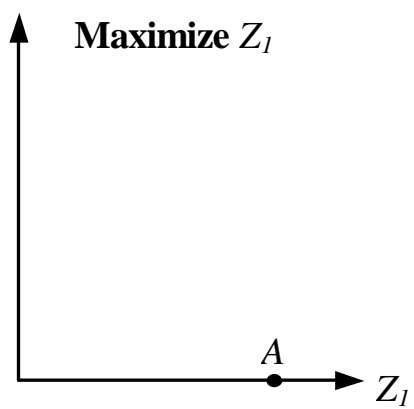

Figure 4.1-5: One Objective

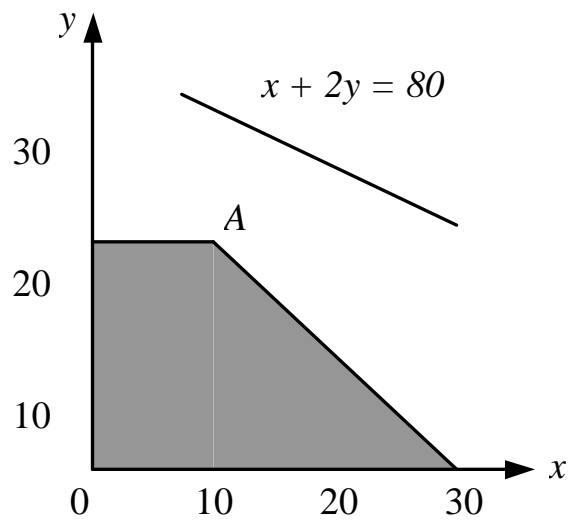

Figure 4.1-7: Decision Space

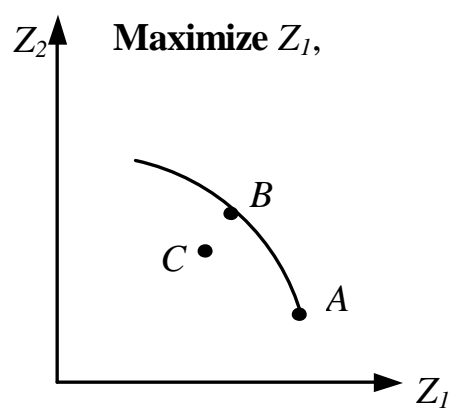

Figure 4.1-6: Two Objectives

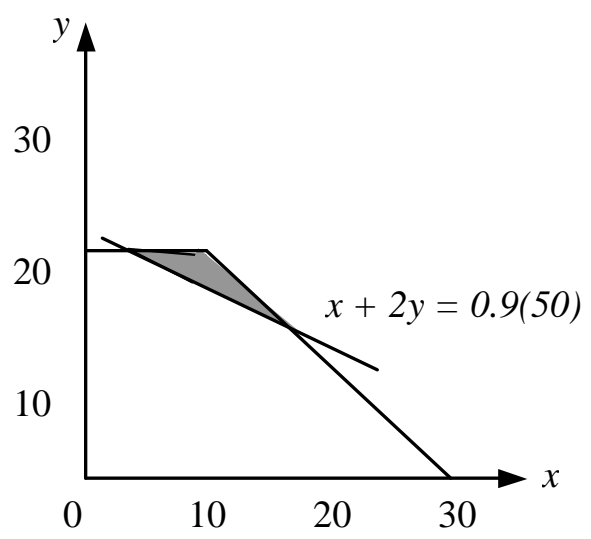

Figure 4.1-8: Reduced Decision Space

\subsubsection{Application of MGA to pipe support optimization}

As noted from the discussion in the previous section, application of MGA requires definitions of a reduced decision space and a difference metric to generate the alternatives. For pipe support optimization, decision space can be reduced by imposing the following constraints: 


$$
C_{m g a} \quad ? \quad k_{1} C_{o p t}
$$

where $k_{1}>1$ and, $C_{m g a}$ and $C_{o p t}$ are the costs associated with the alternative and the optimal solution respectively;

As stated earlier, MGA requires a difference metric that can be maximized to generate alternatives that are "as different as possible." We use the following topology difference metric which is applicable to binary strings and commonly known as "Hamming distance." If the two strings are represented as $\left\langle b_{1}, b_{2}, \ldots, b_{n}\right\rangle$ and $\left\langle c_{1}, c_{2}, \ldots, c_{n}\right\rangle$, we can write,

$$
\text { ? ? } ?_{i ? 1}^{n}\left|b_{i} ? c_{i}\right|
$$

The alternatives are generated by modifying Equation 4.1-1 to maximize $\mathrm{d}$ instead of cost and adding an additional penalty to account for the constraint given by Equation 4.1-4. The value of $k_{l}$ is taken to be 1.3 .

This procedure is used to generate alternatives to the optimal solutions for $n_{\text {req }}=5,6$, and 7 , which are shown in Figures 4.1- 9, 4.1-10 and 4.1-11. The quality of MGA solutions is evaluated with respect to several quantities that were not included in the optimization either explicitly or implicitly. These include the location of supports under lumped masses, the fundamental frequency of vibration, the maximum support load to capacity ratio, the maximum stress ratio, and the shear force and the fixed end moment at the two anchors. The values of these parameters for various alternatives are shown in Tables 4.1-2, 4.1-3, and 4.1-4. It is observed that even though all the solutions are good with respect to cost, some of the solutions are better when compared to others with respect to some or all of the unmodeled issues. 

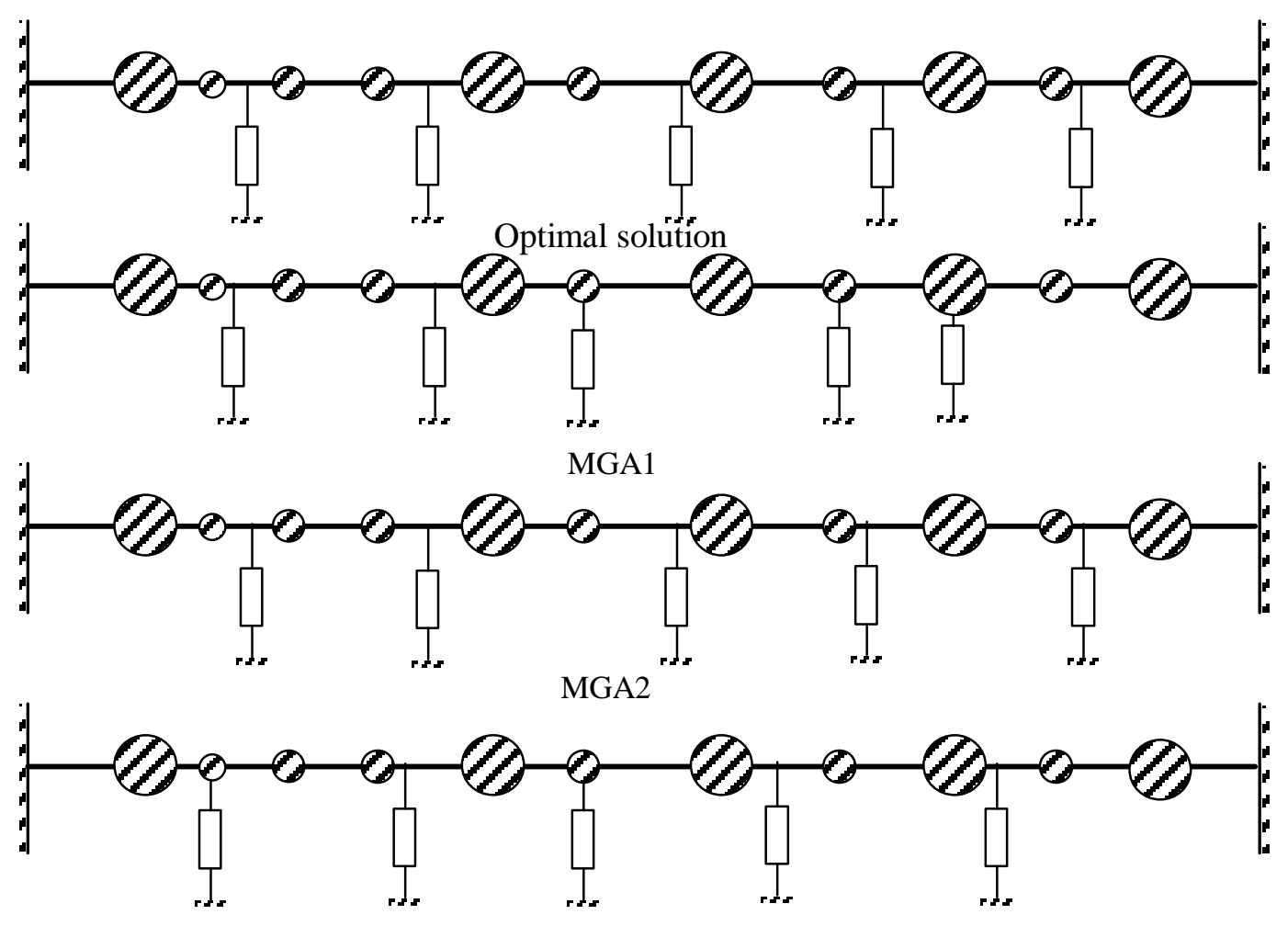

MGA3

Figure 4.1-9: MGA Solutions For Lifetime Cost With $\mathbf{N}_{\text {req }}=5$

It is important to note that these solutions represent only a few "good" but "different" designs for review by the designer. The designer may either select one of these solutions as the final design or use one as a starting point for further exploration. Even though the issues such as the location of supports under lumped masses, frequencies, and the anchor forces and moments are explicitly quantified in Tables 4.1-2, 4.1-3, and 4.1-4 and Figures 4.1-9, 4.1-10, and 4.1-11, establishing the relative quality of various solutions requires the use of professional judgment and experience on part of the designer. For example, a designer may prefer to place a support directly under a lumped mass that represents an active equipment such as a motor even if its active weight is not as large as that of other passive equipment such as valves. Similarly, a designer may wish either to modify a piping design for reducing excessively high anchor loads (forces and anchor moments) transferred to the attached equipment or improve the stability of the equipment. 

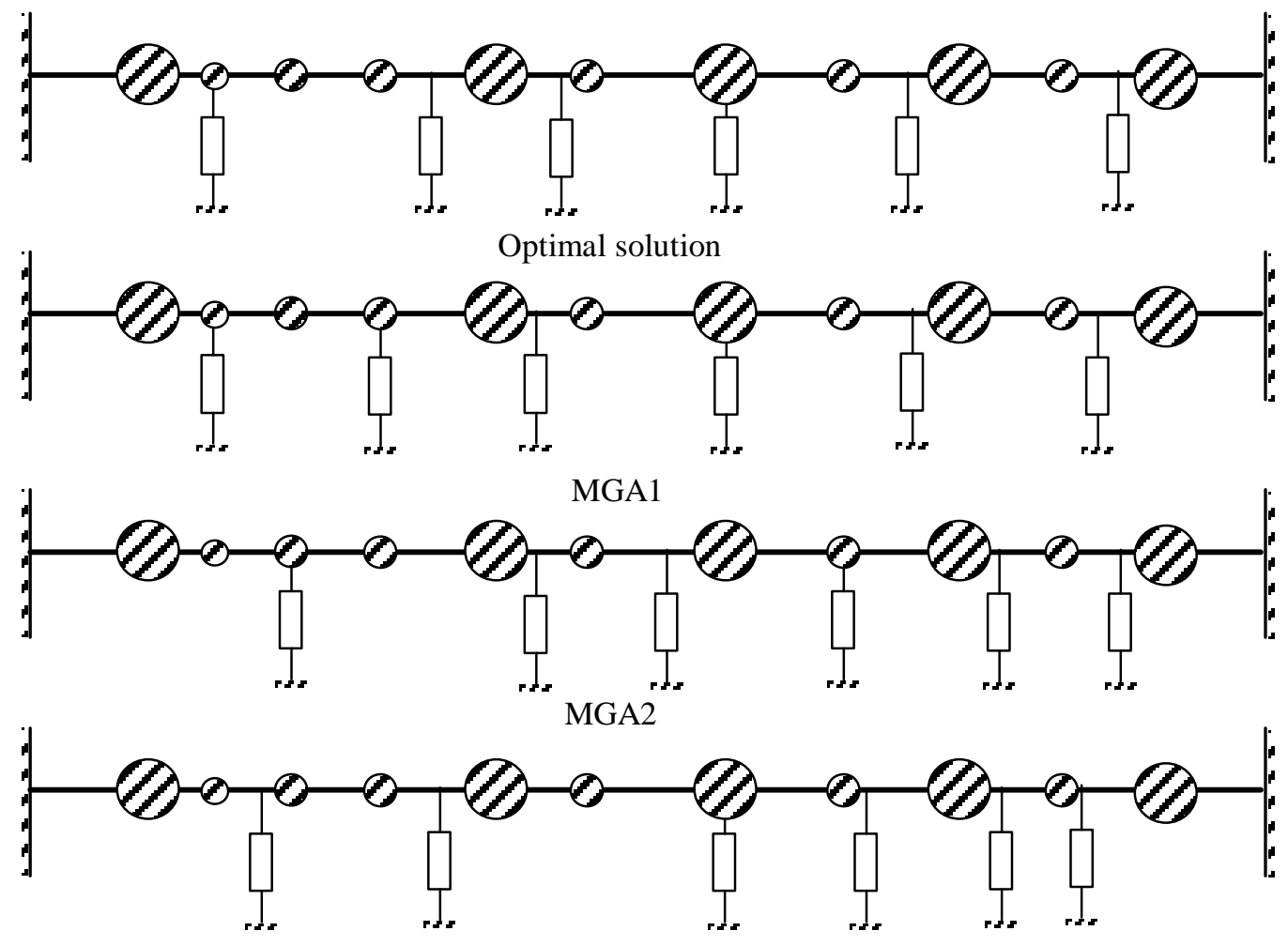

MGA3

Figure 4.1-10: MGA Solutions For Lifetime Cost With $\mathrm{N}_{\text {req }}=6$

For the purpose of illustration, let us examine the support arrangement and response characteristics of the alternatives generated for $n_{\text {req }}=5$, which are shown in Figure 4.1-9 and Table 4.1-3, respectively. It is observed that none of the lumped masses are supported in the optimal solution for lifetime cost (corresponding to $n_{r e q}=5$ ). The first alternative MGAl may appear to be better than the optimal solution as many more lumped masses are supported. On the other hand, it should be noted that the maximum stress, and the forces and moments at the second anchor for this alternative are much higher than the optimal solution. The second alternative $M G A 2$ has lower anchor forces and anchor moments than the optimal solution but none of the lumped masses are supported. The third alternative $M G A 3$ has more lumped masses supported than the optimal solution but fewer than that in $M G A 1$. Further, the anchor forces and anchor moments are only slightly higher than those of the optimal solution. Hence $M G A 3$ may serve as a good compromise. Moreover, it should be noted that the lumped masses that are supported in MGA1 and MGA3 are not the same. The designer may use judgment to evaluate the relative importance of supporting different lumped masses. Finally, the designer can "tinker" with an alternative generated by MGA to arrive at a final design. 

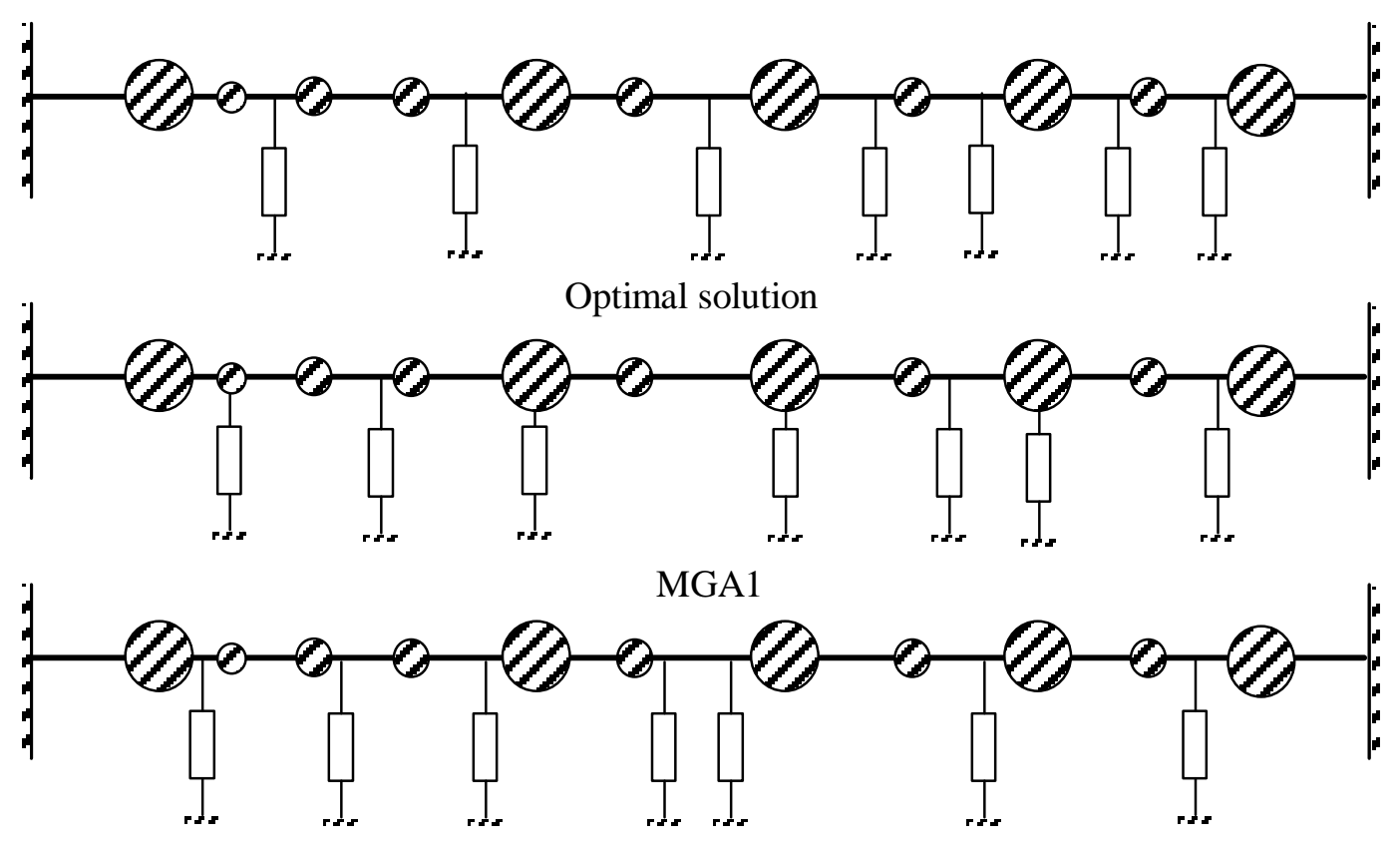

MGA2

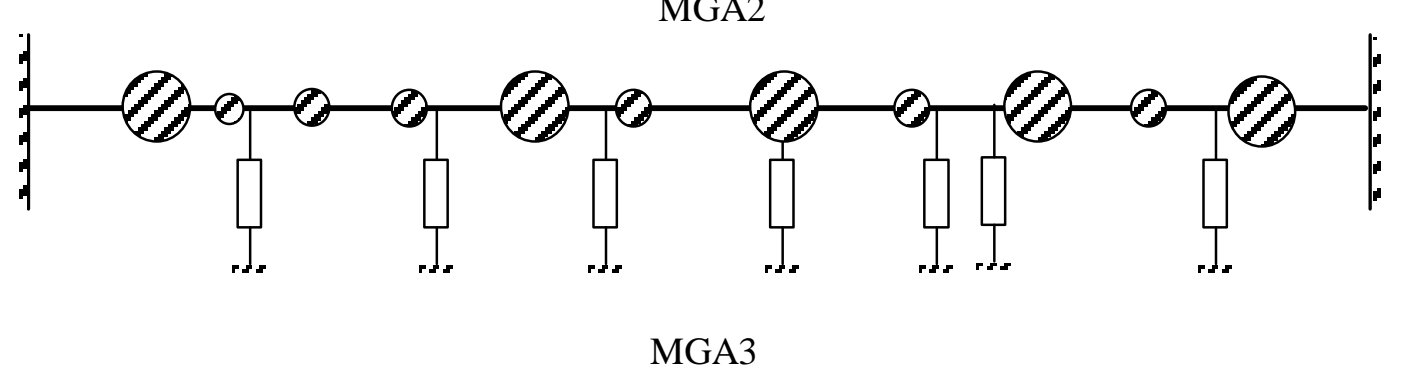

Figure 4.1-11: MGA Solutions For Lifetime Cost With $\mathrm{N}_{\text {req }}=7$

Table 4.1-2: Properties Of Mga Solutions For $\mathrm{N}_{\text {req }}=5$

\begin{tabular}{l|c|c|c|c|c|c|c|c}
\hline \hline Solution & \multirow{2}{*}{$\begin{array}{c}\text { Cost } \\
\end{array}$} & $\left(\frac{\theta_{k}}{\theta_{k c}}\right)_{\max }$ & \multirow{2}{*}{$\begin{array}{c}\text { Frequency } \\
\left(\times 10^{4}\right)\end{array}$} & & $\left(\frac{\sigma_{i}}{\sigma_{\text {all }}}\right)_{\max }$ & \multicolumn{2}{|c|}{ Anchor 1 } & \multicolumn{2}{|c}{ Anchor 2 } \\
\cline { 5 - 9 } & & & & & $F_{y}$ & $M_{2}$ & $F_{y}$ & $M_{z}$ \\
$(\mathrm{kN})$ & $(\mathrm{kNm})$ & $(\mathrm{kN})$ & $(\mathrm{kNm})$ \\
\hline Optimal & 127.0 & 0.95 & 11.0 & 0.24 & 101 & 185 & 139 & 214 \\
MGA1 & 149.7 & 0.99 & 10.0 & 0.55 & 87 & 147 & 237 & 478 \\
MGA2 & 153.1 & 0.96 & 9.7 & 0.19 & 73 & 107 & 121 & 165 \\
MGA3 & 140.0 & 0.99 & 10.5 & 0.34 & 57 & 81 & 151 & 296 \\
\hline \hline
\end{tabular}


Table 4.1-3: Properties Of MGA Solutions For $\mathrm{N}_{\mathrm{req}}=6$

\begin{tabular}{l|c|c|c|c|c|c|c|c}
\hline \hline Solution & \multirow{2}{*}{$\begin{array}{c}\text { Cost } \\
\end{array}$} & $\left(\times 10^{4}\right)$ & $\left(\frac{\theta_{k}}{\theta_{k c}}\right)_{\max }$ & \multirow{2}{*}{$\begin{array}{c}\text { Frequency } \\
(\mathrm{Hz})\end{array}$} & $\left(\frac{\sigma_{i}}{\sigma_{\text {all }}}\right)_{\max }$ & \multicolumn{2}{|c|}{ Anchor 1 } & \multicolumn{2}{|c}{ Anchor 2 } \\
\cline { 5 - 9 } & & & & $F_{y}$ & $\begin{array}{c}M_{z} \\
(\mathrm{kN})\end{array}$ & $\begin{array}{c}F_{y} \\
(\mathrm{kNm})\end{array}$ & $\begin{array}{c}M_{z} \\
(\mathrm{kN})\end{array}$ \\
$(\mathrm{kNm})$
\end{tabular}

Table 4.1-4: Properties Of MGA Solutions For $\mathrm{N}_{\text {req }}=7$

\begin{tabular}{l|c|c|c|c|c|c|c|c}
\hline \hline Solution & \multirow{2}{*}{$\begin{array}{c}\text { Cost } \\
\left(\times 10^{4}\right)\end{array}$} & $\left(\frac{\theta_{k}}{\theta_{k c}}\right)_{\max }$ & $\begin{array}{c}\text { Frequency } \\
(\mathrm{Hz})\end{array}$ & $\left(\frac{\sigma_{i}}{\sigma_{a l l}}\right)_{\max }$ & \multicolumn{2}{|c|}{ Anchor 1 } & \multicolumn{2}{|c}{ Anchor 2 } \\
\cline { 5 - 9 } & & & & $\begin{array}{c}F_{y} \\
(\mathrm{kN})\end{array}$ & $\begin{array}{c}M_{z} \\
(\mathrm{kNm})\end{array}$ & $\begin{array}{c}F_{y} \\
(\mathrm{kN})\end{array}$ & $\begin{array}{c}M_{z} \\
(\mathrm{kNm})\end{array}$ \\
\hline Optimal & 135.5 & 0.99 & 11.7 & 0.26 & 123 & 225 & 61 & 73 \\
MGA1 & 163.0 & 0.98 & 18.3 & 0.09 & 50 & 71 & 53 & 54 \\
MGA2 & 163.9 & 0.99 & 15.0 & 0.10 & 42 & 51 & 52 & 67 \\
MGA3 & 153.8 & 0.99 & 13.9 & 0.19 & 97 & 163 & 66 & 79 \\
\hline \hline
\end{tabular}

\subsubsection{Application To Real Life Piping System}

Application of the proposed GA technique to an actual nuclear power plant piping is illustrated in this section. We consider a feed-water piping shown in Figure 4.1-12. It consists of a $32.4 \mathrm{~cm}$ (12.75 inch) diameter Schedule 100 pipe loop that is anchored to the reactor vessel at nodes 107 and 114. This loop is connected to an $45.7 \mathrm{~cm}$ (18 inch) diameter Schedule 120 pipe that is anchored at the bottom of the concrete shield wall of the power plant at node 100. The finite element model of the piping system has 75 nodes, i.e., 75 possible support locations and each location can have a maximum of three supports, one in each of the three orthogonal directions $x, y$ and $z$. The dynamic analysis is performed for an envelope of the floor response spectrum input shown in Figure 4.1-13.

The objective function given by Equation 4.1-1, the proposed crossover scheme, and tournament selection are used in the GA to generate the trade-off curve between the lifetime cost and the number of supports. It is observed that a feasible solution exists in which only one support is needed. However, it has a very low fundamental frequency thereby making it undesirable. Figure 4.1-14 shows a trade-off curve in which the number of supports varies between 10 and 19. The frequencies, given in Table 4.1-5 for various solutions, vary between 7 and $25 \mathrm{~Hz}$. Other solutions with higher or lower frequencies are not considered. Solutions that have very high frequencies (in the vicinity of $20 \mathrm{~Hz}$ ) are undesirable due to high frequency loads caused by active equipment such as the motors and 
pumps. It should be noted that a solution having its fundamental frequency between either 8 and $11 \mathrm{~Hz}$ or 5 and $7 \mathrm{~Hz}$ lies in a frequency region where the earthquake input varies significantly with small changes in the frequency. Consequently, the feasibility and the quality of a solution can be very sensitive to the changes in frequencies, especially in the context of uncertainties associated with modeling and analysis techniques. However, solutions that have frequencies in the range of $5-8 \mathrm{~Hz}$ will not experience this sensitivity since the earthquake input is the same in this frequency region. Therefore, we generate another trade-off curve in which the solutions with frequencies below $5 \mathrm{~Hz}$ and above $8 \mathrm{~Hz}$ are not considered. This trade-off curve in which the number of supports vary between 10 and 19 is also shown in Figure 4.1-14 and the cost and frequencies of these solutions are given in Table4.1-6. Three of the solutions corresponding to $n_{\text {req }}=13,15$ and 17 supports are shown in Figures 4.1-15, 4.1-16, and 4.1-17 respectively.

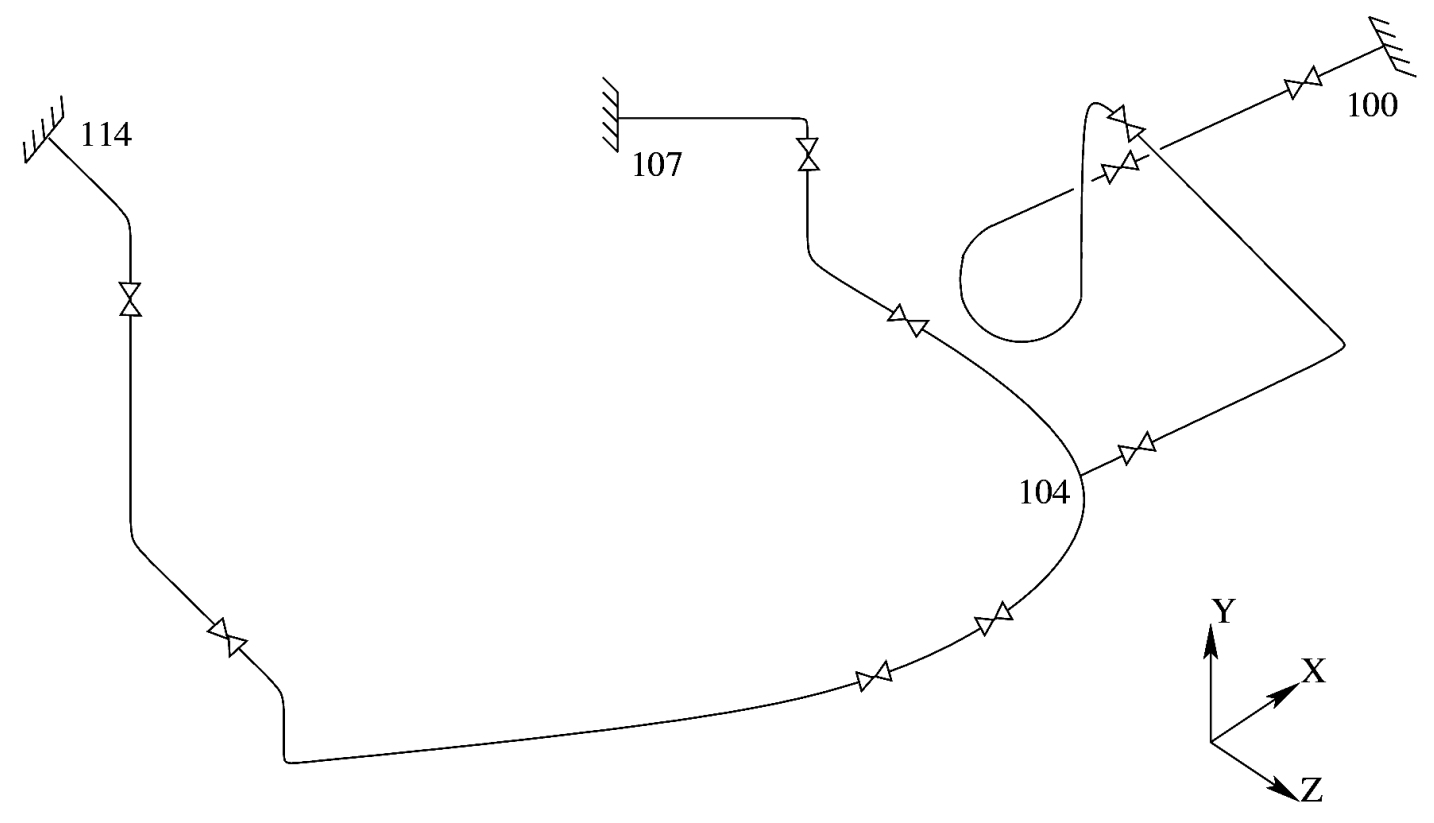

Figure 4.1-12: Real Piping System (Not To Scale) 


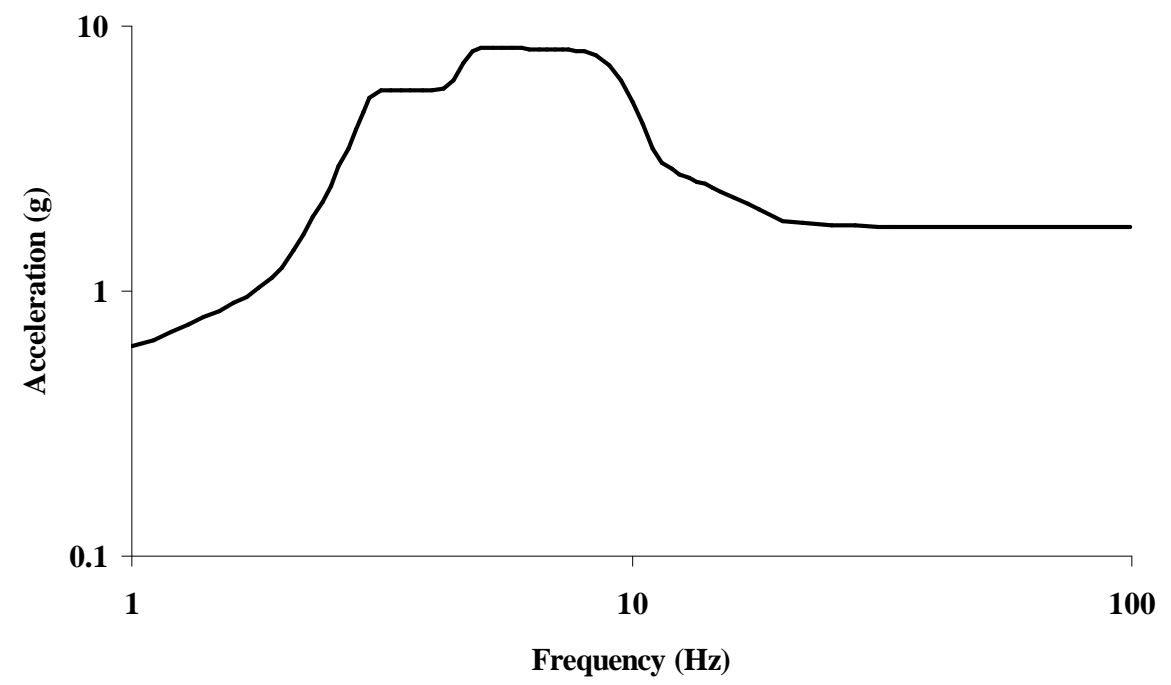

F Igure 4.1-13: Floor Response Spectrum For Real Piping System

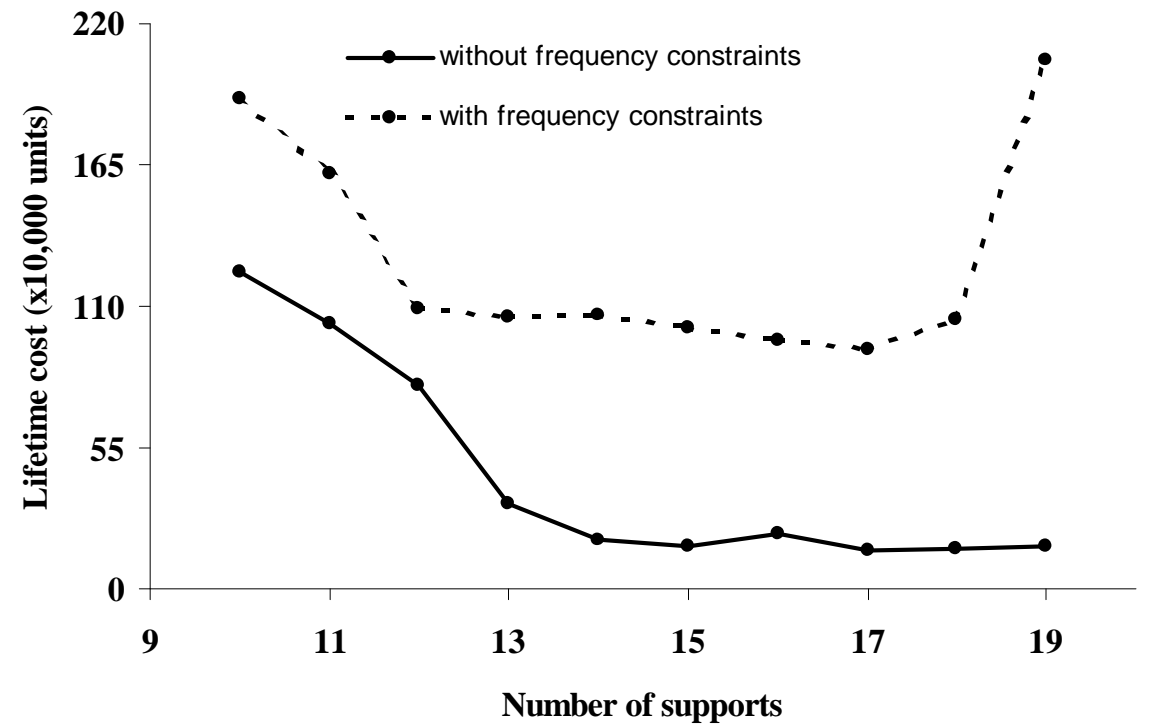

Figure 4.1-14: Trade-Off Curve Between Lifetime Cost And Number Of Supports, Real Life Piping 


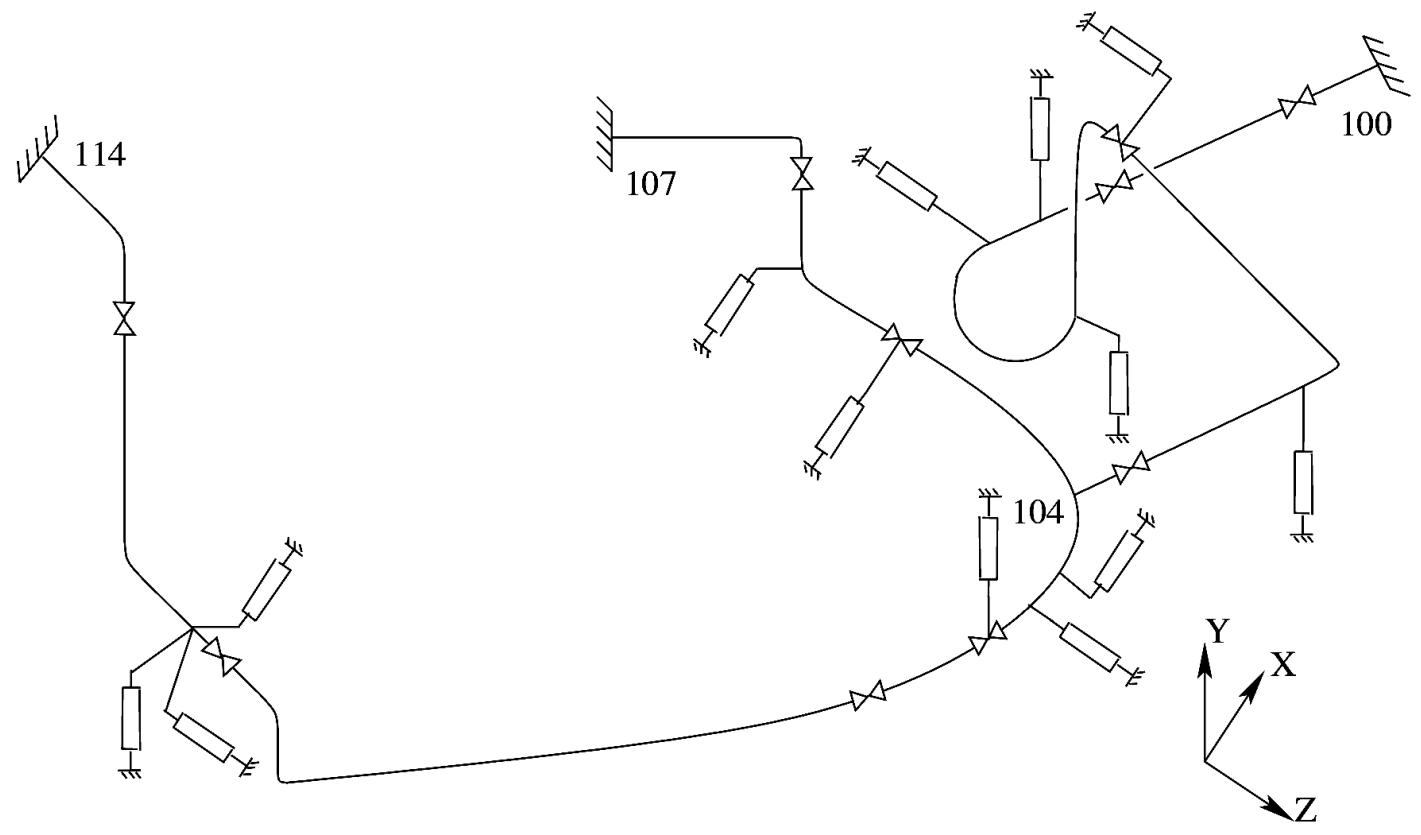

Figure 4.1-15: Optimal Solution For $\mathrm{N}_{\text {req }}=13$, Real Piping System

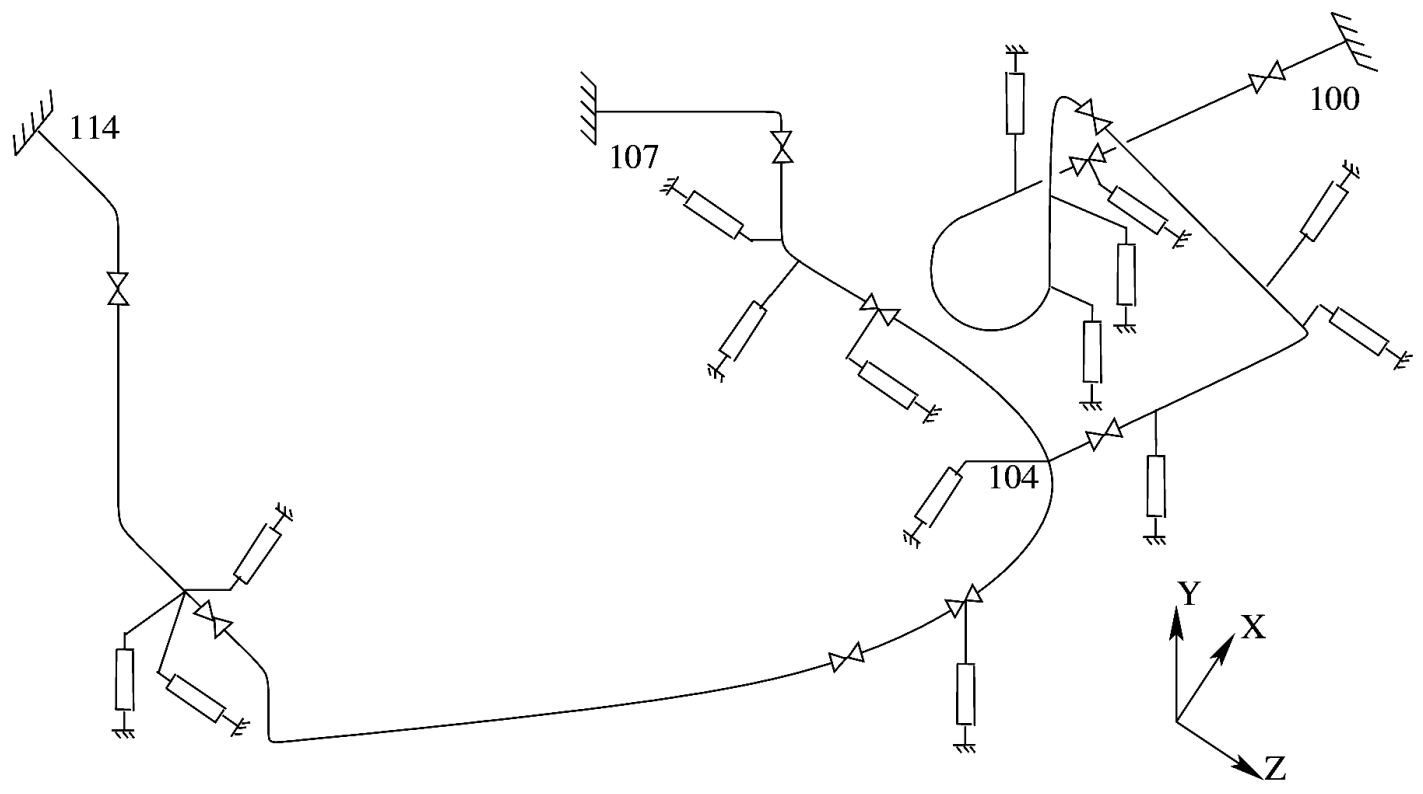

Figure 4.1-16: Optimal Solution For $\mathrm{N}_{\text {req }}=15$, Real Piping System 


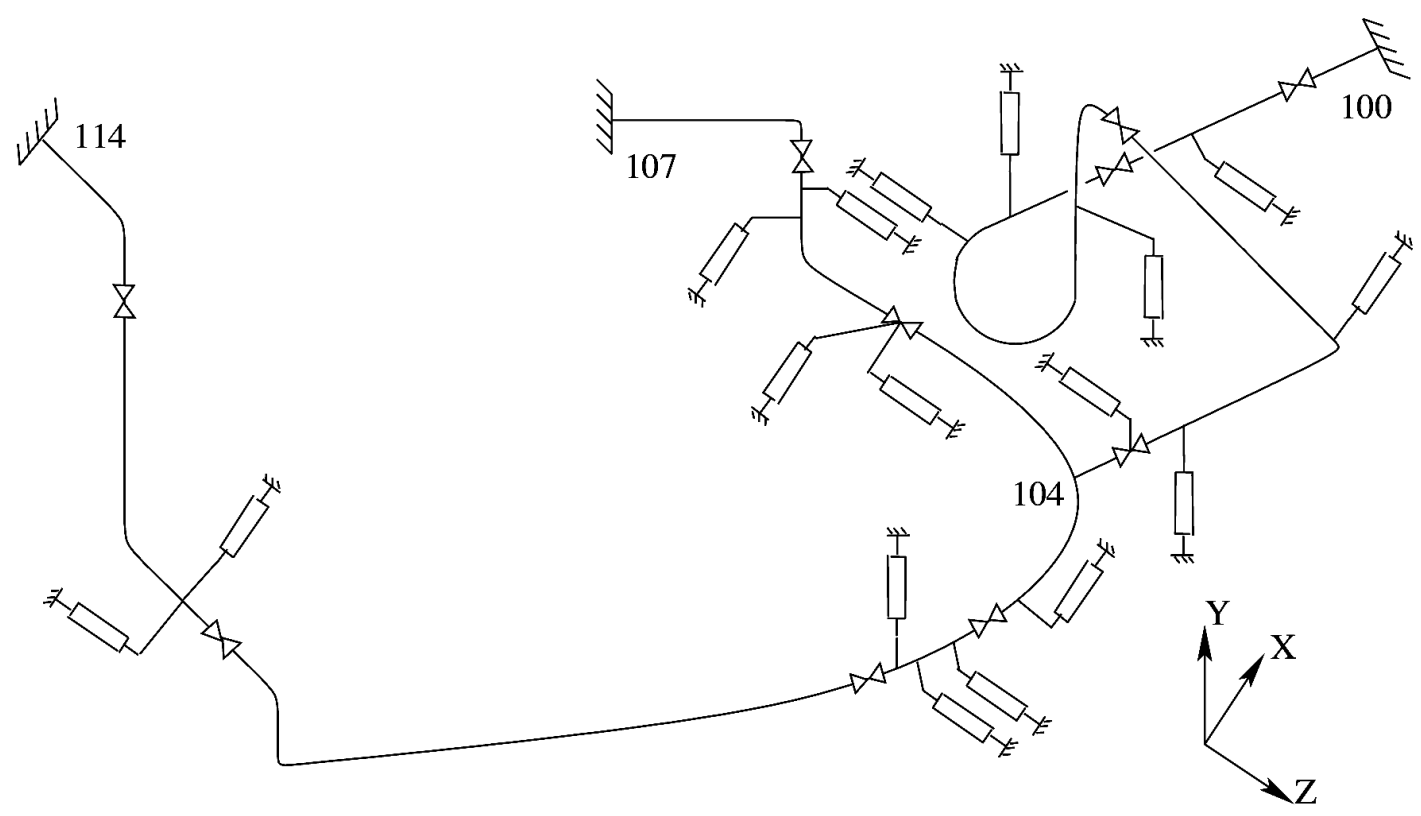

Figure 4.1-17: Optimal Solution For $N_{\text {req }}=17$, Real Piping System

Table 4.1-5: Cost And Frequency Of Optimal Solutions With No Frequency Constraint In Objective Function

\begin{tabular}{c|c|c}
\hline \hline$n_{\text {req }}$ & $\begin{array}{c}\text { Cost } \\
\left(\times 10^{4}\right)\end{array}$ & $\begin{array}{c}\text { Frequency } \\
(\mathrm{Hz})\end{array}$ \\
\hline 10 & 123 & 8.2 \\
11 & 103 & 9.1 \\
12 & 79 & 13.5 \\
13 & 33 & 12.4 \\
14 & 19 & 19.4 \\
15 & 17 & 23.6 \\
16 & 22 & 22.4 \\
17 & 15 & 21.0 \\
18 & 16 & 20.1 \\
19 & 17 & 22.3 \\
\hline \hline
\end{tabular}


Table 4.1-6: Cost And Frequency Of Optimal Solutions With Frequency Constraint In Objective Function

\begin{tabular}{c|c|c}
\hline \hline$n_{\text {req }}$ & $\begin{array}{c}\text { Cost } \\
\left(\times 10^{4}\right)\end{array}$ & $\begin{array}{c}\text { Frequency } \\
(\mathrm{Hz})\end{array}$ \\
\hline 10 & 191 & 7.4 \\
11 & 162 & 7.4 \\
12 & 109 & 7.7 \\
13 & 106 & 7.9 \\
14 & 107 & 7.9 \\
15 & 102 & 7.9 \\
16 & 97 & 7.9 \\
17 & 93 & 7.8 \\
18 & 105 & 7.9 \\
19 & 206 & 7.7 \\
\hline \hline
\end{tabular}




\subsection{BAYESIAN BELIEF NETWORK (BBN)-BASED ADVISORY SYSTEM DEVELOPMENT FOR STEAM GENERATOR REPLACEMENT PROJECT MANAGEMENT}

The growing need for improved project management technique points to the usefulness of a knowledge-base advisory system to help project managers understand current and future project status and optimize decisions based upon the project performances. The work here demonstrates the framework of an advisory system with improved ability in project management. Based upon the literature survey and discussion with relevant experts, the Bayesian Belief Network (BBN) approach was selected to model the steam generator replacement project management problem, where the situation holds inherently large uncertainty and complexities, since it has a superior ability to treat complexities, uncertainty management, systematic decision making, inference mechanism, knowledge representation and model modification for newly acquired knowledge.

Two modes of advisory system have been constructed. As the first mode, the predictive mode has been developed, which can predict future project performance state probability distributions, assuming no intervening management action. The second mode is the advisory mode, which can identify the optimal action among alternatives based upon the expected net benefit values that are incorporating two important components: 1) expected immediate net benefits at post-action time, and 2) the expected long term benefit (or penalty) at scheduled project completion time. During the work, new indices for important variables have been newly developed for effective and efficient project status monitoring. With application of developed indices to the advisory system, the long term benefit (or penalty) found to be the most important factor in determining the optimal action by the project management during the decision making process and was confirmed by the domain experts. As a result, the effort has been focused on incorporating the long term benefit (or penalty) concept in order to provide more reliable and accurate advice to the project managers. In addition, in order to facilitate the communication between the BBN models and the users, an interface program has been developed using the Visual Basic language.

\subsubsection{Advisory system updated from year 2001}

\subsubsection{Identification of two types of cost and benefits}

There are two types of costs and benefits that are occurring as the result of taking an action by management as shown in Figure 4.2.1-1: 1) immediate (or short-term) costs and benefits, and 2) long-term benefits (or penalty). There is certainly some immediate feedback concerning the cost of an action and some immediate mechanism in determining benefit (i.e., short term effect - post action). It is important to be able to determine immediate impact (i.e., expected immediate net benefit), which can be evaluated in terms of the shift up (or down) along the SPI curves (Earned Value Chart) and the post-action project performance state.

In addition to immediate costs and benefits, long-term benefits exist for a company such as gains from an under-spent project budget, an incentive from the project owner company, the company's improved reputation, and so on as a result of being ahead of the project schedule. However, in the case of delayed project completion, there is a cost penalty from the domino effect that originates from failure of timely achievement of deliverables, lost reputation, and so on. Those long-term benefit (or penalty) values are not considered in the 
immediate costs and benefits of an action taken. The project managers can think about the long-term benefit at any time of the decision making process during the project lifetime, but this long-term benefit is mainly concerned with the delay of project completion compared to the scheduled completion time. In other words, although the long-term benefit can be recognized at any time during project lifetime, the time it really occurs will be at the time of project completion. Also, the long-term benefit cannot be determined until scheduled project completion at which time the project completion status (i.e., whether or not the project is completed on time, within budget and performance limits) will be known. Example estimations of immediate costs and benefits and long-term benefit as functions of the SPI state at project completion time are illustrated in Figure 4.2.1-1.

\section{Figure 4.2.1-1. Time Scale Of Two Types Of Costs And Benefits}

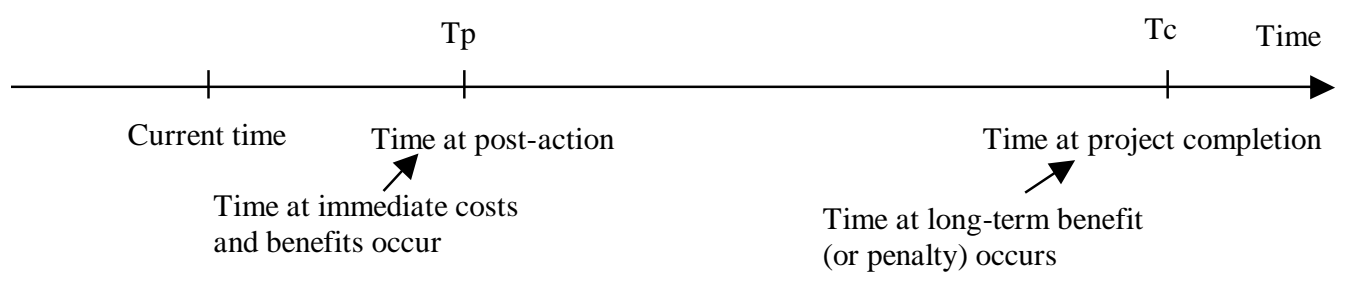

\section{Estimation of immediate costs and benefits}

Suppose, the project SPI is currently running at 0.9 (slightly behind schedule) as in equation (4.2.1-1). The project management then hires an additional person (added cost). It is therefore predicted that the project SPI will be 1.0 - Case 1 or 0.95 - Case 2 within a month. The immediate benefit for hiring an additional person is that the project is back on schedule (no slippage) and new hire needs also the immediate cost. 'No action - Case 0' results in an unchanged status with no immediate cost and no immediate benefit, which would not be good in the long-term (i.e., the time when the project ends) but quite good in the short-term range (i.e., within a few months).

Current SPI $=0.9=\mathrm{BCWP} / \mathrm{BCWS}=\$ 90,000 / \$ 100,000$

In this situation, a project manager takes the action 'Hire more people' with an estimated cost of $\$ 1,000$ and period of one month, bringing the SPI to 1.0.

Case 0

If project manger takes 'No action' (Base assumption)

1) Immediate cost of this action $=\$ 0$

2) Gross immediate benefit of this action $=\$ 0$

3) Net immediate benefit of this action $=$ Gross immediate benefit of this action Immediate Cost of this action $=\$ 0$

Case 1

After 1 month, SPI gets back to 1.0 
Then the immediate part of cost and benefit would be

1) Immediate Cost of this action $=\$ 1,000$

2) Gross immediate benefit of this action $=$ BCWS-BCWP $=\$ 100,000-\$ 90,000=\$ 10,000$

3) Net immediate benefit of this action $=\$ 10,000-\$ 1,000=\$ 9,000$

Case 2

After 1 month, SPI gets back to 0.95

Then the immediate part of cost and benefit would be

1) Immediate Cost of this action $=\$ 1,000$

2) Gross immediate benefit of this action $=0.95 * \mathrm{BCWS}-\mathrm{BCWP}=0.95 * \$ 100,000-$ $\$ 90,000=\$ 5,000$

3) Net immediate benefit of this action $=\$ 5,000-\$ 1,000=\$ 4,000$

\section{Estimation of long-term benefit (or penalty)}

It is possible to estimate the long-term benefit values if we assume to know the project performance state at the scheduled project completion time. For example, suppose that the project performance curve (i.e., SPI) shows a value of 0.85 at the scheduled project completion time, and then the project management has to spend more money to complete the project with extended (or rescheduled) project lifetime. The long-term penalty under this situation would include additional labor cost, additional material cost, lost reputation of company, and so on. In addition, the long-term penalty should include the interest and/or penalty due to delay if written as such in the contract. In summary, long-term penalty means the total amount of additional costs to the company in order to complete the project, contractual penalties and the company's lost reputation due to delay of project completion. While the other elements of additional cost are clear and tangible, the reputation loss may have ambiguous and intangible meaning. Therefore, further discussion about the meaning of company's reputation follows.

If the contracted company fails to complete the project on time, within budget and performance limits, it might result in serious damage on the company's image (i.e., company's reputation), hence customer and stockholders dissatisfaction. Then what is the meaning of reputation in the context? A brief definition of reputation can be found in Milewicz J. and Herbig P.'s paper; "Reputation is the estimation of the consistency over time of an attribute of an entity". In other words, reputation is an aggregation of all previous transactions of the company over its lifetime up to the present and requires consistency of the company's actions over a prolonged time for its formation. The main contributors to reputation are the company's historic performance quality (work performed or products manufactured), credibility (whether the company can be relied upon and do what it says it will do) and integrity (the honesty of its board of directions and management staff), as perceived by potential customers. Reputation is one of the most important factors on the company's future business. For example, in the case of governmental contracting, the company faces a single customer and failure to perform successfully can be catastrophic in terms of obtaining further contracts with the government. Therefore, the term 'reputation value' can be interpreted in two ways: 1) the premium that a customer is willing to pay more because the company has higher quality, credibility and integrity compared to its competitors and 2) the future revenue due to the increased chance of more business because 
of the company's better image of success. Reputation loss, which is intangible, may be the largest component in the penalty suffered by a contracted company, which in most cases is due to delay in project completion.

Suppose, instead, that the SPI of the scheduled project completion time shows value of 1.1. Then some benefits to the company are gains from under-spent project budget, incentives from project owner company, improved reputation, and so on. In this case, the long-term benefit can be positive number (i.e., not penalty). The relationships for a long-term benefit (or penalty) value estimation are illustrated below in equations (4.2.1-2) and (4.2.1-3).

Case 1. SPI at scheduled project completion time is less than 1.0

Total long-term penalty value $=$ Tangible penalty + Intangible penalty

Tangible penalty $=$ Additional labor cost + Additional material cost + Penalty in contract Intangible penalty $=$ Company's reputation loss.

Case 2. SPI at scheduled project completion time is 1.0 or greater than 1.0

Total long-term benefit value $=$ Tangible benefit + Intangible benefit

Tangible benefit $=$ Gains from under-spent project budget + Incentive from project owner company

Intangible benefit $=$ Improved company's reputation.

Once the SPI at scheduled project completion time and the components in above the equations are determined, then the long-term benefit values can be estimated as seen in Table 4.2.1-1.

Table 4.2.1-1. Example Long-Term Benefit (Or Penalty) As Function Of SPI State At Scheduled Project Completion Time

\begin{tabular}{|l|l|l|l|}
\hline SPI state at scheduled project completion time & $0.6 \sim 0.8$ & $0.8 \sim 1.0$ & $1.0 \sim 1.2$ \\
\hline Long-term benefit (or penalty) $(\mathrm{k} \$)$ & $\mathrm{X} 1$ & $\mathrm{X} 2$ & $\mathrm{X} 3$ \\
\hline
\end{tabular}

Note: X1 and X2 are likely to be negative (penalty) and X3 is positive (benefit)

\subsubsection{Updated Predictive Mode}

Due to the computational speed problem, new algorithm to enhance the speed has been developed as described in Figure 4.2.1-2. The main feature of the new algorithm is to repeat the one future-time prediction calculation with repeatedly updated evidence with one time-step model until it reaches the largest future time-step of interest. In the new algorithm, the 1-month future project performance predicted with current evidence is converted into new current evidence in the next time step and then, the 2-month future project performance can be calculated with new current evidence (i.e., 1-month future project performance). This process repeats until the future prediction reaches the largest time step defined by the user; for example, if the user defined the largest future time wanted as 10 months future then the prediction process is repeated ten times with the current evidence updated nine times. The 1-time step model does not need to store 12 different conditional probability sets and hence, it does not need a large size memory to store a large number of probability sets. As a result, the computational time is significantly enhanced with the help of a large size memory allocated for calculation, not for just storing data. For 
example, while it took $2 \sim 3$ minutes to compute one case of prediction with the previous 3time step model, only $3 \sim 5$ seconds are needed for the same case of prediction with the newly developed 1-time step algorithm. The advisory system in this work employs the newly developed 1-time step algorithm for predictive mode BBNs.

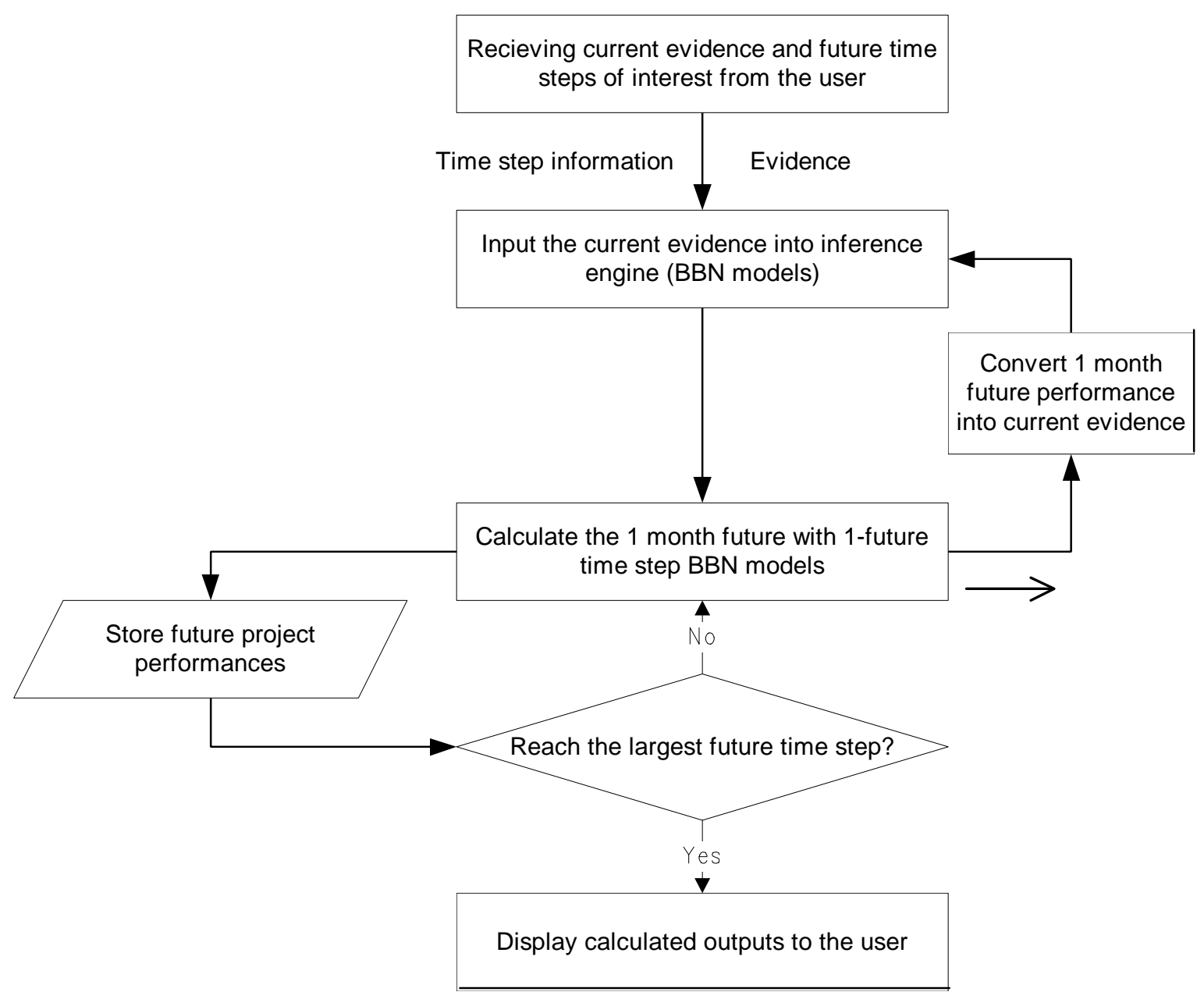

Figure 4.2.1-2. Calculation Algorithm With 1-Time Step Predictive Mode BBN

\subsubsection{Updated advisory mode}

From the preliminary analysis of sensitivity and discussion with domain experts, it is recognized that the long-term benefit plays the most important role in determining optimal action among alternatives. Therefore, for identifying optimal action more accurately and reliably, the long-term benefit should be incorporated into the cost-benefit analysis. As the first step to incorporate the long-term benefit, it should be noted that the times at which two different benefits would occur are not same as illustrated in Figure 4.2.1-1, where immediate cost and benefit occur at time of post-action and long-term benefit (or penalty) occurs at time of project completion. Therefore, it is proposed that the advisory mode of BBN should be developed to consider two different time values of expected immediate net benefits and expected long-term benefit in one mode. The advisory mode BBN developed for incorporating the long-term benefit into the expected net benefit analysis would follow 
the steps in the flowchart as shown in Figure 4.2.1-3. Detailed descriptions of each step can be found subsequent to that figure. It is believed that the optimal action identified based upon two expected net benefits can give more reliable and accurate advice to the project management than by only the expected immediate net benefit.

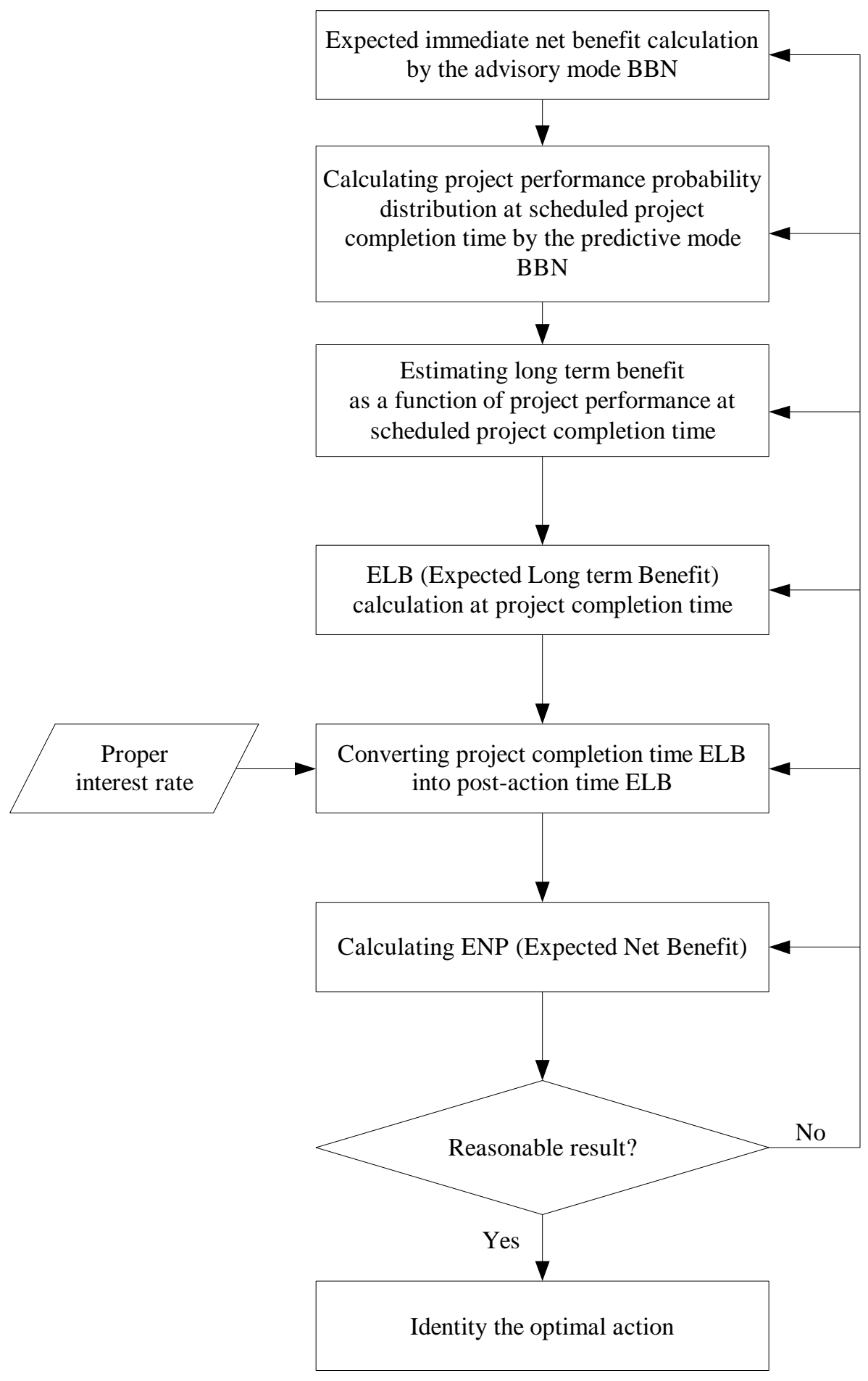

Figure 4.2.1-3. Flowchart For Newly Proposed Advisory Mode BBN 
Step1-Expected immediate net benefit calculation by the advisory mode BBN

The expected immediate net benefit at time of post-action can be calculated automatically using the BBN advisory mode with the immediate cost and benefit information of an action, which can be estimated from the SPI curve change from the pre-action and postaction states. Also the project performance states probability distributions after taking an action are calculated using the advisory mode BBN for SPI, CPI and the important input factors as well.

\section{Step2 - Project performance probability distribution predictions at scheduled project completion time by the predictive mode $\mathrm{BBN}$}

Based upon the identified post-action project performance states (i.e., using the post-action project performance state as current evidence into the predictive mode $\mathrm{BBN}$ ), the project performance probability distributions at scheduled project completion time can be predicted using the predictive mode BBNs.

\section{Step 3 - Estimating the long-term benefit}

With the results of the predictive mode BBNs from step 2 above (i.e., the project performance probability distributions at scheduled project completion time), the expected long-term benefit at project completion time can be estimated if the long-term benefit values as function of the SPI state at that time could be obtained. Estimating the long-term benefit is very difficult during the project work period, and nearly impossible in some cases, because the long-term benefit has so many factors and large uncertainties affecting it. The only time when the estimation of the long-term benefit is possible is at the time of scheduled project completion (i.e., the time when the final project performance is known). Therefore, it is believed that once the prediction values of the project performance status at scheduled completion time are known, then it is not so difficult to estimate the long-term benefit values as a function of the SPI state at scheduled project completion time as illustrated in Table 4.2.1-2 and Figure 4.2.1-4.

Table 4.2.1-2. Example Long-Term Benefit As Function Of SPI StateAt Scheduled Project Completion Time

\begin{tabular}{|c|l|l|l|}
\hline SPI state at project completion time & p.6 0.8 & $0.8 \sim 1.0$ & $1.0 \sim 1.2$ \\
\hline \hline Long-term benefit $(\mathrm{k} \$)$ & $\mathrm{X} 1$ & $\mathrm{X} 2$ & $\mathrm{X} 3$ \\
\hline
\end{tabular}




\section{Long term benefit distribution}

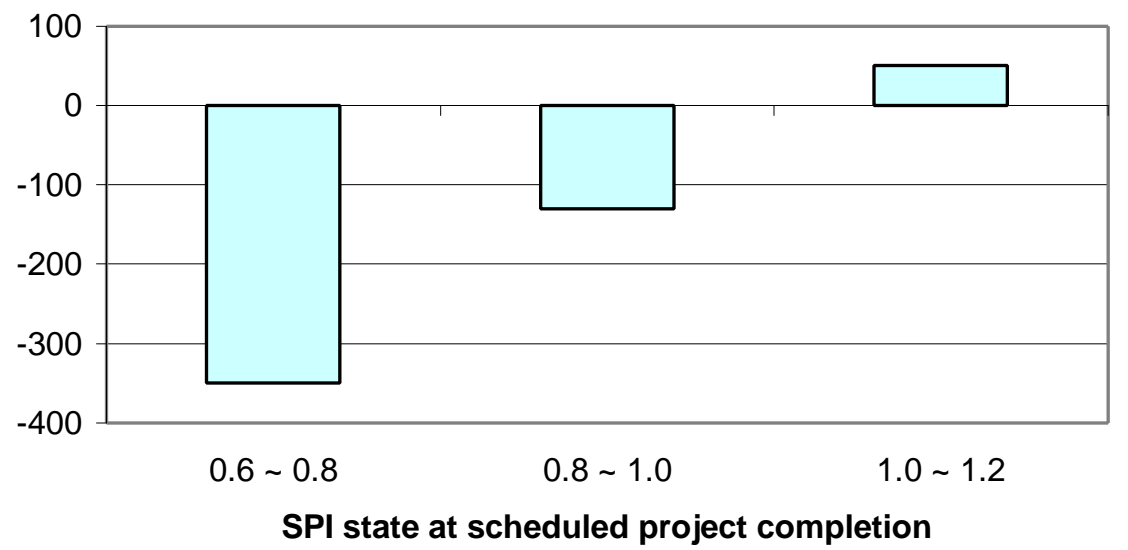

Figure 4.2.1-4. Example Long-Term Benefit As Function Of SPI At Scheduled Project Completion

\section{Step 4 - Calculation of ELB (Expected Long-term Benefit) at scheduled project completion time}

Once the long-term benefit values as a function of scheduled project completion time SPI states and the predictions of the SPI state probability distribution at scheduled project completion time are known, then the ELB (Expected Long-term Benefit (or Penalty)) can be calculated as illustrated in Figure 4.2.1-5 and Equation 4.2.1-4. It should be noted that the scheduled project completion time SPI state probability distribution (i.e., Pi in Figure 4.2.1-5) is automatically calculated by the predictive mode BBN, given the evidence of post-action states of actions, and the long-term benefit values as a function of scheduled project completion time SPI states (i.e., Xi in Figure 4.2.1-5) should be given by the users (or experts).

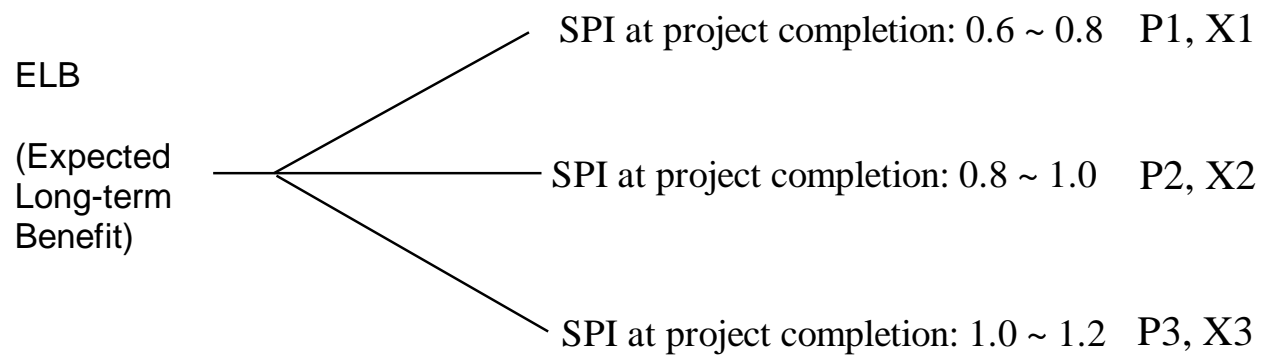

where

$\mathrm{Pi}$ : SPI state probability value of project performance prediction from the predictive mode BBNs

$\mathrm{Xi}$ : Long-term benefit value corresponding SPI state

Figure 4.2.1-5. Tree For Long-Term Benefit Calculation 
$E L B ? ?_{i ? 1}^{3} P_{i} ? X_{i} ? P_{1} ? X_{1} ? P_{2} ? X_{2} ? P_{3} ? X_{3}$

As mentioned in step 3, however, the long-term benefit values have large uncertainties. Therefore, the best way to include such large uncertainties in the analysis is to estimate the long-term benefit values as probability distributions either in the form of a continuous probability distribution (probability density function; pdf) or as discrete probability distribution (probability mass function; pmf). Probability distribution estimation would also reduce difficulties and errors in the long-term benefit values rather than using point values. Once the probability distributions for long-term benefit values are estimated, there would be three different probability distributions: three probability distributions for X1, X2 and $\mathrm{X} 3$ in Table 4.2.1-2 are shown in Figure 4.2.1-6 as example continuous probability distributions.
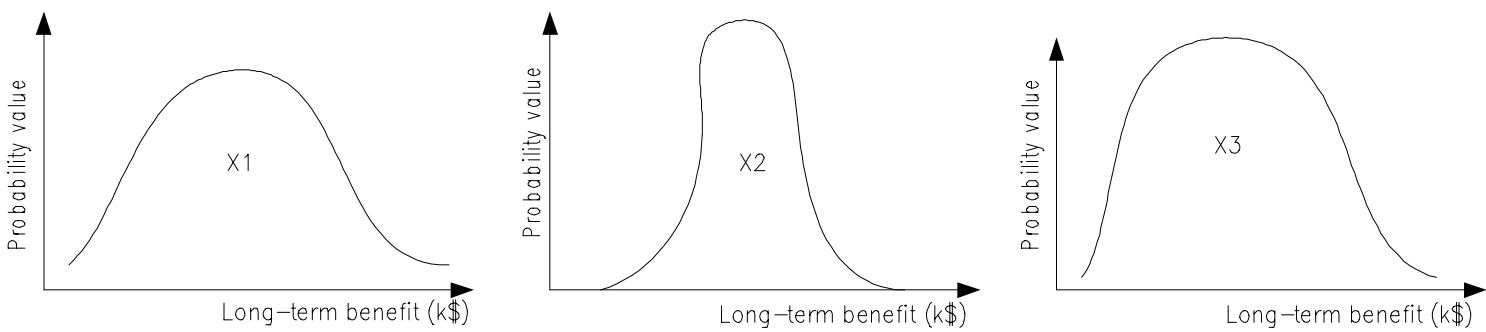

\section{Figure 4.2.1-6. Example Continuous Probability Distributions For Long-Term Benefit Values}

Then calculate the output probability distribution (i.e., the probability distribution for $E L B$ in Equation 4.2.1-4) as a function of the basic component random variables (i.e., X1, X2 and X3) since each of these three variables has its own probability distributions. However, the calculation of output probability distributions from the probability distributions of input component variables is not an easy process. Even though a general approach for this process has been developed, it is not very useful in practice and will not be presented here. For example, in the case of a linear combination of normal distribution variables, the output probability distribution can be computed theoretically. However, this is of very little use in practical problems.

Instead of the theoretical approach, the numerical approach is more widely used in practical areas. In physical problems, there are analytical expressions that are very complex and the input variables may follow any probability distributions. In many cases, there are no analytical expressions for the desired output, but rather, computer programs that can produce the output of interest. In these cases, the output probability distribution can be produced by a random sampling technique from input probability distributions. This approach is the numerical methods for output probability distribution production. One of the widely used numerical approaches is 'Monte Carlo Simulation'. Using this numerical approach on computer, the probability distribution of output variable is calculated without large difficulties in the case of continuous probability distributions. 
In this analysis, these probability distributions are estimated in the form of discrete functions (probability mass functions). The current version of BBN models cannot handle the continuous probability distributions. Therefore, in order to communicate with BBN models, the continuous probability distributions for long-term benefit values cannot be used. In addition, for a practical reason, three discrete probability distributions with three values in each distribution are estimated as illustrated in Figure 4.2.1-7. In the case of three probability distributions for input long-term benefit (i.e., one pmf for X1, one pmf for X2 and one pmf for X3) and three values in each probability distribution, there are 27 (=3?3?3) combination output values in the resultant probability distributions (i.e., 27 ELBs in Equation 4.2.1-4 with 27 corresponding probability values). If the number of values in each probability distribution increases, then the number of expected long-term benefit values in the output distributions would grow exponentially (e.g., $64=4$ ? 4? 4). This rapid growth will result in a big burden to computation time and hence, money.
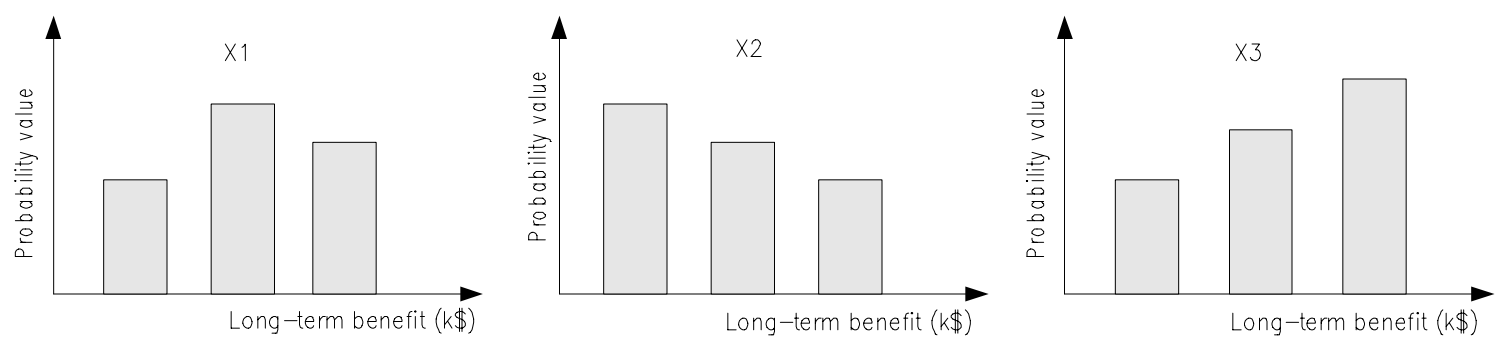

\section{Figure 4.2.1-7. Example Discrete Probability Distributions For Long-Term Benefit Values}

In order to compare among action alternatives, a single expected long-term benefit value for each action alternative (i.e., single value ELB like in Equation 4.2.1-4) is required. In the discrete case with 27 output values (i.e., 27 ELBs from Equation 4.2.1-1-4 with 27 corresponding probability values), the single value ELB can be estimated with following equation (4.2.1-5).

$E L B ? ?_{i ? 1}^{27} P_{i} ? E L B_{i} ? P_{1} ? E L B_{1} ? P_{2} ? E L B_{2} ? \quad ? P_{26} ? E L B_{26} ? P_{27}$ ? ELB $27 \cdot(4.2 .1-5)$

where

$E L B=$ Single value of Expected Long-term Benefit at scheduled project completion time

$E L B_{i}=27$ output Expected Long-term Benefits from equation (4.2.1-4)

$P_{i}=27$ probability values corresponding $E L B_{i}$

\section{Step 5 - Converting the ELB at scheduled project completion time into at post-action} time

At this step, the expected long-term benefit value at the scheduled project completion time should be modified into a value for post-action time (or vice versa), taking into account interest and inflation during the period since two benefit values are needed for different time points. The proper interest rate should be selected, in order to match the one used in 
that project scheduling frame, from two kinds of interest rates: 1) constant interest rate or 2) market interest rate. After choosing the interest rate, the expected long-term benefit at the scheduled project completion time can be converted into the one at another time using the expression in equation (4.2.1-6).

$$
E L B_{p} ? \frac{E L B}{(1 ? i)^{T_{c} ? T_{p}}}
$$

where

$E L B=$ Expected Long-term Benefit at scheduled project completion time

$E L B_{p}=$ Expected Long-term Benefit at post-action time

$i=$ Interest rate

$T c=$ Time at scheduled project completion

$T p=$ Time at post-action

\section{Step 6 - Calculation of Expected net benefit}

After converting the expected long-term benefit into post-action time, the expected net benefit can be calculated from two benefits: 1) expected immediate net benefit from step 1 and 2) expected long-term benefit from step 5. It should be noted that we could estimate the expected net benefit in two ways: 1) expected net benefit at post-action time and 2) expected net benefit at scheduled project completion time. Here, the post-action time is selected because project managers usually prefer to make timely decisions on the net benefit in the near future. The expected net benefit of an action can be calculated using equation (4.2.1-7).

$$
E N B ? E N B_{i} ? E L B_{p}
$$

where

$E N B=$ Expected Net Benefit

$E N B i=$ Immediate Expected Net Benefit from immediate cost and benefit

$E L B p=$ Expected Long-term Benefit at post-action time

\section{Step 7 - Identifying the optimal action}

After calculation of the expected net benefit value for each action alternative, the optimal action under the current situation can be identified through comparison of these expected net benefit values. Under the assumption of risk-neutral preference by the project manager, the optimal one is the action with the largest expected net benefit.

With this approach, both the expected net benefit from immediate cost and benefit and the long-term benefit are included in the analysis. The optimal action identified based upon this approach can give more reliable and accurate advice to the project manager rather than by only the expected immediate net benefit. 


\subsubsection{Simulation Results And Analysis}

\subsubsection{Case Studies With The Predictive Mode BBN}

\section{Case Description}

\section{Project: Nuclear Power Plant Case Study for the predictive mode BBN}

Date: 156 weeks after project starts, in the work package development work phase

The evidence at 156 weeks and of the overall project performance information during the period from 156 to 168 weeks is obtained from real steam generator replacement work. The current evidence of the work package development work phase and SPI and CPI histories for overall project performance is summarized in Table 4.2.2-1 and Figure 4.2.2-1, respectively. As illustrated in Table 4.2.2-1, there is no most difficult to implement group in this case study since the current evidence is for the work package development work phase. Based upon the current evidence, the future project performance predictions are computed by the predictive mode BBN and compared to the obtained real case data for benchmarking the prediction values.

Table 4.2.2-1. Evidence Of Project Performance At 156 Weeks

\begin{tabular}{|l||l|l|l|}
\hline & Most difficult group & Medium difficult group & Least difficult group \\
\hline \hline SPI & N/A & 0.995 & 1.000 \\
\hline CPI & N/A & 1.056 & 1.015 \\
\hline TPI (Time) & N/A & 0.987 & 1.000 \\
\hline CDI (Cost) & N/A & 1.107 & 1.081 \\
\hline SCPI (Scope) & N/A & 0.950 & 1.000 \\
\hline QPI (Quality) & N/A & 0.950 & 1.000 \\
\hline Client satisfaction & N/A & 1.050 & 1.000 \\
\hline
\end{tabular}




\section{Overall project SPI and CPI}

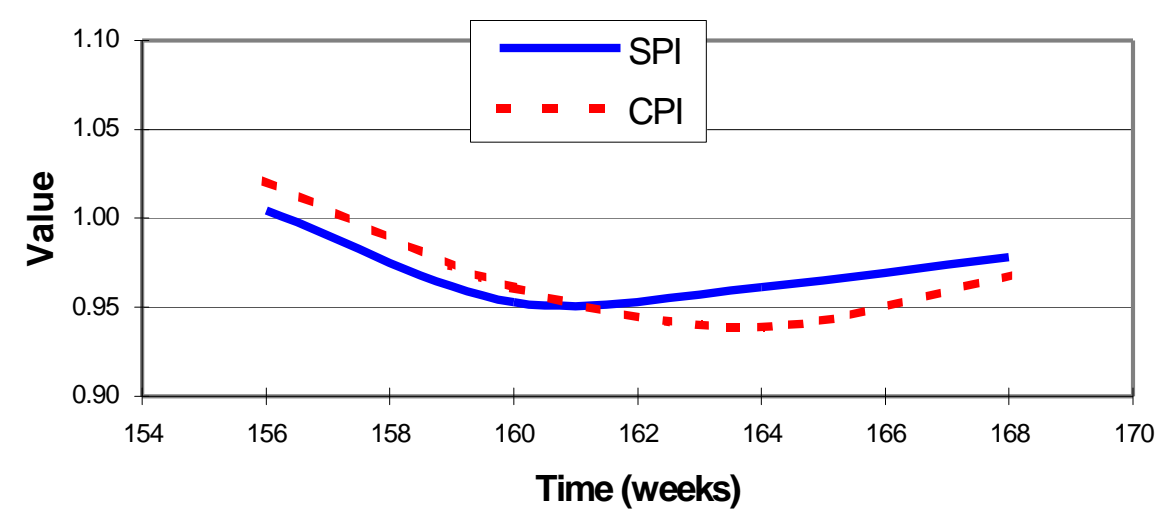

Figure 4.2.2-1. Overall project performances from 156 to 168 weeks

\section{Case Analysis Result With Predictive Mode BBN For Single Work Phase}

Figures 4.2.2-2 and 4.2.2-3 show the predicted overall project performance (SPI and CPI, respectively) probability distributions of 1, 2 and 3 future months based upon the current evidence described in Table 4.2.2-1. In order to benchmark the predictions by the BBN models, the mean values of each probability distribution are calculated and compared to the real case values. As a result, two curves (i.e., real case data vs. prediction value) show good agreement in both SPI and CPI cases as shown in Figures 4.2.2-4 and 4.2.2-5 and the domain experts confirm this agreement. As time goes on, however, the discrepancy between two curves increases as seen in Figure 4.2.2-4 and 4.2.2-5, because the two curves represent different scenarios: 1) the real case data represents the situation of intervening management action and 2) the prediction value is from the situation of no intervening management action.

\section{Future SPI prediction}

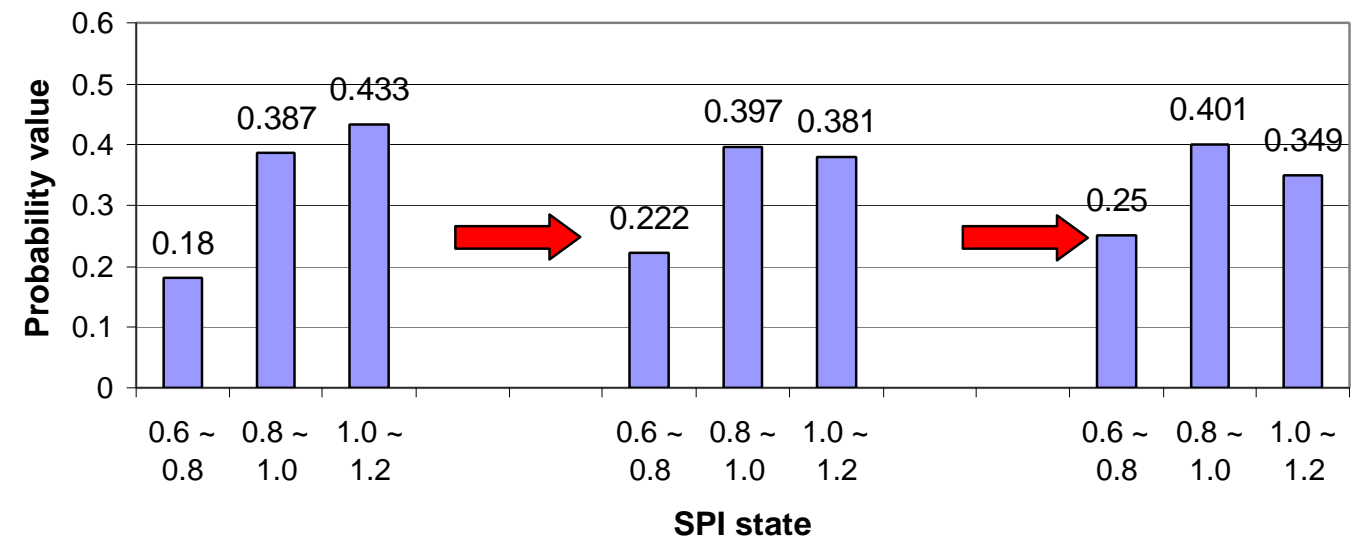

Figure 4.2.2-2. SPI Prediction Probability Distributions For Overall Project Of 1, 2 And 3 Months Future 


\section{Future CPI prediction}

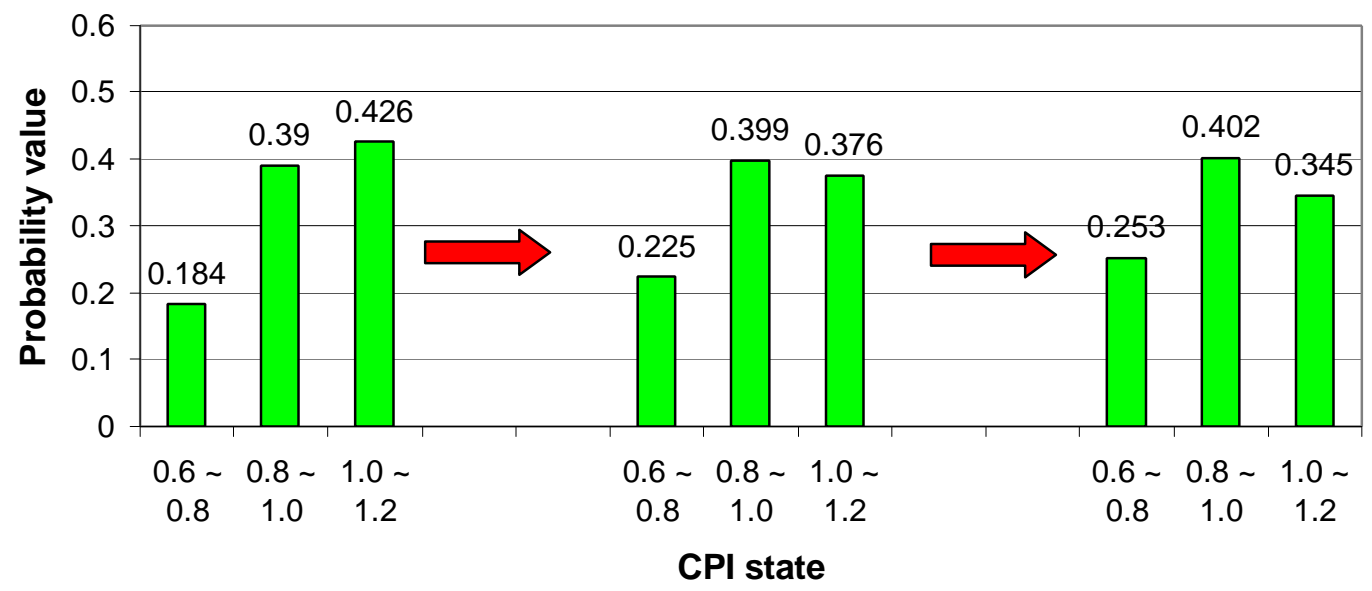

Figure 4.2.2-3. CPI Prediction Probability Distributions For Overall Project Of 1, 2 And 3 Months Future

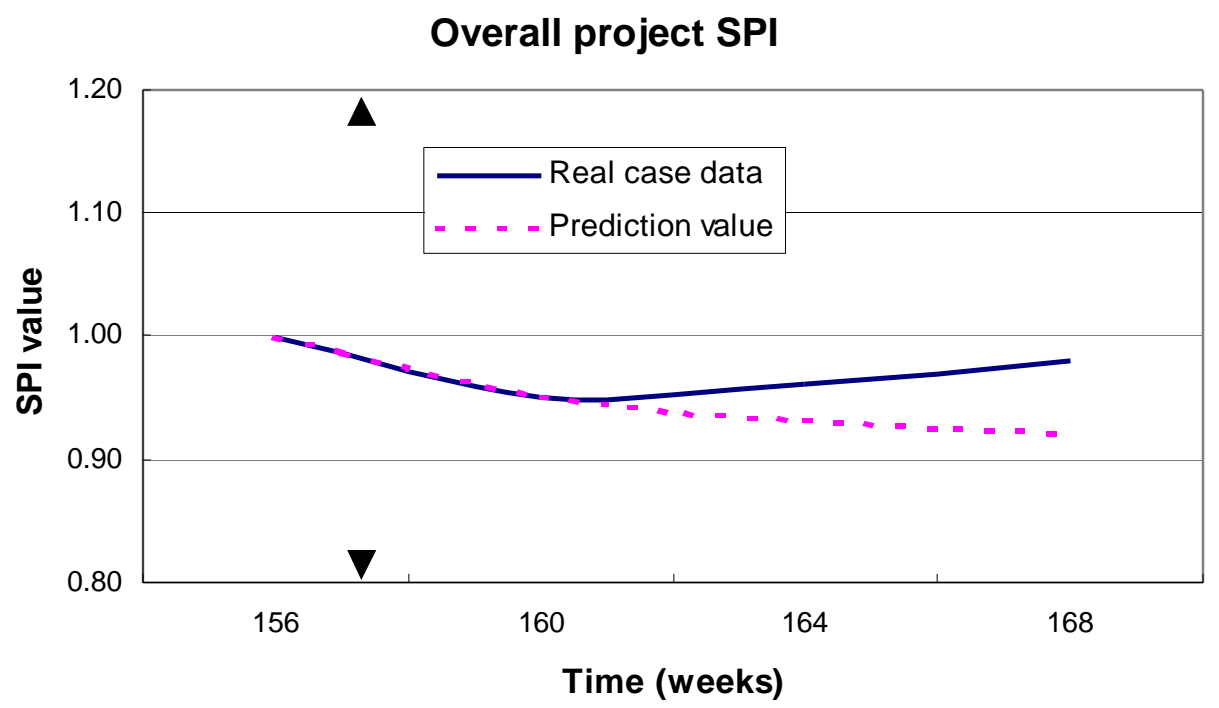

Figure 4.2.2-4. Overall Project SPI Real Case Data Vs. Prediction Value 


\section{Overall project CPI}

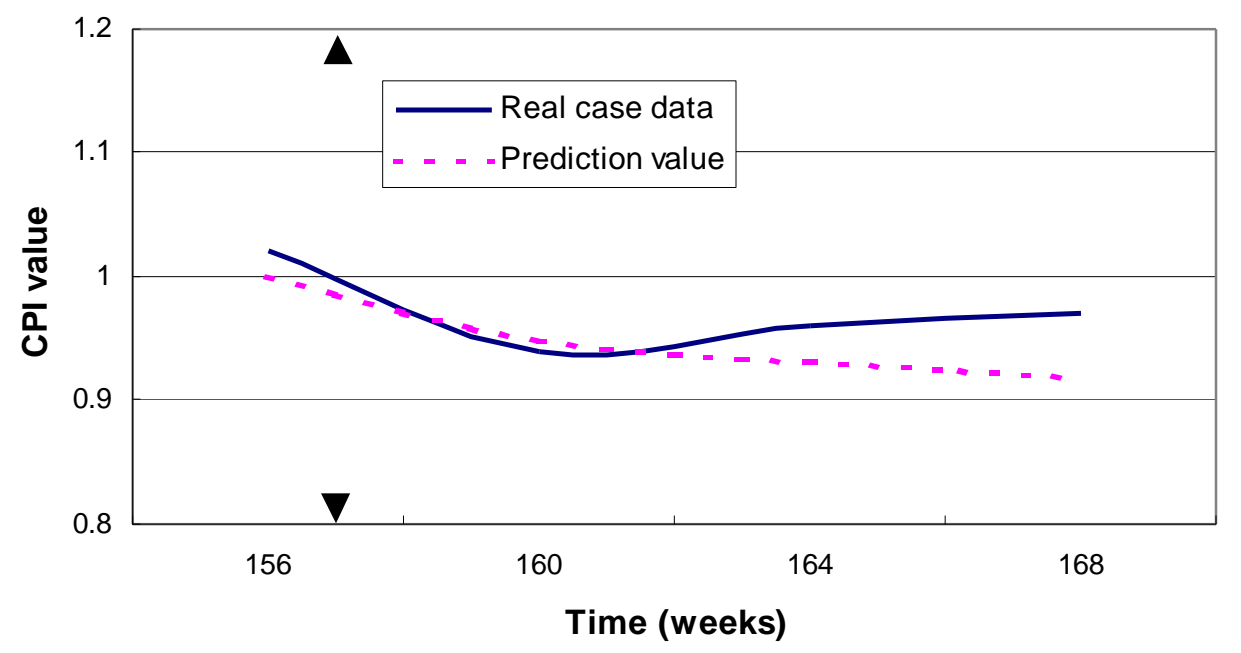

Figure 4.2.2-5. Overall Project CPI Real Case Data Vs. Prediction Value

The prediction probability distribution will eventually go to uniform since uncertainty grows with time. At that time of uniform probability distribution, the discrepancy between two curves will show the maximum value, which indicates the maximum uncertainty. This trend of increasing uncertainty with time is illustrated in Figures 4.2.2-6 for the best scenarios case and 4.2.2-7 for the worst scenario case in the work package development work phase. Both figures show the largest possibility in the medium SPI state even in 12 months future, except the short elapse after current time, since the domain experts believe in the largest likelihood in the medium project performance from their past experiences up to now.

Future overall SPI prediction with the best evidence (WPD phase)

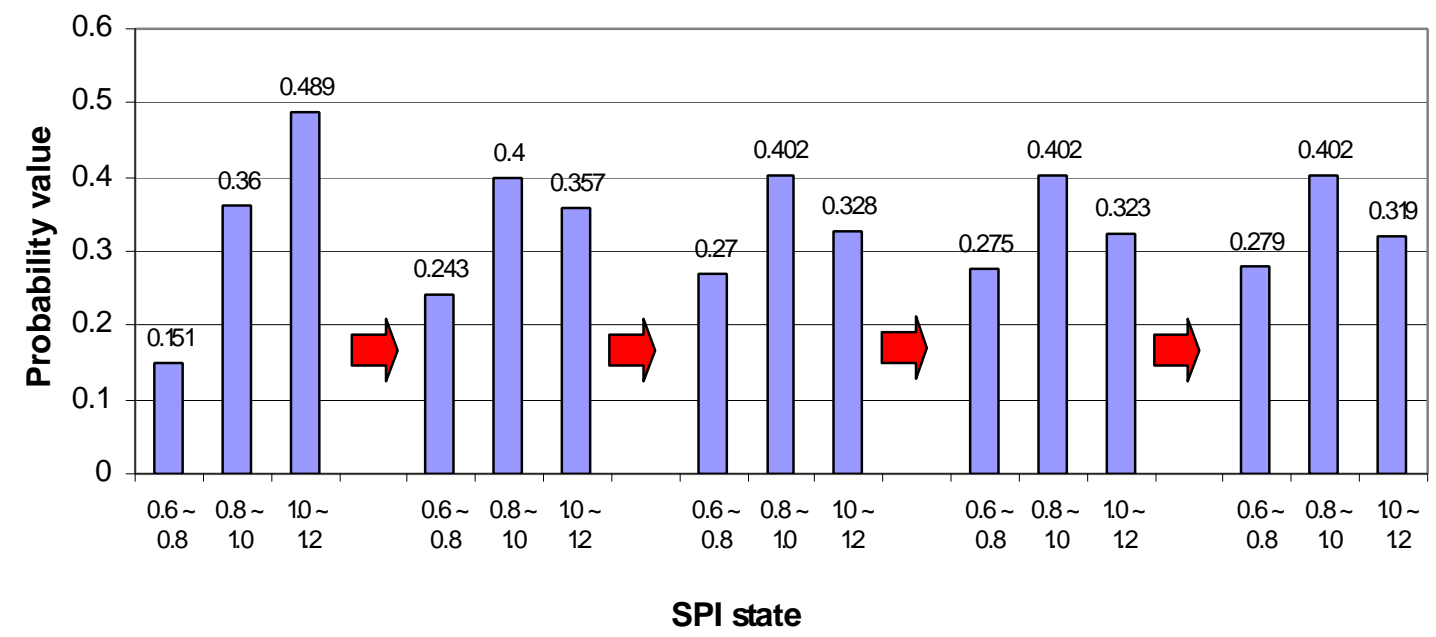

Figure 4.2.2-6. Increasing Uncertainty With Time For The Best Evidence Case 
Future overall SPI prediction with the worst evidence (WPD phase)

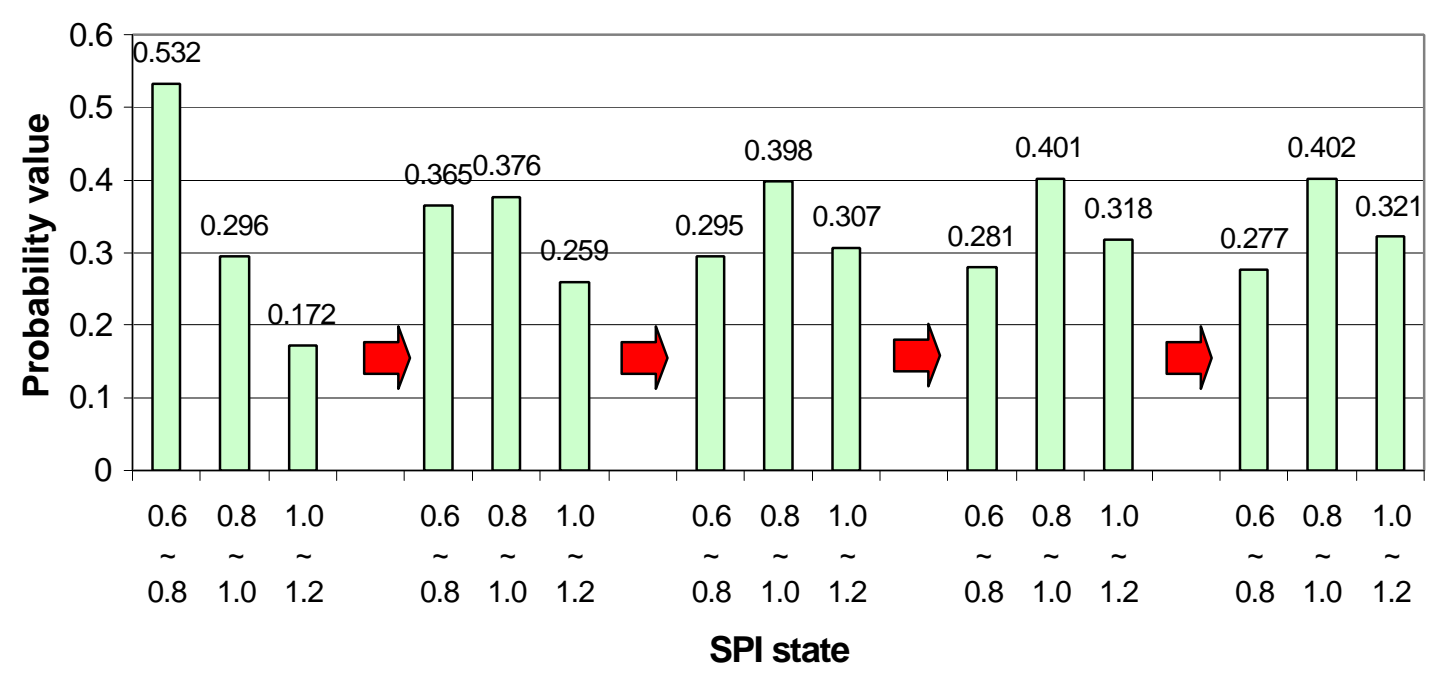

Figure 4.2.2-7. Increasing uncertainty with time for the worst evidence case

With Predictive Mode BBN For Project Lifetime

Figures 4.2.2-8, 4.2.2-9 and 4.2.2-10 show the SPI, CPI and profit probability distribution predictions at scheduled project completion time based upon the current evidence in Table 4.2.2-1. Because there was no real case information for this case, domain experts confirmed these results to be reasonable predictions.

\section{SPI prediction at scheduled project completion}

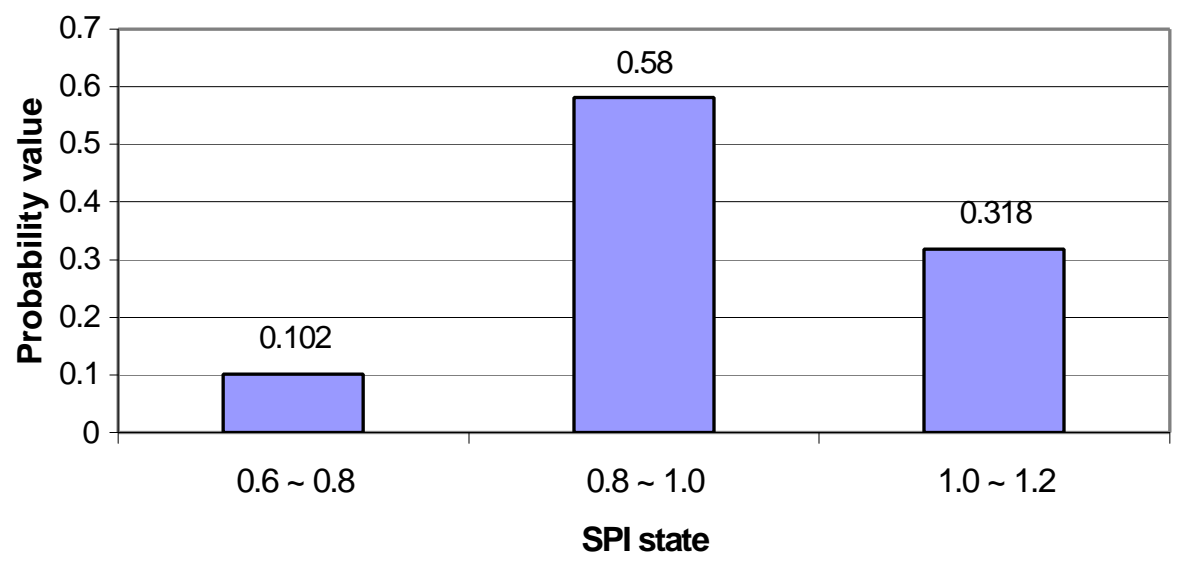

Figure 4.2.2-8. SPI Performance Prediction For Overall Project At Scheduled Completion Time 


\section{CPI prediction at scheduled project completion}

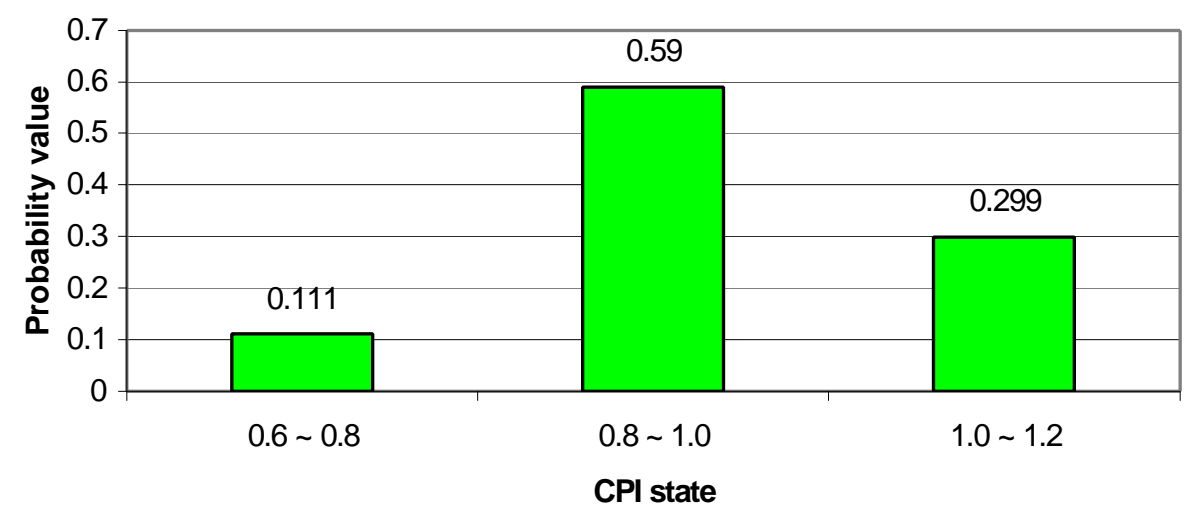

Figure 4.2.2-9. CPI Performance Prediction For Overall Project At Scheduled Completion Time

Profit prediction at scheduled project completion

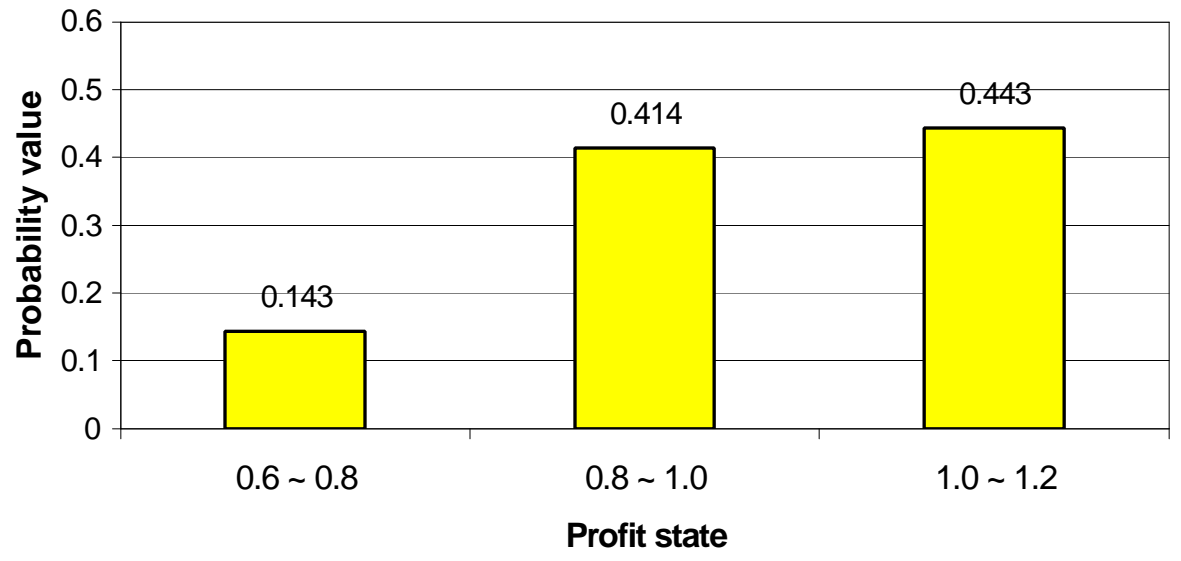

Figure 4.2.2-10. Profit Performance Prediction For Overall Project At Scheduled Completion Time

\subsubsection{Case study with the advisory mode BBN}

\section{Case Description}

\section{Project: Nuclear Power Plant Case Study for the advisory mode BBN}

Date: 149 weeks after project starts, in the work package development work phase

As seen in Figure 4.2.2-11, the overall project shows good performance in SPI and CPI at the time of interest (149 weeks). On a total project basis, the SPI and CPI curves are very good measures to judge project health, but those measures do not tell the status of a specific part (or parts) of the overall project. This case applies to a group of work packages, which 
show the worst case scenario (i.e., all evidence show the worst state performances), not to overall project level in either the medium or the least difficult to implement group.

Although the overall project level performance indicators appear good in Figure 4.2.2-11, the performance of a specific group of work packages may be in bad performance.

\section{Overall project SPI and CPI}

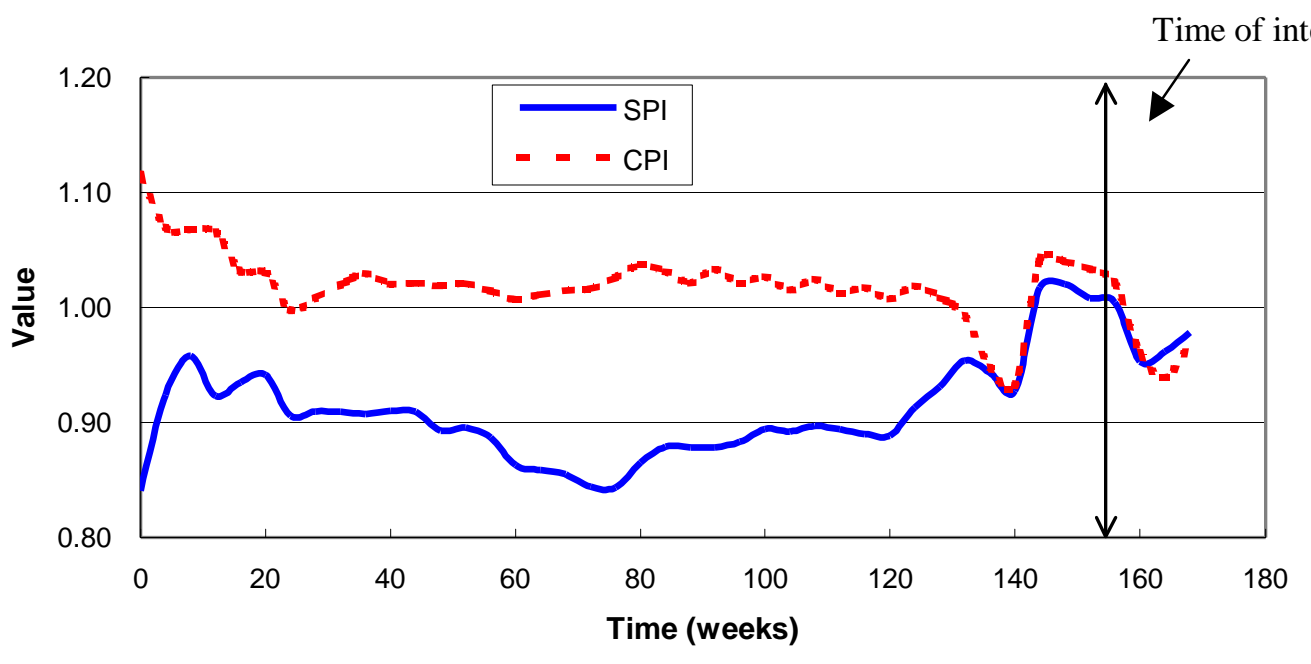

Figure 4.2.2-11. Overall Project Level SPI And CPI History From 0 To 173 Weeks

In fact, the deadline of a specific milestone of those work packages was 161 weeks but their performance did not seem to achieve it. The project managers decided to consider the following management options to achieve this milestone. The present time is at 149 seeks.

\section{Management Options:}

1. Hire Additional Resources - Hire more work forces to achieve the milestone but incur direct and non-direct costs.

2. Overtime Work (OT) - Overtime work with current work forces to obtain the milestone but incur direct and non-direct costs.

3. No action - Most likely misses the milestone and incur penalty.

Option 1 - requires two additional workers for a total of 18 man-weeks (assume 80\$/manhr) as well as indirect cost totaling around $16 \mathrm{k} \$$.

Option 2 - assumes the same amount of man-effort (i.e., 720 man-hours), which results in an absolute cost of 58\$/man-hr as well as indirect cost totaling around $16 \mathrm{k} \$$. Note, however, that this option would also result in an overload of work to the current staff and the necessary expertise is also not in place. Thus, although the possibility of overtime work period extension would be significant, it is not reflected here (i.e., not accounted in costs). 
$\underline{\text { Option } 3}$ - basically assumes no change in project performance status from pre-action time and post-action time and no costs.

In summary, Table 4.2.2-2 shows the alternative action options and corresponding immediate costs in overall project level for the current situation.

Table 4.2.2-2. Action Alternatives And Corresponding Immediate Costs

\begin{tabular}{|l|l|}
\hline Action alternative & Costs $(\mathbf{k} \mathbf{)})$ \\
\hline \hline Hire additional resources & 74 \\
\hline Overtime work & 57 \\
\hline Do nothing & 0 \\
\hline
\end{tabular}

As discussed earlier, the immediate benefits from taken action can be estimated from the resultant BCWS (Budgeted Cost Work Schedule) curve shift and current project SPI state. From discussions with domain experts and the record of SPI and CPI curves (i.e., Earned Value Chart), it was found that a reasonable estimation of the BCWS for that group of work packages, which show bad performance, is 5\% of the total scheduled budget for the work package development work phase, which is $24,764 \mathrm{k} \$$. Based upon $5 \%$ of fraction estimation, BCWS of the Work Package Development work phase and the correlations for immediate benefits, the immediate benefits for every possible SPI curve shift between preaction and post-action time are estimated as seen in Figure 4.2. 2-12. It should be noted that a medium value of each SPI state range (e.g., 0.7 from range of $(0.6 \sim 0.8)$ ) is selected in order for immediate benefit computation. One example calculation is illustrated below.

BCWS for that group of work packages $=0.05 ? 24,764 k \$ ? 1,238 k \$$.

Immediate benefit from Pre-action SPI state, $0.6 \sim 0.8 ?$ Post-action SPI state, $0.8 \sim 1.0$ ? $0.2 ? 1,238 k \$ ? 248 k \$$.

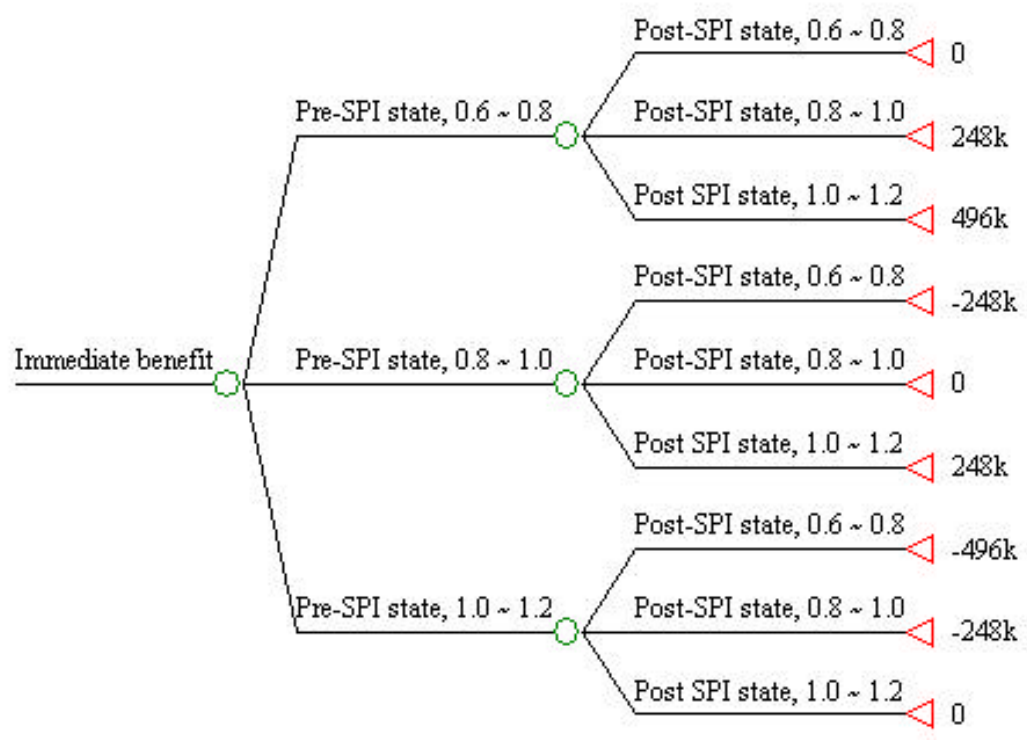

Figure 4.2.2-12. Immediate Benefit Tree For The Advisory Mode Case Study 


\section{Final Management Decision:}

Management eventually chose Option 1 (Hire additional resources). Option 1 may not be an alternative with the most absolute highest expected net benefit, but the benefit of choosing that option allows the current staff to maintain pace and allows the client to gain confidence that Contractor can make their milestones.

\section{$\underline{\text { Assumptions }}$}

It is assumed that the group of work packages that show bad performance in this case study lie in the single-work group, either in the medium or in the least difficult to implement group. Therefore, the case study is performed with both scenarios, that group of work packages in the medium difficult group and in the least difficult group. The immediate benefits as seen in Figure 4.2.2-12 are allocated to either the medium or the least difficult group according to case scenario. Also, it is assumed that the costs in the medium preaction SPI state $(0.8 \sim 1.0)$ have $50 \%$ of those in the worst state $(0.6 \sim 0.8)$ and no cost in the best state $(1.0 \sim 1.2)$, respectively. The costs are allocated in the same way as in the benefits and the immediate cost tree is illustrated in Figures 4.2.2-13.

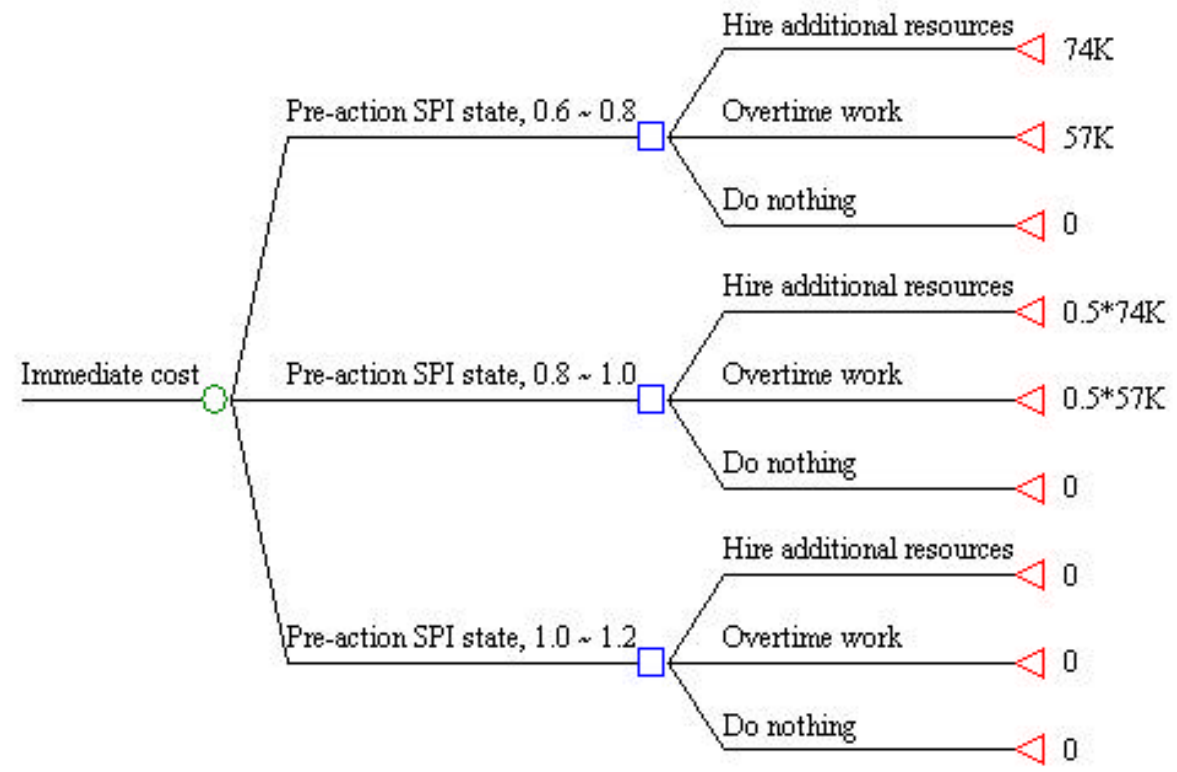

Figure 4.2.2-13. Immediate Cost Tree For The Advisory Mode Case Study

\section{Case analysis results}

In this situation, the project managers can have the following three questions to identify the optimal selection from alternatives. An analysis would be performed in order to answer these three questions.

1. What is the optimal action under this situation?

2. What will be the post-action project performance likelihood, if the project managers take one of action from alternatives? 
3. What will be the project performance likelihood at the scheduled project completion time, if the project managers take one of action from alternatives?

\section{Answer to Question 1.}

Under the expected net benefit criterion - The action with the largest expected net benefit is the optimal action alternative. Since there is no information on long-term penalty (penalty that comes from the costs of the domino effect originating from overdue provision of deliverables, lost reputation and so on), a sensitivity analysis has been performed on long-term benefit (or penalty). Without long-term penalty (e.g., long-term penalty value is 0 in Figures 4.2.2-14 and 4.2.2-15), the 'Do nothing' action is optimal in both scenarios (the medium and the least difficult to implement group), since it gives the largest expected net benefit as seen in Figures 4.2.2-14 and 4.2.2-15. However, as the long-term penalty grows the optimal action changes into 'Overtime work' and then 'Hire additional resources' in the medium difficult group and into 'Hire additional resources' in the least difficult group, respectively. From this result, it is recognized that the long-term penalty (or benefit) of action is the key factor in determining the optimal action under this situation. Therefore the effort is focused on estimating the long-term penalty (or benefit). This will be discussed in a later section.

\section{Expected net benefit of the medium difficult group}

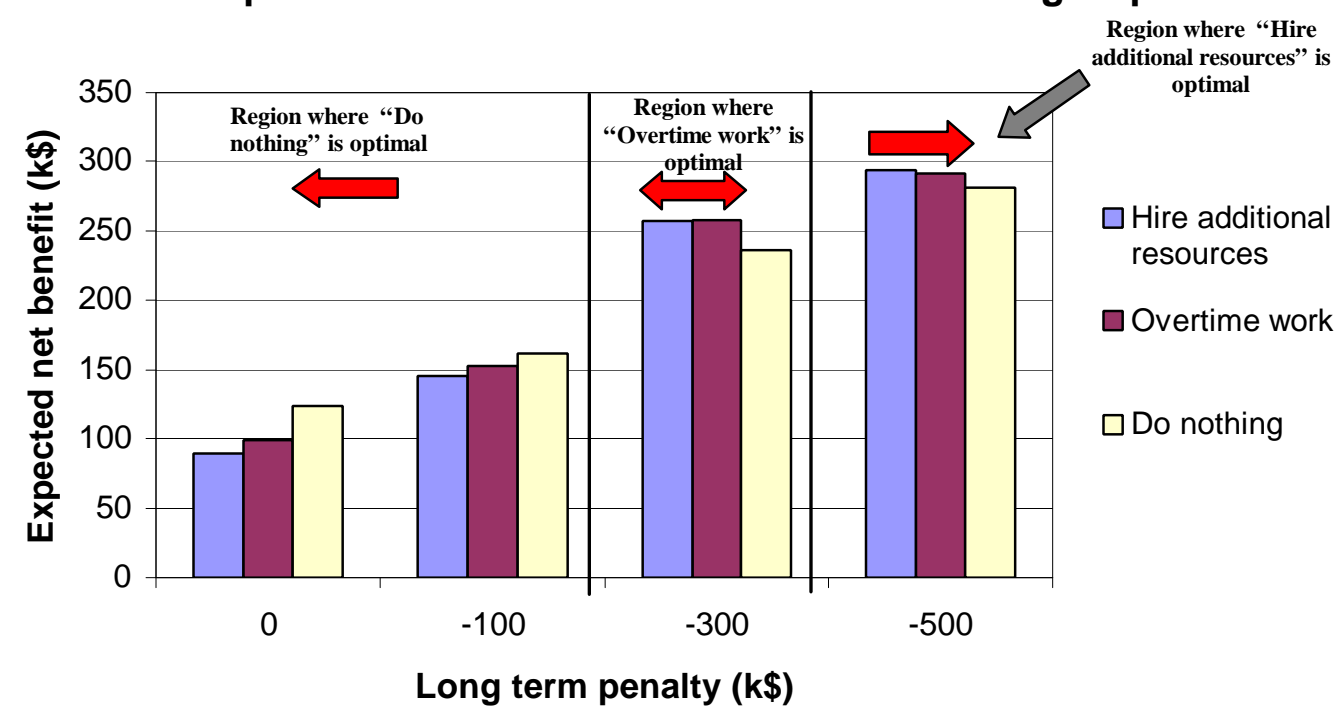

Figure 4.2.2-14. Optimal Action Under Expected Net Benefit Criterion In The Medium Difficult Group Scenario 


\section{Expected net benefit of the least difficult group}

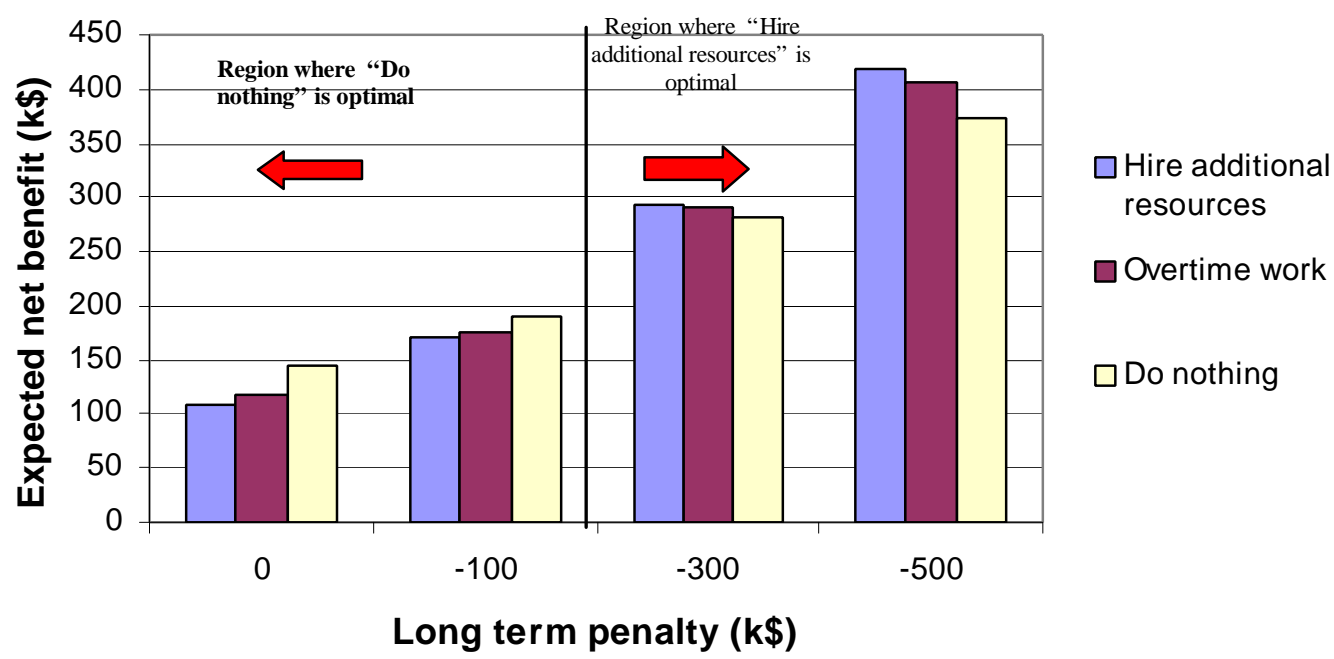

Figure 4.2.2-15. Optimal Action Under Expected Net Benefit Criterion In The Least Difficult Group Scenario

\section{Answer to Question 2.}

One of main concerns of the project managers regarding a bad project performance condition is what will be the project performance after taking one of the actions from the list of alternatives. As discussed in an earlier section, the advisory mode BBN can determine the post-action project performance likelihood corresponding to the taken action. The term 'post-action' is the short time period elapsed that can show the effect of action, generally from 1 to 3 months. In this case study, three action alternatives are considered: 1) Hire additional resources, 2) Overtime work and 3) Do nothing. Figures 4.2.2-16, 4.2.2-17 and 4.2.2-18 show the post-action project performance probability distributions for each action alternative. From the resultant post-action project performance, the action 'Hire additional resources' gives the best project performance likelihood, making it the optimal choice.

Oerall Project Performance - Hire additional resources

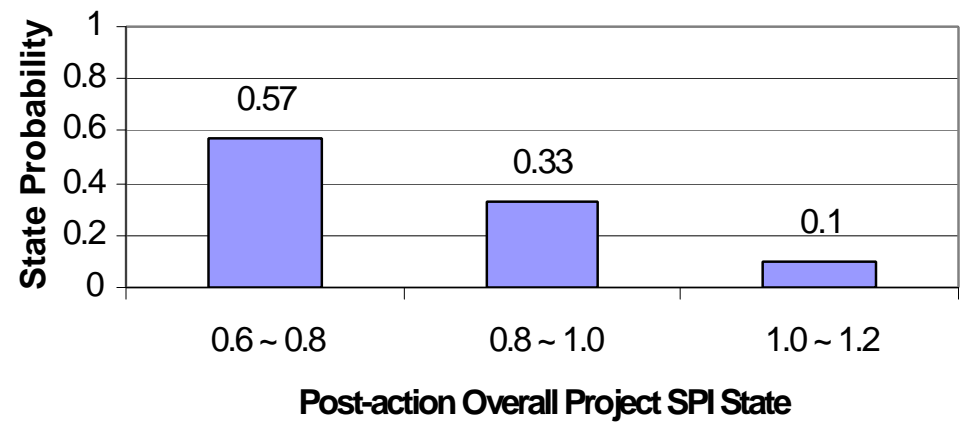

Figure 4.2.2-16. Post-Action Project Performance After Taking 'Hire Additional Resources' 


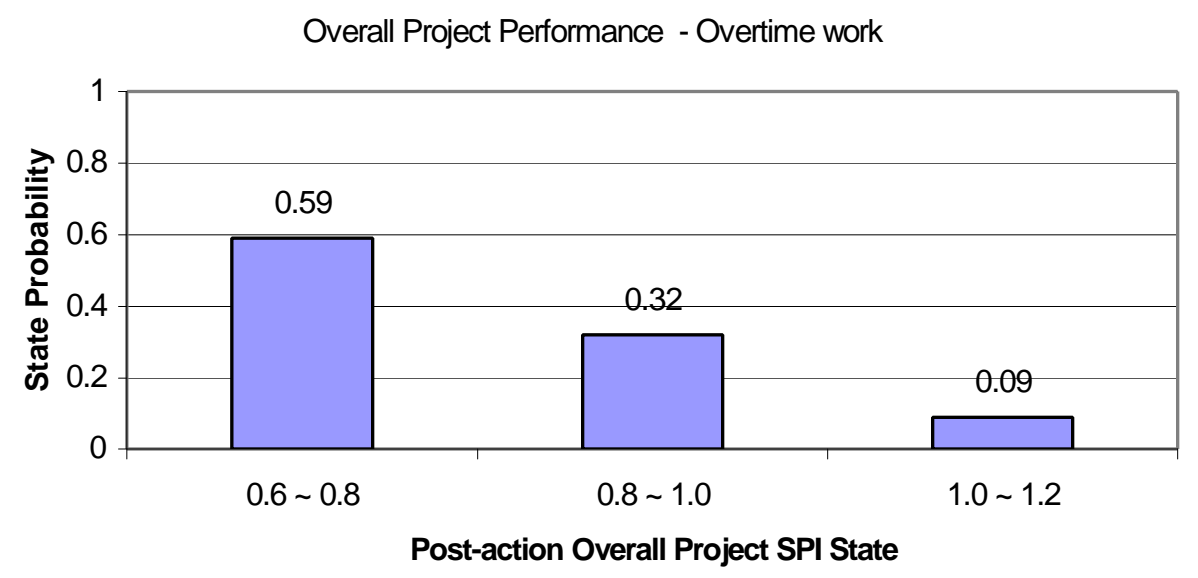

Figure 4.2.2-17. Post-Action Project Performance After Taking 'Overtime Work'

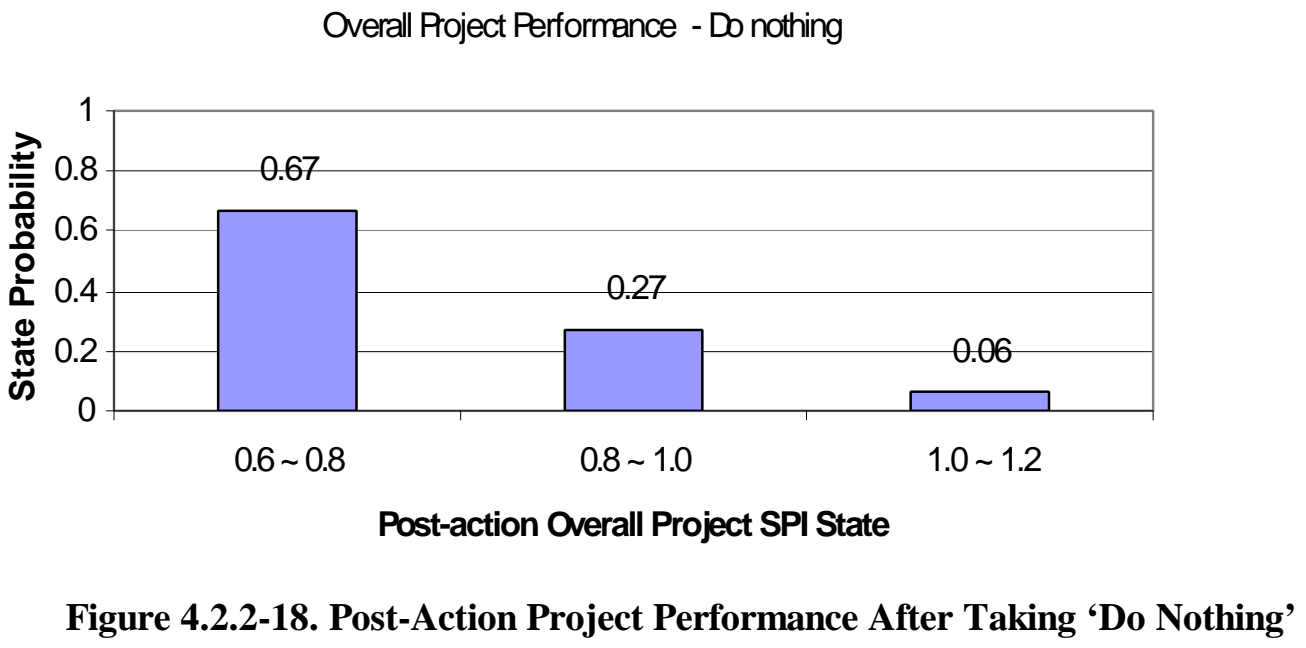

\section{Answer to Question 3.}

Project managers are concerned most about the project performance at the scheduled project completion time since they have to pay a large penalty if they fail to meet the schedule. Especially in the steam generator replacement project of nuclear power plants, the time schedule is a very critical factor because the penalty amount is up to several million dollars per even one-day delay. In addition, they may lose the company's reputation and hence, future business opportunities in the market. Therefore, whether or not they can meet the schedule at the project completion time is one of the most important factors in the decision-making process.

Project performance predictions at scheduled project completion time for each action alternative are shown in Figures 4.2.2-19, 4.2.2-20 and 4.2.2-21. As expected, the action 'Hire additional resources' gives the best project performance prediction since the postaction project performance was found to be the best from answer to question 2 . Therefore, 
the action 'Hire additional resources' is the optimal one based upon the project performance at the scheduled project completion time.

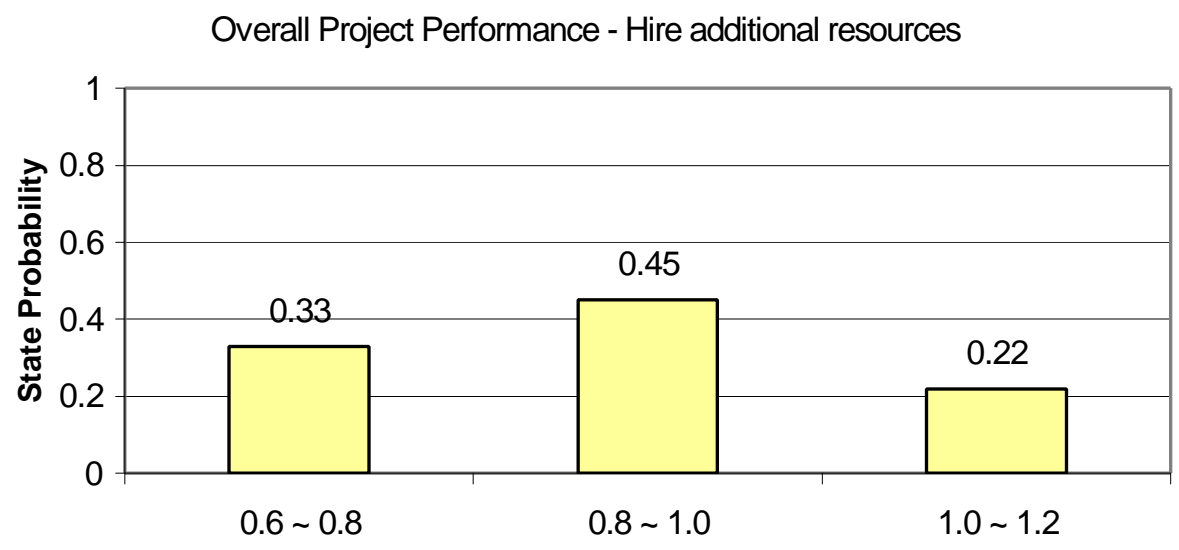

Overall Project SPI State at Scheduled Project Completion Time

Figure 4.2.2-19. Project Performance At Scheduled Project Completion Time After Taking 'Hire Additional Resources'

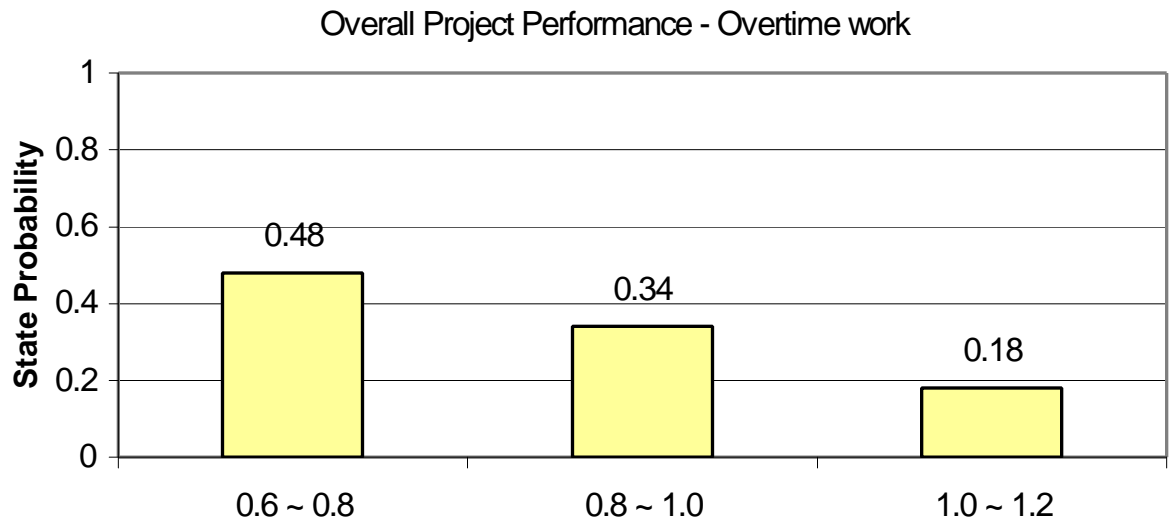

Overall Project SPI State at Scheduled Project Completion Time

Figure 4.2.2-20. Project Performance At Scheduled Project Completion Time After Taking 'Overtime Work' 


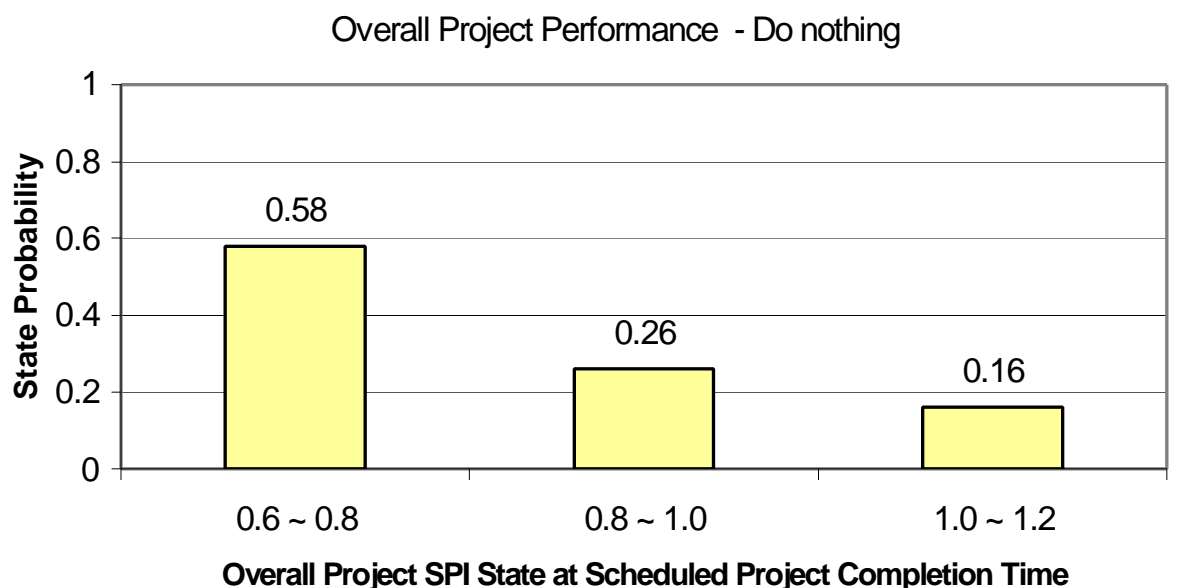

Figure 4.2.2-21. Project Performance At Scheduled Project Completion Time After Taking 'Do Nothing'

Survey for probability distributions for long-term benefit (or penalty)

As discussed in Section 1.1 and illustrated in Figures 4.2.2-14 and 4.2.2-15, there are two types of benefits for project management, immediate benefits (or penalty) and long-term benefits (or penalty), and the long-term benefit is a more important key factor in the management decision-making process than the immediate benefit because the long-term benefit is much larger than the immediate benefit. Therefore, the long-term benefits are estimated under the assumption of a known project performance state at the scheduled completion time and corresponding preference functions of project mangers based upon evaluated long-term benefit values through survey with project managers in real world. The term 'scheduled project completion time' means the time that the project is supposed to be completed in the current project schedule. However, the long-term benefit values have very large uncertainties. As discussed, the best way to include such large uncertainties into the analysis is to estimate the long-term benefit values as probability distributions. Three discrete probability distributions (i.e., one $\mathrm{pmf}^{1}$ for $\mathrm{X} 1$, one pmf for $\mathrm{X} 2$ and one pmf for $\mathrm{X} 3$ - see Table 4.2.3-8 for X1, X2 and X3) with three values in each distribution (i.e., 3 longterm benefit values and corresponding probability values for X1, X2 and X3 in each probability distribution) were estimated, as illustrated in Figure 4.2.2-22. Table 4.2.2-3 shows an example long-term benefit probability distribution for X1.

Table 4.2.2-3. Example Long-Term Benefit Probability Distribution (Pmf) For X1

\begin{tabular}{|l||l|l|l|}
\hline Long-term benefit $(\mathrm{k} \$)$ & $\mathrm{X} 11$ & $\mathrm{X} 12$ & $\mathrm{X} 13$ \\
\hline Corresponding probability value & P1 & P2 & P3 \\
\hline
\end{tabular}

Note: $\mathrm{P} 1+\mathrm{P} 2+\mathrm{P} 3=1.0$

\footnotetext{
${ }^{1}$ Probability mass function (pmf) is another name of discrete probability distribution.
} 

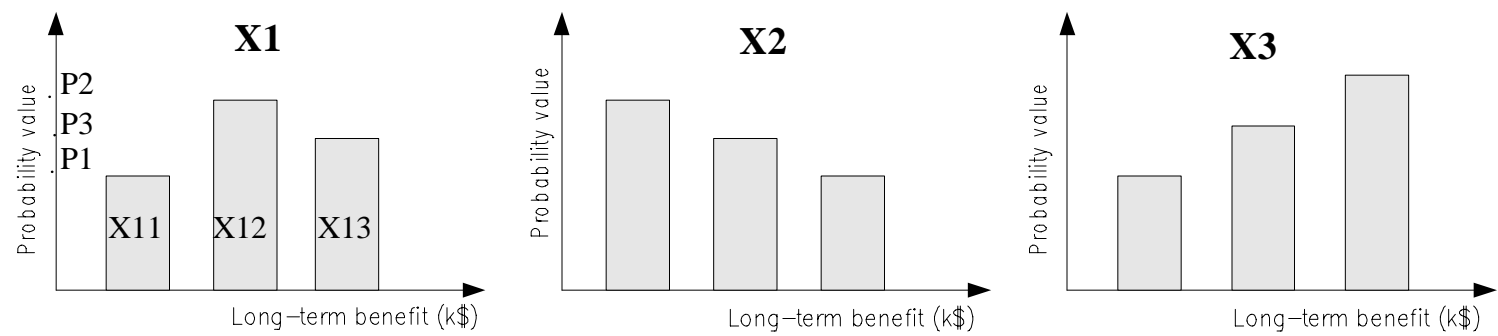

Figure 4.2.2-22. Example Discrete Probability Distributions For Long-Term Benefits

\section{Questionnaire for estimating the long-term benefit distributions}

The next step is to ask several questions in order to estimate your long-term benefit. It should be noted that long-term benefit probability distributions are most likely to be different from one situation to another. Therefore, long-term benefit probability distributions should be reevaluated whenever the situation changes.

\section{Question 1.}

Which components in the following equations are proper to your situation?

Case 1.

SPI at scheduled project completion time is under 1.0

Total long-term penalty value $=$ Tangible penalty + Intangible penalty

Tangible penalty $=$ Additional labor cost + Additional material cost + Penalty in contract

Intangible penalty $=$ Company's reputation loss

Case 2.

SPI at scheduled project completion time is 1.0 or over 1.0

Total long-term benefit value $=$ Tangible benefit + Intangible benefit

Tangible benefit $=$ Gains from under-spent project budget + Incentive from project owner company

Intangible benefit = Improved company's reputation

\section{Question 2.}

What is the range of each component of long-term benefit value, which is identified in Question 1? The range should be evaluated for three different cases: 1) scheduled project completion time SPI of $0.6 \sim 0.8,2$ ) SPI of $0.8 \sim 1.0$ and 3) SPI of $1.0 \sim 1.2$.

\section{Question 3.}

What is the range of the long-term benefit value (i.e., the sum of every components evaluated in Question 2)? The ranges should be evaluated for three different cases: 1) scheduled project completion time SPI of $0.6 \sim 0.8,2$ ) SPI of $0.8 \sim 1.0$ and 3) SPI of $1.0 \sim 1.2$.

\section{Question 4.}

From the three different SPI ranges (i.e., $0.6 \sim 0.8,0.8 \sim 1.0$ and $1.0 \sim 1.2$ ), what is the most likely value from each range of long-term benefit value, which were evaluated in 
Question 3? Then enter those values in Tables 4.2.2-4, 4.2.2-5 and 4.2.2-6 according to proper SPI ranges.

\section{Question 5.}

Please pick up one representing value from upper part and another representing value from lower part of each range of long-term benefit value as seen in Figure 4.2.2-23, which were evaluated in Question 3. We have six representing values from three different SPI ranges (i.e., $0.6 \sim 0.8,0.8 \sim 1.0$ and $1.0 \sim 1.2$ ). Then enter those values in Tables 4.2.2-4, 4.2.2-5 and 4.2.2-6 according to proper SPI ranges.

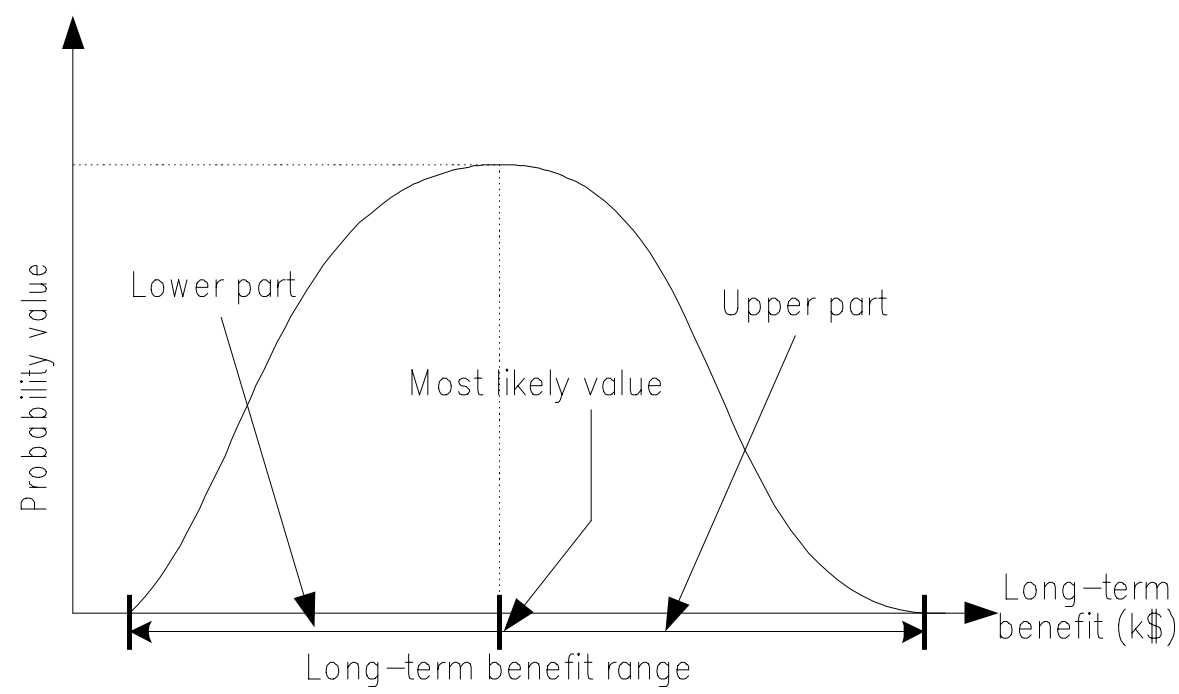

Figure4.2. 2-23. Example Long-Term Benefit Value Range

\section{Question 6.}

Please evaluate the proper probability value to each long-term benefit value in Tables 4.2.2-4, 4.2.2-5 and 4.2.2-6 and enter that probability value into the corresponding Tables. You may use the probability value scale in Figure 4.2.2-24 below. In case of certainty of single value, then enter that single long-term benefit value and corresponding probability value as 1.0 into the tables. The other two spaces for probability values in those tables should be all $0 \mathrm{~s}$.

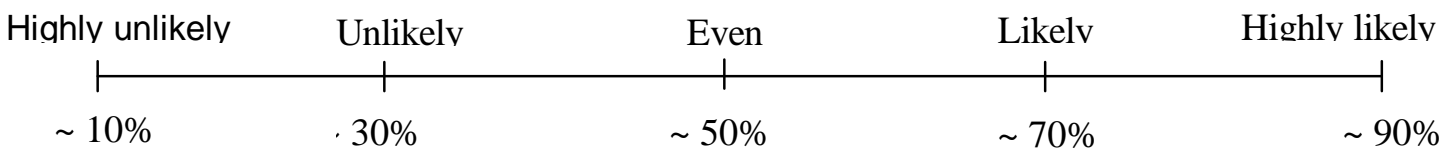

Figure 4.2.2-24. Probability Value Scale 
Table 4.2.2-4. Long-Term Benefit Probability Distribution (Pmf) For SPI - 0.6 0.8

\begin{tabular}{|c||l|l|l|}
\hline Long-term benefit $(\mathrm{k} \$)$ & & & \\
\hline \hline Corresponding probability value & & & \\
\hline
\end{tabular}

Note) Sum of three probability values should be 1.0

Table 4.2.2-5. Long-Term Benefit Probability Distribution (Pmf) For SPI - 0.8 1.0 Long-term benefit $(\mathrm{k} \$)$

Corresponding probability value

Note) Sum of three probability values should be 1.0

Table 4.2.2-6. Long-Term Benefit Probability Distribution (Pmf) For SPI - 1.0 1.2

\begin{tabular}{|l||l|l|l|}
\hline Long-term benefit $(\mathrm{k} \$)$ & & & \\
\hline \hline Corresponding probability value & & & \\
\hline
\end{tabular}

Note) Sum of three probability values should be 1.0

\section{Survey results for probability distributions for long-term benefit (or penalty)}

Two companies are currently performing steam generator replacement work in the market: 1) Bechtel and 2) Framatome ANP Duke Engineering and Service (DE\&S). Since the first steam generator replacement at a U.S. nuclear power plant more than 20 years ago, Bechtel has performed more steam generator replacement work than any other contractor in this market. The other company, Framatome ANP DE\&S began performing steam generator replacement work in 1996 at Catawba Nuclear Station Unit 1 and McGuire Nuclear Station Units 1 and 2. As date of October 2000, Bechtel has performed 24 steam generator replacement projects as the prime contractor, compared to its next closest competitor with four projects. In addition, Bechtel has earned several high achievements such as: 1) shortest overall replacement schedule, 2) lowest U.S. steam generator replacement accumulated radiation exposure, 3) first U.S. one-piece replacement and others. Therefore Bechtel is a leading company in the U.S. steam generator replacement market. Tables 4.2.2-7 and 4.2.28 show the nuclear power plants for which steam generators were replaced by Framatome ANP DE\&S and Bechtel, respectively.

Table 4.2.2-7. U.S. Nuclear Power Plant Of Which SG Replaced By Framatome ANP DE\&S

\begin{tabular}{|l|l|l|l|}
\hline Unit & Owner(s) & Operator & $\begin{array}{l}\text { First commercial } \\
\text { operation date }\end{array}$ \\
\hline \hline Calvert Cliffs 1 & Constellation Energy Group & Constellation Nuclear & $05 / 1975$ \\
\hline Calvert Cliffs 2 & Constellation Energy Group & Constellation Nuclear & $04 / 1977$ \\
\hline Catawba 1 & $\begin{array}{l}\text { NC Eastern Municipal Power Agency } \\
(56.25 \%) \\
\text { Duke Energy Corp. (25\%) } \\
\text { Saluda River Electric Coop (18.75\%) }\end{array}$ & Duke Energy Nuclear LLC & $06 / 1985$ \\
\hline Indian Point 2 & Energy Nuclear Operations & Entergy Nuclear & $08 / 1974$ \\
\hline McGuire 1 & Duke Energy Corp. & Duke Power & $12 / 1981$ \\
\hline McGuire 2 & Duke Energy Corp. & Duke Power & $03 / 1984$ \\
\hline St. Lucie 1 & FPL Group & Florida Power and Light & $12 / 1976$ \\
\hline
\end{tabular}


Table 4.2.2-8. U.S. Nuclear Power Plant Of Which SG Replaced By Bechtel As Of Oct. 2000

\begin{tabular}{|c|c|c|c|}
\hline Unit & Owner(s) & Operator & $\begin{array}{l}\text { First commercial } \\
\text { operation date }\end{array}$ \\
\hline Arkansas Nuclear One 2 & Entergy Arkansas, Inc. & Entergy Nuclear & 03/1980 \\
\hline Braidwood 1 & Exelon & Exelon & $07 / 1988$ \\
\hline Byron 1 & Exelon & Exelon & $09 / 1985$ \\
\hline Cook 1 & American Electric Power & Indiana Michigan Power & $08 / 1975$ \\
\hline Farley 1 & Alabama Power & $\begin{array}{l}\text { Southern Nuclear } \\
\text { Operating Co. }\end{array}$ & $12 / 1977$ \\
\hline Farley 2 & Alabama Power & $\begin{array}{ll}\text { Southern } & \text { Nuclear } \\
\text { Operating Co. } & \end{array}$ & $07 / 1981$ \\
\hline Ginna & RGS Energy Group & $\begin{array}{l}\text { Rochester Gas \& } \\
\text { Electric Corp. }\end{array}$ & $07 / 1970$ \\
\hline Indian Point 3 & Energy Nuclear Operations & Entergy Nuclear & $08 / 1976$ \\
\hline Kewaunee & $\begin{array}{l}\text { Wisconsin Public Service } \\
\text { Alliant Energy }\end{array}$ & $\begin{array}{l}\text { Nuclear Management } \\
\text { Co. }\end{array}$ & $06 / 1974$ \\
\hline North Anna 1 & $\begin{array}{l}\text { Dominion Virginia Power } \\
\text { Old Dominion Electric Coop. }\end{array}$ & Dominion Generation & 06/1978 \\
\hline North Anna 2 & $\begin{array}{l}\text { Dominion Virginia Power } \\
\text { Old Dominion Electric Coop. }\end{array}$ & Dominion Generation & $12 / 1980$ \\
\hline Palisades & CMS Energy Corp. & $\begin{array}{l}\text { Nuclear Management } \\
\text { Co. }\end{array}$ & $12 / 1971$ \\
\hline Palo Verde 2 & $\begin{array}{l}\text { Arizona Public Service } \\
\text { Other } 6 \text { companies }\end{array}$ & $\begin{array}{l}\text { Arizona Nuclear Power } \\
\text { Project }\end{array}$ & 09/1986 \\
\hline Robinson & Progress Energy & $\begin{array}{l}\text { Carolina Power \& Light } \\
\text { Co. }\end{array}$ & 03/1971 \\
\hline Sequoyah 1 & Tennessee Valley Authority & $\begin{array}{ll}\text { Tennessee } & \text { Valley } \\
\text { Authority } & \end{array}$ & $07 / 1981$ \\
\hline Shearon Harris & $\begin{array}{l}\text { Progress Energy } \\
\text { NC Eastern Municipal Power Agency }\end{array}$ & $\begin{array}{l}\text { Carolina Power \& Light } \\
\text { Co. }\end{array}$ & $05 / 1987$ \\
\hline South Texas Project 1 & $\begin{array}{l}\text { Reliant Energy HL\&P } \\
\text { Other } 3 \text { companies }\end{array}$ & $\begin{array}{l}\text { STP Nuclear Operating } \\
\text { Co. }\end{array}$ & $08 / 1988$ \\
\hline South Texas Project 2 & $\begin{array}{l}\text { Reliant Energy HL\&P } \\
\text { Other } 3 \text { companies }\end{array}$ & $\begin{array}{l}\text { STP Nuclear Operating } \\
\text { Co. }\end{array}$ & 06/1989 \\
\hline Summer & $\begin{array}{l}\text { South Carolina Electric \& Gas } \\
\text { South Carolina Public Service Authority }\end{array}$ & $\begin{array}{l}\text { South Carolina Electric } \\
\& \text { Gas Co. }\end{array}$ & $01 / 1984$ \\
\hline Turkey Point 3 & FPL Group & Florida Power \& Light & $12 / 1972$ \\
\hline Turkey Point 4 & FPL Group & Florida Power \& Light & $09 / 1973$ \\
\hline
\end{tabular}

After reviewing the U.S. nuclear power plants whose steam generator has already been replaced in Tables 4.2.2-7 and 4.2.2-8, the first commercial operating date and NEI's (Nuclear Energy Institute) license renewal report, the number of U.S. unclear units considered as candidate future customers to Bechtel and Framatome ANP DE\&S is estimated as 22. Based upon this number and other factors below, the reference reputation value is estimated from the standpoint of future business opportunities as in equation (4.2.2-3).

1. Contract price $=150 \mathrm{M} \$ /$ contract

2. Number of reactors that will replace the $S / G$ in future $=22$

3. $\quad$ Profit margin $=10 \%$ (after taxes)

4. Market share of Framatome ANP DE\&S $=50 \%$

Reputation value (Future profit) ? 150M\$? 22? 0.1? $0.5 ? 165 \mathrm{M} \$$. 
Based upon the reference reputation value in equation (4.2.2-3), the long-term intangible benefit (or penalty) and the long-term tangible benefit (or penalty) as a function of the SPI state at scheduled project completion time are estimated as shown in Figures 4.2.2-25 and 4.2.2-26. The reputation of a company is believed to be very fragile. It can be easily lost and, once lost, takes a much longer time and a larger effort to restore. This fact can be confirmed in this survey result again as shown in Figure 4.2.2-25. In the case of failure to meet the scheduled project completion time (i.e., under SPI value of 1.0), the intangible loss increases very rapidly. However, in the case of being ahead of schedule (i.e., over SPI value of 1.0), the intangible benefit increases slowly.

With the help of estimated long-term intangible and tangible benefits (or penalty) and equations (4.2.3-5) \& (4.2.3-6), the long-term benefit (or penalty) probability distributions as a function of the SPI state at scheduled project completion time are evaluated as illustrated in Figures 4.2.2-27, 4.2.2-28 and 4.2.2-29.

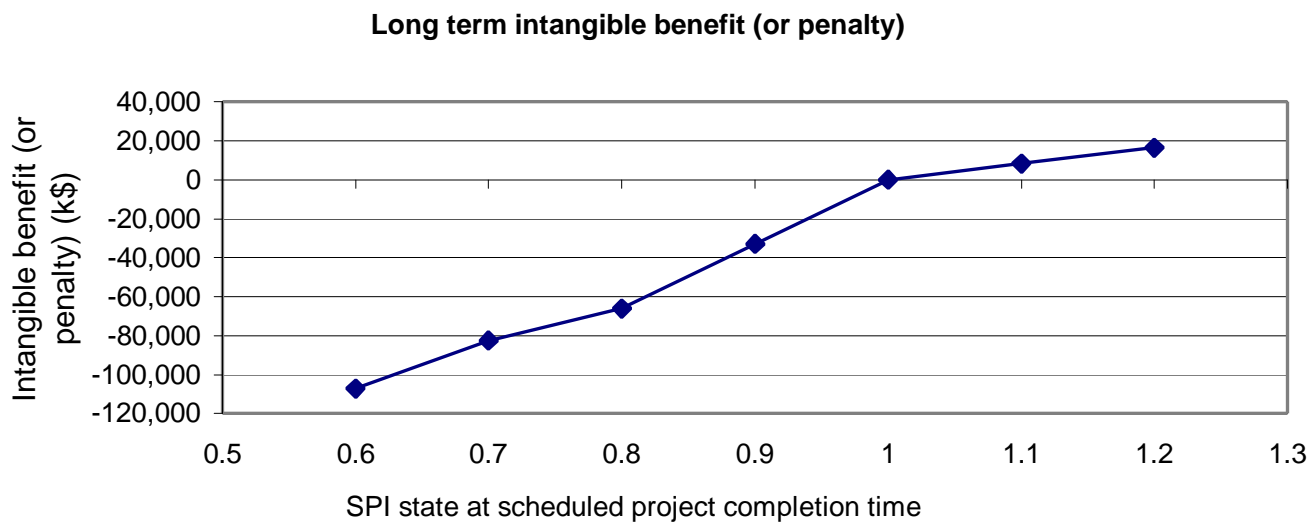

Figure 4.2.2-25. Long-Term Intangible Benefit As Function Of SPI State At Scheduled Project Completion Time

Long term tangible benefit (or penalty)

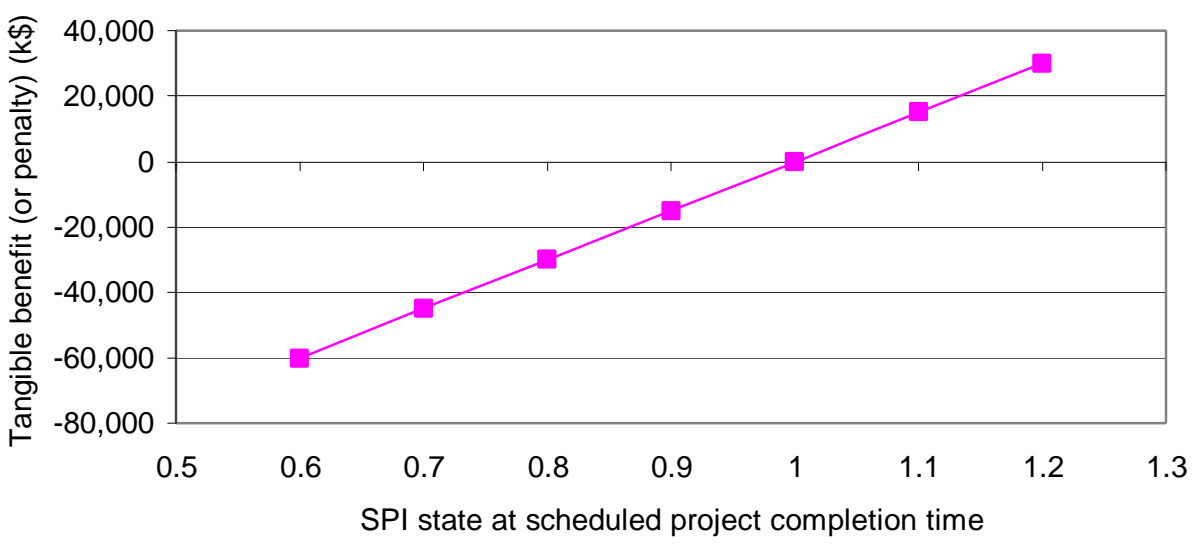

Figure4.2.2-26. Long-Term Tangible Benefit As Function Of SPI State At Scheduled Project Completion Time 
Long term penalty at project completion SPI of $0.6 \sim 0.8$

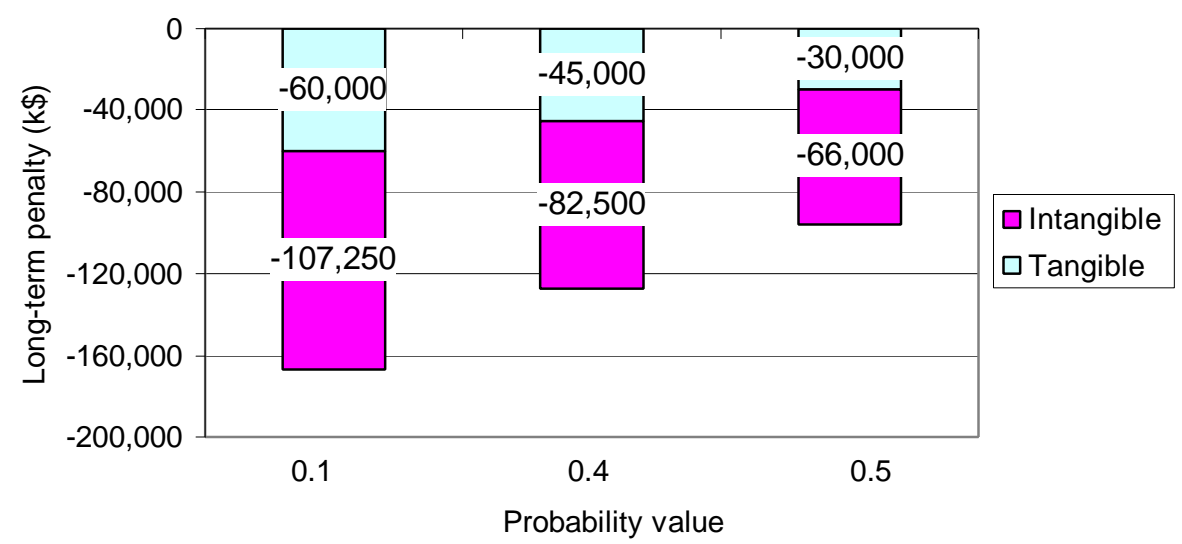

Figure 4.2.2-27. Long-Term Penalty Probability Distribution For SPI Of $0.6 \sim 0.8$ At Scheduled Project Completion Time

Long term penalty at project completion SPI of $0.8 \sim 1.0$

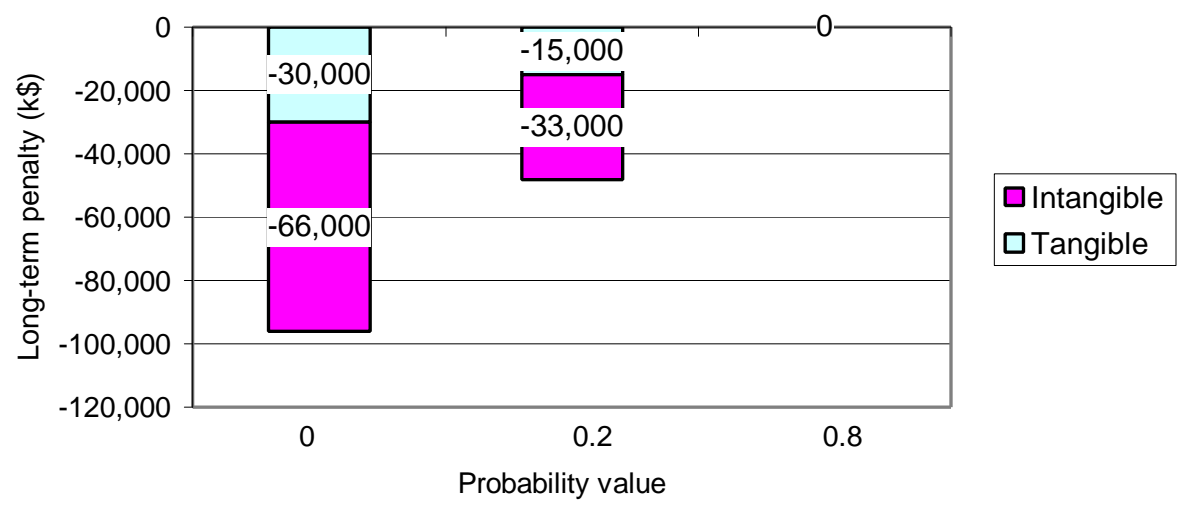

Figure 4.2.2-28. Long-Term Penalty Probability Distribution For SPI Of $0.8 \sim 1.0$ At Scheduled Project Completion Time 
Long term benefit at project completion SPI of $1.0 \sim 1.2$

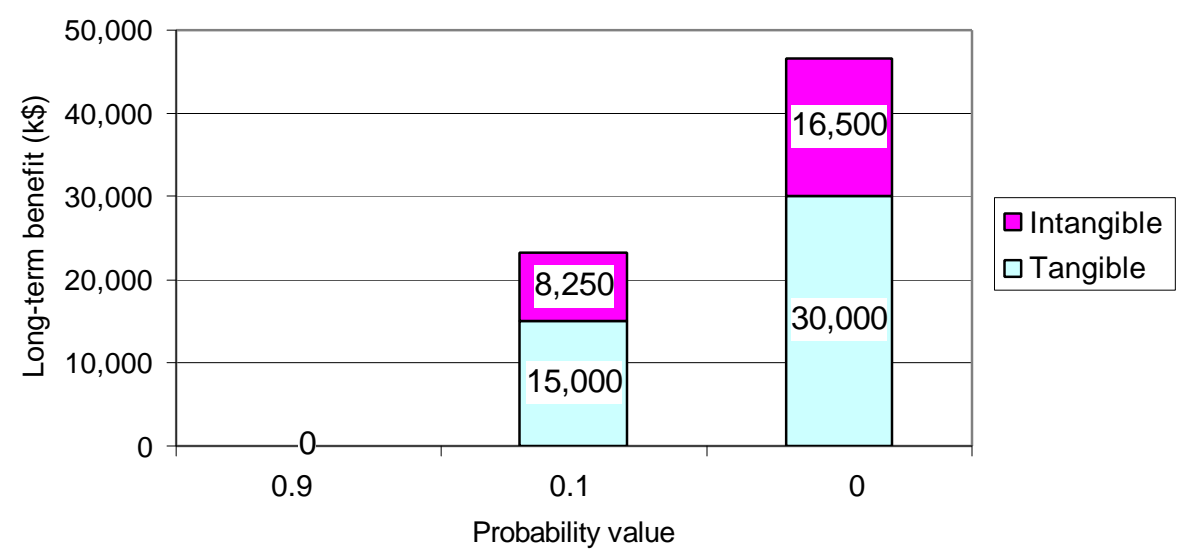

\section{Figure 4.2.2-29. Long-Term Penalty Probability Distribution} For SPI Of $1.0 \sim 1.2$ At Scheduled Project Completion Time

\section{Survey for evaluating preference functions}

Decisions based upon expected monetary values (i.e., expected net benefits) would be convenient, but it can lead to decisions that may not be right. Using expected net benefits to make decisions means that the decision maker is considering only the average or expected payoff. If a long-run frequency approach is used, the expected value is the average amount over many trials. However, the expected net benefit approach does not capture one important point, decision maker's attitude to risk in uncertain situations. In order to overcome this limitation, another decision criterion, the expected utility, based upon the decision theory concerned with measurement and representation of the decision maker's preferences is proposed. Utility theorists focus on accounts of preferences in rational decision making, where an individual's preferences cohere with associated beliefs and actions. Utility refers to the scale on which preference is measured.

There are difficulties in evaluating the utility function in this case because both the large amount of penalties (i.e., negative monetary values) and benefit values (i.e., positive monetary values) should be considered at the same time. Therefore, the preference to risk scale for measuring the decision maker's attitude to risk is developed. With this preference scale, individuals who are afraid of risk or are sensitive to risk show the small values of preference in penalties and the large values of preference in benefits as shown in Figure 4.2.2-30. The other two attitudes to risk are risk-neutral (i.e., no dependency on risk) and risk-seeking. For the person who is risk-neutral, maximizing expected net benefit gives the same result as maximizing expected utility. 


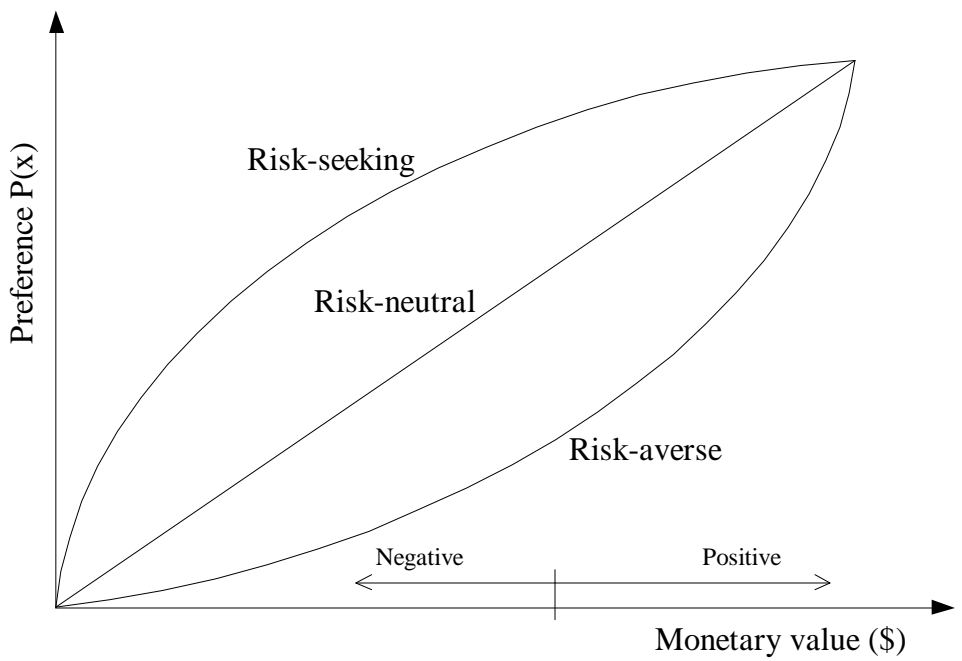

Figure 4.2.2-30. Three Different Preference Functions

Now, in order to evaluate preference functions, several questions are asked. Note that these may be either two of points of view in the risk preference: 1) company point of view and 2) personal (i.e., project manager) point of view. In this case, estimate the risk preference based upon the company point of view. Then, answer the questions and evaluate the risk preference to expected net benefit values in monetary scale.

\section{Questionnaire For Estimating The Preference Functions}

\section{Question 1.}

Please find the maximum and minimum values among the assigned nine long-term benefit values from Table4.2.2-4, 4.2.2-5 and 4.2.2-6 above. Assume that the maximum and minimum values are $\mathrm{X}, \mathrm{Y}$, respectively and then, highly likely, $\mathrm{X}$ would be positive (benefit) and $\mathrm{Y}$ is negative (penalty).

\section{Question 2.}

Please enter those two values (X and $\mathrm{Y}$ ) into Table 4.2.2-9 and then calculate remaining six long-term benefit values (i.e., 3X/4, X/2, X/4, Y/4, Y/2 and 3Y/4) in Table 4.2.2-9. After that, assign your preference to each long-term benefit value according to preference scale in Figure 4.2.2-31.

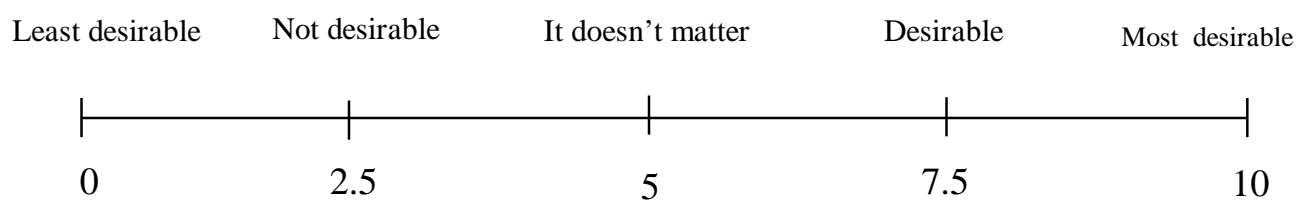

Figure 4.2.2-31. Preference Scale Between 0 And 10 
Table 4.2.2-9. Preference Function Of Long-Term

Benefit Values For Overall Project

\begin{tabular}{|l|l|}
\hline \multicolumn{1}{|c|}{$\begin{array}{c}\text { Long-term benefit value } \\
(\mathbf{k})\end{array}$} & \multicolumn{1}{c|}{ Your preference } \\
\hline Maximum $=\mathrm{X}$ & 10 \\
\hline $3 *$ Maximum $/ 4=3 \mathrm{X} / 4$ & \\
\hline Maximum $/ 2=\mathrm{X} / 2$ & \\
\hline Maximum $/ 4=\mathrm{X} / 4$ & \\
\hline 0 & \\
\hline Minimum $/ 4=\mathrm{Y} / 4$ & \\
\hline Minimum $/ 2=\mathrm{Y} / 2$ & \\
\hline $3 *$ Minimum $/ 4=3 \mathrm{Y} / 4$ & 0 \\
\hline Minimum $=\mathrm{Y}$ & \\
\hline
\end{tabular}

\section{Question 3.}

Please estimate how much fraction of project budget that would be spent on each work group: 1) most difficult work group - ?,2) medium difficult work group - ? and 3) least difficult work group - ?. It should be noted the sum of ?, ?, ? should be 1.0. In case of early three work phases (conceptual design, detailed design and work package development work phases), the most difficult work group does not exist. Therefore, there is no need to consider the most difficult work group (i.e., Table 4.2.2-10 because $\underline{?=0}$ ) and the sum of ?,? should be 1.0.

\section{Question 4.}

Please calculate the long-term benefit values (i.e., such as ?*X/4) in Tables 4.2.2-10, 4.2.2-11 and 4.2.2-12. Then, enter those calculated long-term benefit values $(\mathrm{k} \$)$ into Tables 4.2.2-10, 4.2.2-11 and 4.2.2-12.

\section{Question 5.}

Please assign your preference value for each long-term benefit value in Tables 4.2.210, 4.2.2-11 and 4.2.2-12 according to preference scale in Figure 4.2.2-31 above. 
Table 4.2.2-10. Preference Function Of Long-Term Benefit For Most Difficult Work Group

\begin{tabular}{|l|l|}
\hline Long-term benefit value $(\mathbf{k} \mathbf{\$})$ & Your preference \\
\hline$? *$ Maximum $=? * \mathrm{X}$ & 10 \\
\hline$? * 3 *$ Maximum $/ 4=? * 3 \mathrm{X} / 4$ & \\
\hline \multicolumn{1}{|c|}{$? *$ Maximum $/ 2=? * \mathrm{X} / 2$} & \\
\hline$? *$ Maximum $/ 4=? \mathrm{X} / 4$ & \\
\hline 0 & \\
\hline$? *$ Minimum $/ 4=? * \mathrm{Y} / 4$ & \\
\hline$? *$ Minimum $/ 2=? * \mathrm{Y} / 2$ & \\
\hline$? * 3 *$ Minimum $/ 4=? * 3 \mathrm{Y} / 4$ & \\
\hline$? *$ Minimum $=? * \mathrm{Y}$ & 0 \\
\hline
\end{tabular}

Table 4.2.2-11. Preference Function Of Long-Term Benefit For Medium Difficult Work Group

\begin{tabular}{|l|l|}
\hline Long-term benefit value $(\mathbf{k} \mathbf{)})$ & Your preference \\
\hline \hline$? *$ Maximum $=? * \mathrm{X}$ & 10 \\
\hline$? * 3 *$ Maximum $/ 4=? * 3 \mathrm{X} / 4$ & \\
\hline$? *$ Maximum $/ 2=? * \mathrm{X} / 2$ & \\
\hline$? *$ Maximum $/ 4=? * \mathrm{X} / 4$ & \\
\hline 0 & \\
\hline$? *$ Minimum $/ 4=? * \mathrm{Y} / 4$ & \\
\hline$? *$ Minimum $/ 2=? * \mathrm{Y} / 2$ & \\
\hline$? * 3 *$ Minimum $/ 4=? * 3 \mathrm{Y} / 4$ & \\
\hline$? *$ Minimum $=? * \mathrm{Y}$ & 0 \\
\hline
\end{tabular}




\section{Table 4.2.2-12. Preference Function Of Long-Term Benefit For Least Difficult Work Group}

\begin{tabular}{|l|l|}
\hline Long-term benefit value $(\mathbf{k} \mathbf{\text { ) }}$ & Your preference \\
\hline \hline ?*Maximum $=? * \mathrm{X}$ & 10 \\
\hline ?*3*Maximum $/ 4=? * 3 \mathrm{X} / 4$ & \\
\hline$? *$ Maximum $/ 2=? * \mathrm{X} / 2$ & \\
\hline$? *$ Maximum $/ 4=? * \mathrm{X} / 4$ & \\
\hline 0 & \\
\hline$? *$ Minimum $/ 4=? * \mathrm{Y} / 4$ & \\
\hline$? *$ Minimum $/ 2=? * \mathrm{Y} / 2$ & \\
\hline$? * 3 *$ Minimum $/ 4=? * 3 \mathrm{Y} / 4$ & \\
\hline$? *$ Minimum $=? * \mathrm{Y}$ & 0 \\
\hline
\end{tabular}

\section{Survey results for preference functions}

Based upon the long-term benefit (or penalty) values estimated in the previous survey, the preference functions are evaluated as shown in Figures 4.2.2-32, 4.2.2-33, 2-34 and 4.2.235 . As expected, negative monetary values show low preferences and positive monetary values show high preferences - risk aversion type decision makers.

\section{Preference function for overall project level}

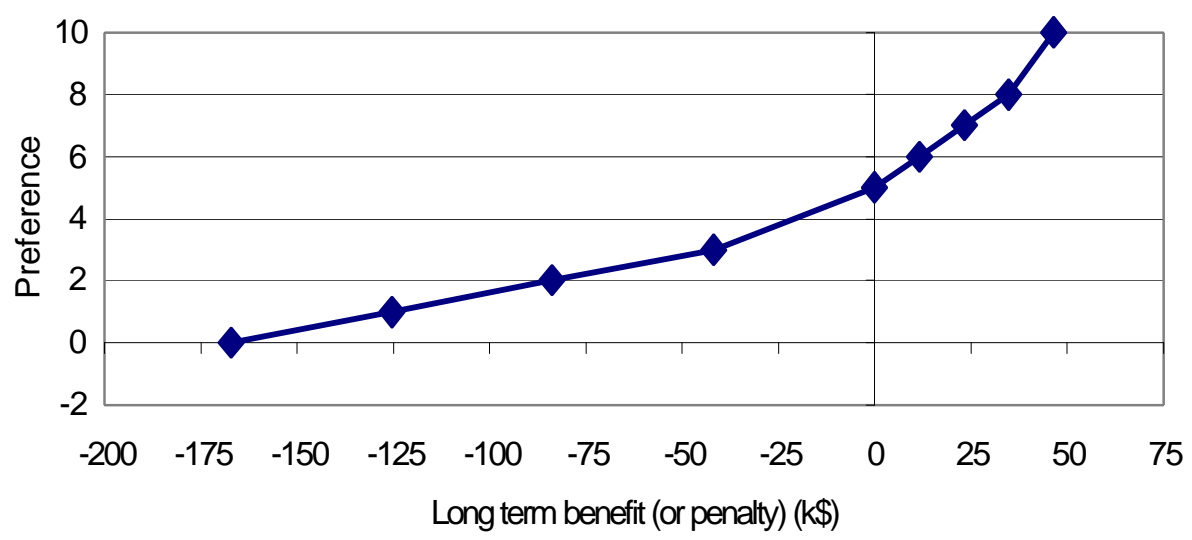

Figure 4.2.2-32. Preference Function For Overall Project Level 


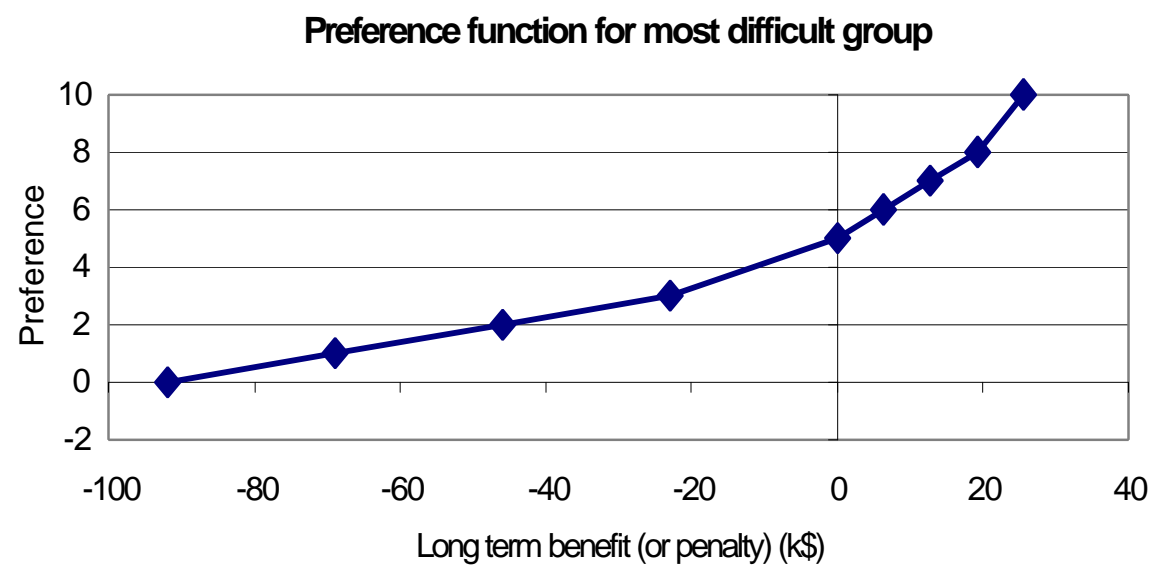

Figure 4.2.2-33. Preference Function For The Most Difficult To Implement Group

Preference function for medium difficult group

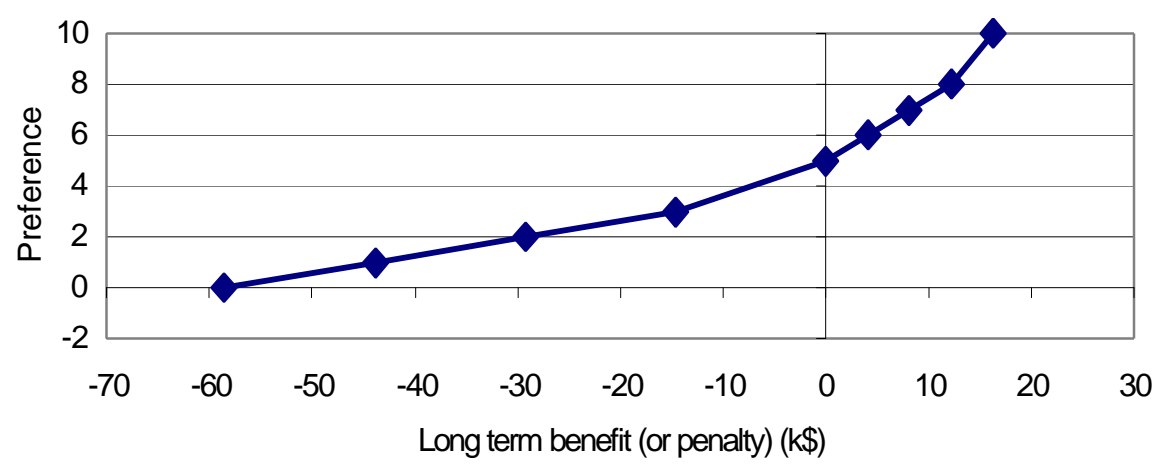

Figure 4.2.2-34. Preference Function For The Medium Difficult To Implement Group

Preference function for least difficult group

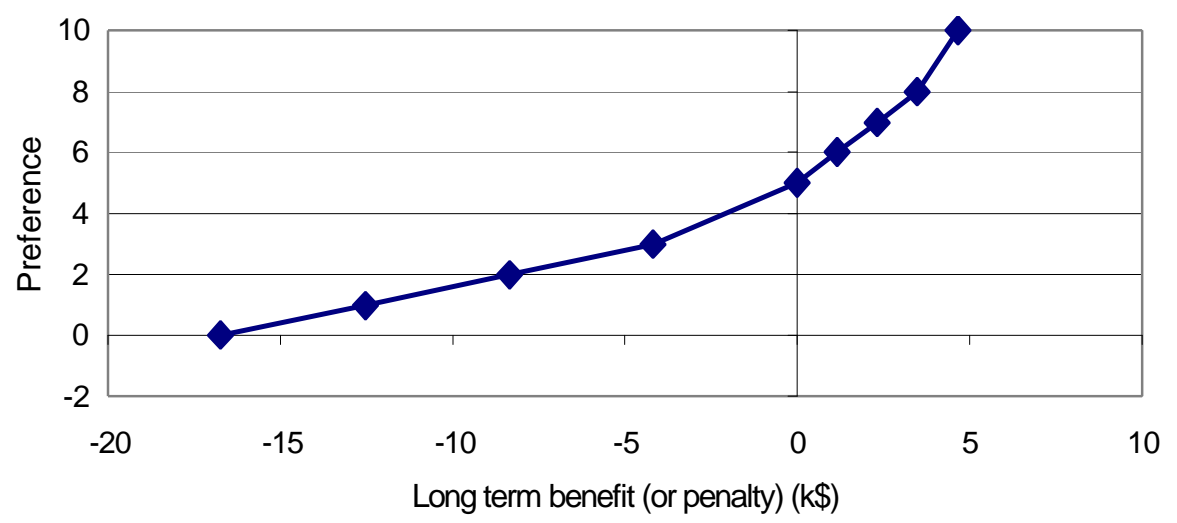

Figure 4.2.2-35. Preference Function For The Least Difficult To Implement Group 
Case analysis results revision with long-term benefit and preference functions

Based upon evaluated long-term benefit probability distributions and corresponding preference functions, the advisory mode BBN case is analyzed again. The optimal action is identified with three criteria as discussed: 1) expected net benefit, 2) expected preference and 3 ) benefit to cost ratio.

1. The Expected Net Benefit Criterion - The action with the largest expected net benefit is the optimal action alternative. Figures 4.2.2-36 and 4.2.2-37 show that 'Hire additional resources' action is the optimal action in both the medium and the least difficult implement group cases.

\section{Expected net benefit of the medium difificult group}

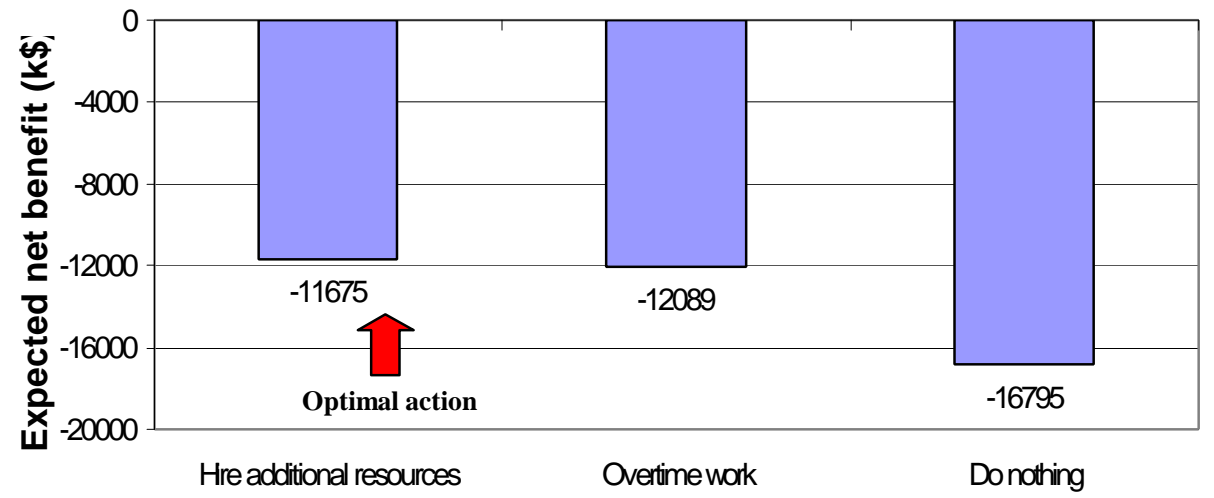

Figure 4.2.2-36. Optimal Action With Expected Net Benefit In The Medium Difficult Group

Expected net benefit of the least difificult group

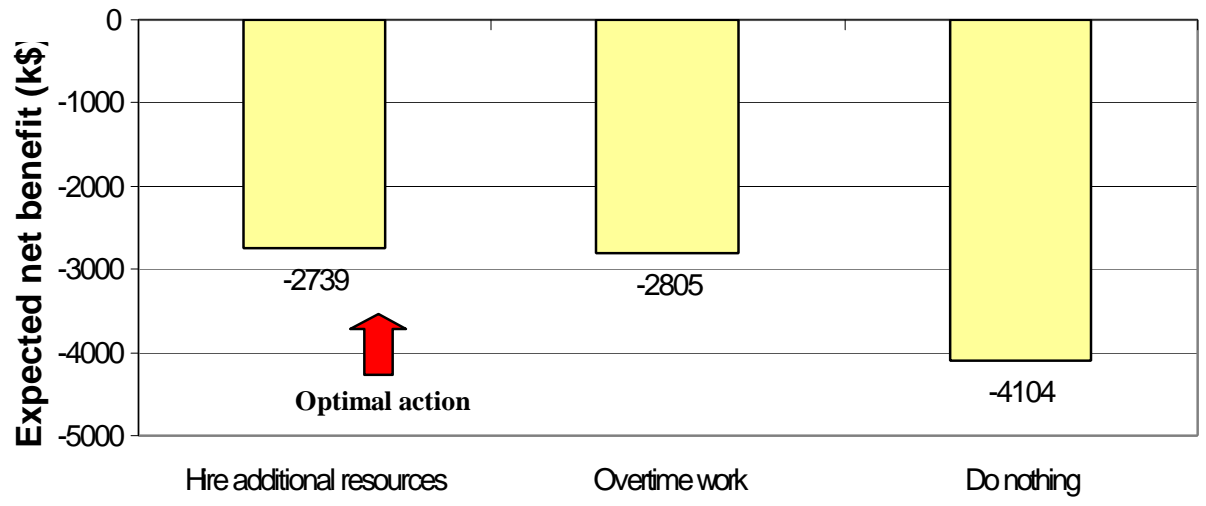

Figure 4.2.2-37. Optimal Action With Expected Net Benefit In The Least Difficult Group

2. The Expected Preference - The action with the largest expected preference value is the optimal action alternative. As discussed in the previous section, the expected preference is the criterion considering the project performance and the decision maker's attitude to 
risk. The 'Hire additional resources' is the optimal action alternative in both the medium and the least difficult groups as shown in Figures 4.2.2-38 and 4.2.2-39.

\section{Expected preference of the medium difficult group}

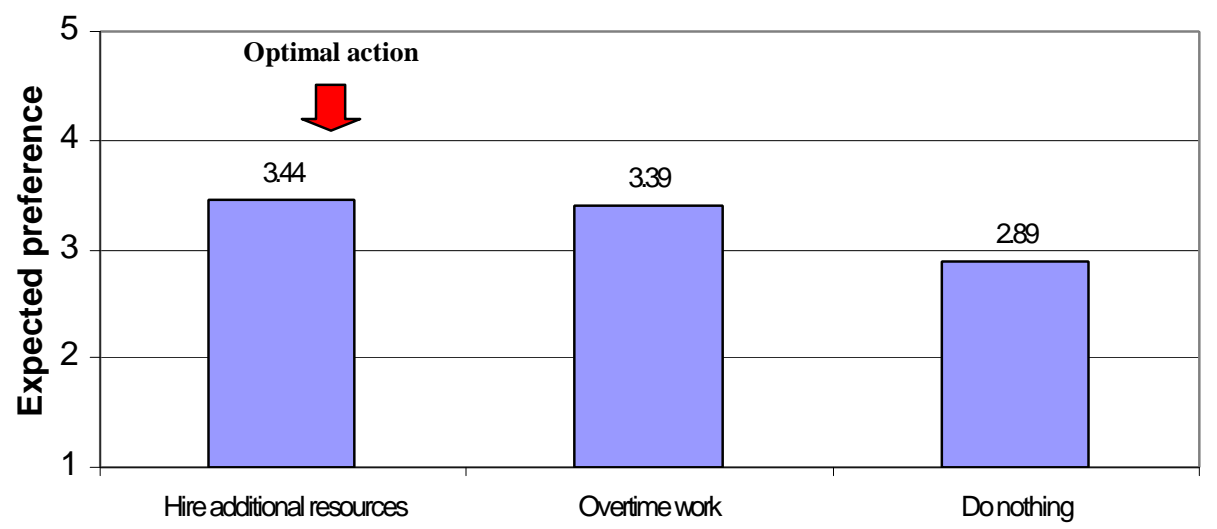

Figure 4.2.2-38. Optimal Action With Expected Preference In The Medium Difficult Group

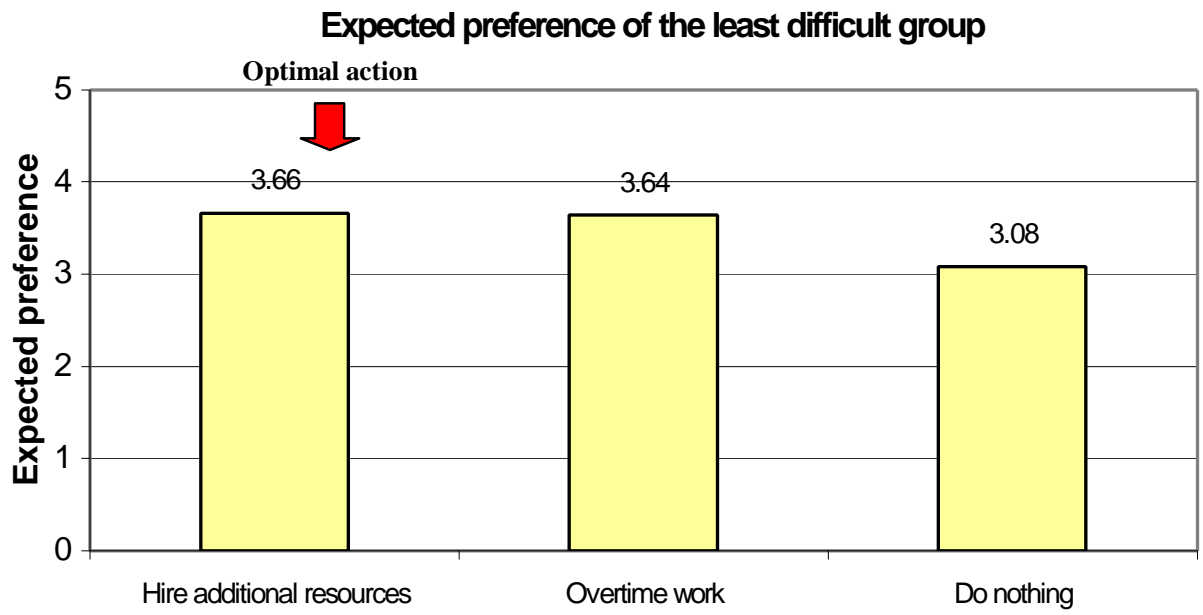

Figure 4.2.2-39. Optimal Action With Expected Preference In The Least Difficult Group

3. The Benefit To Cost Ratio - The action with the largest benefit to cost ratio value is the optimal action. It should be noted that the benefit to cost ratio is not applicable to the action 'Do nothing' since this action has no cost, which means the benefit to cost ratio cannot be calculated. Under the benefit to cost ratio criterion, 'Hire additional resources' is the optimal action alternative in both groups as shown in Figures 4.2.2-40 and 4.2.2-41. 
Benefit to cost ratio of the medium difficult group

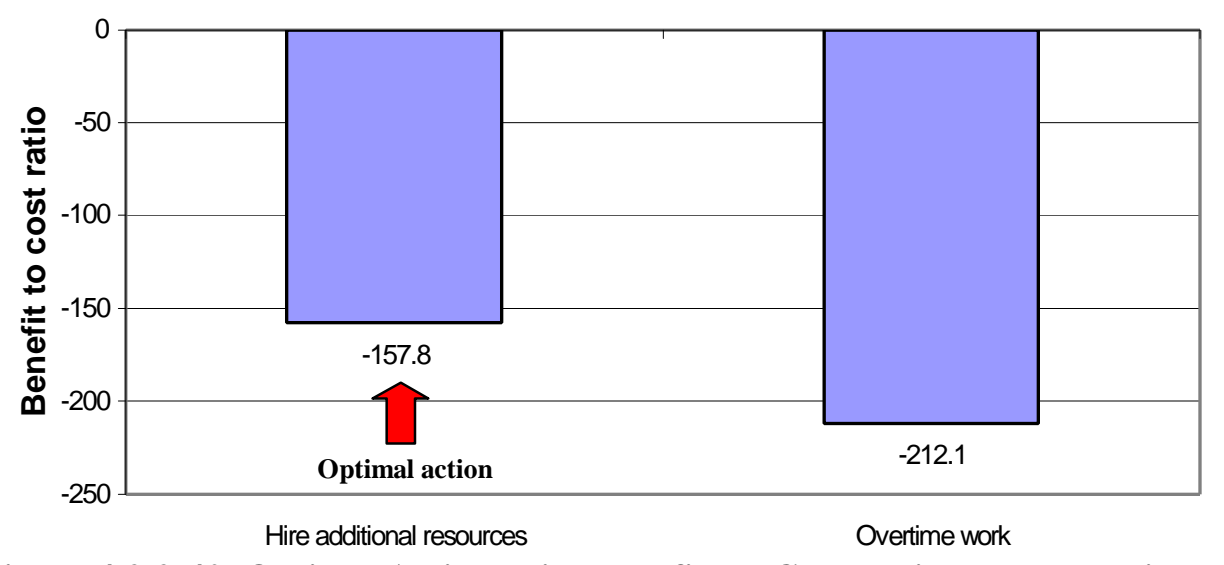

Figure 4.2.2-40. Optimal Action With Benefit To Cost Ratio In The Medium Difficult Group

Benefit to cost ratio of the least difficult group

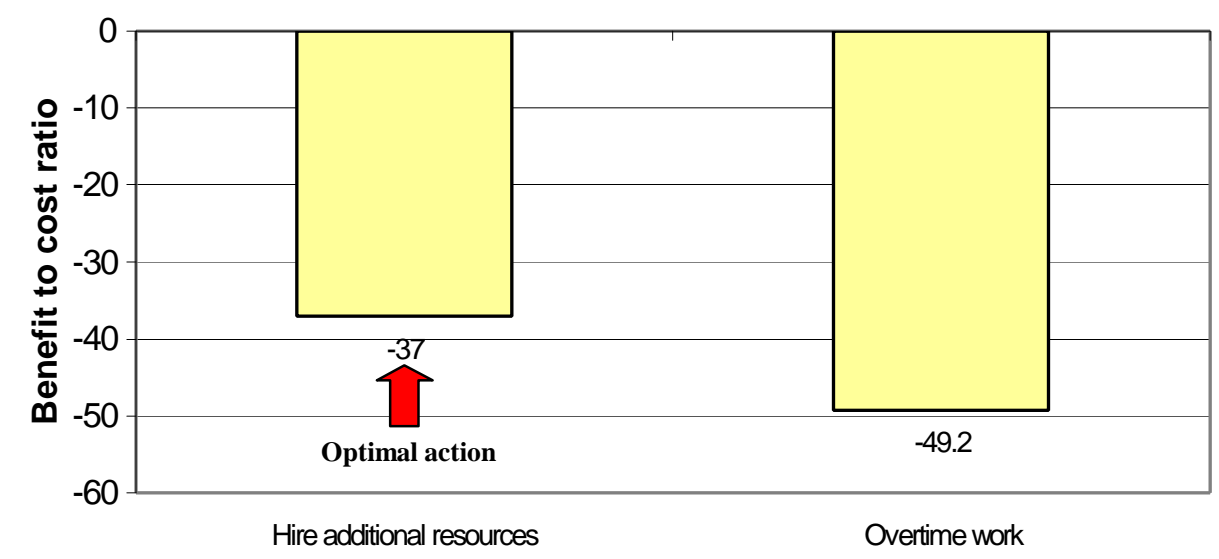

Figure 4.2.2-41. Optimal Action With Benefit To Cost Ratio In The Least Difficult Group

\subsubsection{Applicability Of Current Advisory System To Another Field}

As mentioned earlier, one of problems in the BBN approach is the very large number of conditional probability distributions for describing the strength of dependent relationships among variables. During the process of developing BBN models, obtaining the conditional probability distributions for variables is a very important step since those values govern the behavior of the BBN-based advisory system. In addition, the variables, structure and obtained corresponding conditional probability distributions in the models are likely to be problem specific. As a result, obtaining and adjusting conditional probability distributions are time consuming, slow and require very careful work and continuous iterative communications with domain experts. Therefore, the applicability of currently developed structure, variables and corresponding conditional probability distributions can be an issue when the developed advisory system is applied to other problems or when developing the same type of advisory system for other problems. The application area of the current advisory system in this work is a steam generator replacement project management. When the currently developed advisory system is applied to another problem, such as nuclear 
power plant construction projects or civil construction projects, all steps must be repeated from the very beginning if the level of applicability of currently developed models is not known. Then, it will take a long time and be very cumbersome work. If the level of applicability of current models (i.e., how many or which variables and corresponding conditional probability distributions can be applicable to other problems) is known, then much less time and effort is needed compared to starting from the very beginning. The purpose of this analysis is to identify the variables and corresponding conditional probabilities in currently developed models that can be used in other application fields and hence, to reduce the time and efforts in development of similar advisory systems in the future.

\section{Questionnaire}

1. Following are the input and output variables considered in currently developed advisory system for steam generator replacement project management. For each variable, what is the possibility that a variable would be important in modeling if the current advisory system is applied to specified another area problem? Please enter the estimated possibility in the parenthesis corresponding each variable name below using the scale in Figure 4.2.2-42 at the end of this questionnaire.

\section{For Nuclear Power Plant Construction Project}

- $\quad$ Input variables

1) Time ( )

2) Cost ( )

3) Quality ( )

4) Scope ( )

5) Client satisfaction ( )

6) Human resource ( )

7) Material resource ( )

- $\quad$ Output variables

1) SPI (Schedule Performance Index) ( )

2) CPI (Cost Performance Index) ( ）

\section{$\underline{\text { For Traditional Civil Construction Project }}$}

- $\quad$ Input variables

1) Time $(\quad)$

2) Cost ( ) 

3) Quality ( )
4) Scope ( )
5) Client satisfaction ( )
6) Human resource ( )
7) Material resource ( $)$
- $\quad$ Output variables
1) SPI (Schedule Performance Index $) \quad(\quad$ )
2) CPI (Cost Performance Index) ( $\quad)$

2. Following are the input and output variables considered in a currently developed advisory system for steam generator replacement project management. For each variable, the behaviors are described by corresponding conditional probability values. What is the confidence on those current conditional probability values for each variable can describe the behaviors properly if the current advisory system is applied to another area problem? Please enter the estimated confidence in the parenthesis corresponding each variable name using the scale in Figure 4.2.2-42.

\section{For Nuclear Power Plant Construction Project}

- $\quad$ Input variables

1) Time ( )

2) Cost ( )

3) Quality ( )

4) Scope ( )

5) Client satisfaction ( )

6) Human resource ( )

7) Material resource ( )

- $\quad$ Output variables

1) SPI (Schedule Performance Index) ( $\quad$ )

2) CPI (Cost Performance Index) ( ）

\section{$\underline{\text { For Traditional Civil Construction Project }}$}

- $\quad$ Input variables

1) Time ( )

2) Cost ( )

3) Quality ( ) 

4) Scope ( )
5) Client satisfaction ( $\quad)$
6) Human resource ( )
7) Material resource ( )
- $\quad$ Output variables
1) SPI (Schedule Performance Index $) \quad(\quad$ )
2) CPI (Cost Performance Index) ( )

3. Following are the management actions considered in a currently developed advisory system for steam generator replacement project management. For each management action, what is the possibility that an action would be important if the current advisory system is applied to another area problem? Please enter the estimated possibility in the parenthesis corresponding each action name below using the scale in Figure 4.2.2-42. In addition, if any other actions other than those listed below should be included, please specify them.

\section{For Nuclear Power Plant Construction Project}

1) Hire people from job market ( )

2) Hire people from contractor ( )

3) Overtime work ( )

4) Layoff people ( )

5) Resource transfer ( )

6) Scope change ( )

7) New technology introduction ( )

8) Equipment reduction ( )

9) Do nothing ( )

Actions should be included:

\section{For Traditional Civil Construction Project}

1) Hire people from job market ( )

2) Hire people from contractor ( )

3) Overtime work ( )

4) Layoff people ( )

5) Resource transfer ( )

6) Scope change ( )

7) New technology introduction ( ) 
9) Do nothing

Actions should be included:

Figure 4.2.2-42. Possibility (Or Confidence) Scale Using The Verbal Terms

$\begin{array}{ccccc}\text { Highly unlikely } & \text { Unlikely } & \text { Even } & \text { Likely } & \text { Highly likely } \\ \sim 10 \% & \sim 30 \% & \sim 50 \% & \sim 70 \% & \sim 90 \%\end{array}$

\section{Survey results for applicability level of current model to other fields}

This survey has been given to several human experts so the results in the following figures represent the average value of their answers. In this analysis, the weighted average approach is not selected because it is very difficult to determine the weight of each human expert. Therefore, it is assumed that every human expert has an equal weight to each other.

Levels of applicability of variables considered in the current model to another problem domain are shown in Figure 4.2.2-43 (nuclear power plant construction project) and in Figure4.2.2-44 (traditional civil construction project). As expected, the level of applicability to nuclear power plant construction project shows a higher value than that of the civil project. This is because nuclear power plant construction has more similar aspects with the steam generator replacement work in the same nuclear field, which is the application problem of the current model. Four variables, time, cost, SPI and CPI show around $90 \%$ applicability because time and cost schedules are the factors that the project managers are concerned about most among all types of project: time and cost for input vs. SPI and CPI for output variables. The other variables (i.e., quality, scope, client satisfaction, human resource and material resource) show over $50 \%$ of applicability level in both problem domains.

Applicability to nuclear power plant construction project

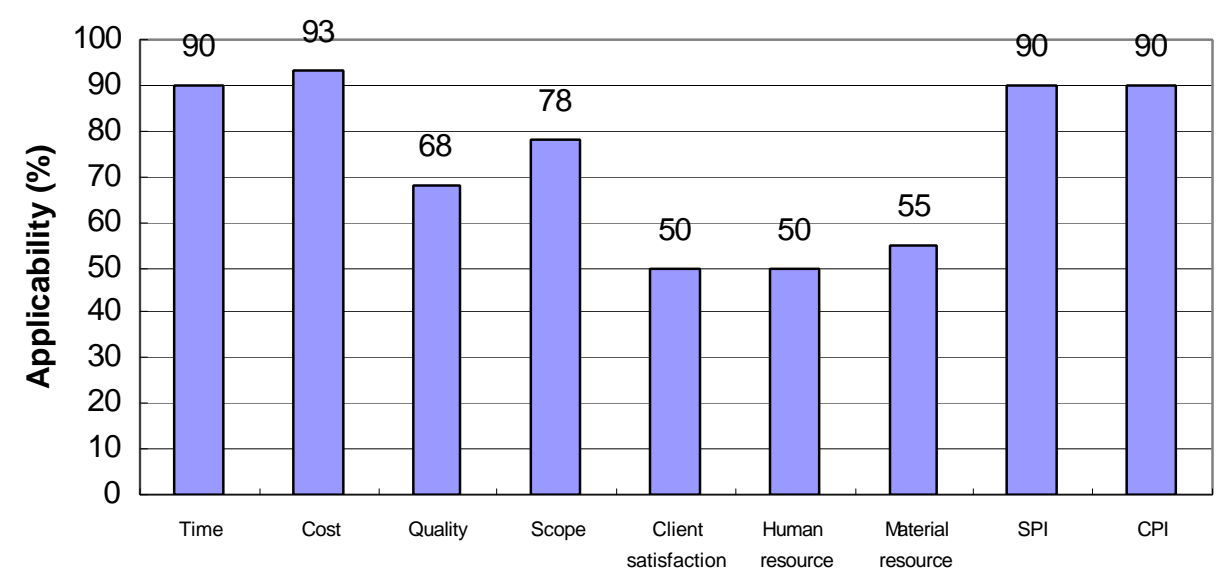

Figure 4.2.2-43. Applicability Of Variables To Nuclear Power Plant Construction Project 


\section{Applicability to civil construction project}

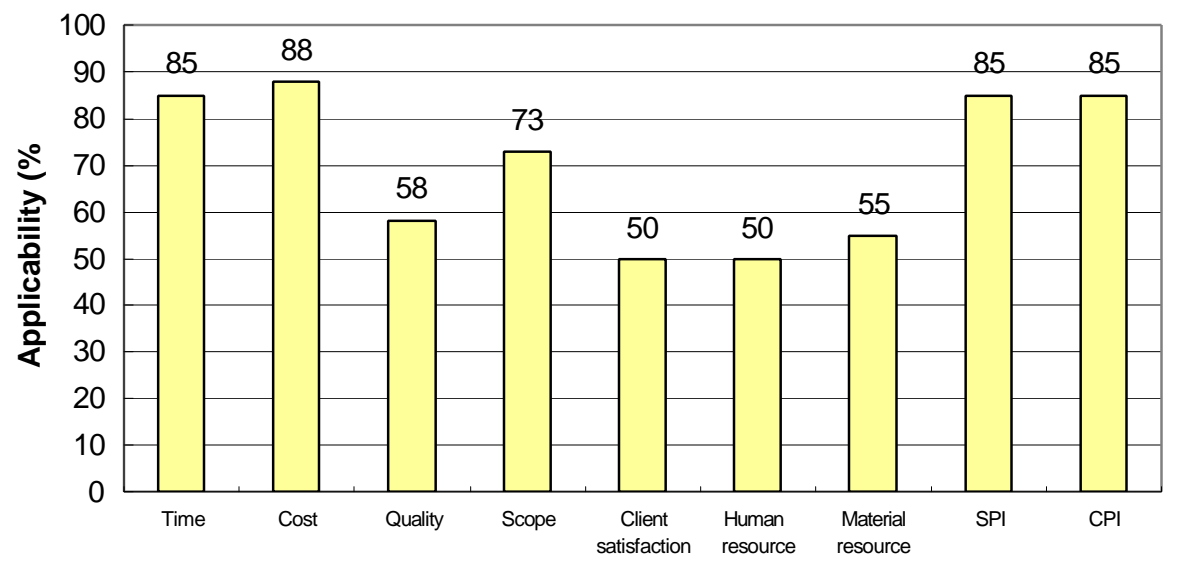

Figure 4.2.2-44. Applicability Of Variables To Civil Construction Project

In both problem domains, the conditional probability values assigned to the current model can be used again with high confidence level, at least over 50\% as seen in Figures 4.2.2-45 and 4.2.2-46. Like applicability of the variables case, the confidence level of current probability values show slightly higher in nuclear power plant construction than that of civil area due to more similar aspects. In addition, the confidence for time and cost schedule variables show higher level than for the other variables (i.e., quality, scope, client satisfaction, human resource and material resource).

\section{Confindence in probability values to nuclear power plant construction} project

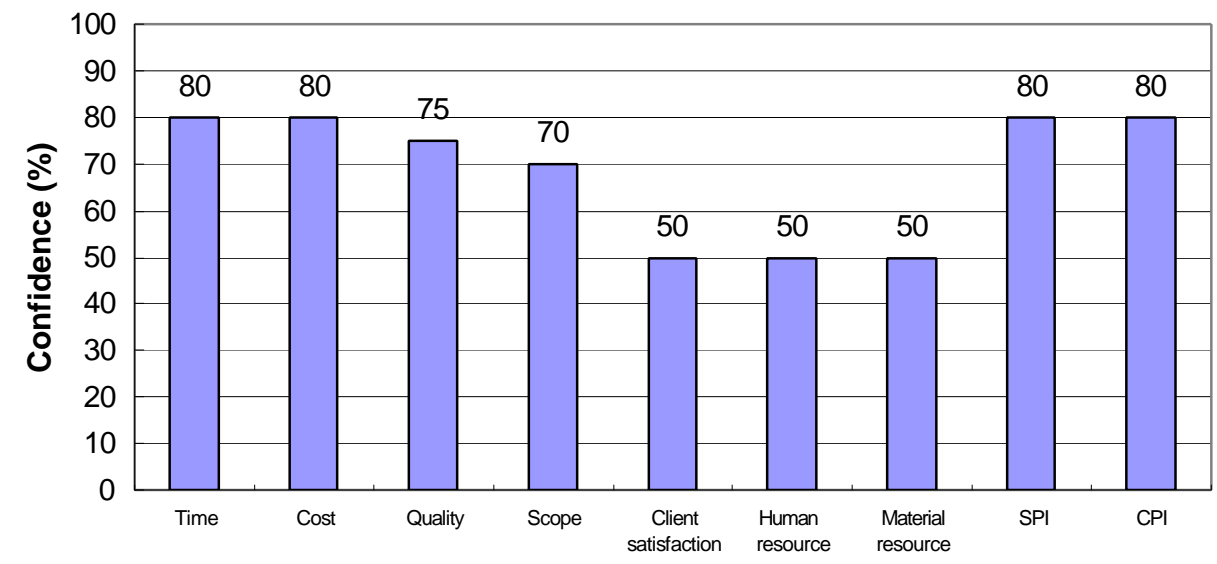

Figure 4.2.2-45. Confidence In Probability Values To Nuclear Power Plant Construction Project 


\section{Confindence in probability values to civil construction project}

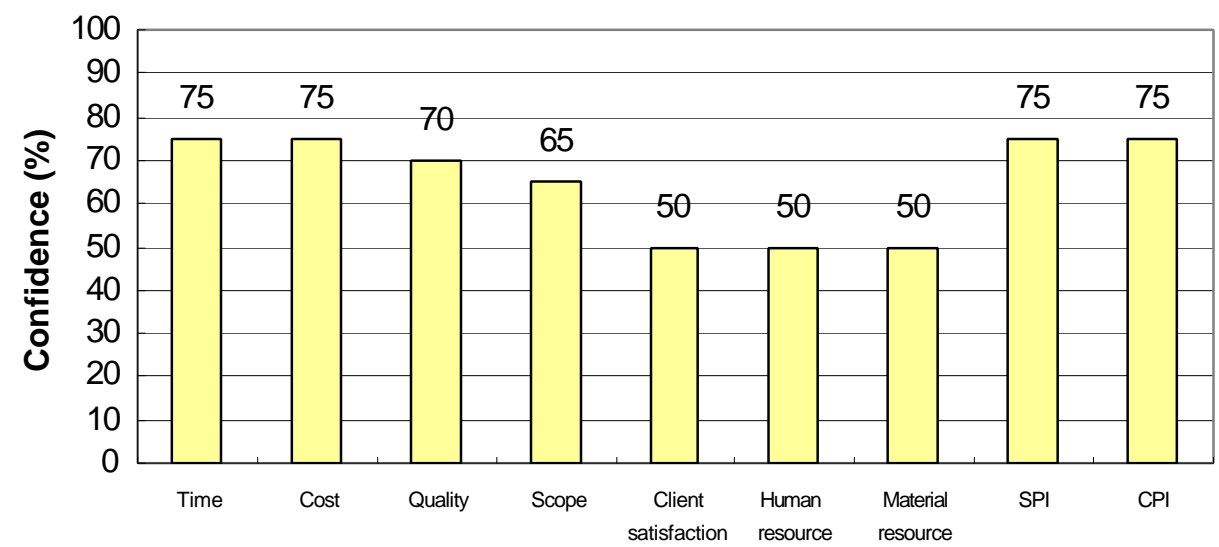

Figure 4.2.2-46. Confidence In Probability Values To Civil Construction Project

All action alternatives considered in the current model show applicability levels over 50\%, except 'New technology introduction'. Among them, the applicability levels for the action alternatives related to resources (i.e., human or material) appear high in both problem domains as seen in Figures 4.2.2-47 and 4.2.2-48: e.g., Hire from contractor, Overtime work, Layoff and Resource transfer. This is because most troubles in the project are from human or materials problems and hence, the action alternatives, which can work effectively and timely, are related to human or material. Note that 'Hire from job market' shows a lower value than 'Hire from contractor' since it takes more time for hiring effect on the project (more time to hire and more time to be familiar to work).

Applicability to nuclear power plant contruction project

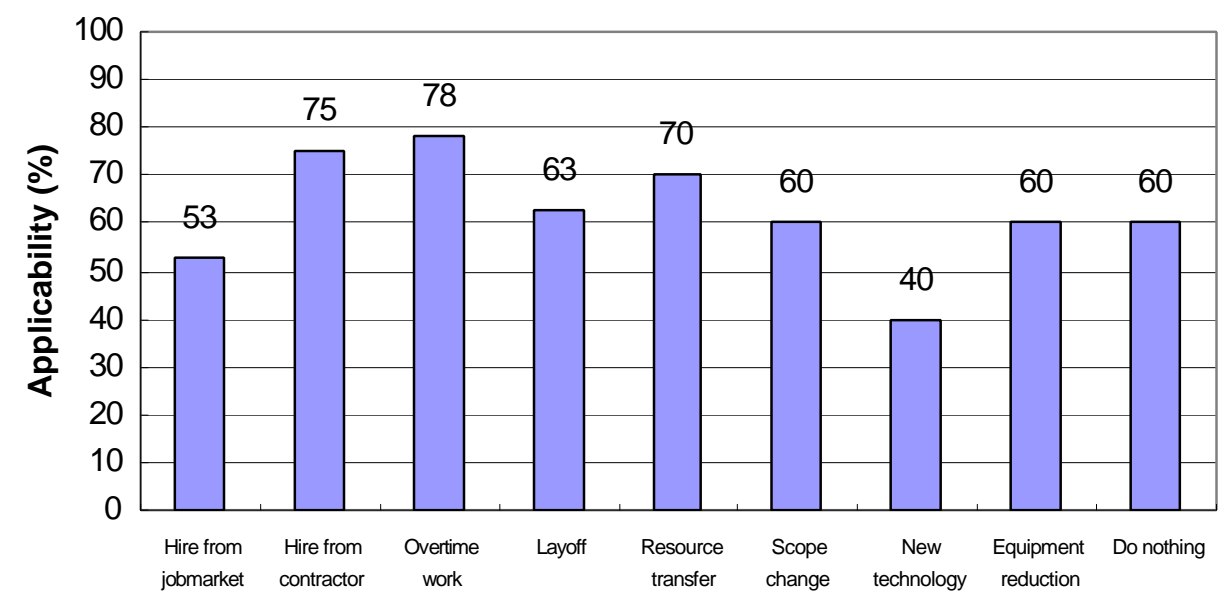

Figure 4.2.2-47. Applicability Of Actions To Nuclear Power Plant Construction Project 


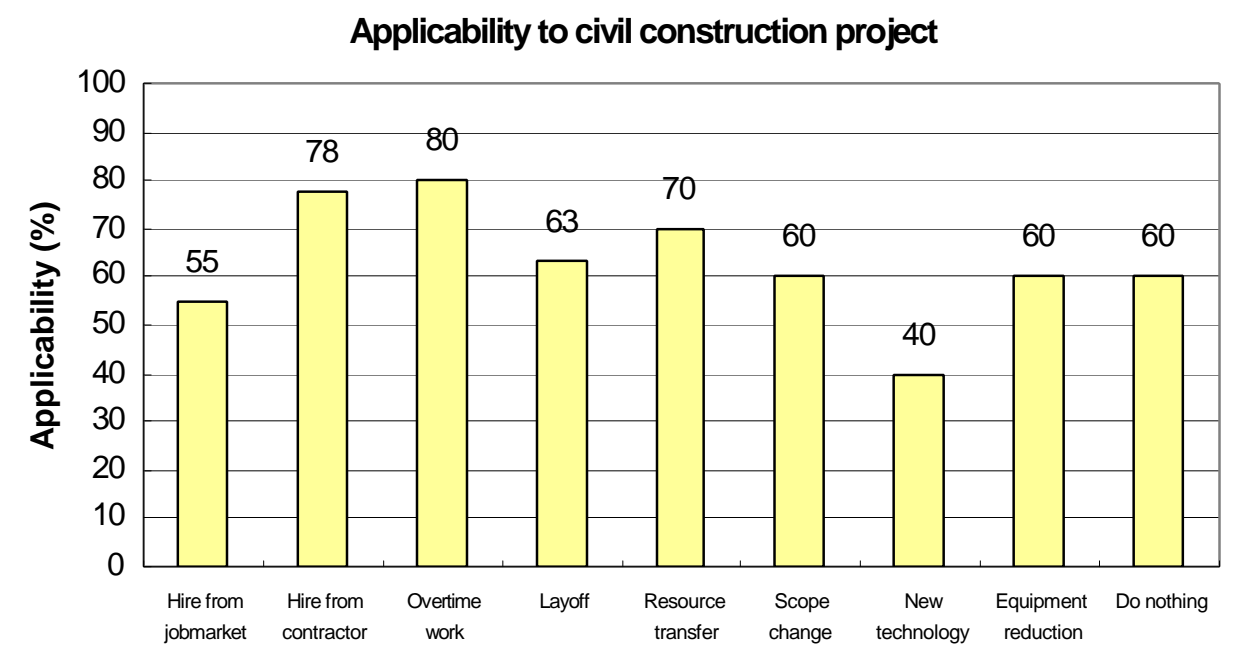

Figure 4.2.2-48. Applicability Of Actions To Civil Construction Project

\subsubsection{Uncertainty incorporation}

Although the advisory system developed using the BBN approach considers many sources of uncertainty in project management problems, there are still remaining uncertainties unresolved, such as the uncertainty in the cost and benefit information and current evidence. The uncertainty in cost and benefit information is already incorporated in the current advisory system. As depicted in long-term benefit (or penalty) probability distributions, the uncertainty in cost and benefit information is expressed in the form of probability distributions and results in the expected average values (i.e., the immediate expected net benefit and the expected long-term benefit). Also, the current advisory system displays the resulting net benefit probability distributions.

For the uncertainty in current evidence, suppose one of the project performance indices shows a single value from the monitoring system. However, this value may not be $100 \%$ certain since there are many sources of uncertainties affecting the project performance index value, such as those in measurements of data, in entering the data into computer systems, in communications among other systems and so on. Therefore, there may be some possibilities that the true state of the performance index value is not that indicated (i.e., there might be a non-zero probability for other state values of the index). The best way to handle this issue is to express the current evidence in the form of a probability distribution function, as in the case of uncertainty in cost and benefit information. Table 4.2.2-13 shows one survey result concerning the uncertainties in our knowledge of the current evidence. It should be noted that this survey was conducted with domain experts, based upon the assumption of use of a very highly reliable monitoring system. This survey has been performed for three states of the project performance index as shown in Table 4.2.2-13: 1) Pessimistic case - index value in range of $0.6 \sim 0.8,2$ ) Neutral case - index value in range of $0.8 \sim 1.0$ and 3) Optimistic case - index value in range of $1.0 \sim 1.2$. For example, the Neutral case in Table 1 shows that there are probabilities of 5\% for the other states of the index (i.e., $0.6 \sim 0.8$ and $1.0 \sim 1.2$ ) although the monitoring system indicates the index to be in state of $0.8 \sim 1.0$ with very high reliability. 
Table 4.2.2-13. Uncertainties In Current Evidence

\begin{tabular}{|l|l|l|l|l|}
\hline \multirow{2}{*}{$\begin{array}{l}\text { Pessimistic } \\
\text { case }\end{array}$} & Index state & $0.6 \sim 0.8$ & $0.8 \sim 1.0$ & $1.0 \sim 1.2$ \\
\cline { 2 - 5 } & Probability & 0.95 & 0.05 & 0 \\
\hline \hline \multirow{2}{*}{$\begin{array}{l}\text { Neutral } \\
\text { case }\end{array}$} & Index state & $0.6 \sim 0.8$ & $0.8 \sim 1.0$ & $1.0 \sim 1.2$ \\
\cline { 2 - 5 } $\begin{array}{l}\text { Optimistic } \\
\text { case }\end{array}$ & Probability & 0.05 & 0.9 & 0.05 \\
\cline { 2 - 5 } & Index state & $0.6 \sim 0.8$ & $0.8 \sim 1.0$ & $1.0 \sim 1.2$ \\
\hline
\end{tabular}

Based upon the uncertainties of current evidence as shown in Table 4.2.2-13 and the 100\% certain evidence value assumption (which was previously used in this work), the error bounds for the predictive mode BBN case example have been calculated again as seen in Figures 4.2.2-49 and 4.2.2-50. The size of the error bound in Figures 4.2.2-49 and 4.2.2-50 expresses the difference between two output project performances, resulting from two cases of current evidence: 1) the $100 \%$ certain current evidence case and 2) the uncertain current evidence case in Table 4.2.2-13. Due to the small uncertainty survey result for the current evidence, the error bounds in future project performance probability distributions are not large; the largest error bound size is around 0.02 (or 2\%). However, the larger uncertainty in current evidence will result in the larger size error bounds in output project performance probability distributions. Also, these error bounds can be a measurement for the uncertainty in conditional probability values in the current BBN advisory system since the resultant project performance probability distributions already incorporate the uncertainty in conditional probability values. Note that the framework of incorporating uncertainty used here can be applied to all analysis results in this work and the predictive mode case example is selected as an example of how to apply this framework to other problems in this work.

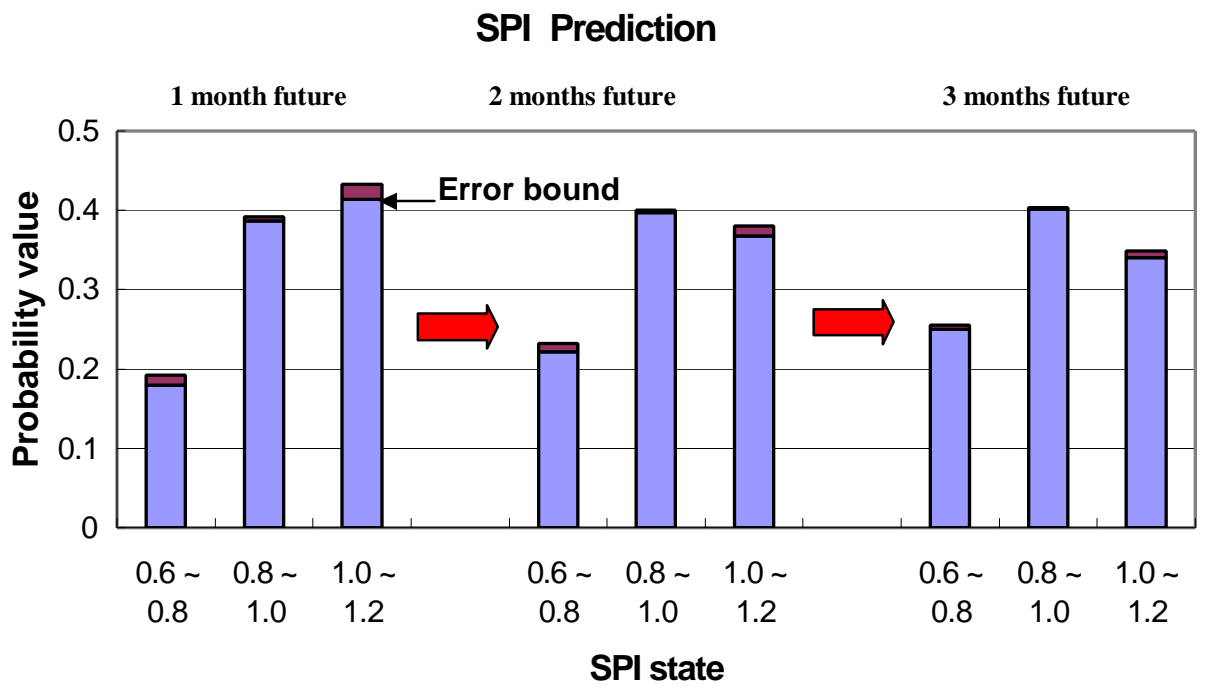

Figure 4.2.2-49. Error Bound In SPI Prediction Probability Distributions 


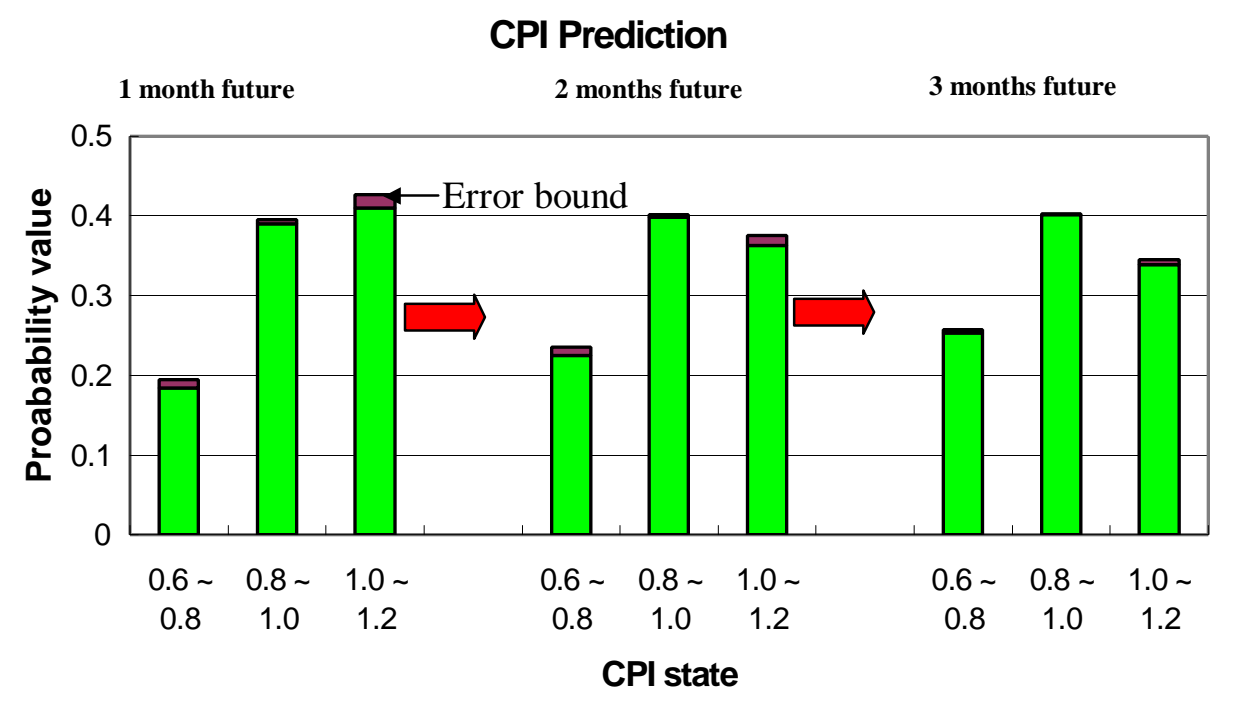

Figure 4.2.2-50. Error Bound In CPI Prediction Probability Distributions

As in the previous predictive mode case study, the mean values of project performance prediction probability distributions are computed and compared to real history values as seen in Figures 4.2.2-51 and 4.2.2-52. From the probability distributions, the maximum and minimum mean values can be computed for each time frame and the difference between these two values represents the size of uncertainties in the predictions. As described in Figures 4.2.2-51 and 4.2.2-52, the uncertainties from the current evidence and the conditional probability values are not large as seen in the small difference between the two curves (i.e., maximum and minimum prediction value curves) since the input uncertainty is not large as shown in Table 4.2.2-13. It should be noted that as time goes on, the discrepancy between the real value and the prediction value without management action increases as seen in Figures 4.2.2-51 and 4.2.2-52, because they represent different scenarios: with management action vs. no management action. However, the predictions with management action by advisory mode BBN in Figures 4.2.2-51 and 4.2.2-52 show good agreement with real values. It is believed that the project manager took action to restore SPI and CPI to normal value (i.e., 1.0) around 161 weeks, because the project performance shows behind schedule (i.e. SPI under 1.0) and over budget (i.e., CPI under 1.0) and the real value curve gets better from that time. Since there is no information on actions really taken, it is assumed that the project managers took an action of 'Hire more people' to restore project performance and the time duration needed to take effect was two months. Therefore, the prediction value curves with management action are computed by the predictive mode BBN from 156 to 161 weeks and by the advisory mode BBN from 161 to 169 weeks. 


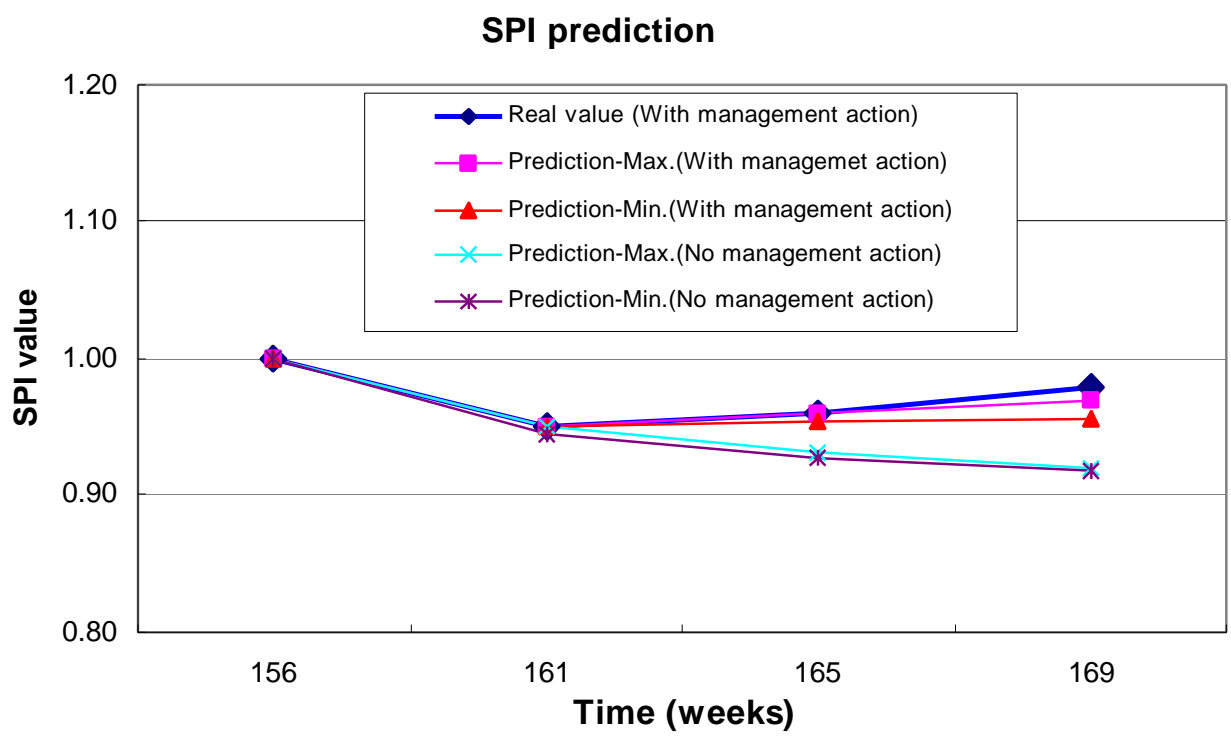

Figure 4.2.2-51. Uncertainty In SPI Prediction In Mean Value Of Probability Distribution

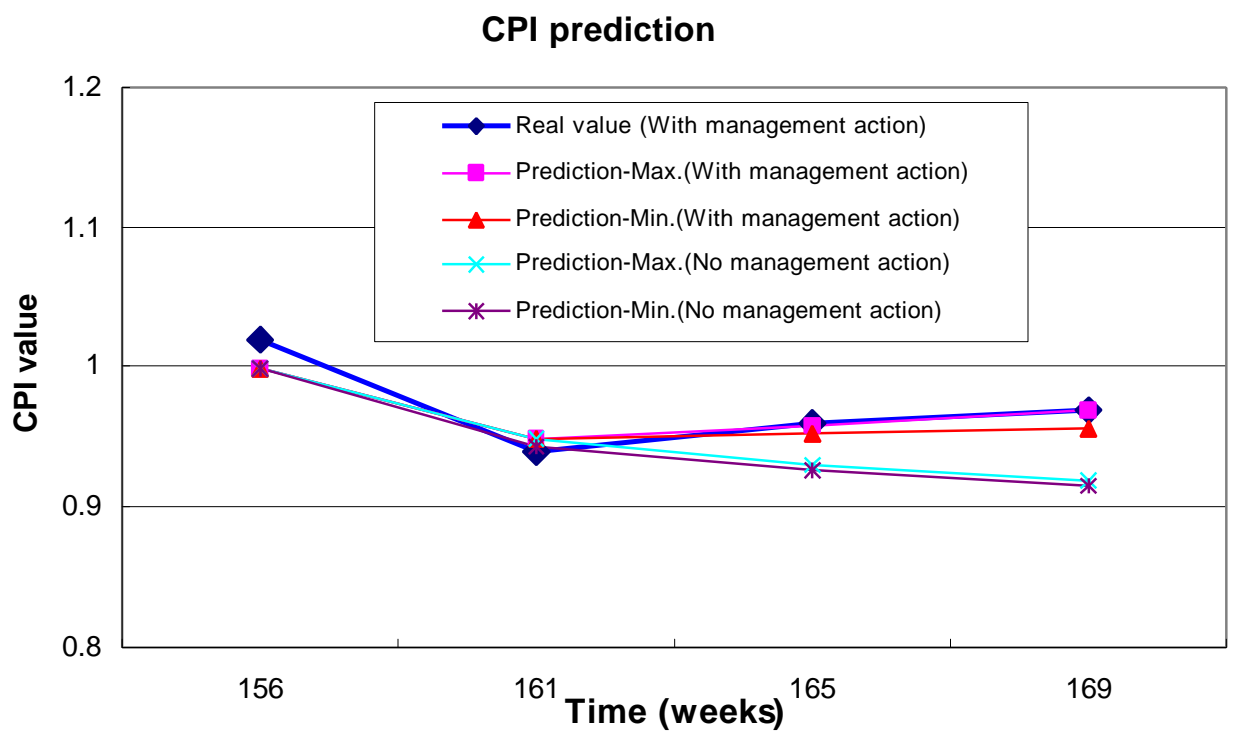

Figure 4.2.2-52. Uncertainty In CPI Prediction In Mean Value Of Probability Distribution

\subsubsection{Conclusions And Future Work}

\subsubsection{Conclusions}

A BBN (Bayesian Belief Network)-based advisory system has been developed in this work. The system will help the Project Managers (PMs) especially in difficult decision-making situations and during the entire project lifetime as well, which is the steam generator 
replacement project that has been selected as an application problem. Based upon the literature survey and discussion with relevant experts, the conclusion is that the BBN approach is superior to artificial neural network or fuzzy-rule approaches in its ability to treat complexities, uncertainty management, systematic decision making, inference mechanism, knowledge representation and model modification for newly acquired knowledge. Therefore, the BBN approach was selected to model the steam generator replacement project management, where the situation holds inherently large uncertainty and complexities.

Different BBN models for the different work phases and the whole project lifetime have been constructed and integrated. During the work process, the communication with the domain experts has been iterated continuously to obtain and to program the expert knowledge base properly. For the BBN models, two modes of advisory system have been constructed for the above purposes. First mode, the predictive mode was developed, which can predict future project performance state probability distributions, assuming no any intervening management action. It is necessary to evaluate whether a future project performance state will be in good shape. If the bad project performances will be predicted in the near future, then the project manager should identify the problems and take proper action promptly to correct them. The second mode is the advisory mode that can identify the optimal action from alternatives based upon the expected net benefit values that incorporate two important components: 1) immediate expected net benefits at post-action time and 2) the expected long term benefit (or penalty) at scheduled project completion time. The long term benefit (or penalty) comes from the costs of the domino effect that originates from overdue provision of deliverables, lost reputation cost, and so on, which are not considered in the immediate benefit of an action taken.

The full accurate costs and benefits of action alternatives (i.e., immediate net benefit and long term benefit (or penalty)) cannot be completely determined until the completion of the project since the time scale for evaluating an action is both short and long terms.

During the work, the new indices for some of the identified important variables (i.e., cost, quality, scope, client satisfaction, human resources and material resources) have been newly developed for effective and efficient project status monitoring. With application of developed indices to the advisory system, the long-term benefit (or penalty) was found to be the most important factor in determining the optimal action by the project management during the decision-making process and domain experts confirmed this fact. As a result, the effort has been focused on incorporating the long-term benefit (or penalty) concept in order to provide more reliable and accurate advice to the project manager. Finding proper input factors in different BBN models for different work phases and entering evidence to them and finding corresponding updated outputs may be cumbersome tasks because there are many factors in many BBN models for a individual work phase and for overall project lifetime. In order to overcome these inconveniences and to facilitate the communication between the BBN models and the users, an interface program has been developed using the Visual Basic language.

With help of the interface programs, a developed advisory system has been benchmarked for both BBN modes (i.e., predictive and advisory modes) with two information sources: 1) real case data and 2) agreement of domain human experts. In the case of real case data, the results of the developed advisory system were compared to real case data. When it was not possible to obtain real case data for benchmarking, the domain human experts confirmed 
the results. The results of currently developed advisory system showed good agreement with either real case data or domain experts' opinions.

When the developed advisory system is applied to the nuclear power plants construction project, the project managers will be able to manage the work more effectively and efficiently because it enhances the control in time, costs, quality and reliability of the projects. Hence, one of the major elements of electricity cost, the capital cost of nuclear power plants will be reduced. As a result, the nuclear power plants will have more competitive power in the electricity market.

\subsubsection{Future Work}

Future efforts should include the four items listed below. Theses are either extension of this work or work needed to complete the efforts undertaken in this study.

First, we need more real case information for benchmarking the advisory system developed in this work. For example, the advisory mode BBN has nine action alternatives but the benchmark has not been performed on the behaviors of all nine actions, only on three action alternatives, because of the insufficient real case data. Since the behavior of three action alternatives showed good agreement with real case data, it was concluded that the structure of BBN model and the conditional probability values for these three action alternatives are good. Nevertheless, more real case data is needed for benchmarking the behaviors of remaining action alternatives.

Second, it was found in this work that the long-term benefit (or penalty) of taken action is the most important key factor in determining the optimal action during the management process. One of the components of long-term benefit (or penalty) is the reputation value, which is very intangible, difficult to estimate and a large part of the long-term benefit (or penalty). In this work, this reputation value has been evaluated through discussions with human experts in the project management domain who may not be experts in reputation and brand issues. Therefore, we believe that estimating this reputation value with brandconsulting firms can provide more reliable and accurate and decision-making processes.

Third, as discussed in the previous section, the ultimate goal of this work was the development of an advisory system for nuclear power plant construction project management. As a result of the advisory system application, it is anticipated that the reduction of the capital cost of nuclear power plant will result in the more competitive power in the electricity market. In order to achieve this ultimate goal, there is need of modification of currently developed advisory system whose application field is the steam generator replacement project management. It is believed that there will not be much need of modification since the applicability level is high.

Lastly, continuous and extensive testing and validation of the advisory system are essential concerning the adequacy and completeness of our developed advisory system. In particular, real field tests are recommended to ensure the advisory system can perform as intended in the actual project management environment. 


\subsection{A SYSTEM DYNAMICS MODEL FOR NUCLEAR POWER PLANT DESIGN AND CONSTRUCTION PROJECT MANAGEMENT}

This section of the report consists of five parts. Part 4.3.1 describes the research motivation, the problem description, and the research approach. Part 4.3.2 introduces the System Dynamics technique and various aspects of the underlying project system; Part 4.3.3 shows in detail the development of the model; In Part 4.3.4, a case study is presented and results are discussed; Conclusion is drawn in Part 4.3.5.

\subsubsection{Introduction}

\subsubsection{Motivation for the Research}

Historically, nuclear plant construction and operation have been vulnerable to costly interruption because of regulation changes, engineering and management problems, and public opposition. Figures 4.3.1-1 and 4.3.1-2 show the comparisons between estimated and actual lead-time and construction costs of nuclear power plants in operation by the end of 1986.

Besides the safety concerns, the high capital cost of nuclear energy is a big contributor to the two-decade hiatus in new plant construction in the U.S. For new nuclear capacity to be constructed, the capital costs must be reduced to be competitive with those of non-nuclear energy sources. One way to reduce the capital cost is to reduce the 'time-to-market' by shortening the construction duration with the aid of information technology and new project management tools. A significant improvement can be expected with shorter 'timeto-market' because of reduced upfront costs. The labor costs will be reduced substantially under shorter duration, and the shorter 'time-to-market' will expose the projects much less to the volatile change environment, which often bring surprises and incur unexpected costs.

The work of this research is aimed to provide a project management tool to help better understand and manage complex projects. With this tool and other technologies available, the project managers hopefully will be able to manage the construction projects more efficiently and more effectively to increase the probability that the project can be completed on schedule and within budget. 


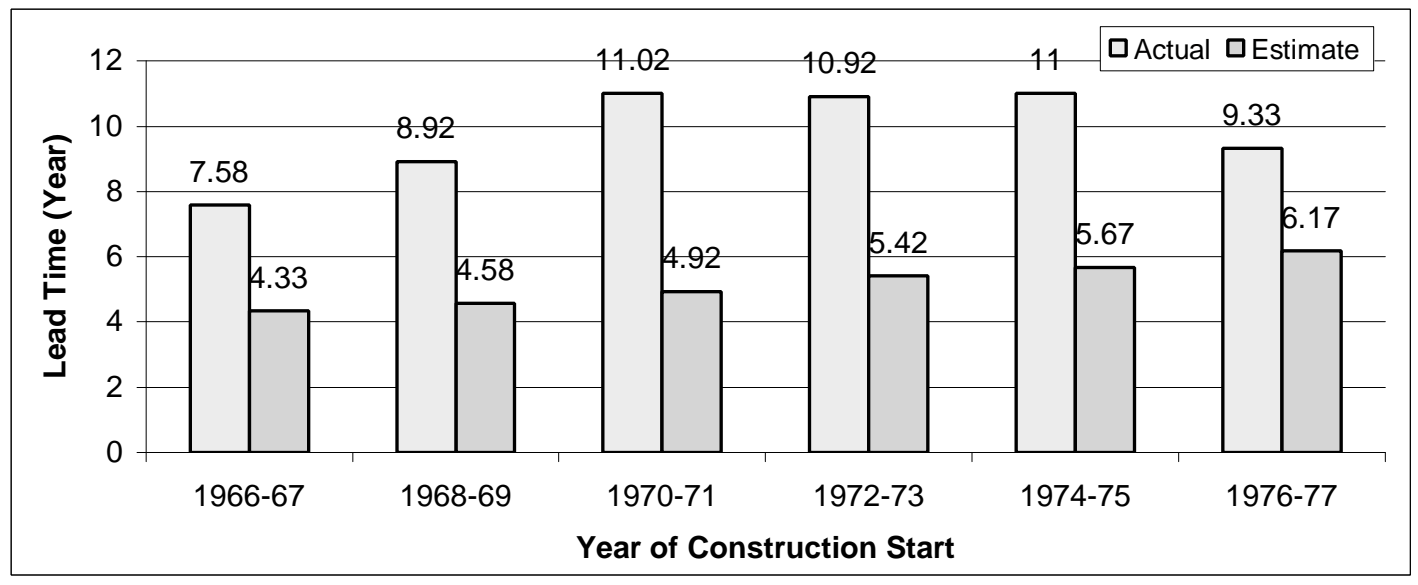

Figure 4.3.1-1: Actual And Estimated Lead-Times Of Nuclear Power Plants In Operation By The End Of 1986 In USA

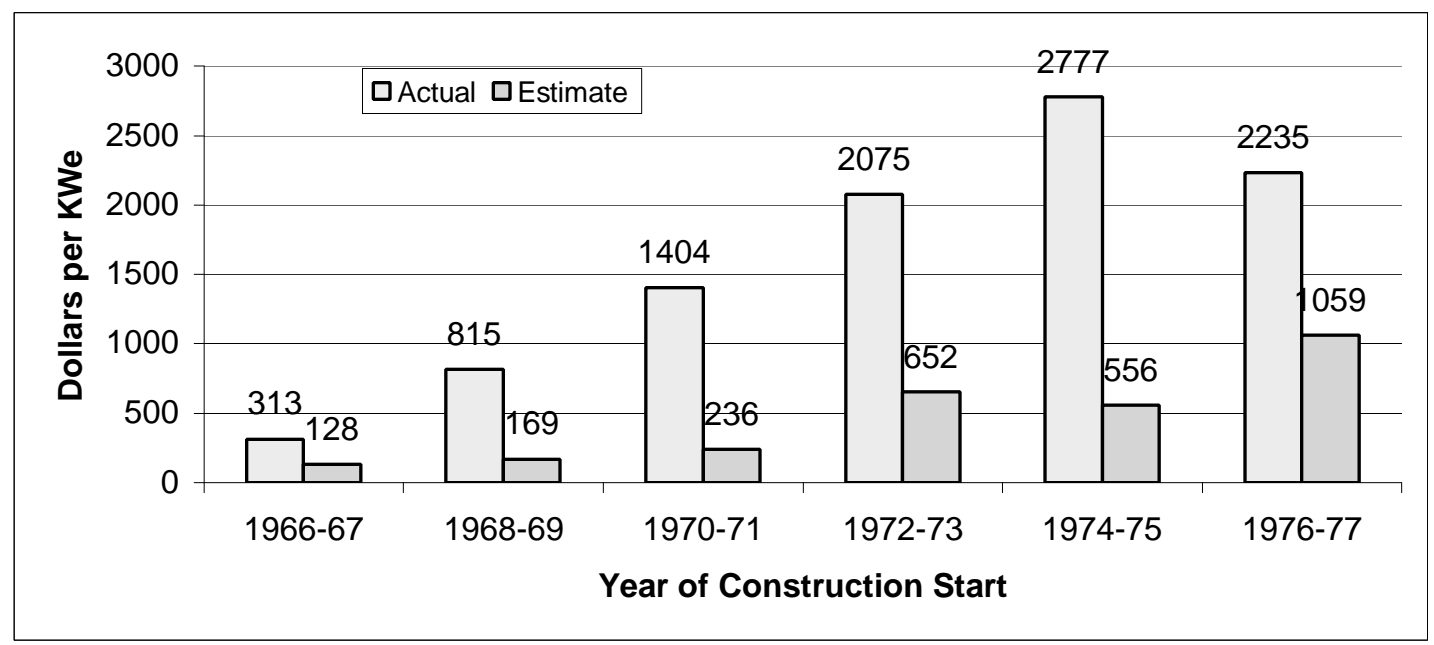

Figure 4.3.1-2: Actual And Estimated Time-Related Costs In Mixed Current Dollars For Nuclear Power Plants In Operation By The End Of 1986 In USA

Source: Computation based upon data from Energy Information Administration, From EIA-254, "Semiannual Progress Report on Status of Reactor Construction," and predecessor survey forms

Note: Estimates made at construction start were used. Estimated time-related costs were computed from data reported by the utility.

\subsubsection{Problem Description}

The underlying problem surveyed by this research is the failure of project managers to fully recognize and utilize the dynamic features of projects, which often drive project performance. All factors external to the project, including changes, impact the project through interaction between elements in the underlying system. 
Much of the cost and schedule overruns involved in nuclear power plant constructions can be attributed to changes. Historically, only a very small fraction of existent nuclear power plants in the USA were constructed as scheduled, even in an approximate sense. One important reason for the overruns is that the project managers were not equipped well enough to deal with problems that were incurred due to changing conditions in these very large and complex construction projects. Because of the large number of feedbacks involved, together with many non-linear, coupled relationships, the implications of these changes were often omitted and generally beyond a person's ability to delineate without any tools.

Figure 4.3.1-3 is an example of a causal loop diagram representing the feedback loops of an 'overtime' management action in response to a possible schedule overrun, which is probably caused by a regulation change that requires removal of part of the completed work and performing it under new specifications.

In causal loop diagrams, causal links (arrows) are labeled positive signs (+) if the variable at the arrowhead move in the same direction as the variable at the arrow's tail, while they are labeled negative signs (-) if the variable at the arrowhead move in the opposite direction as the variable at the arrow's tail, when other factors are held constant. Feedback loops are labeled as balancing (-) if variable values tend to be goal-seeking over repeated passes around the loop or reinforcing $(+)$ if repeated passes accelerate movement in a single direction.

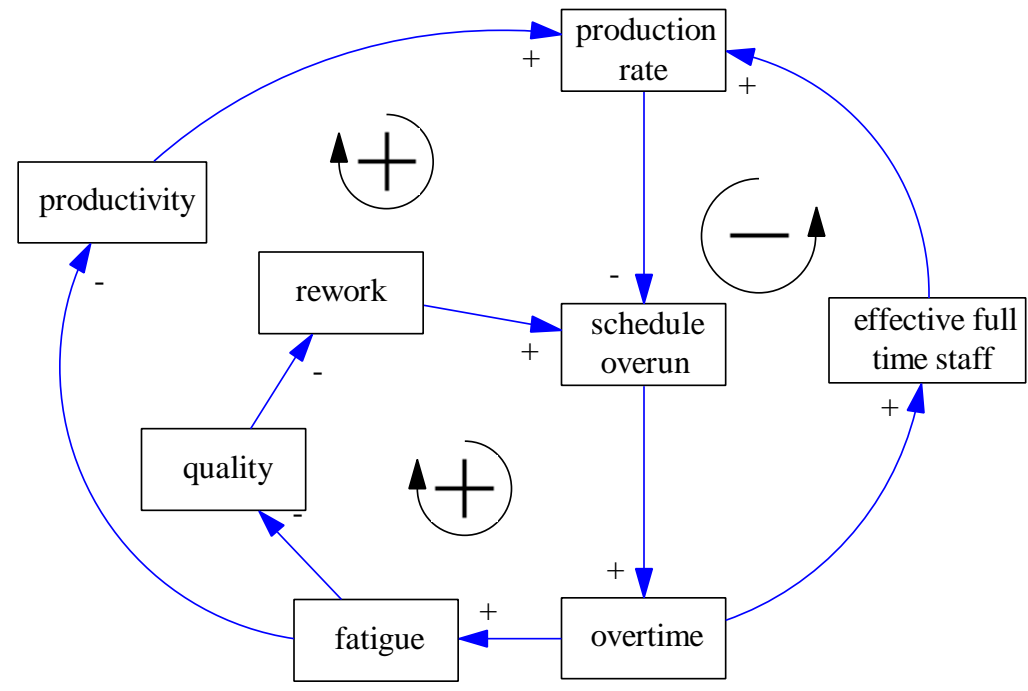

Figure 4.3.1-3: An Example Of A Causal Loop Diagram Representing Three Feedback Loops Following An 'Overtime' Management Action In Response To Schedule Overrun

A common managerial response to the schedule overrun is to use overtime to speed up the production rate and thereby reduces the schedule gap. This is the balancing loop shown on the right part of Figure 4.3.1-3. However, the overtime will cause the fatigue on workers, which tends to lower the productivity and quality of the work. As lower productivity means 
lower production rate, and lower quality means more rework, both of these two feedback loops on the left part of Figure 4.3.1-3 tend to offset part of the beneficial effects of the balancing loop, and sometimes the bad effects can be dominant if the overtime last for a long time, in which case even though more salaries are paid, less work is actually completed.

It is hard, if not impossible, for a person to mentally figure out all the effects shown in Figure 4.3.1-3 in a quantitative way. On the other hand, even with the lack of clear insights upon the implications of their actions, the project managers have to make the decisions. Such uninformed decisions tend to bring many new, unexpected problems in the future through underlying feedback mechanisms that the project managers fail to consider in the first place.

\subsubsection{Research Approach}

The work in this research is to provide insights into the underlying system following management actions and unanticipated events through a system dynamics model so that the project managers are able to make informed decisions in a changing environment. The underlying project system, changes, and their implications are modeled and studied using System Dynamics technique. Using this technique and the expertise of domain experts, a model simulating coupled, non-linear processes and feedback relationships in the construction project of a nuclear power plant is produced. The model was calibrated to a Steam Generator Replacement (SGR) project. Based upon the baseline schedule, baseline budget, changes introduced along the way, and actions response to the change, the model can predict the future schedule and financial performance dynamics, thus allowing the project managers to better understand the full implications of changes, their decisions, and so to avoid making wrong judgments.

\subsubsection{Literature Review}

\subsubsection{System Dynamics}

\subsection{Systems Thinking}

Human beings tend to solve problems in a linear way. This approach works well for simple problems. However, as problems become more complex, for example in addressing project management problems that are cross-functional or strategic, it does not work well.

That is why we need to resort to other approaches. The method of systems thinking, or system dynamics, provides us with a tool to better understand these difficult management problems. The system dynamics approach was introduced in the post World War II era by Jay Forrester, a former electrical engineer at MIT, and has been used for over forty years. This approach requires a shift in the way we think about things. In other words, they require that we move away from looking at isolated events and their causes (usually assumed to be some other events), and start to look at the organization as a system made up of interacting parts.

The term 'system' means an interactively interdependent group of entities. In the work presented here, the objective is to study the management processes, and the focus will be 
on systems of people and technology that are involved in the project design and construction processes.

\subsection{System Representation: Levels, Rates, Auxiliary Variables, Data, and Constants}

In order to study the system behavior, it is necessary to firstly represent the underlying system structure using some notation based upon our knowledge about the system. The notation used in system dynamics modeling is often called 'causal loop diagram'. It defines the causal relationships between the system variables using loop diagrams.

Figure 4.3.2-1 shows an example of a causal loop diagram. It describes a simplified workflow in the construction work implementation process. The project will be finished when all the work flows from 'Work to be Done' to 'Work Completed'. The rate of the flow, the 'work completion rate', is determined by 'workforce' and 'productivity' (assuming perfect quality). When the project lags behind schedule (work completed < scheduled work completed), the schedule pressure increases, requiring to hire more workers in order to catch up with original schedule; However, a large size of workforce can produce congestion problem: workers have to sit-and-wait, and this effectively decrease the productivity.

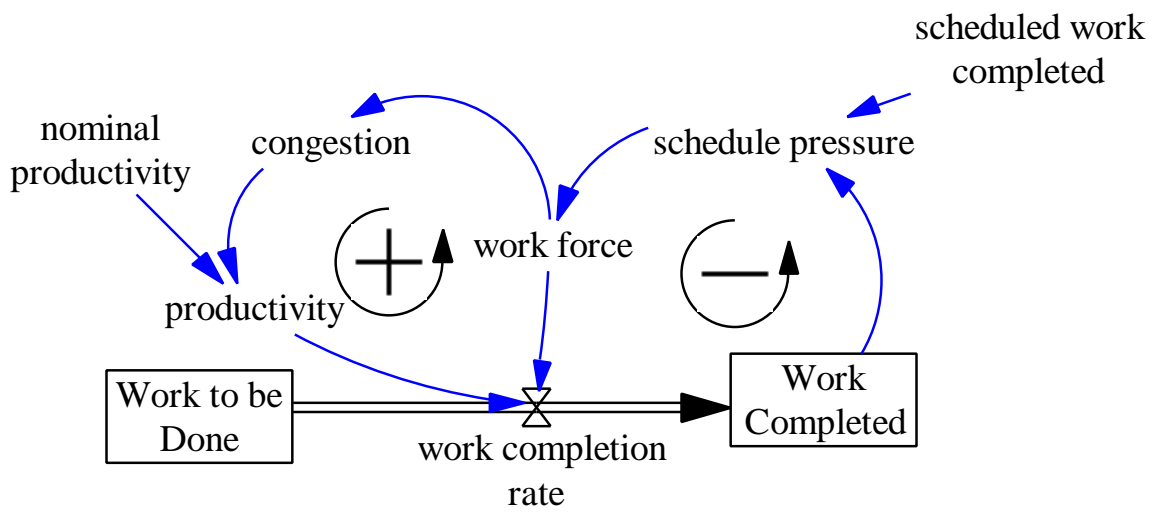

Figure 4.3.2-1: System Structure Example: A Simplified Work Flow Diagram

We will use this typical causal loop diagram to illustrate the different types of variables used in System Dynamics Models: Levels, rates, auxiliary variables, data, and constants.

Levels (also called stocks, accumulations, and states): Levels describe the magnitudes of conserved entities. The magnitudes of the Level variables ultimately determine the dynamic behavior of a system. They have memory and change over time. The values that they take on at any time depend upon the values that they and other variables took at previous time step. The quantities 'Work to be Done' and 'Work Completed' in Figure 4.3.2-1 are Levels. Equation 4.3.2-1 shows how the levels integrate or 'accumulate' based upon the values themselves and other variables in the system:

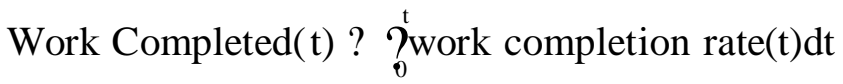

? Work Completed $(\mathrm{t}-\mathrm{dt})$ ? work completion rate $(\mathrm{t}-\mathrm{dt})$ ? $\mathrm{dt}$ 
Rates (also called flows): Rates are the variables that directly change the magnitude of the Levels. They decide the rate of change of the conserved quantities. Rates are essentially the same as Auxiliaries and differ only in the way that they are used in a model. The "work completion rate" in Figure 4.3.2-1 is a rate variable.

Auxiliary: Auxiliary variables provide information needed to calculate the magnitude of the rate variables. They are computed from Levels, Constants, Data, and other Auxiliaries.

Auxiliary variables have no memory, and their current values are independent of the values of such variables at previous times. The "schedule pressure" in Figure 4.3.2-1, for example, is an auxiliary variable. The equation for it is shown in Equation 4.3.2-2.

$$
\text { schedule pressure }(\mathrm{t}) ? \frac{\text { schedule work completed }(\mathrm{t})}{\text { Work Completed }(\mathrm{t})}
$$

Data (also called exogenous variable): Data are input variables that have values that change over time but are independent of anything that happens to other variables. The "scheduled work completed" is a data variable. Its values as a function of time are specified before the simulation. During the simulation, the value of the data variable at current time can be retrieved to calculate other variables.

Constants: These are variables that do not change with time. For example, "nominal productivity" in Figure 4.3.2-1 is a constant variable.

One of the most important features of system thinking is feedback. Feedback is defined as the transmission and return of information. Actually, feedback is no more than a set of causal relationships between some variables, yet as a whole they form a 'loop', or, the information returns to where it originates. Dependent upon the relationships between variables, the returning information can amplify or attenuate the initial information, forming a 'reinforcing' or 'balancing' feedback. For example, in Figure 4.3.2-1, there are two feedback loops. (1) Work Completed (less than scheduled work completed) schedule pressure (increase) work force (increase) completion rate (increase) Work Completed (catch up with the schedule). This is a balancing feedback loop. (2) Work Completed (less than scheduled work completed) es schedule pressure (increase) os work force (increase) es congestion (increase) es productivity (decrease) es completion rate (decrease) $\varangle$ Work Completed (less than scheduled work completed further). This is a 'reinforcing' feedback loop.

The system structure can be constructed with such causal loop diagrams, which link together all the variables involved in the system to indicate the network of their relationships. At the same time, the relationship among the variables is quantified by writing equations for each relationship, which is called "quantification". Equations 4.3.2-1 and 4.3.2-2 are examples of quantification.

When the model is built and quantified, it can then be used as a 'black box'. Based upon inputs entered into the model and its knowledge already built along the process of modeling, the model can (1) provide quantitative, dynamic, and temporal variation of system variables based upon initial conditions, in-process events and corresponding actions input as parameters to the system, and (2) help identify and correct the system defects by testing the system with hypothesized external impacts. Figure 4.3.2-2 shows how system dynamics model works. 


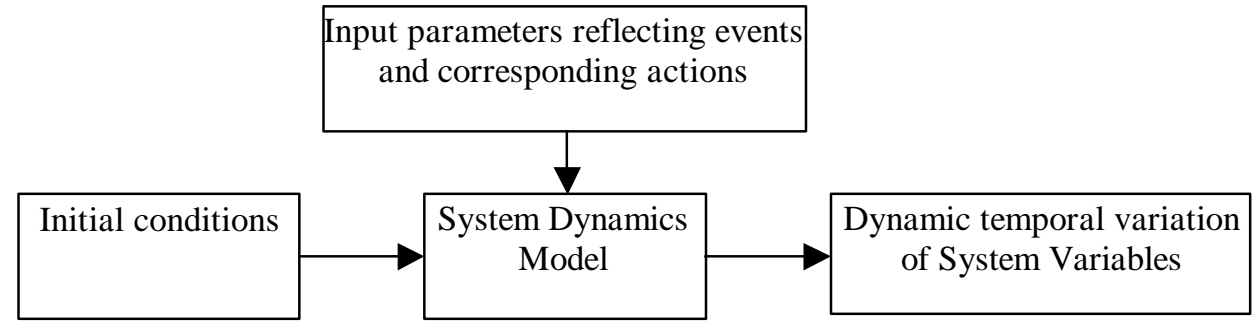

Figure 4.3.2-2: Schematic diagram showing how the system dynamics model works

\subsection{System Dynamics Software}

The methods of system dynamics are general, but their implementation requires the use of specific computer software. A number of different software packages such as Ithink/Stella $^{\circledR}$, DYNAMO $^{\circledR}$, PowerSim $^{\circledR}$, and Vensim ${ }^{\circledR}$ are available to build system dynamics models, and Vensim ${ }^{\circledR}$ is used in the work presented here because: (1) it supports a compact, but informative, graphical notation, (2) the Vensim equation notation is compact and complete, (3) Vensim provides powerful tools for quickly constructing and analyzing process models, and (4) it provides a full Dynamic Link Library functions (Dlls), making it very easy for third-party development platform to integrate its features to develop customized applications.

\subsubsection{Aspects of a Construction Project}

\subsection{Organization}

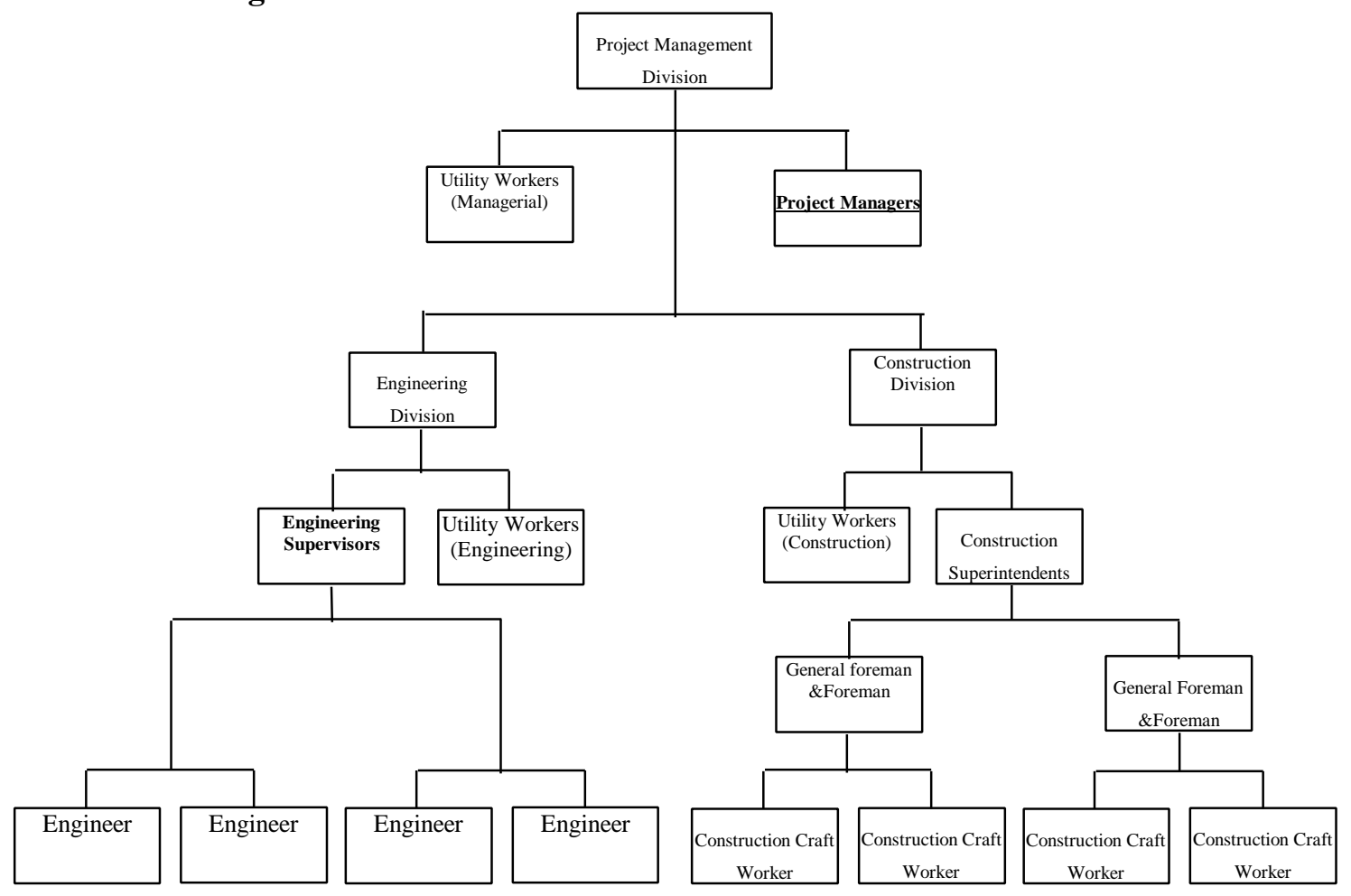

Figure 4.3.2-3: Organization chart 
The organization chart behind the model used in the work presented here is based upon that of a Steam Generator Replacement (SGT) project organization. It is that of the project team of Framatone ANP DE\&S. we also use the management knowledge base and project data from this project team to build and validate the model.

Shown above in Figure 4.3.2-3 are divisions that are directly related to deliverable quantities production (drawings, design specifications, calculations, work packages, construction work units, etc.). Other departments such as those of project control, business, safety, and training are not modeled specifically because these departments only serve as 'helpers' or support of the deliverables production. In this model, their influences on work accomplishment are simply modeled in terms of several factors affecting production. For example, an 'adjustment time' variable, which is defined as the time that it takes to acquire and train a new worker before the worker is permitted to work on site, is used to reflect the efficiency of human resource and up-front training department.

The organization chart therefore consists of three main divisions: The project management, engineering, and construction. The project management division is responsible for the management of the whole project. The engineering management division is responsible for the design phase work and site engineering work. It will be further divided into the design engineering and the field engineering department. The construction division is responsible for all the site preparation, construction, and installation work.

In each department, there are several levels of staff. Management staff has multiresponsibilities competing for the time resources. However, unlike management staff, the responsibilities of the workers and utility workers are sole and straightforward. For workers, their tasks are producing deliverables: drawings, construction units, etc. For utility workers, their tasks are providing help to the staffs in other levels. They include receptionists, secretaries, clerical workers, fire watchers, etc.

Table 4.3.2-1: Routine Management Responsibilities

\begin{tabular}{|l|l|l|l|l|}
\hline \multicolumn{1}{|c|}{ Person } & \multicolumn{1}{|c|}{$\begin{array}{c}\text { External } \\
\text { meetings }\end{array}$} & \multicolumn{1}{c|}{$\begin{array}{c}\text { Internal } \\
\text { meetings }\end{array}$} & \multicolumn{1}{c|}{ Supervision } & \multicolumn{1}{c|}{$\begin{array}{c}\text { Administrative } \\
\text { work }\end{array}$} \\
\hline Project manager & Meet clients & $\begin{array}{l}\text { Meet } \\
\text { subordinates }\end{array}$ & $\begin{array}{l}\text { Plan, Review } \\
\text { subordinates' work }\end{array}$ & Write report \\
\hline Department manager & $\begin{array}{l}\text { Meet clients, } \\
\text { executives from } \\
\text { headquarter }\end{array}$ & $\begin{array}{l}\text { Meet } \\
\text { superintendents } \\
\text { supervisor, and } \\
\text { foremen }\end{array}$ & $\begin{array}{l}\text { Plan, Review work } \\
\text { packages }\end{array}$ & Write report \\
\hline $\begin{array}{l}\text { Construction } \\
\text { superintendent and } \\
\text { engineering } \\
\text { supervisor }\end{array}$ & Meet managers & $\begin{array}{l}\text { Meet foremen } \\
\text { and workers }\end{array}$ & $\begin{array}{l}\text { Plan, Review work } \\
\text { packages }\end{array}$ & $\begin{array}{l}\text { Write work log } \\
\text { and report }\end{array}$ \\
\hline $\begin{array}{l}\text { General foreman and } \\
\text { foreman }\end{array}$ & Meet supervisors & Meet workers & $\begin{array}{l}\text { Plan, Review work } \\
\text { packages }\end{array}$ & $\begin{array}{l}\text { Write work log } \\
\text { and report }\end{array}$ \\
\hline
\end{tabular}

In order to be consistent, a vector of 'management work' (subscripted as 'MgntWork' in the model) is used to represent the responsibilities of management staff using the same term (external meeting, internal meeting, supervision, and administrative work), but each term means different day-to-day responsibilities for the management staff in different level, as listed in Table 4.3.2-1. 
Different types of management work have different priorities when time resource is not enough to do all the work. Work with higher priority is done first or is allocated larger portion of the available time.

\subsection{Project Lifecycle}

The project life cycle has five work phases: conceptual design, detailed design, work package development, mobilization, and outage, with typical duration indicated in Figure 4.3.2-4. Notice the data are provided by Steam Generator Replacement project. For a nuclear power plant construction project, the durations for the work phases are expected to be longer and somewhat different. It should also be noted that the adjacent work phases can have overlaps.

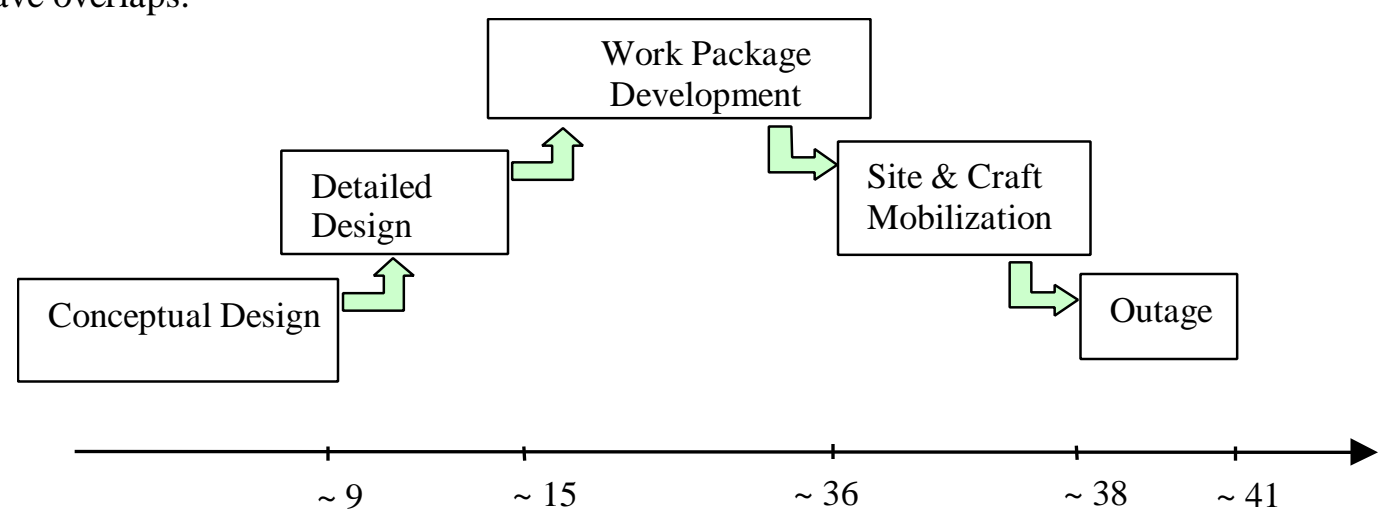

Figure 4.3.2-4: Project Lifecycle Consists Of Conceptual Design, Detailed Design, Work Package Development, Mobilization, And Outage Work Phases

\subsection{Project Work Types}

There are three types of work that will produce deliverables: Design engineering, field engineering, and construction.

Design engineering work consists of two parts. Those performed in conceptual design phase is called 'conceptual design work', while those performed in detailed design phase is called 'detailed design work'. After detailed design phase, the design engineering team will remain in the project but at a smaller size, with their responsibilities changed to providing information concerning the drawings, and to do the possible rework and some additional design work, if needed.

Field engineering department does work package development work, with information from detailed design team. This work may last until the end of outage work phase because construction rework and possible change will require revision of the work package. Nevertheless, most of the work package development work is done in work package development work phase. Besides revision work, field engineers are also responsible to oversee work package implementation from an engineering perspective, which includes procedure adherence, craft assistance, problem resolution, craft training assistance, and work package interpretation. 
Finally, construction work is done by the construction team with help from design engineering team and field engineering team. This is the most important department in the nuclear power plant construction project. Over $80 \%$ of the project costs are spent during the outage phase, which is only about $10 \%$ of the total project duration with high work loads, large size of work force, huge demands for coordination and communication, and many emergencies. As a result, the management of this department turned out to be of significant importance.

\subsubsection{System Dynamics Modeling}

This section describes the representation of the underling system structure, which is broken down to seven dependant sectors: work process sector, labor sector, productivity sector, quality sector, change sector, management actions sector, and performance sector.

\subsubsection{Work Process Sector}

The SGR project has five work phases: conceptual design, detailed design, work package development, mobilization, and outage. All work phases share a common process. Therefore, a single work flow diagram is used to represent all work phases.

\subsection{Rework Cycle}

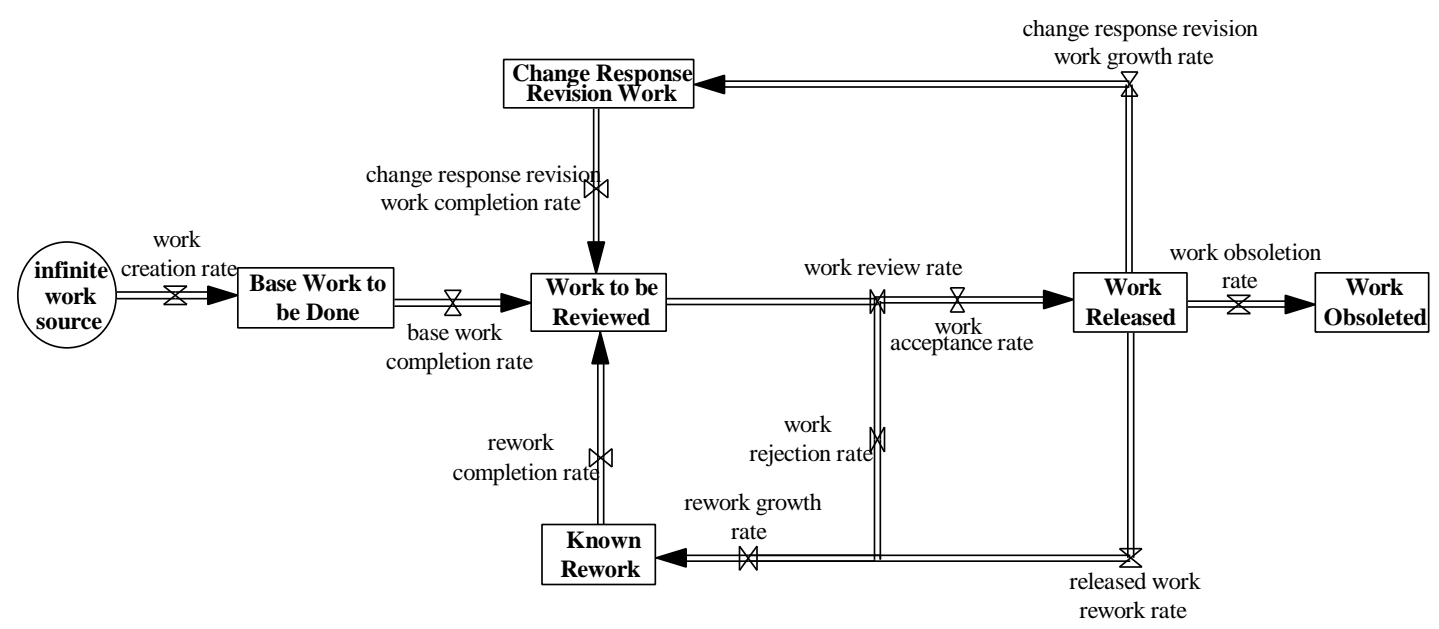

Figure 4.3.3-1: Work process stock and flow diagram

Rework cycle shown in Figure 4.3.3-1 depicts the process by which the work in each work phase flows from work to be done to work released. To be clear, the work in each work phase is disaggregated into three parts: base work, rework, and revision work due to changes. Base work is the work to be performed for the first time, rework is the portion of the completed work that needs correction due of its unacceptable quality, and revision work due to changes is the completed work that needs to be revised in order to incorporate the changes when changes take place.

The current staff level and productivity determine the work completion rates, including 'base work completion rate', 'rework completion rate', and 'change response revision work 
completion rate'. All work completion rates have two components, good and flawed, dependent upon quality of work practice.

The completed work flows to 'Work to be Reviewed', and after that, the work deemed to be acceptable flows to 'Work Released', and the work deemed to be flawed flows to 'Known Rework' otherwise.

It is possible that the reviewer does not identify the errors in the completed work, and accept the flawed work mistakenly. This part is what is called 'Unknown Rework'. Before it is identified, it disguise itself as 'Work Released', yet upon the time it is revealed, it will be rejected and flows to 'Known Rework'. Often this portion of the rework, if identified late, requires also the removal and rework of the relevant 'Good' work, thus timing of error detection is very important.

For project systems exposed to changing environments, there are often unanticipated events. These surprises can increase the work scope, obsolete completed work, or require revision of completed work. The 'work creation rate' between 'Infinite Work Source' and 'Base work to be Done', the 'work obsoletion rate' and 'change response revision work growth rate' out of 'Released Work' reflect these effects upon the project system.

\subsection{Rates}

Figure 4.3.3-2 is the expanded work process stock and flow diagram with all the rates and related auxiliary variables.

Because of the work precedence constraints (see Section 4.3.3.3.3: Staffing Policy), at a specific time, there is only a limited amount of work available to be done. At the same time, the total amount of work that can be performed is constrained by the number of available workers and productivity of the workers. In the cases where not enough resources (labor, materials, etc.) are available to perform all there types of work awaiting, it is assumed that revision work (rework and change response revision work) has a higher priority over base work and will be performed first. 


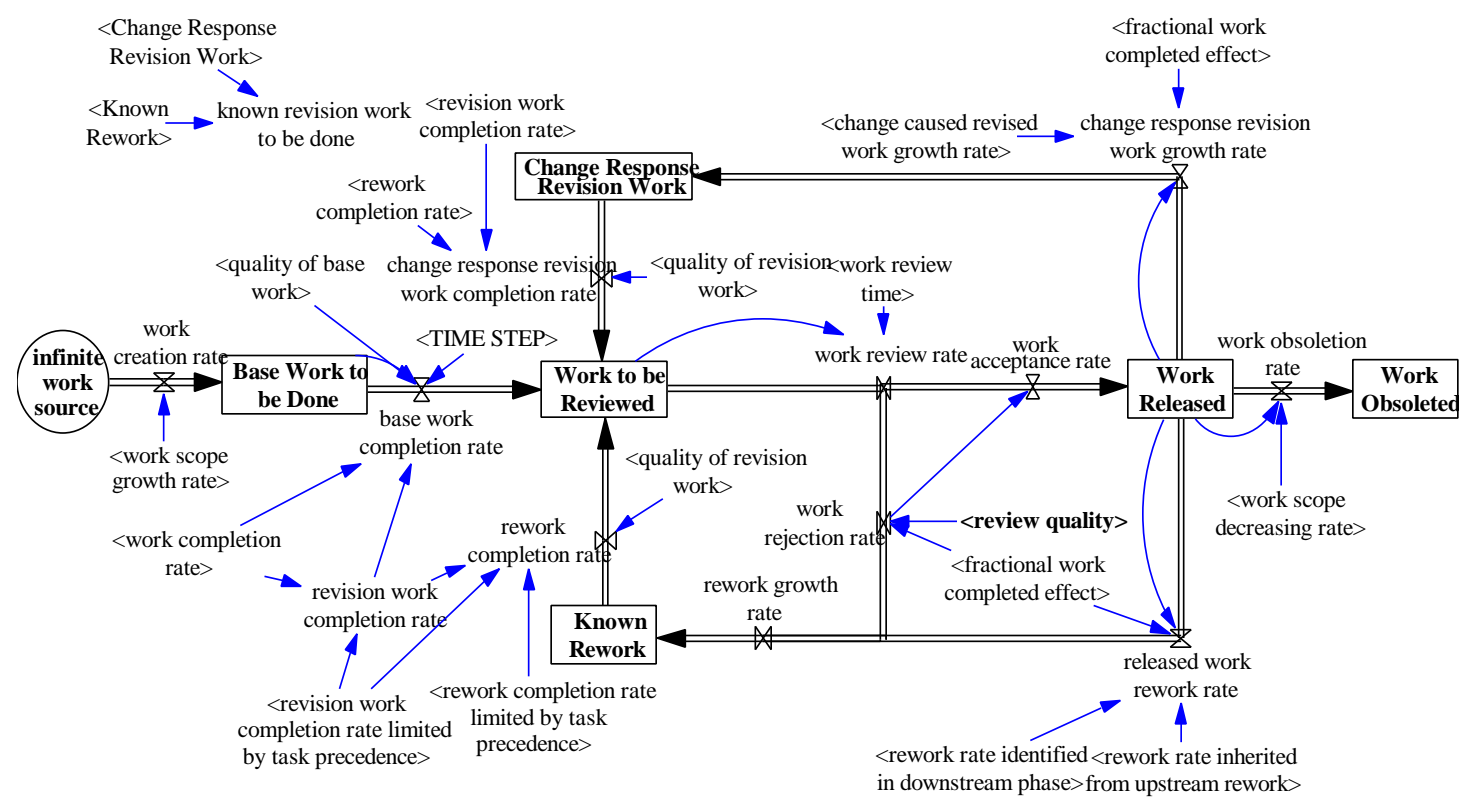

Figure 4.3.3-2: Expanded Work Process Stock And Flow Diagram

In the work review process, it is assumed that there is an average time delay, 'work review time', which may be different in different work phases but which deemed to be constant through a specific phase. The reviewing work is performed subject to a 'review quality', which is defined as the percentage of flawed work identified by the reviewer: stated differently, it is the probability that the 'Flawed' work will be rejected. At the same time, the rejected flawed work will also require some of the related 'Good' work be sent back to rework with it. For example, if a pipe recently installed is found to be in the wrong position, some of the good work in the vicinity may need to be removed in order to reinstall the pipe. As more and more work is completed, this factor will become more and more important. The 'fractional work completed effect' is defined for this purpose.

The work accepted then flows to 'Work Released'.

Besides internal rework rejected in the review process, there are two other external sources of rework, both of them come out of released work (See Figure 4.3.3-3): rework identified in a downstream work phase and rework inherited from a upstream work phase.

For the flawed work mistakenly accepted, it is assumed that it will be identified later in the downstream work phase after a constant delayed time 'accepted flawed work discovery time'. And rework inherited from upstream phase comes as a result of performing the work based upon the accepted flawed work in the upstream work phases. Upon the discovery of the flaws from upstream work phase, all the work based upon them has to be corrected. 


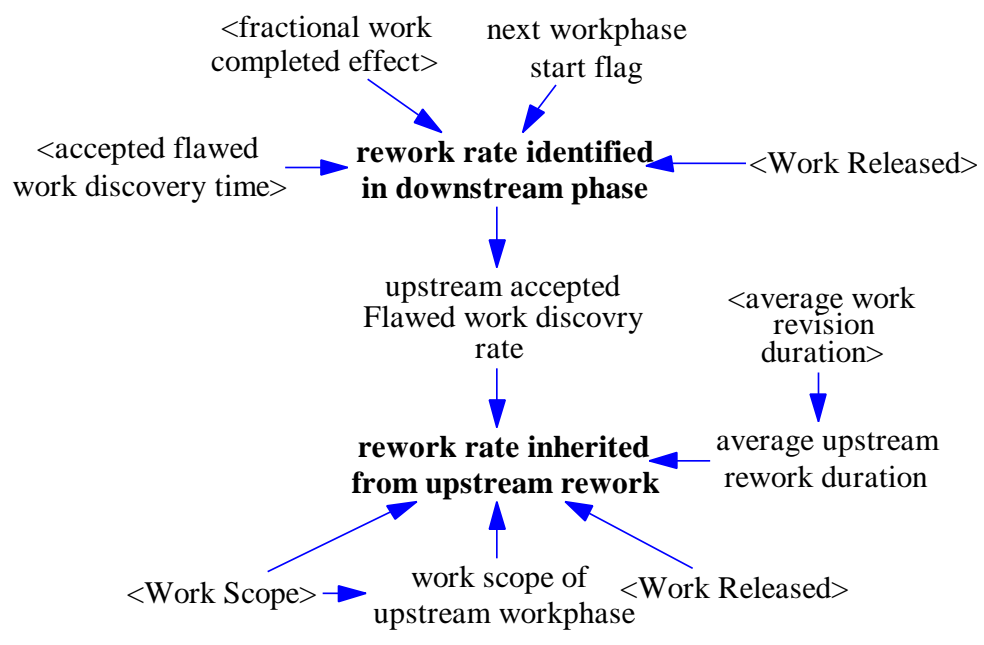
Figure 4.3.3-3: Rework Discovered From Downstream Work Phases And Rework
Inherited From Flawed Released Upstream Work

The work rejected after being reviewed, the accepted work identified as rework in downstream work phase, and the accepted work needed to be reworked because they are based on the accepted flawed upstream work join together to form 'rework growth rate'.

\subsubsection{Labor Sector}

Labor sector models the human resource management in the organization. It starts with a staffing policy that will determine the quantity of the staff expected in the organization, and then needed staffs will be obtained from or laid off to labor market.

In order to evaluate the expertise of the staff population, we divide them into two groups: senior staff and junior staff. We further differentiate each group into unfamiliar staff and familiar staff in order to elaborate the familiarity factor.

\subsection{Staff Flows}

Figure 4.3.3-4 is the staffing stock and flow diagram. Because the diagram is symmetric, we will focus on the lower part of the diagram in the following discussion.

The staffing process begins with determining how many staffs are needed. This process is shown in section 3.3.3. Then, we can come to staff flow chart shown in Figure 4.3.3-4.

The variables in Figure 4.3.3-4 are subscripted to represent different departments and different staff levels. Regardless of their affiliated departments and positions, all staffs share a common staffing flow diagram because they are all staffed and laid off (de-staffed) through a common staffing process. 


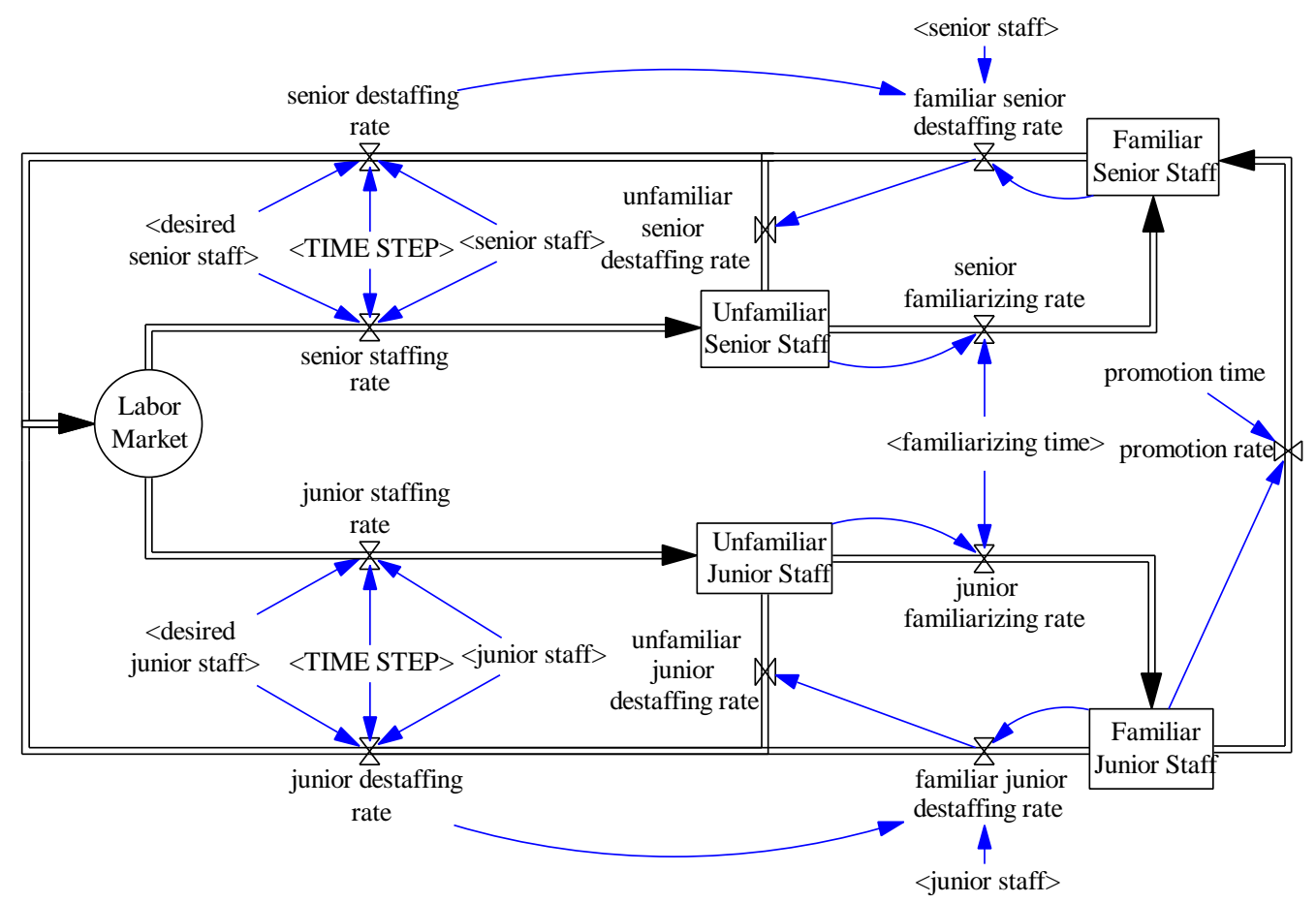

Figure 4.3.3-4: Staffing Stock And Flow Diagram

The desired numbers of senior and junior staffs obtained in 4.3.3.3.3 and current number of senior and junior staffs are used to decide the staffing rate (if desired number is greater than current number) and de-staffing rate (if the desired number is less than current number). In the case of hiring, generally it takes a period of 'ramp-up time' for the new staffs to become familiar with the job. They are called 'unfamiliar staff' during this period, and after that, they become 'familiar staff'. The distinction is important because the former are less productive then the latter.

For a project that lasts for a long time, it is also possible for a junior staff to become a senior via being 'promoted', that is what the 'promotion rate' from familiar junior to familiar senior is for.

\subsection{Staffing Policy}

In order to calculate the quantity of staff required to perform the expected available work, the expected work available to be done should first be determined. The word 'expected' is used in here because it takes time to adjust the workforce. For example, if it takes three days to adjust the workers, then when deciding the number of workers to adjust 'now', we need to know the expected number of staffs needed 'in three days'.

Before getting to that, we will first introduce the concept of 'work precedence', or work sequence. The idea of work precedence comes from critical path method (CPM). Basically, it says: "given that some fraction of work has been completed, not all, but only a portion of the work is available to be done". 


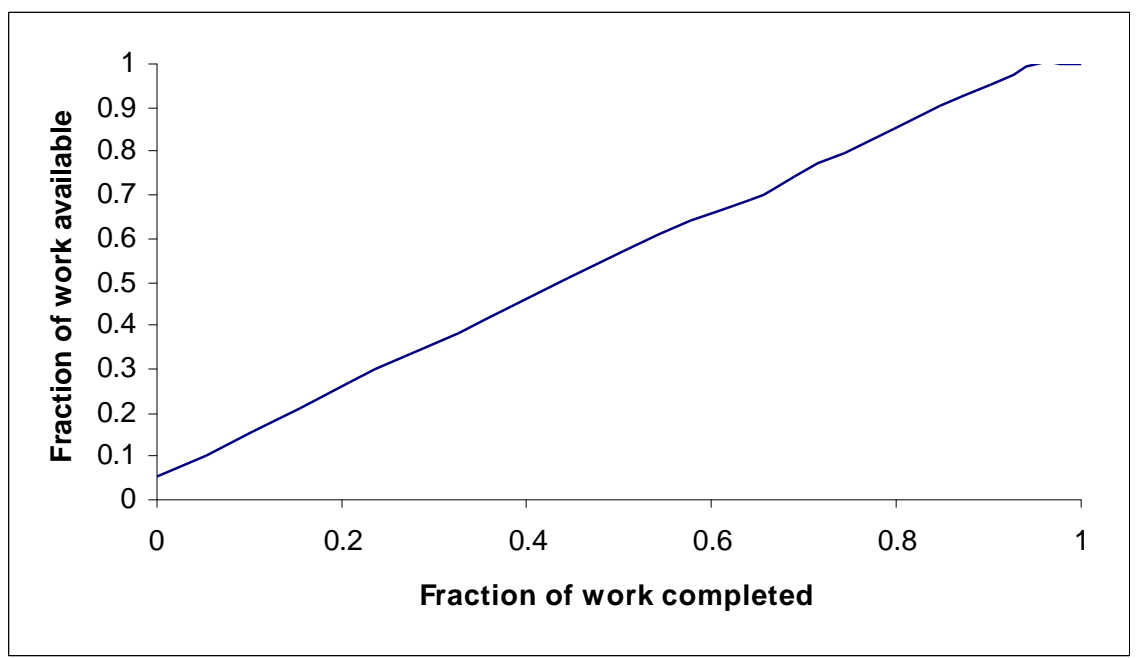

Figure 4.3.3-5: An Example Of Work Precedence Constraints

An example of the precedence constraints may look like Figure 4.3.3-5. It tells how much (in percentage) of the total work is available to be done given some fraction of work has been completed.

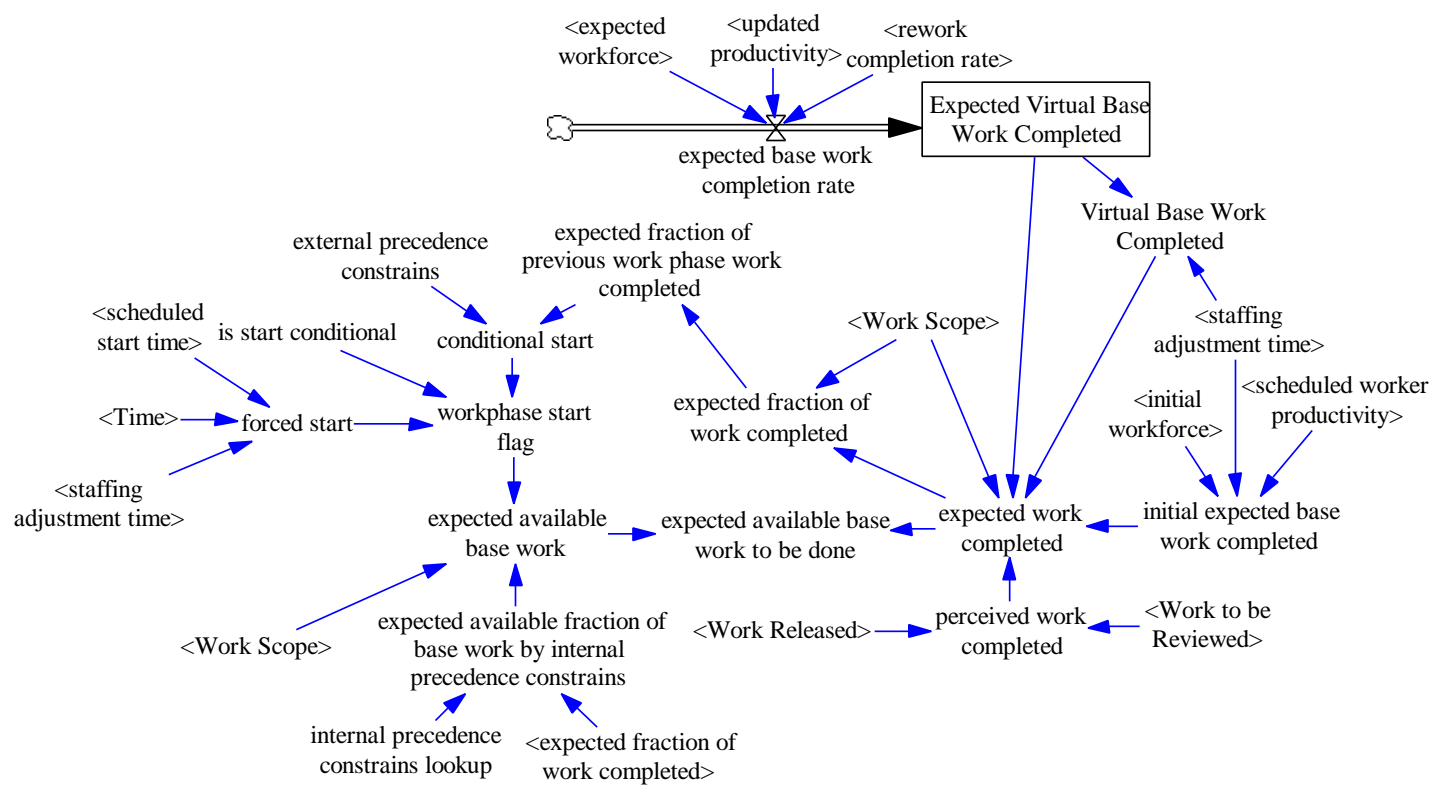

Figure 4.3.3-6: Influence Diagram Showing Available Base Work To Be Done Limited By Internal And External Precedence Constraints

In Figure 4.3.3-6, the internal precedence constraints give the amount of expected base work available to be done when given expected fraction of the base work has been completed in this work phase, and the external precedence constraints decides when the next work phase can start given an expected fraction of work has been completed in the upstream work phase. 
There are two situations concerning phase start: (1) the next work phase will start when a specified fraction of work is completed in the upstream work phase; (2) the work phase will be 'forced' to start when the time is greater than the scheduled start time of the work phase, even though the work completed in the upstream work phase has not reached the desired level. In (2), the out-of-sequence problem will be incurred because of the early start (see 4.3.3.4.3).

After a work phase is started, at any point in time, we look ahead by 'staffing adjustment time' to estimate the number of staff needed, so that we can begin hiring now to have them on site by that time. To do that, first the 'expected work completed' is estimated by summing the current work completed and the work to be completed between now and 'staffing adjustment time' from now. After that, the 'expected available base work' is computed using task precedence constraints, and the 'expected available base work to be done' is obtained by subtracting the 'expected work completed'.

Figure 4.3.3-7 shows how to determine the quantity of work force required in the organization. The limits of work completion rate for base work and revision work can be calculated by dividing the available work to be done with average work duration for these two types of work, where the average work duration is the average time required to finish the work in a specific work phase.

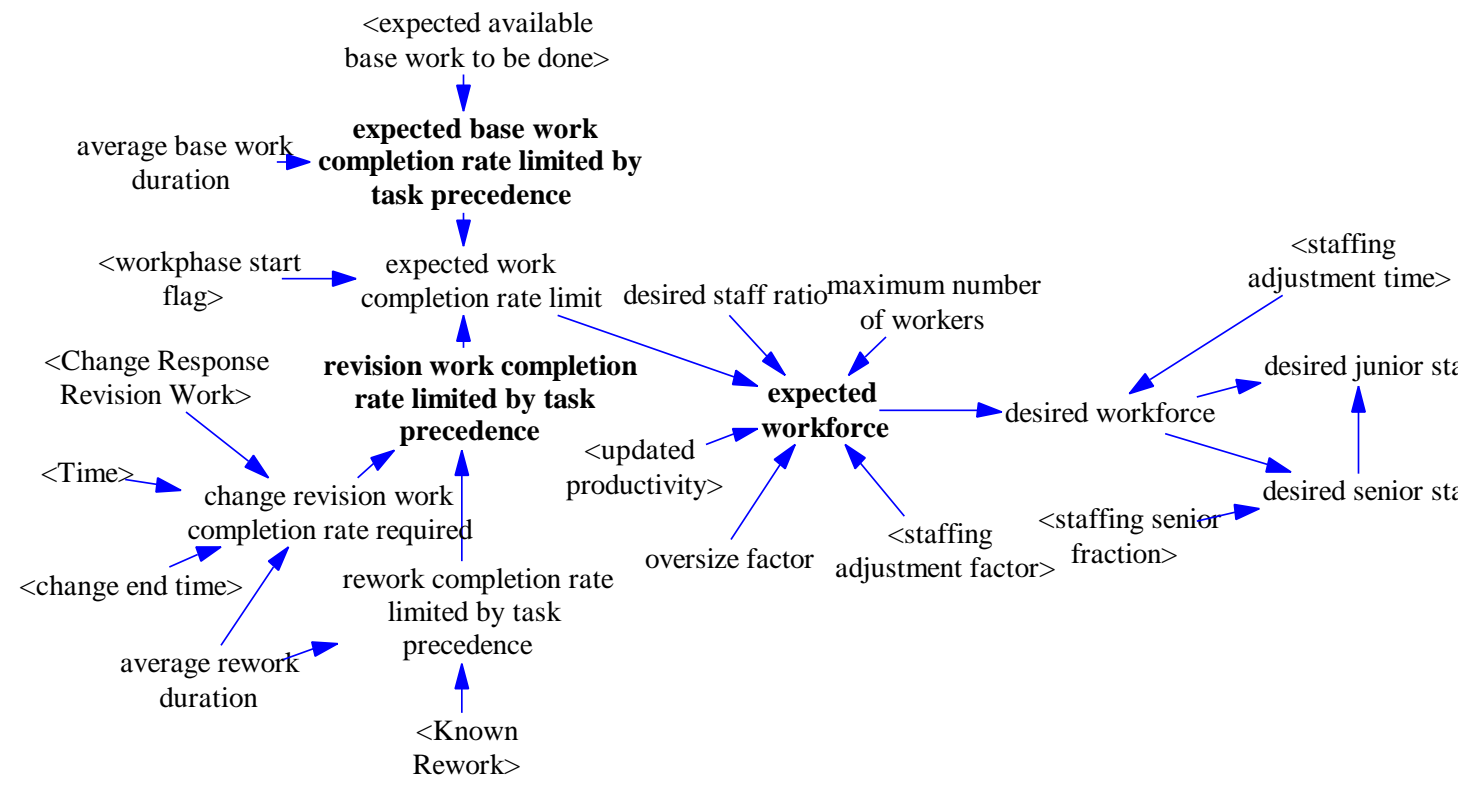

\section{Figure 4.3.3-7: Causal Loop Diagram Showing How To Determine The Quantity Of Work Force Required In The Organization}

If a work phase has started, then the work completion rate limit is the expected work completion rate. Then, with expected work completion rate, productivity, and staffing adjustment factor, etc., the quantity of workers required is determined.

Since the staff ratios are set as constant in this model, the quantity of staff other than workers can be computed by multiplying the number of workers with corresponding ratios. The senior and junior number of the staffs in a specific level can also be determined using the 'desired senior fraction'. 
Finally, since it will take 'staffing adjustment time' to obtain the workforce from the labor market, the desired work force is simply a delayed function of expected workforce.

\subsubsection{Productivity Sector}

Productivity is the state of being productive, which defines how much work a person is able to perform in a given period of time.

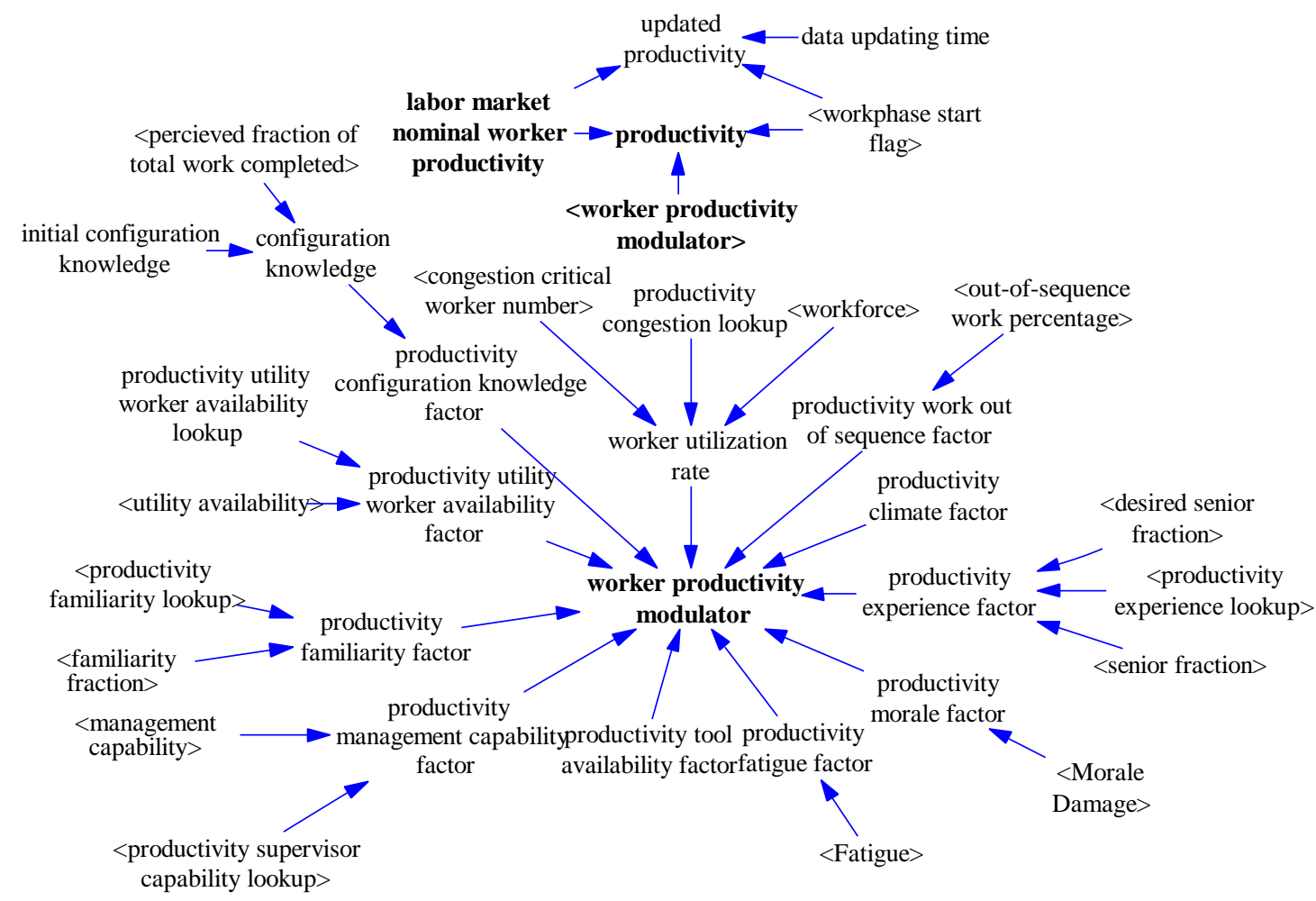

Figure 4.3.3-8: Productivity Factor Model

A factor model is used in the productivity sector. The underlying assumption of such a factor model is that the factors' impacts on the productivity are independent of each other:

productivity? nominal productivity? ? factor $_{i}$

The factors are chosen based upon our conversations with a group of domain experts.

'Updated productivity' is used in staffing, which reflects the fact that the human resource department can at most depend on the productivity obtained recently to made the staffing decision. 'Data updating time' is the time it takes for real productivity data to be obtained by the staffing group. 


\subsection{Congestion Factor}

One person can dig a hole in one minute, but sixty persons will not be able to dig a same hole in one second. Congestion factor reflects the fact that congested working space or too many staff working on a same function produces sit-and-wait problem; in other words, the workers have to wait for resources or work areas to become available. This is a negative impact on productivity because the utilization rate of the workers is reduced.

Figure 4.3.3-9 shows the work utilization rate as a function of degree of congestion. The degree of congestion is defined as the quotient of the actual number of workers to the 'congestion critical workers number', which is the smallest number of workers that begins to cause congestion.

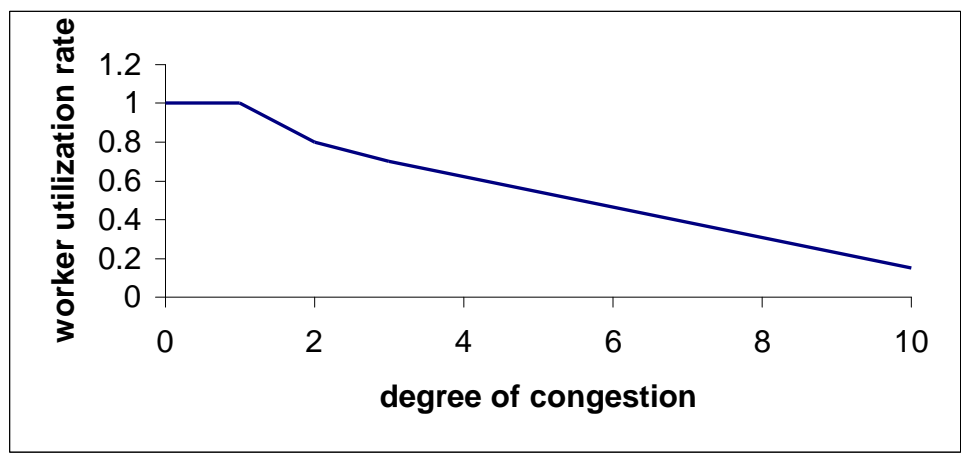

Figure 4.3.3-9: Work Utilization Rate As A Function Of Degree Of Congestion

\subsection{Work-out-of-sequence Factor}

This factor reflects the effect of out-of-sequence work, which causes slow down in the workers' pace because the work that starts with upstream work uncompleted tend to entail assumptions, guesses, and revisions due to lack of information.

The degree of out of sequence is represented by the fraction of work that is out of sequence. It is decided by two factors: (1) the amount of information needed; and (2) the availability of needed information.

Since all the work is assumed to be performed with regard to the internal precedence constraints, only (1) downstream work of current revision work, and (2) early-start portion of the work will be 'out-of-sequence work' (see Figure 4.3.3-10). 


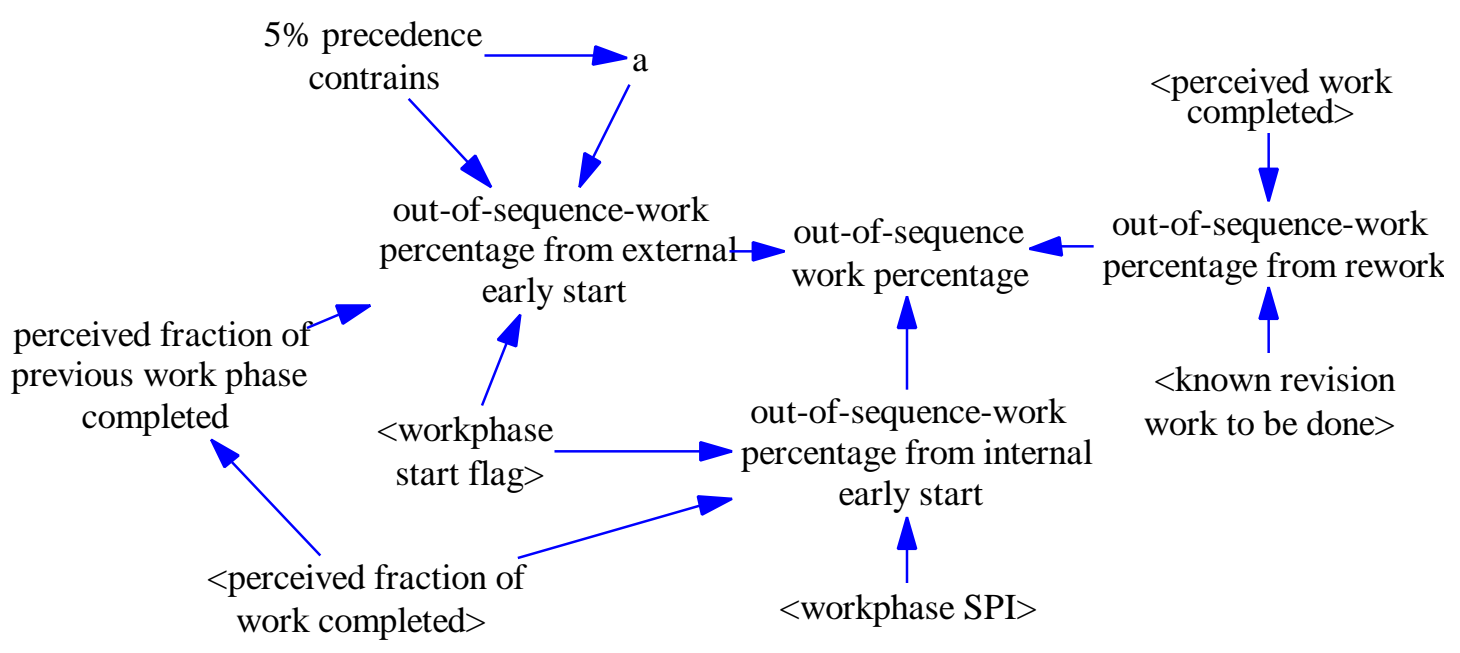

Figure 4.3.3-10: Out-Of-Sequence-Work Components

For the second part, there are two scenarios: (1) early start of downstream phase work before a necessary portion of the upstream phase work has been completed due to schedule pressure, and (2) in a same work phase, early start of downstream work before its precedent work has been completed. The second scenario mostly happens when the work phase is behind schedule, where downstream tasks are started at scheduled time when the precedent work is scheduled to be, but has not been, completed (see Figure 4.3.3-11).

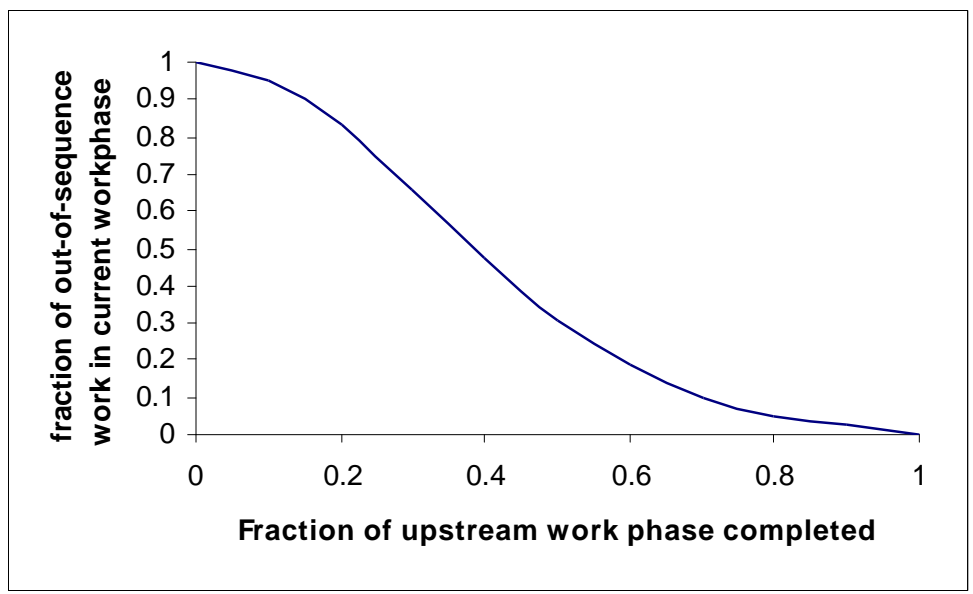

Figure 4.3.3-11 Out-Of-Sequence From Early Start

\subsection{Climate Factor}

The climate factor models the impact of weather on the productivity. Severe weather such as heavy rain and snow can have an adverse impact on the duration and cost of construction activities. 


\subsection{Experience Factor}

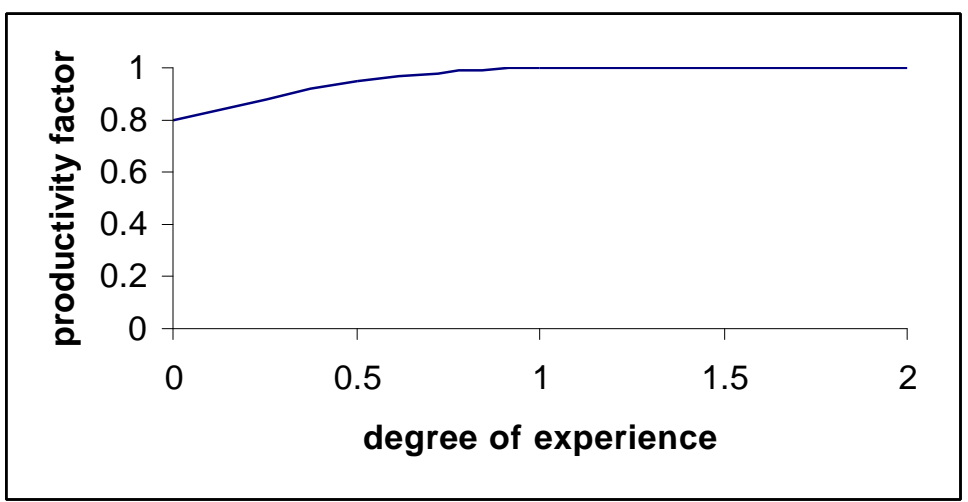

Figure 4.3.3-12: Productivity Experience Factor As A Function Of Degree Of Experience That Is Defined As The Ratio Of The Actual Senior Faction To The Desired Senior Fraction

The experience factor reflects the impact of the experience of the worker on productivity. In this model, the fraction of senior staff decides the experience level.

\subsection{Morale Factor}

Anything which adversely affects morale and which lessens cooperative and positive attitudes toward the work, the employer, and the customer will result in lowered output.

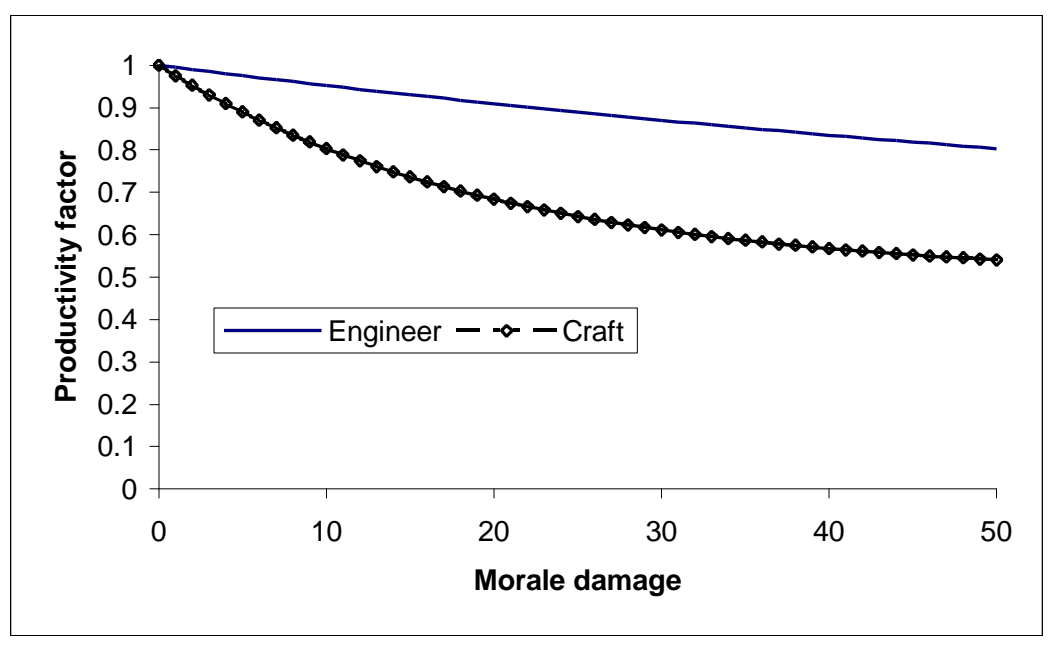

Figure 4.3.3-13: Productivity Morale Factor As A Function Of Morale Damage

Morale damage is an abstract concept. In this model, a unit of morale damage is defined as the morale loss equivalent to the morale loss due to one 8-hour working day's work. It is also assumed that 8-hour's rest at night will recover one unit of morale loss, so that in the normal condition, there is no morale problem. 


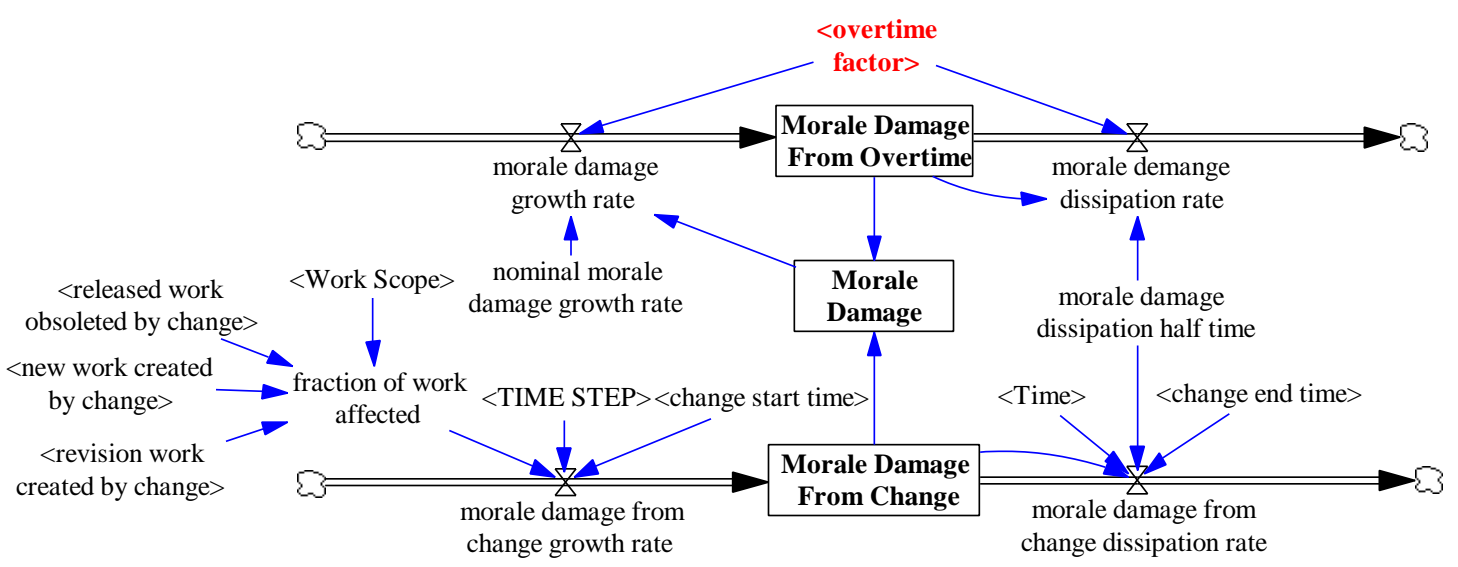

Figure 4.3.3-14: Morale Damage Model

In the work presented here, two sources of morale damage are modeled: one is the morale damage from overtime, and the other from changes.

If the working time exceeds eight hours per day, then the normal rest won't be able to recover the morale loss. The morale damage accumulates at an accelerating rate because as the length of the overtime grows, the rate of morale damage also grows. For example, at the beginning, every eight hours working time only adds one unit of morale damage, but after several weeks' of overtime, an additional eight hours working time will adds more than one units of morale damage (see Figure 4.3.3-15).

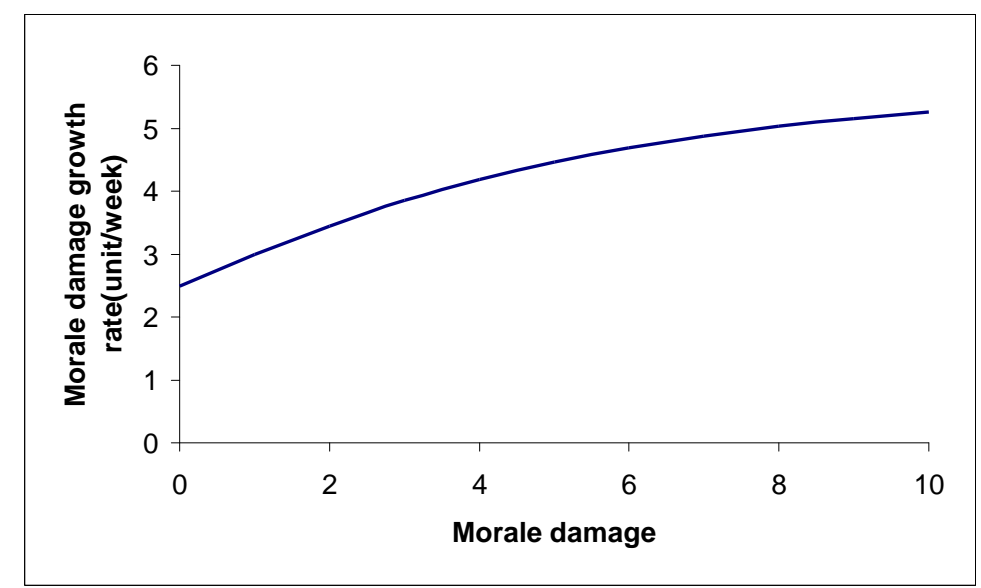

Figure 4.3.3-15: Morale Damage Growth Rate As A Function Accumulated Morale Damage When 50\% Overtime (Or 12 Hours/Day) Is Used

The morale damage keeps accumulating until the overtime returns to normal. After that, the accumulated morale damage will be restored to zero at a rate characterized by 'morale damage dissipation halftime', which means the time it needs to restore half of the morale damage (similar to decaying halftime of nucleus).

Another source of the morale damage is changes. Changes generally cause addition, revision, and obsolescence of the released work, which is very depressing to the project 
team. It is assumed that morale damage will accumulate between 'change start time' and 'change end time', and then the morale will be restored in 'average time to restore morale'.

\subsection{Fatigue Factor}

Fatigue factor models the loss of productivity due to overtime. Bureau of Labor Statistics Bulletin 917 states: "But, whatever the reason, one fact stands out clearly in the survey: the longer the hours, the more scheduled working lost through absenteeism." Absenteeism is promoted when the effects of cumulative fatigue, desires of worker to spend more time with their families, and need for time away from the job to take care of personal business combine with lack of economic incentives to work all available hours because of the high pay received during overtime weeks.

The productivity-killing effects of accidents are widely acknowledged. The BLS Bulletin states: "Injuries also increased as hours increased, not only in absolute number, but also in rate of incidence. In most of the observed instances, the number of injuries per million hours worked was very much higher at the longer hours."

Therefore, fatigue obviously reduced productivity during all hours worked (Figure 4.3.316). It usually takes more overtime hours than normal working hours to produce a same output.

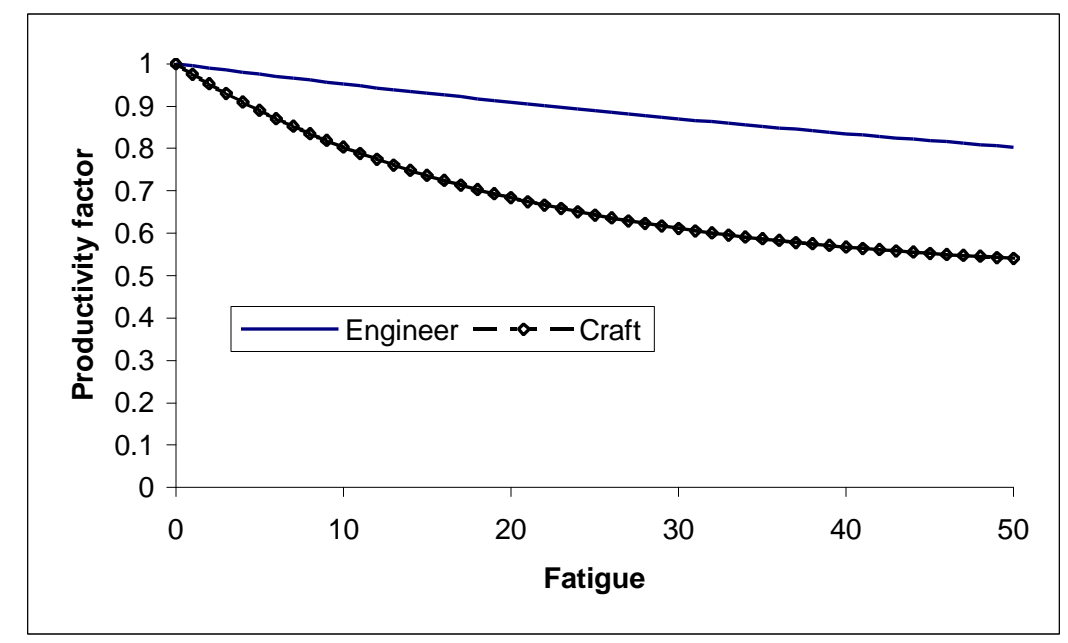

Figure 4.3.3-16: Productivity Fatigue Factor

Similar to morale loss, one unit of fatigue is defined as the equivalent fatigue after one normal working day's work, and it can be restored after one night's rest. As overtime begins, the fatigue starts to build up at an accelerating rate similar to that of morale loss. 


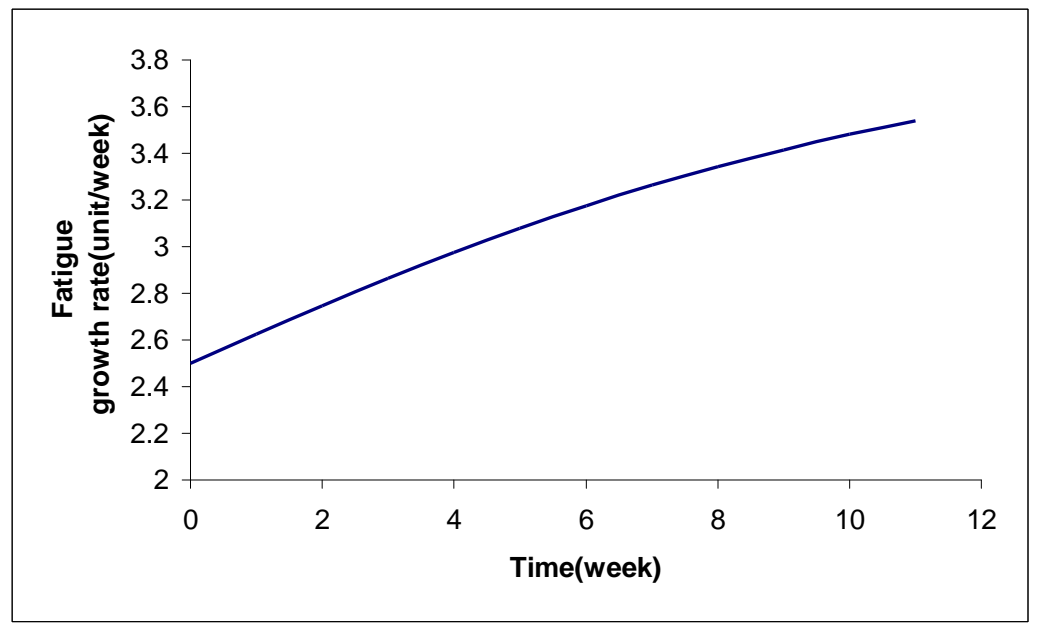

Figure 4.3.3-17: Fatigue Growth Rate As A Function Of Time When 50\% Overtime (12-Hour Working Time) Is Used

The accumulation continues until the end of overtime, and then the fatigue begins to restore to zero at a rate characterized by 'fatigue dissipation halftime'.

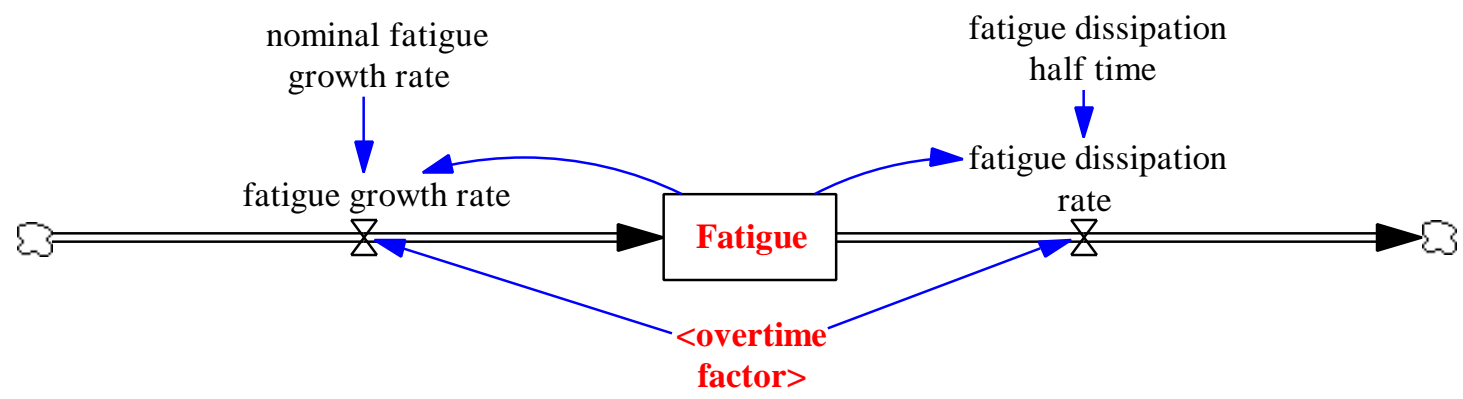

Figure 4.3.3-18: Fatigue Model

\subsection{Tool Availability Factor}

The tool availability factor models the effect of tools on productivity. Dependent upon technologies available, the relevant costs, and the schedule pressure, the project managers can choose to add new machines such as cranes and to deploy new technologies in order to increase the productivity; they can also draw tools out in order to cut high rents. Both of these will affect the productivity, either positively or negatively.

\subsection{Management Capability Factor}

Management leadership is another important factor on the productivity. 


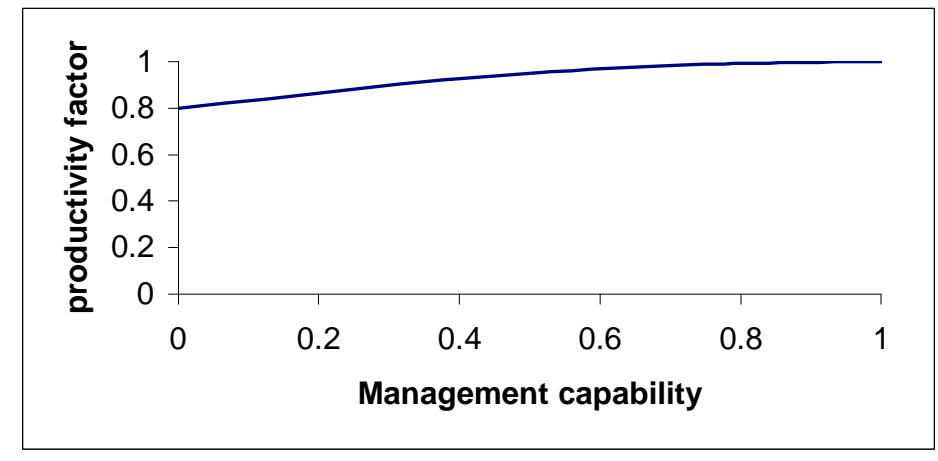

Figure 4.3.3-19: Productivity management capability factor

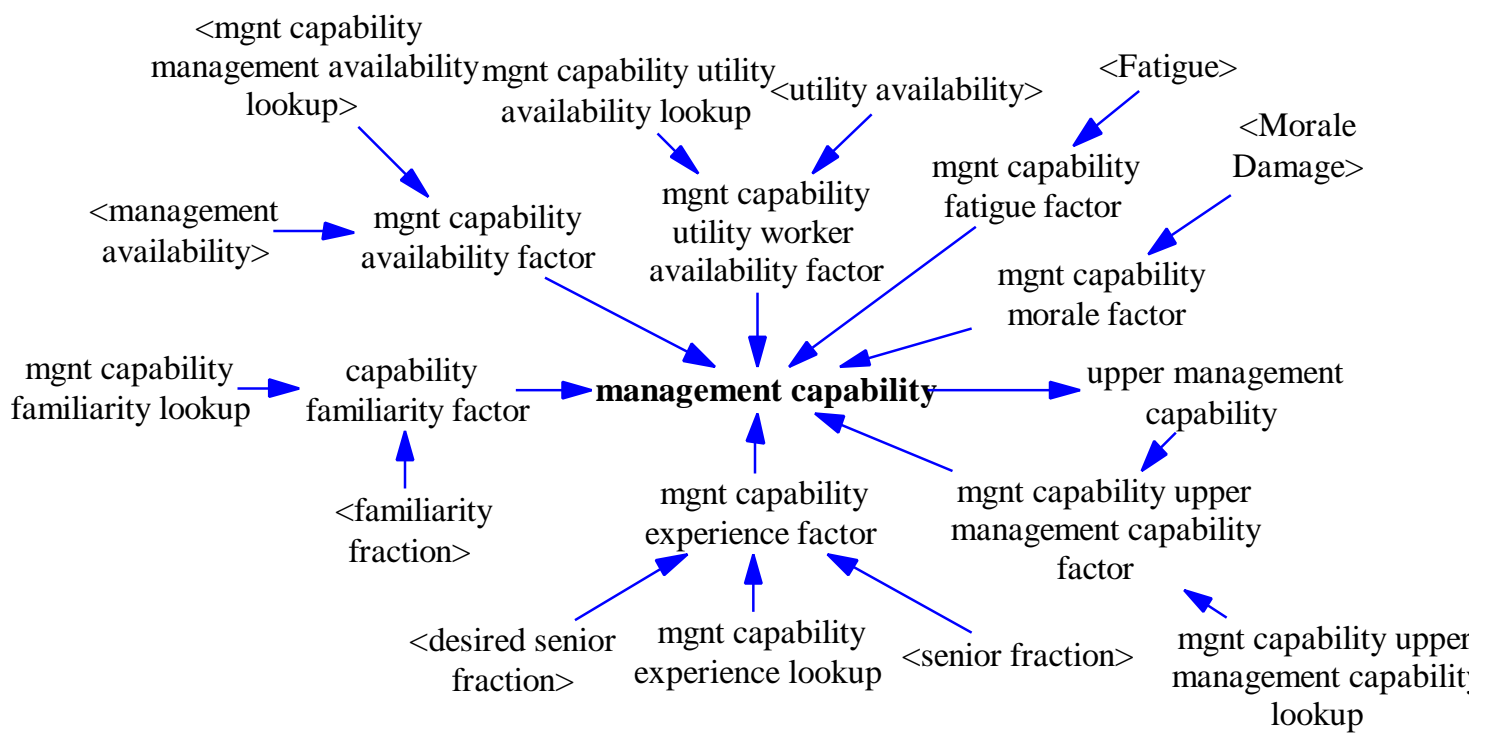

Figure 4.3.3-20: Management capability model

In this model, 'management capability' is defined somewhat different from what it is defined in a dictionary. Basically, it reflects how available, efficient, experienced, familiar, and motivated a management is. It is the product of all these factors, which have maximal values of unity. They are listed in Figure 4.3.3-21. 
Figure 4.3.3-21: Factors That Affects The Management Capability
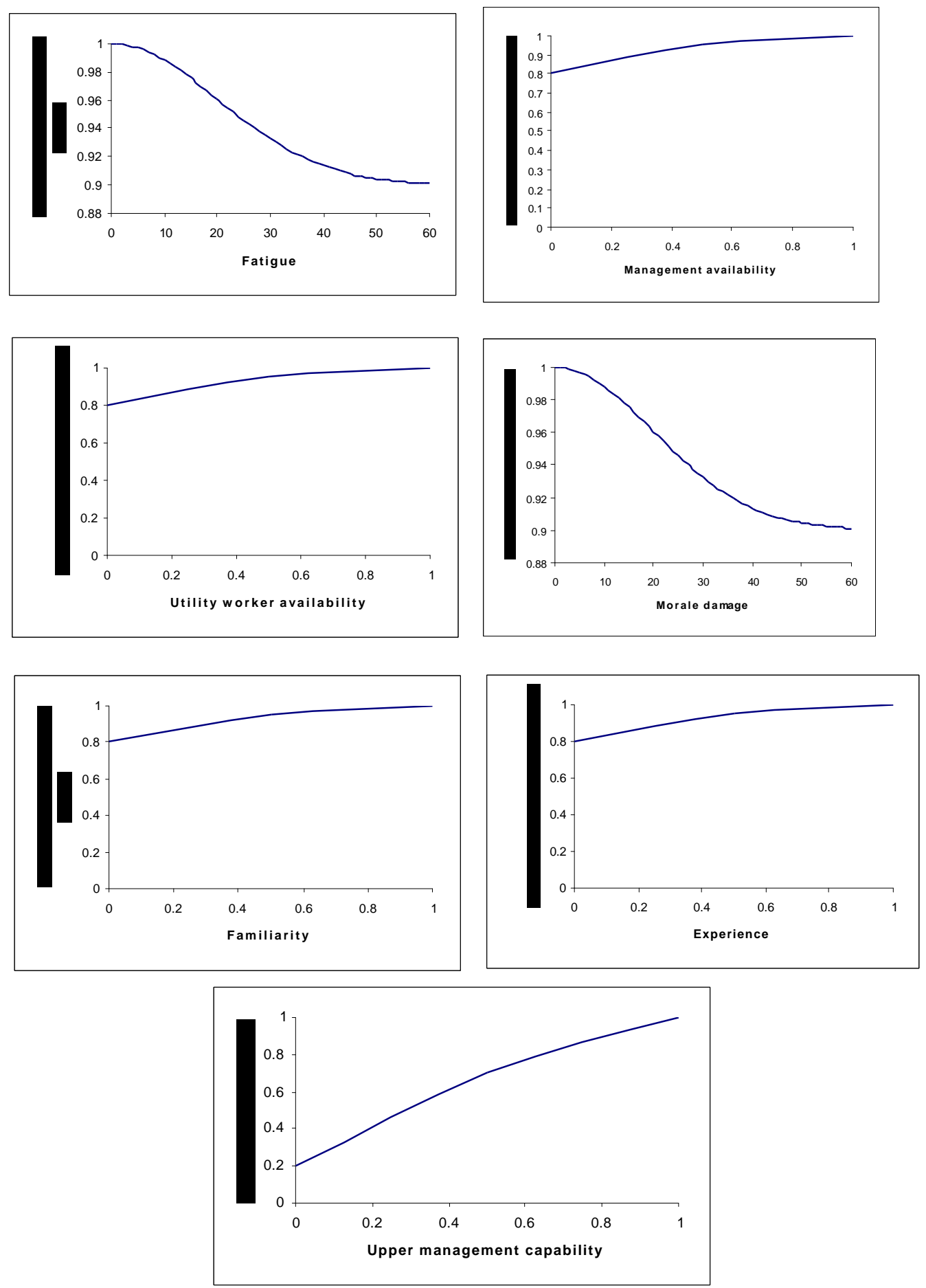


\subsection{Familiarity Factor}

Familiarity with the project, work scope, and people working around can promote productivity. Generally it takes some time for the people new to the project to be familiar with the working environment, for which a jargon 'ramp-up time' is what the project mangers refer to it.

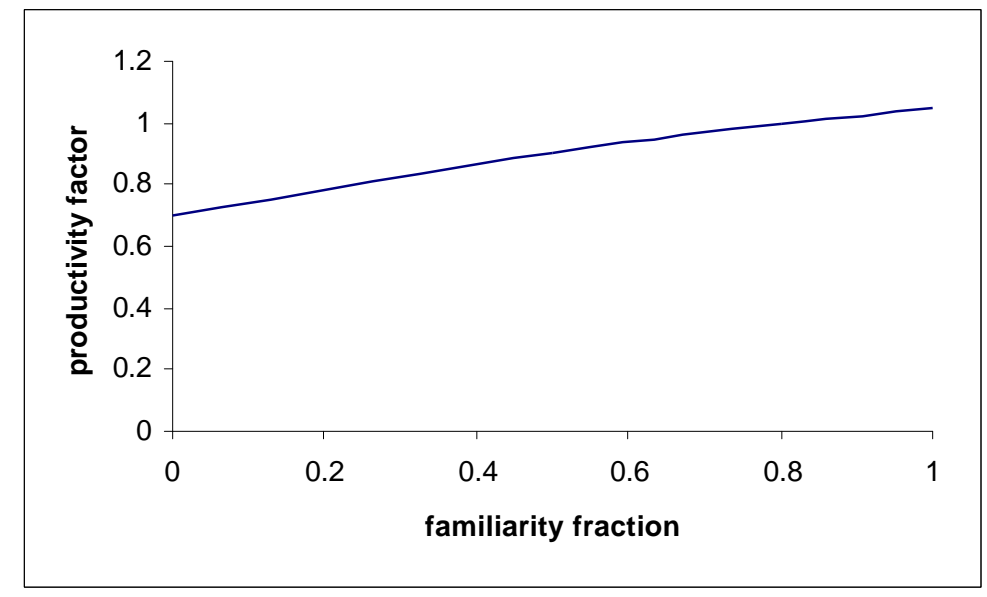

Figure 4.3.3-22: Productivity Familiarity Factor

In the model, the familiarity of the staff is quantified by a quotient of familiar staff number to the total staff number in the staff level.

\subsection{Overtime Factor}

Overtime is one of the management actions taken with the purpose to increase the production rate, so that schedule pressure can be lessened. Apart from the negative effect it brings through fatigue and morale loss, it increases the production rate by adding efficient working time per day.

In the model, the overtime factor for workers are supposed to be input by the model user as a management action during the 'game' simulation, but calculated automatically in a normal simulation dependent upon the SPI of the project. 


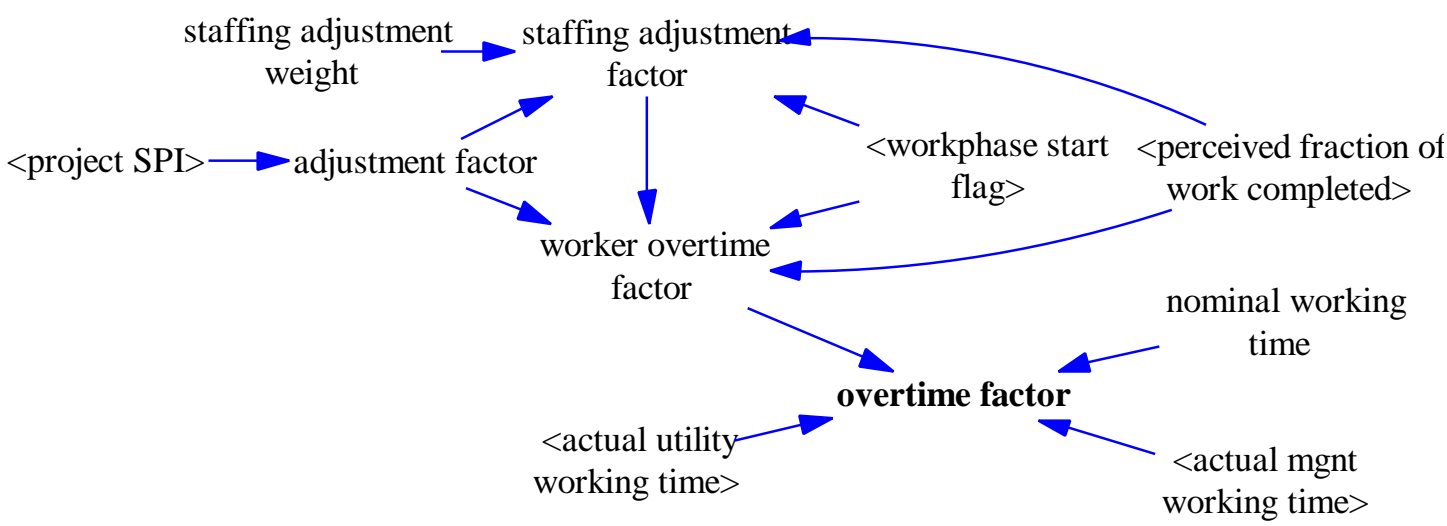

Figure 4.3.3-23: Overtime And Overtime Length Model

Given SPI, the project will need to be speeded up or slowed down by 1/project SPI; There are two ways to adjust the implementation rate: (1) adjusting the working time; and (2) adjusting the workforce size. Project manager can take either one, or both with different allocated weights to satisfy the adjustment need (see Figure 4.3.3-23).

The overtime factors of the other staff in the organization are calculated by first calculating the actual needed working time (See section 4.3.3.4.13: 'Utility Worker Availability Factor'), then dividing it with nominal working time.

\subsection{Configuration Knowledge Factor}

Configuration knowledge about the details of the working site and working procedures turns out to be very important, especially during the design phase. It can be improved by detailed facility walk downs or by computer-aided visualization tools such as a fourdimensional graphic scheduling tool. Under normal condition, configuration knowledge will accumulate like knowledge built in the learning process. With more and more work completed and information revealed, the configuration knowledge naturally increases according to a learning curve.

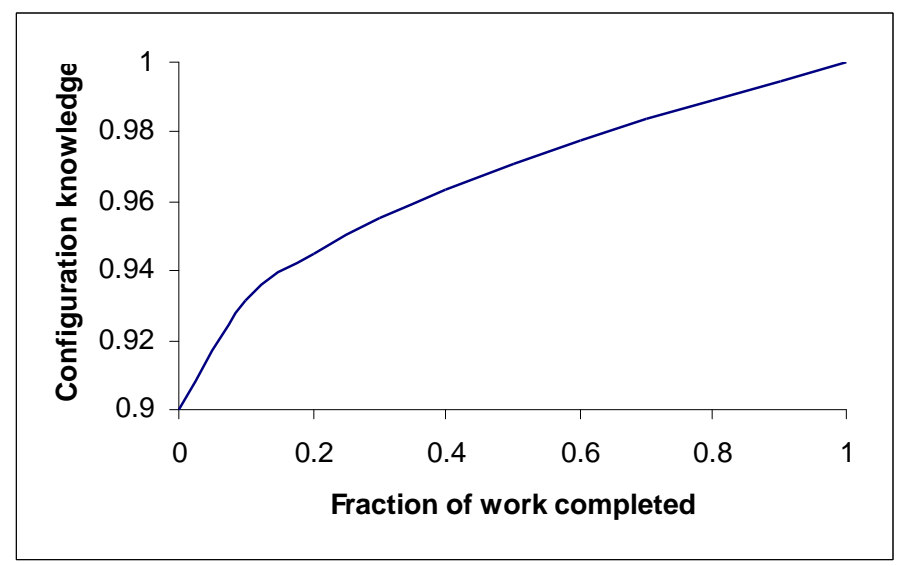

Figure 4.3.3-24: Configuration Knowledge As A Function Of Fraction Of Work Completed 
The configuration knowledge can have impact on many project variables, such as 'ramp-up time', but the most important impacts are on productivity and quality. In the model, configuration knowledge is assumed to have an initial value based upon the similar experience built on previously projects the engineering company undertook. It then accumulates as the project proceeds to a maximum value of unity. It is also assumed that, with configuration knowledge of 0.9 , the productivity is equal to nominal productivity given other things equal, but will increase as configuration knowledge increases as shown in Figure 4.3.3-25.

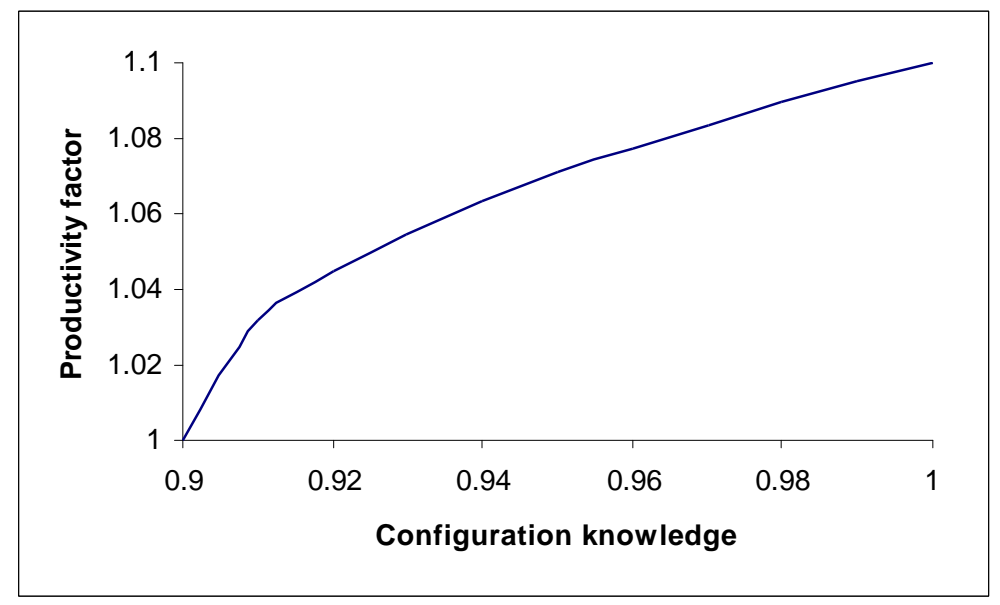

Figure 4.3.3-25: Productivity Configuration Knowledge Factor

\subsection{Utility Worker Availability Factor}

Utility workers refer to support workers such as secretary, receptionist, Xeroxing worker, fire watcher, etc. Their responsibilities are to provide helps to staffs in the other levels, namely, managers, supervisors, foremen, and workers. If there are not enough utility workers, the efficiency of the other staff will be affected.

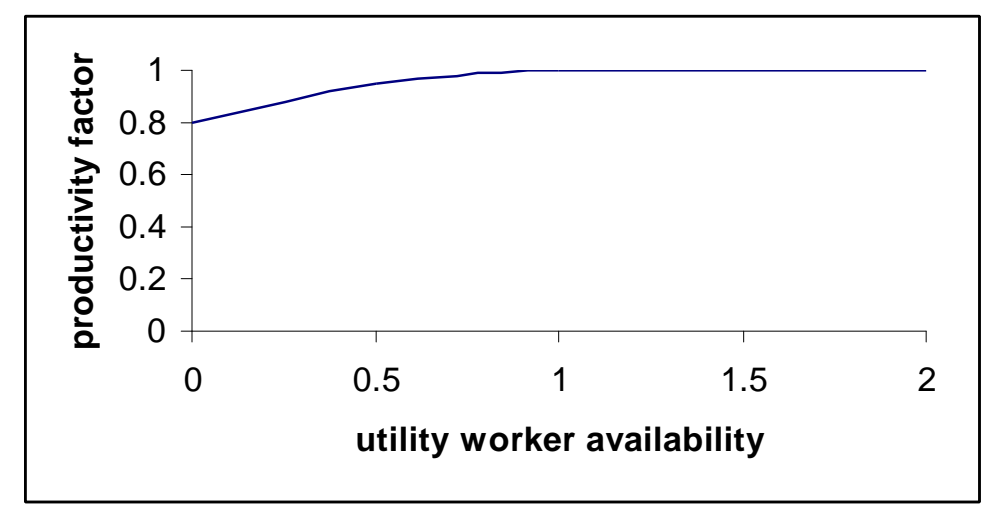

Figure 4.3.3-26: Productivity Utility Worker Availability Factor 
Availability is defined in this model as the quotient of actual working time to desired working time. For management, since they have an array of tasks, their availability is defined as the actual time available for supervision (or oversight) divided by the desired time available for supervision.

In order to calculate the desired working time and actual working time, let's look at Figure 4.3.3-27. It is assumed that if the workers work overtime, all the staffs in other level also work overtime by a same factor. For management, they may need to work for longer time in the case of changes, because in order to deal with the changes, they need to take time to negotiate with the clients and facilitate communications within the organization.

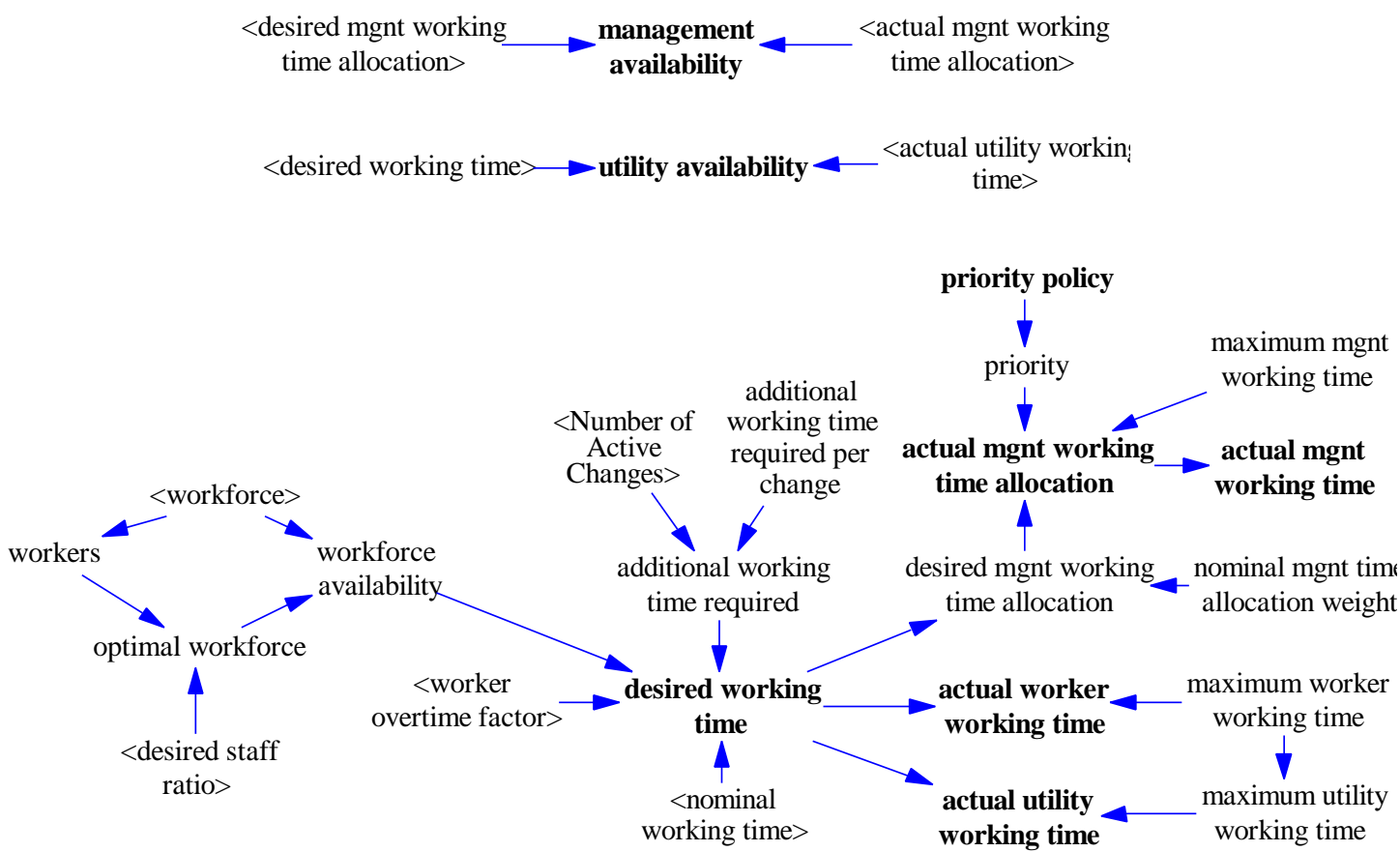

\section{Figure 4.3.3-27: Availability, Desired Working Time, And Actual Working Time Model}

The actual working time is roofed by a maximum working time set up by the company policy. For workers and utility workers, the smaller one of the desired working time and maximum working time is the actual working time.

But for management staff, it is very common that the working time is not enough to handle all the activities, where the working time will be allotted to each activity based upon different priority rules. In the model, two priority rules are provided: with rule No. 1, resources are allocated to the higher priority activities first, and the lower priority activities will get what is left; with rule No. 2 the resources are allocated to all the activities based on their relative weights. For example, if eight hours are available, but 6, 4, 4, 2 hours are required for task 1, 2, 3, 4, with priority from high to low, and with weights $3,2,2,1$, respectively. Under rule No. 1, the first two activities get what they required, while the 
third gets only 2 hours and the fourth gets nothing. Under rule No. 2, however, all the activities get $50 \%$ of what they ask for, or 3,2,2,1 hour(s), respectively.

\subsubsection{Quality Sector}

In this model, quality is defined as the percentage of the total work completed that complies with specifications. Similar to productivity in previous section, it is also built on a group of factors, with the same underlying assumption that the factors' impacts on quality are independent of each other.

$$
\text { quality ? nominal quality? ? } \text { factor }_{i}
$$

The definitions of all the factors in the quality model can be found in Section 4.3.3.4. From Section 4.3.3.2.2: Rework Cycle, we know that there are two flows of work: base work that is to be performed for the first time, and the revision work that is to be performed for the second or nth time. Generally, because of the learning effect, the 'quality of revision work' is often higher than the quality of first-time-implemented work.

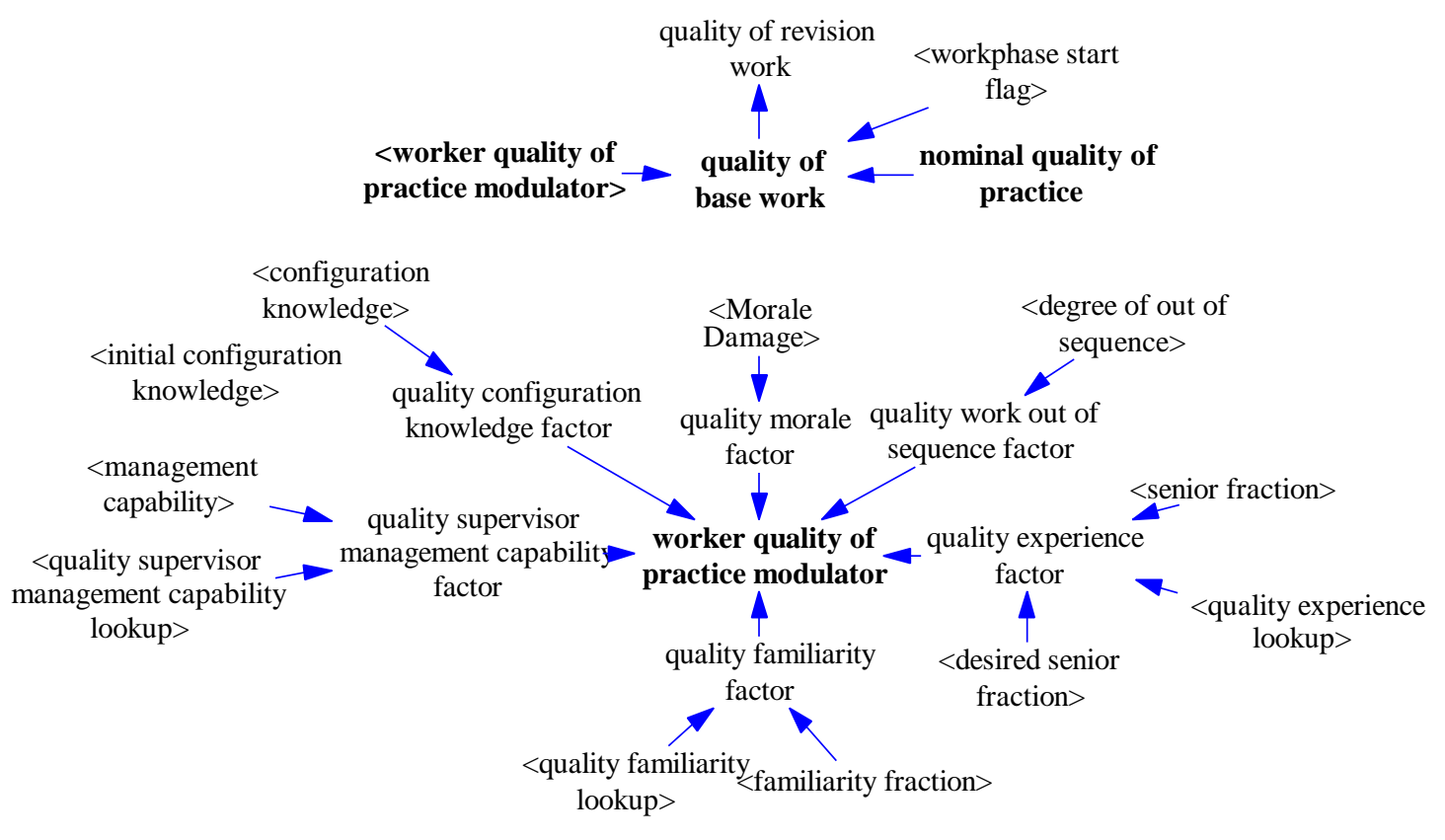

Figure 4.3.3-28: Quality Factor Model

\subsection{Work-out-of-sequence Factor}

Out-of-sequence work requires assumptions and guesses due to unavailability or incompleteness of information, therefore they may not be correct. When the information 
become available, the portion of the work that is based upon invalid assumptions will need to be reworked.

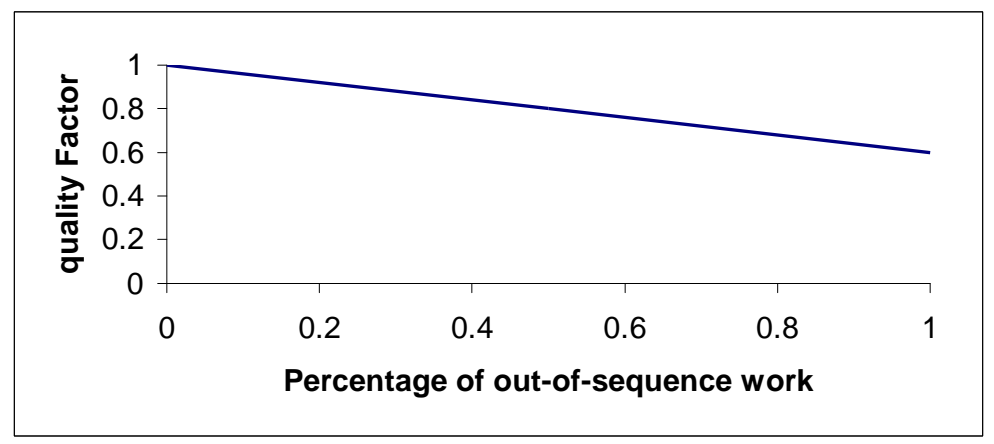

Figure 4.3.3-29: Quality Out-Of-Sequence Factor As A Function Of Out-Of-Sequence Work Percentage

\subsection{Experience Factor}

Senior staff generally performed better than the junior staff. This reflects in quality as a learning curve in which quality increases at a decreasing rate as experience grows. In this model, experience is quantified as the fraction of senior staff.

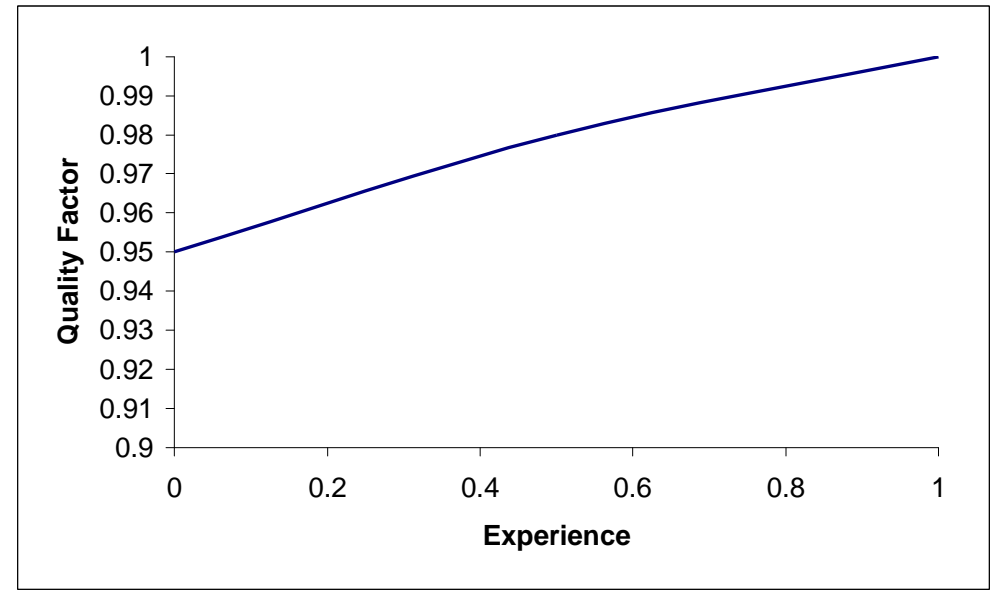

Figure 4.3.3-30: Quality Experience Factor

\subsection{Morale Factor}

People are generally distracted when the morale is low. This will bring in errors in their work and create more rework. As morale decreases, the quality follows an anti-S curve to decrease. 


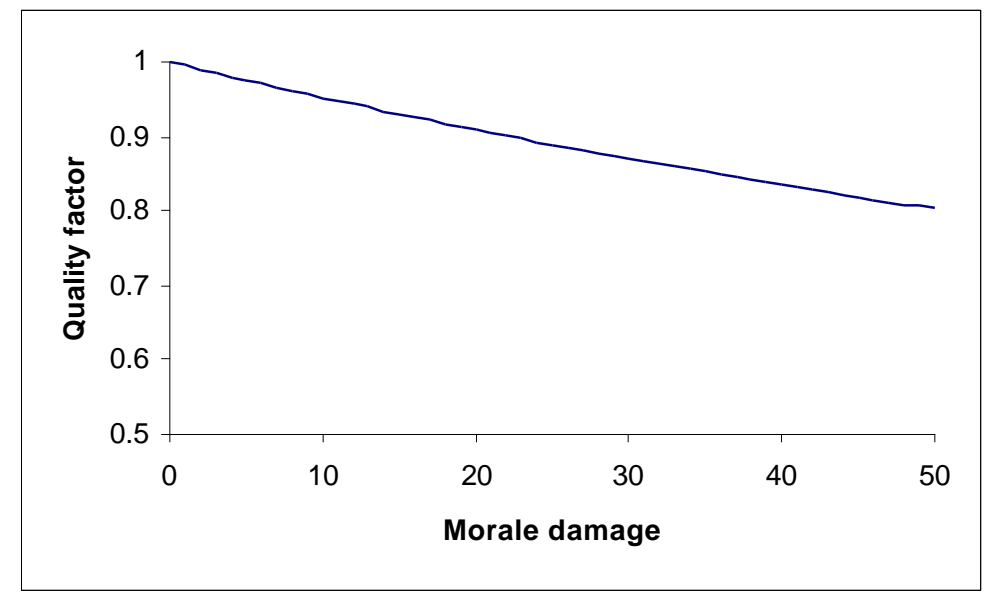

Figure 4.3.3-31: Quality Morale Factor As A Function Of Morale Damage

\subsection{Management Capability Factor}

The availability of the management on work supervision will enhance the quality of the work, and the management's experience, familiarity, efficiency, morale, and the availability of their secretaries, etc. will decide how effective the management is able to work. Management capability, which combines all these factors, is positively related to quality as shown in Figure 4.3.3-32. As management capability increases, the quality increases at a decreasing rate.

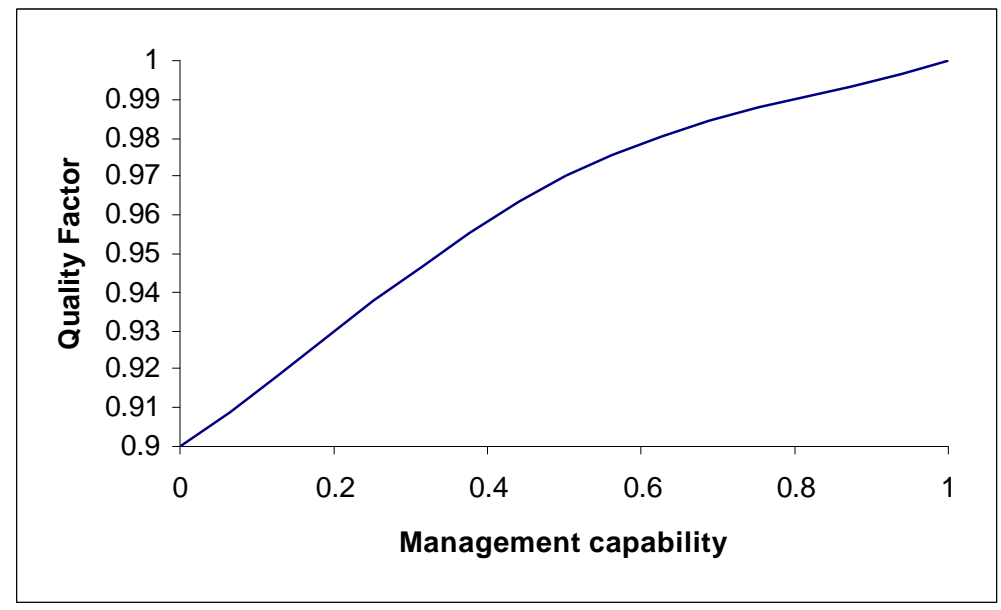

Figure 4.3.3-32: Quality Management Capability Factor 


\subsection{Familiarity Factor}

Worker's familiarity with the tasks, working site, and co-workers around can lead to less mistakes, because they have more information about their work, and they have better communication and coordination with the co-workers.

The relationship follows a learning curve. As familiarity increase, the quality increases at a decreasing rate.

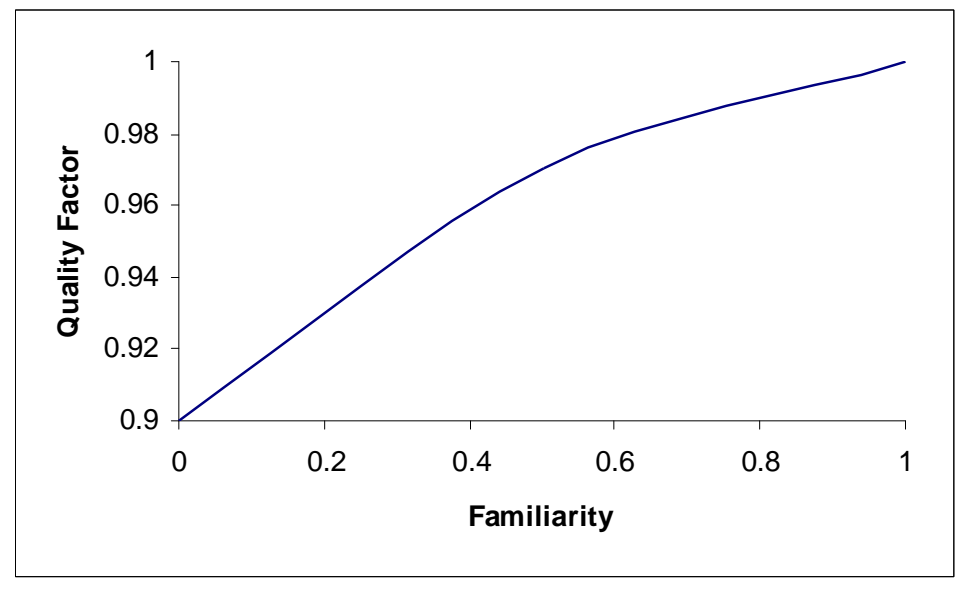

Figure 4.3.3-33: Quality Familiarity Factor

\subsection{Configuration Knowledge Factor}

Knowledge about the details of the configuration is very important in the planning and design phase. The visual knowledge about the work procedure, however, turns out to be important in the construction phase. In general, a better configuration knowledge will improve the quality of the work, as shown in Figure 4.3.3-34.

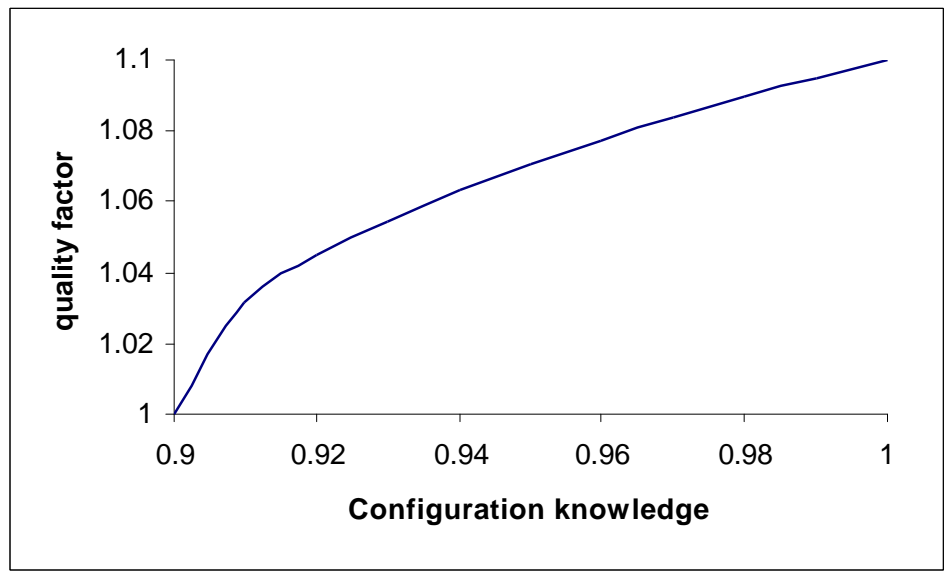

Figure 4.3.3-34: Quality Configuration Knowledge Factor 


\subsubsection{Change Sector}

Historically, changes, most importantly regulatory changes, had played an important role in most of the nuclear power plant construction projects. These changes brought many upsets, sometimes turmoil to the projects. They could create a huge amount of unexpected new work such as construction of auxiliary safety facilities, and they could require significant revision of released work in order to meet the new safety standard, for instance. Morale of the whole organization is impaired when changes occur, and a large amount of management's time has to be dedicated to dealing with the changes, thus becoming less available to oversight the project and harm the productivity and quality of other work; what's more, sometimes they made misjudgments when dealing with changes, which turned the already bad situation into even worse.

\subsection{Active Changes}

Figure 4.3.3-35 models how many changes are currently 'active', or, unfinished. It is assumed that, as long as a change is 'active', the management needs to allocate time to deal with it. Potentially, this will suck up the management's time and make them less available for work supervision, which then will affect the productivity and quality of the project.

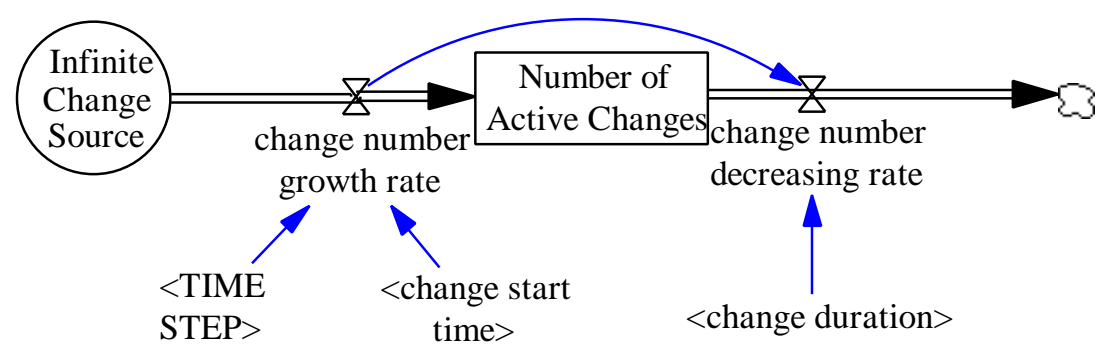

Figure 4.3.3-35: Model For Number Of Active Changes

The number of active changes will accumulate as new changes come in, and decrease when the changes are finished.

\subsection{Scope Change}

Scope change reflects the unexpected work recognized and the work made obsolete after the baseline is made. The change of scope is not necessary approved by the clients, or, in other words, the scope change defined here is broader. In fact, any change in scope, whatever the source and whoever initiates it, is included here. 


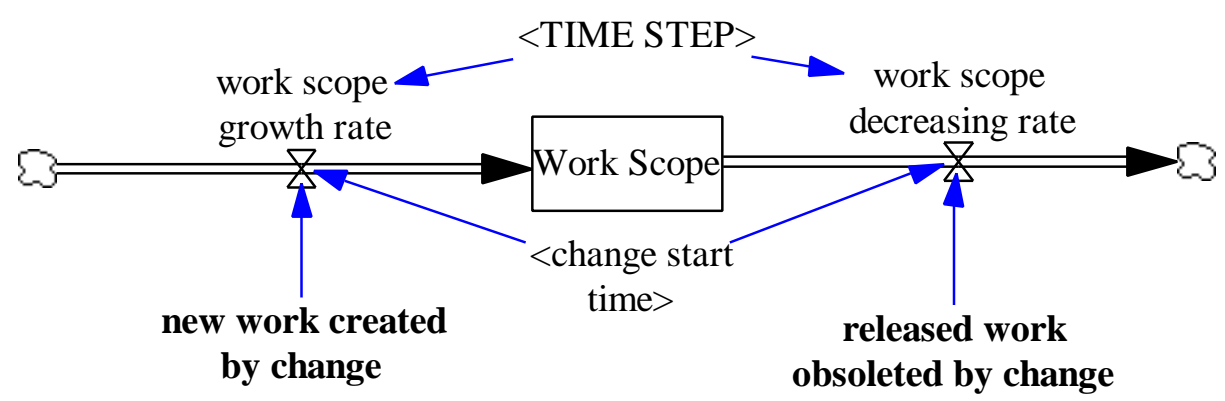

Figure 4.3.3-36: Work Scope Change Model

Note that the work that requires revision because of changes will not change the work scope because there is no new work flows into the system, but only that some work flows from stock to another stock within the system (See Figure 4.3.3-1).

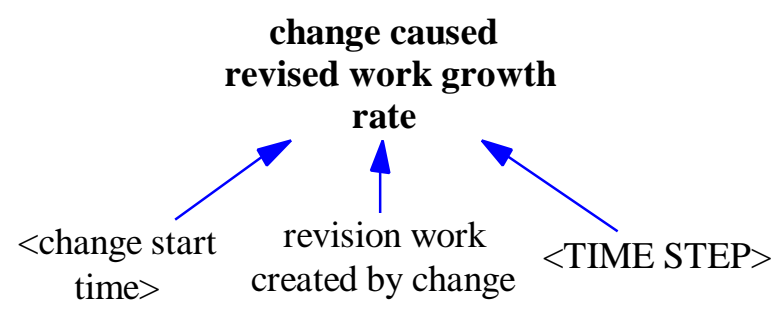

Figure 4.3.3-37: Revision Work Caused By Change

\subsection{Budget Change}

A scope change is generally accompanied with a budge for the change, especially if it is initialed or approved by the clients.

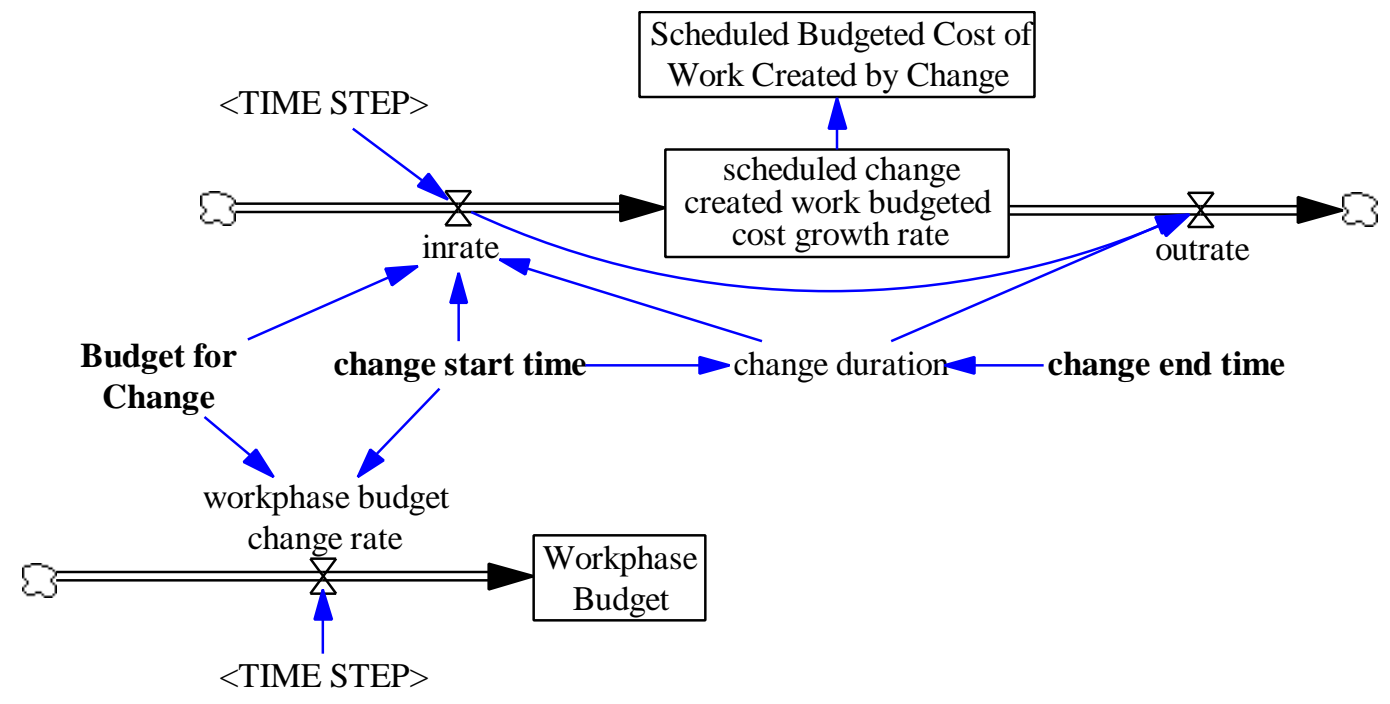

Figure 4.3.3-38: Budget Change Model 
At the time the change starts, the budget for the change flows into the system immediately.

\subsubsection{Management Action Sector}

In order to maintain schedule and budget, project managers often need to take corresponsive actions based upon current project performances or to deal with unanticipated changes. In a SGR project, the typical management actions are shown in Table 4.3.3-1, where 'resource change' and 'scope or schedule change' are actually modeled in the other subsystem. We generalize the remaining actions into three categories: overtime, work force adjustment, and equipment adjustment.

Table 4.3.3-1. Action Alternatives For SGR Project

\begin{tabular}{|l|l|}
\hline \multicolumn{1}{|c|}{ Overall project level } & \multicolumn{1}{c|}{ Individual work task level } \\
\hline \hline Resource change & Resource transfer \\
\hline \multirow{4}{*}{ Human resource change } & Hire from job market \\
\cline { 2 - 2 } & Hire from contractor \\
\cline { 2 - 2 } & Overtime work \\
\cline { 2 - 2 } & Layoff person \\
\hline Scope or schedule change & Scope reduction \\
\hline \multirow{2}{*}{ Process change } & Introduction new technology \\
\cline { 2 - 2 } & Equipment reduction \\
\hline Do thing & Do thing \\
\hline
\end{tabular}

\subsection{Overtime}

People who insist on overtime seldom realize the associated hidden costs, which can be more significant than premium pay for the overtime hours. Premiums affect only overtime hours, but continuing scheduled overtime drastically affects the costs of all work hours and makes all work hours more expensive (American Subcontractors Association, 1995). Loop 1 in Figure 4.3.3-39 is the expect effect that most project managers see. Overtime is used to promote the effective full-time work force. Given other things unchanged, the production rate can be expected to increase, thus diminishing the schedule pressure. However, the hidden effects of loops 2, 3, and 4 are generally overlooked or underestimated. In fact, these effects can be very significant, especially when fatigue and morale problems accumulate after many overtime weeks. Both fatigue and morale problems will damage the productivity and lowered the production rate, which offsets the effect from loop 1. At the same time, the quality lost due to low morale will incur more rework. This in fact also counters part of the desired effects. 


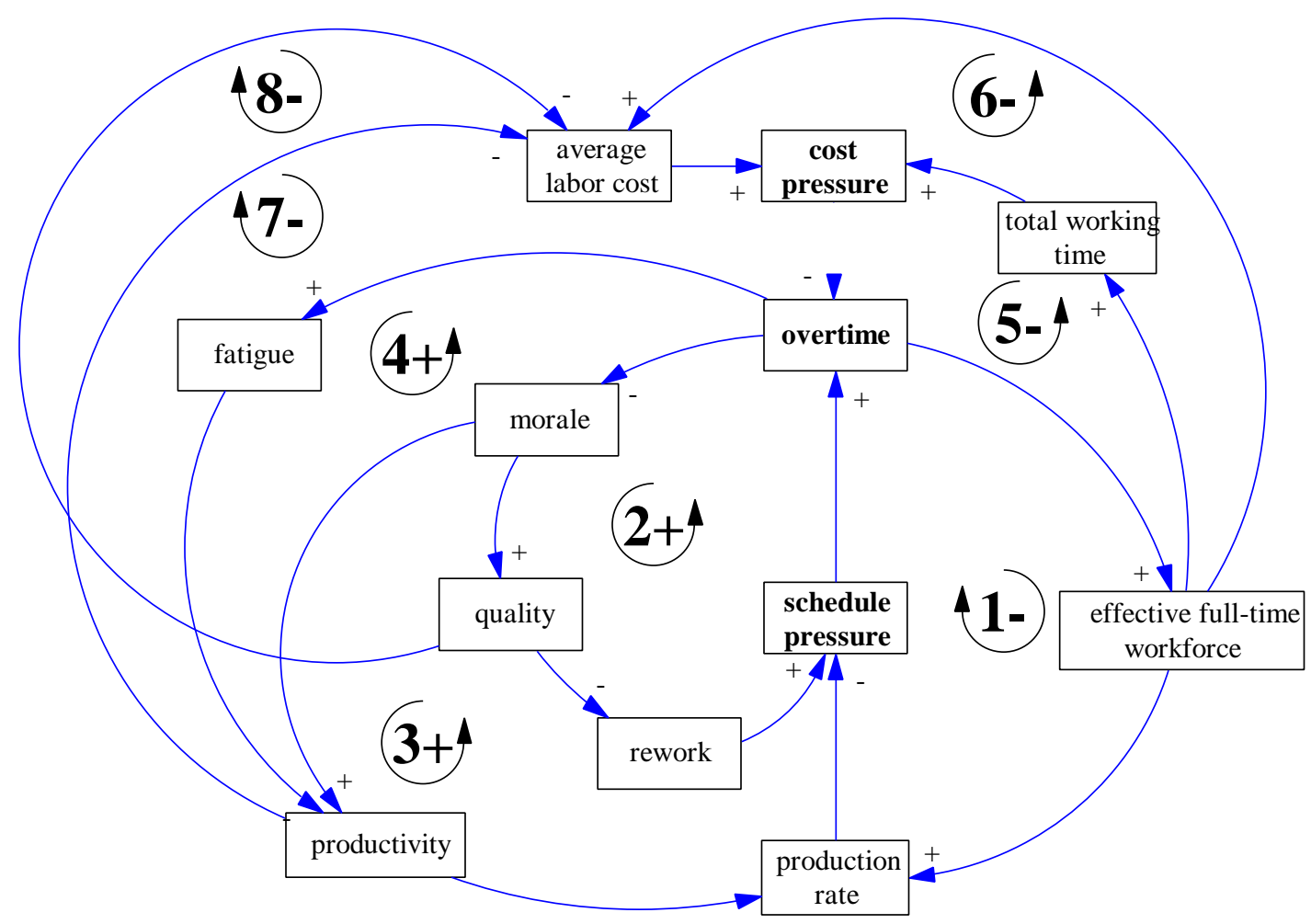

Figure 4.3.3-39: Feedbacks Related To Overtime Management Action

Loops 5, 6, 7, and 8 demonstrate the costs effects of the use of overtime. Since workers work longer hours per day, more money needs to be paid to the workers compared to what they are normally paid. However, as loop 6 indicates, since overtime hours are paid at a premium, the total hours are paid at a higher than normal rate. On the other hand, because of the lowered productivity and quality, the work completed for every dollar paid decreases, leading the labor costs more expensive (see loop 7 and 8) for not only the overtime hours, but also normal hours.

\subsection{Workforce Adjustment}

If overtime is not the right answer, then what can the project management do to reduce schedule pressure? A straightforward answer is to increase the size of the staff. However, due to the unavailability of labor in some situations, this is not always a valid option; even when the labor is fairly available, the project manager may still need to provide some economic stimulus to induce them to the project from other employers, which makes the cost unacceptable.

Yet there are other implications: a high level of work force can bring many side effects. First of all, the needs for ramp-up training and coordination rise. Productivity-reducing accidents are more likely, and more sit-and-wait congestion-related problems can hardly be avoided, especially when the working site is a confined place such as nuclear island. 
As shown in Figure 4.3.3-40, loop 1 depicts the expected picture of increasing the size of the work force. Compared to overtime work, an increase in size of the work force is not at the price of a premium payment. Therefore the costs will increase at steady rate if the level of work force size is below the permitted ceiling, while the schedule pressure is reduced (see loop 1); However, the large size of work force, even though below the root, means more ramp up training and coordination. At the beginning, the effect of increasing manpower may not be as expected because of the unfamiliarity of new workers.

The negative effect will not become a phenomenal problem until the size of the staff reaches its limit. At this time, because of the adverse effects following congestion problem, the productivity of the workers', and, thus, the production rate will be lowered (see loop 2), offsetting part of the expected feedbacks in loop 1. At the same time, the lowered productivity results in less amount of work produced with a same budget, and subsequently the average labor cost from the perspective of products produced is higher (see loop 3). As a result, costs will increase at a higher than normal rate.

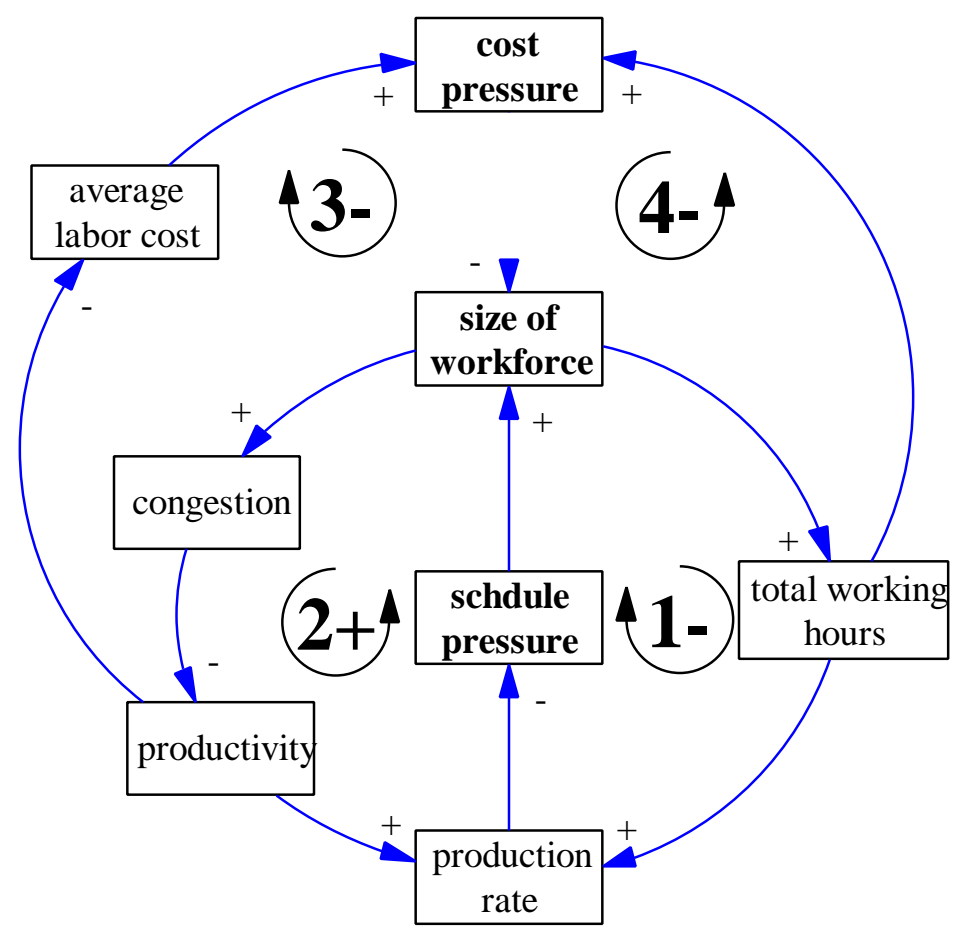

Figure 4.3.3-40: Feedbacks Related To Workforce Adjustment 


\subsection{Tool Adjustment}

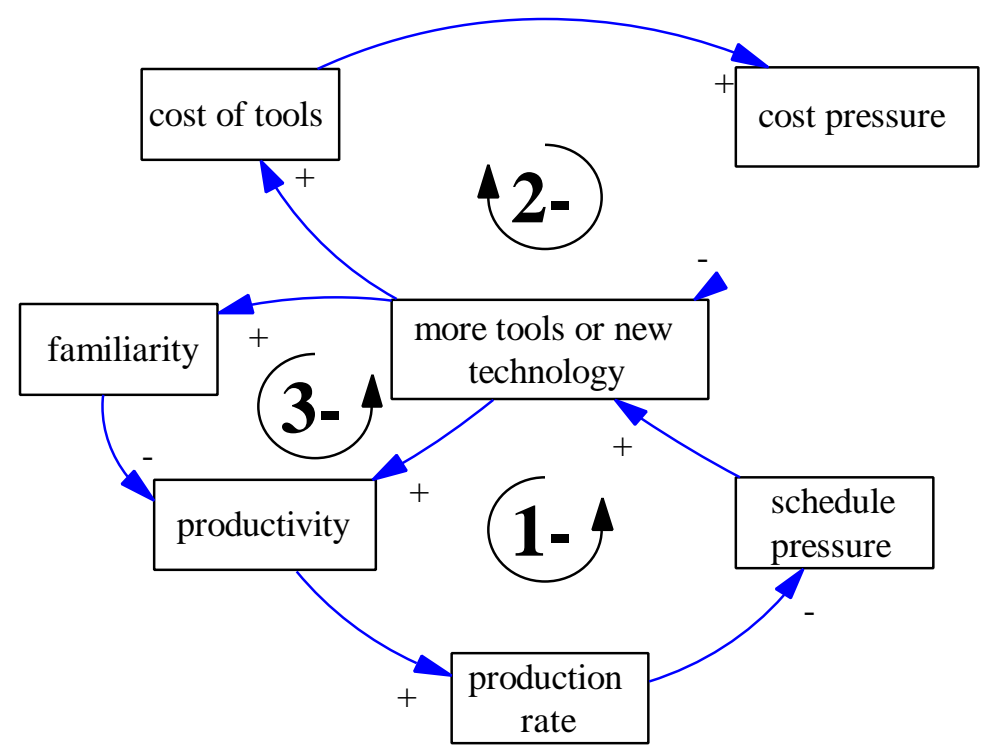

Figure 4.3.3-41: Feedbacks Related To Tool Adjustment

Machine and new technologies can greatly improved productivity, but sometimes this benefit is accompanied at a forbidding cost for obtaining the equipment or new technology. Also the training is necessary, or otherwise the killing-effect of low familiarity will make expected benefits less likely. Figure 4.3.3-41 shows the feedback of tool adjustment process.

\subsubsection{Performance Sector}

All the efforts are aimed to achieve good project performance. It is about economic return, it is about reputation, and it is about the company's survival in the future. In this model, three performance indicators are modeled to show the health of the project: quality, schedule, and cost.

\subsection{Quality Performance}

Concerns about nuclear plant safety have permeated the design and construction of nuclear plants. Quality performance of the construction is critically important for engineering company's success in this market. 


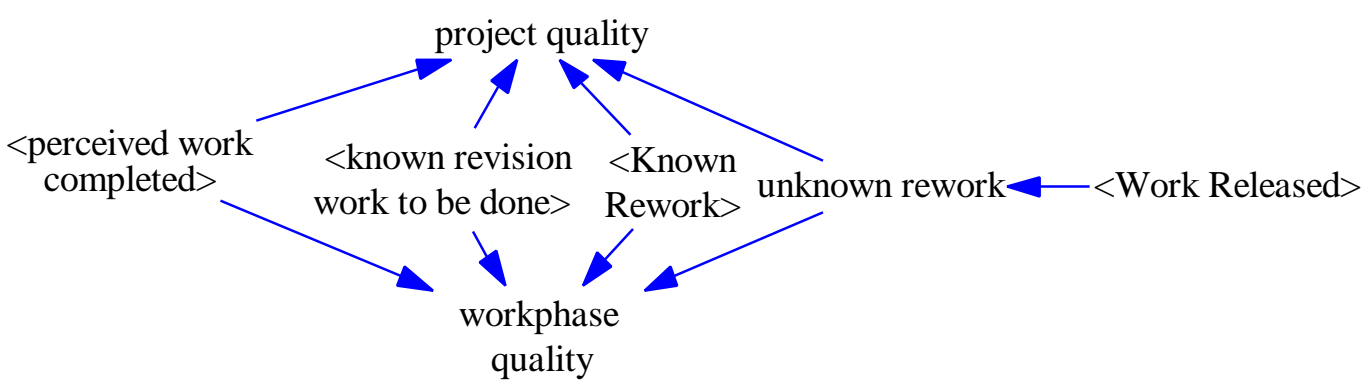

Figure 4.3.3-42: Project Quality Model

In this model, quality is defined as the ratio of all rework, both known and unknown, to all the work completed. This is somewhat different from what the project managers define it, where only known rework is considered. Technically speaking, it is almost impossible to evaluate the unknown portion of the flawed work, but that does not mean the project quality is what the project managers claim it to be, unless they can prove there is no unknown rework.

\subsection{Schedule Performance and Cost Performance}

Nuclear power plant generally has a very large capacity. Everyday it can generate the revenue in millions of dollars. In other words, time is very precious for a nuclear power plant. An early wrap-up of the project not only brings covenant bonus to the engineering firm, but also generates goodwill value to the company for its good reputations.

At the same time, in order to create returns, the project managers have to try their best to keep the costs under control. In Project Management Institution's book, 'roject Management Body of Knowledge, a set of schedule and cost indices are introduced, schedule performance index (SPI) and cost performance index (CPI) are the two that we are going to use in this model. 


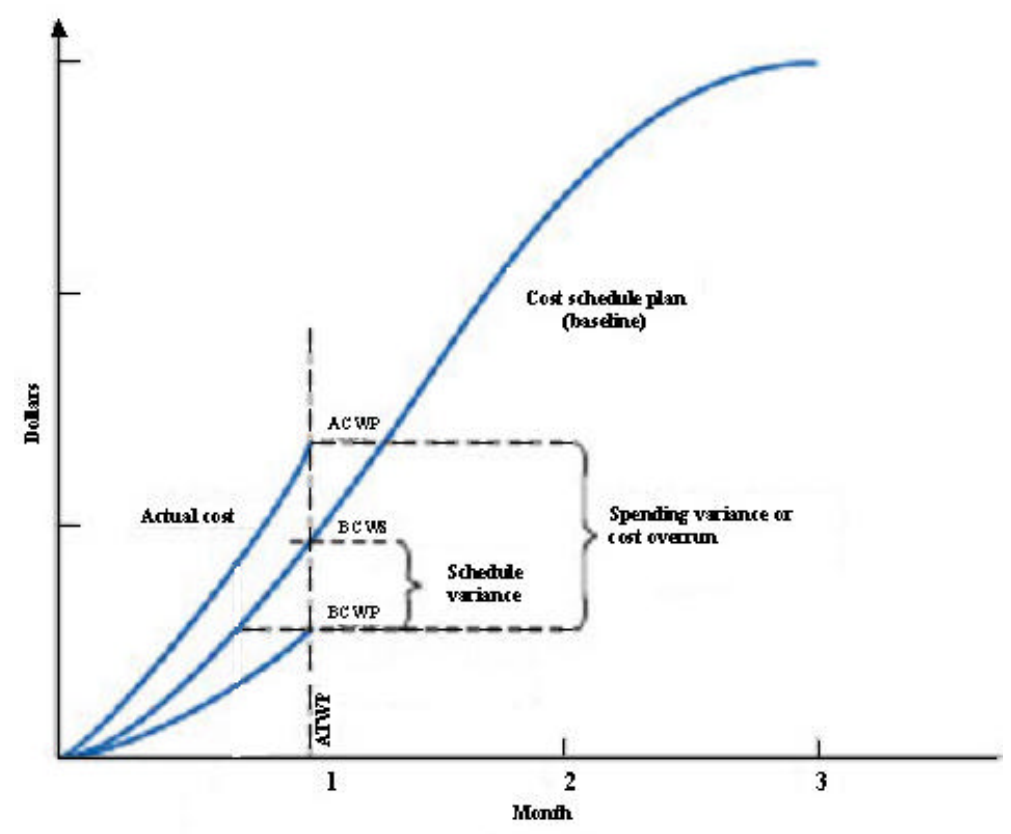

Figure 4.3.3-43: Project Schedule And Cost Performance Indices

Table 4.3.3-2: Some Cost And Schedule Terminologies In Project Management

\begin{tabular}{|c|c|}
\hline Acronym & Definition \\
\hline BCWS & Budgeted cost of work scheduled \\
\hline BCWP & Budgeted cost of work performed \\
\hline ACWP & Actual cost of work performed \\
\hline
\end{tabular}

See Figure 4.3.3-43. There are a few project managers' jargons. The definitions are listed in Table 4.3.3-2. In a schedule and cost overrun project, the BCWS, BCWP, and ACWP will look like Figure 4.3.3-43: BCWP is less then BCWS because of lagging behind, and ACWP is greater than BCWP because of cost overrun.

With these three parameters, the SPI and CPI are defined as:

$$
\begin{aligned}
& \text { SPI } ? \frac{B C W P}{B C W S} \\
& \text { CPI } ? \frac{\text { BCWP }}{A C W P}
\end{aligned}
$$

For both SPI and CPI, a value of unity means exactly on schedule or cost, while the larger the value, the better. If we assume every unit of work cost a same amount, then SPI and CPI will be:

SPI $? \frac{\text { actual work performted }}{\text { scheduled work performed }}$ 


$$
\text { CPI ? } \frac{\text { budgeted cost per work unit performed }}{\text { actual cost per work unit performed }}
$$

Before the description of how the SPI and CPI are calculated, let's look at how the costs are determined. It is assumed that project costs are linearly associated with labor cost; therefore project costs can be computed by scaling up the labor cost with a multiplier called 'total cost/labor cost ratio'.

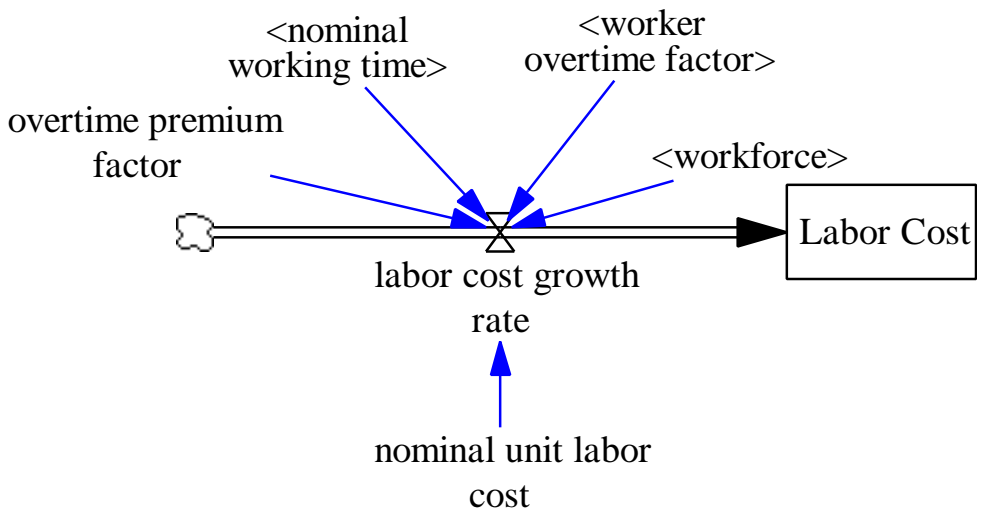

Figure 4.3.3-44: Labor Cost Model

The fact that reduced productivity and quality leads to higher unit labor cost is considered in accumulated person hours needed to finish the work. At the same time, the overtime hours paid in premium are also accounted for by the 'overtime premium factor'.

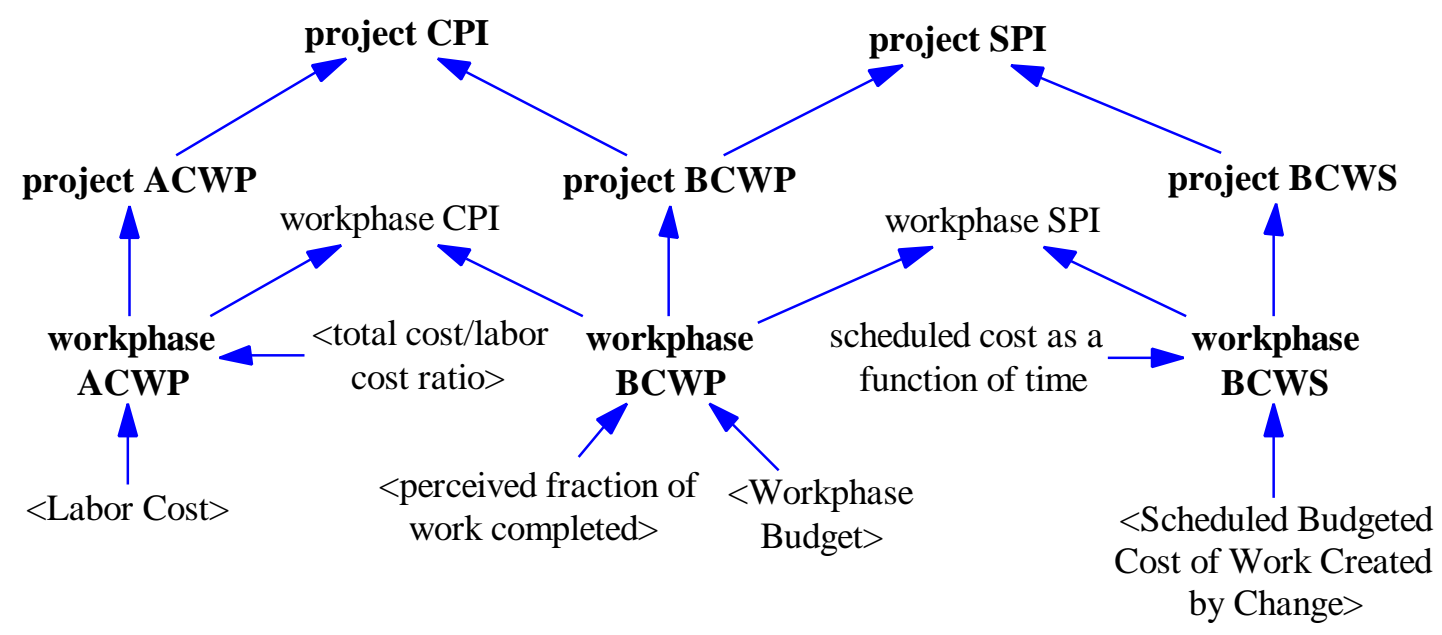

Figure 4.3.3-45: Project Schedule Performance And Cost Performance

In order to compute SPI and CPI, the three parameters ACWP, BCWP, and BCWS need to be first determined. In the work phase level, ACWP is calculated by multiplying the labor cost with the multiplier, 'total cost/labor cost ratio'; BCWP is calculated by multiplying the scheduled total budget with the fraction of work completed; BCWS, at a specific time, 
equals to the sum of scheduled cost for the original work scheduled to be completed and the scheduled cost for the change-created work scheduled to be completed by this time.

\subsubsection{SYSTEM BEHAVIOR ANALYSIS}

In the project level, ACWP, BCWP, and BCWS are simply the sum of those in each work phase.

In this section, the baseline schedule typical of a Steam Generation Replacement project is used as basis to analyze the behavior of the model under some imaginary events and management actions. The behaviors will be shown as temporal plots of several key parameters representing project progress and performance.

Table 4.3.4-1 lists the basic information about the baseline schedule.

Table 4.3.4-1: Basic Information About The Baseline Schedule

\begin{tabular}{|c|c|c|c|c|c|}
\hline Items & $\begin{array}{l}\text { Conceptual } \\
\text { Design }\end{array}$ & Detailed Design & \begin{tabular}{|c|} 
Work \\
Package \\
Develop- \\
ment
\end{tabular} & $\begin{array}{l}\text { Mobiliza- } \\
\text { tion }\end{array}$ & Outage \\
\hline $\begin{array}{l}\text { Initial Work to be Done (work } \\
\text { unit) }\end{array}$ & 177 & 153 & 515 & 975 & 2320 \\
\hline unit labor $\operatorname{cost}(\$ /$ PersonHour $)$ & 40 & 40 & 40 & 30 & 30 \\
\hline total cost/labor cost ratio & 1 & 1.2 & 1.4 & 1.4 & 2.75 \\
\hline Scheduled total $\operatorname{cost}(\$)$ & $8.09 \mathrm{E}+05$ & $1.22 \mathrm{E}+06$ & $2.56 \mathrm{E}+06$ & $1.72 \mathrm{E}+06$ & $8.06 \mathrm{E}+06$ \\
\hline Schedule Start Time(week) & 0 & 31 & 57 & 143 & 151 \\
\hline $\begin{array}{l}\text { Scheduled Productivity (work } \\
\text { unit/person*week) }\end{array}$ & 0.5 & 0.3 & 0.5 & 1 & 1 \\
\hline Scheduled Quality of practice & 0.7 & 0.8 & 0.9 & 0.95 & 0.95 \\
\hline Review Quality & 0.8 & 0.9 & 0.9 & 0.95 & 0.99 \\
\hline $\begin{array}{l}\text { accepted flawed work } \\
\text { discovery time (week) }\end{array}$ & 20 & 10 & 5 & 2 & 1 \\
\hline Maximum number of workers & 50 & 50 & 50 & 500 & 500 \\
\hline $\begin{array}{l}\text { Congestion critical worker } \\
\text { number }\end{array}$ & 18 & 30 & 18 & 154 & 275 \\
\hline $\begin{array}{l}\text { Labor Market Productivity } \\
\text { (work unit/person*week) }\end{array}$ & 0.5 & 0.3 & 0.5 & 1 & 1 \\
\hline $\begin{array}{l}\text { Labor Market Quality of } \\
\text { practice }\end{array}$ & 0.7 & 0.8 & 0.9 & 0.95 & 0.95 \\
\hline $\begin{array}{l}\text { Phase starts when upstream } \\
\text { phase finishes }(\%)\end{array}$ & 1 & 0.953 & 0.921 & 0.979 & 0.999 \\
\hline
\end{tabular}

\subsubsection{Baseline System Behavior}

Baseline is scheduled based upon expected information, e.g. expected productivity, expected quality, expected work scope, etc.: not better, not worse. The actual values of these variables will never by the same as expected, though the deviations may be small. Yet 
in the baseline simulation, it is assumed that all the input information is the same as expected.
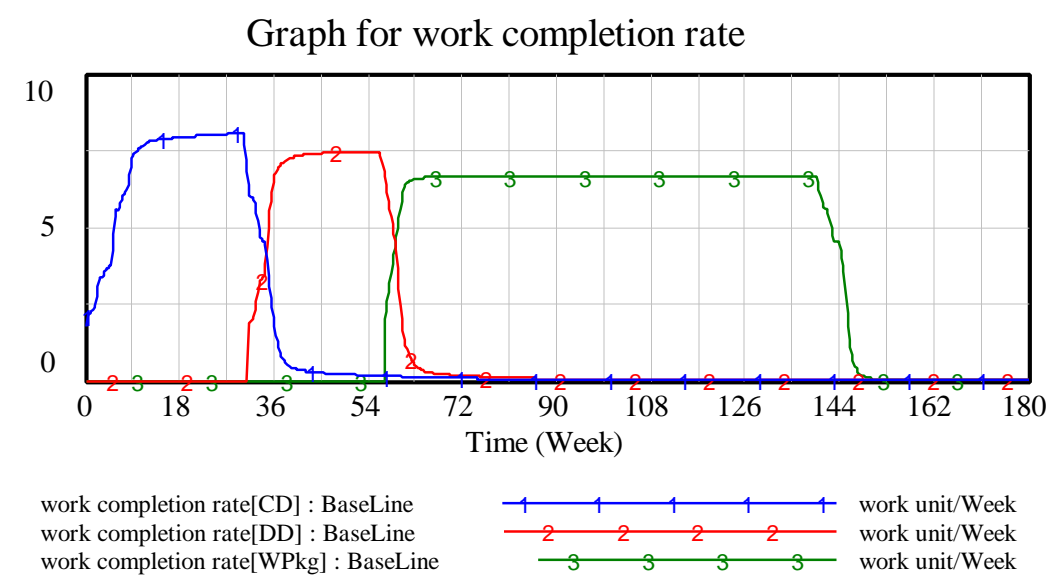

work completion rate[WPkg] : BaseLine

Graph for work completion rate

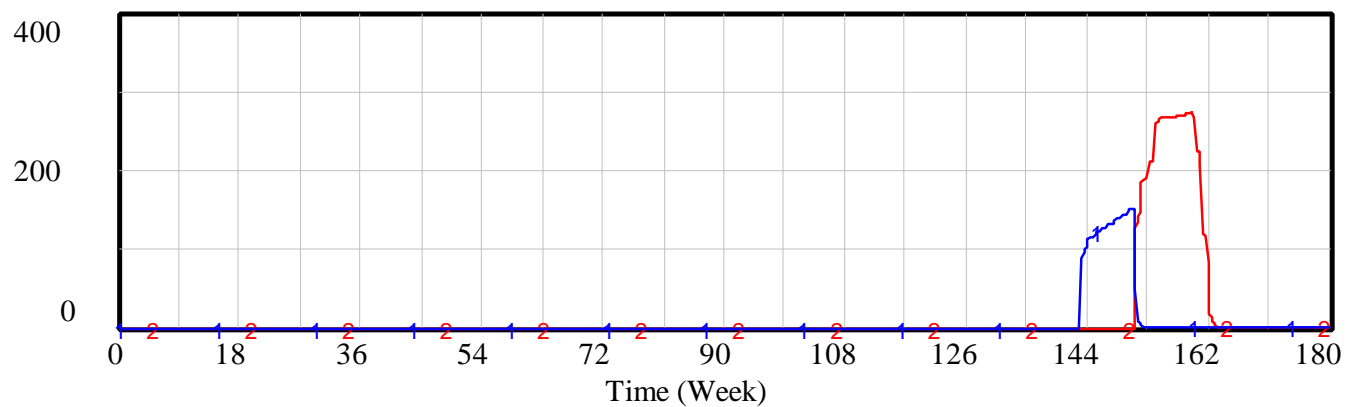

work completion rate[Mob] : BaseLine work completion rate[Outage] : BaseLine

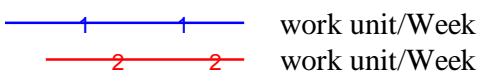

Figure 4.3.4-1: Baseline Work Completion Rate For Each Work Phase

Figure 4.3.4-1 shows the baseline work completion rates for each work phase. Each of them follows a similar pattern: starts low, grow quickly, sustain in some level, and drops to zero quickly in the end. This is the typical, or ideal pattern that most schedule makers strive to work out. In this way, the resources are levelized, and resources management is eased. Resource leveling (often referred to as resource smoothing) attempts to reduce the sharp variations among the peak sand valleys in the resource demand histogram while maintaining the original project duration (Moselhi, a., and Lorterapong, P. 1993).

If we look at the ' $y$ ' axis, it is not difficult to notice that the work completion rates in construction work phases are much larger than those in design and work package development work phases. In fact, $60 \%$ of the work (in terms of work hours) is performed in the last two phases, while the duration of last two phases is only $12 \%$ of the total time. This is why it is so important and difficult to management the construction phases, which has little flexibility and little room to allow changes and mistakes. 
Since expected quality of practice can never be perfect, there will be rework associated with it. As an example, Figure 4.3.4-2 shows the rework growth rate in the outage phase. Basically, it has a pattern close to the work completion rate pattern, but skew toward the latter end.

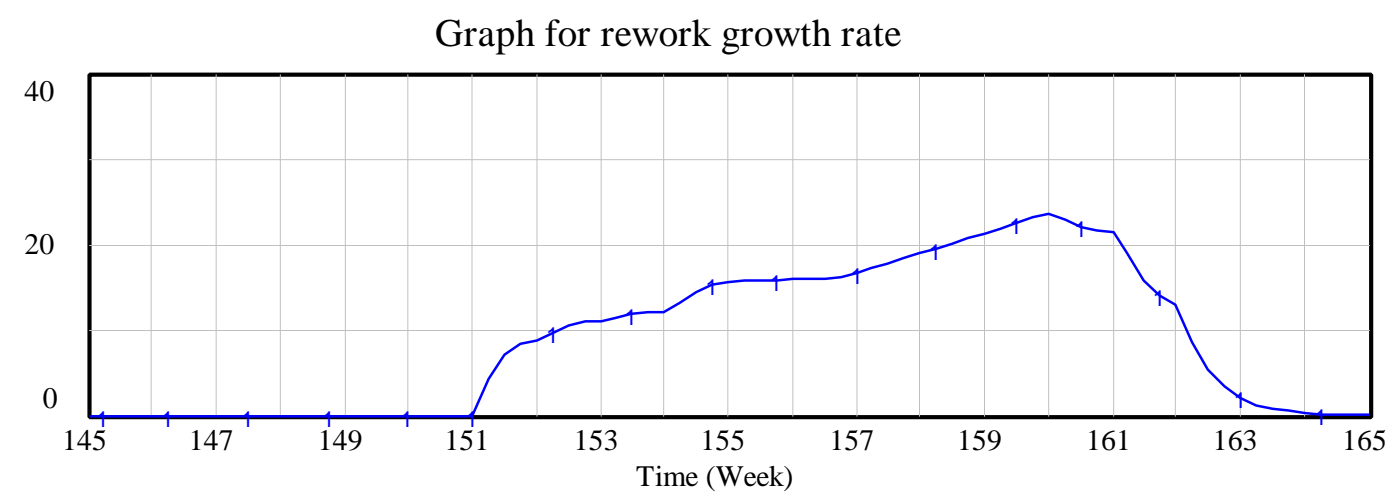

rework growth rate[Outage] : BaseLine

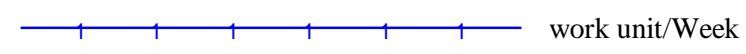

Figure 4.3.4-2: Rework Rate In Outage Work Phase

The reason for the skew is explained in Figure 4.3.4-4. Three are three sources of rework (see Section 4.3.3.2.3): rework rejected in the work review process, accepted flawed work discovered in downstream tasks, and accepted work inherited from upstream flawed work on which it is based. At the same time, there are two components in each part of the rework: flawed work and good work, by their nature. While flawed work should unquestionably be sent to rework, the good work is incriminated because they are associated with the flawed work and needs to be done together (see Section 4.3.3.2.3). The ratio of incriminated good work to associated flawed work grows as more and more work is completed (see Figure 4.3.4-3). It makes sense since at the end, a small change to a device would require an overhaul of the system, while at the beginning it does not matter that much.

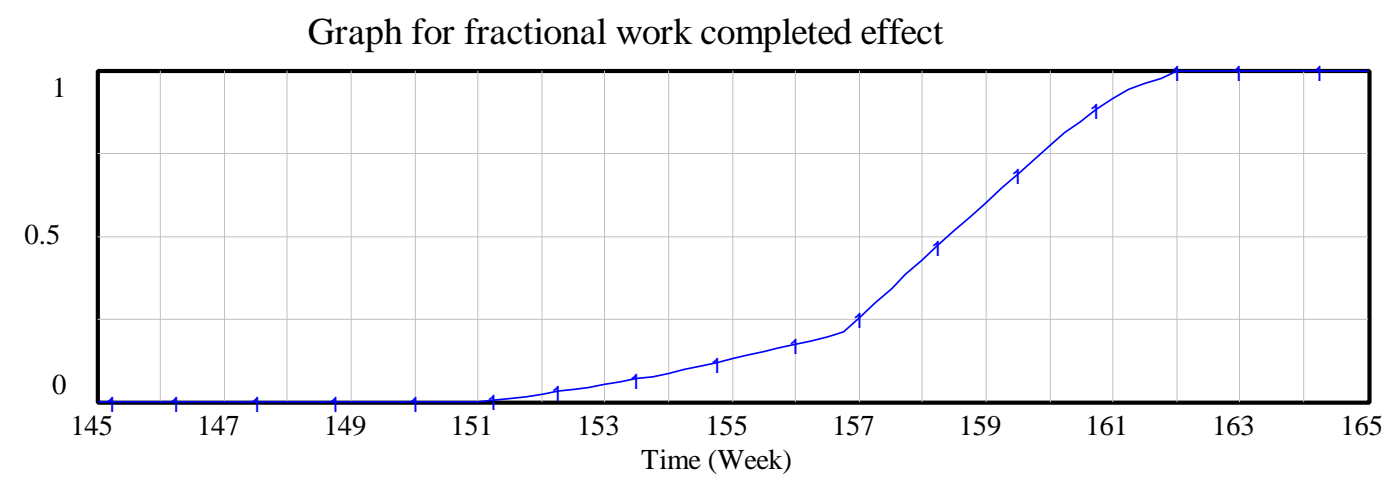

fractional work completed effect[Outage] : BaseLine

Figure 4.3.4-3: Good Work That Is Forced To Be Reworked By Flawed Work Identified As A Function Of Fractional Work Completed, Expressed In Ratio Of Incriminated Good Work To Flawed Work 
Because of this effect, the incriminated good work grows in such a pattern as in Figure 4.3.4-4. And the total rework, good plus flawed, aggregate to the shape as shown in Figure 4.3.4-2, with a peak at the latter half of the curve, then drops down to zero primary because much less work is performed at that time.
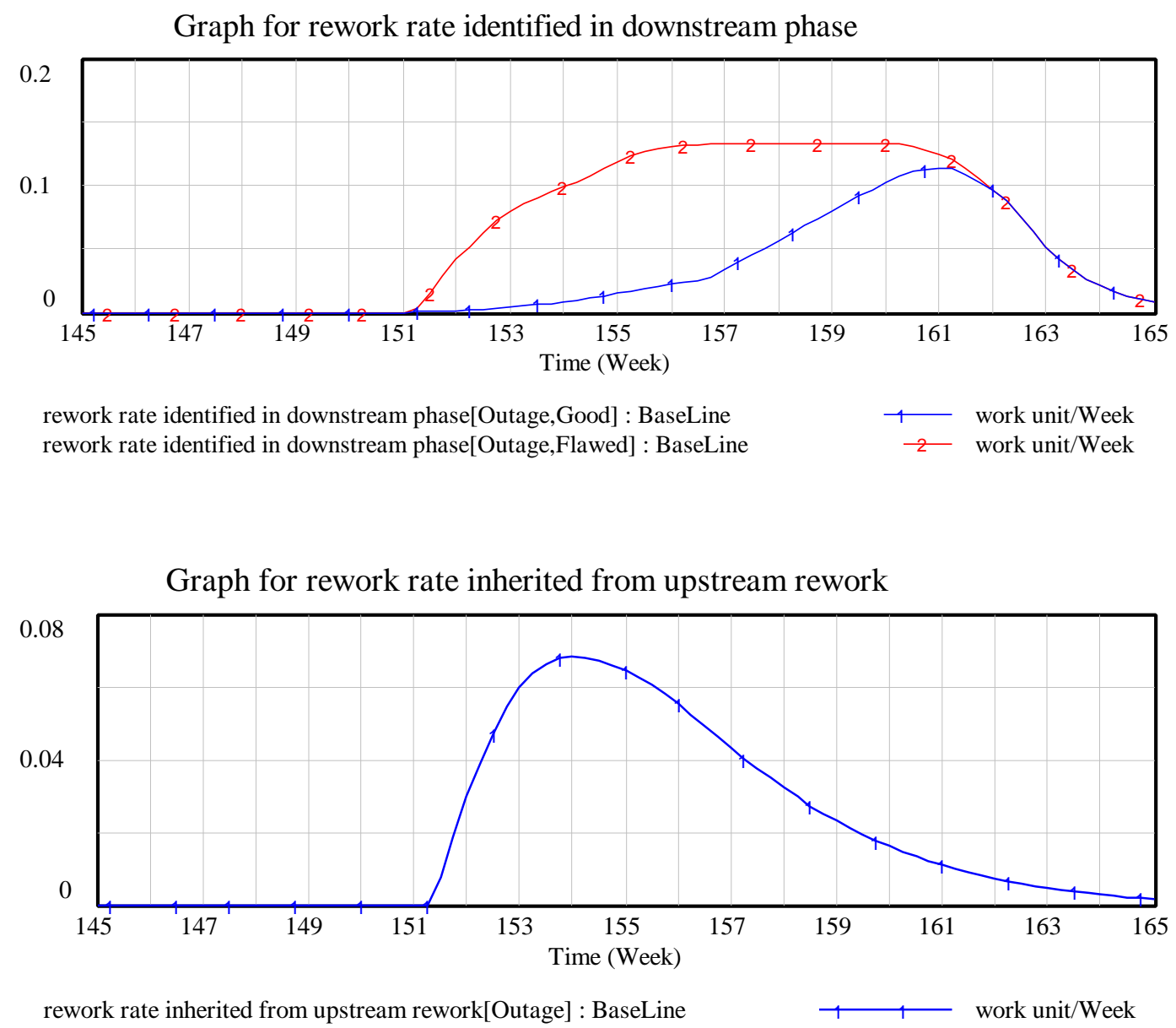

Figure 4.3.4-4: Components Of The Rework Rate In Outage Phase

One important implication of rework to human resource management is that, unlike base work that can be foreseen and make the hiring plan prior to the time it is actually performed, rework is assumed to be realized only when it is identified. The consequence is a delay with regard to staffing because of the time required to adjust workforce.

\subsubsection{System Behavior Under Lower-than-expected Productivity}

One surprise that often happens to the construction project is that the average productivity of the workers it hires is lower than what it expected in the baseline schedule. For instance, some of the states in America have regulations that require the employers to hire craft workers within the state. If the schedule maker assumes productivity attainable in his/her 
own state, while the project is conducted in another state that has such regulations, it is very likely that the assumptions are not correct, either because of unavailability of information, or because of incomplete information.

While it is possible to have higher than expected productivity, which is perfect and the management will be happy about, it is sometimes a tragedy if the actual available productivity is lower than expected. The reason for that is obvious: when making the schedule, especially an optimal one that most engineering company is seeking and most scheduling software claim they can provide, makes use of every possible room to complete the project in as shorter time as possible. In other words, the room left for adjustments to deal with surprises is very small, where when something bad really happens, the whole project goes into a mess, making in very hard to reverse it onto the right track because of a lot of ripple effects, some of them illustrated in the imaginary case below.

In here, an actual nominal (average) productivity that is $10 \%$ lower than the expected nominal productivity for the craft workers is assumed in the outage phase, with everything the same as the baseline case for the rest of the work phases.

It is also assumed that management will always take such a single action through out the project: adjust the size of the workforce by a factor of $\frac{1}{\text { SPI }}$, that is to say, to decrease the size of workforce when the project is ahead of schedule (SPI>1) in order to achieve better cost performance (pay less salary), and to increase the size of workforce when the project is lagging behind schedule $(\mathrm{SPI}<1)$ in order to catch up with the schedule. Although increasing the size of workforce will immediately increase the labor cost, making cost performance worse off, catching up with the schedule at such immediate cost is still worthwhile pursuing when considering the possible reputation loss and penalties associated with failure to make the deadline.

Figure 4.3.4-5 shows the comparison of actual productivities between baseline case and lower average productivity case. Because of unfavorable ripple effects described soon in this section (see also Section 4.3.3.7.3), the actual productivity attainable in the lower average productivity case is lower than $90 \%$ of its baseline peer.

It is assumed that there is a time delay when site data are available to planning departments such as human resource department. It is also assumed that the staffing decisions are made base upon available productivity and quality data. At the beginning of the outage, productivity is therefore deemed to be as expected, therefore the size of workforce is the same as in baseline, as shown in Figure 4.3.4-6. As project moves forward, the planning group will come to realize that the actual average productivity is lower than expected, as shown in Figure 4.3.4-7. By this time, new data are used in deciding the size of workforce, which is expected to be 1.11 times of that in baseline case---by linear thinking; however, since the productivity is lower than the one used in staffing prior to this point, the project is obviously lagging behind schedule at this point. According to the management actions described a short time ago, the size of the workforce should be even greater than 1.11 times of that in baseline case. 


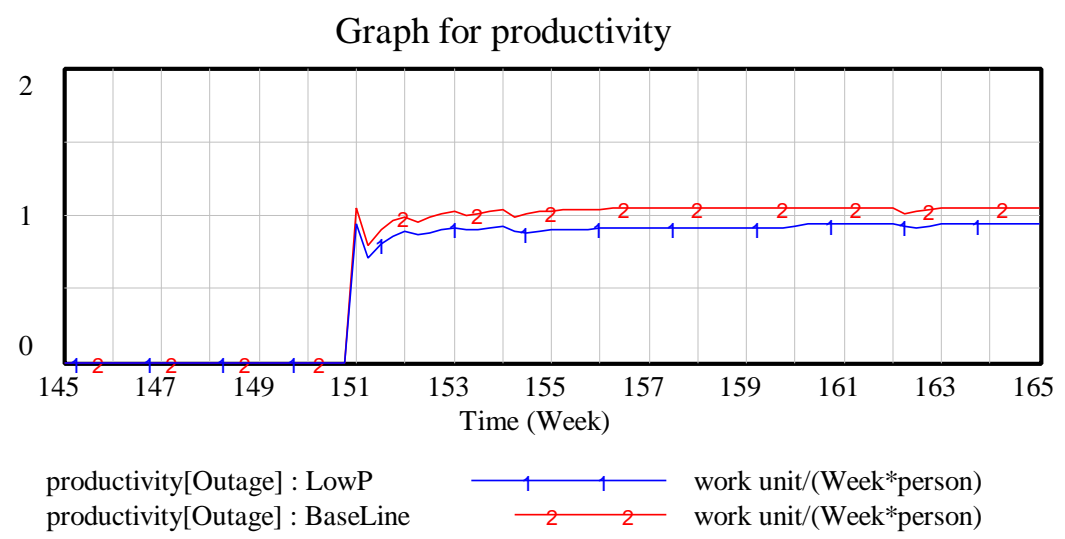

Figure 4.3.4-5: A Comparison Of Actual Productivities Between Baseline Case And Lower Average Productivity Case

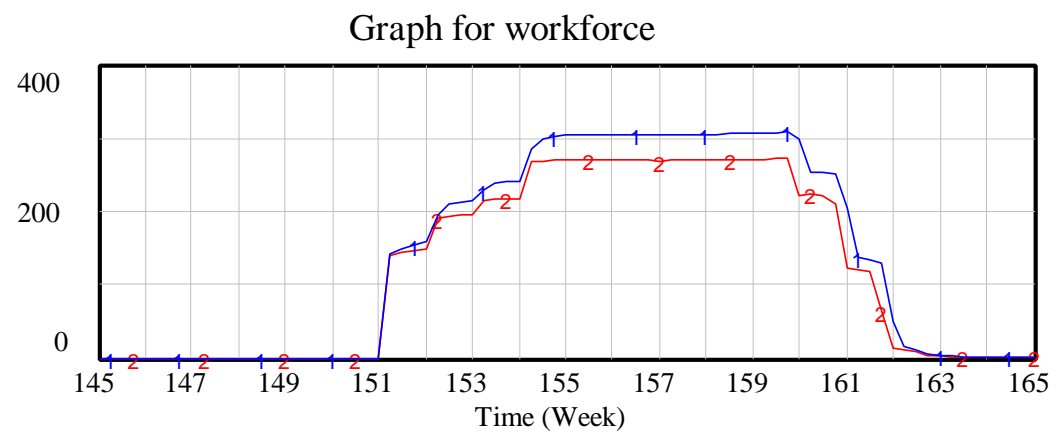

workforce[Outage,Worker] : LowP workforce[Outage,Worker] : BaseLine

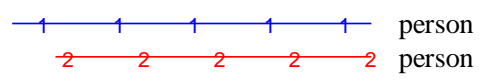

Figure 4.3.4-6: A Comparison Of Actual Workforce Between Baseline Case And Lower Average Productivity Case

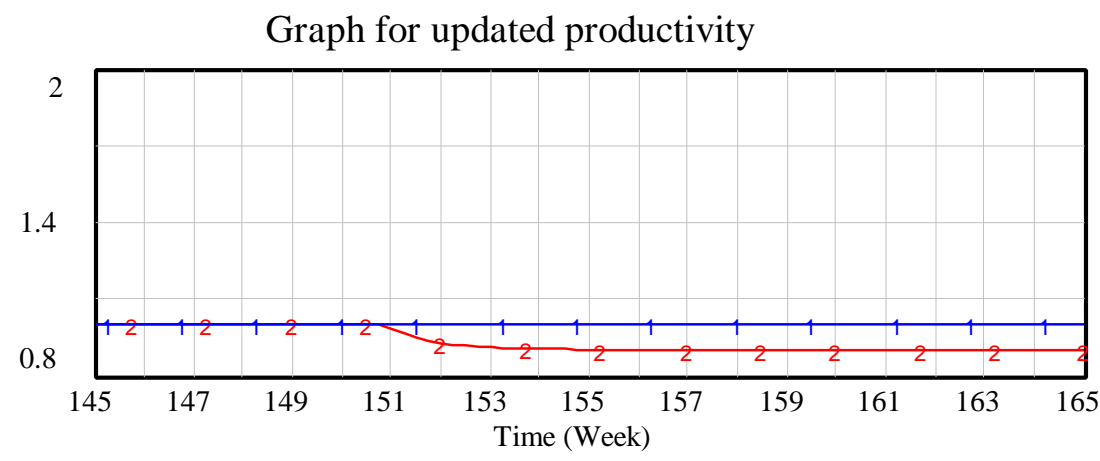

updated productivity[Outage] : BaseLine updated productivity[Outage] : LowP

-1 work unit/(Week*person)
-2 work unit/(Week*person)

Figure 4.3.4-7: A Comparison Of Realized Productivity Between Baseline Case And Lower Average Productivity Case 
Yet this is still not the end of the story. Since the schedule is an optimal one, as we mention earlier, there is little room left for adjustment. Now we are adding $11 \%+$ more workers, which will inevitably cause the congestion problem (see also Section 4.3.3.4.2). As shown in Figure 4.3.4-8, because of the sit-and-wait, the actual worker utilization rate is lower, making the actual productivity averaged among workers lower. This effect will digress the project from its reverse-back path to some extent.

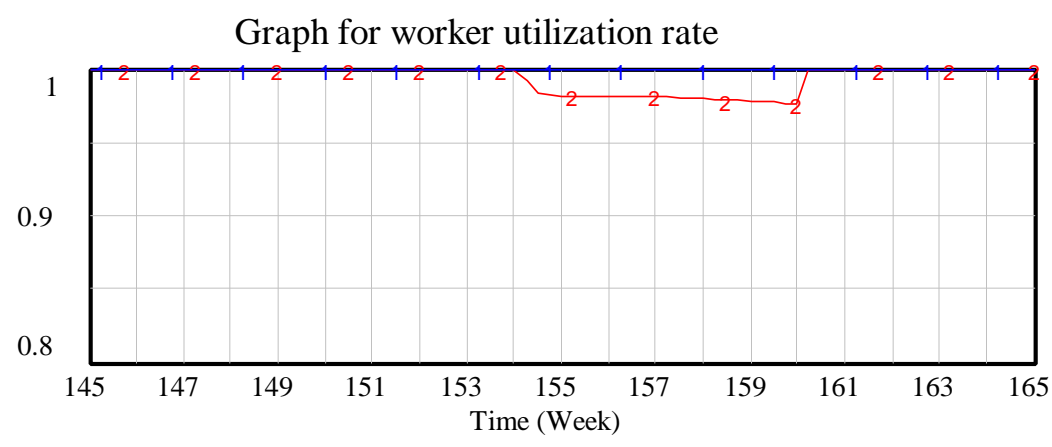

worker utilization rate[Outage] : BaseLine worker utilization rate[Outage] : LowP

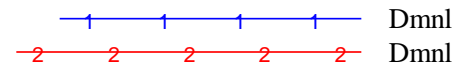

Figure 4.3.4-8: A Comparison Of Worker Utilization Rate Between Baseline Case And Lower Average Productivity Case

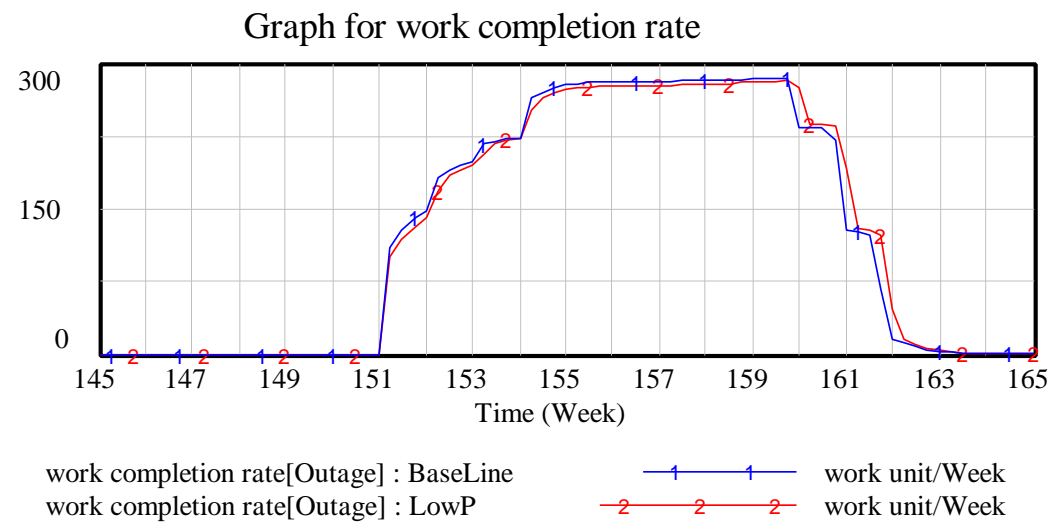

\section{Figure 4.3.4-9: A Comparison Of Work Completion Rate Between Baseline Case And Lower Average Productivity Case}

And for this reason, though the workforce is adjusted to reflect the lower average productivity, the actual work completion rate (workforce times productivity) of lower average productivity case is still lower than that of the baseline case, as shown in Figure 4.3.4-9. For project SPI, it cannot be recovered as expected because of the same reason, and it actually follows the path as shown in Figure 4.3.4-10. 


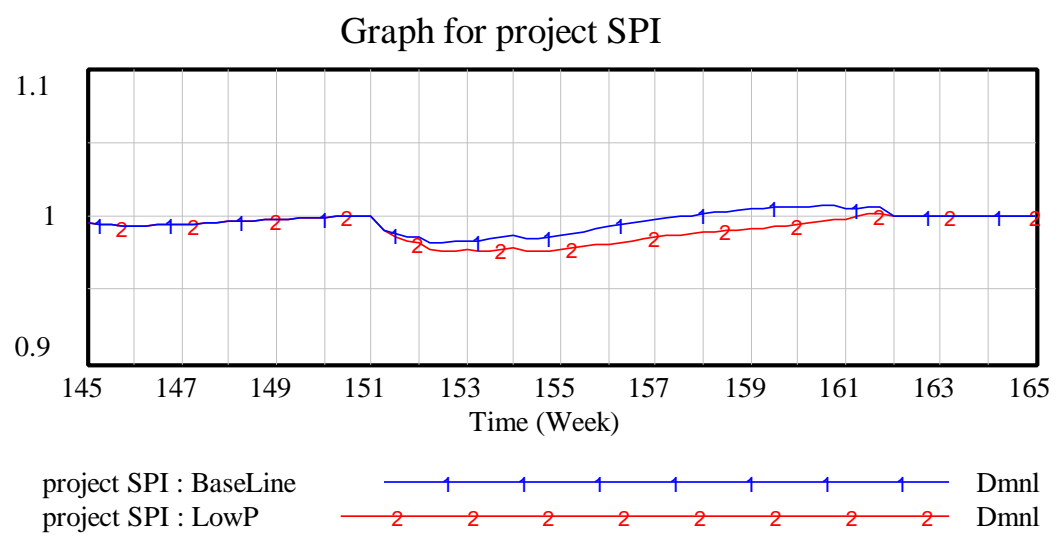

\section{Figure 4.3.4-10: A Comparison Of Project SPI Between Baseline Case And Lower Average Productivity Case}

As to cost, not surprisingly, the final actual cost for lower average productivity case is higher than baseline case, by a factor greater than 1.11, of course (see Figure 4.3.4-11).

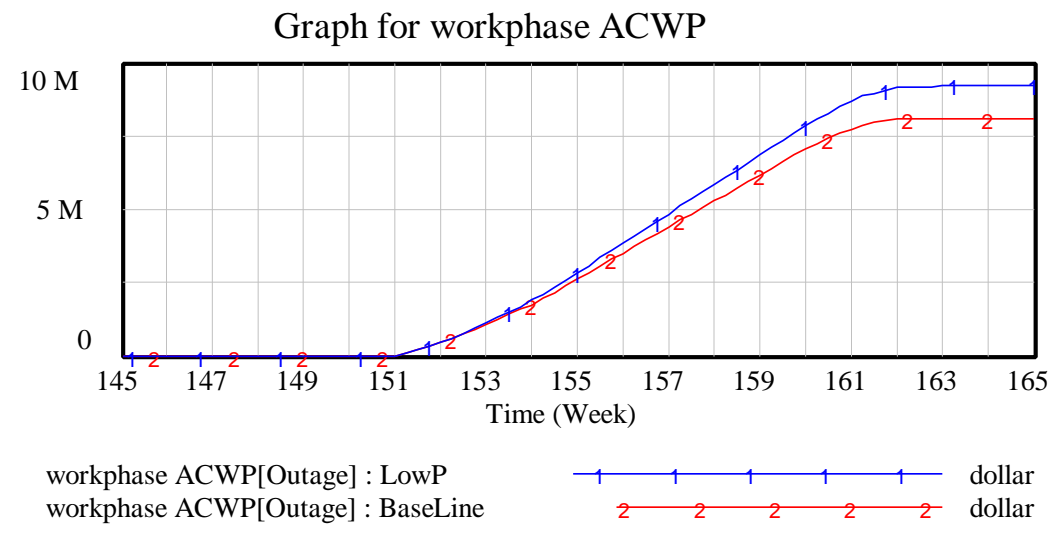

\section{Figure 4.3.4-11: A Comparison Of Actual Cost For Work Performed (ACWP) Between Baseline Case And Lower Average Productivity Case}

It should be noted that the actual feedbacks involved are much more than simply a congestion feedback. For example, since project is lagging behind schedule, more tasks will be early started in order to catch up, which cause more out-of-sequence work problem. All these feedbacks are reflected in the curves we just showed. Congestion feedback, nevertheless, is the one that has most significant impact under our management strategy in this case.

\subsubsection{System Behavior Under Lower-than-expected Quality}

Another surprise that project managers often face is that the average quality of the workers is lower than what it expected in the baseline schedule. As will be shown soon in below, over-estimation on quality will bring the project into a worse situation than a same degree of over-estimation on productivity. 
In this case, the actual nominal (average) quality is assumed to be $10 \%$ lower than the expected nominal quality for the craft workers in the outage phase, with everything the same as the baseline case for the rest of the work phases.

It is also assumed that management will take a same action through out the project as in the previous case: adjust the size of the workforce by a factor of $\frac{1}{\text { SPI }}$.

Figure 4.3.4-12 shows the quality comparison between baseline case and lower quality case, which is roughly yet lower than $90 \%$ of its baseline peer. Although there are feedbacks that tend to move actual quality lower than $90 \%$ of the baseline level, for example, the more out-of-sequence work because of more rework, the feedbacks are not very strong, therefore the deviation is not very phenomenal.

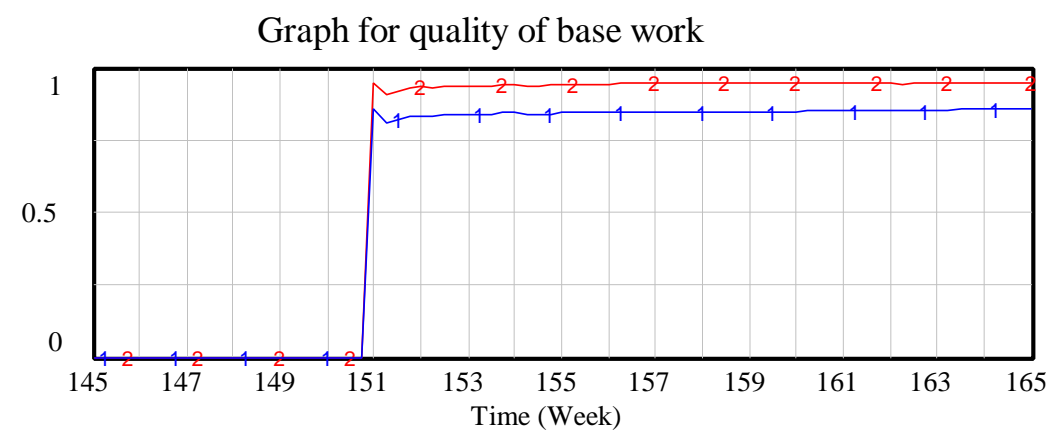

quality of base work[Outage] : LowQ quality of base work[Outage] : BaseLine

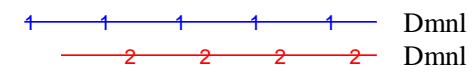

\section{Figure 4.3.4-12: A Comparison Of Quality Between Baseline Case And Lower Quality Case}

Work completion rate comparison is shown in Figure 4.3.4-13. Since in lower quality case, the productivity is higher, while the size of workforce is similar (see Figure 4.3.4-14), work completion rate in lower quality case is higher. However, remember completed work does not equal to good work, in lower quality case, a larger portion of the work completed is flawed. 


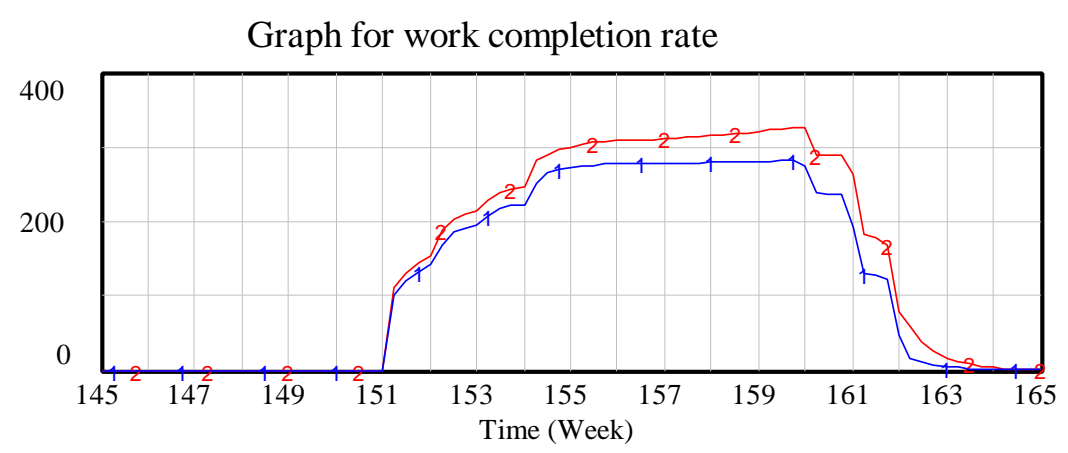

work completion rate[Outage] : LowP work completion rate[Outage] : LowQ

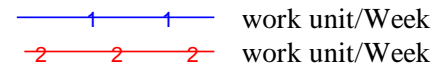

\section{Figure 4.3.4-13: A Comparison Of Actual Work Completion Rate Between Lower Productivity Case And Lower Quality Case}

The assumption that rework will not be considered in the staffing planning until it is discovered results in the system behavior in Figure 4.3.4-14. At the beginning, the rework, especially the part that is to be discovered in the downstream tasks, does not show up at the level that it actually reaches, while lower productivity in the other case has been realized. As a result, for the first half of the work phase, low productivity case has a larger size of workforce than the low quality case. However, as time goes on, more and more accumulated flawed work will be discovered, plus rework that is rejected in the review process and the portion of good work that is incriminated, which as a ratio to flawed work grows as more and more work is completed, these aggregate to form a curve as in Figure 4.3.4-15. The resultant size of workforce will be therefore higher than that of in the lower productivity case in the latter half of the staffing curve.
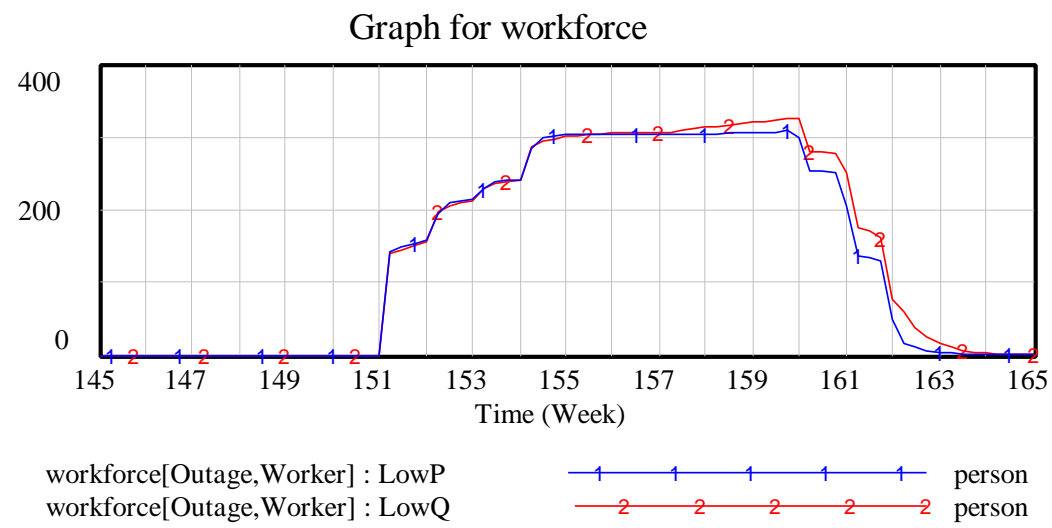

Figure 4.3.4-14: A Comparison Of Workforce Between Lower Productivity Case And Lower Quality Case 


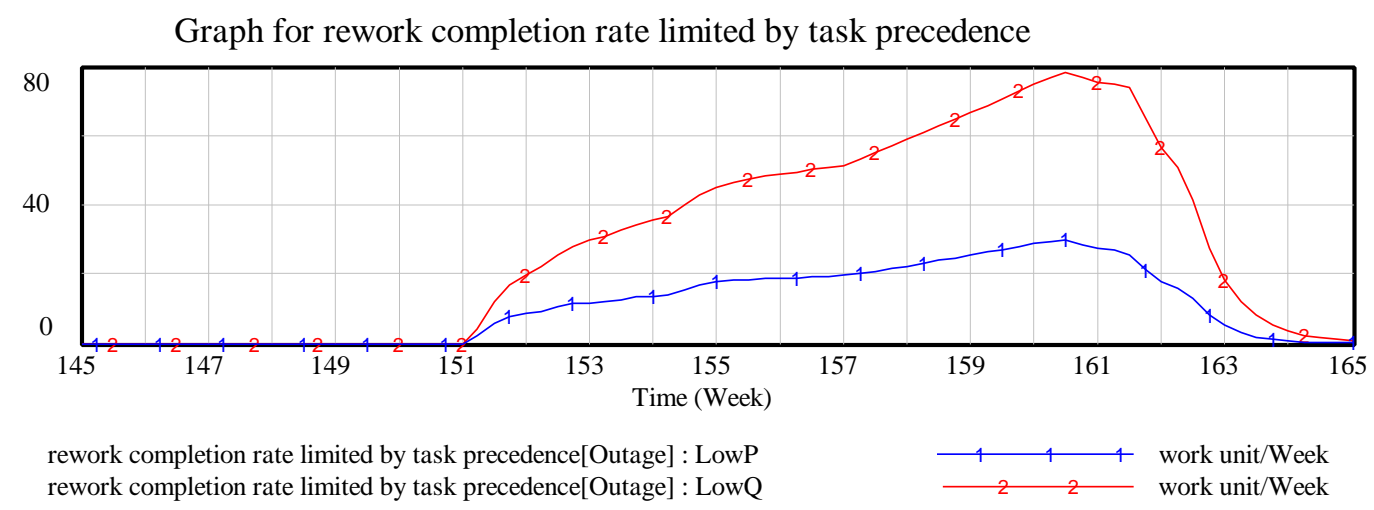

\section{Figure 4.3.4-15: A Comparison Of Rework Completion Rate Between Lower Productivity Case And Lower Quality Case}

For the same reason, project SPI and CPI behaves in a similar pattern as in workforce (see Figures 4.3.4-16 and 4.3.4-17). Because the flawed work is not realized, or, not discovered at the beginning, the work 'perceived' to be completed is an optimistic one compared to what it should be, making SPI and CPI better than the lower productivity case; Upon discovery of these hidden rework, all the over-estimation will disappear; what's more, because of the 'fractional work completed effect', SPI and CPI will actually be worse than lower productivity case because effectively more work (base work plus rework) has to be performed.
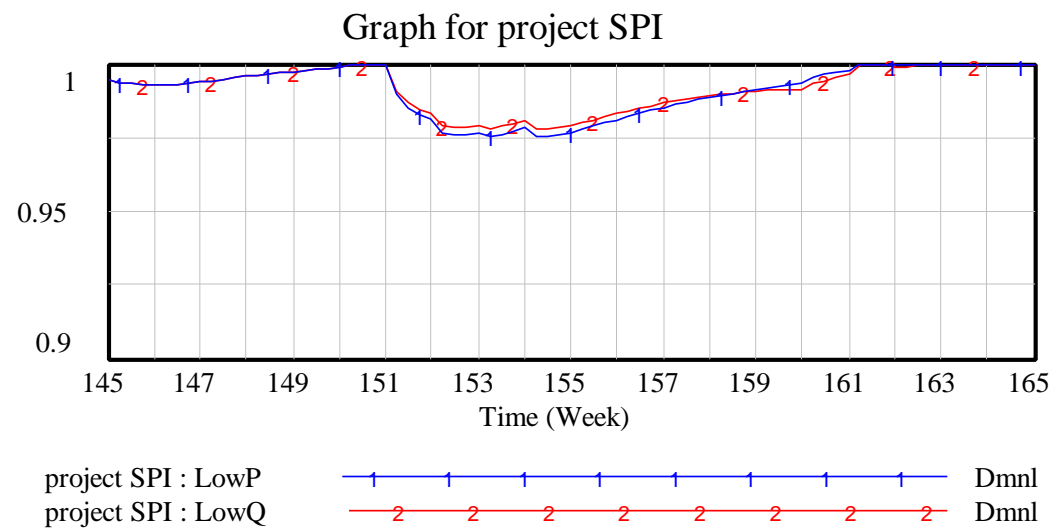

\section{Figure 4.3.4-16: A Comparison Of Project SPI Between Lower Productivity Case And} Lower Quality Case 


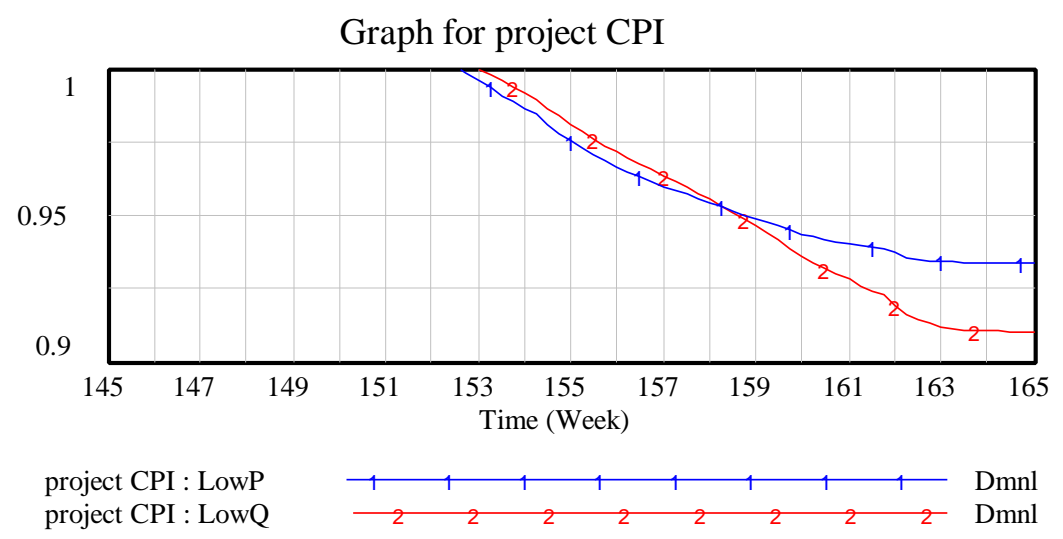

Figure 4.3.4-17: A Comparison Of Project CPI Between Lower Productivity Case And Lower Quality Case

\subsubsection{System Behavior With a Change}

For those projects that span months and years, changes are daily meals, thought not taste good, rather than abnormal. They happen all the time. Sometimes it is because of regulation change; sometimes it is because the client wants to add or remove part of the project; sometimes it is an organization change within the engineering company.

In this case, a regulation change is assumed to take place during the outage phase at week 154 , or the fourth week after the start of outage phase, and is scheduled to be finish within four weeks, or week 158 . The change adds 100 work units to the project. It also requires removal and redoing of 100 released work units. The total work units in this phase before the change is 2320 work units, with a budget of $\$ 8.06 \mathrm{M}$. Since the project team needs to do 200 more work units, a 'fair' budget of $\$ 695 \mathrm{~K}$ is added to the project. Once again, the management action is adjusting the size of workforce by a factor of 1/SPI.

The most direct impact of this change to the project, of course, is the change in work scope (see Figure 4.3.4-18). Since only 100 new work units, or new scope, is added to the project, while the other 100 work units are already in the original work scope and are simply removed and redone, the work scope for the outage phase will increase by 100 work units at week 154, when the change begin to take effect. 


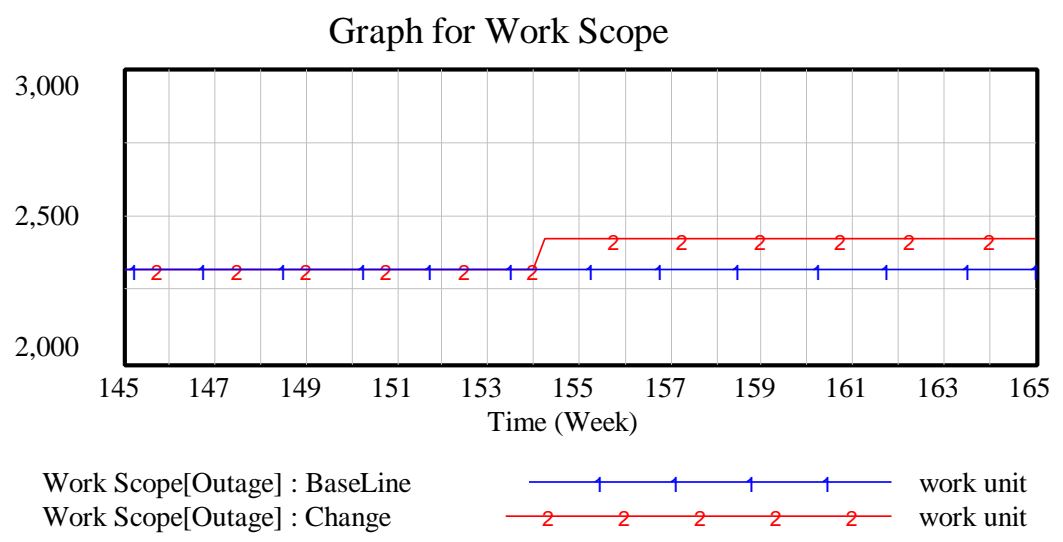

\section{Figure 4.3.4-18: A Comparison Of Work Scope Between Change Case And Baseline Case}

Though has no impact on the project scope, the removal of 100 work units effectively decrease the amount of work perceived to be completed all of a sudden, as shown in Figure 4.3.4-19.

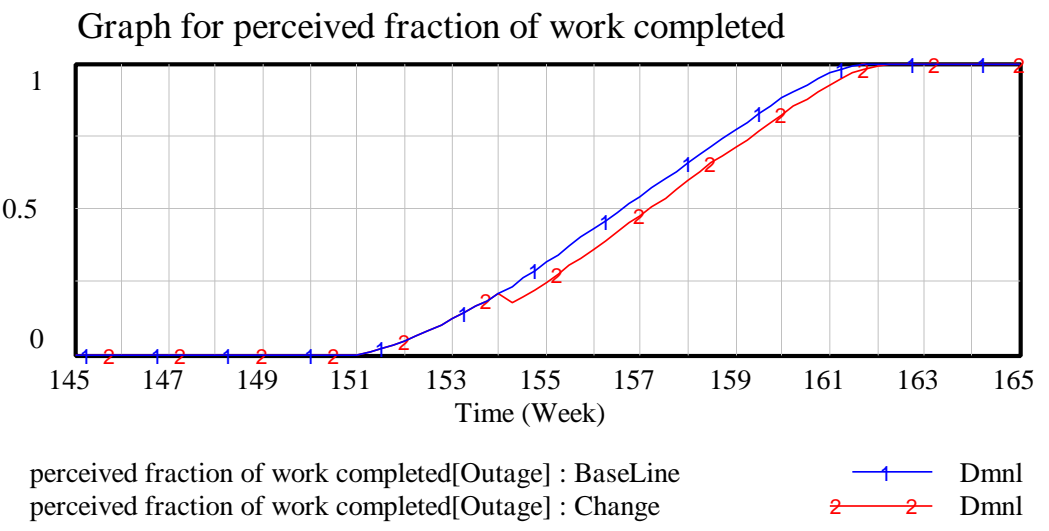

\section{Figure 4.3.4-19: A Comparison Of Work Completed Between Change Case And Baseline Case}

One impact of a sudden decrease in completed work is to cause out-of-sequence problem for the downstream work based upon the work that is removed. This can be seen in Figure 4.3.4-20, where when the change kicks in, there is an abrupt increase in out-of-sequence work percentage. Though as work removed is reinstalled, the out-of-sequence problem is fixed gradually, it hurts the productivity all along the way, which causes ripple effects. 


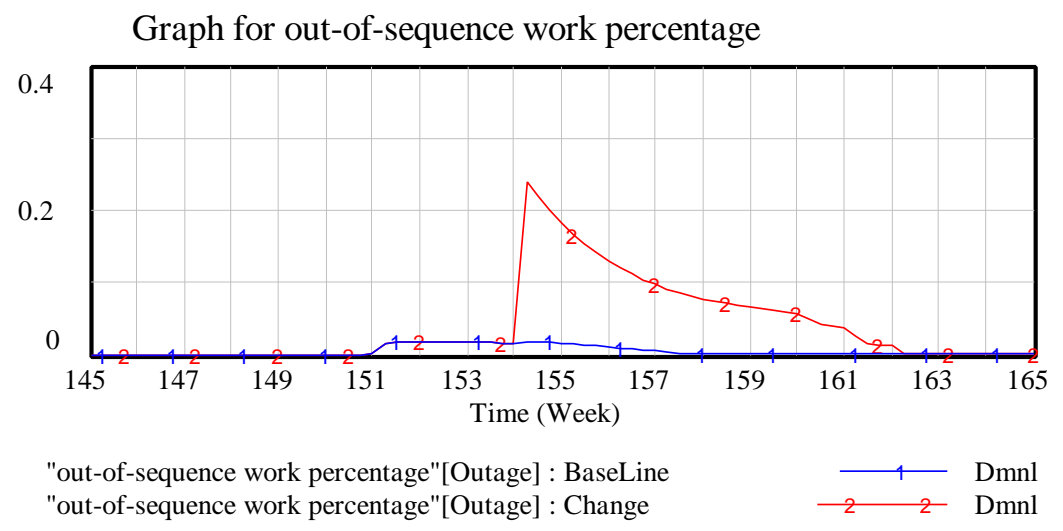

\section{Figure 4.3.4-20: A Comparison Of Out-Of-Sequence Work Percentage Between Change Case And Baseline Case}

Another impact of the completed work removal is to draw down the project SPI suddenly at week 154. This will introduce, according to the management action assumed, an overstaffing effort hoping to catch up. At the same time, the realization of 100 work units of new work scope also required sizing up the workforce. This is shown in Figure 4.3.4-21. Notice that the workforce is not sized up at week 154, but shortly after that because of the time needed to hire labors from the market.

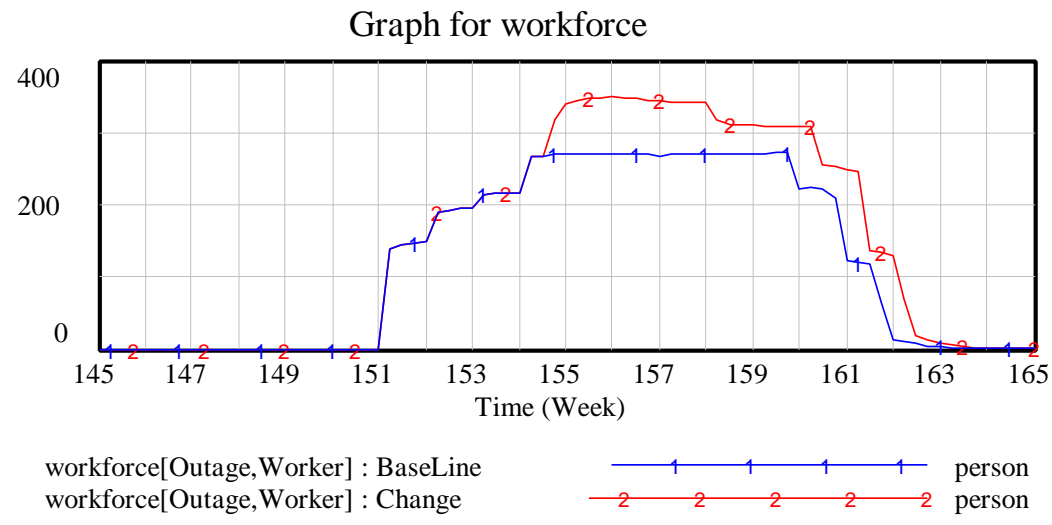

Figure 4.3.4-21: A Comparison Of Workforce Between Change Case And Baseline Case

Also notice that after week 158, when the work associated with the change is supposed to be completed, the workforce does not return to its baseline level. The reason is because the productivity and quality of the work other than those associated with the change are damaged by the change, and they are lower than their peers in the baseline case, making situations worse. 


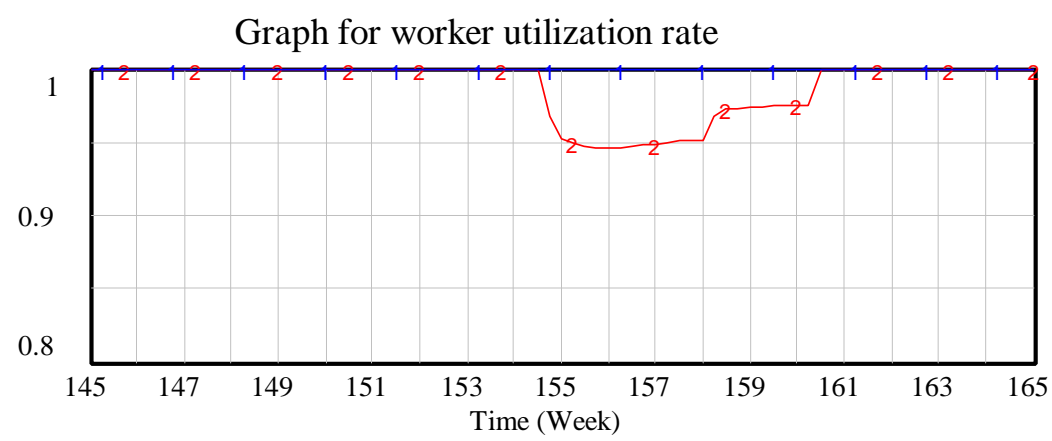

worker utilization rate[Outage] : BaseLine worker utilization rate[Outage] : Change

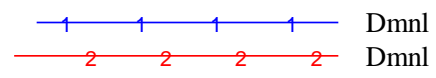

Figure 4.3.4-22: A Comparison Of Worker Utilization Rate Between Change Case And Baseline Case

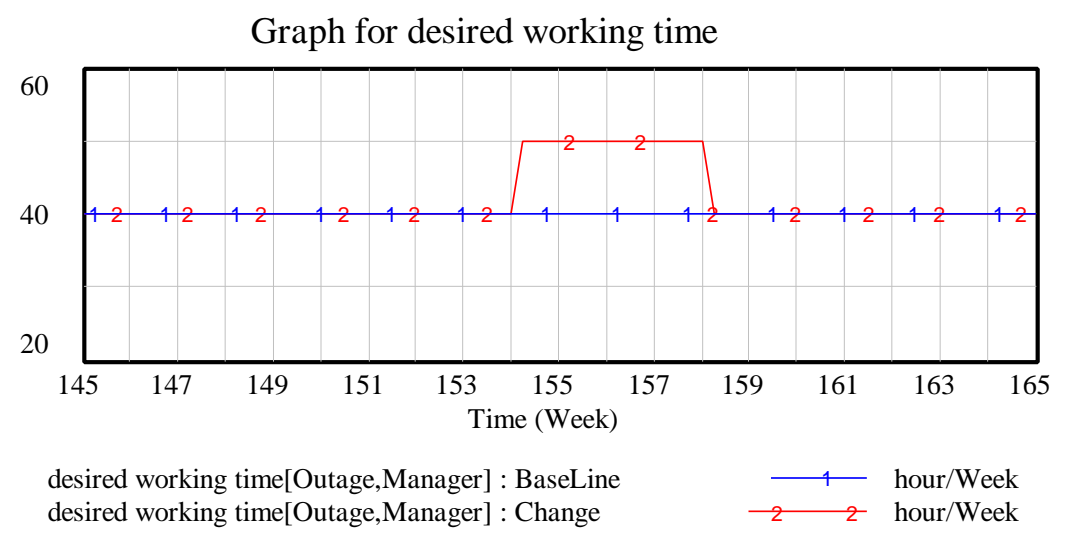

\section{Figure 4.3.4-23: A Comparison Of Desired Working Time Between Change Case And Baseline Case}

Besides the out-of-sequence problem that we discuss earlier, there are other factors that hurt productivity and quality. One important one is the congestion following the oversized workforce, as shown in Figure 4.3.4-22. At the same time, since change demands times resources, sometimes a big chunk, from management staffs to take care of interests of parties involved (see Figure 4.3.4-23), the availability for them on project oversight becomes limited, producing a negative impact on the productivity and quality of the project. Even if the management has room to work overtime to accommodate the increased working time demand, the fatigue caused by the overtime may also adversely affect their efficiency to work, and at last hurt the productivity and quality of the project.

Figures 4.3.4-24 and 4.3.4-25 shows the plot of productivity and quality of the workers. As have been indicated earlier, this is not a once through but a dynamics process, involving a series of feedbacks, with information transmitted back and forth. 


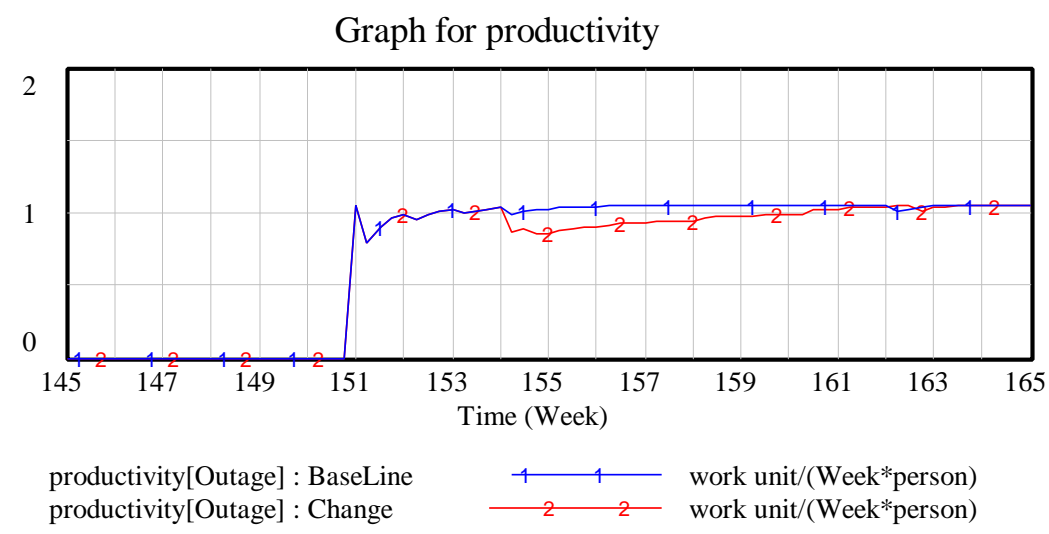

Figure 4.3.4-24: A Comparison Of Productivity Between Change Case And Baseline Case

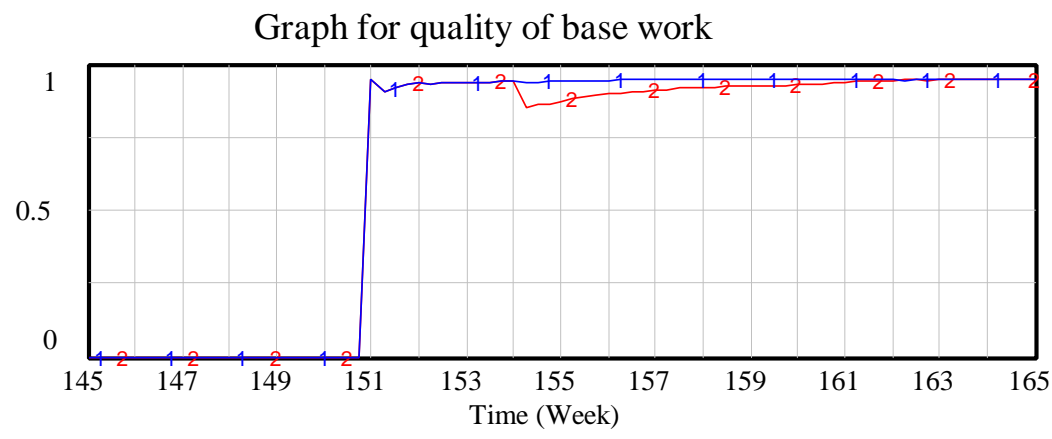

quality of base work[Outage] : BaseLine quality of base work[Outage] : Change

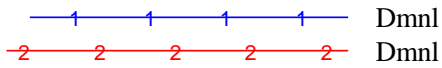

Figure 4.3.4-25: A Comparison Of Worker's Quality Between Change Case And Baseline Case

Graph for project SPI

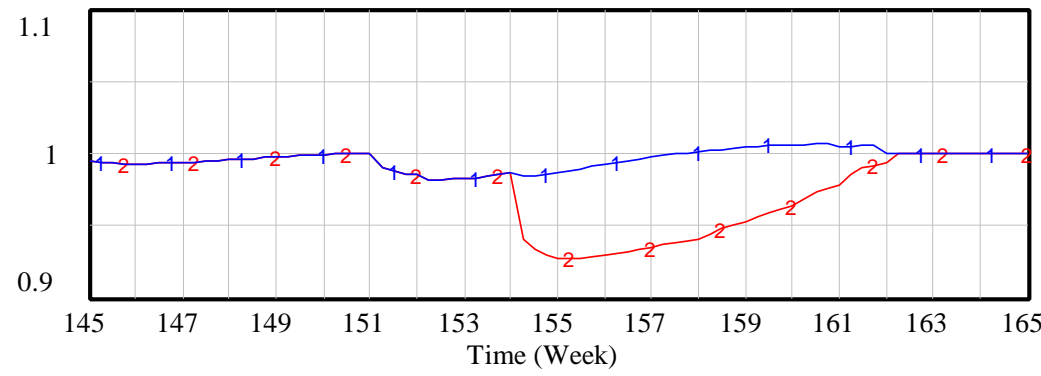

project SPI : BaseLine project SPI : Change

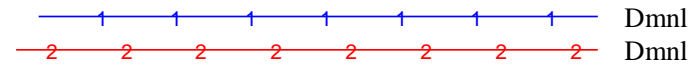

Figure 4.3.4-26: A Comparison Of Project SPI Between Change Case And Baseline Case 


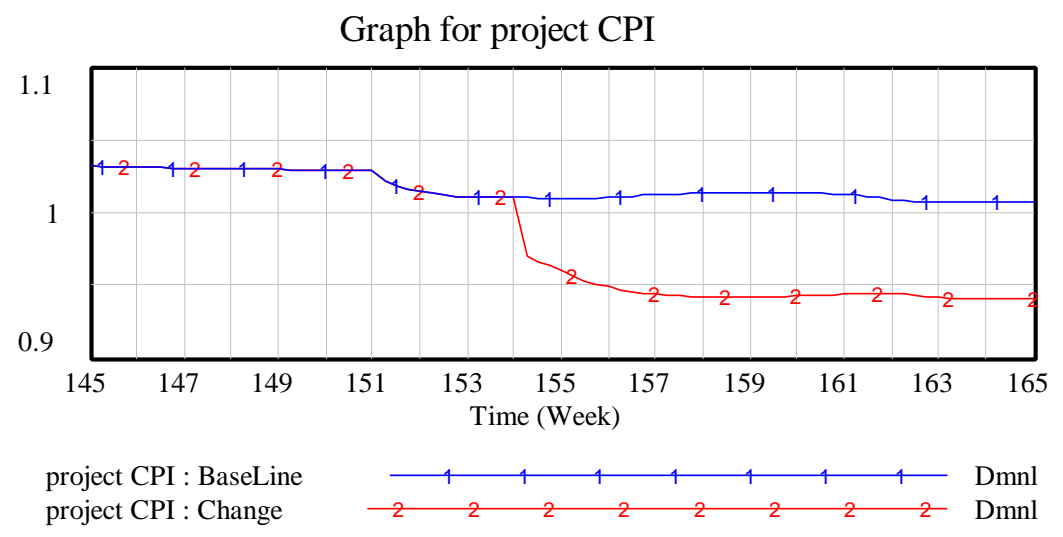

\section{Figure 4.3.4-27: A Comparison Of Project CPI Between Change Case And Baseline Case}

As productivity and quality are lower than the baseline case, project SPI and CPI will consequently be lower, as shown in Figures 4.3.4-26 and 4.3.4-27. Especially, even though a 'fair' budget is added to the project, it turns out that other parts of the project will be adversely affected by the change, and at last, if looking at the project as a whole, the 'fair' budget is not justified. In other words, the budget for this change should be much more than merely $\$ 695 \mathrm{~K}$ to cover all the associated loses. According to the data obtained from the simulation, the budget for this change should be $\$ 1.7 \mathrm{M}$, or $\$ 1 \mathrm{M}$ more than the 'fair' budget, in order to match the cost performance to that of the baseline.

\subsubsection{System Behavior With a Management Firedrill}

This section studies the domino effect in the management system. The event surveyed is the lost of construction manager at week 154 due to some reason, and the new manager does not come in until week 156.

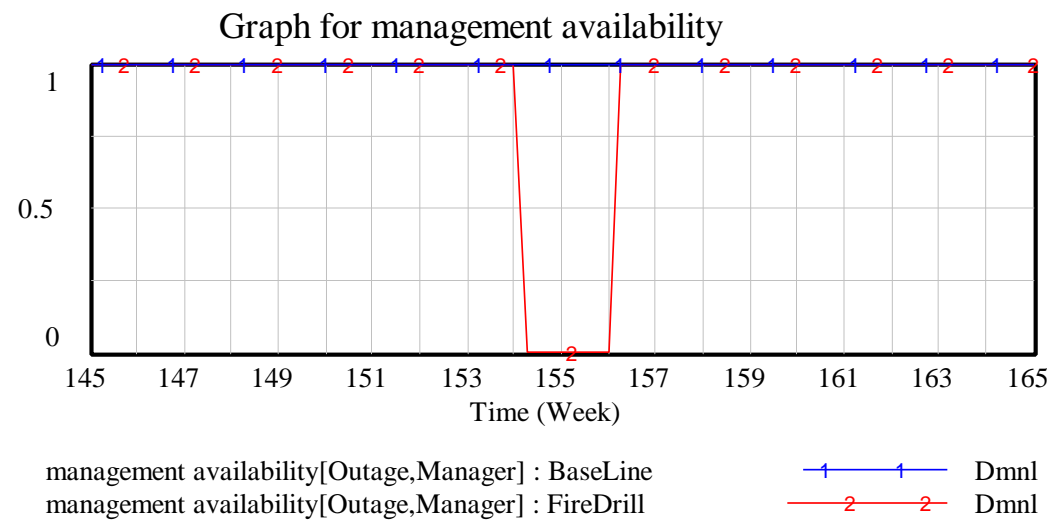

Figure 4.3.4-28: A Comparison Of Management Availability Between Management Firedrill Case And Baseline Case 


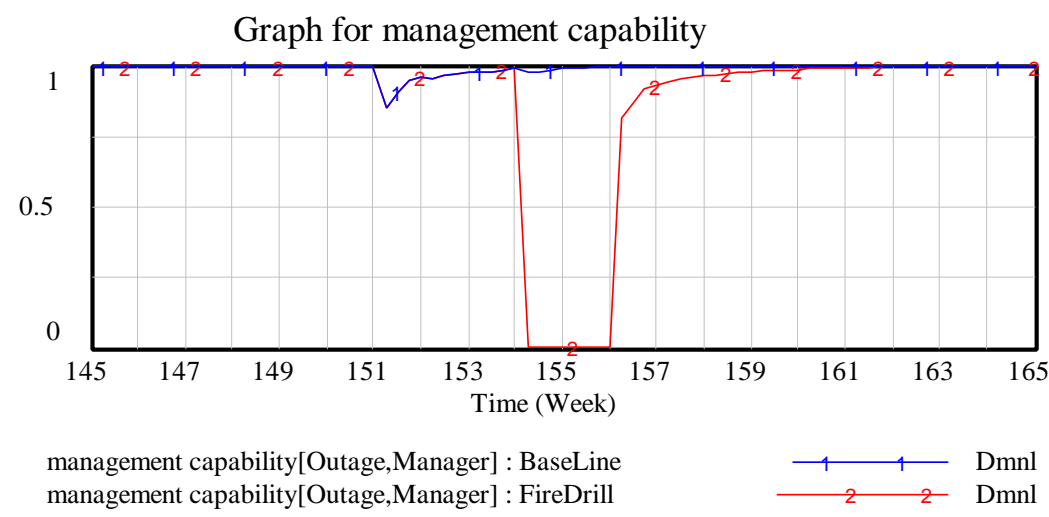

Figure 4.3.4-29: A Comparison Of Management Capability Between Management Firedrill Case And Baseline Case

Upon the lost of the manager, the availability of manager to the supervisors plummets to zero, and obviously its 'capability' (see Section 4.3.3.4.9) is down to zero, as can be seen in Figures 4.3.4-28 and 4.3.4-29. When the new manager comes on post, however, the management capability does not recover at once because the newcomer needs a period of time to become familiar with the job. This is shown in Figure 4.3.4-30.

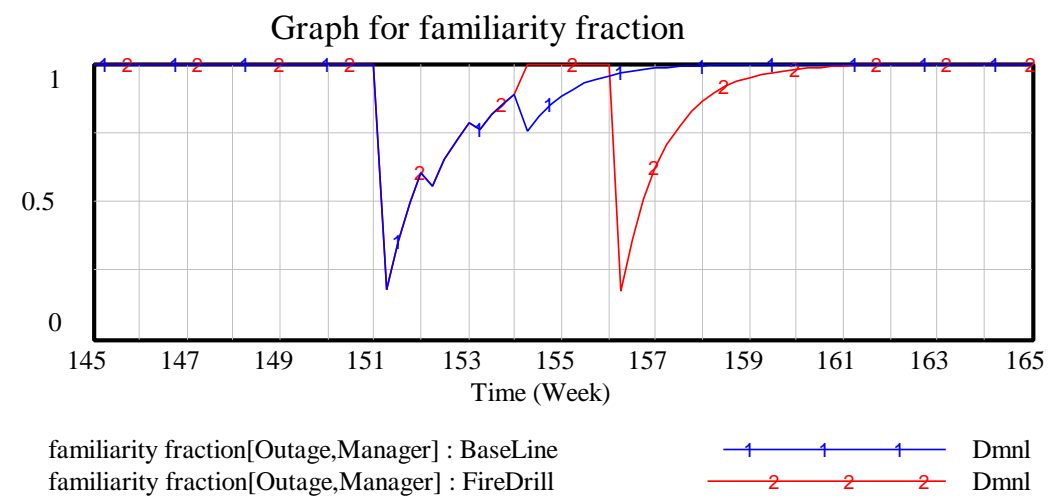

\section{Figure 4.3.4-30: A Comparison Of Manager Familiarity Between Management Firedrill Case And Baseline Case}

The domino effect is shown in Figure 4.3.4-31. With no manager, the supervisors will lose general directions, and most importantly, lose their coordination and communications with other departments. As a result, their capabilities will be much lower, but not zero since they can still manage to practice their functions, though not as efficient as normal. Same thing happen to next levels, general foremen and foremen. 


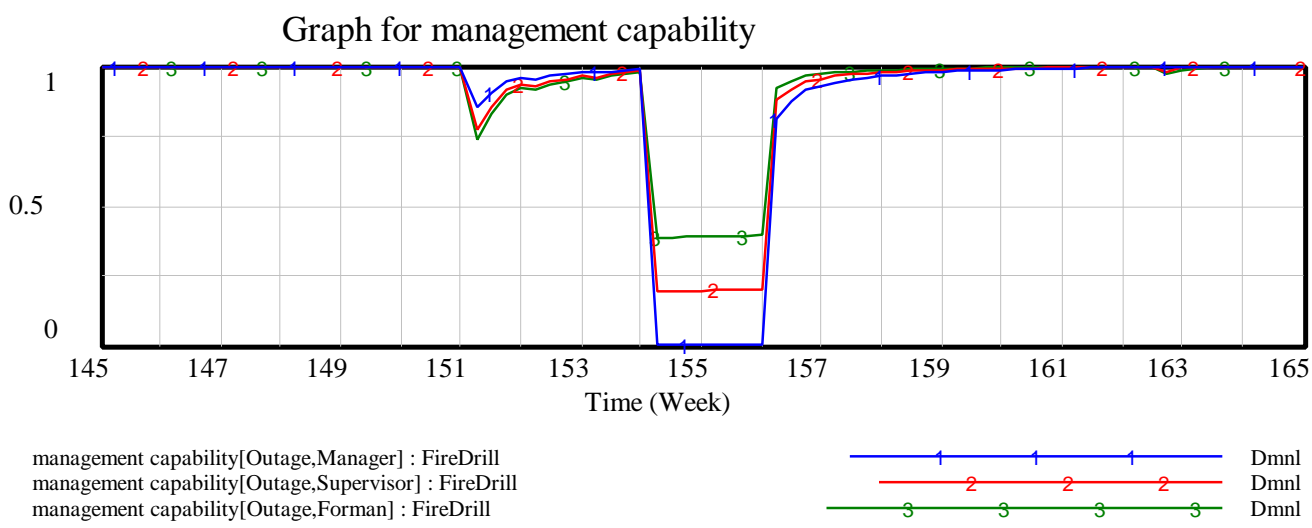

Figure 4.3.4-31: A Comparison Of Management Capability Between Management Firedrill Case And Baseline Case

Graph for productivity management capability factor

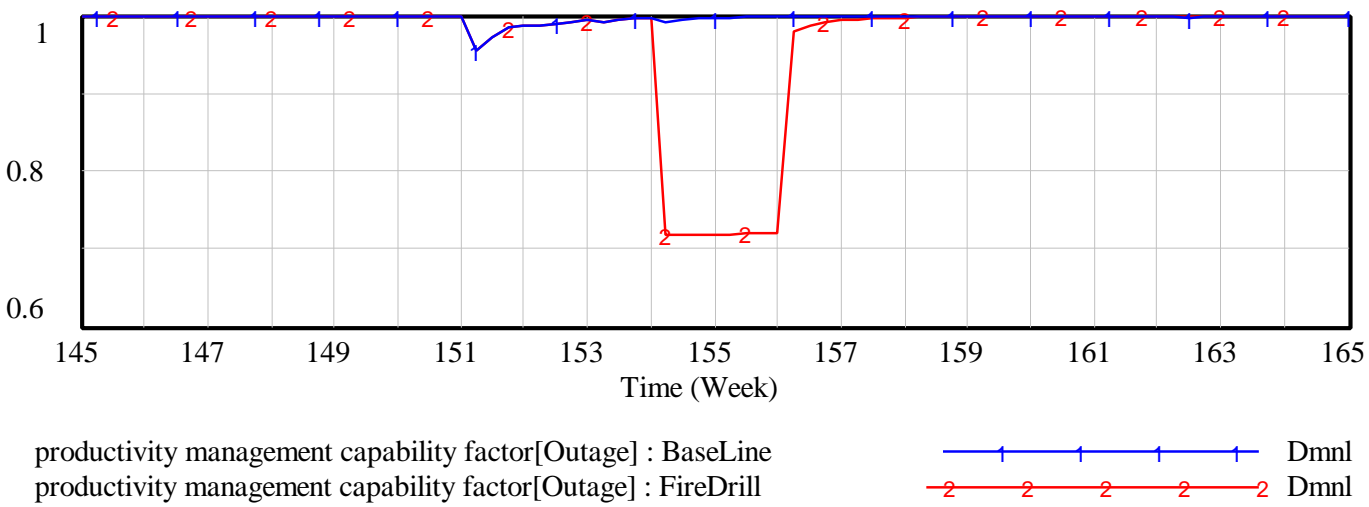

Figure 4.3.4-32: A Comparison Of Productivity Management Capability Factor Between Management Firedrill Case And Baseline Case

Graph for quality supervisor management capability factor

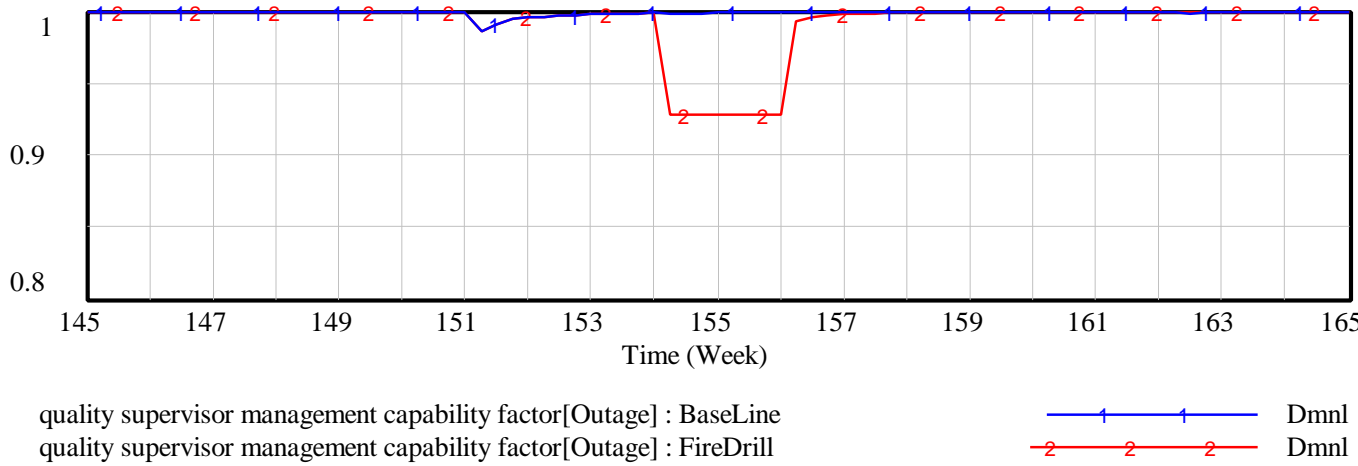

Figure 4.3.4-33: A Comparison Of Quality Management Capability Factor Between Management Firedrill Case And Baseline Case 
At last, workers' productivity and quality are affected because of the unclear instructions conveyed to them via foremen. This is shown in Figures 4.3.4-32 and 4.3.4-33.

Of course, with lower productivity and quality, other feedback will begin to kick in. For instance, out-of-sequence work percentage will be higher than baseline, as shown in Figure 4.3.4-34. Also, workforce will need to be sized up, as in Figure 4.3.4-35, and congestion will kick in, etc. Detailed descriptions of these feedbacks are no longer elaborated here.

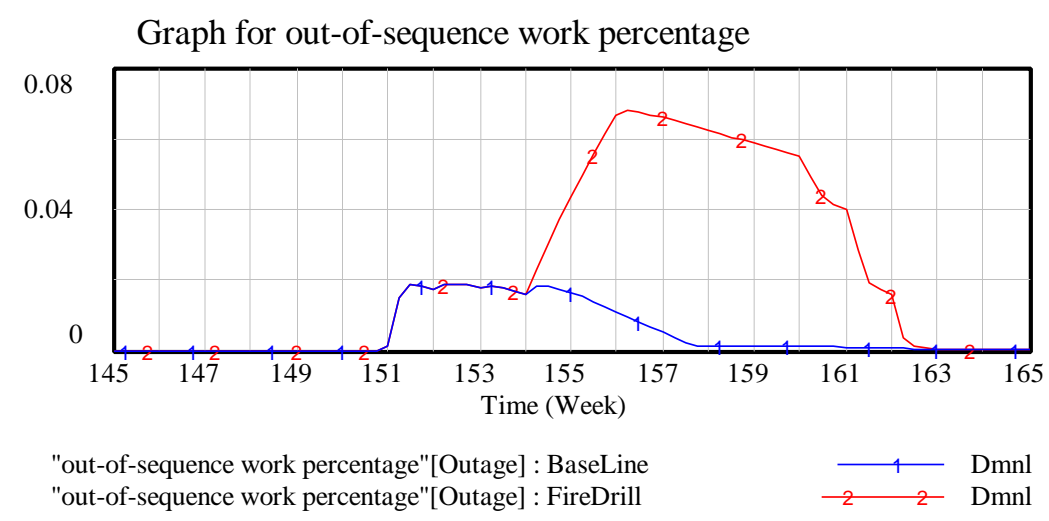

\section{Figure 4.3.4-34: A Comparison Of Out-Of-Sequence Work Percentage Between Management Firedrill Case And Baseline Case}

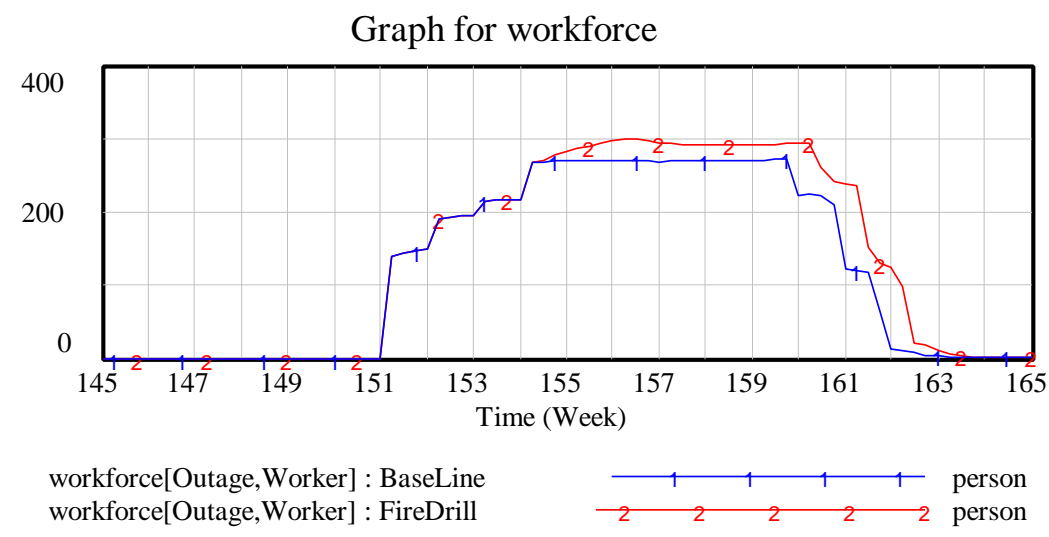

\section{Figure 4.3.4-35: A Comparison Of Workforce Between Management Firedrill Case And Baseline Case}

At last, Figures 4.3.4-36 and 4.3.4-37plots the project SPI can CPI, from which it can be seen that this firedrill delays the project completion date by about three days, which is not that bad, but cost the project $\$ 1 \mathrm{M}$ more than the baseline because of the negative feedbacks on productivity, quality, and size of workforce. 


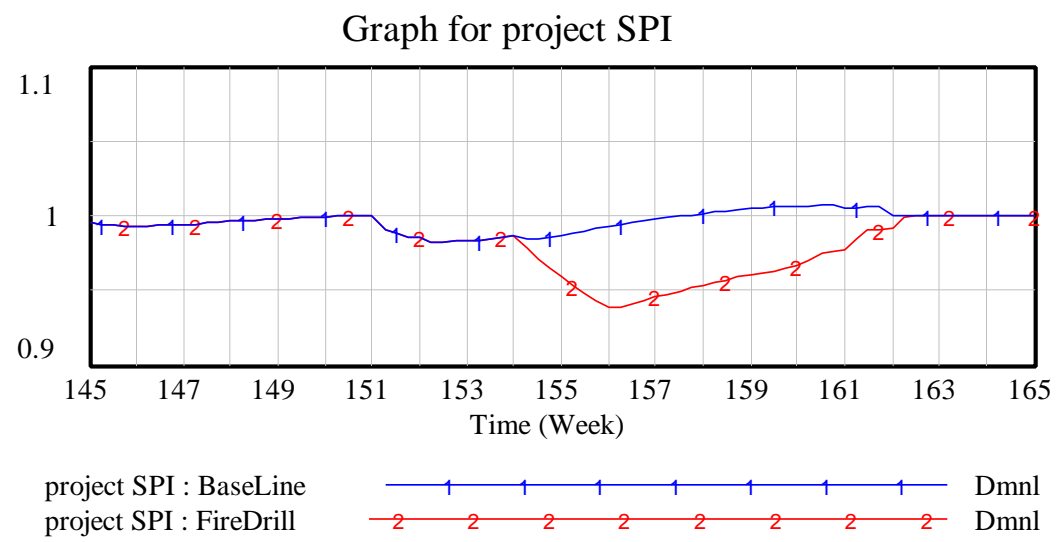

Figure 4.3.4-36: A Comparison Of Project SPI Between Management Firedrill Case And Baseline Case

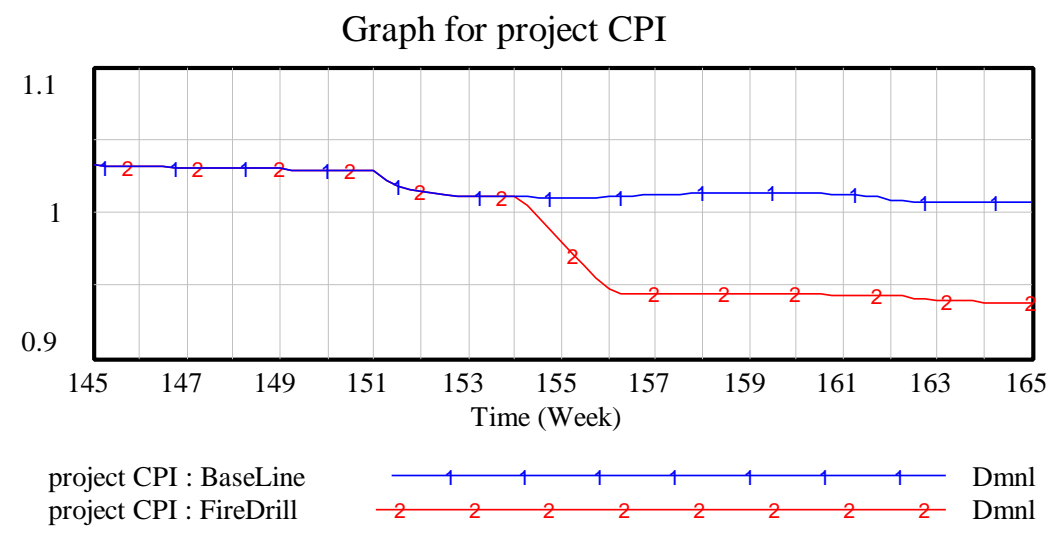

Figure 4.3.4-37: A Comparison Of Project CPI Between Management Firedrill Case And Baseline Case

\subsubsection{System Behavior Under Change and Overtime}

In the previous cases, we have used a same management action: adjust the work force by a factor of 1/project SPI. However, more than often, project managers may use overtime as their management actions, especially in the cases where overtime is asked for by the workers in order to get better paid, or where the additional work force is unavailable.

In this case, a same regulation change as in Section 4.5 is assumed to take place. However, the management action switches from adjusting the size of workforce by a factor of 1/SPI to adjusting the working time by a factor of $1 / \mathrm{SPI}$.

Figure 4.3.4-38 shows the comparison of fatigue due to overtime under these two management actions. In the overtime case, fatigue keeps growing until the project is completed at about week 163. 


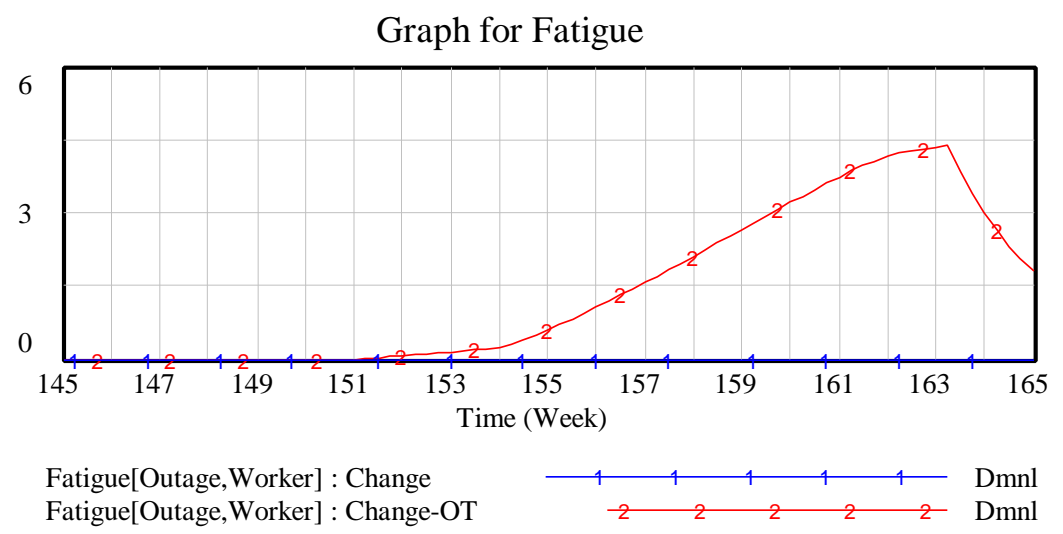

Figure 4.3.4-38: A Comparison Of Fatigue Between Workforce Adjusting Case And Overtime Adjusting Case

The fatigue will cause a decrease in productivity as shown in Figure 4.3.4-39.

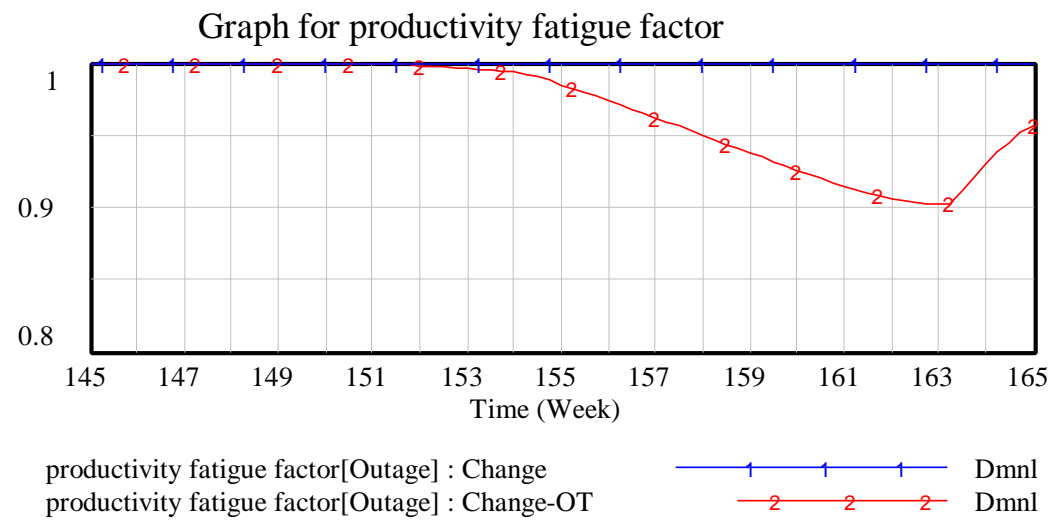

Figure 4.3.4-39: A Comparison Of Productivity Fatigue Factor Between Workforce Adjusting Case And Overtime Adjusting Case

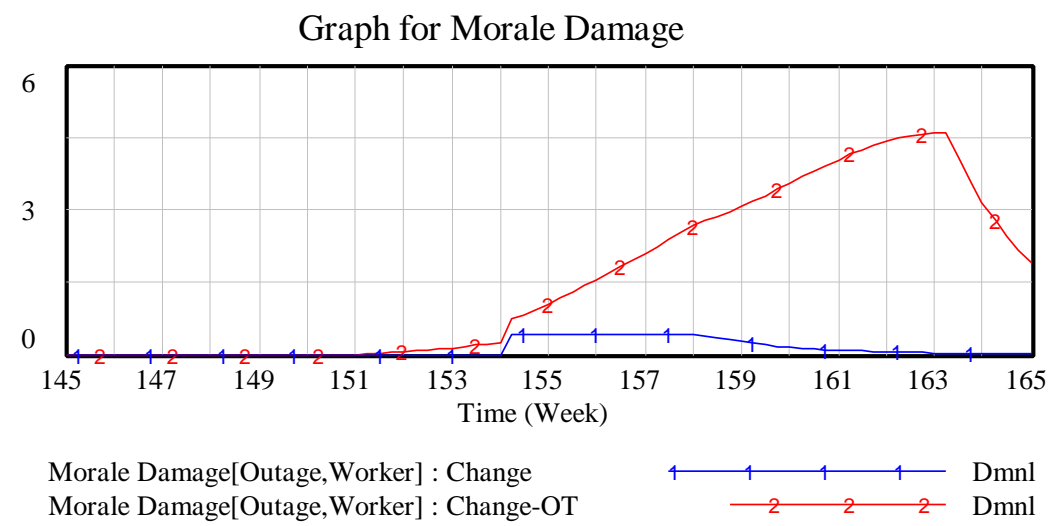

Figure 4.3.4-40: A Comparison Of Morale Damage Between Workforce Adjusting Case And Overtime Adjusting Case 
Overtime not only causes fatigue, but also drives down the morale of the project team. In addition, the change itself also contributes to part of the moral damage, as shown in Figure 4.3.4-40: morale damage in the workforce adjusting case is resulted from the change, and morale damage in the overtime adjusting case is the sum of the two.

And the low morale will do harm to both productivity and quality, as can be seen in Figures 4.3.4-41 and 4.3.4-42.

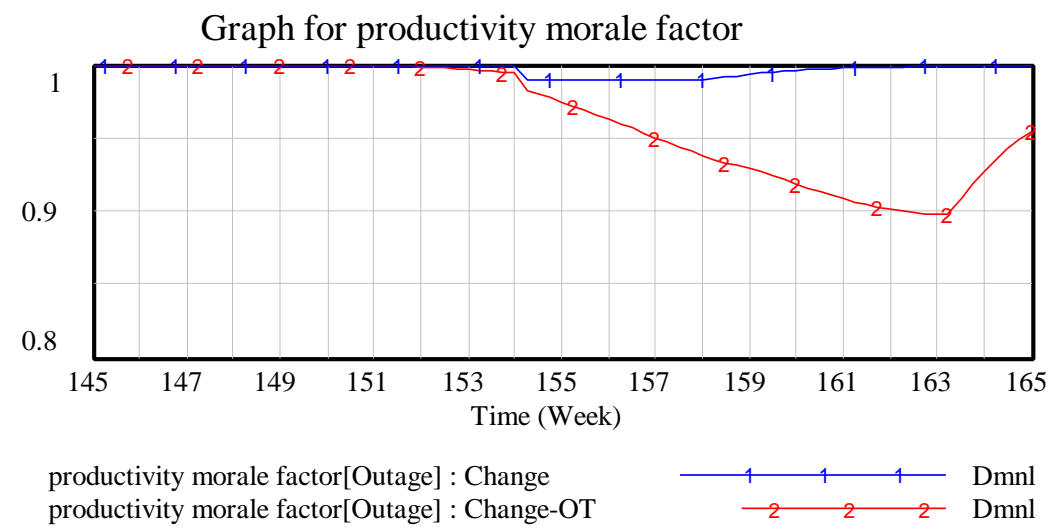

Figure 4.3.4-41: A Comparison Of Productivity Morale Factor Between Workforce Adjusting Case And Overtime Adjusting Case

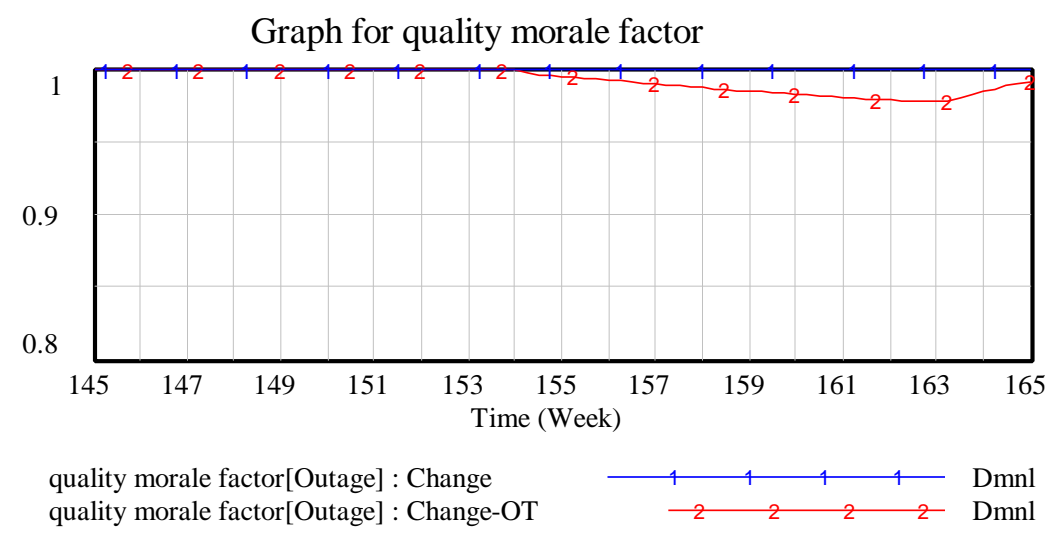

\section{Figure 4.3.4-42: A Comparison Of Quality Morale Factor Between Workforce Adjusting Case And Overtime Adjusting Case}

As a result, the worker's productivity and quality look like Figures 4.3.4-43 and 4.3.4-44. As fatigue and morale damage accumulate, the negative effects on productivity and quality grow, making them depart more and more from the non-overtime case. 
Graph for productivity

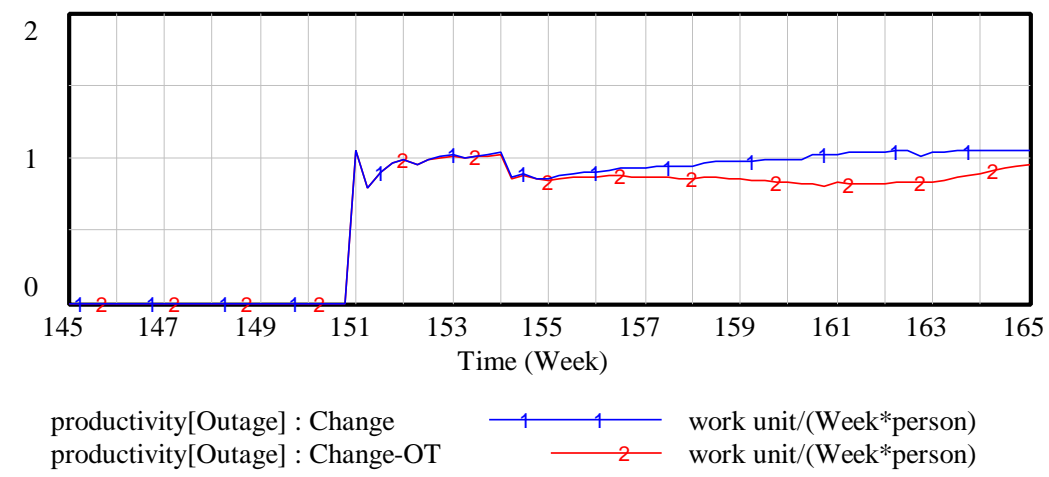

Figure 4.3.4-43: A Comparison Of Productivity Between Workforce Adjusting Case And Overtime Adjusting Case

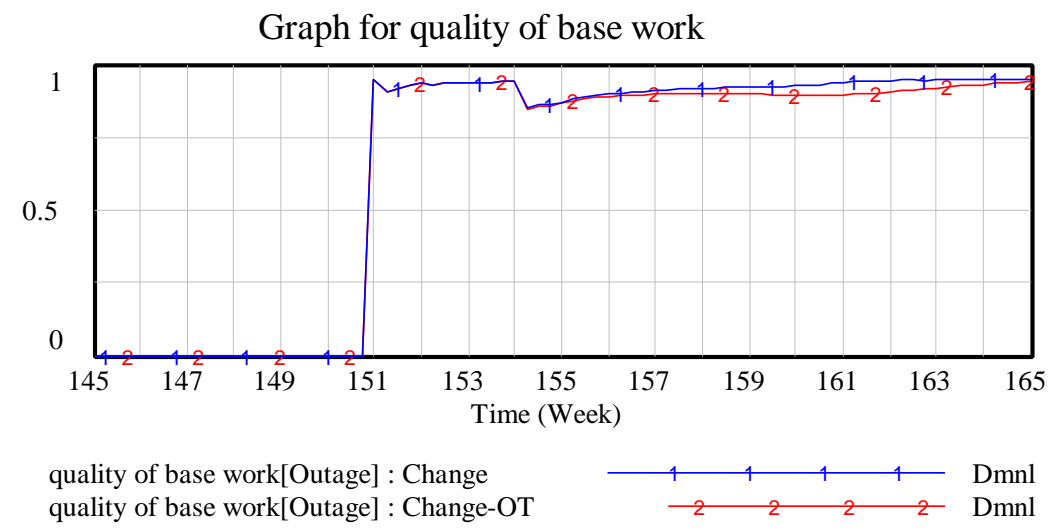

Figure 4.3.4-44: A Comparison Of Worker's Quality Between Workforce Adjusting Case And Overtime Adjusting Case

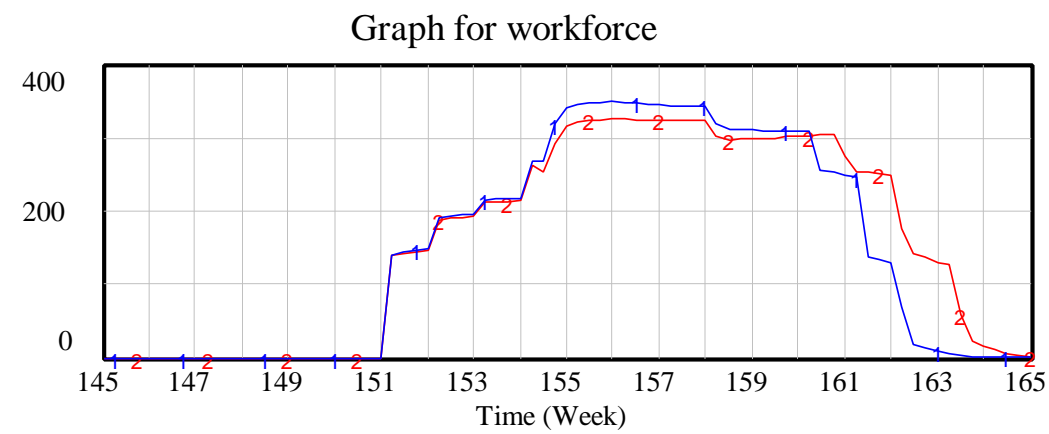

workforce[Outage,Worker] : Change workforce[Outage,Worker] : Change-OT

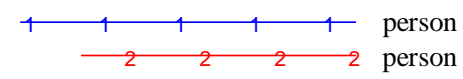

Figure 4.3.4-45: A Comparison Of Workforce Between Workforce Adjusting Case And Overtime Adjusting Case 
The lower quality means more rework. This part of the work becomes significant when the base work is mostly completed, or latter part of the outage phase. As shown in Figure 4.3.445 , the workforce needed in overtime case is larger in the tail because of this reason.

Figures 4.3.4-46 and 4.3.4-47 prove how expensive the overtime is. Since the productivity and quality keep deteriorating, the overtime hours are not only inefficient, but also hurt the normal working hours, making the working hours more expensive with regard to dollar per work unit. As a result, compared to non-overtime case, the work is completed more behind schedule, and the price paid is higher.
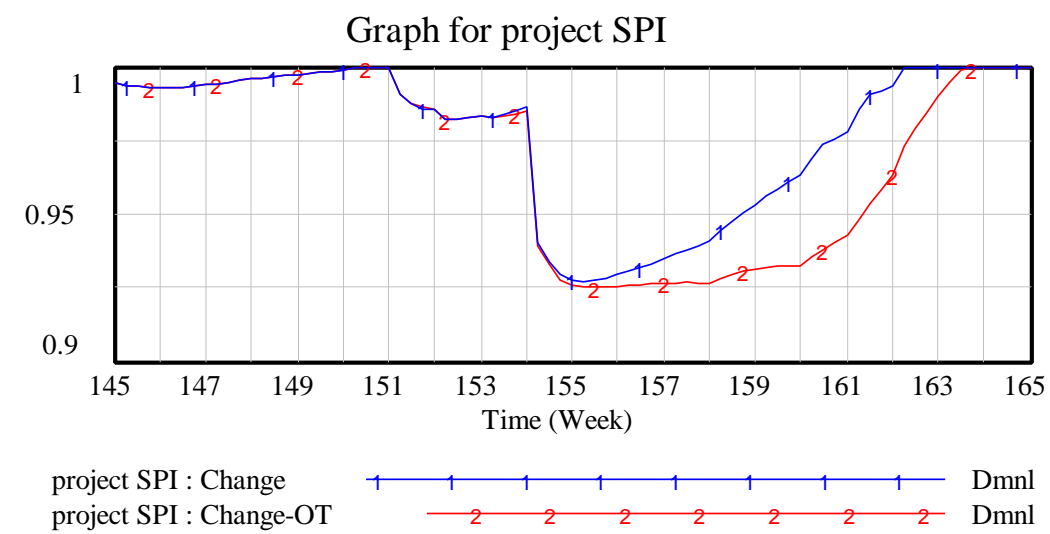

Figure 4.3.4-46: A Comparison Of Project SPI Between Workforce Adjusting Case And Overtime Adjusting Case

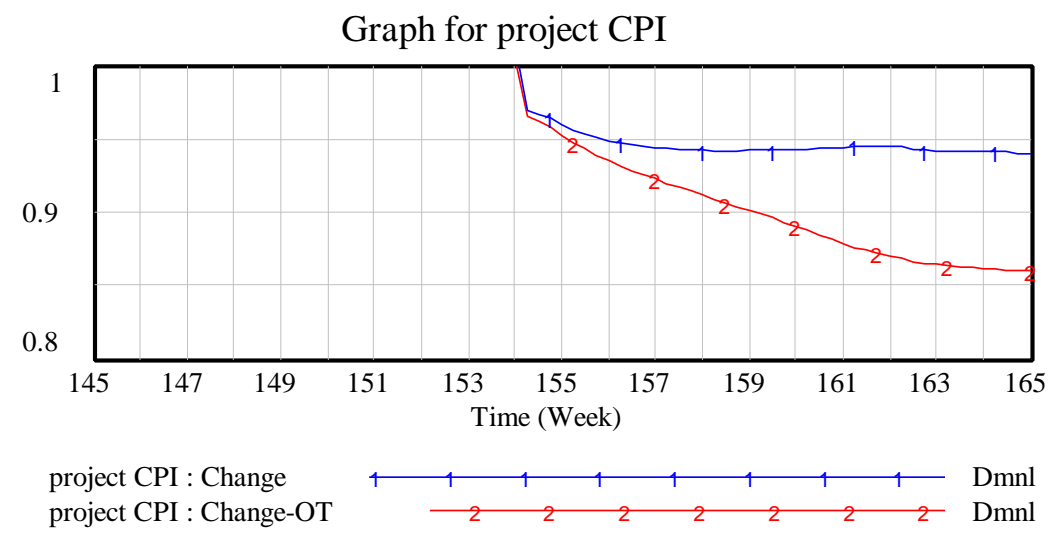

Figure 4.3.4-47: A Comparison Of Project CPI Between Workforce Adjusting Case And Overtime Adjusting Case 


\subsubsection{Conclusion}

\subsubsection{Recapitulation}

In the history of nuclear power plant construction projects, schedule and cost overruns had been rule rather than exception. One of the reasons for this phenomenon is due to the fact that, in these very large and complex projects, the project managers failed, or were not equipped well enough, to fully recognize and utilize the dynamic, cross-functional features of projects, which often drive project performance.

Using a computer simulation technique known as System Dynamics, a system dynamics model has been built in the work presented here based upon detailed project system analysis and extensive project management information collected from long-time line managers to study the dynamic behaviors of non-linear, coupled, multi-phases design and construction projects. The model was calibrated to a multi-phase Steam Generator Replacement (SGR) project. With baseline data typical of a SGR project, simulations were performed and analyzed under imaginary scenarios such as lower than expected productivity, lower than expected quality, unexpected change, and management firedirll. Management actions such as overtime and workforce adjustment were also studied, compared, and discussed.

The research finds that 'ripple effects', or feedbacks, play an important role on the project performance. Upsets following unexpected changes or events such as regulatory change or loss of important employees can derail a normal project, while management actions in response to these events can have negative impacts that may not only offset, but also surpass the expected positive effects.

It was also found that system dynamics models are able to capture and quantify these 'ripple effects' and provide clear insights into the dynamics of the project, which will help project managers to foresee the unfavorable implications and consequently, to negotiate with regulatory agencies and clients, and to act accordingly to foreclose them.

\subsubsection{Major Findings}

\subsection{Role of Underlying Systems}

Underlying system structure is the underlying source of the problems that surface in the project. The coupled, non-linear relationships between system variables decide the mechanism by which the impacts of external events and management actions are propagated and ultimately show up as project performances. Correcting the underlying system inefficiencies instead of tracing problems in an 'event causes event' fashion is the only way to eradicate the problems associated with the system.

A number of important feedback loops are identified in the underlying systems. These include productivity-overtime-fatigue feedback, productivity-overstaffing-congestion feedback, ...etc. The flows of information in these either reinforcing or balancing feedback 
loops, combining the non-linear relationship between system variables, are dynamic and critical to the system behaviors when project deviates from its normal track.

Delays such as time needed to hire workforce play another important role in determining the behavior of the system. Project variables do not move as fast as we want them to, and they do not move at a same pace, either. This produces coordination and out-of-sequence problems. A better management of these delays may be able to improve the efficiencies.

\subsection{Implications of Changes and Unexpected Events}

The research shows that changes and unexpected events impact the project performance in a more than complex way. The indirect part of the impacts, which is a result of feedbacks, turns out to be significantly important compared to the direct impacts. A 'fair' budget associated with a change order proves to be far from being fair after accounting for its impacts on the rest of the project; and the loss of key employees bring disastrous consequences through the internal organizational structure.

\subsection{Implications of Management Actions}

More than often, the actual outcomes of management actions taken in response to changes and unexpected events are found to be substantially worse than expectations due to the multi-dimensional features of the management actions, which always involve intertwined feedbacks, many of them are adverse.

For overtime management action, the associated hidden costs can be more significant than premium pay. Continuing overtime drastically affects costs of all hours and makes all hours more expensive because, as a result of overtime, fatigue and morale loss grow exponentially, lowering the productivity and quality of all the hours.

For workforce adjustment management action, there are also many adverse feedbacks due to a high level of work force. The delays produced by necessary ramp up training, the coordination needs within a large body of workforce, more likely productivity-killing accidents, and more sit-and-wait due to congestion problem can hardly be avoided.

The recognition of these impacts will help to design combo-management actions to not only address the central issues, but also prepare to mitigate the side effects for a more efficient execution of the actions.

\subsection{System Dynamics as a Tool}

System dynamics models are able to capture and quantify the 'ripple effects' and provide clear insights into the dynamics of the project, which is demonstrated in the System Behavior Analysis under imaginary scenarios. It helps project managers to foresee the ramifications of changes and consequently, to negotiate with regulatory agencies and clients, and to act accordingly to foreclose them. Although this is not a tool that will relieve the managers of the need to be an expert on such projects, it serves as an information 
source to facilitate the decision-making process and improve the quality of the management actions.

\subsubsection{Limitations}

In reality, a System Dynamics Model is never complete, as each specific application can reveal important, incompletely treated phenomena and interactions.

In this model, the project organization is not complete. Only four departments are included: design engineering department, field engineering department, construction department, and QA/QC department. In reality, other department such as Safety, Project Control, Business, Training, and Public Relation are also important with regard to flows of information, material, and deliverables. They all interact with each other and as a whole form an interrelated network.

While vertical communications from top managers to workers are modeled in detail, the coordination across different departments is only modeled as some simplified factors, except the task precedence relationship between departments. But in fact, for instance, the unavailability of design engineers in the construction phase can cause delays of the construction tasks in a dynamic way, which cannot be fully captured by an 'engineering worker availability factor'.

In the work presented here, only labor costs are modeled, with the overall project cost estimated by scaling up from labor costs by a constant factor for each work phase. A significant difference between this estimated project costs and real project costs is possible if other costs such as materials, tools, and equipment costs do not move together with labor costs.

The inputs from outside the model boundary are assumed to be independent of what happened inside the system, which is not always true. For instance, change orders are assumed to be random events in this model, but it is not difficult to notice that the better communication between clients and project managers (inside the system) can in effect reduce the chances of client-initiated change orders (from outside the system).

For many effects modeled in the work presented here, the quantitative relationships still need to be verified. For example, by how much the productivity is lowered by some degree of morale loss? Many such relationships may never be quantified given their abstract, uncertain, and complex properties.

\subsubsection{Future Work}

To better understand and capture the dynamics characteristics of a very large and complex project, a more comprehensive and complete model is necessary. This integrated model shall incorporates all the departments involved and clearly define the relationships between them with regard to information flows, material flows, and monetary flows. Not only feedbacks within the system, but also feedbacks between internal and external variables (inputs) shall be considered to catch the full implications of events and actions. More extensive investigations shall be conducted to calibrate and relationships between variables under specific situations. 
To improve management performance, investigations of possible management actions will be beneficial. More fundamentally, studies on altered underlying systems will be helpful to improve the system structure to promote the efficiency of the system.

\subsection{Modeling of Nuclear Power Plant Refurbishment Project}

In addition to the modeling described above, work will continue independently of this effort with a Systems Dynamics method. The System Dynamics method is being used to create a model based upon the SGT project model, discussed previously, of the engineering and construction process which is now ongoing at an operating nuclear power plant. The construction is a refurbishing process to make sure the three-unit plant can operate effectively until the end of its operating license.

To-date, a deterministic System Dynamics model has been constructed and data have been collected in raw form. The plant model was created by modifying an earlier model (SGT) which was used to model the steam generator replacement process. Some changes had to be made to the SGT model in order to incorporate all the differences in the SGT projects and the refurbishment project. The major ones are listed below:

? Client relationship: In all construction projects, the client-contractor relationship is very important due to the time spent with the client and the policies invoked to carry out the client's wishes. However, in this particular project, the client is responsible for the construction part of the project, while a consulting engineering firm is responsible for the engineering of the project. Therefore, the client must efficiently provide the engineering project managers with documentation and feedback if the project is to run smoothly. Thus, client interaction is probably even more crucial in this project than most.

? Organizational structure: The organizational structure of the construction staff is still the same as that in the SGT model. However, the structure has been flattened somewhat for the engineering part of the project. This is due to the organizational structure of consulting engineering firm being somewhat flatter than that of the client.

Some of the scenarios being modeled include:

? Low workforce: The work force characteristics are important because there may be a shortage of qualified workers in the field in this particular geographic region.

? Low quality work: The work quality is important for same reason as previous problem.

? Change of scope: The possibility of scope changes is one of the major reasons for creating a System Dynamics model of a project. For a large project, a change in scope can have a dramatic effect. This is very important because it is hard to predict the consequences of changes in such a project. These effects are nonlinear and have no known functional relationship. That is, if the change in scope is doubled, there will not necessarily be only twice the consequence, etc.

The next step of the work is to reformulate the data so it can be entered into the model. Once the data has been entered, discussion with project managers at the consulting engineering firm will be necessary in order to evaluate the model and adjust the parameters affecting the outcome. This will be finished by the end of 2002 . 


\subsection{DEVELOPMENT OF DESIGN-TO-ANALYSIS TOOL (DTA) FOR NUCLEAR POWER PLANTS}

\subsubsection{Summary}

As one of the possible ways to accomplish the project objective, Sandia National Laboratories (SNL) is leading a research effort to investigate the feasibility of developing a design-to-analysis tool to enhance the efficiency of design/analysis cycle. This tool is conceptually planned to facilitate an efficient interaction between designers and analysts by linking the plant design database to a suite of computer software that performs finite element mesh generation and model simulation. When this tool is fully implemented, it will provide a significant cost saving in design, construction, and operation of future nuclear power plants by: (1) creating a single database management system that contains accurate and updated plant information, (2) allowing analysts to provide timely feedback on structural response to optimize designs, and (3) streamlining management of design changes.

A pressurizer, which is a component in the reactor coolant system of the System 80+ pressurized water reactor design, with its connecting piping system, was selected as an example for demonstrating the feasibility of developing the design-to-analysis tool. SNL was primarily responsible for developing this tool, with support from the staff at Westinghouse Electric Company (WEC) to provide the design database of pressurizer and Framatome-ANP \& DE\&S, to supply the details of piping configurations and supports.

One of the important products from the design-to-analysis tool is the creation of coupled finite element models that connect components to their piping system and surrounding building structures. The demonstration effort generated a coupled model of the pressurizer assembly and its piping system, thus permitting the investigation of the technical merits in performing integrated analyses of this coupled model. Integrated seismic analyses of this coupled model were performed to demonstrate the possibility of optimizing the structural design, in particular, the piping supports, while upholding the code requirements of safe operations.

This report documented all individual steps and the associated computer software in developing the design-to-analysis tool. A list of identified technical difficulties was discussed and the resolution methods were proposed to improve this tool to become more efficient, robust and user-friendly.

\subsubsection{Background}

With the advancement in computer hardware and software technology, there are ongoing revolutionary changes in the practices of designing structures and performing analyses to evaluate these designs. The model-based design (MBD) process has been developed using sophisticated computer software to handle huge volumes of design data. Significant improvements in the analytical solver programs have also been made to evaluate performance of structures with dynamic and integrated analyses. These technologies help pave the way to revolutionize the practice of designing and analyzing new generation nuclear power plants, not only to improve their performance efficiency, but more 
importantly, to make them more economically competitive in the power generation industry.

The design-to-analysis tool is one of the new methodologies designed to accomplish these goals for the next generation nuclear power plants. This tool serves to provide a collaborative interaction between designers and analysts to work as partners to improve the efficiency of design/analysis cycle, to optimize plant design, and to streamline management of design changes. More importantly, this tool facilitates a single database management system to help reduce the plant life costs of operation and maintenance by promoting communication of accurate and updated information, and by decreasing rework and redundancies.

Generally, designers and analysts in commercial nuclear industry do not interact in a collaborative manner. This practice is based on the fact that the process of generating analysis models and performing calculations usually requires an elaborate effort, leading to producing analysis results on the performance of the plant/system long after the completion of the design cycle. Therefore, designers usually tend to use very conservative, hence expensive approaches because analysis results based on advanced technology have not been readily available to identify the range of uncertainties to support design optimization. In addition, structures, systems and components have been generally designed and analyzed independent of their connecting items in the currently operating nuclear plants in the United States. In order to ensure the adequacy of these designs, conservative conditions were assumed and applied to represent the effects of the connecting structural elements. These conditions can lead to increased costs to implement the resulting design during construction. The design-to-analysis tool is designed to alleviate these problems by allowing analysts to provide timely and informative feedback in an iterative manner to improve designs, instead of just providing information to confirm the completed designs.

Conceptually, the design-to-analysis tool links the plant design database to a package of computer software that performs finite element mesh generation and model simulation.

The details of the plant design are represented by 3D solid models using the MBD process. By applying the design-to-analysis tool, analysts can go through systematic steps of simplification, decomposition, and translation of 3D solid models of all parts, to generate meshes of finite element models, and to perform model analyses in an organized and efficient manner.

In the process of developing the design-to-analysis tool, major technical issues were encountered and identified. It was discovered that most of the different design and analysis programs used in the development process are not compatibility with each other. In addition, finite element models require precise definitions of joint connectivity and geometry compatibility of gap and space interference. However, these requirements are not present in 3D solid models, which usually serve as space holders of individual parts.

There are a few companies in aircraft and auto industries that have developed and applied the design-to-analysis tool to design and analyze their products composed mainly of mechanical parts. However, the design configuration of nuclear power plants presents two unique challenges in developing this tool: (1) the plant design configuration is usually massive and complicated, and (2) most systems consist of mechanical components connected to piping systems and buildings of concrete structures. Some of the tool development efforts in resolving these challenges are addressed in details in the following sections. 


\subsubsection{Development of Design-to-Analysis Tool}

\subsubsection{Description of Design-to-Analysis Tool}

Sandia has been performing a research effort to investigate the feasibility of developing a design-to-analysis tool, as a part of a DOE NERI project, to reduce the overall total life costs of future nuclear power plants. As an objective, this tool can be used to enhance the efficiency of design/analysis cycle and fine tune safety margins in order to reduce the capitol costs of future plants. A pressurizer, a component in the reactor coolant system of the System 80+ containment vessel, has been selected as the example for demonstrating the feasibility in developing this tool. The selected structural subsystem includes the pressurizer assembly and its connecting piping system.

The design-to-analysis tool usually involves five steps that are described schematically in Figure 4.4-1:

(1) Creating solid models to represent/define the design geometry,

(2) Translating the solid model representation to a format compatible with a selected finite element meshing tool,

(3) Generating a mesh for a finite element analysis model,

(4) Translating the mesh files to a format compatible with a selected finite element solver, and

(5) Performing the analytical simulations of the finite element model with the numerical solver.

The identified processes in Figure 4.4-1 are defined as below:

erocess 1 - Pro/E (Pro/ENGINEER) [1] for pressurizer assembly, and SolidWorks [2] or Pro/E Piping Module for piping system. In this project, SolidWorks was used to generate the solid models for piping system.

Process 2 - Pro/E to ACIS Translator

Process 3 - CUBIT [3]

Process 4 - PATRAN [4] was used to translate the Exodus II files from CUBIT to a format compatible to the ABAQUS [5] code format.

Process 5 - ABAQUS finite element solver 


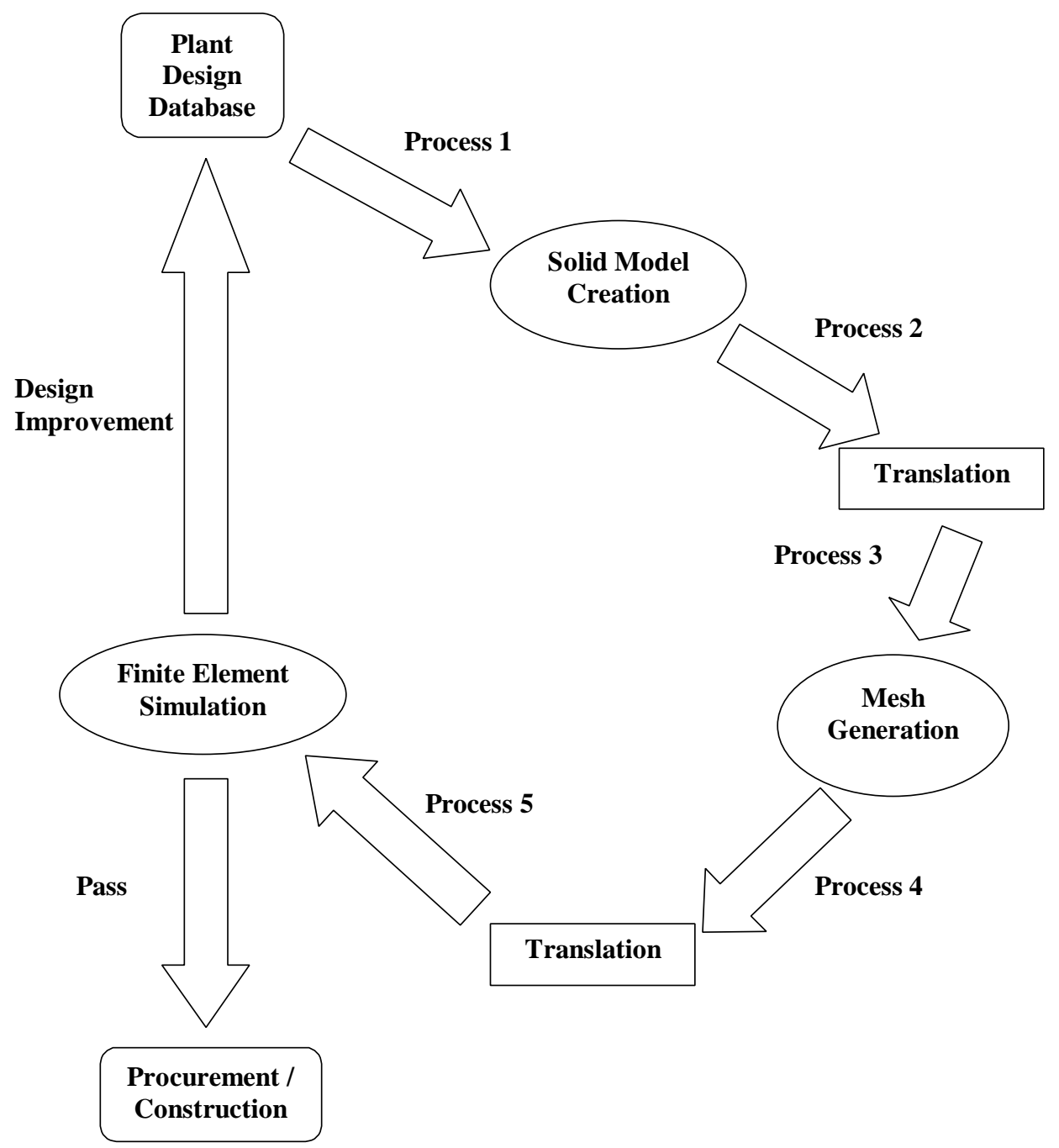

Figure 4.4-1. Schematic of Five Steps in Design-to-Analysis Tool

\subsubsection{Finite Element Model of Pressurizer Assembly with Shell Elements}

The tool development process started with generating individual solid models of all parts of the pressurizer assembly using the Pro/E software package. Westinghouse Electric Company provided the detailed design database of the assembly. A solid model of the pressurizer assembly was developed by placing the individual solid Westinghouse Electric Company models of all parts at specified locations, as shown in Figure 4.4-2. The next step involved a translator package that converts the contents of the solid model to a format compatible to a selected mesh generator, CUBIT. After eliminating space interference and 
resolving joint connectivity issues, a finite element mesh with shell elements of the pressurizer assembly was generated, as shown in Figure 4.4-3.

After importing the translated file into CUBIT, the utilities in CUBIT were used to verify the quality of design geometry, and to assist the process of fixing identified invalid geometry. The approach to the mesh generation and the selection of finite element types are dependent on the analysis objectives and the analysis solver codes. It is advisable to have a clear understanding of the mesh requirements, such as element size, mesh density and element type when creating the design solid models. The mesh of finite element models consist of shell or solid (tetrahedral or hexahedral) elements, or may include a hybrid of them.

The process of geometry decomposition becomes more involved with the complexity of large finite element analysis models that require a contiguous mesh throughout the model. It is important to exercise careful planning to balance the mesh quality, density and contiguity issues when creating the design solid models. The level of decomposition depends on the model geometry and the mesh requirements coupled with the performance of software and hardware packages. In addition, the degree of decomposition should be weighted with model management issues. It is recommended to perform decomposition within practical limits of computer time in order to maintain the file sizes at a manageable level to facilitate meshing operations.

Once a quality mesh is completed, the next step is to assign sidesets and/or nodesets to the model. In CUBIT, sidesets are a mechanism by which constraints may be applied to the model. They usually represent a grouping of element sides with which a constraint must be associated to address the physics of the problem. The nodesets are typically used to specify load or boundary conditions on portions of the CUBIT model or to identify a group of nodes for a special output request in the finite element analysis solver code. The entire finite element model data is then exported and incorporated into the appropriate input file for the various solvers.

\subsubsection{Finite Element Model of Piping System}

There was a parallel effort on gathering the design layout of a piping system connected to the pressurizer assembly from Duke Engineering \& Services. Sequential steps included generating a finite element mesh for this system and combining the meshes for the pressurizer assembly and its piping system. In the process of creating a solid model for the piping system, it was found that the format in the majority of piping software packages is not compatible with that in Pro/E, which is designed for constructing solid models of structural components and was used for generating the solid model for pressurizer assembly. After an extensive research effort, the SolidWorks package was selected to produce the 3D-CAD model of piping system. Since shell elements were used to simulate the pressurizer assembly, the current code capability dictates that the piping system should also be represented by shell elements in order to satisfy the connectivity requirements at joint locations.

\subsubsection{Coupled Model of Pressurizer Assembly and Piping System}

The initial finite element model of the pressurizer assembly consisted entirely of about 10,000 shell elements. To perform a dynamic seismic analysis in a practical amount of 
time ( $<24 \mathrm{hrs})$, it is necessary to keep the number of elements of the coupled model to a reasonably low level. Consequently, the element sizes of the pressurizer assembly were increased in order to reduce the total number of elements. The coarse model of the pressurizer assembly with larger element sizes is shown in Figure 4.4-4. A closer view of the top head is shown in Figure 4.4-5. This figure reveals the locations of the piping on the top head and two of the four shear lugs near the top of the pressurizer assembly. These shear lugs were modeled with 3D solid elements. A view of the bottom head and support skirt is shown in Figure 4.4-6.

The next task was to assemble all connecting piping with the pressurizer assembly. The piping system consists of the surge line, the spray line and two depressurization lines as shown in Figure 4.4.-7. Initially, all piping was modeled with shell elements. However, when the total number of elements in the model with shell elements exceeded 30,000, it was determined that most of the piping should be modeled using beam elements to greatly reduce the number of elements. It is important to select the location for the transition points from shell to beam elements. It was determined that these transition points should not be located near discontinuities such as nozzles. Instead they were all located in the middle of long straight sections of the piping. These transition locations can be seen for all piping at the top of the pressurizer assembly in Figure 4.4-8. A closer view showing the pipe-beam element ending at the center of the pipe-shell section is shown in Figure 4.4-9.

The seismic analysis of the coupled model was performed using the ABAQUS finite element code. The final model as shown in Figure 4.4-7 consisted of 2302 elements using 9054 nodes. Several different types of elements were used in this model. The pressurizer assembly itself and the support skirt were modeled with 8-node shell elements. In addition, the piping sections exiting the top of the pressurizer and one at the bottom were also modeled using 8-node shell elements. The four key shear lugs were modeled using 20node solid brick elements. Most of the piping, except that modeled with shell elements, was modeled using 3-noded beam-pipe elements. These beam-pipe elements in the ABAQUS code allow hoop strains to develop as a result of internal or external pressure loading on the pipes. 


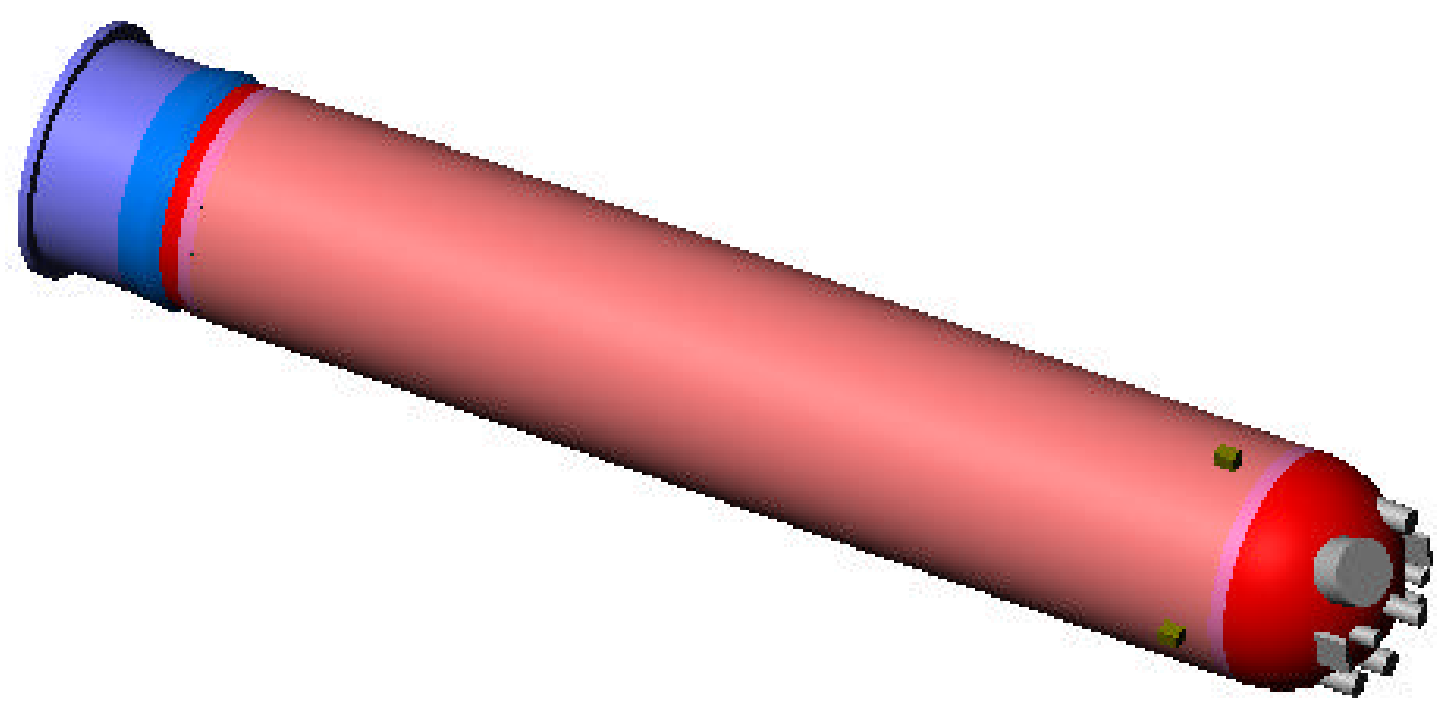

Figure 4.4-2. Solid Model of Pressurizer Assembly

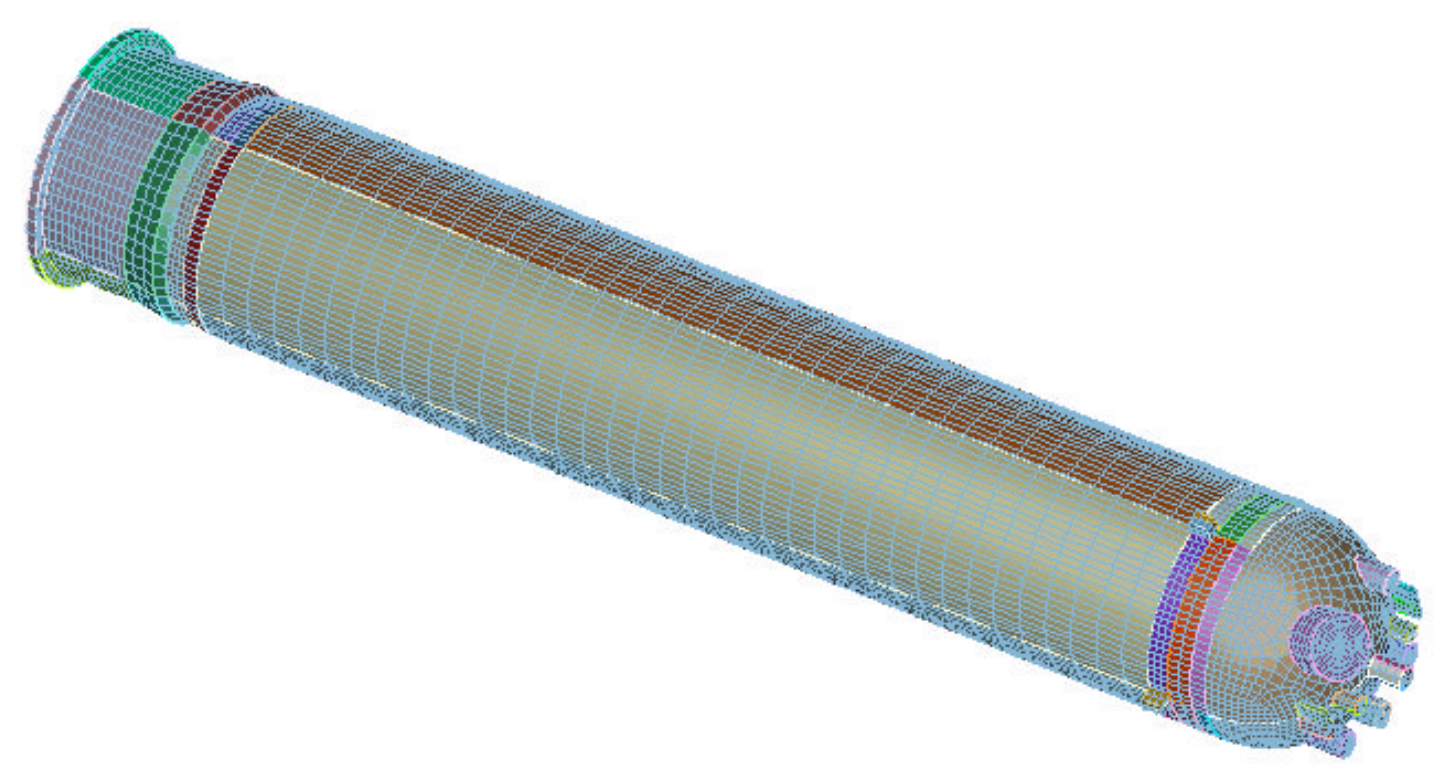

Figure 4.4-3. Initial Meshed Pressurizer Assembly 


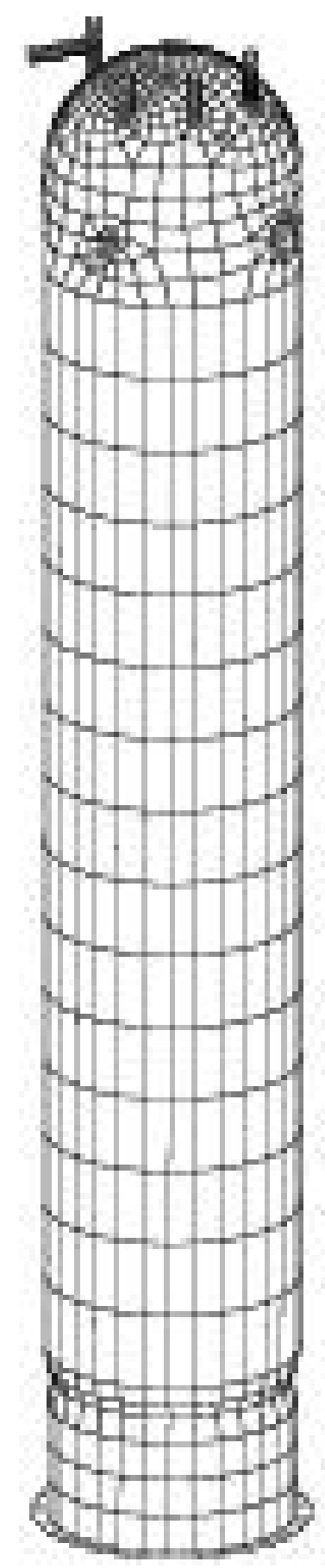

Figure 4.4-4. Final Model of Pressurizer Assembly 


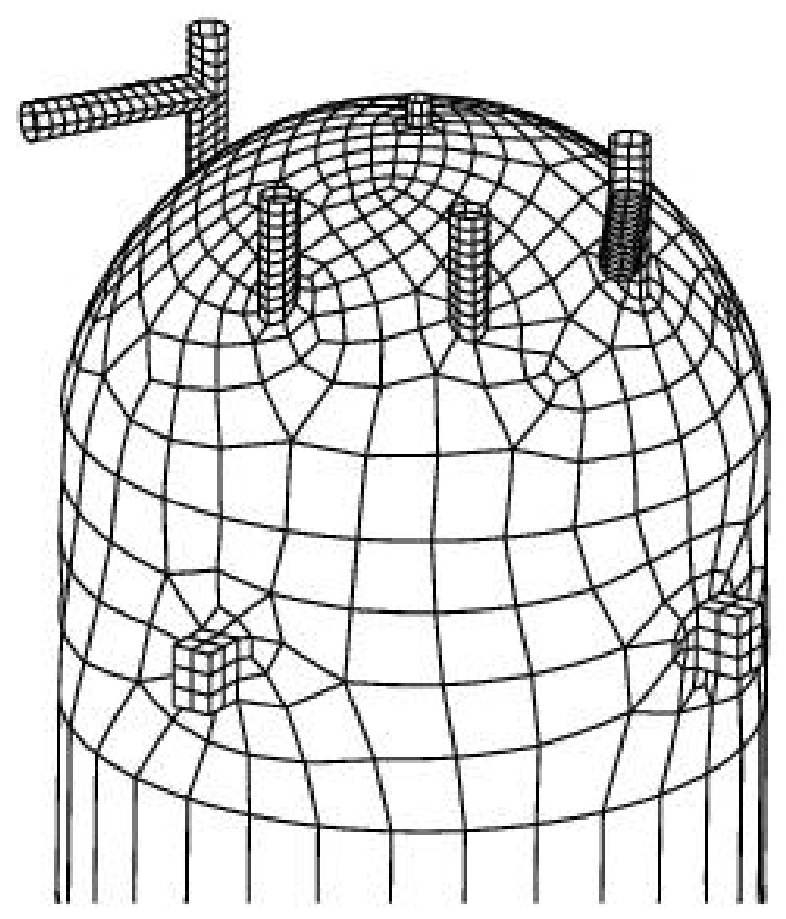

Figure 4.4-5. Top Head of Pressurizer Assembly

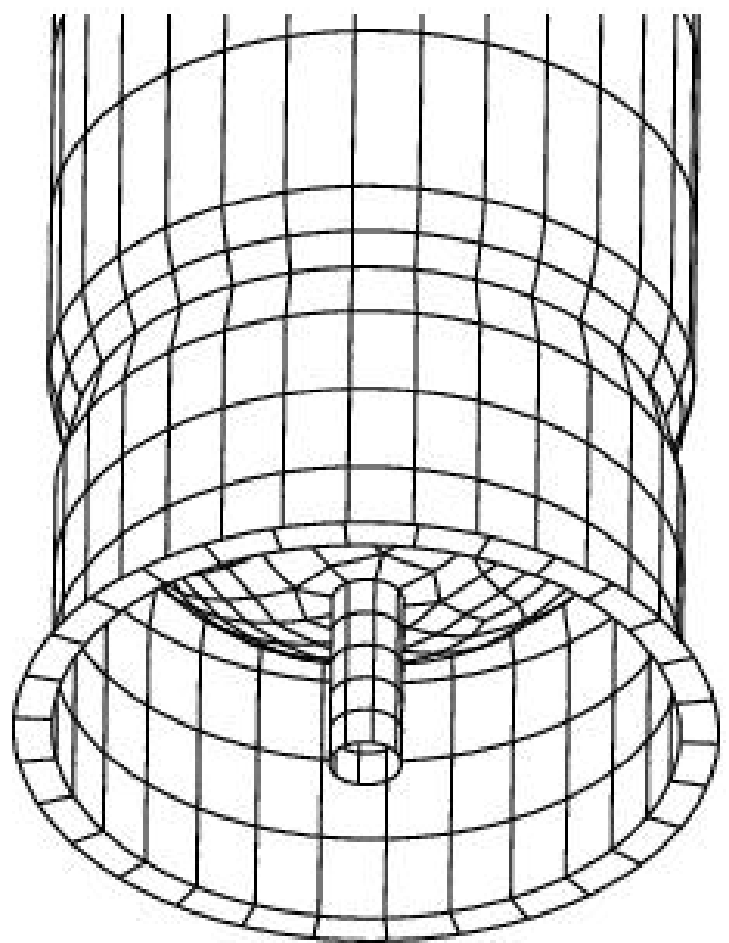

Figure 4.4-6. Bottom Head and Pressurizer Skirt 
A special element type, the distributing coupling element in the ABAQUS code, was used to connect the beam-pipe elements to the shell-pipe elements. The distributing coupling elements distribute loads and mass from the end node on the beam-pipe element to a collection of coupling nodes on the shell-pipe elements.

Framatome provided the details of pipe supports in terms of support types and locations. The pipe supports consisted of rigid connections, springs and snubbers. Rigid connections were modeled with fixed support points, spring supports were modeled with 1D spring elements, and the snubbers were modeled using 1D dashpot elements.

\subsubsection{Modeling Problems}

Some of the modeling problems stemmed from attempting to minimize the number of elements in the coupled model of the pressurizer assembly and its piping system in order to reduce the computational penalty in executing analyses. A significant reduction in the number of elements was accomplished by using beam elements to represent most of the piping system. It should be reminded that the accuracy of analysis results in the piping system will be reduced by using beam elements instead of shell elements, but the overall results are not expected to be greatly affected in a seismic analysis.

Since two different element types were used, it was necessary to determine suitable locations to make the transition and find an appropriate connection method that assures all forces, moments and masses are transferred correctly from one element type to another. The chosen method is to use beam-pipe elements transitioning to shell-pipe sections. These transitions are chosen in the straight section of the pipes using the distributing coupling elements. No sensitivity analyses have been performed to check the validity of this approach and the adequacy of these distributing coupling elements because of budget and schedule constraints.

After the final input file was developed from PATRAN, a few additional modifications and checks were made before the coupled model could be executed using the ABAQUS code. It is required in the ABAQUS code that a normal to the beam element be defined for any beam-pipe section. The current version of CUBIT and PATRAN does not contain this provision and it is therefore impossible to pass the necessary information into the ABAQUS code. The simplest way to specify this information is to define an additional point for each beam element in the direction of its normal. This procedure was performed for each of the approximately 200 beam elements.

For the purpose of pressure application, it was necessary to define the normal direction of all shell elements on the pressurizer assembly and the shell-pipe sections. The normal is determined by the order in which the nodes defining each shell element are specified in CUBIT. Most of these normals were identical for each different section of shell elements (i.e., top head, cylinder, bottom head, etc.). However, in some cases, CUBIT changed the nodal numbering and thereby reversed the normal to the surface direction. When all these normals were properly determined, a positive pressure could be applied to the inside surface of the pressurizer assembly and shell-pipe sections. 


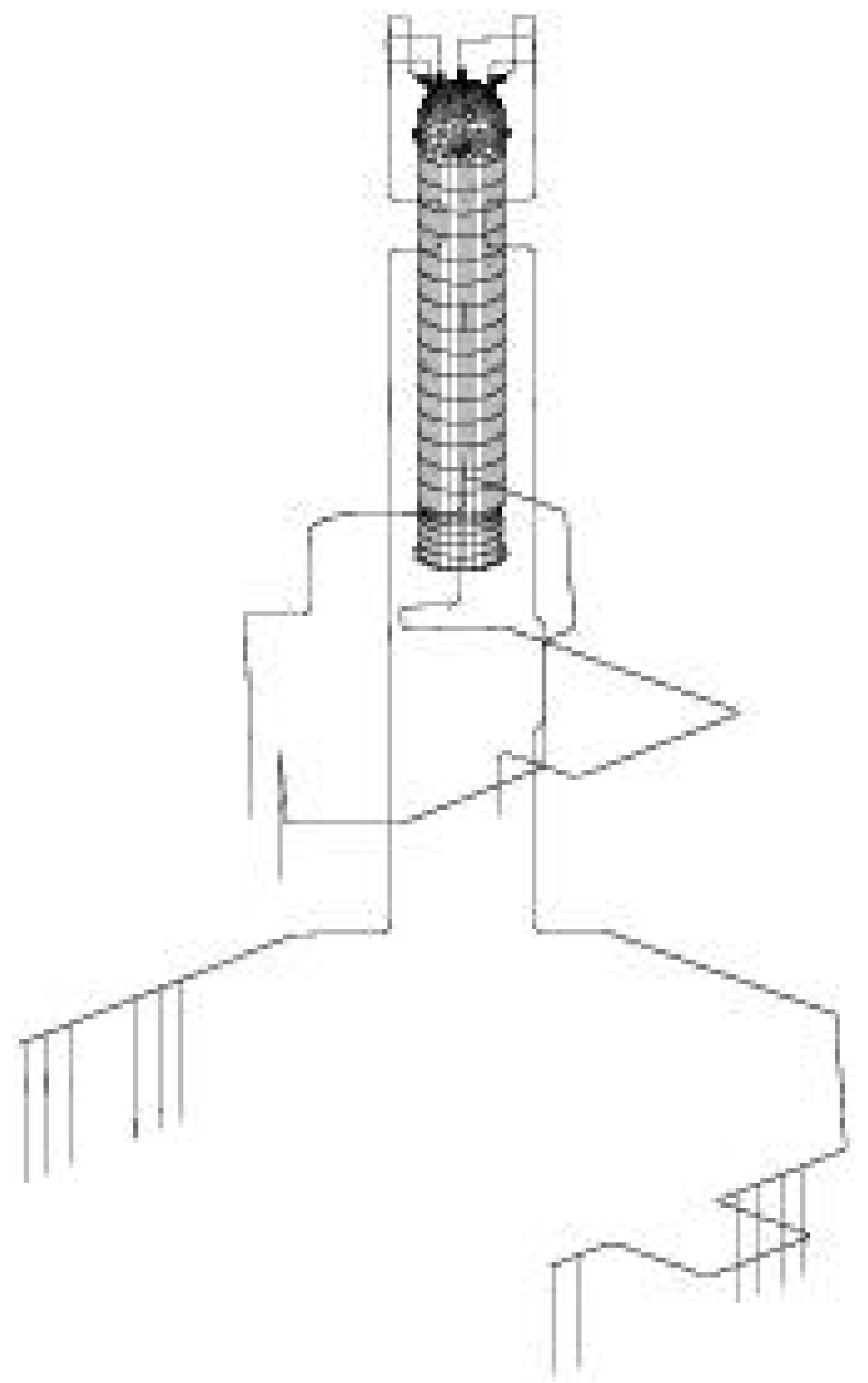

Figure 4.4-7. Coupled Model of Pressurizer Assembly and Piping System 


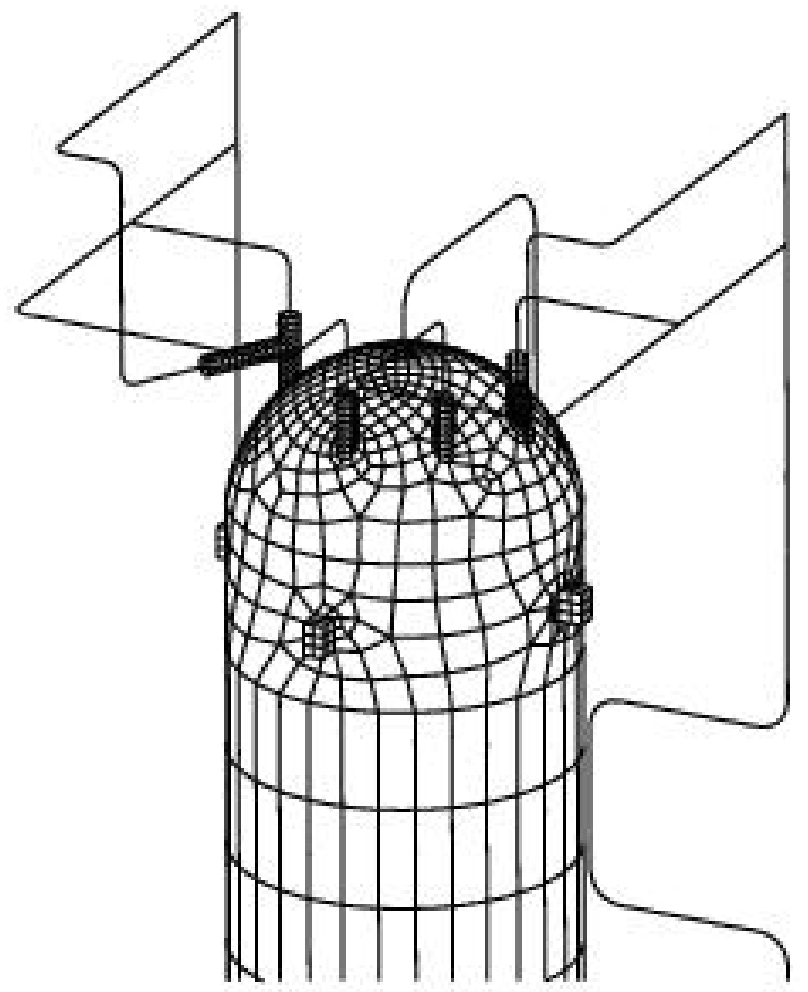

Figure 4.4-8. Top of Pressurizer with Piping

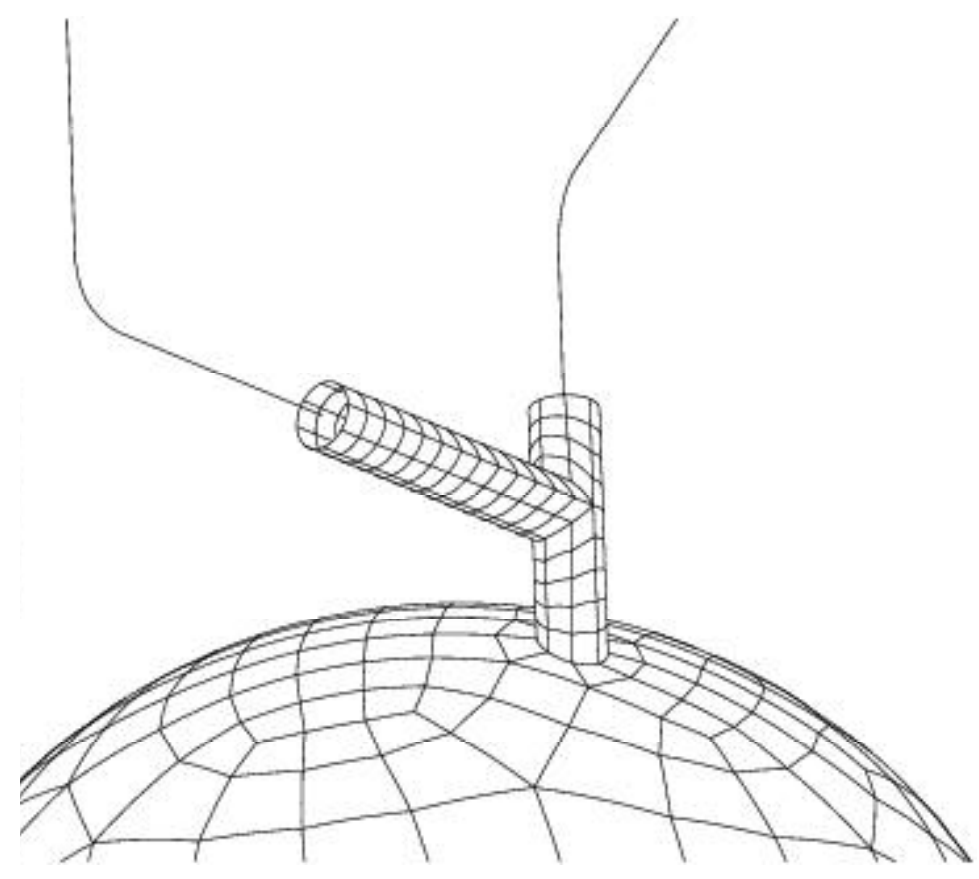

Figure 4.4-9. Piping Transition (Shell elements to Beam elements) 
After specifying the pipe anchor location information, a final mesh generation was performed with CUBIT assuring that nodes would be located where all supports connected to the piping system. However, there is currently no way to pass the information on the support orientation and the material properties for the springs and snubbers from solid models to the analysis model, so this information was entered directly into the ABAQUS input file.

For the rigid supports, the appropriate nodes were fixed only in the specified direction(s) of the rigid support. For the spring supports, a 1D spring element was inserted from the appropriate node on the piping to a fixed node. Each 1D spring element was also oriented in the direction specified, usually in the vertical direction. The material properties for the spring such as stiffness coefficient were also input. For the snubber supports, a 1D dashpot element was inserted from the appropriate node on the piping to a fixed node. Each 1D dashpot element was also oriented in the direction specified, usually in the horizontal direction. The material properties for the dashpot such as dashpot coefficient were input in the same manner.

\subsubsection{Integrated Seismic Analyses of Coupled Model}

\subsubsection{Analysis Inputs}

Seismic analyses were performed with the coupled model after it was completely developed. The first step in the analysis was to apply the gravity load. This is an important step due to the large size of the pressurizer assembly and the significant amount of large pipes. An operating pressure of $2250 \mathrm{psi}$, in a normal operating mode, was applied to the entire system, including the pressurizer assembly as well as all shell-pipe and beam-pipe sections.

For this implicit seismic analysis of the coupled model, the time history of accelerations from the El Centro earthquake was chosen as the ground motion. This earthquake time history has two different horizontal components and one vertical component, as shown in Figures 4.4-10, 4.4-11, and 4.4-12, respectively. The maximum horizontal acceleration is $0.31 \mathrm{~g}$ 's and the maximum vertical acceleration is $0.20 \mathrm{~g}$ 's. These peak ground accelerations are near the upper bound for most Safe Shutdown Earthquake (SSE) at most nuclear power plant sites. The analysis covered the entire recorded time history of 40 seconds with a time step of 0.02 seconds. It took about 9 hours to complete the entire analysis on a single processor, out-of-date $180 \mathrm{MHz}$ workstation. On the more efficient processors typically in the $750-1000 \mathrm{MHz}$ ranges, the entire seismic analysis would probably complete in less than 2 hours.

The seismic accelerations were applied at all pressurizer supports at the skirt base, horizontally at the shear lug locations, at each of the rigid supports of the piping system, and at the ends of each of the spring and dashpot element locations. In this analysis, it was decided to apply the acceleration components instead of displacement components uniformly at each support location because application of displacements could be nonconservative if there were significant differential displacements in the structure supporting the entire model. Developing a detailed model of the entire structure enclosing and supporting the pressurizer assembly and piping system could have been performed but would have taken much more effort. However, a simplified 1D model of the building 
structure could be developed with nodes at various floor elevations tied to the support points of the coupled model. Ground accelerations would then be applied only at the base of the simple structure model. Nodes on this structure would then transfer accelerations and displacements to the various support points on the pressurizer assembly and piping system during the seismic analysis.

Another analysis assumption was also made to locate the pressurizer assembly and its associated piping system in the lower section of a rigid containment structure. In the lower floors of a very rigid structure, the amount of differential displacement between elevations should be minimal.

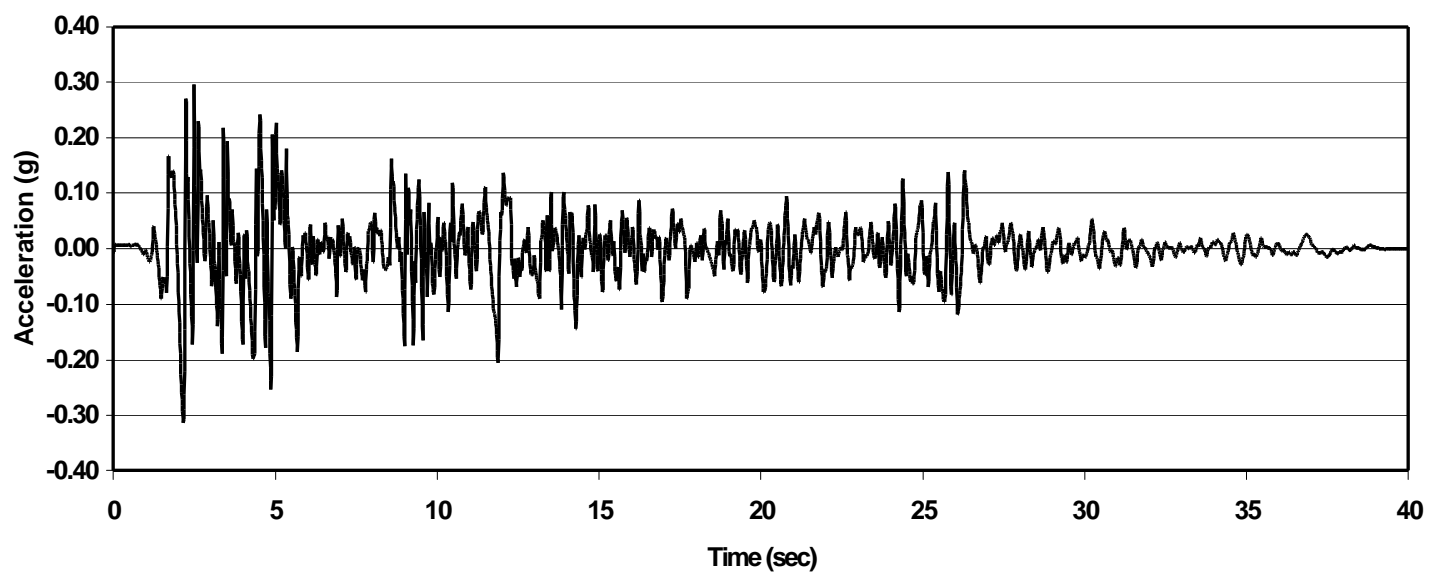

Figure 4.4-10. El Centro Earthquake Time History (Horizontal X-direction)

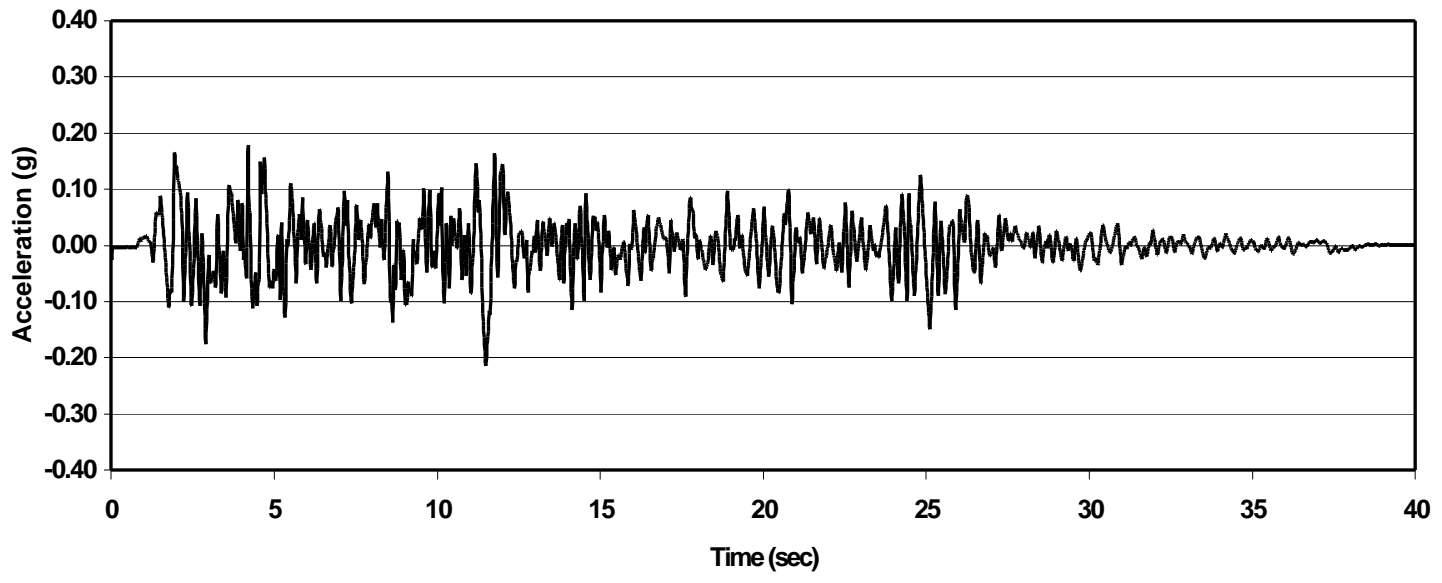

Figure 4.4-11. El Centro Earthquake Time History (Horizontal Y-direction) 


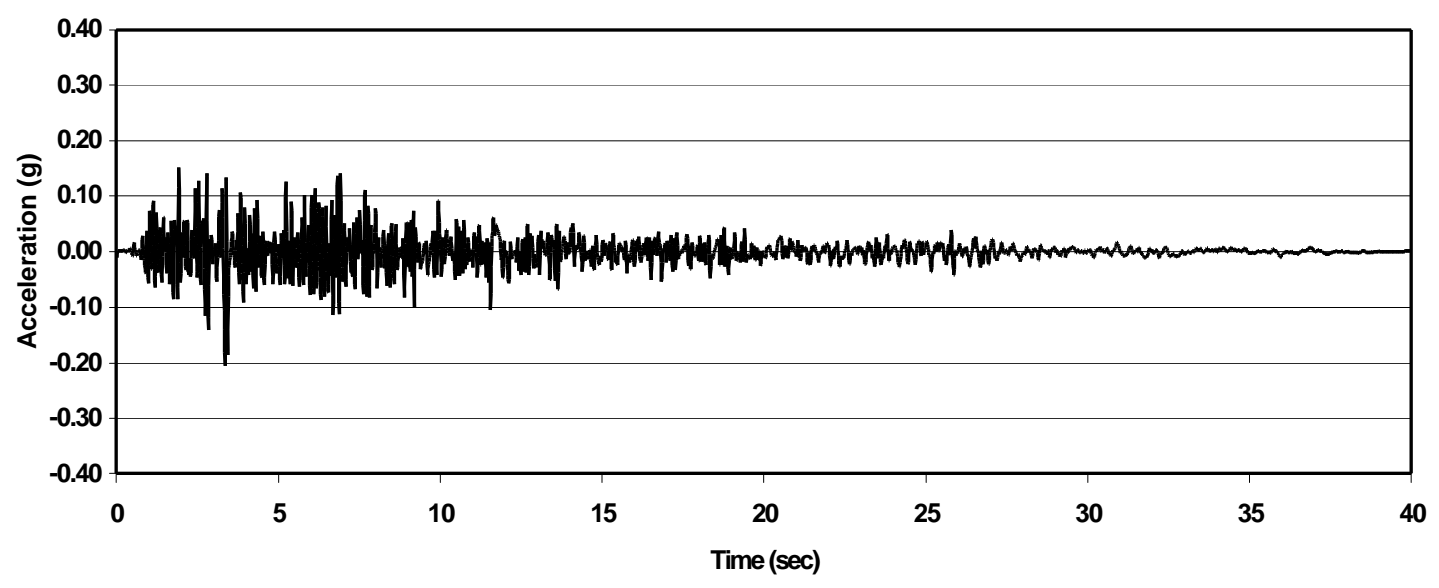

Figure 4.4-12. El Centro Earthquake Time History (Vertical Z-direction)

\subsubsection{Seismic Analysis Results}

The first seismic analysis was performed with all the 'original' specified design conditions (i.e., pressurizer wall thickness and pipe support locations). The design wall thickness of the pressurizer cylinder is nominally 5 inches and the dome and bottom head are nominally 4 inches. After the analysis was completed, peak stresses in the pressurizer wall and the piping system were searched and identified. Results of the maximum principal stresses in the pressurizer wall are shown in Figure 4.4-13. The average principal stress in the cylinder wall is approximately $23.0 \mathrm{ksi}$. Peak stresses reach $41.6 \mathrm{ksi}$ in the cylinder near the shear lugs and $42.5 \mathrm{ksi}$ in the dome near the nozzle locations. The peak stresses appear high around these areas because local thickened sections were not simulated in the model.

A closer look at the maximum stresses near the shear lug locations is provided in Figure 4.4- 14. Although principal stresses reach $41.6 \mathrm{ksi}$ in the cylinder wall near the shear lugs, these are very localized and can be attributed to the analytical model not accurately reflecting the actual detail of the shear lug as shown in Figure 4.4-15. The analysis model used shell elements to represent the cylinder walls and solid 3D elements to represent the shear lugs. Therefore the gradual tapering of the actual shear lug does not occur in the analysis model, resulting in a higher stress concentration at the interface. To improve the accuracy of this local stress, a much finer mesh of solid 3D elements could be used for the cylinder wall / shear lug interface. In addition, a local detailed analysis could be performed using the analysis results from the coupled model at the boundaries of the local model.

A similar response also occurs in the pressurizer dome at the piping nozzle connections. The maximum principal stresses in pressurizer dome are shown in Figure 4.4-16. The maximum principal stress in the dome shell elements is $42.5 \mathrm{ksi}$ and that in the piping shell elements is $73.6 \mathrm{ksi}$. Again these peak stresses are very localized at the interface between the dome and the piping. These peak stresses are also the result of a simplified local representation in the coupled model. The actual detail of the piping nozzles attaching to the pressurizer dome is shown in Figure 4.4-17. The piping is much thicker near the interface at each of the nozzles and also includes a small amount of tapering at the connection. Once 
again, a finer mesh or more detail local model would provide more accurate and much smaller actual peak stresses at the pressurizer / nozzle interface.

It is interesting to note that the principal stresses are very nominal near the ends of the shell pipes as they transition into beam-pipe elements, as shown in Figure 4.4-16. Therefore, it was appropriate to select the transition from shell to beam elements in the straight section of piping away from the nozzle and no abnormal stresses were induced from this modeling approach.

There are four primary piping lines attached to the pressurizer: the surge line, the spray line, and two depressurization lines. An isometric of each of these lines are shown in Figures 4.4-18 - 4.4-21 along with the spring and snubber support locations. These isometrics do not identify the rigid support location. 

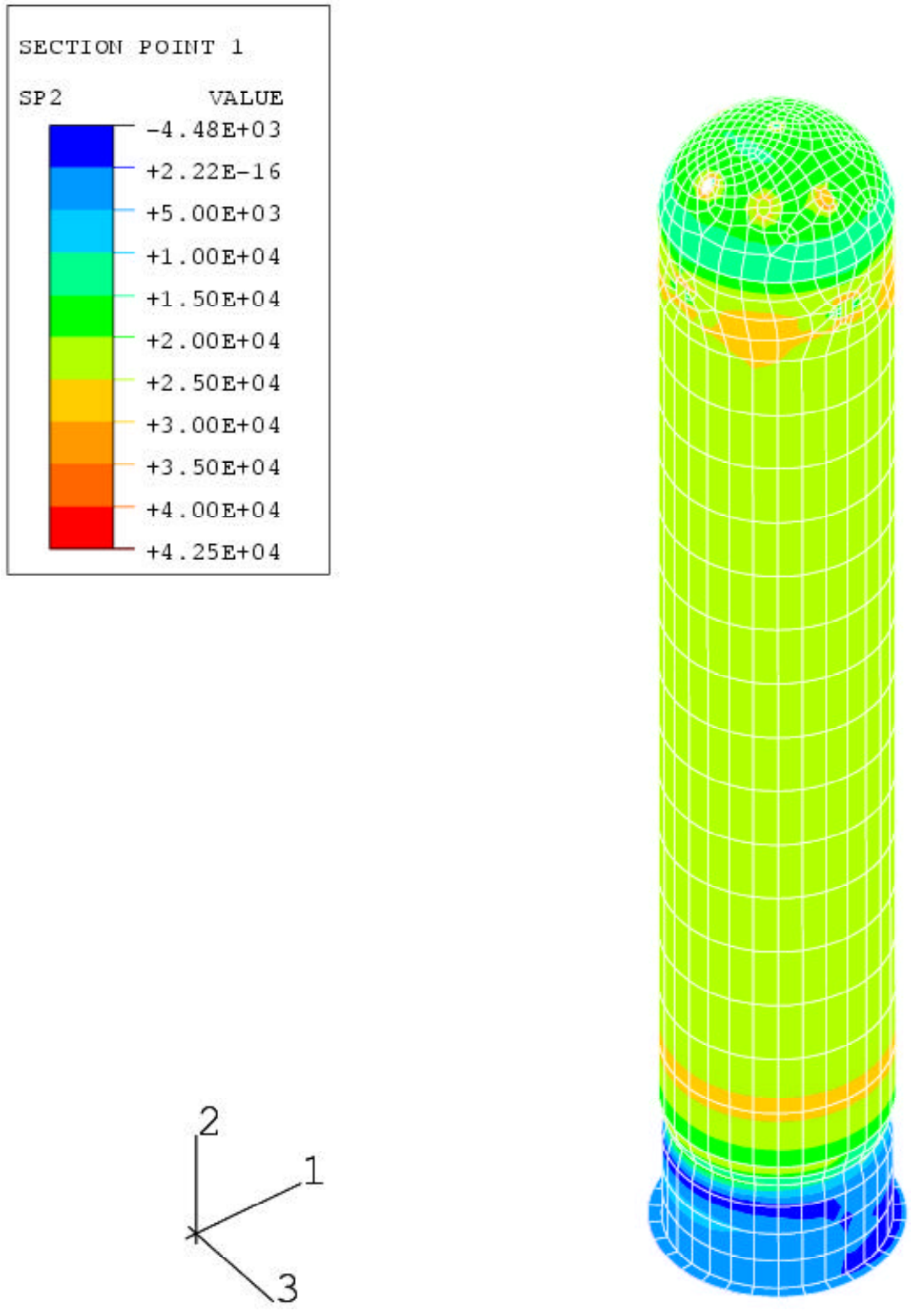

Figure 4.4-13. Maximum Principal Stresses in Pressurizer and Skirt (original) 

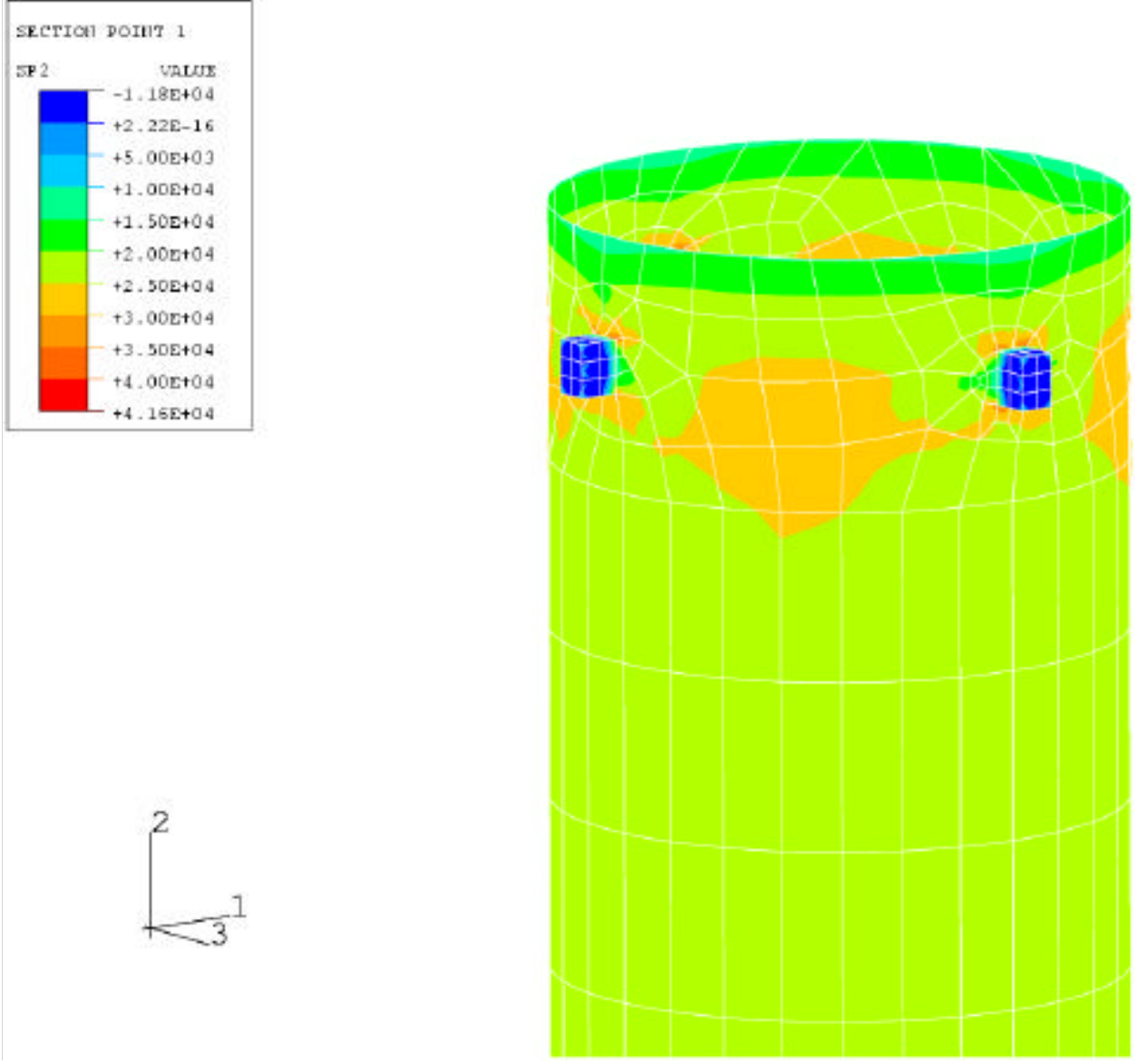

Figure 4.4-14. Maximum Principal Stresses in Pressurizer near Shear Lugs (original)

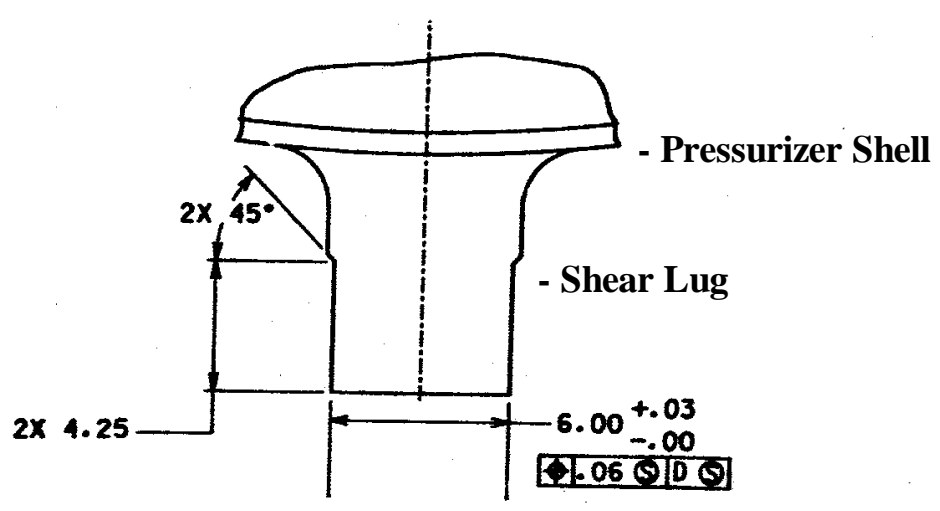

Figure 4.4-15. Pressurizer Shear Lug Detail 


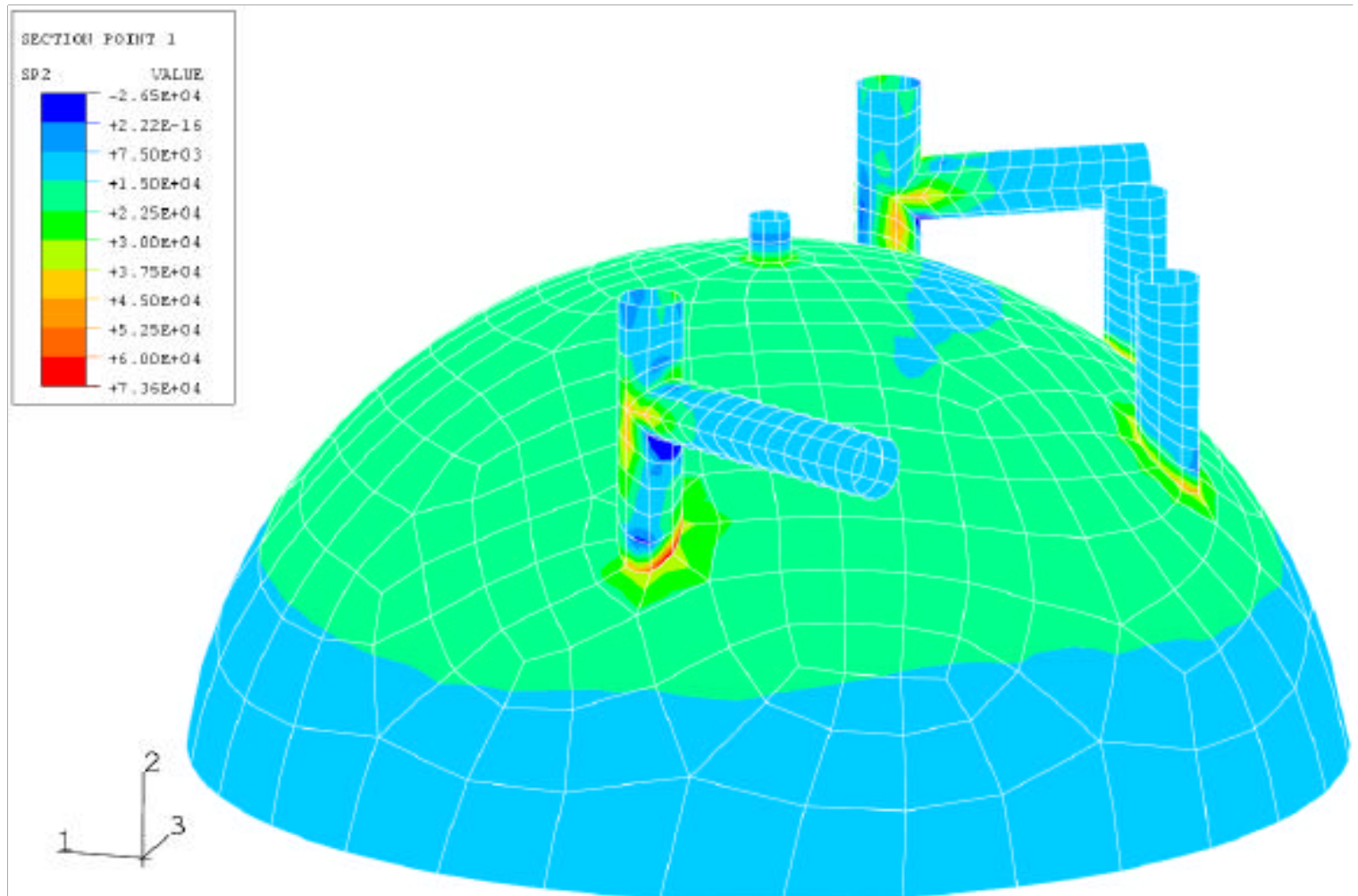

Figure 4.4-16. Maximum Principal Stresses in Dome (original)

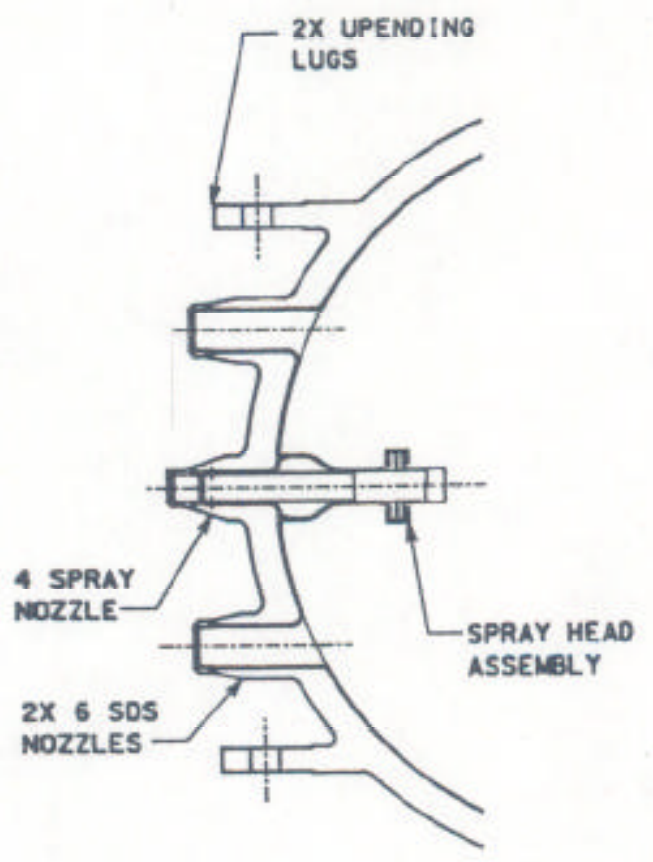

Figure 4.4-17. Pressurizer Dome Nozzles Detail 


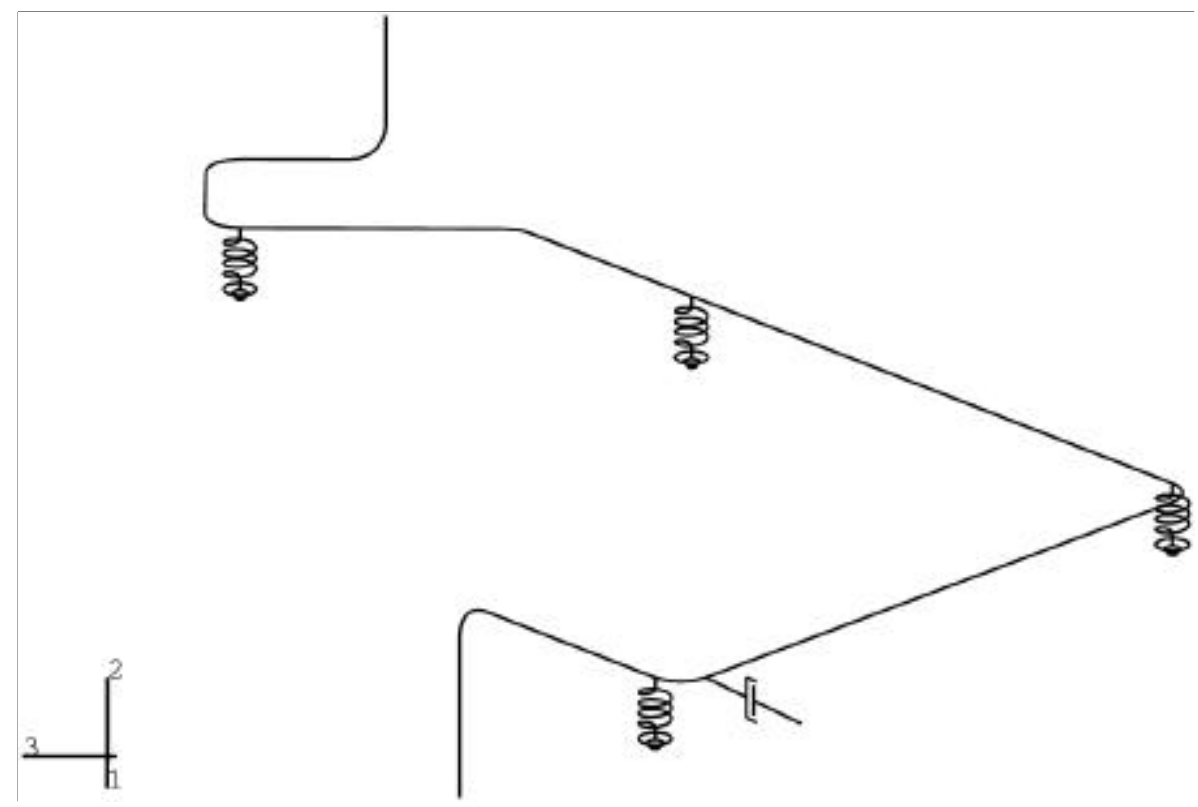

Figure 4.4-18. Surge Line Piping with Spring and Snubber Supports (original)

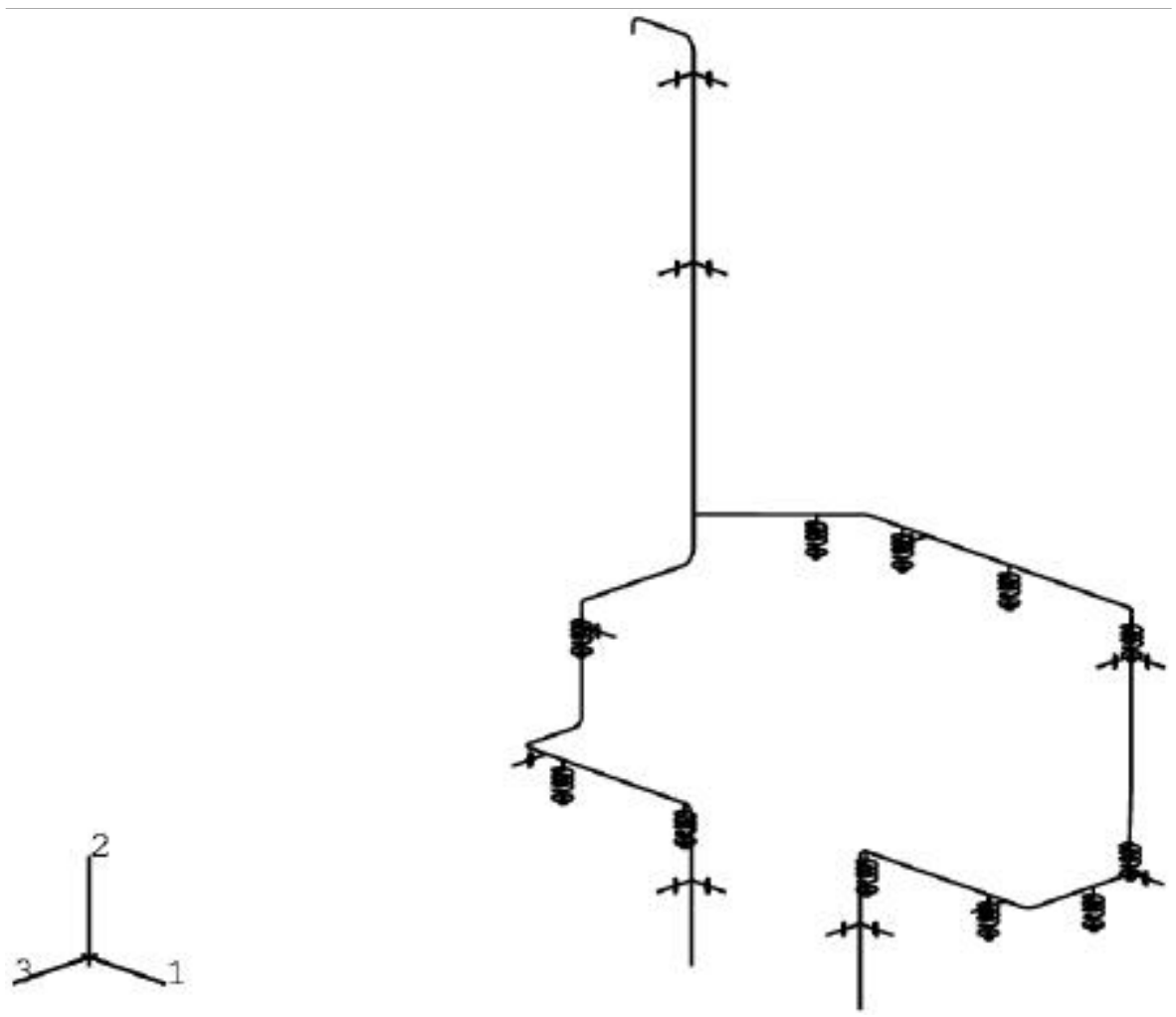

Figure 4.4-19. Spray Line Piping with Spring and Snubber Supports (original) 


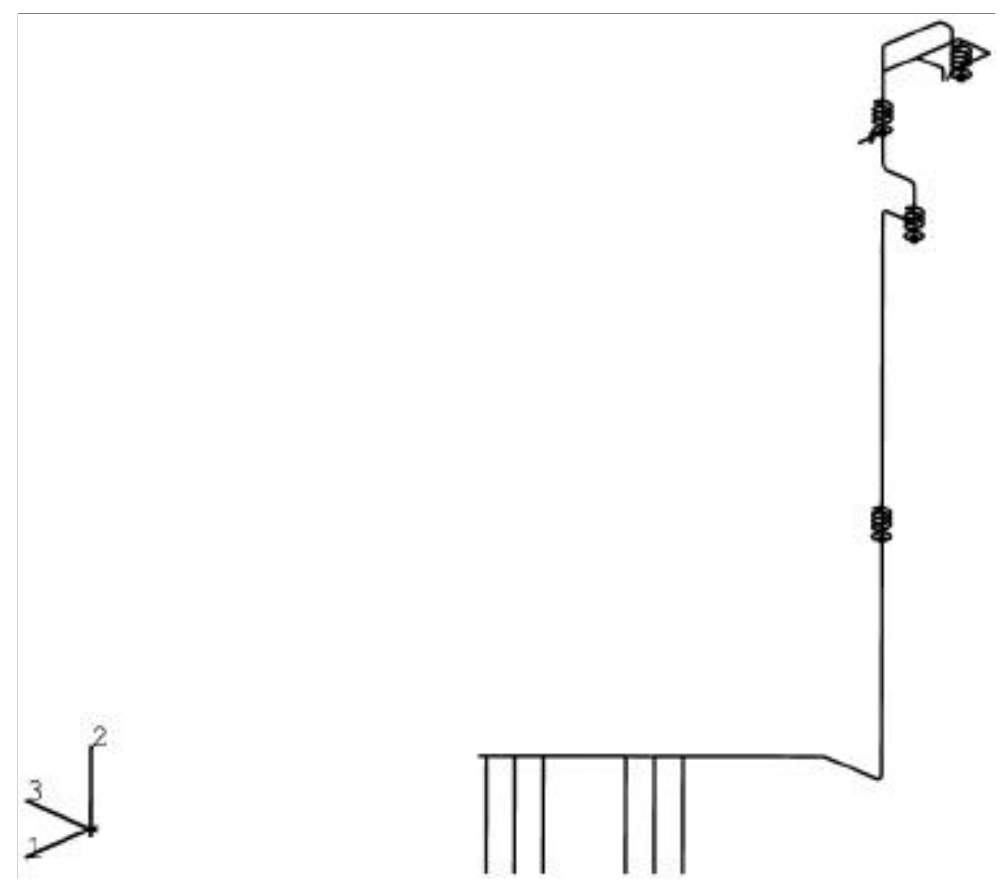

Figure 4.4-20. Depressurization Line \#1 Piping with Spring and Snubber Supports (original)
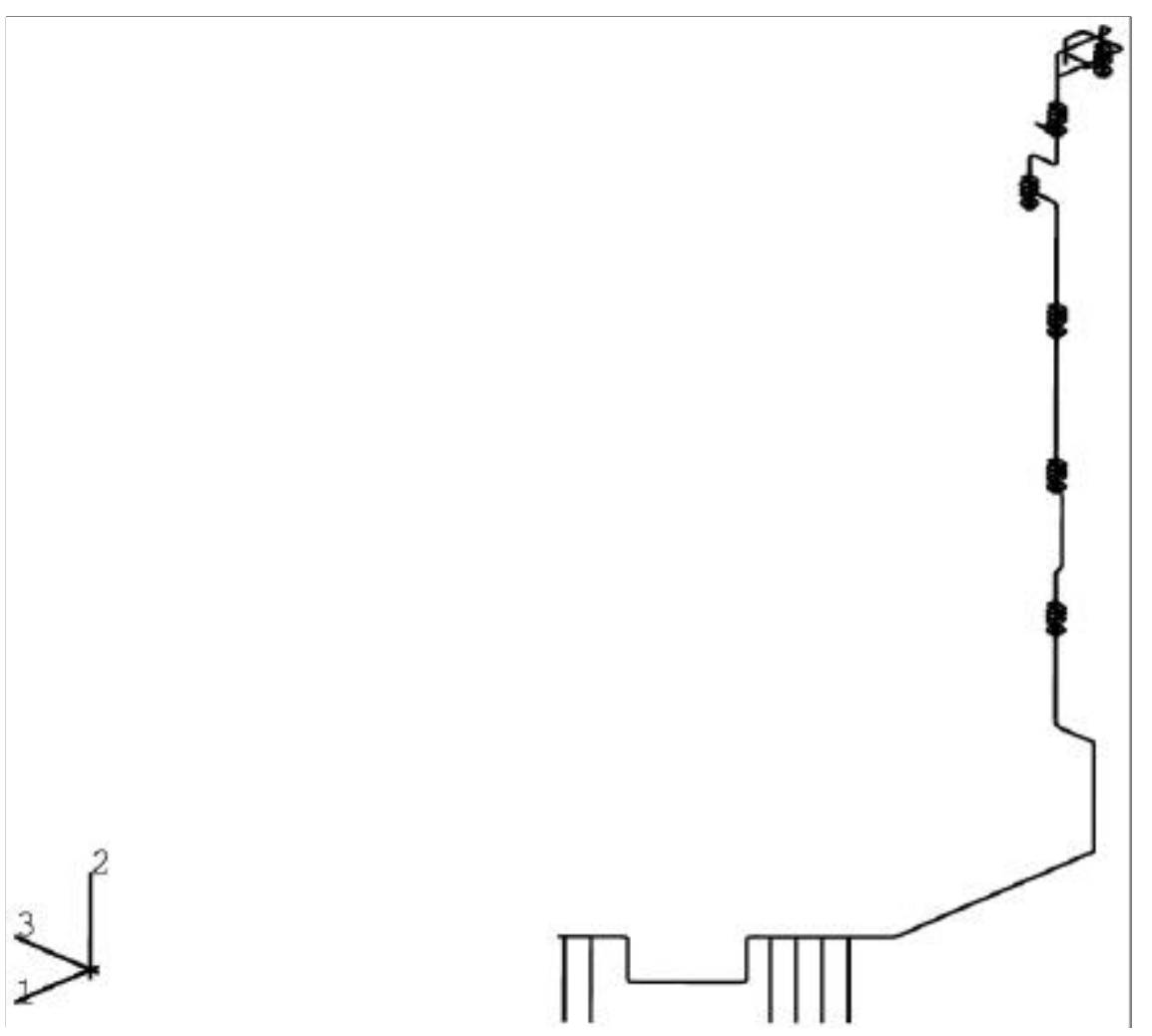

Figure 4.4-21. Depressurization Line \#2 Piping with Spring and Snubber Supports (original) 
The maximum principal stresses in each of the piping lines are shown in Figures 4.4-22 4.4-25. The peak stresses in each of the piping lines using the original configuration of supports are listed as follows:

Surge Line:

Spray Line:

Depressurization Line \#1:

Depressurization Line \#2:
$12.3 \mathrm{ksi}$

$10.3 \mathrm{ksi}$

$13.8 \mathrm{ksi}$

$15.9 \mathrm{ksi}$

While all these stresses are well below the yield stresses for these piping materials, the spray line appears to be the most balanced design. Although there may have been more than enough supports, all principal stresses are nearly in the 9 - 10 ksi range. Conversely, the depressurization line \#2 appears to have a few 'hot spots', with peak stresses reaching $16 \mathrm{ksi}$ in one spot and $15 \mathrm{ksi}$ in two other locations.

After the first analysis was complete using all the "original" as-designed conditions, a second analysis was performed by changing the wall thickness of the pressurizer assembly. For this design change, it was not necessary to repeat the entire design-to-analysis mesh generation procedure. Since the pressurizer assembly was modeled entirely with shell elements and changing the wall thickness involves only making a simple change in the input file.

The wall thickness of the pressurizer assembly was reduced by 0.5 inches for the second analysis. The cylinder wall is therefore 4.5 inches and the dome and bottom head are 3.5 inches. By reducing the wall thickness, some useful insight into its effect on peak stresses could become available in determining the amount of design margin and if a safe operation could still be upheld with a reduced wall thickness.

This second analysis was performed in an identical procedure to the first 'original' analysis. The gravity load and internal pressure were initially applied. Then the same El Centro earthquake time history was applied to the coupled model of pressurizer assembly and its piping system and support configurations. After the analysis was complete, the maximum principal stresses were computed and are shown for the pressurizer assembly in Figure 4.426.

The peak maximum principal stresses near the shear lugs are shown in Figure 4.4-27. The average principal stress in the cylinder wall is approximately $26.0 \mathrm{ksi}$. Peak stresses reached $44.2 \mathrm{ksi}$ in the cylinder wall near the shear lugs as compared to $41.6 \mathrm{ksi}$ using the original design wall thickness. Peak stresses in the dome and associated piping are shown in Figure 4.4-28. The peak stresses were $48.5 \mathrm{ksi}$ in the dome near the nozzle locations, which compares to $42.5 \mathrm{ksi}$ in the first analysis. Although these peak stresses did increase, they are primarily due to modeling effects of simplifying local geometry details. 


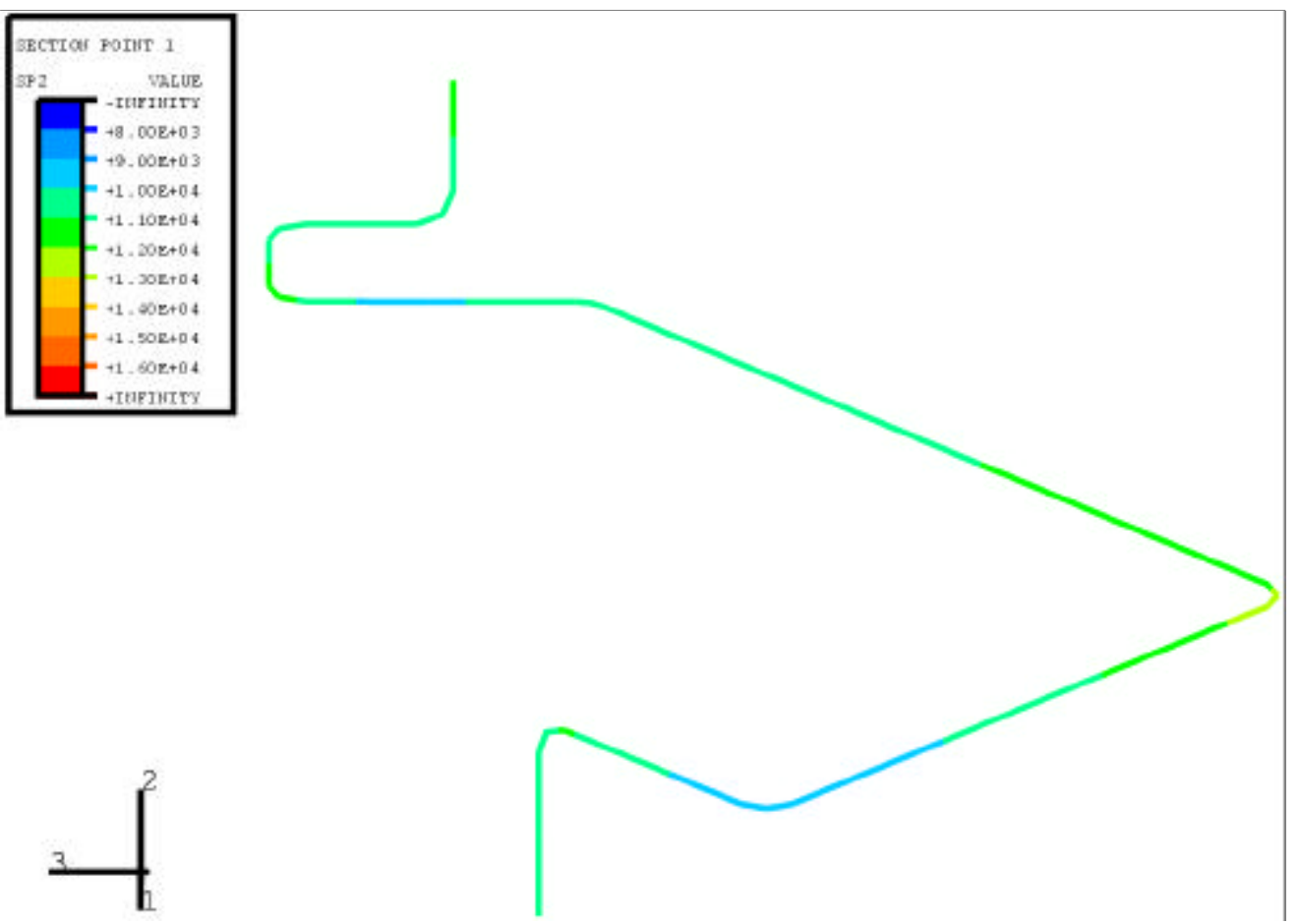

Figure 4.4-22. Maximum Principal Stresses in Surge Line (original)
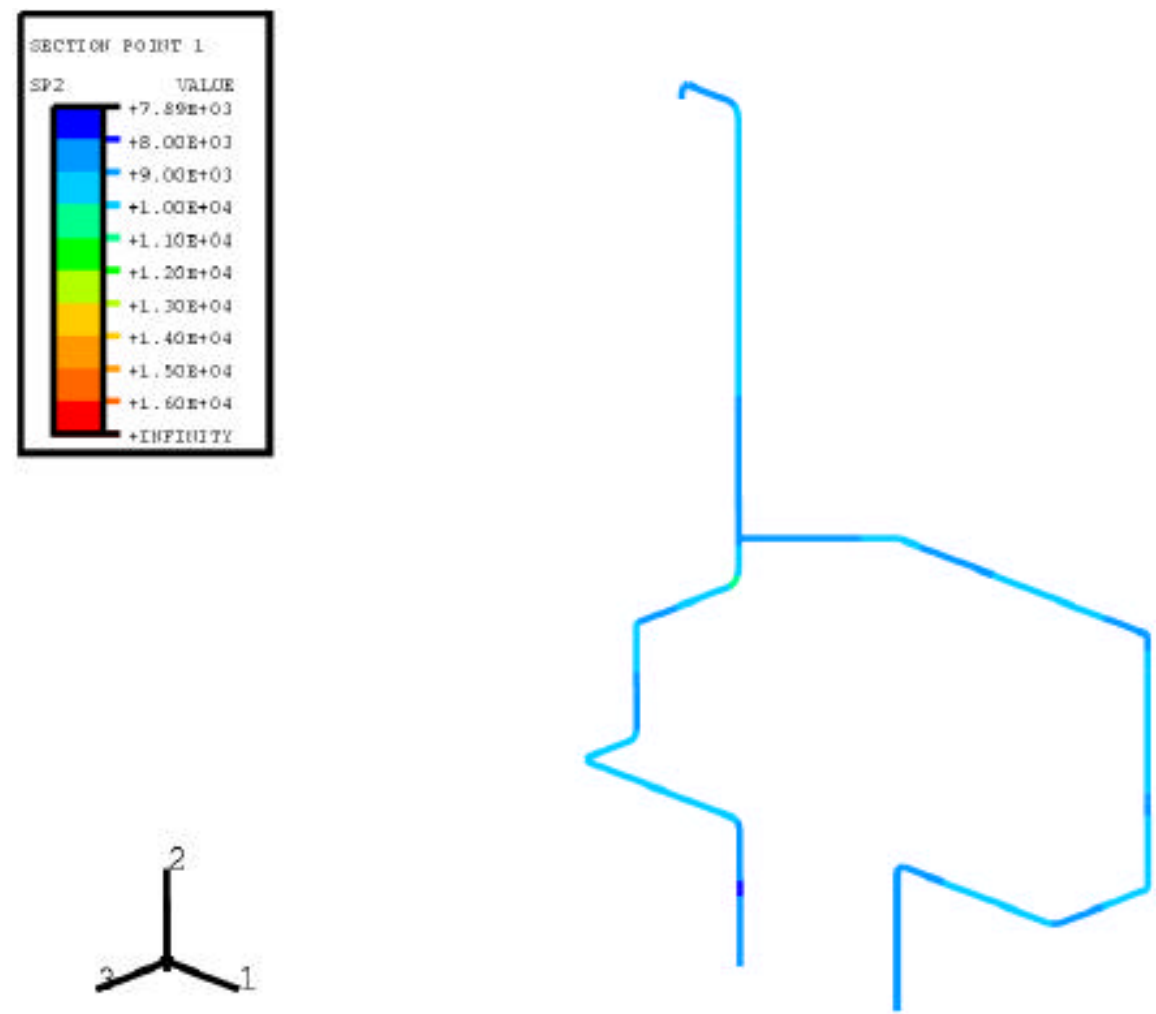

Figure 4.4-23. Maximum Principal Stresses in Spray Line (original) 

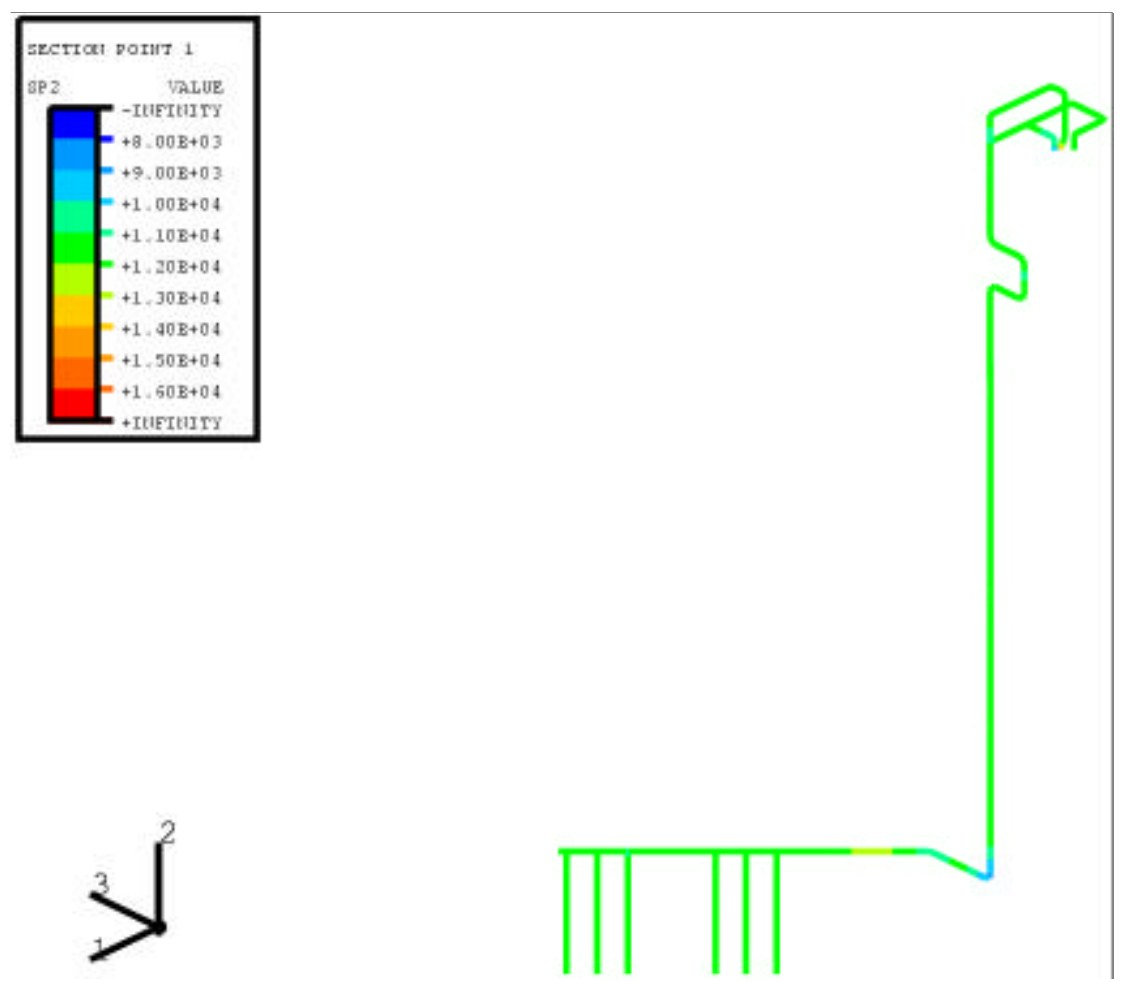

Figure 4.4-24. Maximum Principal Stresses in Depressurization Line \#1 (original)
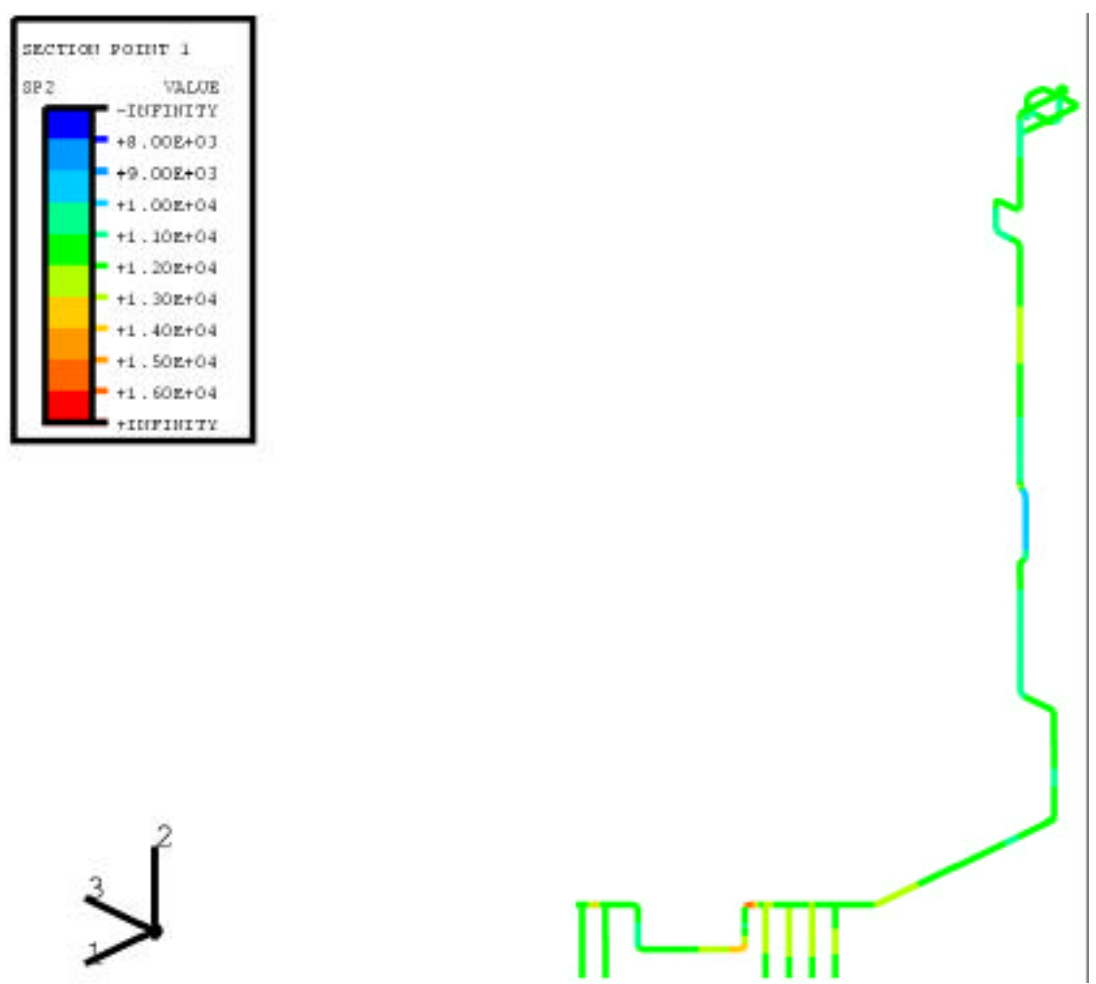

Figure 4.4-25. Maximum Principal Stresses in Depressurization Line \#2 (original) 

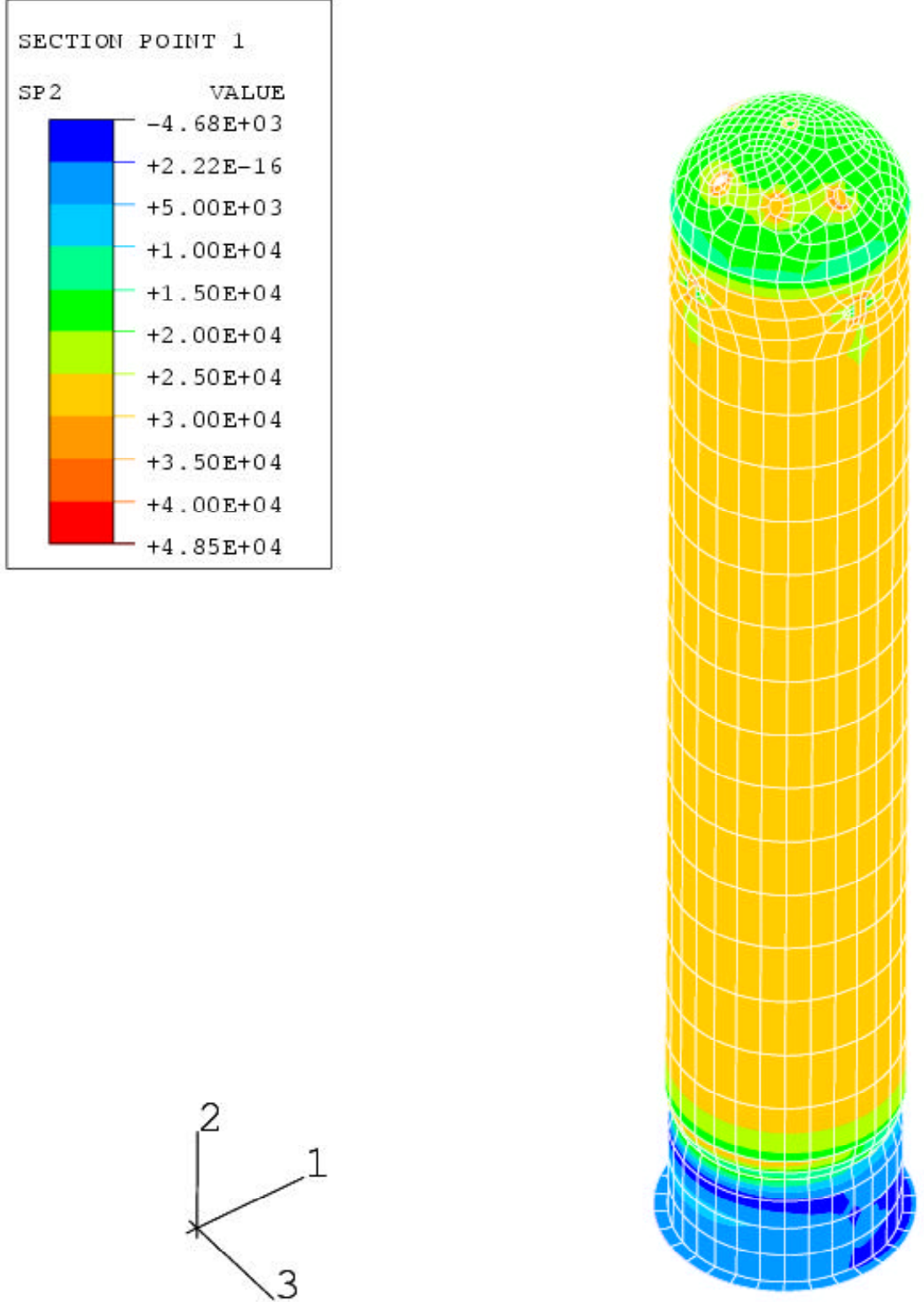

Figure 4.4-26. Maximum Principal Stresses in Pressurizer and Skirt (reduced thickness) 

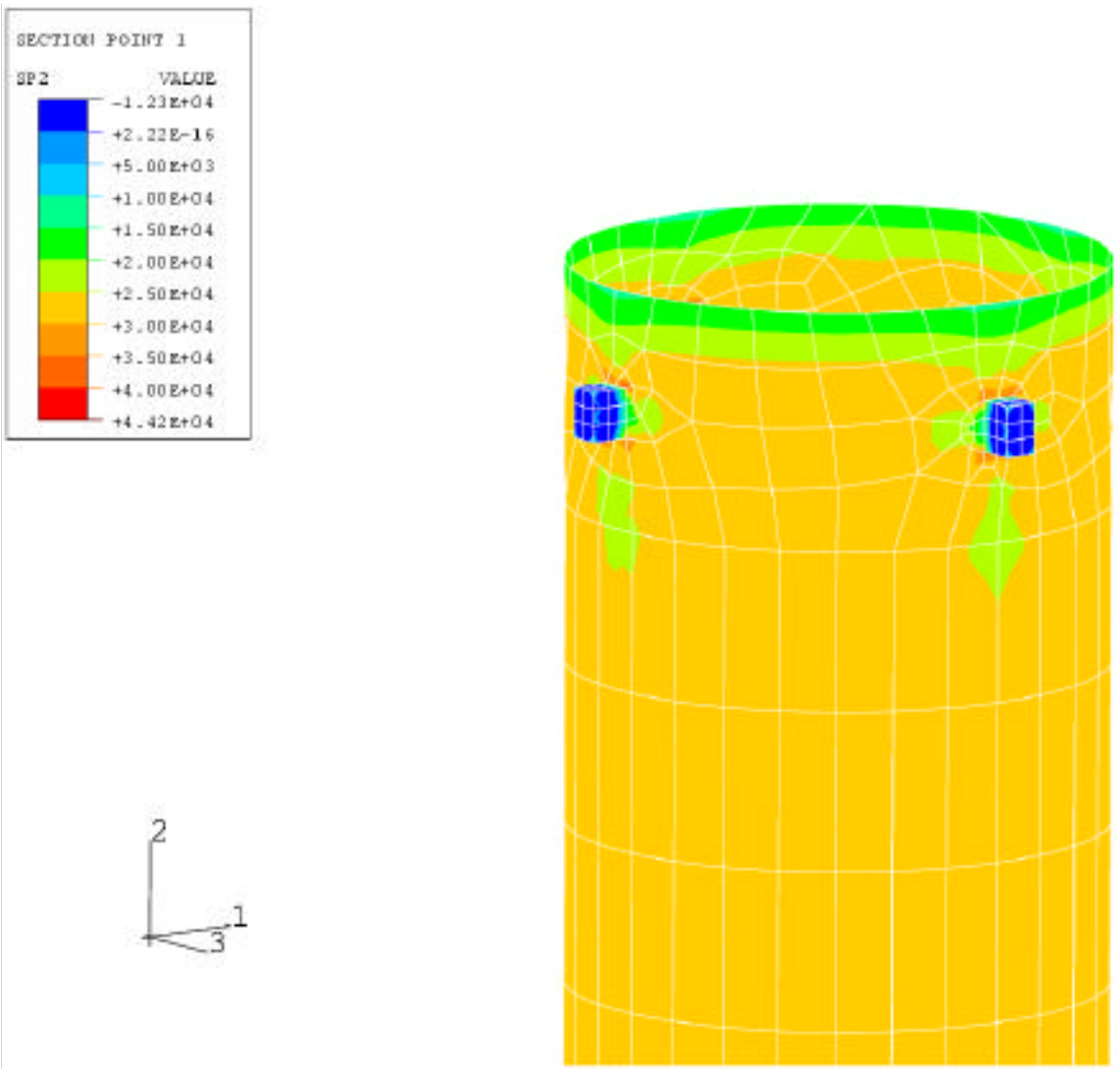

Figure 4.4-27. Maximum Principal Stresses near Shear Lugs (reduced thickness) 


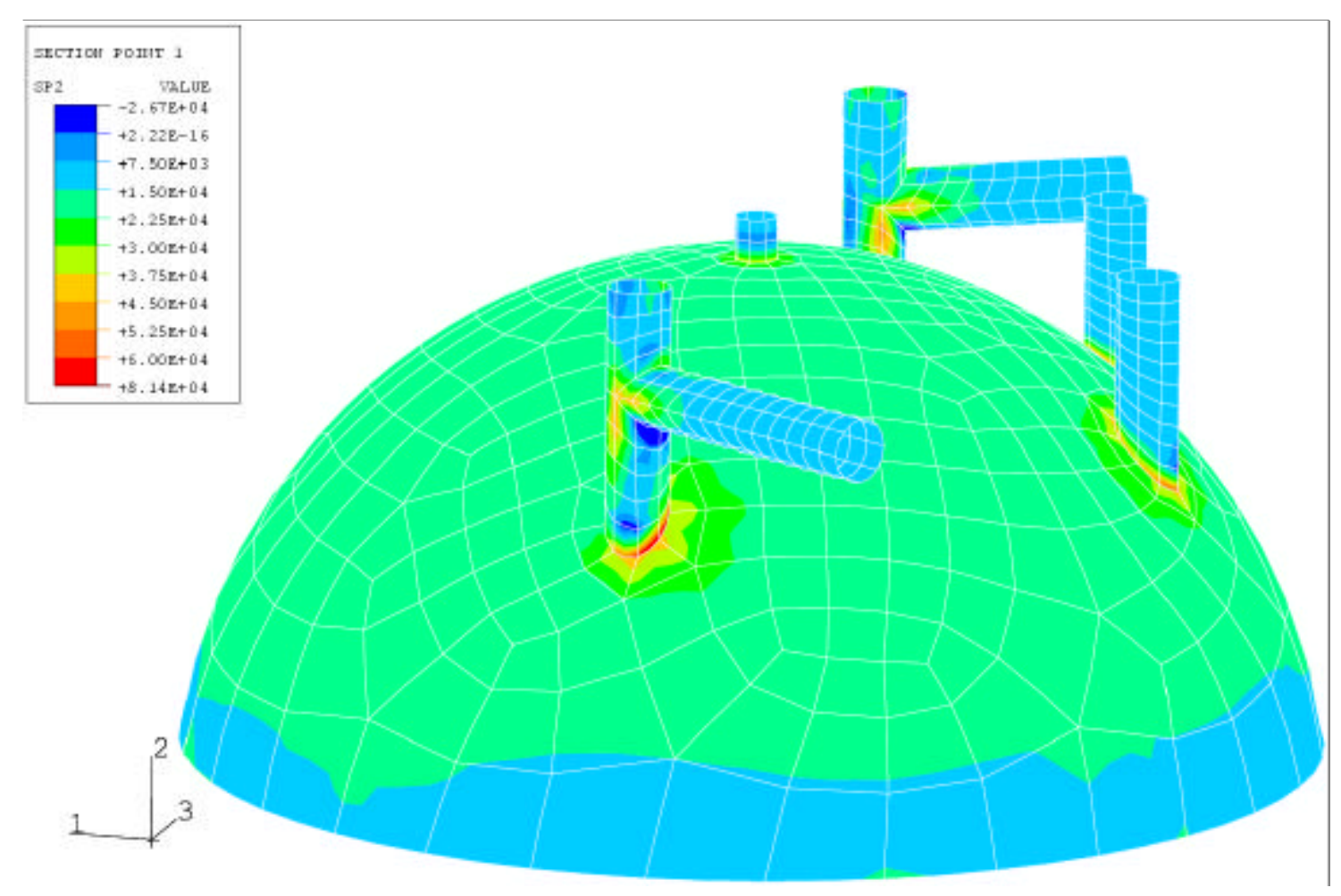

Figure 4.4-28. Maximum Principal Stresses in Dome (reduced thickness)

Since the only modification in this second analysis involved a reduced pressurizer wall thickness, the peak stresses in the piping segments remained largely unchanged. A more flexible pressurizer could have induced larger motions and loads on the piping system. However, this did not occur because the pressurizer assembly is fixed at the base skirts and is horizontally attached near the top at each of the four shear lug locations.

A third analysis was performed to investigate modifications to the piping configuration. In this analysis, approximately $50 \%$ of piping supports were removed. This reduction applied to all spring supports, snubbers, and rigid pipe attachments. The purpose of the third analysis with a reduced number of piping supports was to investigate its effect on peak stresses to provide an insight in assessing the amount of design margin and whether a safe operation could be upheld.

Isometrics of each of the four primary piping lines are shown in Figures 4.4-29 - 4.4-32 along with the spring and snubber support locations. These isometrics did not label the rigid support locations.

The maximum principal stresses in each of the pressurizer lines are shown in Figures 4.4$33-4.4-36$. The peak stresses in each of the lines from the third analysis using fewer supports are listed as follows along with the peak stresses from the first analysis: 


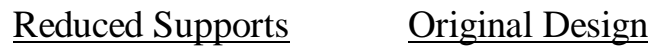

$\begin{array}{lll}\text { Surge Line: } & 16.9 \mathrm{ksi} & 12.3 \mathrm{ksi} \\ \text { Spray Line: } & 13.3 \mathrm{ksi} & 10.3 \mathrm{ksi} \\ \text { Depressurization Line \#1: } & 15.4 \mathrm{ksi} & 13.8 \mathrm{ksi} \\ \text { Depressurization Line \#2: } & 16.3 \mathrm{ksi} & 15.9 \mathrm{ksi}\end{array}$

While the peak stresses did increase with the reduced number of supports, these stresses are still well below the yield stresses for these piping materials. In some sections of piping system, the peak stresses actually went down.

The third analysis did not involve an organized method to optimize the effect on modifying the peak stresses in piping system due to a reduction of piping supports. Analysis results indicate that reducing piping supports might increase peak stresses by a small amount in some locations but it could also reduce them in other locations. With a maximum allowable principal stress as a guide, it would not be difficult to perform sensitivity analyses with different combinations of supports in order to determine the most optimum cost effective piping design with the fewest piping supports.

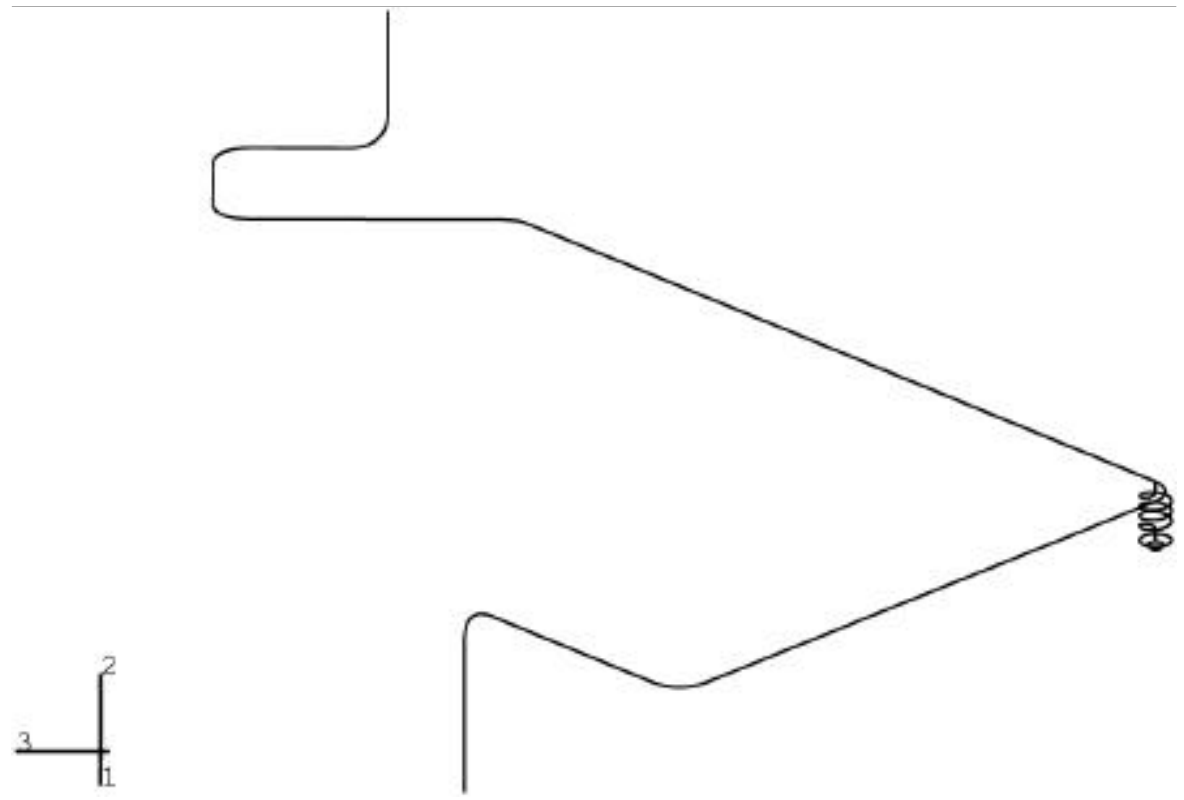

Figure 4.4-29. Surge Line Piping with Spring and Snubber Supports (reduced supports) 


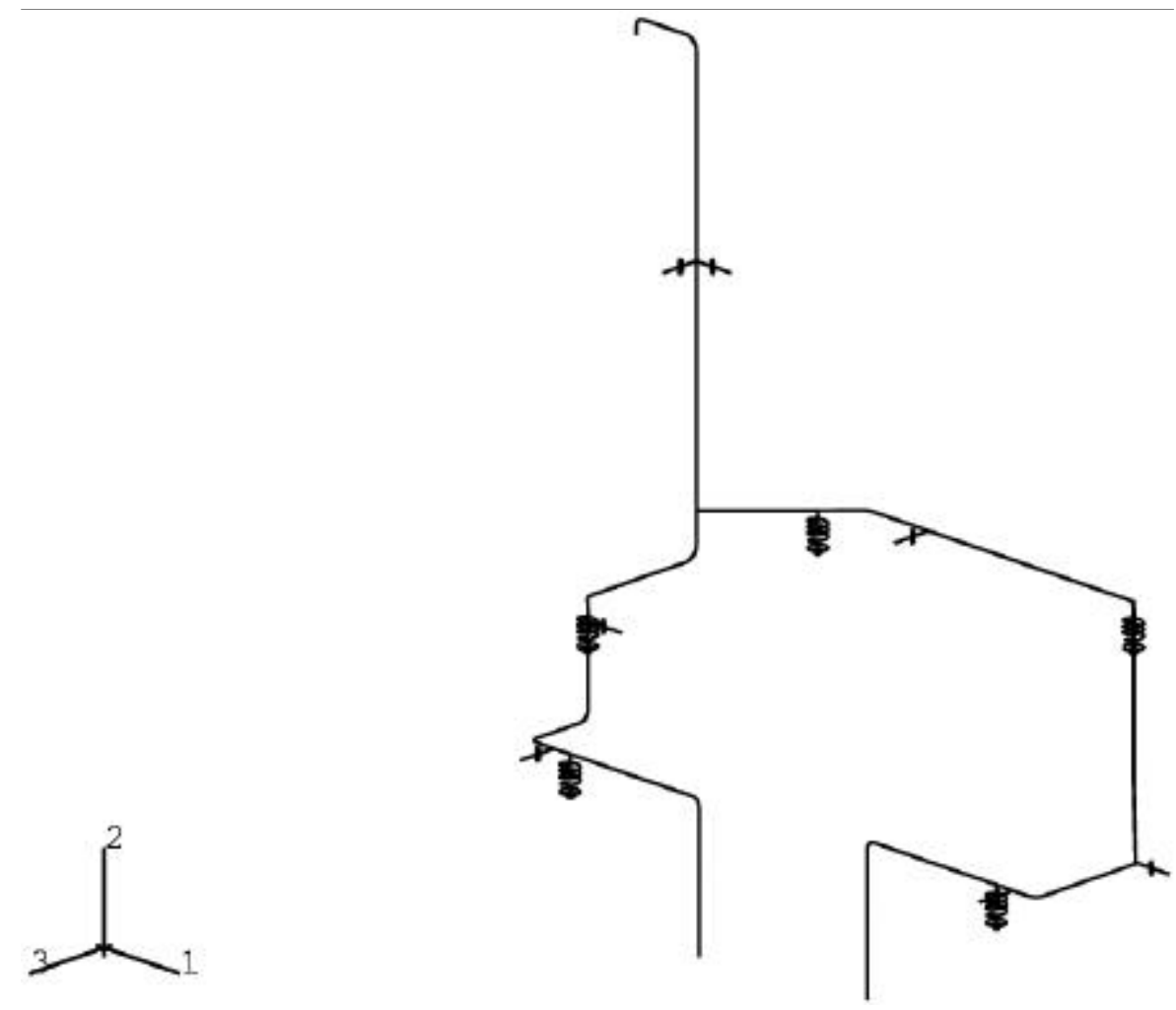

Figure 4.4-30. Spray Line Piping with Spring and Snubber Supports (reduced supports)
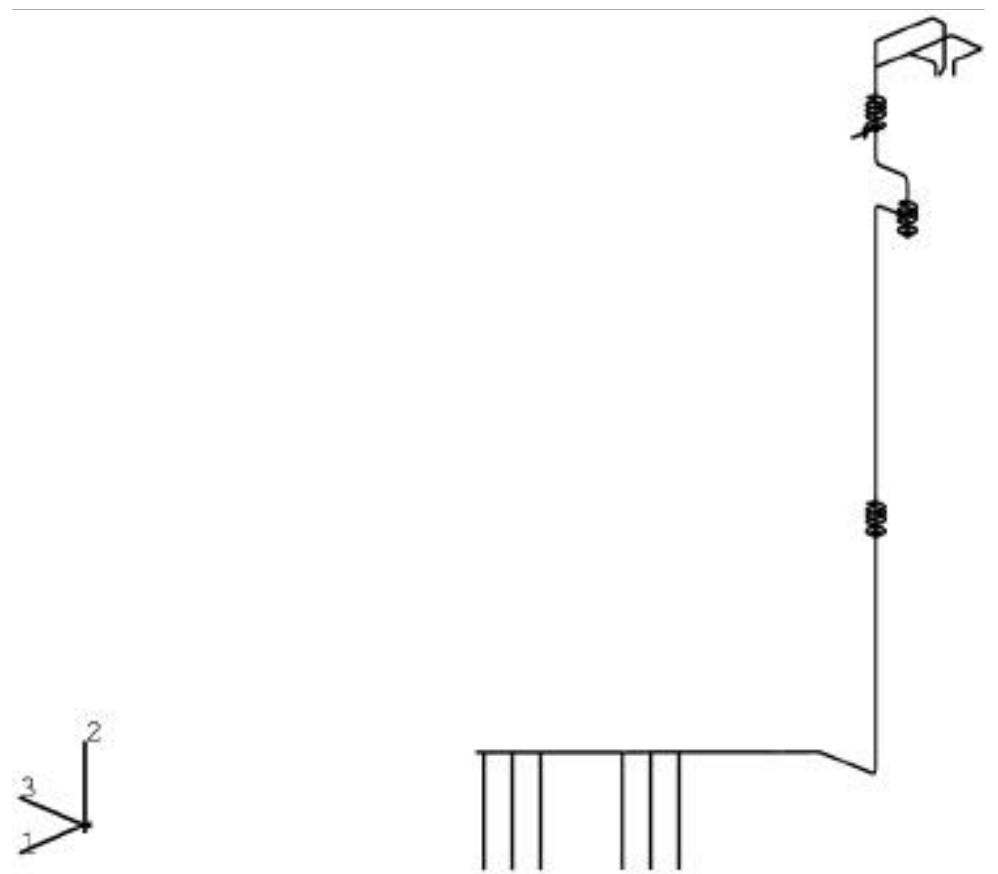

Figure 4.4-31. Depressurization Line \#1 Piping with Spring and Snubber Supports 
(reduced supports)

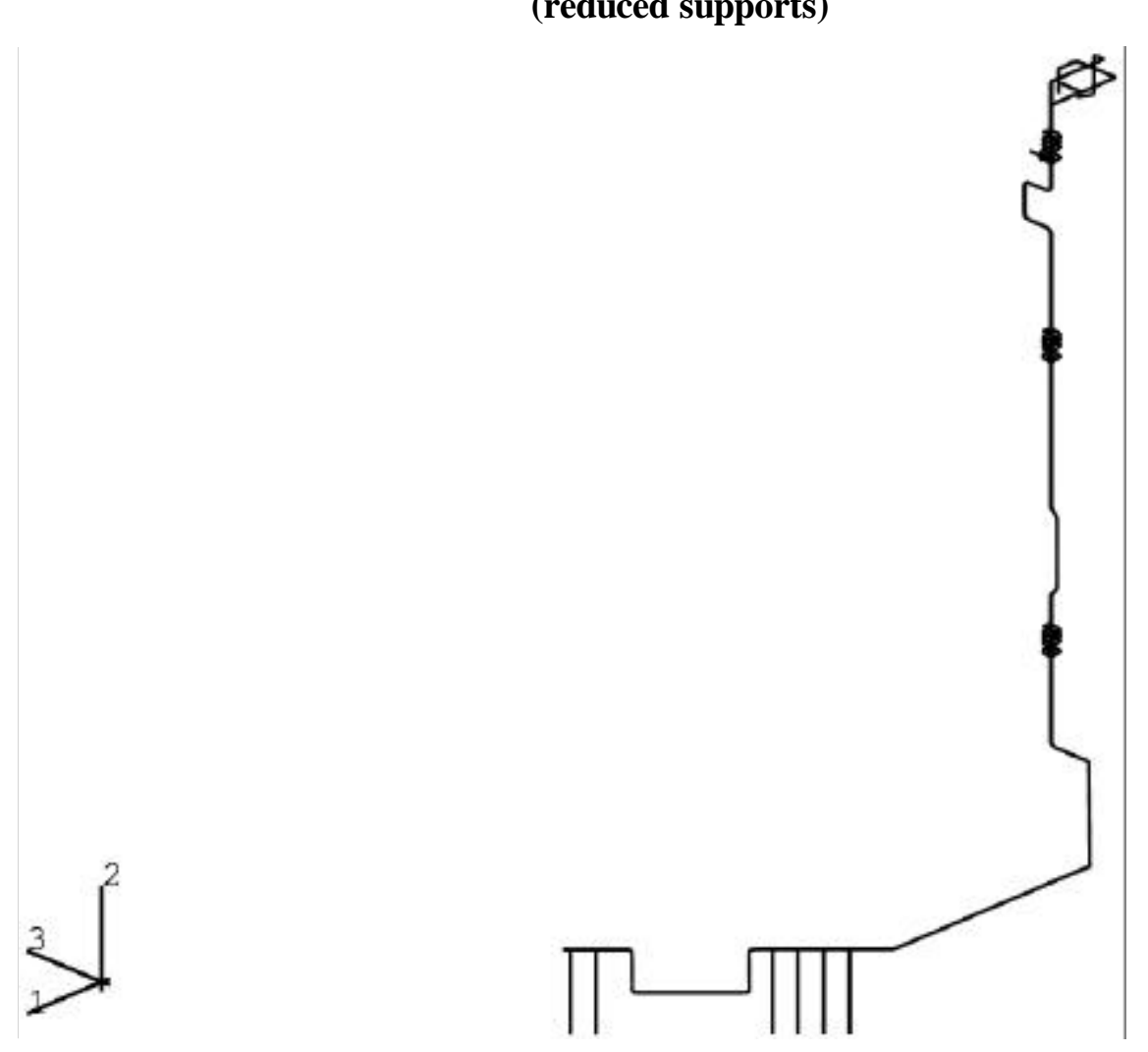

Figure 4.4-32. Depressurization Line \#2 Piping with Spring and Snubber Supports (reduced supports) 

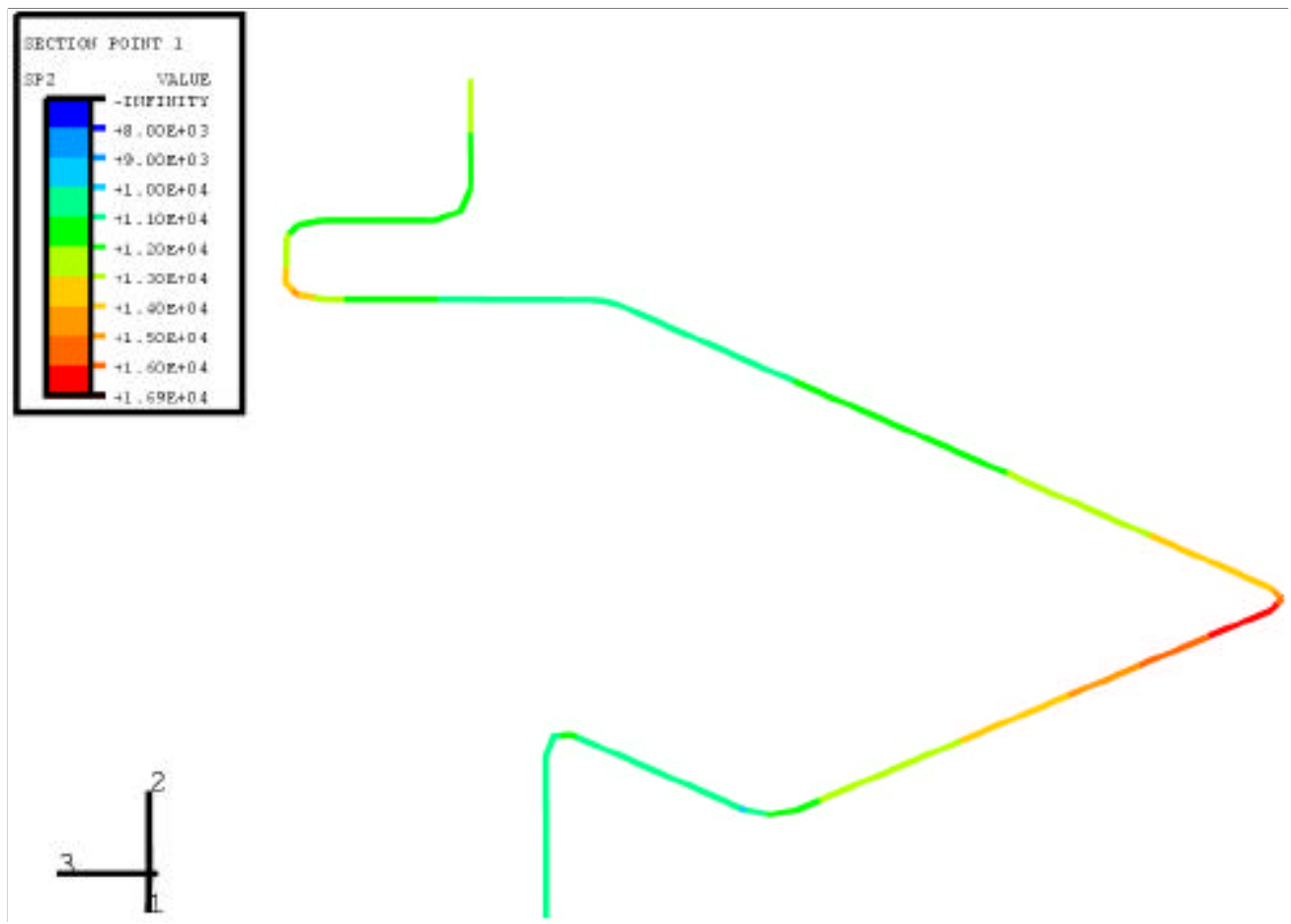

Figure 4.4-33. Maximum Principal Stresses in Surge Line (reduced supports)
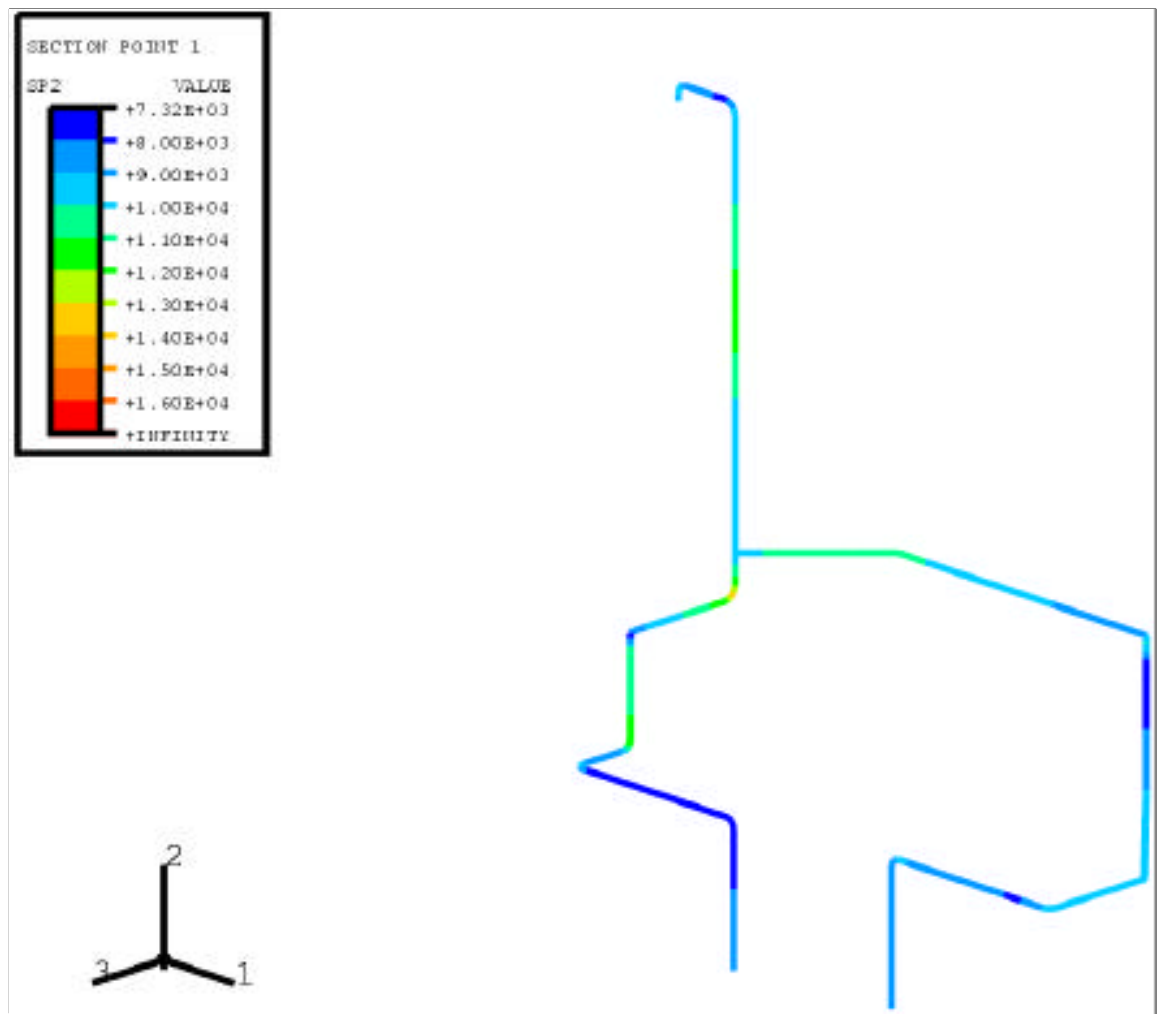

Figure 4.4-34. Maximum Principal Stresses in Spray Line (reduced supports) 

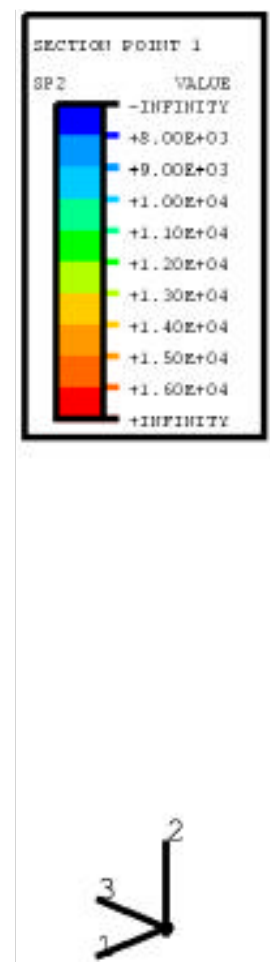

Figure 4.4-35. Maximum Principal Stresses in Depressurization Line \#1 (reduced supports)
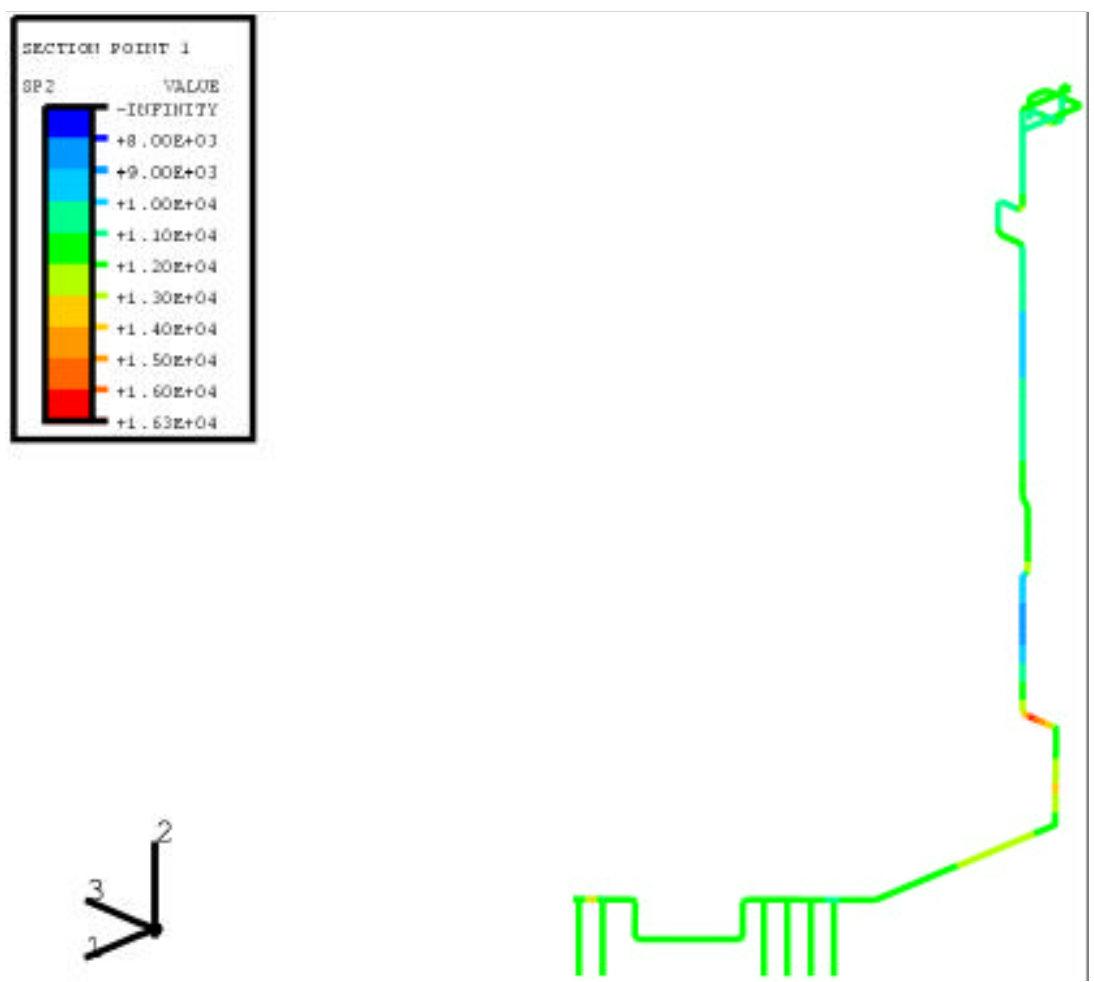

Figure 4.4-36. Maximum Principal Stresses in Depressurization Line \#2 (reduced supports) 


\subsubsection{Technical Issues}

\subsubsection{Hybrid elements connecting shell and beam elements}

In assembling the finite element model of the pressurizer assembly with its connecting piping system, it was found desirable and computationally efficient to simulate the pressurizer assembly with shell elements and the piping system with beam elements. However, the current capability of finite element codes does not produce a high level of accuracy in the analysis results at joints where these mixed elements are used. As a compromise, the transition locations of mixed elements were not selected at the nozzles of the pressurizer assembly where the piping is physically connected. Instead, the transition locations were selected at the straight portion of piping in order to avoid probable inaccurate analysis results at the pressurizer nozzles.

It would be beneficial to develop a hybrid element that consists of shell elements (for portions of pressurizer assembly) and beam elements (for portions of piping system) at the nozzle locations. In performing integrated analyses of a coupled model composed of components and piping system, this hybrid element with mixed elements will not only provide the necessary ingredient for resolving the connectivity issues at joints with different structural elements, but also enhances the computational efficiency. The current scope of work for this project does not include the development of this hybrid element, but there are plans to pursue this development effort as a part of a continuing $R \& D$ program to develop and benchmark the design-to-analysis tool in the near future.

\subsubsection{2 .Compatibility of computer software}

In the process of developing the design-to-analysis tool, it was discovered that most design and analysis computer software involved in this development process was not compatible with each other. This major technical hurdle led to the development of special algorithms to translate files to compatible formats in progressing through three principal steps of creating solid models, generating analysis meshes, and assembling analysis models. These undesirable processes are not only time consuming to develop and execute, but also reduce the efficiency and broad-based acceptance of the design-to-analysis tool.

\subsubsection{Robustness of design-to-analysis tool}

One of the major objectives of developing the design-to-analysis tool is to establish a single design database management system to facilitate communication between designers and analysts and to streamline design changes. It is therefore important to develop a very robust tool to maintain an updated and accurate database system that permits upstream design changes incorporated in solid models to be investigated and evaluated in the downstream analysis models. 


\subsubsection{User-friendliness of design-to-analysis tool}

This project fulfills the deliverable milestone by completing a development process of the design-to-analysis tool and demonstrating its feasibility of application. However, it was found that it is not a simple and easy task to exercise this tool. As a matter of fact, the users are required to have a specialized skill and knowledge base prior to exercising this tool. It is well understood that much research should be conducted to improve the userfriendliness of the tool before it can receive broad-based acceptance for its application. 


\subsubsection{Summary}

The design-to-analysis tool for future generation nuclear power plants attempts to take full advantages of advanced computer software and hardware in support of managing a huge design database system, assembling a very complicated plant design, and analyzing the design details. This tool serves to provide a single database management system to facilitate communication of accurate and updated information and to improve the efficiency of design/analysis cycle to optimize plant design and to streamline design changes. All of these features are necessary ingredients to help reduce the overall life cost of future generation nuclear power plants and improve their economical competitiveness in the power generation industry.

An added advantageous feature of the design-to-analysis tool is that it paves the way to performing integrated analyses of coupled models. The nuclear power plant designs consist of unique system configurations of mechanical components connected to piping systems and buildings of concrete structures. Current modeling practices involve analyzing isolated subsystems with added conservative assumptions to safeguard their safe operations. Performing integrated analyses of coupled models may help optimize the design of subsystems by eliminating or reduce uncertainty ranges and conservative assumptions. This desirable feature offered by the design-to-analysis tool will help reduce the design and construction costs of future generation nuclear power plants.

In this project, a pressurizer, which is a component in the reactor coolant system of the System $80+$ pressurized water reactor design, with its connecting piping system, was selected as an example for demonstrating the feasibility of developing the design-toanalysis tool. Sandia National Laboratories was primarily responsible for developing this tool, with support from the staff at Westinghouse Electric Company to provide the design database of pressurizer and Duke Engineering \& Services, which was later merged with Framatome, to supply the details of piping configurations and supports.

This project completed the development process of the design-to-analysis tool by assembling the solid models of the pressurizer assembly and the piping system, generating the finite element mesh of the coupled model, and performing integrated seismic analyses of the coupled model. The "original" design of the coupled model was modified by decreasing the wall thickness of pressurizer assembly and by reducing the number of piping supports by $50 \%$. Seismic analyses were also performed of the coupled models with modified configurations to evaluate their effects on stress response of the pressurizer assembly and the piping system. Analysis results indicate that these modifications did not cause significant increase in their stress response. It can therefore be concluded that the pressurizer assembly and the piping system are able to uphold their safe operation with these design modifications. 


\subsection{D VISUALIZATION MODELING}

\subsubsection{Introduction}

The purpose of this study is to develop new ideas to reduce the capital cost and construction duration for future nuclear power plant, through participating in US DOE's Nuclear Energy Research Initiative (NERI) program under the collaborative research and development agreement between KOPEC and Westinghouse Electric Company. This study covers 3D CAD model development, 4D-simulation development, construction process improvement, and NERI task study result summarization.

\subsubsection{3D Model and 4D Simulation Development}

The 3D Model was developed for 4D simulation by reforming the 3D CAD design model of unit 3 of the Ulchin nuclear power plant. 4D simulation was implemented and modified by integrating the 3D Model with selected construction activities.

\subsubsection{Construction Schedule Review}

The Critical path of nuclear plant construction was identified and several studies on construction schedule reduction and new construction methods were thoroughly compared. This review is applicable to the Korean Standard Nuclear Plant (KSNP) currently operating at the Ulchin and Yangwang sites, the improved Korean Standard Nuclear Plant (KSNP+) and the APR1400 next generation plant that is currently completing detailed design.

\subsubsection{Construction Process Improvement}

A strategy and a technical road map for nuclear power plant productivity improvement were identified and various ideas for construction process improvement were identified.

\subsubsection{Tracking DPCIT Task Study Result}

KOPEC has kept pace with US DPCIT team and has participated in annual team meeting since 1999. DPCIT task study status and results were tracked and summarized.

\subsubsection{Overview of KSNP (Korean Standard Nuclear Plant)}

When the KSNP was initially designed, the basic design concepts were the following:

?? Utilize the most up-to-date proven technologies

?? Incorporate design improvements reflecting operating experience

?? Meet enhanced regulatory requirements

?? Standardize the design for future plants 
?? Gradually improve safety and performance

\subsubsection{Design Characteristics}

Following are the general design characteristics:
?? Reactor type
PWR
?? Power Rating
1,000 MWe (1,050 MWe, 2,825 MWt) class
?? Plant lifetime
40 years
?? Seismic design basis
$0.2 \mathrm{~g}(\mathrm{SSE}), 0.1 \mathrm{~g}(\mathrm{OBE})$
?? Refueling cycle $2 \sim 18$ months
?? Load follow capability : daily load follow operation

\subsubsection{Safety Concept}

The safety concept of the KSNP is based on the multiple level defense-in-depth approach; prevention of accidents or deviations from normal operation, detection of accidents through monitoring, control of accidents to prevent propagation into severe accidents, and mitigation of severe accidents.

This safety concept is maintained through compliance with design, manufacturing and operating requirements devised to maintain safety. The safety objective is pursued in compliance with deterministic requirements and supplemented by probabilistic methods. The use of improved passive and active engineered safety features further reduces the possibility of occurrence of the severe accidents.

\subsubsection{Highlights of the KSNP Design and Development Approach}

?? Adoption of safety depressurization system

?? Enhancement of plant safety during mid-loop operation

?? Decrease in the possibility of the interfacing system loss of coolant accident

?? Application of the leak-before-break concept in reactor coolant system piping, pressurizer surge-line piping, shutdown cooling system piping and safety injection system piping

?? Application of human factors engineering in the main control board design

?? Adoption of Inconel-690 steam generator tube material for later units

?? Improvement of chemical and volume control system

?? Adoption of digital balance-of-plant control system

?? Incorporation of digital plant protection system for later units

?? Installation of generator circuit breaker 
?? Installation of Alternate Alternating Current(AAC) diesel generator

?? Provision for control of hydrogen generation

?? Increase in the floor areas of the reactor cavity

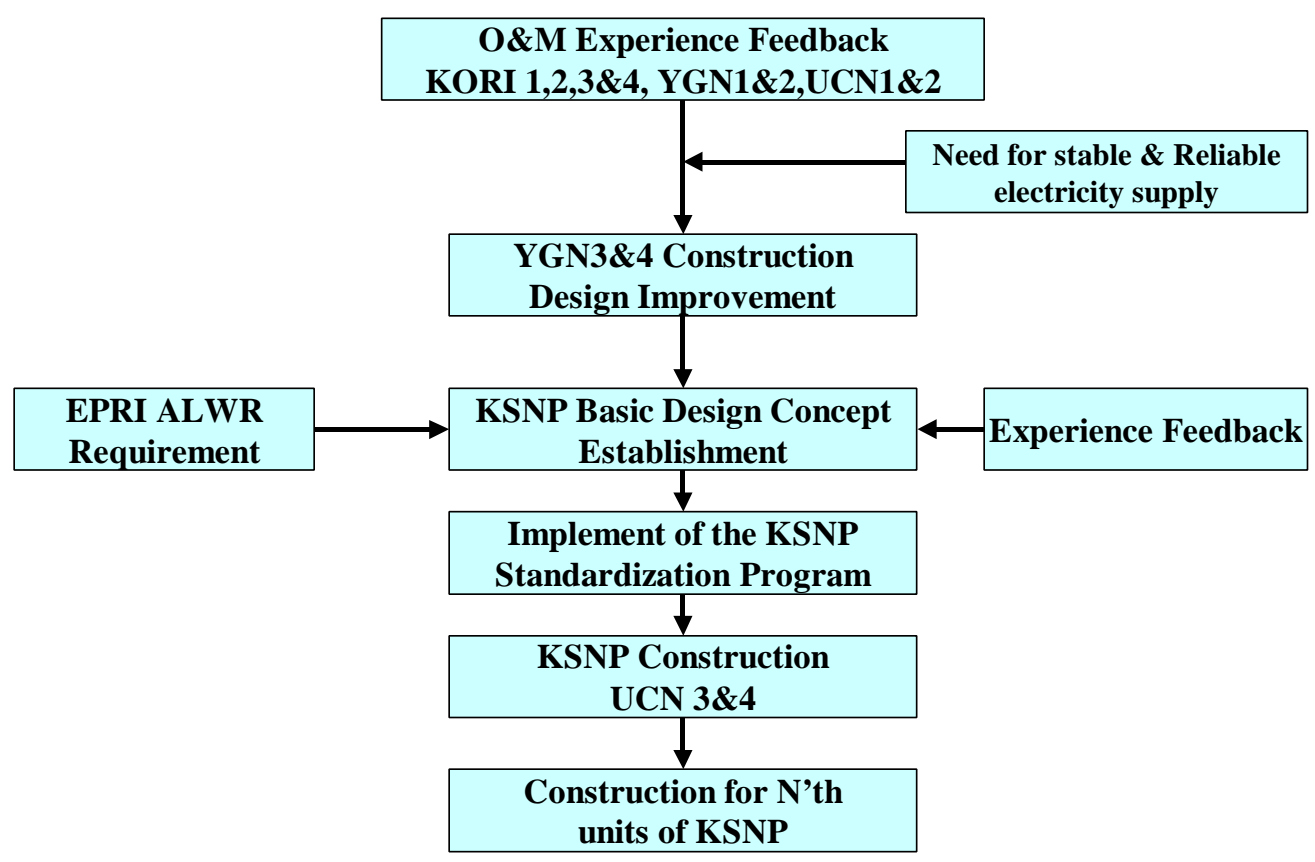

Figure 3..2-1 KSNP Development Approach

\subsubsection{Development 3D CAD Model}

The major tasks for the development of the 3D CAD Model and 4D Visualization are comprised of following work activities:

estevelopment of a 3D CAD Model for KSNP

Review of the construction schedule and related work activity

esedentification and extraction of construction sub models with construction area

Establishing links between the 3D CAD component object and the construction activity eompile 4D visualization simulation from activity sequence and generate a movie clip 


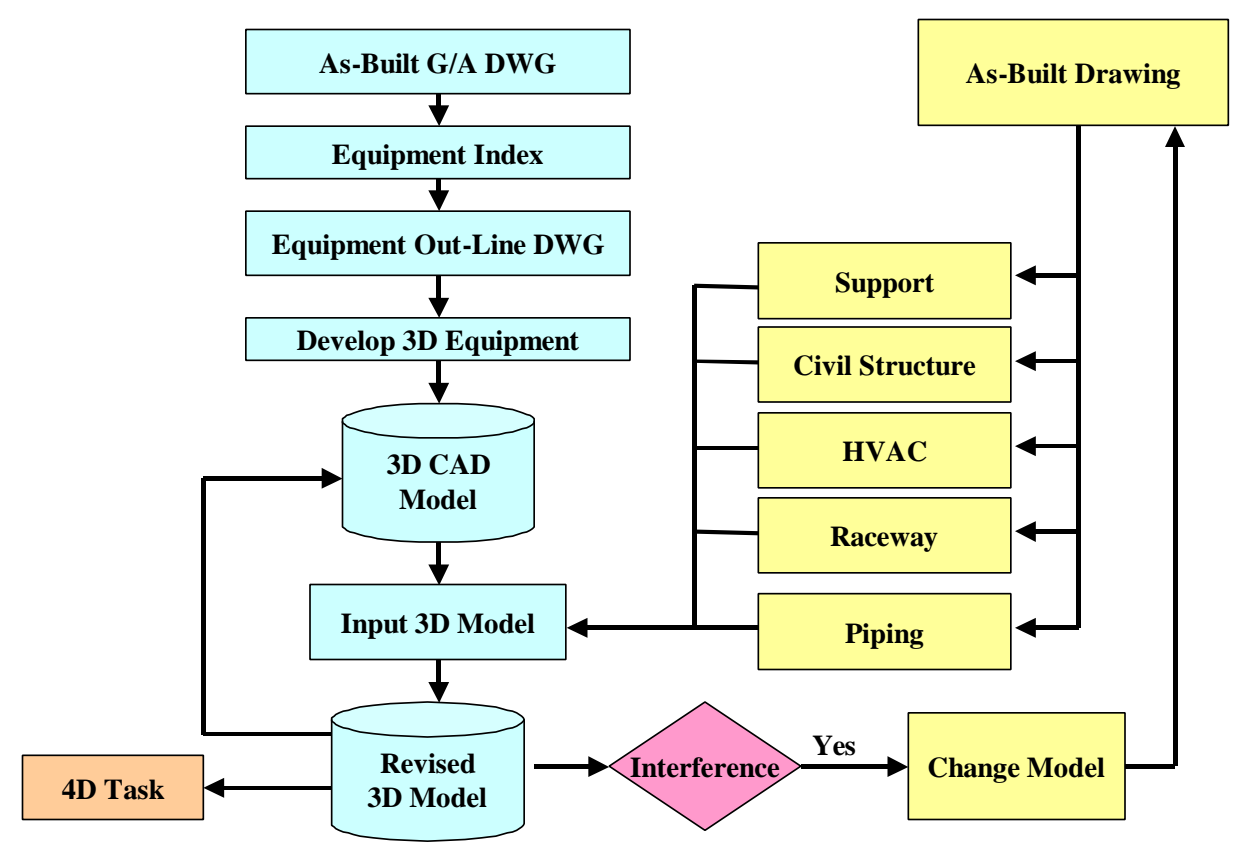

Figure 4.5.3-1 Work Process for KSNP 3D CAD Model Development

\subsubsection{Development for KSNP 3D CAD Model}

There several purpose for development of 3D CAD Model during design phase, the major reason for developing 3D CAD Model are space control between different design component, review of plant accessibility in O\&M Phase, and etc. Followings are the purpose for 3D CAD Model development.

??Optimization plant space arrangement

??By designing real plant component configuration, enhance design quality from plane base design to spatial base

??Eliminate drafting error between drawing by generating construction drawing not only plan but also section from one single database

??By eliminating the design crash, reduce the construction and design rework

??Extract quantity for construction material and commodity

??Interface 3D design configuration to analysis program

??Review and enhance construction schedule by developing 4D visualization model 


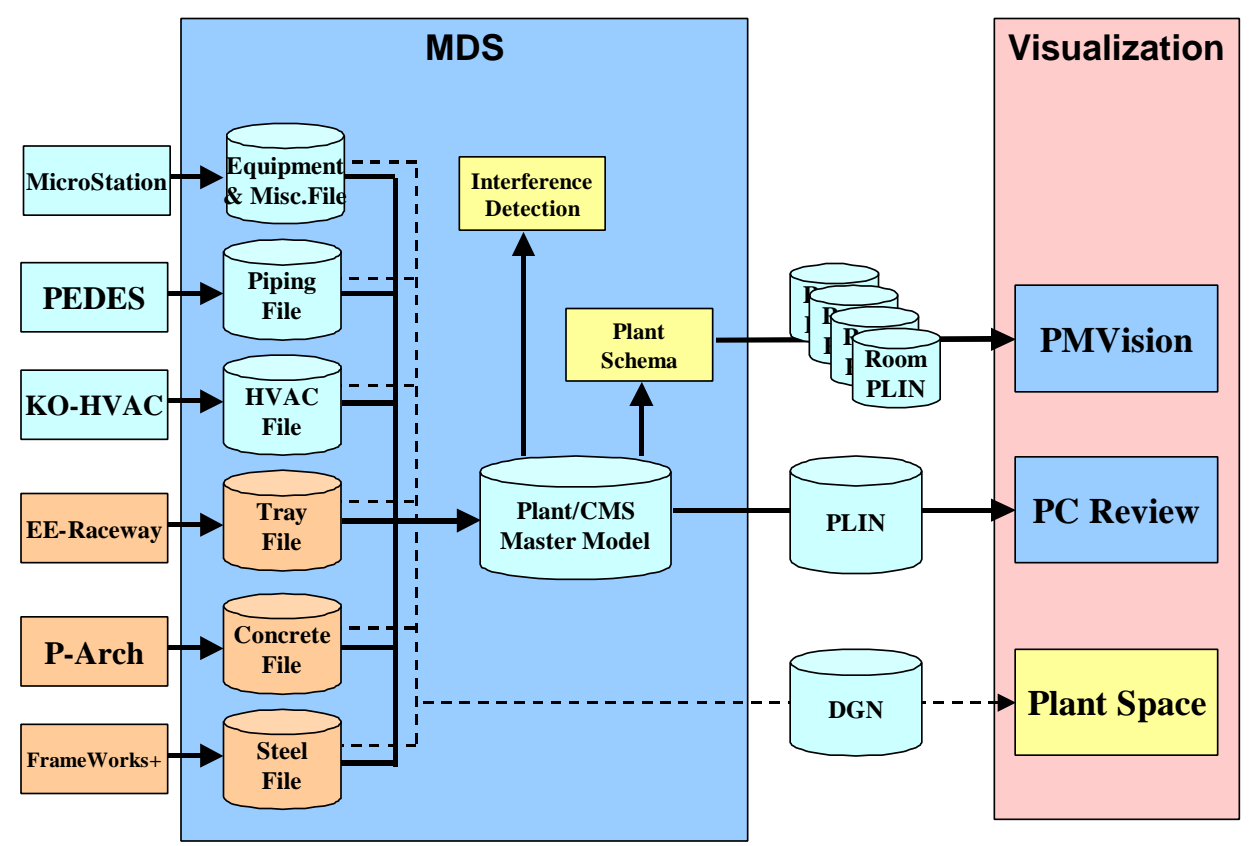

Figure 4.5.3-2 3D CAD Software Structure of IPIMS

\subsubsection{Structure for CAD Software}

The IPIMS (Integrated Plant Information Management System), which was developed by KOPEC for engineering information technology, was used. IPIMS is a comprehensive system that is composed of four functional application tools:

? Intelligent 2D CAD System

? 3-D CAD System

? Engineering Data Base (EDB) System

? Drawing \& Document Management (DDMS) System

The IPIMS is the primary software used by engineers and project managers for accessing the plant information database and utilized in the design of KSNP, KSNP+ and APR1400 project. IPIMS can be used to supplement not only physical plant information database but also Engineering Data Base (EDB) System to transfer commodity data to cost estimating software.

This IPIMS was developed for design engineering and to support construction management requirements. Nuclear power plants are very complex structures with large concrete structures. The construction sequence is very important for the pouring of concrete, the sequence of equipment installation and concrete slab, piping installation and penetration, various support and embedded plate, etc. 
The capability of construction simulation can review this kind of construction sequences and support to develop optimized construction schedule. Figure 4.5.3-2 is the 3D CAD system structure of IPIMS for your reference.

Left sides of above system structure are modeler software to develop 3D design component configuration. The design configuration data are transferred to Plant/CMS software that combines all design data, and the 3D spatial information are controlled and managed in MDS (Model Database System) environment. From integrated 3D CAD spatial database Plant/CMS Database, the construction sub-model can be extracted and link to construction activity. The design crash checks are processed in Plant/CMS environment

\subsubsection{Development for KSNP 4D CAD Concept Model}

Originally, the development of 3D CAD model should be executed in design phase with coordinate different engineering discipline. We should develop model develop engineering procedure and organization. But, the KSNP construction projects had already been completed and in O\&M phase now. So we developed KSNP 3D CAD model by using UCN $3 \& 4$ as-built drawing and design document.

Figure 4.5.3-1 is the work procedure for KSNP 3D CAD model development task. Skilled 3D CAD input engineers were taking part in 3D CAD model development work for better work process and quality. After completion of thee 3D CAD model development, we converted 4D visualization model for construction simulation work. The detail developed 3D CAD component are as following

??Concrete and Steel Structure

?? Base Mat

?? Containment Dome

?? Containment Outside Wall

?? Liner Plate

?? Concrete Beam

?? Concrete Column

?? Concrete Slab

?? Concrete Wall

?? Inside Wall

?? Steel Structural Beam

?? Steel Structural Column

?? Steel Brace

?? Steel Grating

?? Stair

?? Equipment Foundation

??Architecture Component

?? Architecture Partition Wall

?? Door 
?? Window

??Miscellaneous Concrete and Steel Structure

?? Embedded Plate

?? Misc. Beam

?? Misc. Monorail

?? Misc. Plate

?? Penetration Opening

?? Sleeve

?? Steel Hand Rail

?? Steel Ladder

?? Steel Plate

?? Structure Wall

?? Equipment

?? Equipment (HVAC)

?? Equipment (Electrical)

?? Equipment (Mechanical)

?? Instrument Panel

?? Local Panel

?? System Component

? HVAC Duct

?? Cable Tray

?? Piping (Large Bore)

?? Piping (Small Bore)

?? Instrument \& Tubing

??Support

?? Support for I\&C

?? Support for Instrument

?? Support for Pipe

?? Support for HVAC DUCT

?? Support for RCP lateral

?? Support for Tray 


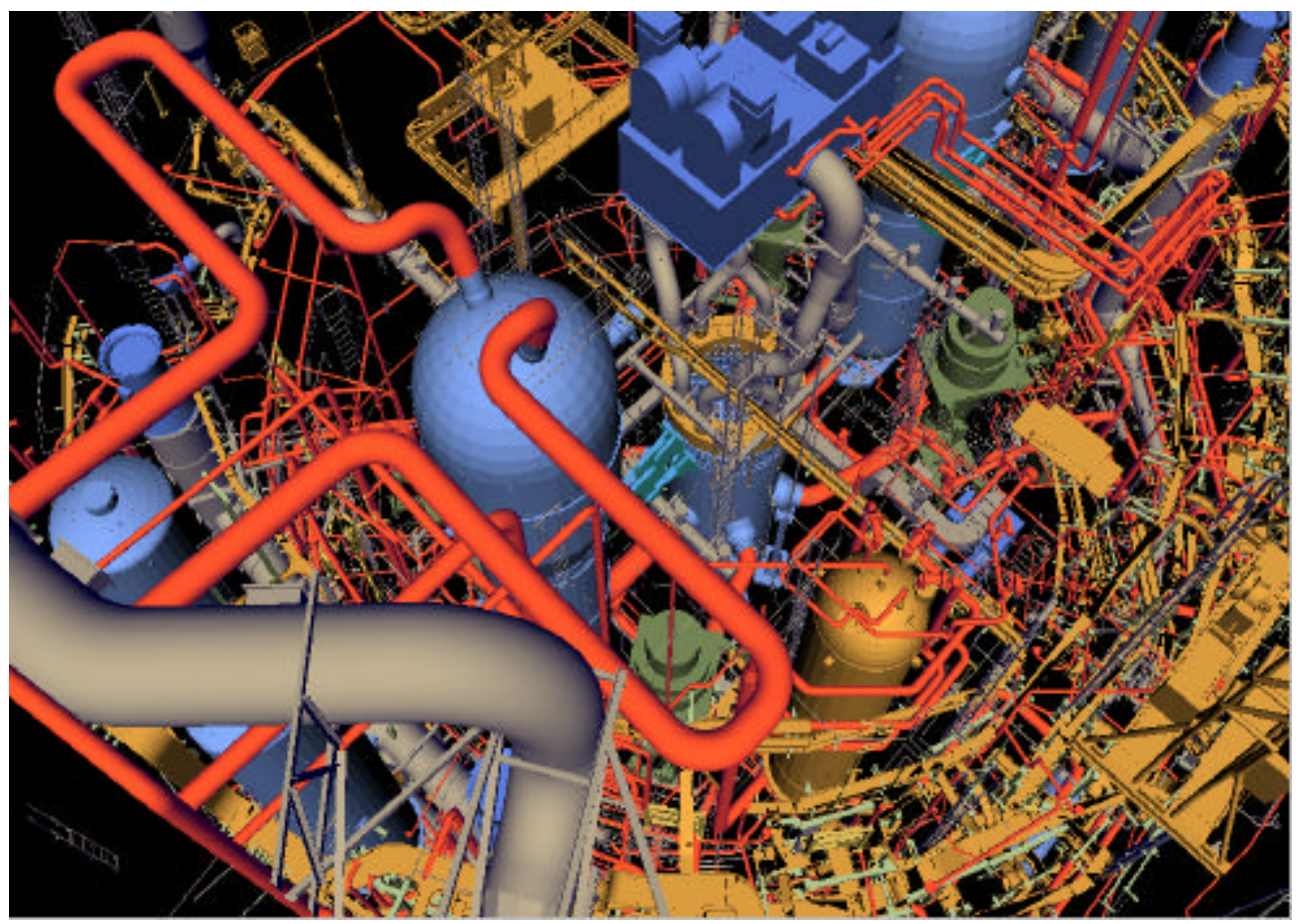

Figure 4.5.3-3 Inside of KSNP Containment Building

\subsubsection{Development 4D Construction Model with Construction Activity}

KOPEC has already completed to develop 3D CAD Model and to transform it from UNIX Version to the PC-based. The level III Construction schedule has been revised based on Ulchin $3 \& 4$ actual datum, being completed in a way that minimize the error by way of establishing Database and its related works down to the detail level under the situation not having PMVision yet. Followings are covering the synopsis of the study results on this review.

\subsubsection{Improvement of Construction Plan/Schedule}

When it comes to the construction schedule, its status of the Critical Path Activities for the Containment Building was identified and several alternatives for Construction Reduction Studies in-and-out of KOPEC were fully reviewed and compared. The state-of-the-art technologies and items to reduce construction duration of Nuclear Plants were finally analyzed on the bases of those study results.

\subsubsection{3D Model and 4D Visualization}

The 4D Visualization has been developed as a model by adding 3D model of KSNP (Korea Standard Nuclear Plants) Ulchin $3 \& 4$ up to the construction schedule reviewed. Since the models for design and construction are having inherently inherent figures, they shall be modified to comply with. For instances the design model refers to the design point of view, whereas the Construction model defines the physical constructability in-situ. With this 
study, 4D Visualization was developed for Standard Nuclear Plants.

The 4D Visualization were linked with the level III construction schedule into databases, which builds up the information by classifying the construction activities necessary for the 4D visualization, based upon the actual construction schedule analyses. The construction plan, which has no affect on the 4D, such as architectural interior design works, has not been included.

The established 3D Model graphic objects and construction activities were logically linked to be suitable for 4D visualization. Being linked the 3D model and 4D with the construction activities, KOPEC has kept on studying 4D operation by continuously improving 3D model and its functional studies.

\subsubsection{NERI DPCIT Collaborative Studies}

The Korean Team's study was mainly concerned in 4D visualization reflecting KOPEC's know-why and experiences feedback processes in the area of Nuclear plants construction such as its status, strategies, and evaluation which are cutting-edge technologies.

\subsection{Status}

The 4D visualization is aimed to perform the design tasks by 3D design configuration model, taking the place of existing 2D drawings in a way that 3D model is linked with construction activities and realizing simulation techniques for Virtual Construction.

The 4D is not become a real technology in the field yet for its functional requirements and application needs have not been clearly defined. Through the study herein, KOPEC team has approached having 3D model construction-simulated in utilization of the Ulchin $3 \& 4$ design information and applied 62 months plan form the First concrete to Commercial operation of the plant.

\subsection{Completed Work}

Eonstruction schedule review and activities come-out

The schedules for the plants are generally composed of four level of hierarchy from level I to level IV. The level I schedule, Top Level Schedule (Project Milestone Schedule) provides the overall plan of key milestones of the project such as construction completion date, major equipment installation dates, etc. The schedules and activities for each level of hierarchy are as follows.

Level 1 ; Provides the overall project plan for each construction phase : Design, Procurement, Construction/Installation, and Test (Start-up)

\&s Level 2 ; Provides more developed tasks and their relations for each discipline (Summary Schedule)

Level 3; Provides breakdown activities from Level 2 schedule, their duration and relation in a format of CPM (Critical Path Method) Logic Net. It will be played a role as a schedule analyses standard.(Integrated Project Schedule)

\& Level IV: Provides a detailed date and plan based on Level III IPS, directly being applied in-situ. 
KOPEC's study for 3D model and 4D visualization are basically based on Level III schedule of which line activities are generally 1,500 to 2,000 for construction phase. The activities that are not directly related with the purpose of the study were excluded. Finally among the Level III construction activities of 1,437, the activities of which numbered 390 for Containment building, Turbine hall, and Auxiliary buildings were selected to be used in simulation.

estice and Extract of The 3D model

Construction simulation was mainly concerned on reviewing the activities in Critical Path. Therefore the following lists were picked up between the Civil/Structural and Equipment installation tasks.

\begin{tabular}{ll} 
Object Name & \multicolumn{1}{c}{ Description } \\
CA & Architecture Partition Wall \\
CB & Beam \\
CC & Containment Outside Wall \& Column \\
CD & Containment Dome \\
CE & Equipment Foundation \\
CM & Base Mat \\
CO & Penetration Opening \\
CS & Slab \\
CT & Gallery Access Shaft \\
CV & RCP lateral Support \\
CW & Inside Wall \\
E & Electrical Equipment \\
F & Mechanical Equipment \\
J & Instrument Tubing \& Panel \\
SA & Equipment Anchor Bolt \\
SB & Steel Beam \\
SE & Embedded Plate \\
SG & Plate \\
SI & Liner Plate \\
SM & Misc. Plate \\
SP & Steel Platforms \\
SR & Misc. Monorail \\
ST & Stair \\
SX & Brace \\
SY & Bolt \\
V & HVAC Equipment \\
&
\end{tabular}

\subsection{Linking Design and Construction activity}

As the above-mentioned, the design and construction objects for model were linked to each other. The ways to link them are:

Civil/Structural works including general equipment: to be linked by building/level-wise Major equipment (Reactor Vessel, Steam Generator, etc.) : to be linked by component base, which directly link component to component 


\subsection{Operating The Construction Sequences And Generating Animation Files}

Using a PMVsion of Plant/CMS software. the 3D model had been constituted and their configuration files were generated necessary for the simulations. These 3D configuration files are -called the "PLIN", with attached deliverables (refer to 4.5.5).

\section{E.PLINNERIS.DAT}

The construction plan prepared with the Microsoft Access of Micro Soft Co., were established and named in compliance with the agreement under the Plant/CMS software articles for file name.

QSMVISIONNERIS.MDB

In this work the following two tasks were performed for the Power Block of Ulchin Nuclear Power Plant Unit 3 \& 4 in which the Containment building, Turbine hall, and Auxiliary buildings were located.

Concrete and Steel Structural construction simulation \&quipment installation simulation

Though the relevant software and adequate hardware are required in order to confirm the simulation results, the results were generated as a animation file to get a easy access as a most popular format of MPEG, which could be operated, in Windows Media Player of the Micro Soft Windows.

\subsubsection{Deliverables}

Following are the deliverables for 4D visualization modeling task

\subsubsection{Construction Schedule Database file}

?? PMVisionNeris.MDB

\subsubsection{Construction Simulation Movie Clip}

?? CONT_TOTAL.MPG : $\quad$ Containment Building Movie Clip

?? AUXB_TOTAL.MPG : $\quad$ Auxiliary Building Movie Clip

?? TURB_TOTAL.MPG : $\quad$ Turbine Building Movie Clip

?? TOTAL.MPG : $\quad$ Whole Power Block Movie Clip 


\subsection{Product Development}




\subsection{Product Model}

The vision of the product model evolved considerably the three year course of the project. The fundamental concept of a single composite model that would provide an easily viewable and queriable representation of the design, as it passes through all phases of DPCIT, endured. However, the perception of the make up of this model and how it would transition from phase to phase progressed with development experience and advances made in Information Technology. In particular, both FRAMATOME ANP DE\&S and Westinghouse have been engaged in an EPRI funded project to develop a template for future information and configuration management systems for advanced nuclear plants, based on the application of commercial software. The EPRI project concluded its initial work in November 2000 with a proof of principle demonstration of the concepts. At that time the Product (or Plant) Model was viewed as an object representation of the physical design. Vital elements of the product model for advanced nuclear plants were to be managed in Product Data Management (PDM) system as described in the EPRI report ${ }^{1}$. The primary objects in the model are the plant "parts" and "assemblies" of parts. This is essentially the same model used in most commercial Product Data Management (PDM) Systems. Each part or assembly has a set of associated attributes that describe the part. One of the key attributes is cost of the part. A roll up through the Model allows determination of equipment costs. The Product model was visualized with a 3D CAD application that is linked to the model.

Subsequently, Westinghouse elected to proceed with the implementation of the ideas and concepts for their AP1000 reactor design. This follow on effort is partially funded by EPRI as well. As this work has taken place the information technology applied has progressed from the original PDM application to what is now refered to as Product Lifecycle Management and Collaborative Product Commerce. Ongoing work by EPRI is examining the implementation of this technology to contain the product model for the AP1000 reactor design. The project developed requirements and interfaces for a broad set of technical, licensing and business needs as well as the existing AP600 information data management systems. The backbone of the resulting top-level architecture is a commercially available Collaborative Product Commerce (CPC) system. CPC solutions are built on a Web architecture where all project personnel at various locations as well as customers and suppliers can work together and exchange information. CPC solutions are typically based on relational database managers and provide data management and retrieval, workflow and other capabilities to support the entire product development cycle as well as follow-on service during product operations. A key element of this solution will be to ensure integrated logical access and retrieval all the data to support lifecycle activities.

Thus, the product model as it has evolved represents much more than simply the collection of $2 \mathrm{D}$ and $3 \mathrm{D}$ representations of plant and system layout. In the aggregate, the product model is the complete data warehouse of plant information whether it is used for procurement, design or licensing. Multiple views of the data will be supported in the plant model to accommodate the various user needs and differing perspectives. Figure 5-1 below illustrates the concept and links to specific software packages for the AP1000 plant. $^{2}$

\footnotetext{
${ }^{11}$ EPRI Report 1000056, Development of Advanced Plant Information System (AIMS) for Future Nuclear Plants, EPRI, Palo Alto, CA, September 2000

2 AIMS Architectural Description and Implementation Plan, EPRI, Palo Alto, CA: 2001. 1003176
} 


\section{Figure 5-1 - Concepts and Links to Specific Software Packages for the AP1000 Plant}

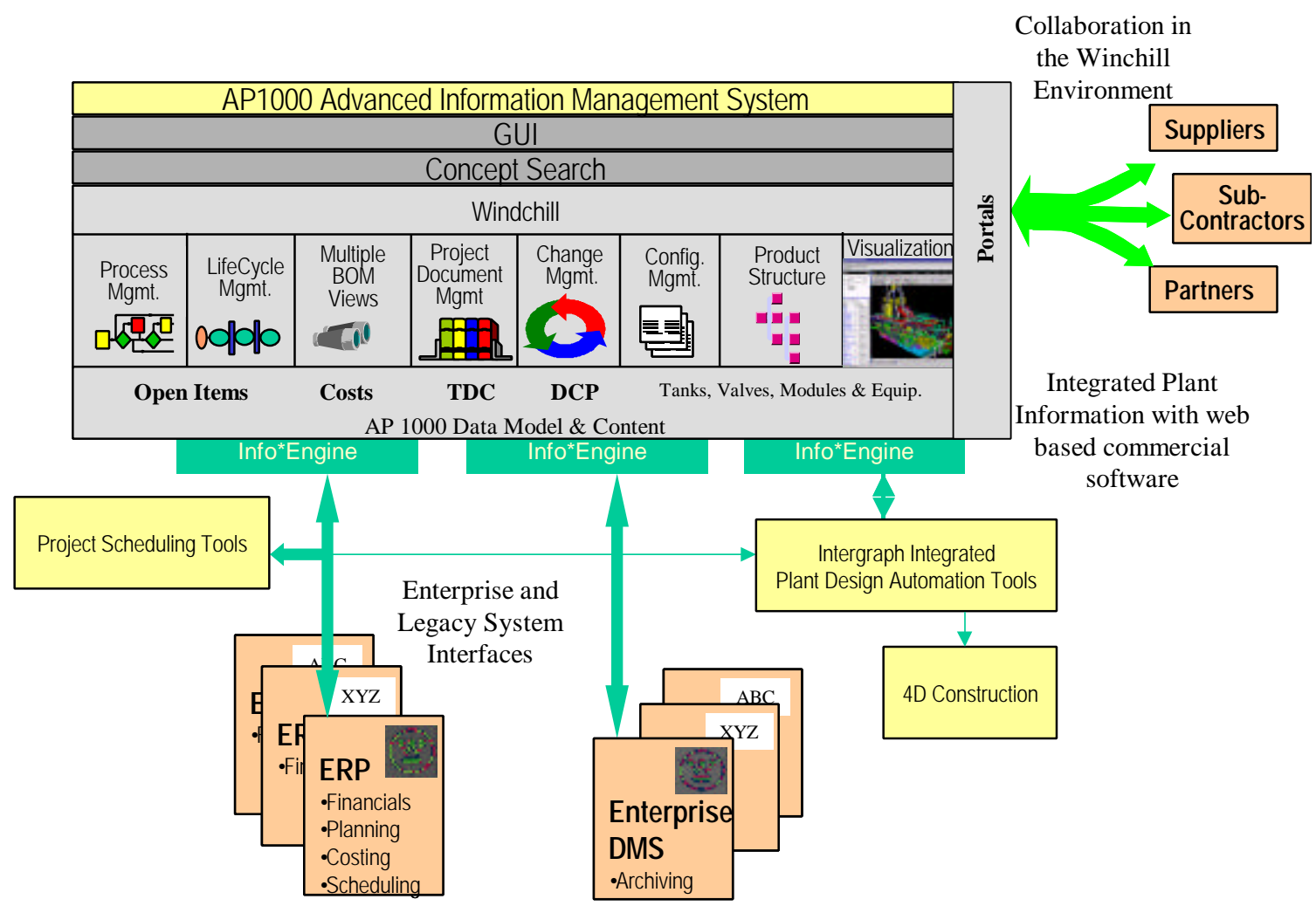

The document database associated with the plant model can serve as the directory for and link to the design and construction documentation. The database also could serve as the means for sharing other engineering models, such as, structural models of component, equipment and structures among the project participants. Using the document database in the plant model, links can be developed to project management, supply management and construction management data that is required for the execution of the project. By associating inspection and installation data recorded during construction with the product model database, the data will be stored in a manner that it is readily available during plant operation and maintenance.

With all of the plant information readily available through the Product Model, a construction supervisor can assemble all the information (drawings, procedures), verify material availability, plan and schedule material movement, identify and location special equipment required, and record the completion status (including quality assurance) of each work package that is assigned to him.

\subsection{Productivity Model}

The Productivity Model is represented by the 4D model described in the Year One report. Thus, the Productivity Model incorporates both the 3D-design information and the sequence of the construction schedule. The construction schedule typically considered as 
the plan for conducting the plant construction and startup. In many cases for previous plants, the plant design is well underway before detailed construction scheduling occurs. It is generally agreed that there are benefits to be gained from early development of the construction schedule during the preliminary design of the plant. The design can be checked for constructability considerations. Changes that could simplify the construction activities to decrease both the cost and duration of the construction period can be factored into the design if they are discovered early in the process.

Based on this year's work by Westinghouse in the domain of construction productivity, we have added the construction productivity measures described in Section 4.2 to further augment our productivity model. Since design and construction are intimately linked, this represents a logical concentration of potential improvements to a power plant design.

Many of the delays and cause of rework for both design and construction are the result of the interfaces between the structures and equipment. Electronic aided design is becoming common place for component and equipment design, as well as plant layout and structure design. The ability to integrate equipment and component models into the plant model is an area that requires improvement to achieve the maximum benefit of electronic aided design.

We expect that visualization technologies will become embedded components of the product model in the future rather than after the fact software add ons. In particular the visualization technology will be a vital tool in reducing plant construction time by allowing new work sequences to be examined in detail and to anticipate and thereby schedule around congested areas and interferences.

This year's work on the productivity model includes the insights for information technology application into the fabrication, construction and installation processes as detailed in Section 4.0.

\subsection{Process Model-Management Tools for Complex Projects}

The process model is intended to be a method of capturing and implementing best practices found in other industries as well as adapting new tools and practices to meet the needs for an improved schedule at a reduced cost and with a modest risk. The following sections cover the current thinking on what ought to be contained in the process model. The model will evolve over time as new practices are identified and validated.

The risk of undertaking large capital projects is another barrier to new advanced nuclear plant construction. While companies like Intel are undertaking one to two billion dollar semi-conductor wafer plants on a fast track schedule with expected completion times within two years, the nuclear industry's ability to meet a much faster schedule remains to be proven. For companies like Intel, speed to market is a key factor in their success, thus the rework and field changes that are characteristic of fast track projects are tolerated within the overall cost of the project since time is the key variable. Our confidence in building new nuclear units will need to be improved to support new plant orders in the United States. Thus, the philosophy of the new nuclear power construction will be one of a fairly high assurance that cost and schedule can be met. To this end, and because of the licensing process needs, a complete plant design down to very fine levels of detail for safety systems will be required. These prerequisites will help ensure that the project plan can be undertaken. However no plan is immune from change and experience has shown 
that change will occur anyway. Therefore our confidence in building from a complete set of plans and a predicted schedule will need to be reinforced with new project management methods. In the sections that follow, a few applications of new technology to the project management domain are described.

\subsubsection{Process Model Element-Bayesian Belief Networks}

Bayesian Belief Networks (BBNs) will be incorporated into the Process Model as the work progresses in Year Two. The value of BBNs is in their representation of conditional probabilities, that is if event $\mathrm{A}$ occurs and there is a relationship to event $\mathrm{B}$, then we can know what the probability of B is given that we already know that A has occurred. Our application for this type of technology will be to develop project management models capable of updating themselves once the project commences and we have captured the productivity data and can make comparisons to the project's assumptions. In this way, we would expect to update our project schedule and cost estimates and thereby have a better understanding of the outcomes being achieved. More importantly, this type of model updating gives us a chance to intervene earlier and more effectively than waiting for a large set of cost of schedule data to accumulate. It can be said that no project plan survives first contact with the real world situation once the project starts. Therefore, we wish to have a plan that can be updated and modified using our performance information with the goal of being better able to meet the cost and schedule requirements.

\subsubsection{Process Model Element—Systems Dynamics}

The method of systems thinking, or system dynamics, provides us with a tool to better understand difficult management problems. The system dynamics approach was introduced in the post World War II era by Jay Forrester, a former electrical engineer at MIT, and has been used for over forty years. This approach requires a shift in the way we think about things. In other words, they require that we move away from looking at isolated events and their causes (usually assumed to be some other events), and start to look at the organization as a system made up of interacting parts.

Our interest in system dynamics is from two perspectives, how do I deal with changes to my project and can we predict how things will continue to turn out once a project is underway. For example the following graphic illustrates the modeling that we do for system dynamics to represent some of the processes within a nuclear construction team.

Figure 5-2 -

Systems Dynamics Modeling Within a Nuclear ConstructionTeam

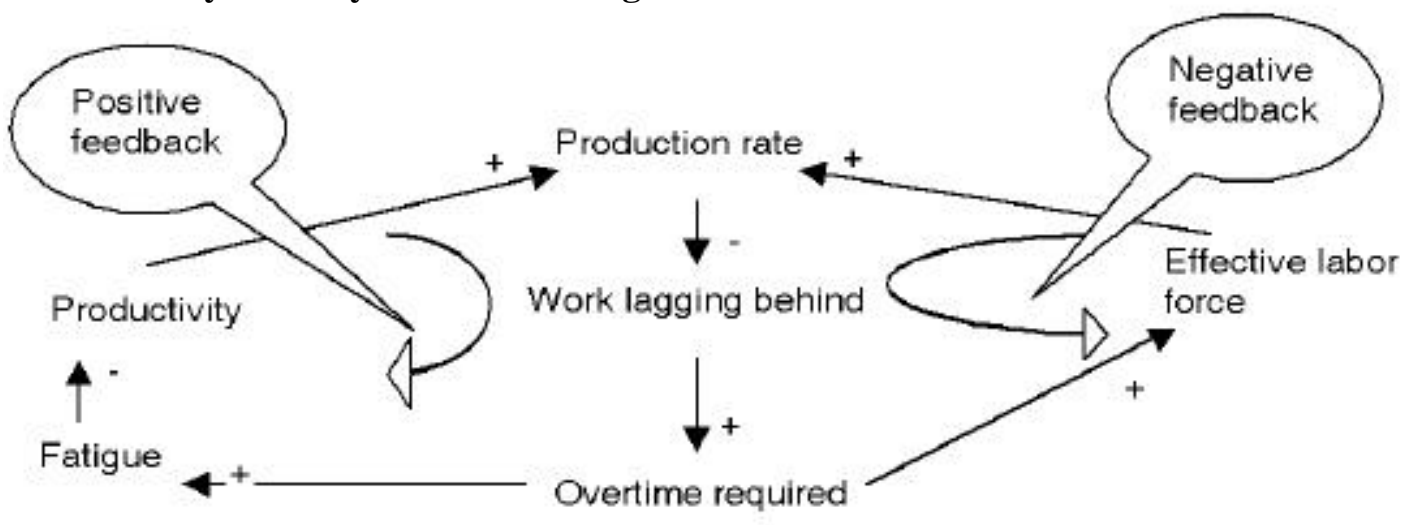


This type of modeling is intended to capture the complexity of situations like the construction of nuclear power plants.

\subsubsection{Process Model Element-Project Coordination Tools}

A new element this year is the advent of project coordination tools. These tools represent technology to be applied as a project is being formed with a host of original equipment manufacturers, subcontractors and the major equipment suppliers as well as the construction team. Relying upon a centralized project database $\mathrm{e}^{3}$ the specification and procurement of equipment will be managed and sequenced to the construction schedule for example. Project coordination technologies such as the Stanford University iRoom concept ${ }^{4}$ pictured below.

\section{Figure 5-3 - iRoom with Multiple Perspectives of Construction Project ${ }^{5}$}

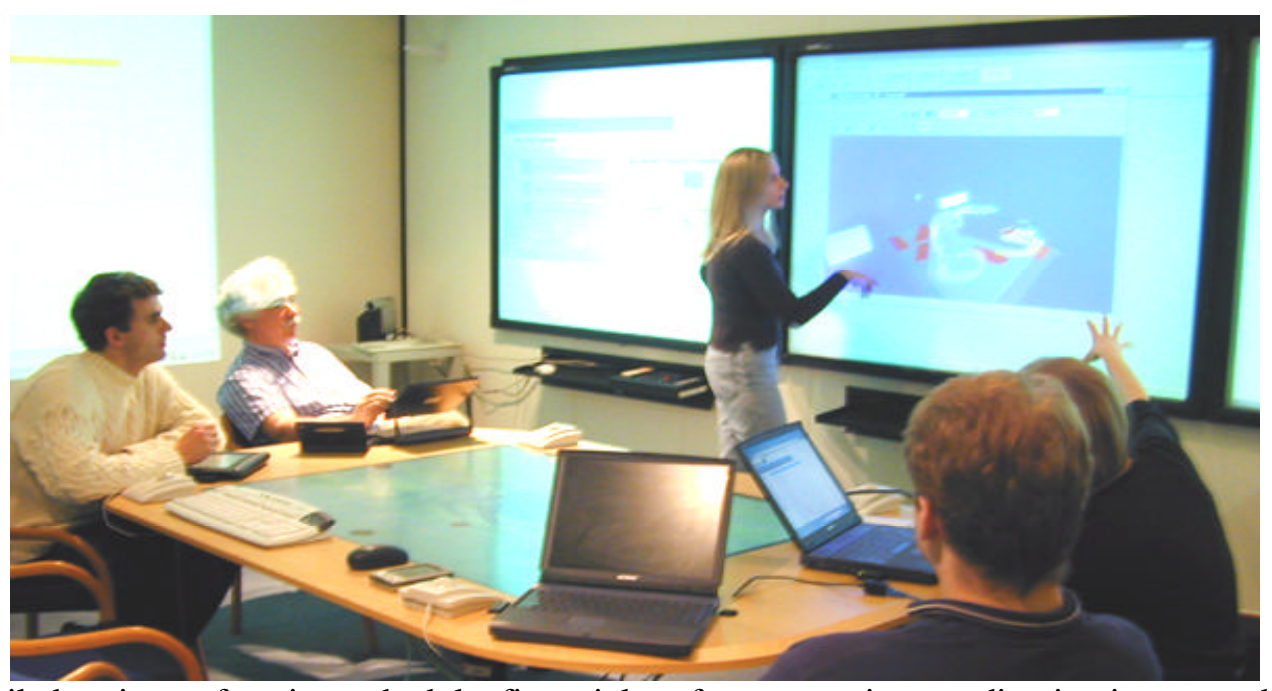

Detailed reviews of project schedule, financial performance, site coordination issues such as equipment laydown, staging for major lifts and other significant critical path activities can be well coordinated using the iRoom approach. For the most part the iRoom concept consists of commercial off the shelf technology arranged in a dedicated space. Thus this approach can be applied to any construction project in the future.

An example of the multiple views of the project can be seen in Figure 5-4 below that shows information that is off to the side of Figure 5-3 above.

\footnotetext{
3 As documented in EPRI Report 1000056, Management of the Licensed Bases of Advanced Nuclear Plants: Proof of Approach, EPRI, September 2000.

4 Fox, Armando; Johanson, Brad; Hanrahan, Pat; Winograd, Terry (2000). "Integrating information appliances into an interactive workspace." IEEE Computer Graphics and Applications, 20(3), 54-65.

5 Schwegler, Benedict R.; Fischer, Martin A.; O'Connell, Michael Benefits of Information Technology in Construction." Proceedings International Conference on Construction: Construction for Tomorrow's City, Volume 1, Hong Kong, June 19-21, 2001, pages xxiii to xxxviii
} 


\section{Figure 5-4 - Additional iRoom View with Integrated Project Schedule, Visualization}

and Contract Information ${ }^{6}$

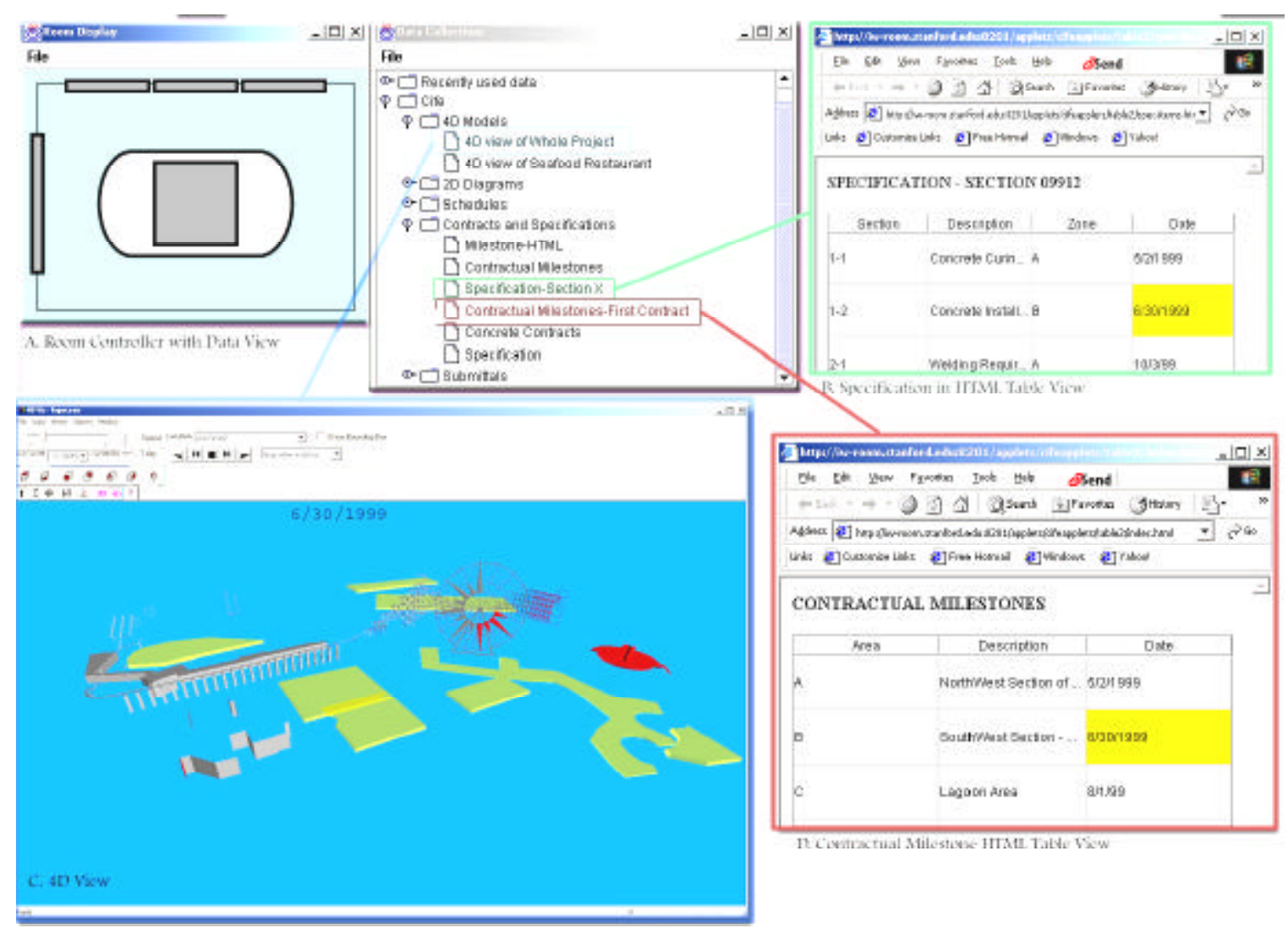

Summarizing work in the Process Model area, we envision that complex projects such as future nuclear plants will have augmented methods of assessing project progress, the ability to determine alternatives when faced with schedule disruptions and a robust capability to engage all stakeholders in resolving issues to ensure on time and on budget performance. Thus, confidence in achieving the capital cost expenditures will be generated thereby improving the outlook for new nuclear plant construction. improving the outlook for new nuclear plant construction. 


\section{$\boldsymbol{A}_{\text {Appendix A }}$}

Framatome ANP DE\&S

Camillo A. DiNunzio

James Flaherty

Bijon Mahnoori

Dimitrios Antonopoulos

Larsen, William

Guy Riggio

Maurice Sills

Richard Parlor

William Fox, III

Parlor, Richard

Kurt Merdick

Michael Terrell

Massachusetts Institute of Technology

Michael Golay

Dohyoung Kim

Haibo Chen

Bobby Middleton

\section{North Carolina State University}

Abhinav Gupta

A. K Gupta.

B Choi.

S. M. Rahman

R. K. Saigal

\section{Sandia National Laboratories}

Eric Klamerus

Vincent Luk

Gary Rochau

\section{Project Participants}

Principal Investigator

Manager, Engineering

Mechanics

Senior Engineer

Supervisor, Engineering

Mechanics

Senior Project Manager

Senior Consultant

Engineering Manager

Senior Consultant

Vice President, Steam Generator

Team

Senior Consultant

Project Management

Project Management

Professor, Nuclear Engineering

Graduate Research Assistant

Graduate Research Assistant

Graduate Research Assistant

Assistant Professor

Professor \& Director of Center

for Nuclear Power Plant

Structures, Equipment \& Piping

Graduate Student

Graduate Student

Graduate Student

Principal Member of Technical

Staff

Principal Member of Technical

Staff

Principal Member of Technical

Staff 
Korea Power Engineering Company

Gum Taek Jin

Kyung Hwa.Chung

Project Manager

Wan il Jung

Jae Min Yim

IT and 3D CAD System

Project Control Technology

Scheduler

Westinghouse Electric Company-Nuclear Systems

Teresa Ann Aldous

Maria E. Assard.

Scheduling Coordinator

Steven A.Austin

Senior Engineer

George P. Berntsen.

Consulting Engineer

Robert E. Bryan.

Dreyfuss Colin

George A.Davis Jr.

Senior Consulting Engineer

Senior Consulting Engineer

Engineer

Lyle D. Gerdes.

John F. Kielb.

Michael T. .Luedke

Director Government Programs

Senior Consulting Engineer

Principal Nuclear Engineer

Donn M Matteson.

Consulting Engineer

John F. Mullooly

Senior Consulting Engineer

Manager-Mechanical Design

Engineering

Richard S.Turk

Engineering Manager-Systems

Michael Volodzko

Development

Consulting Engineer

Bernd E.Von Kutzleben

Senior Consulting Engineer 


\title{
$\boldsymbol{B}_{\text {APPENDIX }}$
}

\section{System Dynamics Model Model Variables, Definitions, And Equations}

\author{
Variable $\quad 5 \%$ precedence contrains \\ Definition This is the fraction that, if a workphase starts when this fraction of upstream work phase \\ has been completed, the percentage of out-of-sequence work in the workphase upon \\ starting is only $5 \%$. \\ Equation $5 \%$ precedence contrains [WorkPhase] $=$ GET XLS \\ CONSTANTS('NppInput.xls','General','I16') \\ Unit Dmnl \\ Variable a \\ Definition This is a coefficient of a exponential function which determines the percentage of out-of- \\ sequence work when a given fraction of upstream work has been completed. \\ Equation a[WorkPhase] $=\mathrm{LN}(0.05) /$ "5\% precedence contrains"[WorkPhase] / "5\% precedence \\ contrains"[WorkPhase] \\ Unit Dmnl \\ Variable accepted flawed work discovery time \\ Definition This is the average time it takes for the Flawed accepted work to be discovered after it is \\ accepted. \\ Equation accepted flawed work discovery time[WorkPhase] = GET XLS \\ CONSTANTS('NppInput.xls','General','I10') \\ Unit Week \\ Variable actual mgnt working time \\ Definition This is the actual number of hours the management staff works per day. \\ Equation actual mgnt working time[Dep,MgntLevel] = SUM ( actual mgnt working time \\ allocation[Dep,MgntLevel,MgntWork!] ) \\ Unit hour/Week \\ Variable actual mgnt working time allocation \\ Definition This is the actual allocation of management staff's working time on their various tasks \\ (meeting, supervision, administrative work,etc.). See MgntWork. \\ Equation actual mgnt working time allocation[Dep,MgntLevel,MgntWork] = ALLOCATE BY \\ PRIORITY ( desired mgnt working time allocation[Dep,MgntLevel,MgntWork] , \\ priority[MgntWork], Admin, 1, maximum mgnt working time ) \\ Unit hour/Week
}




\begin{tabular}{|c|c|}
\hline Variable & actual utility working time \\
\hline Definition & This is the acutal working time per day of the utility workers during next period. \\
\hline Equation & $\begin{array}{l}\text { actual utility working time[Dep] = MIN ( desired working time[Dep,Utility] , maximum } \\
\text { utility working time ) }\end{array}$ \\
\hline Unit & hour/Week \\
\hline Variable & additional working time required \\
\hline Definition & This is the additional working time needed by the management staff to handle the change. \\
\hline Equation & $\begin{array}{l}\text { additional working time required[Dep,StaffLevel] }=\text { Number of Active Changes[Dep] } * \\
\text { additional working time required per change[StaffLevel] }\end{array}$ \\
\hline Unit & hour/Week \\
\hline Variable & additional working time required per change \\
\hline Definition & $\begin{array}{l}\text { This is the input reflecting the time neede by the management staff to handle ONE } \\
\text { average-scale change. }\end{array}$ \\
\hline Equation & additional working time required per change[StaffLevel] = GET XLS \\
\hline & CONSTANTS('NppInput.xls','General','b20') \\
\hline Unit & hour/Week \\
\hline Variable & adjustment factor \\
\hline Definition & $\begin{array}{l}\text { This is the adjustment factor (either by taking overtime adjustment or over-staffing } \\
\text { adjustment, or both) that is required to turn the project back to schedule. }\end{array}$ \\
\hline Equation & adjustment factor $=$ XIDZ $(1$, project SPI, 1$)$ \\
\hline Unit & Dmnl \\
\hline Variable & average base work duration \\
\hline Definition & This is the average work duration for the base work tasks. \\
\hline Equation & average base work duration[WorkPhase] = GET XLS \\
\hline & CONSTANTS('NppInput.xls','General','B8') \\
\hline Unit & Week \\
\hline Variable & average rework duration \\
\hline Definition & This is the average work revision duration for the rework tasks. \\
\hline Equation & average rework duration[WorkPhase] = GET XLS \\
\hline & CONSTANTS('NppInput.xls','General','I9') \\
\hline Unit & Week \\
\hline Variable & average upstream rework duration \\
\hline Definition & This is the average work duration of one unit of upstream rework. \\
\hline Equation & average upstream rework duration[WorkPhase] $=2,2,2,1,1$ \\
\hline Unit & Week \\
\hline
\end{tabular}


Definition Equation

Unit

Variable Definition

Equation

Unit

Variable

Definition

Equation

Unit

Variable

Definition

Equation

Unit

Variable

Definition

Equation

Unit

Variable Definition

Equation

Unit
This is the amount of base work completed per unit time. base work completion rate[WorkPhase, Good] $=$ MIN ( Base Work to be Done[WorkPhase] / TIME STEP , work completion rate[WorkPhase] - revision work completion rate[WorkPhase] )* quality of base work[WorkPhase]base work completion rate[WorkPhase,Flawed] = MIN ( Base Work to be Done[WorkPhase] / TIME STEP , work completion rate[WorkPhase] - revision work completion rate[WorkPhase] )* ( 1 quality of base work[WorkPhase] )

work unit/Week

Base Work to be Done

This is the base work scheduled to be done. A work unit is defined as the amount of work that a worker can finish in a unit time under normal condition.

Base Work to be Done[WorkPhase] = INTEG( work creation rate[WorkPhase] - SUM ( base work completion rate[WorkPhase, xQuality!] ), initial work to be done[WorkPhase] )

work unit

Budget for Change

This is the user specified amount of budget that is to be obtained for a change that occurs unexpectedly.It can be positive or negative. If it's positive, it means new work is created (increase scope); If it's negative, it means some of the work to be done doesn't have to be done any more. (decrease scope )

Budget for Change[WorkPhase] $=0,0,0,0,694737$

dollar

capability familiarity factor

This is the factor that specifies the effect of management staff's familiarity on management staff's capability.

capability familiarity factor[Dep,MgntLevel] = mgnt capability familiarity lookup ( familiarity fraction[Dep,MgntLevel] )

Dmnl

change caused revised work growth rate

This is the amount of accepted work that requires revision per unit time because of changes.

change caused revised work growth rate[WorkPhase] = revision work created by change[WorkPhase] / TIME STEP * PULSE ( change start time[WorkPhase], TIME STEP )

work unit/Week

change duration

This is the active time of the change. (The scheduled time period between its occurrence and it is completed.)

change duration[WorkPhase] $=$ change end time[WorkPhase] - change start

time[WorkPhase]

Week 

Variable change end time
Definition This is the user specified time that the change will ends (scheduled finish time).
Equation change end time[WorkPhase] $=\operatorname{GAME}(158)$
Unit Week

Variable change number decreasing rate

Definition This is the number of changes that are completed per unit time.

Equation change number decreasing rate[WorkPhase] = DELAY FIXED ( change number growth rate[WorkPhase] ,change duration[WorkPhase] , 0)

Unit $\quad$ 1/Week

Variable change number growth rate

Definition This is the number of changes occur per unit time.

Equation change number growth rate[WorkPhase] = IF THEN ELSE ( change start

time[WorkPhase] > 0, PULSE ( change start time[WorkPhase] , TIME STEP ) / TIME

STEP , 0)

Unit $\quad$ 1/Week

Variable Change Response Revision Work

Definition This is the uncompleted work that requires revision because of changes.

Equation Change Response Revision Work[WorkPhase] = INTEG( SUM ( change response revision work growth rate[WorkPhase, $\mathrm{xQ}$ Quality!] - change response revision work completion rate[WorkPhase, $\mathrm{XQuality!]} \mathrm{),0)}$

Unit work unit

Variable change response revision work completion rate

Definition This is the change-caused revision work completed per unit time.

Equation change response revision work completion rate[WorkPhase,Good] $=$ revision work

completion rate[WorkPhase] * quality of revision work[WorkPhase] - rework completion rate[WorkPhase,Good]change response revision work completion

rate[WorkPhase,Flawed] $=$ revision work completion rate[WorkPhase] $*$ ( 1 - quality of revision work[WorkPhase] ) - rework completion rate[WorkPhase,Flawed]

Unit $\quad$ work unit/Week

Variable change response revision work growth rate

Definition This is the amount of accepted work that requires revision per unit time because of changes.

Equation change response revision work growth rate[WorkPhase,Good] $=$ change caused revised work growth rate[WorkPhase] * ZIDZ ( Work Released[WorkPhase,Good] , SUM (

Work Released[WorkPhase, xQuality!] ) ) * ( 1 + fractional work completed effect[WorkPhase] )change response revision work growth rate[WorkPhase,Flawed] $=$ change caused revised work growth rate[WorkPhase] * ZIDZ ( Work

Released[WorkPhase,Flawed], SUM ( Work Released[WorkPhase,xQuality!] ) )* ( $1+$ fractional work completed effect[WorkPhase] )

Unit work unit/Week 


\begin{tabular}{|c|c|}
\hline Variable & change revision work completion rate required \\
\hline Definition & This is the rate at which the revision work caused by changes is required to be completed. \\
\hline Equation & $\begin{array}{l}\text { change revision work completion rate required[WorkPhase] = Change Response Revision } \\
\text { Work[WorkPhase] / MAX ( average rework duration[WorkPhase], change end } \\
\text { time[WorkPhase] - Time ) }\end{array}$ \\
\hline Unit & work unit/Week \\
\hline Variable & change start time \\
\hline Definition & This is the user specified time that the change occurs. \\
\hline Equation & change start time[WorkPhase] $=-1,-1,-1,-1,154$ \\
\hline Unit & Week \\
\hline Variable & conditional start \\
\hline Definition & $\begin{array}{l}\text { This indicator is used to start a workphase to when the pre-specified fraction of upstream } \\
\text { work phase has been completed, yet the time has not come to the point that the current } \\
\text { workphase is scheduled to start. }\end{array}$ \\
\hline Equation & $\begin{array}{l}\text { conditional start[WorkPhase] = IF THEN ELSE }(\text { expected fraction of previous work } \\
\text { phase work completed[WorkPhase] >= external precedence constrains[WorkPhase] }, 1 \text {, } \\
\text { 0) }\end{array}$ \\
\hline Unit & Dmnl \\
\hline Variable & configuration knowledge \\
\hline Definition & $\begin{array}{l}\text { This is the accumulated configuration knowledge as a funciton of fraction of work } \\
\text { completed. Generally, an engineering company has some degree of configuration } \\
\text { knowledge based upon its previous experience and working site walkdowns. This } \\
\text { knowledge will accumulate as more and more information about the working site is } \\
\text { revealed, which is represented by the fraction of work completed for the whole project. }\end{array}$ \\
\hline Equation & $\begin{array}{l}\text { configuration knowledge }=\text { initial configuration knowledge }+ \text { SQRT }(\text { percieved fraction } \\
\text { of total work completed }) *(1-\text { initial configuration knowledge })\end{array}$ \\
\hline Unit & Dmnl \\
\hline Variable & congestion critical worker number \\
\hline Definition & This is the minimum number of workforce that starts to cause congestion problem. \\
\hline Equation & congestion critical worker number[Dep] = GET XLS \\
\hline & CONSTANTS('NppInput.xls','General','I12') \\
\hline Unit & person \\
\hline Variable & data updating time \\
\hline Definition & This is the time it takes to update the project status, for example, productivity. \\
\hline Equation & data updating time $=1$ \\
\hline Unit & Week \\
\hline Variable & Dep \\
\hline
\end{tabular}



Definition These are the three departments we consider in the model Design Engineering
$\begin{array}{ll}\text { Equation } & \text { Department, Field En }<->\text { WorkPhase }\end{array}$
Unit

Variable desired junior staff

Definition This is the desired number of junior staff.

Equation desired junior staff[Dep,StaffLevel] $=$ controller[StaffLevel] $*($ desired

Unit person

Variable desired mgnt working time allocation

Definition This is the desired allocation of management staff's working time on their various tasks (meeting, supervision, administrative work,etc.). See MgntWork.

Equation desired mgnt working time allocation[Dep,MgntLevel,MgntWork] $=$ desired working time[Dep,MgntLevel] * nominal mgnt time allocation weight[MgntLevel,MgntWork]

Unit hour/Week

Variable desired senior fraction

Definition This is the desired senior staff fraction. The engineering companies generally don't hire $100 \%$ senior staff because of the higher labor cost, and they can not hire $100 \%$ junior staff because of the concern about the productivity and quality.

Equation desired senior fraction[Dep,StaffLevel] = GET XLS

CONSTANTS('NppInput.xls','General','I25')

Unit Dmnl

$\begin{array}{ll}\text { Variable } & \text { desired senior staff } \\ \text { Definition } & \begin{array}{l}\text { This is the desired number of senior staff. } \\ \text { desired senior staff[Dep,StaffLevel] = controller[StaffLevel] } * \text { desired }\end{array} \\ \text { Equation } & \begin{array}{l}\text { workforce[Dep,StaffLevel] } * \text { staffing senior fraction[Dep,StaffLevel] } \\ \text { person }\end{array}\end{array}$

Variable desired size of workforce

Definition This is the desired number of workforce based upon the number of workers and the desired staff ratios.

Equation desired size of workforce[Dep,Manager] = workers[Dep] $*$ desired staff

ratio[Dep,Manager] / desired staff ratio[Dep,Worker]desired size of

workforce[Dep,Supervisor] = workers[Dep] $*$ desired staff ratio[Dep,Superviror] /

desired staff ratio[Dep,Worker]desired size of workforce[Dep,Forman] $=$ workers[Dep] $*$

desired staff ratio[Dep,Forman] / desired staff ratio[Dep,Worker]desired size of

workforce[Dep,Worker] = workers[Dep]desired size of workforce[Dep,Utility] =

Unit person

Variable desired staff ratio 
Definition This is the average staff ratio in each department of the stafflevels to the workers in that department.

Equation desired staff ratio[Dep,StaffLevel $]=$ GET XLS

CONSTANTS('NppInput.xls','General','B25')

Unit Dmnl

Variable desired workforce

Definition This is the desired size of workforce.

Equation desired workforce[Dep,StaffLevel] = DELAY FIXED $($ expected

workforce[Dep,StaffLevel] ,staffing adjustment time[Dep,StaffLevel] , initial

workforce[Dep,StaffLevel] )

Unit person

Variable desired working time

Definition This is the desired working time for the next time period in order to finish the amount of work in the next period.

Equation desired working time[Dep,StaffLevel] $=$ XIDZ $($ worker overtime factor[Dep] $*$ nominal working time + additional working time required[Dep,StaffLevel], workforce

availability[Dep,StaffLevel] , 1e+006)

Unit hour/Week

Variable expected available base work

Definition This is the amount of base work available to done give some fraction of the upstream work has been completed.

Equation expected available base work[WorkPhase] = workphase start flag[WorkPhase] * Work Scope[WorkPhase] * expected available fraction of base work by internal precedence constrains[WorkPhase]

Unit work unit

Variable expected available base work to be done

Definition This is the expected base work available to be done, which is the difference of 'expected available base work' and 'expected work completed'

Equation expected available base work to be done[WorkPhase] = expected available base work[WorkPhase] - expected work completed[WorkPhase]

Unit work unit

Variable expected available fraction of base work by internal precedence constrains

Definition This is the total fraction of base work that is available to be done based upon the fraction of current work phase's work that has been completed.

Equation expected available fraction of base work by internal precedence constrains[WorkPhase] = internal precedence constrains lookup[WorkPhase] ( expected fraction of work completed[WorkPhase] )

Unit Dmnl

Variable expected base work completion rate

Definition 


\begin{abstract}
Equation $\quad$ expected base work completion rate[WorkPhase] $=$ expected workforce[WorkPhase,Worker] * updated productivity[WorkPhase] - SUM ( rework completion rate[WorkPhase,xQuality!] )

Unit work unit/Week
\end{abstract}

Variable expected base work completion rate limited by task precedence

Definition This is the amount of work in each department that is produced by each type of the base work. It is scheduled to be done during next period. Note the next period means time t to $\mathrm{t}+\mathrm{dt}$, not all the time left.

Equation expected base work completion rate limited by task precedence[WorkPhase] $=$ expected available base work to be done[WorkPhase] / average base work duration[WorkPhase]

Unit work unit/Week

Variable $\quad$ expected fraction of previous work phase work completed

Definition This is the perceived fraction of work of previous work phase to be completed.

Equation expected fraction of previous work phase work completed[CD] $=1$ expected fraction of previous work phase work completed[DD] $=$ expected fraction of work completed[CD]expected fraction of previous work phase work completed[WPkg] $=$ expected fraction of work completed[DD] expected fraction of previous work phase work completed $[\mathrm{Mob}]=$ expected fraction of work completed[WPkg]expected fraction of previous work phase work completed[Outage] $=$ expected fraction of work

Unit Dmnl completed[Mob]

Variable expected fraction of work completed

Definition This is the perceived fraction of work completed.

Equation expected fraction of work completed[WorkPhase] = expected work completed[WorkPhase] / Work Scope[WorkPhase]

Unit Dmnl

Variable $\quad$ Expected Virtual Base Work Completed

Definition This is the estimated work that will be completed by next time step (now+workforce adjustment time) based upon staffing plan and updated nominal productivity.

Equation Expected Virtual Base Work Completed[WorkPhase] = INTEG( expected base work completion rate[WorkPhase] , 0)

Unit work unit

Variable expected work completed

Definition

Equation

expected work completed[WorkPhase] $=$ MIN ( Work Scope[WorkPhase] , Expected Virtual Base Work Completed[WorkPhase] + initial expected base work completed[WorkPhase] - Virtual Base Work Completed[WorkPhase] + perceived work

Unit work unit 


\begin{tabular}{|c|c|}
\hline $\begin{array}{l}\text { Variable } \\
\text { Definition }\end{array}$ & expected work completion rate limit \\
\hline Equation & $\begin{array}{l}\text { expected work completion rate limit[WorkPhase] = workphase start flag[WorkPhase] * ( } \\
\text { expected base work completion rate limited by task precedence[WorkPhase] + revision } \\
\text { work completion rate limited by task precedence[WorkPhase] ) } \\
\text { work unit/Week }\end{array}$ \\
\hline Variable & expected workforce \\
\hline Definition & This is the disired work force to finish the work required. \\
\hline Equation & $\begin{array}{l}\text { expected workforce[Dep,Manager] = expected workforce[Dep,Worker] * desired staff } \\
\text { ratio[Dep,Manager] / desired staff ratio[Dep,Worker] expected } \\
\text { workforce[Dep,Supervisor] = expected workforce[Dep,Worker] * desired staff } \\
\text { ratio[Dep,Supervisor] / desired staff ratio[Dep,Worker]expected workforce[Dep,Forman] } \\
=\text { expected workforce[Dep,Worker] * desired staff ratio[Dep,Forman] / desired staff } \\
\text { ratio[Dep,Worker] expected workforce[Dep,Worker] = expected workforce[Dep,Worker] } \\
\text { expected workforce[Dep,Utility] = expected workforce[Dep,Worker] * desired staff } \\
\text { ratio[Dep,Utility] / desired staff ratio[Dep,Worker] }\end{array}$ \\
\hline Unit & person \\
\hline Variable & external precedence constrains \\
\hline Definition & $\begin{array}{l}\text { This is the lookup table indicating what fraction of work is available to be done if some } \\
\text { fraction of the unstream work phase's work has been completed. }\end{array}$ \\
\hline Equation & $\begin{array}{l}\text { external precedence constrains[WorkPhase] = GET XLS } \\
\text { CONSTANTS('NppInput.xls','General','I17') }\end{array}$ \\
\hline Unit & Dmnl \\
\hline Variable & familiar junior destaffing rate \\
\hline Definition & This is the number of familiar junior workers that are destaffed per unit time. \\
\hline Equation & $\begin{array}{l}\text { familiar junior destaffing rate[Dep,StaffLevel] = IF THEN ELSE ( junior } \\
\text { staff[Dep,StaffLevel] }>0 \text {, junior destaffing rate[Dep,StaffLevel] } * \text { ( Familiar Junior } \\
\text { Staff[Dep,StaffLevel] / junior staff[Dep,StaffLevel] ), 0) }\end{array}$ \\
\hline Unit & person/Week \\
\hline Variable & Familiar Junior Staff \\
\hline Definition & This is the current number of familiar junior workers. \\
\hline Equation & $\begin{array}{l}\text { Familiar Junior Staff[Dep,StaffLevel] = INTEG }(\text { junior familiarizing } \\
\text { rate[Dep,StaffLevel] - familiar junior destaffing rate[Dep,StaffLevel] - promotion } \\
\text { rate[Dep,StaffLevel] , 0) }\end{array}$ \\
\hline Unit & person \\
\hline Variable & familiar senior destaffing rate \\
\hline Definition & This is the number of familiar senior workers that are destaffed per unit time. \\
\hline Equation & $\begin{array}{l}\text { familiar senior destaffing rate[Dep,StaffLevel] }=\text { IF THEN ELSE }(\text { senior } \\
\text { staff[Dep,StaffLevel] > 0, senior destaffing rate[Dep,StaffLevel] } *(\text { Familiar Senior } \\
\text { Staff[Dep,StaffLevel] / senior staff[Dep,StaffLevel] }), 0)\end{array}$ \\
\hline Unit & person/Week \\
\hline
\end{tabular}




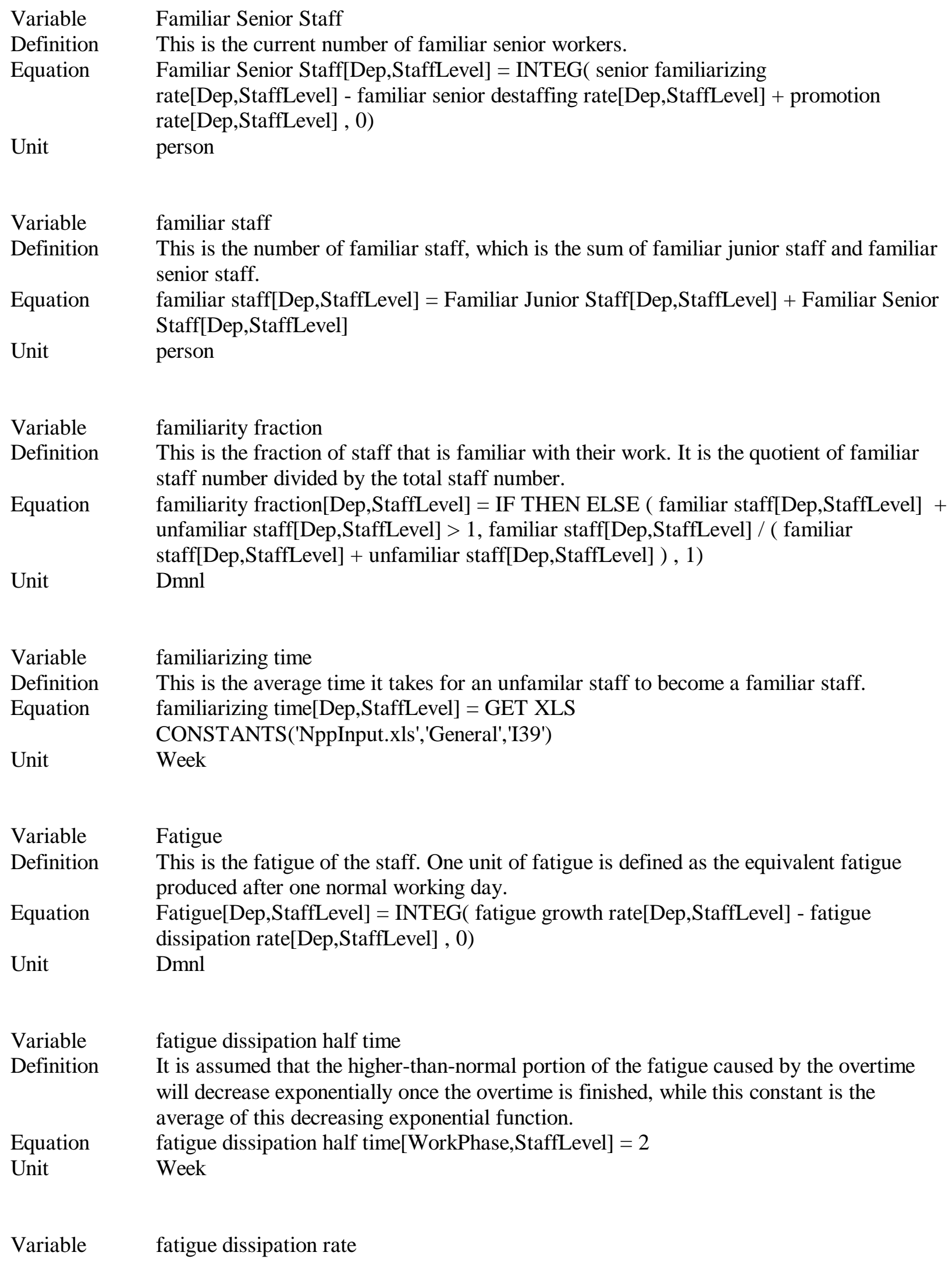

Variable fatigue dissipation rate 

Definition This is the rate at which the fatigue is decreasing.
Equation fatigue dissipation rate[WorkPhase,StaffLevel] = IF THEN ELSE ( overtime factor[WorkPhase,StaffLevel] > 1, 0, Fatigue[WorkPhase,StaffLevel] / fatigue dissipation half time[WorkPhase,StaffLevel] )
Unit 1/Week

Variable fatigue growth rate

Definition This is the fatigue growth rate due to overtime.

Equation fatigue growth rate[WorkPhase,StaffLevel] = nominal fatigue growth rate[WorkPhase,StaffLevel] * ( overtime factor[WorkPhase,StaffLevel] - 1) * ( $1+$ ARCTAN ( Fatigue[WorkPhase,StaffLevel] / 5) )

Unit $\quad$ 1/Week

Variable

Definition

Equation

Unit

Variable

Definition

Equation

Unit

Variable

Definition

Equation

Unit

Variable

Definition

Equation

Unit

Variable Definition
FINAL TIME

The final time for the simulation.

FINAL TIME $=180$

Week

forced start

This indicator is used to forced a workphase to start when the pre-specified fraction of upstream work phase has not been completed, yet the time comes to the point that the current workphase is scheduled to start.

forced start[WorkPhase] = IF THEN ELSE $($ Time $>=$ scheduled start time[WorkPhase] staffing adjustment time[WorkPhase,Worker] , 1, 0)

Dmnl

fraction of work removed

This is the fraction of completed work removed (either obsoleted or requires revision) because of changes.

fraction of work removed[WorkPhase] $=($ new work created by change[WorkPhase] + released work obsoleted by change[WorkPhase] + revision work created by change[WorkPhase] ) / Work Scope[WorkPhase]

Dmnl

fractional work completed effect

This is the effect reflecting the fact that the more the work has been completed, the more good work will be 'incriminated', or brought to rework together with the flawed work. For example, the rework of a pipe may require rework of the structures in the vincinity. fractional work completed effect[WorkPhase] $=$ fractional work completed effect lookup[WorkPhase] ( perceived fraction of work completed[WorkPhase] )

Dmnl

fractional work completed effect lookup

Rework of Flawed work may bring some of the relevant good work also goes to rework, and the more work that has been completed, the more good work will be affected. The 
lookup specifies the multiplier that is used to multiply the Flawed rework to give the total rework (Flawed rework+good rework) as a function of fraction of work completed.

Equation fractional work completed effect lookup[CD] $([(0,0)-(2,1)],(0,0),(1,0))$ fractional work completed effect lookup[DD] ( $[(0,0)-(2,1)],(0,0),(1,0))$ fractional work completed effect lookup[WPkg] ( $[(0,0)-(2,1)],(0,0),(1,0))$ fractional work completed effect lookup[Mob] $([(0,0)-(2,1)],(0,0),(0.5,0.1),(1,0.5))$ fractional work completed effect lookup[Outage]

Unit $([(0,0)-(2,1)],(0,0),(0.5,0.1),(1,0.5))$

Variable initial configuration knowledge

Definition Generally, an engineering company has some degree of configuration knowledge based upon its previous experience and working site walkdowns.

Equation initial configuration knowledge = GET XLS CONSTANTS('NppInput.xls','General','B3')

Unit Dmnl

Variable initial expected base work completed

Definition This is the work that initial $(\mathrm{t}=0)$ workforce can complete within 'staffing adjustment time'.

Equation initial expected base work completed[WorkPhase] $=$ initial workforce[WorkPhase,Worker] * scheduled worker productivity[WorkPhase] * staffing adjustment time[WorkPhase,Worker]

Unit work unit

Variable initial junior staff

Definition This is the number of junior staff put on the project at $t=0$.

Equation initial junior staff[Dep,StaffLevel] = initial workforce[Dep,StaffLevel] - initial senior staff[Dep,StaffLevel]

Unit person

Variable initial senior staff

Definition This is the number of senior staff put on the project at $t=0$.

Equation initial senior staff[Dep,StaffLevel] = initial workforce[Dep,StaffLevel] * staffing senior fraction[Dep,StaffLevel]

Unit person

Variable INITIAL TIME

Definition The initial time for the simulation.

Equation INITIAL TIME $=0$

Unit Week

Variable initial work to be done

Definition This is the initial (base) work to be done for each work phase.

Equation initial work to be done[WorkPhase] = GET XLS

CONSTANTS('NppInput.xls','General','B9')

Unit work unit 


\begin{tabular}{|c|c|}
\hline Variable & initial workforce \\
\hline Definition & This is the number of staff put on the project at $\mathrm{t}=0$. \\
\hline \multirow{2}{*}{ Equation } & initial workforce[CD,StaffLevel $]=$ GET XLS \\
\hline & $\begin{array}{l}\text { CONSTANTS('NppInput.xls','General','b21')initial workforce[DD,StaffLevel] = 0initial } \\
\text { workforce[WPkg,StaffLevel] = 0initial workforce[Mob,StaffLevel] = 0initial } \\
\text { workforce[Outage,StaffLevel] = } 0\end{array}$ \\
\hline Unit & person \\
\hline Variable & inrate \\
\hline \multicolumn{2}{|l|}{ Definition } \\
\hline Equation & $\begin{array}{l}\text { inrate[WorkPhase] = Budget for Change[WorkPhase] / change duration[WorkPhase] / } \\
\text { TIME STEP } * \text { PULSE ( change start time[WorkPhase], TIME STEP ) }\end{array}$ \\
\hline Unit & dollar/(Week*Week) \\
\hline Variable & internal precedence constrains lookup \\
\hline Definition & $\begin{array}{l}\text { This is the lookup table indicating what fraction of work is available to be done given } \\
\text { some fraction of the work of current work phase has been completed. }\end{array}$ \\
\hline Equation & internal precedence constrains lookup[CD] $([(0,0)-$ \\
\hline & $(1,1)],(0,0.0112994),(0.0112994,0.0225989),(0.0225989,0.0338983),(0.0338983,0.05084$ \\
\hline & $75),(0.0508475,0.0677966),(0.0677966,0.0847458),(0.0847458,0.112994),(0.112994,0.1$ \\
\hline & $41243),(0.141243,0.169492),(0.169492,0.20339),(0.20339,0.237288),(0.237288,0.27118$ \\
\hline & $6),(0.271186,0.305085),(0.305085,0.338983),(0.338983,0.372881),(0.372881,0.40678),($ \\
\hline & $0.40678,0.440678),(0.440678,0.474576),(0.474576,0.508475),(0.508475,0.542373),(0.54$ \\
\hline & $2373,0.576271),(0.576271,0.610169),(0.610169,0.644068),(0.644068,0.677966),(0.6779$ \\
\hline & $66,0.711864),(0.711864,0.745763),(0.745763,0.779661),(0.779661,0.813559),(0.813559$, \\
\hline & $0.847458),(0.847458,0.881356),(0.881356,0.915254),(0.915254,0.937853),(0.937853,0.9$ \\
\hline & $60452),(0.960452,0.977401),(0.977401,0.99435),(0.99435,1),(1,1))$ \\
\hline Unit & Dmnl \\
\hline Variable & is start conditional \\
\hline Definition & $\begin{array}{l}\text { This is to indicate whether a work phase can start before its scheduled starting time when } \\
\text { a pre-specified fraction of its upstream work has been completed. }\end{array}$ \\
\hline Equation & is start conditional[WorkPhase] $=1,1,1,0,0$ \\
\hline Unit & Dmnl \\
\hline Variable & junior destaffing rate \\
\hline Definition & This is the number of junior workers that are destaffed per unit time. \\
\hline Equation & $\begin{array}{l}\text { junior destaffing rate[Dep,StaffLevel] = IF THEN ELSE ( desired junior } \\
\text { staff[Dep,StaffLevel] < junior staff[Dep,StaffLevel] , ( junior staff[Dep,StaffLevel] - } \\
\text { desired junior staff[Dep,StaffLevel] ) / TIME STEP , 0) }\end{array}$ \\
\hline Unit & person/Week \\
\hline
\end{tabular}




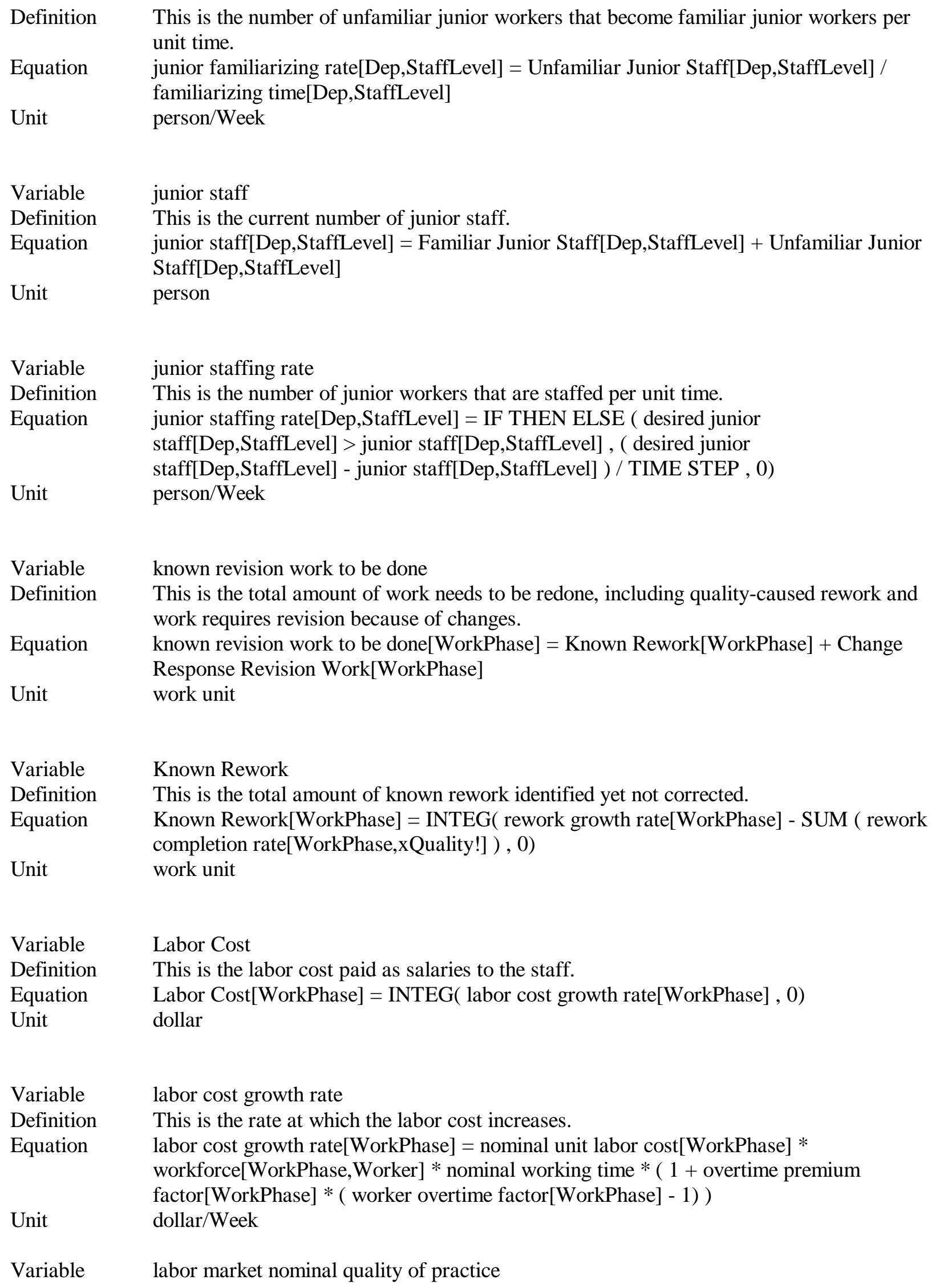



Definition This is the nominal quality of practice, which is define as the fraction of work performed correctly if the work is performed for the first time.
Equation labor market nominal quality of practice[WorkPhase] = GET XLS
CONSTANTS('NppInput.xls','General','I15')
Unit Dmnl

Variable labor market nominal worker productivity

Definition This is the nominal productivity of the base worker in each work phase.

Equation labor market nominal worker productivity[WorkPhase] = GET XLS

Unit work unit/(Week*person)

Variable management availability

Definition This is the availability of the management staff. one is the normal value.

Equation management availability[Dep,MgntLevel] = ZIDZ ( actual mgnt working time allocation[Dep,MgntLevel,OverSight] , desired mgnt working time

Unit Dmnl

Variable management capability

Definition This is the 'capability' of management staff, considering the experience, familiarity, efficiency, morale, and so on.

Equation management capability[Dep,MgntLevel] = mgnt capability upper management capability factor[Dep,MgntLevel] * mgnt capability availability factor[Dep,MgntLevel] * mgnt capability experience factor[Dep,MgntLevel] * capability familiarity factor[Dep,MgntLevel] * mgnt capability utility worker availability factor[Dep] * mgnt capability morale factor[Dep,MgntLevel] * mgnt capability fatigue

Unit Dmnl

Variable maximum mgnt working time

Definition This is the maximum working time per day that a management staff may be assigned to work under engineering company's policy or other guidelines.

Equation maximum mgnt working time = GET XLS CONSTANTS('NppInput.xls','General','I3')

Unit hour/Week

Variable maximum number of workers

Definition This is the maximum number of worforce allowed in the project for each phase and each staff level.

Equation maximum number of workers[Dep] = GET XLS

CONSTANTS('NppInput.xls','General','I11')

Unit person

Variable maximum utility worker working time 

Definition This is the maximum working time per day that a worker may be assigned to work under engineering company's policy or other guidelines.
Equation maximum utility worker working time $=$ GET XLS
CONSTANTS('NppInput.xls','General','I4')
Unit hour/Week

Variable maximum utility working time

Definition This is the maximum working time per day that a utility worker may be assigned to work under engineering company's policy or other guidelines.

Equation maximum utility working time $=$ maximum utility worker working time

Unit hour/Week

Variable mgnt capability availability factor

Definition This is the factor that reflects the availability of the management staff on the capability.

Equation mgnt capability availability factor[Dep,MgntLevel] = mgnt capability management

availability lookup ( management availability[Dep,MgntLevel] )

Unit Dmnl

Variable mgnt capability experience factor

Definition This is the factor that specifies the effect of management staff's experience on management staff's capability.

Equation mgnt capability experience factor[Dep,MgntLevel] = mgnt capability experience lookup ( senior fraction[Dep,MgntLevel] / desired senior fraction[Dep,MgntLevel] )

Unit Dmnl

Variable mgnt capability experience lookup

Definition This lookup specifies the management capability experience factor as a function of management staff's experience. 1 is the normal value of experience (in which case the actual senior fraction equals to the desired senior fraction)

Equation mgnt capability experience lookup ( $[(0,0)-(10,10)],(0,0.8),(0.5,0.95),(1,1),(2,1))$

Unit Dmnl

Variable mgnt capability familiarity lookup

Definition This lookup specifies the management capability familiarity factor as a function of management staff's familiarity. Familiarity ranges from 0 to 1 , corresponding to the worst and best situation.

Equation mgnt capability familiarity lookup ( $[(0,0)-(10,10)],(0,0.8),(0.5,0.95),(1,1),(2,1))$

Unit Dmnl

Variable mgnt capability fatigue factor

Definition This reflects the influence of fatigue on management's capability.

Equation mgnt capability fatigue factor[Dep,MgntLevel] $=$ EXP $(-$ Fatigue[Dep,MgntLevel] *

Unit Dmnl 


\begin{tabular}{|c|c|}
\hline Variable & mgnt capability management availability lookup \\
\hline Definition & $\begin{array}{l}\text { This lookup specifies the management capability management availability factor as a } \\
\text { function of management staff's availability. Availability is defined as the actual available } \\
\text { person hours divided by desired person hours.।!!! }\end{array}$ \\
\hline Equation & mgnt capability management availability lookup $([(0,0)-(10,10)],(0,0),(1,1),(2,1))$ \\
\hline Unit & Dmnl \\
\hline Variable & mgnt capability morale factor \\
\hline Definition & $\begin{array}{l}\text { This is the factor that specifies the effect of management staff's morale on management } \\
\text { staff's capability. }\end{array}$ \\
\hline Equation & $\begin{array}{l}\text { mgnt capability morale factor[Dep,MgntLevel] }=\text { EXP }(- \text { Morale } \\
\text { Damage[Dep,MgntLevel] } * 0.01) / 10+0.9\end{array}$ \\
\hline Unit & Dmnl \\
\hline Variable & mgnt capability upper management capability factor \\
\hline Definition & $\begin{array}{l}\text { This factor reflects the influence of the upper management's capabilities on the } \\
\text { subordinates' capabilities. }\end{array}$ \\
\hline Equation & $\begin{array}{l}\text { mgnt capability upper management capability factor[Dep,MgntLevel] = mgnt capability } \\
\text { upper management capability lookup ( upper management capability[Dep,MgntLevel] ) }\end{array}$ \\
\hline Unit & Dmnl \\
\hline Variable & mgnt capability upper management capability lookup \\
\hline Definition & $\begin{array}{l}\text { This lookup specifies the function that describes how the upper management's } \\
\text { capabilities affect the subordinates' cabapilities. }\end{array}$ \\
\hline Equation & $\begin{array}{l}\text { mgnt capability upper management capability lookup ( }[(0,0)- \\
(10,10)],(0,0.2),(0.5,0.7),(1,1),(2,1))\end{array}$ \\
\hline Unit & Dmnl \\
\hline Variable & mgnt capability utility availability lookup \\
\hline Definition & $\begin{array}{l}\text { This lookup specifies the management staff's capability utility worker availability factor } \\
\text { as a function of utility worker's availability. Availability is defined as the actual available } \\
\text { person hours divided by desired person hours. }\end{array}$ \\
\hline Equation & mgnt capability utility availability lookup $([(0,0)-(10,10)],(0,0.8),(0.5,0.95),(1,1),(2,1))$ \\
\hline Unit & Dmnl \\
\hline Variable & mgnt capability utility worker availability factor \\
\hline Definition & $\begin{array}{l}\text { This is the factor that specifies the effect of utility worker's availability on management } \\
\text { staff's capability. }\end{array}$ \\
\hline Equation & $\begin{array}{l}\text { mgnt capability utility worker availability factor }[\mathrm{Dep}]=\text { mgnt capability utility } \\
\text { availability lookup ( utility availability[Dep] ) }\end{array}$ \\
\hline Unit & Dmnl \\
\hline
\end{tabular}


Equation MgntLevel Manager,Supervisor,Forman

Unit

Variable MgntWork

Definition These are the categories of work for the management staff. MeetEx for manager means meeting with clients, executives or deputy from headquarters; for superintendent and supervisor means meeting with the managers and superintendents, respectively. MeetIn means meeting with the subordinates. Oversight means the supervision work. Admin means the logistic work such was writing the progress report and planning.

Equation MgntWork MeetEx,MeetIn,OverSight,Admin

Unit

Variable Morale Damage

Definition This is the morale of the staff of each level in each department. One unit of morale damage is defined as the equivalent damage of morale by one normal working day's work.

Equation $\quad$ Morale Damage[Dep,StaffLevel $]=$ Morale Damage From Overtime[Dep,StaffLevel $]+$ Morale Damage From Change[Dep,StaffLevel]

Unit Dmnl

Variable morale damage dissipation half time

Definition It is assumed that the higher-than-normal portion of the morale loss caused by the overtime will decrease exponentially once the overtime is finished, while this constant is the average of this decreasing exponential function.

Equation morale damage dissipation half time[WorkPhase,StaffLevel] $=2$

Unit Week

Variable Morale Damage From Change

Definition This is the morale loss due to changes.

Equation Morale Damage From Change[Dep,StaffLevel] = INTEG( morale damage from change growth rate[Dep,StaffLevel] - morale damange from change dissipation rate[Dep,StaffLevel] , 0)

Unit Dmnl

Variable morale damage from change growth rate

Definition It is assumed that if all the work completed up until now is obsoleted, then the morale loss will be 20. If only a portion is obsoleted, then the morale loss will proportionally less than 20 .

Equation morale damage from change growth rate[Dep,StaffLevel] $=5 /$ TIME STEP $*$ fraction of work removed[Dep] * PULSE ( change start time[Dep] , TIME STEP )

Unit $\quad$ 1/Week

Variable $\quad$ Morale Damage From Overtime

Definition This is the morale loss of the staff because of overtime. One unit of fatigue is defined as the equivalent morale loss produced after one normal working day. 

Equation $\quad$ Morale Damage From Overtime[Dep,StaffLevel] = INTEG( morale damage growth rate[Dep,StaffLevel] - morale demange dissipation rate[Dep,StaffLevel] , 0)
Unit Dmnl

Variable morale damage growth rate

Definition This is the fatigue growth rate due to overtime.

Equation morale damage growth rate[Dep,StaffLevel] = nominal morale damage growth rate[Dep,StaffLevel] * ( overtime factor[Dep,StaffLevel] - 1) * (1 + ARCTAN ( Morale Damage[Dep,StaffLevel] / 5) )

Unit $\quad$ 1/Week

Variable morale damange from change dissipation rate

Definition This is the rate at which the morale loss due to changes decreases.

Equation morale damange from change dissipation rate[Dep,StaffLevel] = IF THEN ELSE ( Time < change end time[Dep] , 0, Morale Damage From Change[Dep,StaffLevel] / morale damage dissipation half time[Dep,StaffLevel] )

Unit $\quad$ 1/Week

Variable morale demange dissipation rate

Definition This is the rate at which the morale loss due to overtime decreases.

Equation morale demange dissipation rate[Dep,StaffLevel] = IF THEN ELSE ( overtime factor[Dep,StaffLevel] > 1, 0, Morale Damage From Overtime[Dep,StaffLevel] / morale damage dissipation half time[Dep,StaffLevel] )

Unit $\quad$ 1/Week

Variable

Definition

Equation

Unit work unit

new work created by change

This is the user specified amount of work that is created by a change that occurs unexpectedly. It can be positive as well as negative. If it's positive, it means new work is created (increase scope); If it's negative, it means some of the work to be done doesn't have to be done any more. (decrease scope )

Variable nominal fatigue growth rate

Definition This is the nominal fatigue growth rate under normal situations. It is assumed that each 8hour working day wil add one unit of fatigue to the staff.

Equation nominal fatigue growth rate[WorkPhase,StaffLevel] $=5$

Unit $\quad 1 /$ Week

Variable nominal mgnt time allocation weight

Definition This is the fraction of time assigned to each category of management work under normal conditions.

Equation nominal mgnt time allocation weight[MgntLevel,MgntWork] = GET XLS

Unit Dmnl 
Variable nominal morale damage growth rate

Definition This is the nominal morale loss rate under normal situations. It is assumed that each 8hour working day wil add one unit of morale loss to the staff.

Equation nominal morale damage growth rate[WorkPhase,StaffLevel] $=5$

Unit $\quad 1 /$ Week

Variable nominal review quality

Definition This is the nominal review quality, a value of one means $100 \%$ of the Flawed completed work will be identified in the review process, while a value of $95 \%$ means $5 \%$ of the Flawed completed work will be accepted mistakenly because we are not able to identified them in the review process.

Equation nominal review quality[WorkPhase] $=$ GET XLS

CONSTANTS('NppInput.xls','General','B16')

Unit Dmnl

Variable nominal unit labor cost

Definition This is the nominal cost per work unit in each work phase.

Equation nominal unit labor cost[WorkPhase] = GET XLS

Unit dollar/(hour*person)

Variable nominal working time

Definition This is the nominal hours of working time per day.

Equation nominal working time = GET XLS CONSTANTS('NppInput.xls','General','I2')

Unit hour/Week

Variable Number of Active Changes

Definition This is the number of active(ongoing) changes.

Equation Number of Active Changes[WorkPhase] = INTEG( change number growth

rate[WorkPhase] - change number decreasing rate[WorkPhase] ,0)

Unit Dmnl

Variable out-of-sequence work percentage

Definition This is a varialbe reflecting the degree of out of sequence problem. In this model, both

the rework and change-caused revision work will lead to work out of sequence problem.

Equation out-of-sequence work percentage[WorkPhase] = MIN ( 1, "out-of-sequence-work percentage from external early start"[WorkPhase] + "out-of-sequence-work percentage from internal early start"[WorkPhase] + "out-of-sequence-work percentage from rework"[WorkPhase] )

Unit Dmnl

Variable out-of-sequence-work percentage from external early start

Definition It is assumed that, when the current work phase starts, if the previous work phase is $100 \%$ completed, there is no work-out-of-sequence from early start; if a '5\% point' is completed, 

the rest of the points are obtan from an exponential derived from these three points.
Equation out-of-sequence-work percentage from external early start[WorkPhase] $=$ workphase start flag[WorkPhase] * IF THEN ELSE ( perceived fraction of previous work phase completed[WorkPhase] $<$ "5\% precedence contrains"[WorkPhase], EXP ( a[WorkPhase] * perceived fraction of previous work phase completed[WorkPhase] * perceived fraction of previous work phase completed[WorkPhase] ), 0.05 / ( "5\% precedence contrains"[WorkPhase] - 1) * ( perceived fraction of previous work phase completed[WorkPhase] - 1) )
Unit Dmnl

Variable out-of-sequence-work percentage from internal early start

Definition This is the out-of-sequence work percentage due to early start of tasks when precedent tasks in the same workphase has not been completed.

Equation out-of-sequence-work percentage from internal early start[WorkPhase] $=$ workphase start flag[WorkPhase] * perceived fraction of work completed[WorkPhase] * ( 1 - MIN ( 1, workphase SPI[WorkPhase] ) )

Unit Dmnl

Variable out-of-sequence-work percentage from rework

Definition This is the out-of-sequence work percentage due to rework, where those work that based upon these rework will be out-of-sequence.

Equation out-of-sequence-work percentage from rework[WorkPhase] = ZIDZ ( known revision work to be done[WorkPhase], known revision work to be done[WorkPhase] + perceived work completed[WorkPhase] )

Unit Dmnl

Variable oversize factor

Definition This factor indicates a staffing policy. A value of one means exactly the number of workforce to finish the work will be hired. Sometimes project managers takes in more workforce that it actually need to accomadate surprises, at which time this factor will be greater than one.

Equation oversize factor[Dep] $=1,1,1,1,1$

Unit Dmnl

Variable overtime factor

Definition actual working time divided by nominal working time

Equation overtime factor[Dep,Manager] = MAX ( 1, actual mgnt working time[Dep,Manager] $/$

nominal working time )overtime factor[Dep,Supervisor] $=$ MAX ( 1, actual mgnt working time[Dep,Supervisor] / nominal working time )overtime factor[Dep,Forman] = 
Unit

Variable

Definition

Equation

Unit

Variable

Definition

Equation

Unit

Variable

Definition

Equation

Unit

Variable

Definition

Equation

Unit

Variable

Definition

Equation

Unit

Variable

Definition
MAX ( 1, actual mgnt working time[Dep,Forman] / nominal working time )overtime factor[Dep, Worker] = worker overtime factor[Dep]overtime factor[Dep,Utility] = MAX ( 1 , actual utility working time[Dep] / nominal working time )

$$
\text { Dmnl }
$$

overtime premium factor

This is the factor considering the fact that the overtime portion of the work may obtain better paid compared to the normal situation. 1 is the normal value.

$$
\text { overtime premium factor[WorkPhase] = GET XLS }
$$

CONSTANTS('NppInput.xls','General','I13')

Dmnl

perceived fraction of previous work phase completed

This is the perceived fraction of work completed in the upstream workphase.

perceived fraction of previous work phase completed[CD] = 1perceived fraction of previous work phase completed[DD] $=$ perceived fraction of work

completed[CD]perceived fraction of previous work phase completed[WPkg] $=$ perceived fraction of work completed[DD]perceived fraction of previous work phase

completed[Mob] $=$ perceived fraction of work completed[WPkg]perceived fraction of previous work phase completed[Outage] $=$ perceived fraction of work completed[Outage]

Dmnl

perceived fraction of work completed

This is the perceived fraction of work completed in each workphase.

perceived fraction of work completed[WorkPhase] = perceived work

completed[WorkPhase] / Work Scope[WorkPhase]

Dmnl

perceived work completed

This is the perceived work completed, ie. work accepted plus the work completed but awaiting review.

perceived work completed[WorkPhase] = SUM ( Work Released[WorkPhase, $x$ Quality!]

+ Work to be Reviewed[WorkPhase,xQuality!] )

work unit

percieved fraction of total work completed

This is the perceived fraction of work of the whole project completed. In ordet to add up the work from different work phases, some converstion may be needed to translate the possible different work units used in different work phases.

percieved fraction of total work completed $=$ SUM ( perceived work completed[WorkPhase!] ) / SUM ( Work Scope[WorkPhase!] )

Dmnl

priority

This is the mathematical representation of 'priority policy', which is only a $0 / 1$ switch. 

Equation $\quad$ priority $[$ MeetEx $]=10$ priority $[$ MeetIn $]=$ IF THEN ELSE $($ priority policy $=0,10$, 8)priority $[$ OverSight $]=$ IF THEN ELSE $($ priority policy $=0,10,6)$ priority $[$ Admin $]=$ IF THEN ELSE ( priority policy $=0,10,4$ )
Unit Dmnl

Variable priority policy

Definition This is the priority policy to allocate the management staff's working time when not enough time is available to finish all the job awaiting. 0 weighted allocation (Each category of work will be done in proportion to its amount 1 exclusive priority, higher priority first (Time is assigned to the higher priority work frist, and the lower priority work may not be done if the time is used up by the higher priority work).

Equation $\quad$ priority policy = GET XLS CONSTANTS('NppInput.xls','General','b4')

Unit Dmnl

Variable productivity

Definition This is the productivity of the base work.

Equation productivity[WorkPhase] $=$ workphase start flag[WorkPhase] $*$ labor market nominal worker productivity[WorkPhase] * worker productivity modulator[WorkPhase]

Unit work unit/(Week*person)

Variable productivity climate factor

Definition This is the factor that reflects the effect of climate, especially Flawed weather, on productivity.

Equation $\quad$ productivity climate factor[Dep $]=\operatorname{GAME}(1)$

Unit Dmnl

Variable productivity configuration knowledge factor

Definition This is the improvement on productivity because of better configuration knowledge.

Equation productivity configuration knowledge factor[CD] = SQRT ( configuration knowledge / 0.9)productivity configuration knowledge factor[DD] = SQRT ( configuration knowledge / 0.9)productivity configuration knowledge factor[WPkg] = SQRT ( configuration knowledge / 0.9)productivity configuration knowledge factor[Mob] = 1productivity configuration knowledge factor[Outage] $=1$

Unit Dmnl

Variable productivity congestion lookup

Definition This is the lookup that specifies the productivity congestion factor as a funtion of congestion, which is defined as the actual number of workers divided by the scheduled number of workers.

Equation productivity congestion lookup $[\mathrm{CD}]([(0,0)-$ $(10,2)],(0,1),(0.5,1),(1,1),(2,0.9),(3,0.8),(10,0.5))$ productivity congestion lookup[DD] ( $[(0,0)-(10,2)],(0,1),(0.5,1),(1,1),(2,0.9),(3,0.8),(10,0.5))$ productivity congestion lookup[WPkg] $([(0,0)-(10,2)],(0,1),(0.5,1),(1,1),(2,0.9),(3,0.8),(10,0.5))$ productivity congestion lookup [Mob] $([(0,0)$ $(10,2)],(0,1),(0.5,1),(1,1),(2,0.8),(3,0.7),(10,0.15))$ productivity congestion lookup[Outage] $([(0,0)-(10,2)],(0,1),(0.5,1),(1,1),(2,0.8),(3,0.7),(10,0.15))$ 
Unit Dmnl

Variable productivity experience factor

Definition This is the factor that reflects the effect of worker's experience on productivity. Here the experience is calculated as actual senior fraction divided by desired senior fraction, and 1 is the nomal value.

Equation productivity experience factor[Dep] = productivity experience lookup ( senior fraction[Dep,Worker] / desired senior fraction[Dep,Worker] )

Unit Dmnl

Variable productivity experience lookup

Definition This is the lookup that specifies the productivity experience factor as a function of actual senior worker fraction and desired senior worker fraction. 1 is the normal value of experience (in which case the actual senior fraction equals to the desired senior fraction)

Equation productivity experience lookup $([(0,0)-(10,10)],(0,0.8),(0.5,0.95),(1,1),(2,1))$

Unit Dmnl

Variable productivity familiarity factor

Definition This is the factor that reflects the effect of workers' familiarity on productivity.

Equation productivity familiarity factor[Dep] = productivity familiarity lookup ( familiarity fraction[Dep,Worker] )

Unit Dmnl

Variable productivity familiarity lookup

Definition This is the lookup that specifies the productivity familiarity factor as a function of worker's familiarity fraction. Familiarity ranges from 0 to 1 , corresponding to the worst and best situation.

Equation productivity familiarity lookup ( $[(0,0)-(2,2)],(0,0.7),(0.5,0.9),(0.8,1),(1,1.05),(2,1.05))$

Unit Dmnl

Variable productivity fatigue factor

Definition This is the factor that reflects the effect of fatigue on productivity.

Equation productivity fatigue factor $[\mathrm{CD}]=\mathrm{EXP}(-$ Fatigue $[\mathrm{CD}$, Worker $] * 0.01) / 2+$ 0.5 productivity fatigue factor[DD] $=\mathrm{EXP}(-$ Fatigue $[\mathrm{CD}$, Worker $] * 0.01) / 2+$

0.5 productivity fatigue factor[WPkg] $=\mathrm{EXP}(-$ Fatigue $[\mathrm{CD}$, Worker $] * 0.01) / 2+$

0.5 productivity fatigue factor[Mob] $=\operatorname{EXP}(-$ Fatigue$[M o b$, Worker] $* 0.05) / 2+$

0.5 productivity fatigue factor[Outage $]=\operatorname{EXP}(-$ Fatigue $[\mathrm{CD}$, Worker $] * 0.05) / 2+0.5$

Unit Dmnl

Variable productivity management capability factor

Definition This is the factor that reflects the effect of supervisor's leadership on productivity.

Equation productivity management capability factor[Dep] = productivity supervisor capability

Unit Dmnl 
$\begin{array}{ll}\text { Variable } & \text { productivity morale factor } \\ \text { Definition } & \text { This is the factor that reflects the effect of morale on productivity. } \\ \text { Equation } & \text { productivity morale factor }[\mathrm{CD}]=\mathrm{EXP}(- \text { Morale Damage }[\mathrm{CD}, \text { Worker }] * 0.01) / 2+ \\ & 0.5 \text { productivity morale factor[DD] }=\mathrm{EXP}(- \text { Morale Damage }[\mathrm{CD}, \text { Worker }] * 0.01) / 2+ \\ & 0.5 \text { productivity morale factor }[\mathrm{WPkg}]=\mathrm{EXP}(- \text { Morale Damage[CD,Worker] } * 0.01) / 2 \\ & +0.5 \text { productivity morale factor }[\mathrm{Mob}]=\mathrm{EXP}(- \text { Morale Damage[CD,Worker }] * 0.05) / 2 \\ & +0.5 \text { productivity morale factor[Outage }]=\operatorname{EXP}(- \text { Morale Damage }[\mathrm{CD}, \text { Worker }] * 0.05) / \\ & 2+0.5\end{array}$

Unit Dmnl

Variable productivity supervisor capability lookup

Definition This is the lookup that specifies the productivity supervisor capability factor as a function of supervior's capability.Capability ranges from 0 to 1, corresponding to the worse and best situation.

Equation productivity supervisor capability lookup[Dep] ( $[(0,0)$ -

$(10,10)],(0,0.6),(0.5,0.9),(1,1),(2,1))$

Unit Dmnl

Variable productivity tool availability factor

Definition This is the factor that reflects the effect of tool availability on productivity.

Equation productivity tool availability factor[Dep] = GAME( 1 )

Unit Dmnl

Variable productivity utility worker availability factor

Definition This is the factor that reflects the effect of availability of utility worker on productivity.

Equation productivity utility worker availability factor[Dep] = productivity utility worker availability lookup ( utility availability[Dep] )

Unit Dmnl

Variable productivity utility worker availability lookup

Definition This is the lookup that gives productivity utility worker availability factor as a funtion of availability of utility workers. Availability is defined as the actual available person hours divided by desired person hours.

Equation productivity utility worker availability lookup ( $[(0,0)$ -

$(10,10)],(0,0.8),(0.5,0.95),(1,1),(2,1))$

Unit Dmnl

Variable productivity work out of sequence factor

Definition This is the factor that consider the effect of out of sequence problem, which is reflected by the ratio of the known rework to total completed work. out of sequence fraction of the work is assumed to be cimpleted at a rate of $50 \%$ of the nominal.

Equation productivity work out of sequence factor[WorkPhase] $=1$ - "out-of-sequence work percentage"[WorkPhase] $* 0.5$

Unit Dmnl 


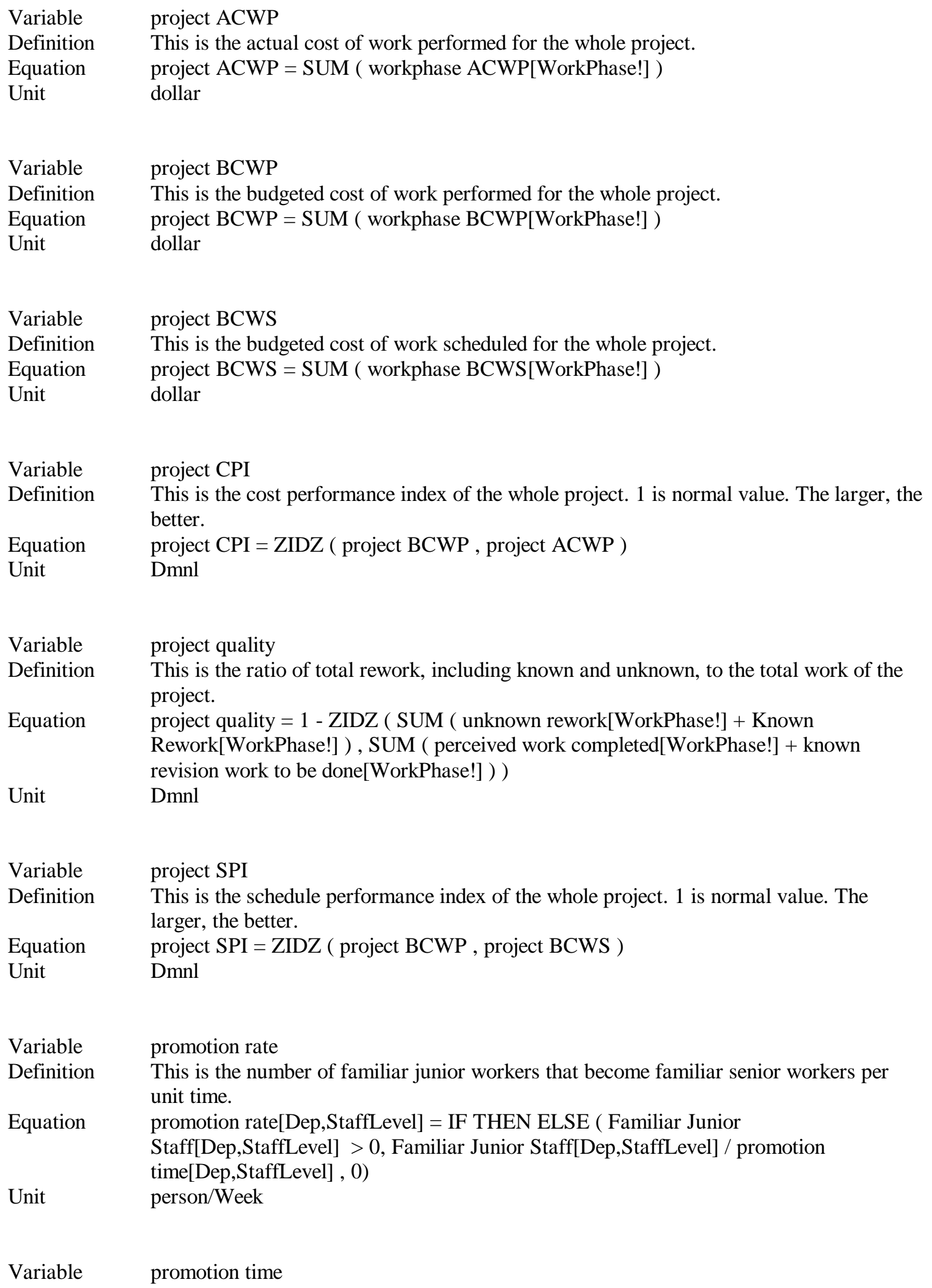




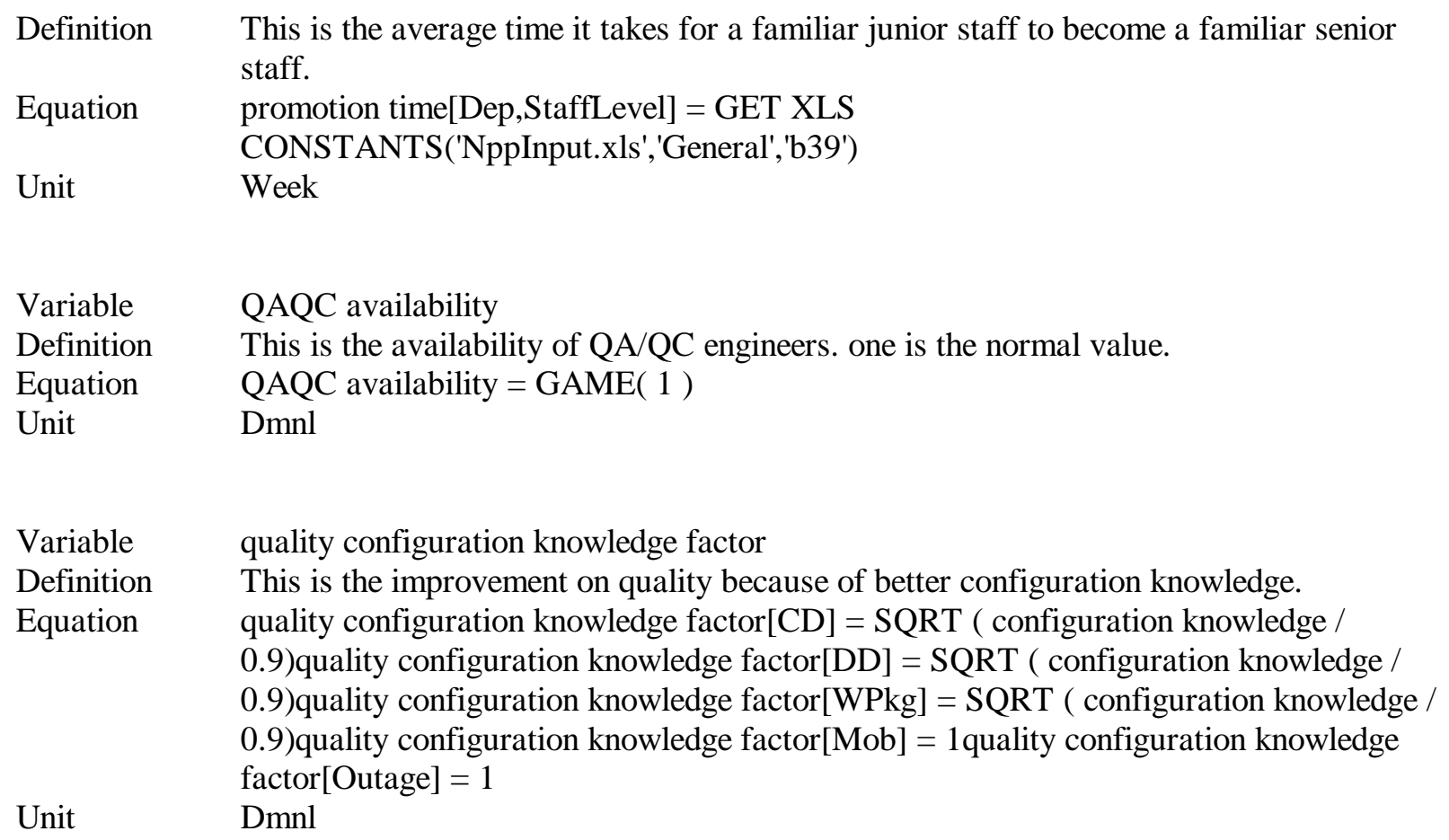
quotient of the senior worker fraction divided by the desired senior worker fraction. 1 is the normal value of experience (in which case the actual senior fraction equals to the desired senior fraction)

Equation $\quad$ quality experience lookup $([(0,0)-(10,10)],(0,0.95),(0.5,0.98),(1,1))$

Unit Dmnl

Variable quality familiarity factor

Definition This is the factor that reflects the effect of worker's familiarity on the quality.

Equation quality familiarity factor[Dep] = quality familiarity lookup ( familiarity

Unit Dmnl

Variable quality familiarity lookup

Definition This is the lookup that specifies the quality familiarity factor as a function of the fraction of the workers that are familiar workers.Familiarity ranges from 0 to 1 , corresponding to the worst and best situation.

Equation quality familiarity lookup ( [(0,0)-(10,10)],(0,0.95), $(0.5,0.98),(1,1))$ 
Unit Dmnl

$\begin{array}{ll}\text { Variable } & \text { quality morale factor } \\ \text { Definition } & \text { This is the factor that reflects the effect of worker's morale on the quality. } \\ \text { Equation } & \text { quality morale factor[Dep] = EXP ( - Morale Damage[Dep,Worker] } * 0.01) / 2+0.5 \\ \text { Unit } & \text { Dmnl }\end{array}$

Variable quality of base work

Definition This is the quality of the base work performed.

Equation quality of base work[WorkPhase] $=$ workphase start flag[WorkPhase] $*$ MIN ( 1, labor market nominal quality of practice[WorkPhase] $*$ worker quality of practice modulator[WorkPhase] )

Unit Dmnl

Variable quality of revision work

Definition quality of revison work generally is higher than the quality of work performed for the first time since more information is available and because of the learning curve effect. In this model, a square root function is used to reflect this fact.

Equation quality of revision work[WorkPhase] = SQRT ( quality of base work[WorkPhase] )

Unit Dmnl

Variable quality supervisor management capability factor

Definition This is the factor that reflects the effect of supervisor's leadership on the quality.

Equation quality supervisor management capability factor[Dep] = quality supervisor management capability lookup ( management capability[Dep,Supervisor] )

Unit Dmnl

Variable quality supervisor management capability lookup

Definition This is the lookup that specifies the quality supervisor management capability factor as a function of supervisor's capability whose normal value is one.Capability ranges from 0 to 1 , corresponding to the worst and best situation.

Equation quality supervisor management capability lookup ( $[(0,0)$ -

$(2,10)],(0,0.9),(0.5,0.97),(1,1),(2,1))$

Unit Dmnl

Variable quality work out of sequence factor

Definition This is the factor that consider the effect of out of sequence problem, which is reflected by the ratio of the known rework to the total completed work. Assume the out of seqence work can be performed with a quality of 0.6 times nominal value.

Equation quality work out of sequence factor[WorkPhase] = 1 - "out-of-sequence work percentage"[WorkPhase] $* 0.4$

Unit Dmnl

Variable released work obsoleted by change 
Definition This is the user specified amount of work that is to be made obsolete by a change that occurs unexpectedly.

Equation $\quad$ released work obsoleted by change[WorkPhase] $=0,0,0,0,0$

Unit

work unit

Variable released work rework rate

Definition This is the amount of accepted work that goes back to rework per unit time. For the Flawed work that is mistakenly accepted, it goes to rework when it is discovered; The rework of Flawed accepted work may also cause some of the good work to be redone again, and therefore some of the good accepted work also goes back to rework. The ratio of good accepted work and Flawed accepted work that goes to rework is a function of fractional work completed the more the work has been completed, the more good accepted work need to be redone in order to do the rework of Flawed accepted work. At the same time, the work following the undiscovered flawed upstream work will also goes to rework.

Equation released work rework rate[WorkPhase,Good] = rework rate identified in downstream phase[WorkPhase,Good] + rework rate inherited from upstream rework[WorkPhase] * ZIDZ ( Work Released[WorkPhase,Good], SUM ( Work

Released[WorkPhase,xQuality!] ) )* ( 1 + fractional work completed effect[WorkPhase] ) released work rework rate[WorkPhase,Flawed] = rework rate identified in downstream phase[WorkPhase,Flawed] + rework rate inherited from upstream rework[WorkPhase] * ZIDZ ( Work Released[WorkPhase,Flawed] , SUM ( Work

Released[WorkPhase,xQuality!] ) ) * ( 1 + fractional work completed effect[WorkPhase] )

Unit $\quad$ work unit/Week

Variable

Definition

Equation

Unit

Variable

Definition

Equation

Unit

Variable Definition

Equation Unit review quality

This is the fraction of Flawed work that can be identified and then be rejected in the review process. Some of the Flawed completed work may be accepted by mistake. review quality[WorkPhase] $=$ nominal review quality[WorkPhase] $*$ review quality QAQC availability factor

Dmnl

review quality QAQC availability factor

This is the factor that reflects the effect of QA/QC engineers' availability on the review quality.

review quality QAQC availability factor = review quality QAQC availability lookup ( QAQC availability )

Dmnl

review quality QAQC availability lookup

This is the lookup that specifies the review quality QAQC availability factor as a function of QA/QC availability. Availability is defined as the actual available person hours divided by desired person hours.

review quality QAQC availability lookup $([(0,0)-(2,10)],(0,0.9),(0.5,0.97),(1,1),(2,1))$

Dmnl 


\begin{tabular}{|c|c|}
\hline Variable & revision work completion rate \\
\hline Definition & $\begin{array}{l}\text { This the rate at which the work that required to be revised because of changes is } \\
\text { completed. }\end{array}$ \\
\hline Equation & $\begin{array}{l}\text { revision work completion rate[WorkPhase] = MIN ( work completion rate[WorkPhase], } \\
\text { revision work completion rate limited by task precedence[WorkPhase] ) }\end{array}$ \\
\hline Unit & work unit/Week \\
\hline Variable & revision work completion rate limited by task precedence \\
\hline Definition & This is the amount of derived work to be done that is caused by the rework per unit time. \\
\hline Equation & $\begin{array}{l}\text { revision work completion rate limited by task precedence[WorkPhase] }=\text { rework } \\
\text { completion rate limited by task precedence[WorkPhase] }+ \text { change revision work } \\
\text { completion rate required[WorkPhase] }\end{array}$ \\
\hline Unit & work unit/Week \\
\hline Variable & revision work created by change \\
\hline Definition & This is the amount of work that requires to be revised because of the change. \\
\hline Equation & revision work created by change[WorkPhase] $=0,0,0,0,100$ \\
\hline Unit & work unit \\
\hline Variable & rework completion rate \\
\hline Definition & This is the amount of quality-caused rework completed per unit time. \\
\hline Equation & rework completion rate[WorkPhase, Good] = revision work completion rate[WorkPhase] \\
\hline & * ZIDZ ( rework completion rate limited by task precedence[WorkPhase] , revision work \\
\hline & completion rate limited by task precedence[WorkPhase] ) * quality of revision \\
\hline & work[WorkPhase]rework completion rate[WorkPhase,Flawed] = revision work \\
\hline & completion rate[WorkPhase] $*$ ZIDZ ( rework completion rate limited by task \\
\hline & precedence[WorkPhase] , revision work completion rate limited by task \\
\hline & precedence[WorkPhase] ) *( 1 - quality of revision work[WorkPhase] ) \\
\hline Unit & work unit/Week \\
\hline Variable & rework completion rate limited by task precedence \\
\hline Definition & $\begin{array}{l}\text { This is the upper limit of the rate at which rework can be completed because of the task } \\
\text { precedence relationship. }\end{array}$ \\
\hline Equation & rework completion rate limited by task precedence[WorkPhase] = Known \\
\hline & Rework[WorkPhase] / average rework duration[WorkPhase] \\
\hline Unit & work unit/Week \\
\hline Variable & rework growth rate \\
\hline Definition & This is the amount of work that goes to rework per unit time. \\
\hline Equation & $\begin{array}{l}\text { rework growth rate[WorkPhase] }=\text { SUM }(\text { work rejection rate[WorkPhase, } x \text { Quality!] + } \\
\text { released work rework rate[WorkPhase, } x \text { Quality!] })\end{array}$ \\
\hline Unit & work unit/Week \\
\hline
\end{tabular}


Definition This is the amount of accepted work identified as being 'flawed' per unit time. The identified flawed work also bring some of the 'good' work back to rework. see 'fractional work completed effect'.

Equation rework rate identified in downstream phase[WorkPhase,Good] = rework rate identified in downstream phase[WorkPhase,Flawed] * fractional work completed effect[WorkPhase]rework rate identified in downstream phase[WorkPhase,Flawed] $=$ Work Released[WorkPhase,Flawed] / accepted flawed work discovery time[WorkPhase]

Unit work unit/Week

Variable rework rate inherited from upstream rework

Definition This is the rate at which the completed work is sent back to rework because the upstream work that it is based upon turned out to be flawed.

Equation rework rate inherited from upstream rework[WorkPhase] = DELAY3 ( upstream accepted Flawed work discovry rate[WorkPhase] * Work Scope[WorkPhase] / work scope of upstream workphase[WorkPhase] * ZIDZ ( SUM ( Work

Released[WorkPhase,xQuality!] ), Work Scope[WorkPhase] ), average upstream rework duration[WorkPhase] )

Unit work unit/Week

Variable SAVEPER

Definition The frequency with which output is stored.

Equation $\quad$ SAVEPER $=0.25$

Unit Week

Variable Scheduled Budgeted Cost of Work Created by Change

Definition This is the total accumulative scheduled budgeted cost of the work that is created by the changes up until now.

Equation Scheduled Budgeted Cost of Work Created by Change[WorkPhase] = INTEG( scheduled change created work budgeted cost growth rate[WorkPhase] ,0)

Unit dollar

Variable scheduled change created work budgeted cost growth rate

Definition This is the scheduled budgeted cost growth rate for the work created by the changes.

Equation scheduled change created work budgeted cost growth rate[WorkPhase] = INTEG( inrate[WorkPhase] - outrate[WorkPhase] , 0)

Unit dollar/Week

Variable scheduled cost as a function of time

Definition This is the cost (labor+others) schedule table as a function of time.

Equation scheduled cost as a function of time[CD] = GET XLS

DATA('NppInput.xls','CD','A','F3')scheduled cost as a function of time[DD] = GET XLS DATA('NppInput.xls','DD','A','F3')scheduled cost as a function of time[WPkg] = GET XLS DATA('NppInput.xls','WPkg','A','F3')scheduled cost as a function of time[Mob] = GET XLS DATA('NppInput.xls','Mob','A','F3')scheduled cost as a function of time[Outage] = GET XLS DATA('NppInput.xls','Outage','A','F3')

Unit dollar 


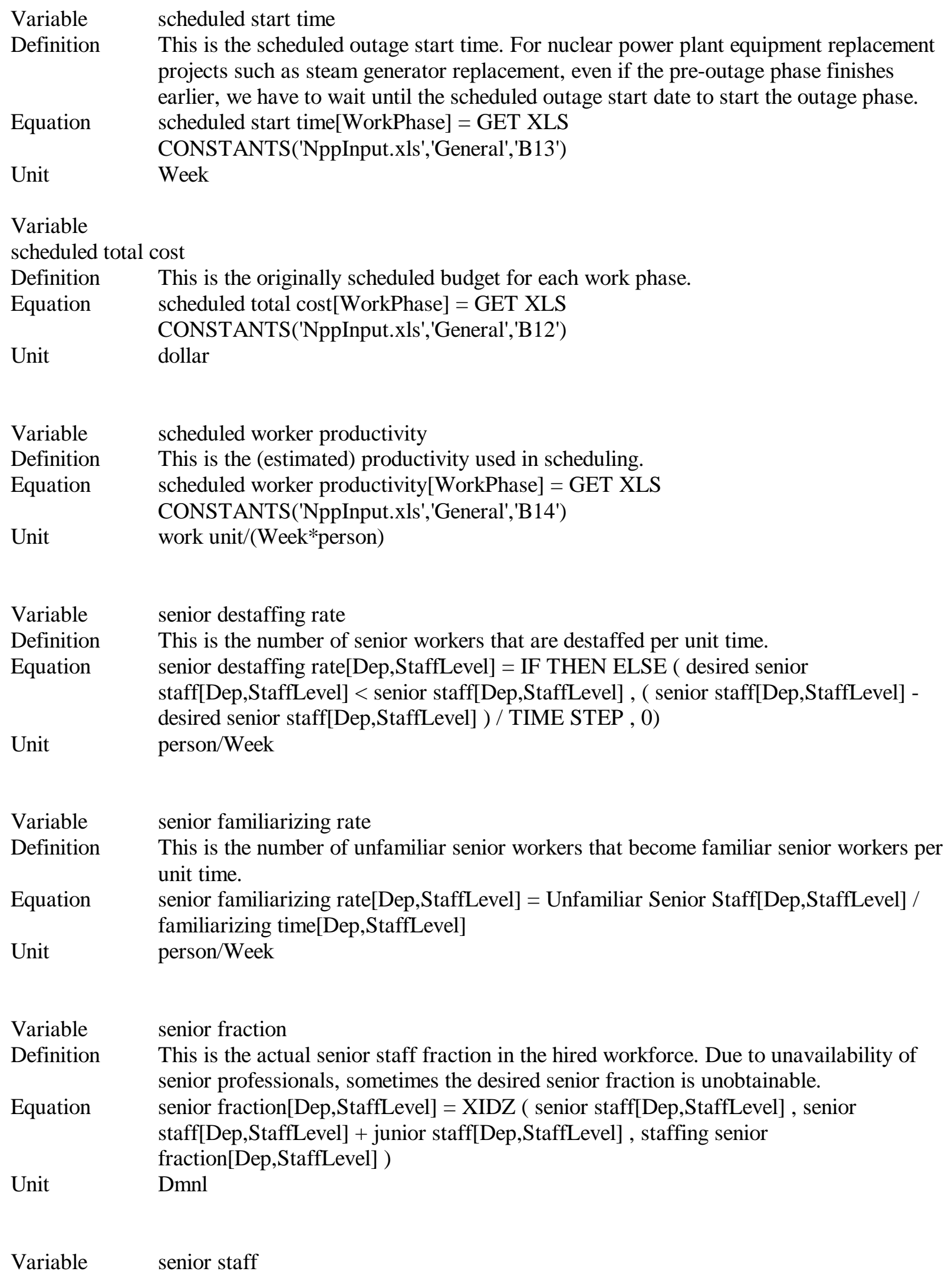


Definition

Equation

Unit

Variable

Definition

Equation

Unit

Variable

Definition

Equation

Unit

Variable

Definition

Equation

Unit

Variable

Definition

Equation

Unit

Variable

Definition

Equation

Unit

Variable

Definition
This is the current number of senior staff.

senior staff[Dep,StaffLevel] = Familiar Senior Staff[Dep,StaffLevel] + Unfamiliar Senior Staff[Dep,StaffLevel]

person

senior staffing rate

This is the number of senior workers that are staffed per unit time.

senior staffing rate[Dep,StaffLevel] = IF THEN ELSE ( desired senior

staff[Dep,StaffLevel] > senior staff[Dep,StaffLevel] , ( desired senior

staff[Dep,StaffLevel] - senior staff[Dep,StaffLevel] ) / TIME STEP , 0)

person/Week

staffing adjustment factor

This is the factor to adjust the workforce when the project deviates from baseline, either behind or ahead of schedule, in order to turn the project onto the right track.

staffing adjustment factor[Dep] $=$ IF THEN ELSE ( workphase start flag[Dep] $=0$ OR perceived fraction of work completed[Dep] > 0.99, 1, POWER ( adjustment factor , staffing adjustment weight[Dep] ) )

Dmnl

staffing adjustment time

This is the time required to adjust the staff, i.e. the time to acquire plus the upfront training time before a staff is put on his or her position to work.

staffing adjustment time[Dep,StaffLevel] = GET XLS

CONSTANTS('NppInput.xls','General','b32')

Week

staffing adjustment weight

This specifies the weight between overtime and over-hiring management actions when the project is behind schedule.

staffing adjustment weight [Dep] = GET XLS

CONSTANTS('NppInput.xls','General','I18')

Dmnl

staffing senior fraction

This is the actual senior staff fraction used in the hiring. Due to unavailability of senior professionals, sometimes the desired senior fraction is unobtainable.

staffing senior fraction[Dep,StaffLevel] = GET XLS

CONSTANTS('NppInput.xls','General','I32')

Dmnl

StaffLevel

This is the organizational staff level hierarchy. Manager, Supervisor, and Forman are management staff, Worker are those who actually do the work, and Utility workers are those such as secretary and fire watchers to help the other poeple in the organization. 
Equation StaffLevel Manager,Supervisor,Forman,Worker,Utility

Unit

$\begin{array}{ll}\text { Variable } & \text { Time } \\ \text { Definition } & \text { Internally defined simulation time. } \\ \text { Equation } & \text { Time = INTEG( 1, INITIAL TIME ) } \\ \text { Unit } & \text { Week }\end{array}$

Variable TIME STEP

Definition The time step for the simulation.

Equation TIME STEP $=0.0625$

Unit Week

Variable total cost/labor cost ratio

Definition This is the ratio of total cost to labor cost. It is used as a multiplier to estimate the total cost based upon the labor cost.

Equation total cost/labor cost ratio[WorkPhase] = GET XLS

CONSTANTS('NppInput.xls','General','B11')

Unit Dmnl

Variable unfamiliar junior destaffing rate

Definition This is the number of unfamiliar junior workers that are destaffed per unit time.

Equation unfamiliar junior destaffing rate[Dep,StaffLevel] = junior destaffing rate[Dep,StaffLevel] - familiar junior destaffing rate[Dep,StaffLevel]

Unit person/Week

Variable Unfamiliar Junior Staff

Definition This is the current number of unfamiliar junior workers.

Equation Unfamiliar Junior Staff[Dep,StaffLevel] = INTEG( junior staffing rate[Dep,StaffLevel] junior familiarizing rate[Dep,StaffLevel] - unfamiliar junior destaffing rate[Dep,StaffLevel] , initial junior staff[Dep,StaffLevel] )

Unit person

Variable unfamiliar senior destaffing rate

Definition This is the number of unfamiliar senior workers that are destaffed per unit time.

Equation unfamiliar senior destaffing rate[Dep,StaffLevel] = senior destaffing rate[Dep,StaffLevel] - familiar senior destaffing rate[Dep,StaffLevel]

Unit person/Week

Variable Unfamiliar Senior Staff

Definition This is the current number of unfamiliar senior workers.

Equation Unfamiliar Senior Staff[Dep,StaffLevel] = INTEG( senior staffing rate[Dep,StaffLevel]

- senior familiarizing rate[Dep,StaffLevel] - unfamiliar senior destaffing

rate[Dep,StaffLevel] , initial senior staff[Dep,StaffLevel] ) 


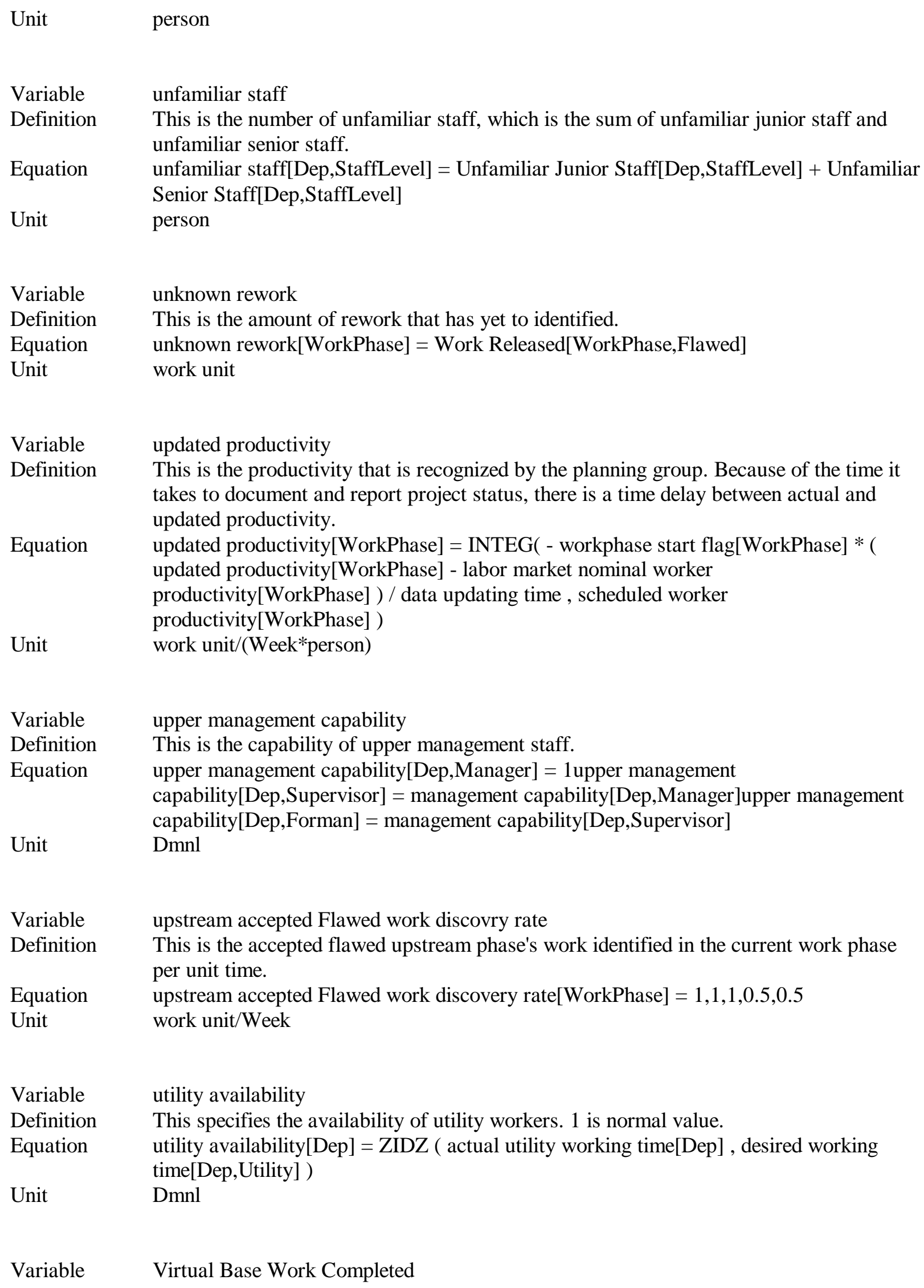

Variable Virtual Base Work Completed 

Definition This is the estimated work that will be completed now based upon staffing plan and updated nominal productivity. It is simply a delayed function of 'Expected Virtual Base Work Completed'.
Equation Virtual Base Work Completed[WorkPhase] = DELAY FIXED ( Expected Virtual Base Work Completed[WorkPhase] ,staffing adjustment time[WorkPhase,Worker] , 0)
Unit work unit

Variable work acceptance rate

Definition This is the amount of work that is accepted per unit time.

Equation work acceptance rate[WorkPhase, $x Q u a l i t y]=$ work review rate[WorkPhase, $x$ Quality] work rejection rate[WorkPhase, $\mathrm{xQuality]}$

Unit work unit/Week

Variable work completion rate

Definition This is the work completion rate limited by the current number of workforce and productivity.

Equation $\quad$ work completion rate[WorkPhase] $=$ workforce[WorkPhase,Worker] $*$ productivity[WorkPhase] * overtime factor[WorkPhase,Worker]

Unit $\quad$ work unit/Week

Variable work creation rate

Definition This is the amount of work created by change per unit time.

Equation

Unit work creation rate[WorkPhase] $=$ work scope growth rate[WorkPhase] work unit/Week

\begin{tabular}{|c|c|}
\hline Variable & Work Obsoleted \\
\hline Definition & This is the total amount of work obsoleted by changes. \\
\hline Equation & $\begin{array}{l}\text { Work Obsoleted[WorkPhase] = INTEG( SUM ( work obsoletion } \\
\text { rate[WorkPhase,xQuality!] ), 0) }\end{array}$ \\
\hline Unit & work unit \\
\hline Variable & work obsoletion rate \\
\hline Definition & This is the amount of work that is made obsolete by changes per unit time. \\
\hline Equation & $\begin{array}{l}\text { work obsoletion rate[WorkPhase,Good] }=\text { work scope decreasing rate[WorkPhase] } * \\
\text { ZIDZ ( Work Released[WorkPhase,Good]. SUM ( Work }\end{array}$ \\
\hline & $\begin{array}{l}\text { Released[WorkPhase,xQuality!] ) )work obsoletion rate[WorkPhase,Flawed] = work } \\
\text { scope decreasing rate[WorkPhase] * ZIDZ ( Work Released[WorkPhase,Flawed] , SUM } \\
(\text { Work Released[WorkPhase,xQuality!] ) ) }\end{array}$ \\
\hline Unit & work unit/Week \\
\hline Variable & work rejection rate \\
\hline Definition & This is the amount of work that is rejected after reviewing. \\
\hline Equation & $\begin{array}{l}\text { work rejection rate[WorkPhase,Good] }=\text { work rejection rate[WorkPhase,Flawed] } * \\
\text { fractional work completed effect[WorkPhase]work rejection rate[WorkPhase,Flawed] = } \\
\text { work review rate[WorkPhase,Flawed] * review quality[WorkPhase] }\end{array}$ \\
\hline Unit & work unit/Week \\
\hline
\end{tabular}




\begin{tabular}{|c|c|}
\hline Variable & Work Released \\
\hline Definition & This is the accepted portion of the completed work. \\
\hline \multirow[t]{3}{*}{ Equation } & $\begin{array}{l}\text { Work Released[WorkPhase,Good] = INTEG( work acceptance rate[WorkPhase,Good] - } \\
\text { released work rework rate[WorkPhase,Good] - work obsoletion rate[WorkPhase,Good] - } \\
\text { change response revision work growth rate[WorkPhase,Good], 0)Work }\end{array}$ \\
\hline & $\begin{array}{l}\text { Released[WorkPhase,Flawed] = INTEG(work acceptance rate[WorkPhase,Flawed] - } \\
\text { released work rework rate[WorkPhase,Flawed] - work obsoletion }\end{array}$ \\
\hline & rate[WorkPhase,Flawed] - change response revision work growth \\
\hline Unit & work unit \\
\hline Variable & work review rate \\
\hline Definition & This is the amount of work that is reviewed per unit time. \\
\hline Equation & $\begin{array}{l}\text { work review rate[WorkPhase, } x Q \text { Quality] = Work to be Reviewed[WorkPhase, } x \text { Quality] / } \\
\text { work review time[WorkPhase] }\end{array}$ \\
\hline Unit & work unit/Week \\
\hline Variable & work review time \\
\hline Definition & This is the time it takes to review an average amount of work. \\
\hline Equation & work review time[WorkPhase] = GET XLS CONSTANTS('NppInput.xls','General','I8') \\
\hline Unit & Week \\
\hline Variable & Work Scope \\
\hline Definition & This is the work scope of each work phase. \\
\hline Equation & $\begin{array}{l}\text { Work Scope[WorkPhase] = INTEG( work scope growth rate[WorkPhase] - work scope } \\
\text { decreasing rate[WorkPhase], initial work to be done[WorkPhase] ) }\end{array}$ \\
\hline Unit & work unit \\
\hline Variable & work scope decreasing rate \\
\hline Definition & $\begin{array}{l}\text { This is the amount of accepted work that is made obsolete by changes per unit time. If the } \\
\text { work has not been performed, you should apply a negative value to 'work scope growth } \\
\text { rate'. }\end{array}$ \\
\hline Equation & $\begin{array}{l}\text { work scope decreasing rate[WorkPhase] = released work obsoleted by } \\
\text { change[WorkPhase] / TIME STEP } * \text { PULSE ( change start time[WorkPhase], TIME } \\
\text { STEP ) }\end{array}$ \\
\hline Unit & work unit/Week \\
\hline Variable & work scope growth rate \\
\hline Definition & $\begin{array}{l}\text { This is the amount of work created by change per unit time. It may be negative, for } \\
\text { example when a change requires removal of some unperformed work. }\end{array}$ \\
\hline Equation & $\begin{array}{l}\text { work scope growth rate[WorkPhase] = new work created by change[WorkPhase] / TIME } \\
\text { STEP } * \text { PULSE ( change start time[WorkPhase], TIME STEP ) }\end{array}$ \\
\hline Unit & work unit/Week \\
\hline
\end{tabular}




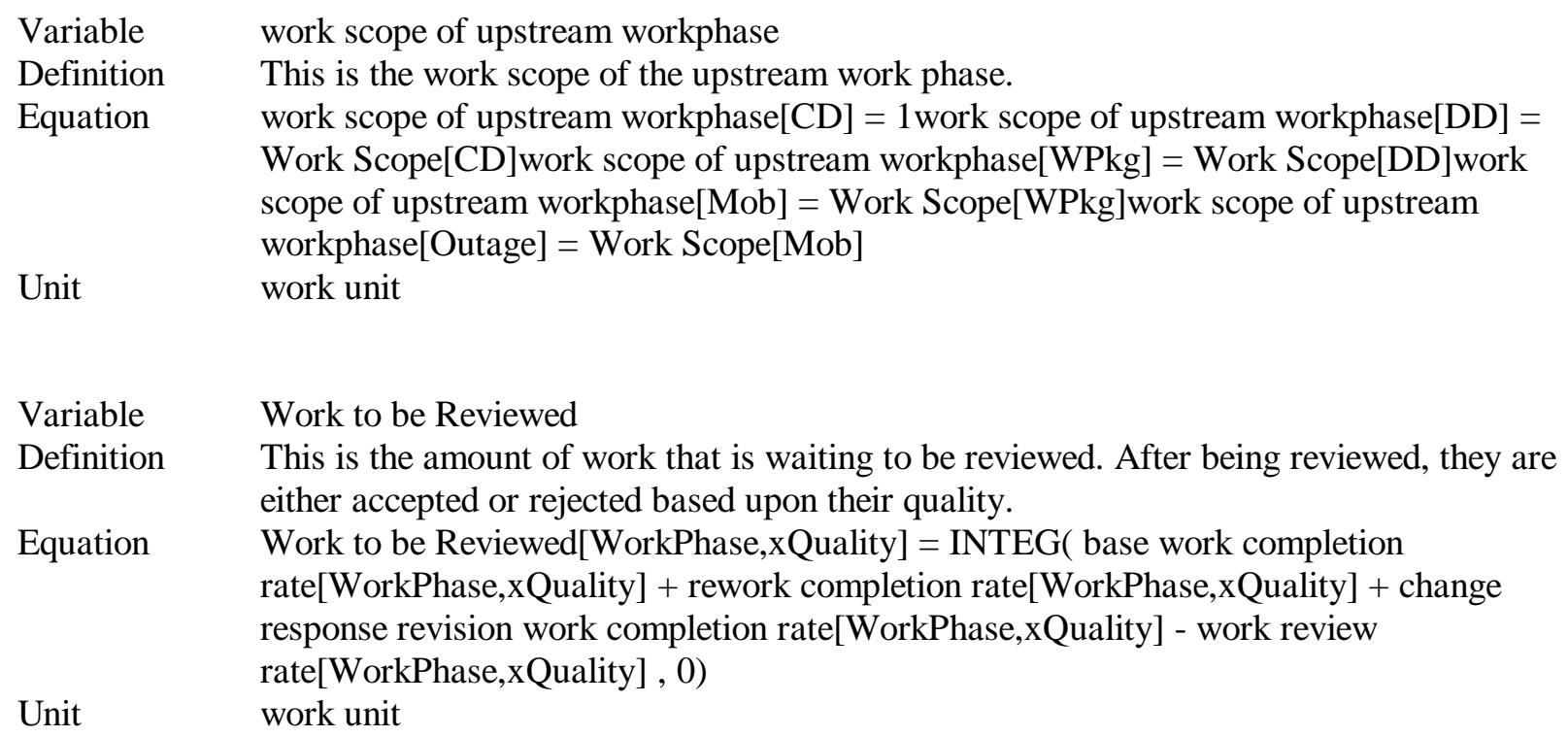

Unit Dmnl

Variable worker quality of practice modulator

Definition This is the modulator to the quality considering worker's experience, familiarity,morale, and so on.

Equation worker quality of practice modulator[Dep] = quality configuration knowledge factor[Dep] * quality experience factor[Dep] * quality familiarity factor[Dep] * quality morale factor[Dep] * quality supervisor management capability factor[Dep] * quality work out of sequence factor[Dep]

Unit Dmnl

Variable worker utilization rate 


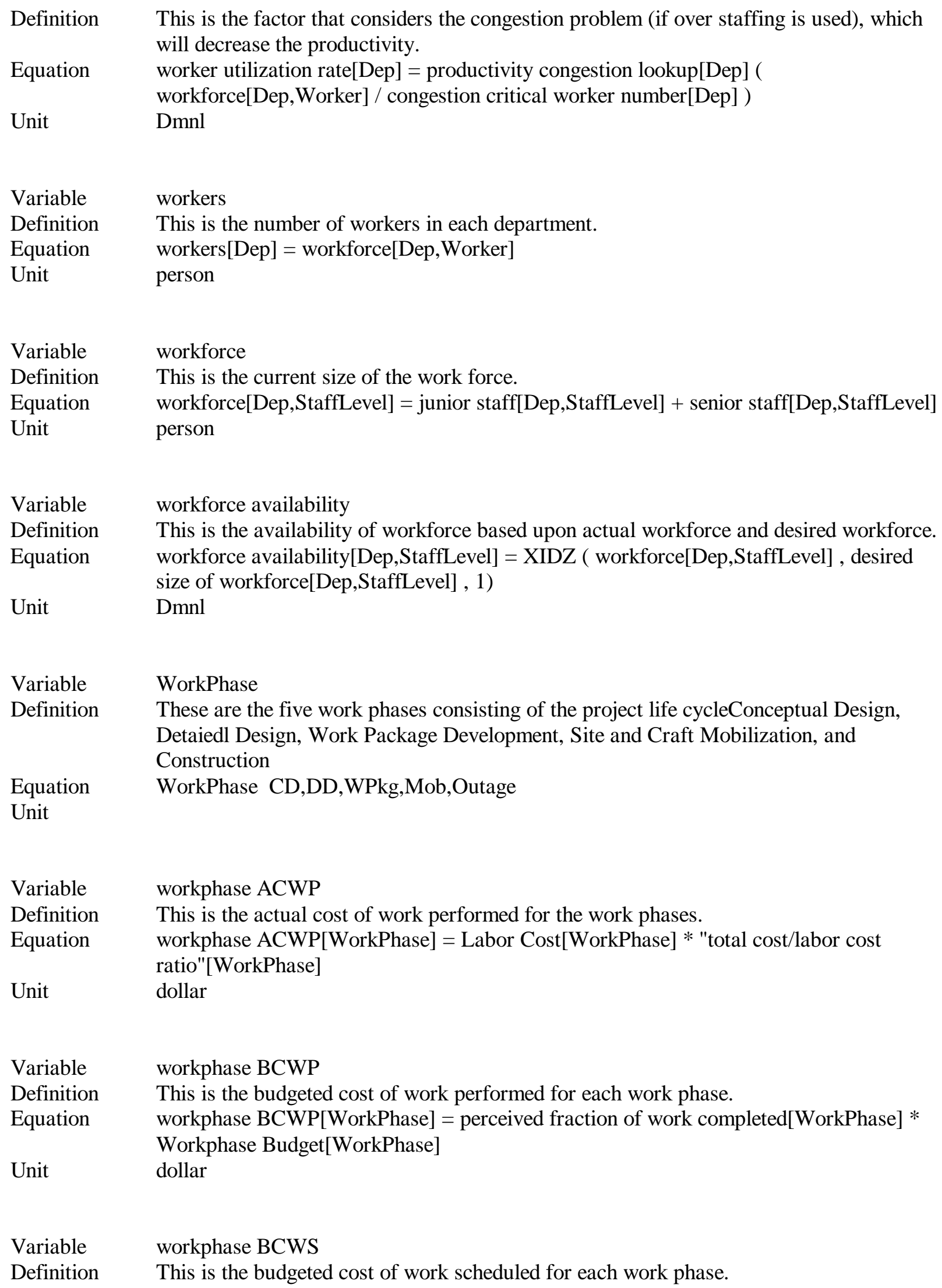




\begin{tabular}{|c|c|}
\hline Equation & $\begin{array}{l}\text { workphase BCWS[WorkPhase] = scheduled cost as a function of time[WorkPhase] + } \\
\text { Scheduled Budgeted Cost of Work Created by Change[WorkPhase] } \\
\text { dollar }\end{array}$ \\
\hline Variable & Workphase Budget \\
\hline Definition & This is the total budget for the work phases. \\
\hline Equation & $\begin{array}{l}\text { Workphase Budget[WorkPhase] = INTEG( workphase budget change rate[WorkPhase], } \\
\text { scheduled total cost[WorkPhase] ) }\end{array}$ \\
\hline Unit & dollar \\
\hline Variable & workphase budget change rate \\
\hline Definition & This is the rate at which the budget of each work phase is increased. \\
\hline Equation & $\begin{array}{l}\text { workphase budget change rate[WorkPhase] = Budget for Change[WorkPhase] / TIME } \\
\text { STEP } * \text { PULSE ( change start time[WorkPhase], TIME STEP ) }\end{array}$ \\
\hline Unit & dollar/Week \\
\hline Variable & workphase CPI \\
\hline Definition & $\begin{array}{l}\text { This is cost performance index of each workphase.If a workphase has not started, this } \\
\text { variable takes } 1 \text { as its value. }\end{array}$ \\
\hline Equation & $\begin{array}{l}\text { workphase CPI[WorkPhase] = ZIDZ ( workphase BCWP[WorkPhase] , workphase } \\
\text { ACWP[WorkPhase] ) }\end{array}$ \\
\hline Unit & Dmnl \\
\hline Variable & workphase quality \\
\hline Definition & $\begin{array}{l}\text { This is the ratio of a work phase's rework, including known and unknown, to the total } \\
\text { work of the work phase. }\end{array}$ \\
\hline Equation & $\begin{array}{l}\text { workphase quality[WorkPhase] = } 1-\text { ZIDZ ( Known Rework[WorkPhase] + unknown } \\
\text { rework[WorkPhase], perceived work completed[WorkPhase] + known revision work to } \\
\text { be done[WorkPhase] ) }\end{array}$ \\
\hline Unit & Dmnl \\
\hline Variable & workphase SPI \\
\hline Definition & $\begin{array}{l}\text { This is current schedule performance index of each workphase.If a workphase has not } \\
\text { started, this variable takes } 1 \text { as its value. }\end{array}$ \\
\hline Equation & $\begin{array}{l}\text { workphase SPI[WorkPhase] = ZIDZ ( workphase BCWP[WorkPhase] , workphase } \\
\text { BCWS[WorkPhase] ) }\end{array}$ \\
\hline Unit & Dmnl \\
\hline Variable & workphase start flag \\
\hline Definition & This is the flag indicating whether or not a work phase has started. \\
\hline Equation & $\begin{array}{l}\text { workphase start flag[WorkPhase] = MAX ( conditional start[WorkPhase] } * \text { is start } \\
\text { conditional[WorkPhase], forced start[WorkPhase] ) }\end{array}$ \\
\hline Unit & Dmnl \\
\hline
\end{tabular}




\section{$\boldsymbol{C}_{\text {Appendix }}$}

\section{REFERENCES}

American Subcontractors Association(1995). Guideline on overtime, construction costs and productivity. http://www.constructionguidelines.org/pdf-prot/overtime.pdf

ASME (1995). "Rules for construction of nuclear facility components, Boiler \& Pressure VesselCode Section III, ASME.

Baugh Jr., J.W. S.C. Caldwell, and E.D. Brill Jr., (1997), "A mathematical programming approach for generating alternatives in discrete structural optimization," Engineering Optimization, vol.28, pp.1-31.

Brill Jr., E.D. J.M. Flach, L.D. Hopkins, and S. Ranjithan, (1990), "MGA: A decision support for complex, incompletely defined problems," IEEE Transactions on Systems, Man, and Cybernetics, vol.20, no.4,pp.745-757.

Cravens K. S. and Guilding C., "Brand Value Accounting: an International Comparison of Perceived Managerial Implications", Journal of International Accounting, Audition \& Taxation, Vol. 10, pp. 197221,2001

CUBIT Mesh Generation Environment Volume 1: Users Manual, SAND94-1100, April 18, 2000, Sandia National Laboratories, Albuquerque, New Mexico.

Development Of Advanced Technologies to Reduce Design, Fabrication and Construction for Future Nuclear Power Plants, Year One Activities Report, DOE Project DE-FCO-99SF21898/A002

Development Of Advanced Technologies to Reduce Design, Fabrication and Construction for Future Nuclear Power Plants, Year Two Activities Report, DOE Project DE-FCO-99SF21898/A002

DST, (1997), "PIPESTRESS User's Manual," DST Computer Services, Geneva, Switzerland, Version 3.4.09.

Flavin, Christopher and Lenssen, Nicholas (1999). Worldwatch News Brief: Nuclear Power Nears Peak. http://www.worldwatch.org/alerts/990304.html

Ford, David N. (1995). The Dynamics of Project Management: An Investigation of the Impacts of Project Process and Coordination on Performance. Doctoral thesis. Massachusetts Institute of Technology. Cambridge, MA

Gupta, A. P. Kripakaran, G.M. Kumar, and J.W. Baugh, Jr. (2002), “GA-Based Decision Support for Optimizing Seismic Response of Piping Systems,” Journal of Structural Engineering, ASCE, submitted

Hibbitt, D. et al., ABAQUS/Standard, Version 5.8 User's Manual, Hibbitt, Karlsson, and Sorensen, Inc., Pawtucket, Rhode Island, 1998. 
Kirkwood, Craig W. (1998). System Dynamics Methods: A Quick Introduction. http://www.public.asu.edu/ kirkwood/sysdyn/SDIntro/SDIntro.htm

Milewicz J. and Herbig P., "Evaluating the Brand Extension Decision Using a Model of Reputation Building”, Journal of Product \& Brand Management, Vol. 3 No. 1, pp. 39-47, 1994

Moselhi, A., and Lorterapong, P. (1993). Least impact algorithm for resource allocation. Can. J. Civ. Engrg., CSCE, 20(2), 180-188

Park, Mooseo (1999). Robust Control of Cost Impact on Fast-Tracking Building Construction Projects. Master thesis. Massachusetts Institute of Technology. Cambridge, MA

PATRAN, developed by MSC, Version 2001R², 2002.

Pro/ENGINEER, developed by Parametric Technology Corporation (PTC), Version 2000i, 2000.

Project Management Institute (PMI) Standards Committee (1996). A Guide To The Project Management Body Of Knowledge. PMI. Newtown Square, PA

Randolph, Thomas, H. and Napolitan, Carmen L (1994). The Effects of Changes on Labor Productivity: Why and How Much. Construction Industry Institute. Austin, TX

SolidWorks, developed by SolidWorks Corporation, Version 2000, 2000.

Sterman, John D. (1992). System Dynamics Modeling for Project Management. http://web.mit.edu/jsterman/www/SDG/project.html

Stout, Daniel S. (1998). Project Management Model of a Nuclear Facility Renovation. Master thesis. Massachusetts Institute of Technology. Cambridge, MA

Uranium Information Center Ltd. (2001). Nuclear Issues Briefing Paper 8: The Economics of Nuclear Power. http://www.uic.com.au/nip08.htm

Ward R. and Perrier R., "Brand Valuation: the times are a changing”, The Journal of Brand Management, 5(4), pp. 283-289, 1998 